\title{
Stereodivergent Catalysis
}

\author{
Irina P. Beletskaya, ${ }^{\mathrm{a}}$ Carmen Nájera, ${ }^{* \mathrm{~b}}$ and Miguel Yus ${ }^{\mathrm{b}}$ \\ ${ }^{a}$ Chemistry Department, M. V. Lomonosov Moscow State University, Leninskie Gory 1, 119992 Moscow, \\ Russia \\ ${ }^{b}$ Departamento de Química Orgánica and Centro de Innovación en Química Avanzada \\ (ORFEO-CINQA), Universidad de Alicante, Apdo. 99, E-03080 Alicante, Spain
}

This review covers diastereo- and enantiodivergent catalyzed reactions in acyclic and cyclic systems using metal complexes or organocatalysts. Among them, nucleophilic addition to carbon-carbon and carbon-nitrogen double bonds, $\alpha$-functionalization of carbonyl compounds, allylic substitutions, and ring opening of oxiranes and aziridines are considered. The diastereodivergent synthesis of alkenes from alkynes is also included. Finally, stereodivergent intramolecular and intermolecular cycloadditions and other cyclizations are also reported.

\section{Contents}

1. Introduction

2. Stereodivergence in Acyclic Systems

2.1. Nucleophilic Additions to Carbonyl Compounds

2.1.1. Addition of Organometallic Reagents

2.1.2. Aldol Reactions

2.1.2.1. Aldol Reactions Involving Metal Enolates

2.1.2.2. Organocatalyzed Aldol Reactions

\subsubsection{Nitroaldol Reactions}

\subsubsection{Addition of Other Nucleophiles}

\subsubsection{Reduction of Ketones}

\subsubsection{Addition of Hydrides}

\subsubsection{Hydrogenation}

\subsubsection{Transfer Hydrogenation}

\subsubsection{Enzymatic Reduction}

\footnotetext{
* To whom correspondence should be addressed. Phone: +34 965903728. Fax: +34 965903549. E-mail: cnajera@ua.es.URL: www.ua.es/dqorg
}

\subsection{Nucleophilic Addition to $\mathrm{C}=\mathrm{N}$ Bonds}

\subsubsection{Addition of Organometallic Reagents}

\subsubsection{Imines}


2.2.1.2. Nitrones

2.2.2. Mannich Reactions

2.2.2.1. Metal-Catalyzed Mannich Reactions

2.2.2.2. Organocatalyzed Mannich Reactions

2.2.3. Addition of Other Nucleophiles

2.2.4. Reduction of Ketimines

2.3. Addition to Alkenes

2.3.1. Conjugate Additions

2.3.1.1. Metal-Catalyzed Conjugate Additions

2.3.1.2. Organocatalyzed Conjugate Additions

2.3.2. Hydrogenation of Alkenes

2.3.2.1. Asymmetric Hydrogenation of Functionalized Alkenes

2.3.2.2. Asymmetric Hydrogenation of Unfunctionalized Alkenes

2.3.3. Hydroformylation of Alkenes

2.3.4. Other Addition Reactions

2.4. $\alpha$-Functionalization of Carbonyl Compounds

2.5. Oxiranyl and Aziridinyl Ring Opening

2.6. Allylic Substitution Reactions

2.6.1. Carbon Nucleophiles

2.6.2. Nitrogen Nucleophiles

2.6.3. Oxygen Nucleophiles

2.6.4. Other Nucleophiles

2.7. Diastereodivergent Synthesis of Alkenes

2.7.1. Hydrometallation of Alkynes

2.7.1.1. Hydroboration of Alkynes

2.7.1.2. Hydrosilylation of Alkynes

2.7.1.3. Hydrostannation of Alkynes

2.7.2. Reduction of Alkynes

2.7.3. Other Reactions

3. Stereodivergence in Cyclic Systems

3.1. Addition to $\mathrm{C}=\mathrm{C}$ Bonds

3.1.1. Conjugate Additions

3.1.2. Hydrogenation

3.1.3. Hydroboration

3.2. $\alpha$-Functionalization of Carbonyl Compounds 
3.3. Allylic Substitution Reactions

3.4. Other Reactions

4. Stereodivergence in Intramolecular Cyclizations

4.1. Intramolecular Nucleophilic Additions

4.1.1. Addition to Carbonyl Compounds

4.1.2. Addition to $\mathrm{C}=\mathrm{N}$ Bonds

4.2. Intramolecular Conjugate Additions

4.3. Intramolecular Nucleophilic Substitution

4.4. Other Intramolecular Cyclizations

5. Stereodivergence in Intermolecular Cyclizations

5.1. [4+2] Cycloadditions

5.1.1. Diels-Alder Reactions

5.1.2. Hetero Diels-Alder Reactions

5.2. 1,3-Dipolar Cycloadditions

5.2.1. Nitrones

5.2.2. Azomethine Ylides

5.2.3. Diazo Compounds

5.2.4. Other [3+2] Cycloadditions

5.3. [2+2] Cycloadditions

5.4. Other Intermolecular Cyclizations

5.4.1. Five-Membered Rings

5.4.2. Six-Membered Rings

6. Conclusions

Author Information

Corresponding Authors

Notes

Biographies

Acknowledgments

Abbreviations

References

\section{INTRODUCTION}

Chemists have already synthesized ca. 40 million organic compounds and are able to synthesize almost any possible structure. Obviously, without solving selectivity 
problems, no kind of chemistry is sufficiently efficient. However, there is also a problem of another kind, even more difficult to tackle: creating with minimum possible expenditure two stereoisomers, especially enantiomeric pure compounds. This problem is of paramount practical importance for pharmaceutical applications in order to evaluate the biological activity of both enantiomers. Normally, one can use separate reactions and starting compounds to prepare each diastereomer or enantiomer, but the simplest strategy is to use the same starting material and small variation of reaction conditions, including small changes in the structure of reagents, to obtain both stereoisomers in the individual form. Probably, one of the best solutions to the problem is provided by asymmetric catalysis by small changes of the catalyst and the reaction conditions as well.

The concept of stereodivergence considers reactions which generate different stereoisomers starting from the same substrate, or practically the same, just by changing usually the catalyst, reagents or reaction conditions. Stereodivergence in organic synthesis has become in the last 30 years a fundamental strategy for the stereocontrol in synthetic processes. The synthetic potential of stereodivergent reactions has been demonstrated in the last 30 years in many fundamental reactions and synthetic strategies. This effect should be included in the concept of efficiency of a reaction as well as in atom economy process. According to the evolution of synthetic organic chemistry over the years, catalytic stereodivergent methods have become very important especially in the last 20 years. Metal-catalyzed and organocatalyzed processes are the main strategies in stereodivergent catalysis. Approaches to different diastereomers face the challenge of the relative configuration control of several stereocenters by a simple synthetic operation. Enantiodivergent reactions face the challenge of affording different enantiomers avoiding the use of opposite sources of chirality. These strategies have been applied to acyclic and cyclic substrates not only in inter- but also in intramolecular transformations and applied to the total synthesis of numerous natural products and related stereoisomeric compounds as well as bioactive molecules.

For stereodivergent catalysis, structural modifications of the ligand or the metal in the case of metal complexes and in the organocatalysts are crucial. In addition, changes in the reaction conditions such as solvent, temperature, pressure, and additives, especially Lewis and Brønsted acids or bases, can also determine the stereodivergence. In the case of metal catalysis not only the ligand but also the metallic precursor used to form the catalytic species, even with the same element, can be an important strategy. The ligand-to-metal ratio has been also observed to switch the stereoselectivity. Chiral and achiral counteranions play an important role in the case of cationic metal complexes. Different diastereoselectivity has been also observed in the presence and in the absence of a catalyst and/or additive. Some small structural modifications in the substrate have been also observed to influence in the stereodivergence.

Although important stereodivergent developments have been achieved, still there are not well-defined strategies, serendipity being the most important factor on these findings. However, this topic has been only partially covered. ${ }^{1-11}$ The aim of this review 
is to overview, for the first time, this efficiency concept in synthetic organic chemistry to get some light about which reactions are good candidates for stereodivergent catalytic transformations. Although, asymmetric catalytic reactions have been mainly presented, some asymmetric processes using chiral substrates and achiral catalysts are also considered. We think that it would be very useful to have a comprehensive overview about this subject considering that it has a big impact in the development of stereocontrolled reactions in total synthesis of natural products and in general of biologically active compounds. We expect that this comprehensive information will be of interest for all the chemical community, both at the academia and at the industrial level.

\section{STEREODIVERGENCE IN ACYCLIC SYSTEMS}

Acyclic systems bearing a functional group susceptible of synthetic transformation are widely applied in organic synthesis. Multiple $\mathrm{C}=\mathrm{X}$ bonds such as carbonyl compounds and imines are able to generate a new stereocenter by nucleophilic addition of different types of nucleophiles through many fundamental processes for the acyclic control of several stereocenters. In addition, carbonyl compounds can also be functionalized at the $\alpha$-position. In the case of carbon-carbon multiple bonds, conjugate additions to electrondeficient olefins is considered the most important reaction for the functionalization of the $\beta$-position of carbonyl compounds and related systems. Enantioselective hydrogenation and hydroformylation of alkenes by means of chiral complexes are important industrial processes for the synthesis of $\alpha$-amino acids and aldehydes, respectively. The ring opening of epoxides by nucleophiles allows the synthesis of 1,2difunctionalized compounds. Pd- and Ir-catalyzed allylations of carbon- and heteronucleophiles are excellent strategies for the enantio- and diastereodivergent synthesis of acyclic systems bearing a $\mathrm{C}=\mathrm{C}$ bond in the aliphatic chain. Finally, in this section diastereodivergent methods for the synthesis of alkenes mainly based on the hydrometallation, and reduction of alkynes will be considered.

\subsection{Nucleophilic Additions to Carbonyl Compounds}

In this section, the stereodivergent addition of different nucleophiles such as organometallic reagents, enolates, nitroalkanes, cyanides, and others to carbonyl compounds catalyzed by Lewis acids are considered. In the case of enantiodivergent strategies, chiral metal complexes, usually chiral Lewis acids, or organocatalyzed nucleophilic additions are the main strategies. Enantiodivergent addition of organometallics can be carried out using chiral Lewis acids with the same source of chirality. Diastereodivergent aldol reactions have been observed using either different metal in the enolate or different Lewis acids, especially in the Mukaiyama aldol reaction. In addition, also the temperature and the solvent can play an important role in 
the diastereoselectivity. In enantiodivergent aldol reactions by using the same ligand, the metal or the metal counteranion can be crucial. Enantiodivergent effects have been detected in organocatalyzed aldol reactions by the presence of different additives or solvents. The enantiodivergent addition of other nucleophiles to carbonyl compounds such as nitroalkanes, cyanide and phosphites or phosphinates has been mainly performed under asymmetric catalysis using metal complexes as catalysts. Stereodivergent reductions of ketones have been mainly performed by the addition of hydrides to ketones and by the hydrosilylation using chiral metal complexes as catalysts either with the same chiral ligand or by a different metal. Enantiodivergent hydrogenation of ketones has been mainly carried out under heterogeneous conditions with platinum on alumina modified by Cinchona alkaloids. Enzymatic reduction can be carried out enantiodivergently by changing the strain of the enzyme.

2.1.1. Addition of Organometallic Reagents. Organometallic reagents have been used as strong carbonucleophiles for diastereodivergent synthesis of alcohols by changing the metal, additives and reaction conditions such as temperature. In the case of diastereodivergent additions of $\alpha$-alkoxy organometallic compounds derived from Li, $\mathrm{Mg}$ and $\mathrm{Cu}$ to aldehydes, 1,2-diols were obtained with low syn-diastereoselectivity. However, the corresponding organolead compounds 2, accessible from stannanes 1, allowed the diastereodivergent synthesis of syn- and anti-diols $\mathbf{3}$ in the presence of different Lewis acids. ${ }^{12}$ Thus, in the presence of 1.2 eq of $\mathrm{TiCl}_{4}$ syn-3 compounds were mainly formed, whereas with 2.5 eq of $\mathrm{BF}_{3} \cdot \mathrm{OEt}_{2}$, anti-diols were preferentially obtained according to intermolecular chelation $\mathbf{A}$ and nonchelation $\mathbf{B}$ control models, respectively (Scheme 1). The $\mathrm{TiCl}_{4}$-mediated reaction proceeds through a $\mathrm{S}_{\mathrm{E}} 2$ retention mechanism as it has been demonstrated by enantiomerically enriched $(S)-\mathbf{1}\left(\mathrm{R}^{1}=\mathrm{Me}\right)$.

Scheme 1. Diastereodivergent Addition of $\alpha$-Alkoxy Organolead Compounds to Aldehydes Catalyzed by Lewis Acids 


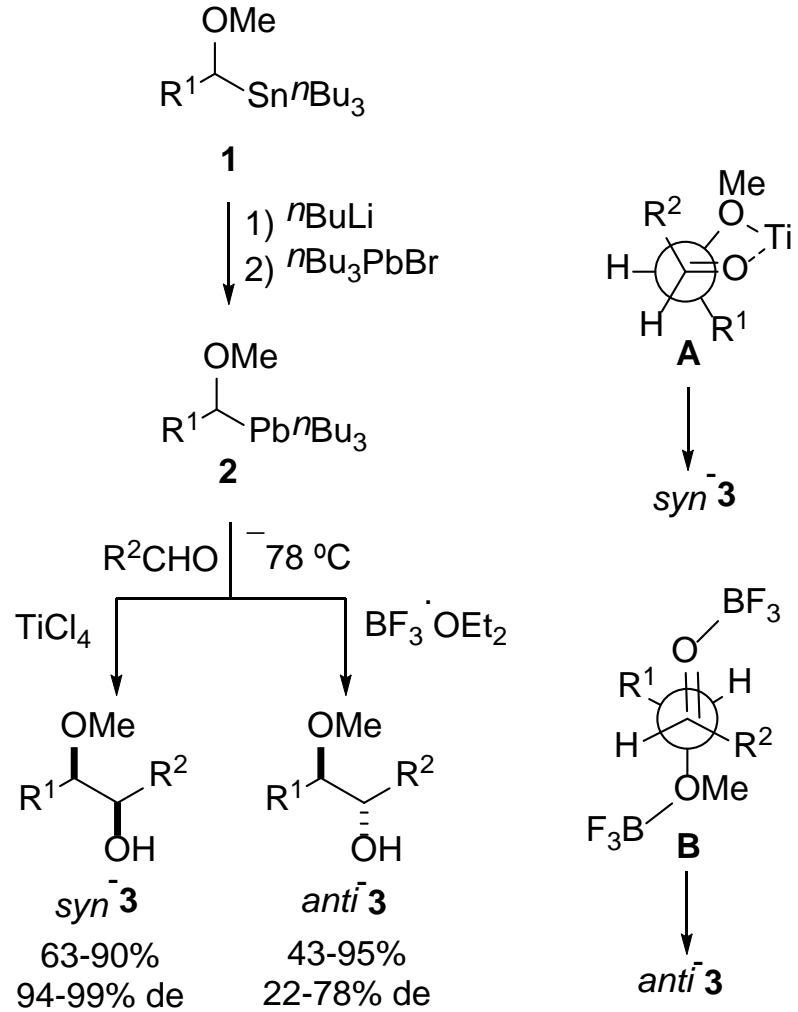

A diastereodivergent addition of vinylmagnesium bromide to aldehyde $\mathbf{4}$, derived from D-xylose, has been achieved in the absence or presence of a Lewis acid (Scheme 2). ${ }^{13}$ In the absence of Lewis acid, alcohol syn-5 was obtained at $-78{ }^{\circ} \mathrm{C}$ in $62 \%$ de, whereas in the presence of stoichiometric amounts of $\mathrm{MgBr}_{2} \cdot \mathrm{OEt}_{2}$ anti-5 was formed in ca. $80 \%$ de. In the first case, due to the chelating ability of $\mathrm{Mg}^{2+}$, the transition state (TS) 5A is operating. The syn to anti switching of diastereoselectivity in the presence of $\mathrm{MgBr}_{2} \cdot \mathrm{OEt}_{2}$ can be rationalized by the TS 5B with the participation of $\alpha$ - and $\gamma$-alkoxy groups. Products 5 were further submitted to ring-closing metathesis and transformed into derivatives of conduritol B and F as well as meso-inositol and chiro-inositol.

Scheme 2. Diastereodivergent Addition of Vinylmagnesium Bromide to Aldehyde 4 in the Absence or Presence of $\mathrm{MgBr}_{2} \cdot \mathrm{OEt}_{2}$ 


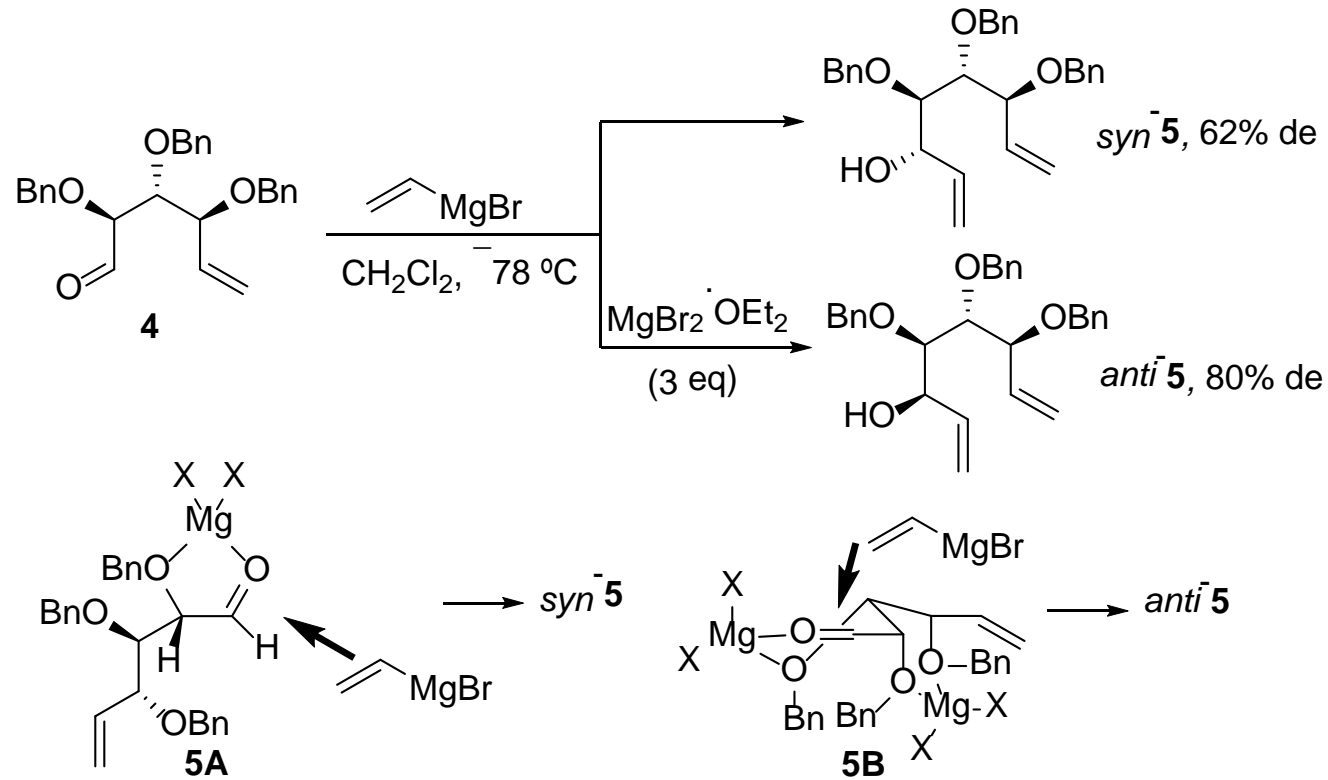

Allylic organometallic reagents add to carbonyl compounds through different strategies depending on the metal. Organolithium and magnesium reagents are very reactive and therefore the presence of a Lewis acid is not necessary like in the case of other less reactive allylmetals. ${ }^{14,15}$ These processes became very popular as acyclic stereocontrolled methodologies either in stoichiometric or catalytic additions. However, stereodivergent allylation of aldehydes has been achieved with allylstannanes in the presence of a Lewis acid. An example has been observed in the case of aldehydes with differently protected 3-hydroxytetrahydropyranyl groups, which were introduced by Charette and co-workers as chiral auxiliaries for the diastereodivergent addition of organometallic reagents to $\alpha$-alkoxy aldehydes 6. ${ }^{16-18}$ These chiral auxiliaries bear two potential chelating units able to act as tridentate ligands with a Lewis acid. In the case of the benzyloxytetrahydropyranyl group the $\mathrm{MgBr}_{2} \cdot \mathrm{OEt}_{2}$-mediated addition of allyltributyltin gave the $(R)$-alcohol 7 with de up to $95 \%$ (Scheme 3). ${ }^{18}$ On the other hand, using the tris(isopropyl)silyl protecting group resulted the (S)-alcohol 8 in 86\% de. This diastereodivergence was attributed to a remote protecting group effect in the substrate.

\section{Scheme 3. Diastereodivergent Addition of Allyltributyltin to Differently Protected $\alpha$-Alkoxy Aldehydes 6 Mediated by $\mathrm{MgBr}_{2} \cdot \mathrm{OEt}_{2}$}

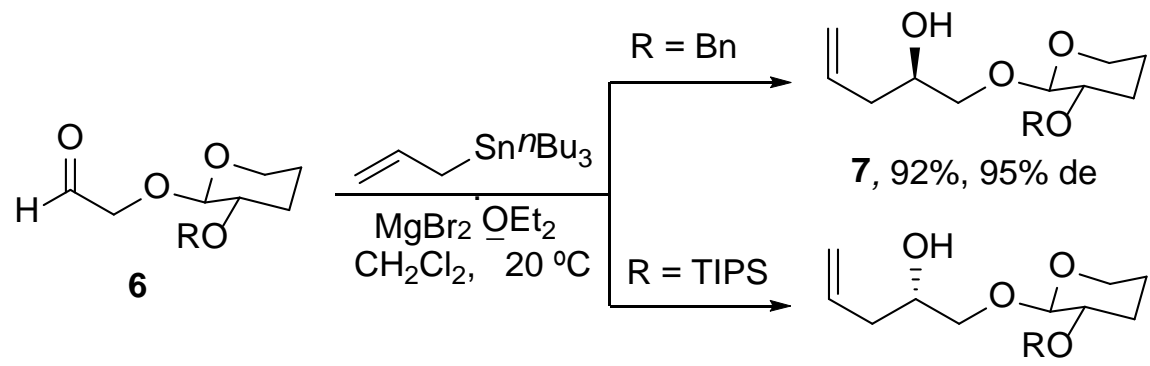

8, $90 \%, 86 \%$ de 
Dynamic solvation effects have been observed by Cainelli and co-workers in the ethylmagnesium bromide addition to (S)-2-(tert-butyldimethylsilyloxy)propanal. ${ }^{19}$ The diastereofacial switch of selectivity syn/anti depends from the ethereal solvent and the temperature but with low diastereoselectivity levels. Addition of tertiary amines (5 mol\%) to hexane for the addition of $n$-butyllithium to 2-phenylpropanal and to $(S)$-2(tert-butyldimethylsilyloxy)propanal strongly influences syn/anti ratios. ${ }^{20}$ Preferential formation of anti-isomers with de up to $93 \%$, took place in the presence of $n \mathrm{Bu}_{3} \mathrm{~N}$ at -75 to $-65{ }^{\circ} \mathrm{C}$ for the addition of $n$ BuLi to 1-phenylpropanal.

Studies from Coleman and Carpenter ${ }^{21}$ about the diastereodivergent addition of vinylmetals to Garner's aldehyde 9 demonstrated that vinyllithium and vinylmagnesium bromide gave mainly anti-10 alcohols (with drup to 5:1), whereas in the case of vinylzinc chloride in nonpolar solvents, syn-10 (with $\mathrm{dr}$ up to 6:1) were the major isomers. In agreement with the Felkin-Anh model and through the coordination of vinylzinc chloride with the Boc group, the observed diastereofacial bias could be explained. The diastereodivergent synthesis of erythro- and threo-sphingoshine derivatives has been achieved by addition of 1-alkenylmetals to $(S)$-Garner's aldehyde 9. ${ }^{22}$ In the presence of $\mathrm{ZnBr}_{2}$, natural erythro-(anti)-10 were the major isomers using 1alkenylzirconocene in THF, whereas 1-alkenylethylzinc without Lewis acid gave threo(syn)-10 in DCM (Scheme 4).

\section{Scheme 4. Diastereodivergent Addition of Alkenylmetals to Garner's Aldehyde 9}

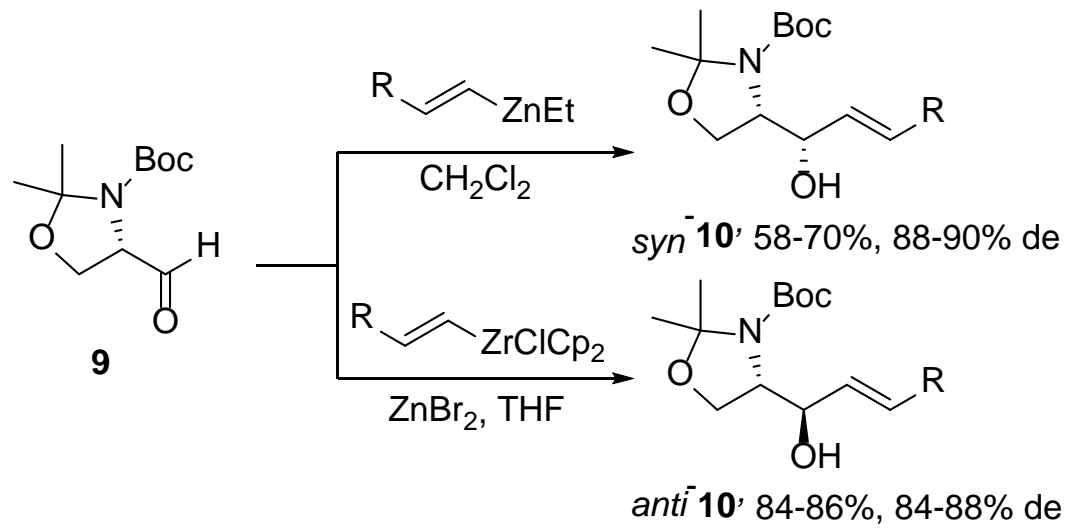

Diastereomeric 3-amino-2,3,6-trideoxyhexoses, key components of anthracycline antibiotics, have been synthesized from protected $\alpha$-hydroxy- $\beta$-amino hex-5-enals. Diastereodivergent addition of lithium dimethylcuprate to cis-11 gave syn-alcohol 12, whereas the addition of methyllithium in the presence of $\mathrm{TiCl}_{4}$ afforded anti-12 (Scheme 5). ${ }^{23}$ Similar stereodivergence was observed with trans-aldehyde $\mathbf{1 1 .}$

Scheme 5. Diastereodivergent Addition of $\mathrm{Me}_{2} \mathrm{CuLi}$ and MeLi to Aldehyde 11 


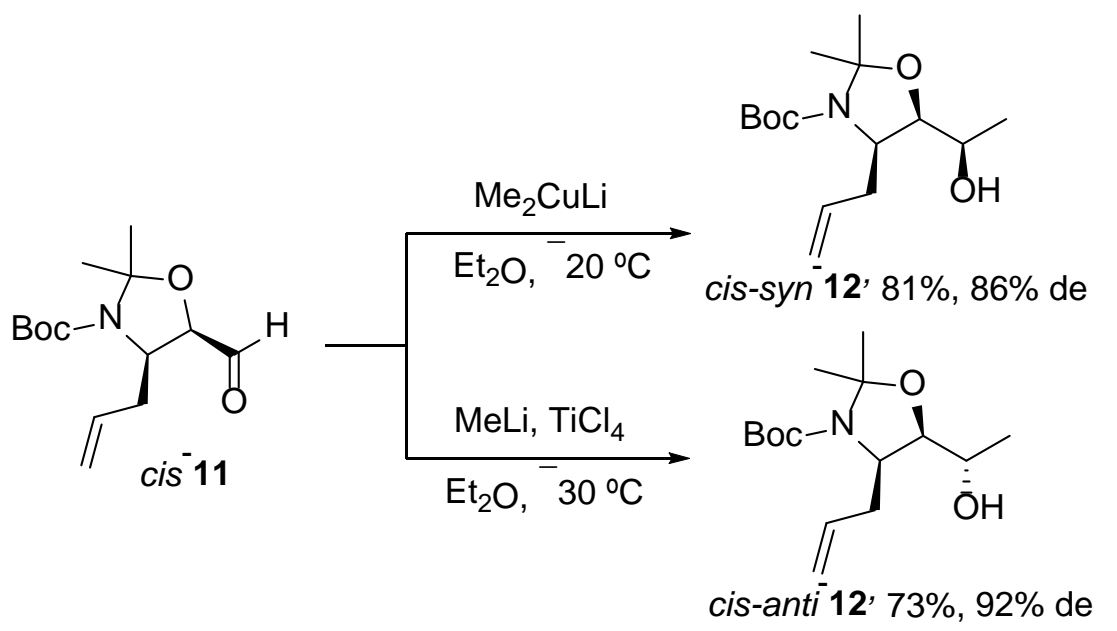

Enantiodivergent additions of organometallic reagents to carbonyl compounds can be controlled by the nature of the metal but also by the reaction conditions, as in the case of diastereodivergent processes. In this case, the substituents of the ligand can be also critical for the inversion of asymmetric induction. ${ }^{1-11,24}$ Enantiocatalyzed addition of dialkylzinc compounds to aldehydes to give secondary alcohols has become a classical reaction in asymmetric catalysis serving as a benchmark reaction for new catalysts. ${ }^{25-}$ ${ }^{28}$ However, only few examples have been reported to achieve enantiodivergent catalysis. Reversal of enantioselectivity was first observed when different substitutions at the nitrogen of $\beta$-aminoalcohols 13 were screened as catalysts. ${ }^{29}$ In the case of $N, N$ dimethyl derivative 13a, the resulting (S)-1-phenyl-1-propanol, obtained by addition of diethylzinc to benzaldehyde, was isolated in $96 \%$ ee. In contrast, the enantiomeric $(R)-$ alcohol was formed in $98 \%$ ee by means of the $N$-sulfonyl derivative $\mathbf{1 3 b}$ (Scheme 6).

Scheme 6. Enantiodivergent Addition of Diethylzinc to Benzaldehyde Catalyzed by $\beta$-Amino Alcohols 13

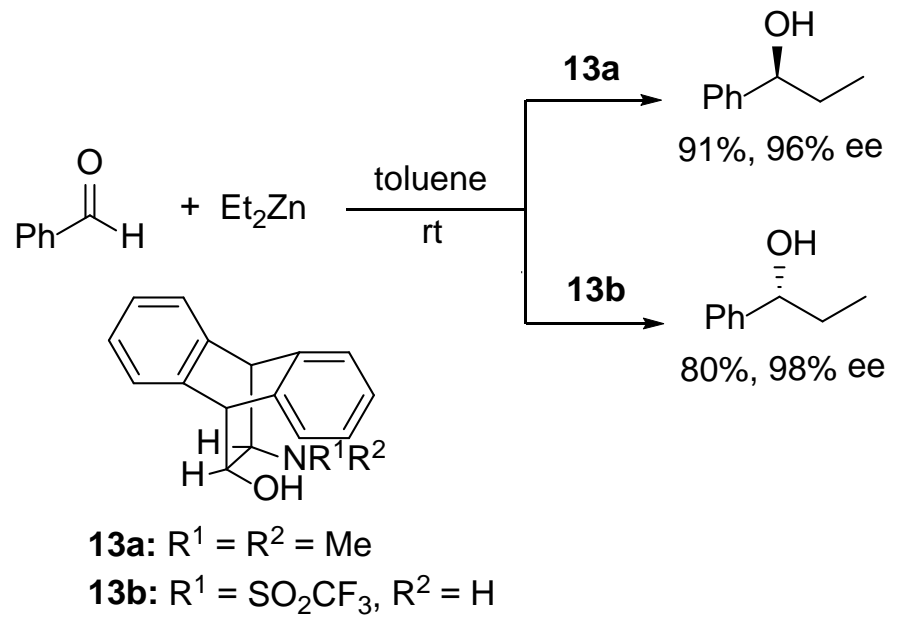

Changes in the backbone substituents of different $\beta$-aminoalcohols had the same reversal effect in the enantioselective addition of diethylzinc to aldehydes. For instance, in the case of L-serine derived ligands $\mathbf{1 4 a}$ and $\mathbf{1 4 b},{ }^{30}$ a change from phenyl to $n$-butyl and of the secondary to a tertiary amine, afforded $(S)$-alcohols in $68-83 \%$ ee and the $(R)$ - 
alcohols in 60-79\% ee, respectively, in the addition of diethylzinc to aldehydes (Figure 1). Fenchone-based ligands $\mathbf{1 5 a}$ and $\mathbf{1 5 b},{ }^{31}$ gave (S)-1-phenyl-1-propanol in $26 \%$ ee and (R)-1-phenyl-1-propanol in 63\% ee, respectively (Figure 1). $\beta$-Amino alcohols 16a and $\mathbf{1 6 b},{ }^{32,33}$ afforded the best results as enantiodivergent ligands providing $(R)$ - and $(S)$-1phenyl-1-propanol in $80 \%$ and $92 \%$ ee, respectively (Figure 1). D-Fructose derived ligands 17a and 17b, ${ }^{34}$ produced $(R)$ - and $(S)$-1-phenyl-1-propanol in $82 \%$ and only $15 \%$ ee, respectively (Figure 1 ). Chiral $C_{2}$-symmetric trans-2,5-disubstituted pyrrolidines 18a and 18b, ${ }^{35,36}$ induced moderate enantiodivergence with aromatic aldehydes bearing 3-fluoro and 3- and 4-chloro substituents (Figure 1). Similar enantioselectivities have been observed using pipecolic acid derived aminoalcohols 19a and $\mathbf{1 9 b} \mathbf{b}^{37}$ (Figure 1): (S)-1-phenyl-1-propanol was obtained by means of 19a (5 mol\%) in $83 \%$ ee, whereas the diphenyl substituted ligand $\mathbf{1 9 b}$ gave the $(R)$-alcohol in $82 \%$ ee.

Figure 1. Homochiral Ligands Used in the Enantiodivergent Addition of Diorganozinc Reagents to Aldehydes 
<smiles>[14CH3]C([18OH])(c1ccccc1)C(O)(c1ccccc1)[C@@H](N)COc1ccccc1</smiles><smiles>Cc1cccc(C[C@]2(O)OC3(CCCCC3)OC23COC(C)(C)O3)n1</smiles>

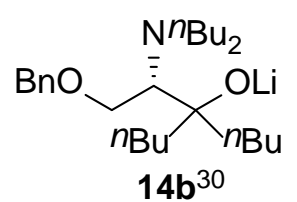<smiles>[X]c1cccc2c1OC1(OC)C3CCC(C3)C21CO</smiles>

15a: $X=H^{31}$

15b: $X=\mathrm{SiMe}_{3}{ }^{31}$

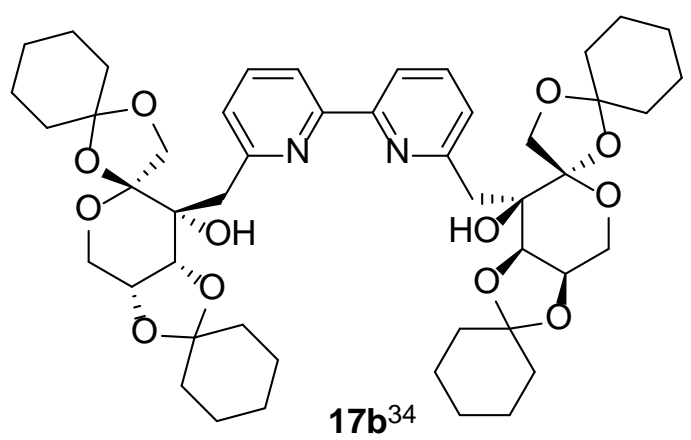<smiles>[R]N[C@]1(N[C@H]2CCCC[C@@H]2O)CCCC[C@H]1O</smiles>

16a: $\mathrm{R}=\mathrm{H}^{32.33}$

16b: $\mathrm{R}=\mathrm{Me}^{32,33}$<smiles>COC[C@@H]1CC[C@@H](COC)N1CC(O)(c1ccccc1)c1ccccc1</smiles>

18a $\mathbf{a}^{35,36}$<smiles>[13CH2]N1C(C(O)(c2ccccc2)c2ccccc2)CCC1C(O)(c1ccccc1)c1ccccc1</smiles><smiles>[R]C([R])(O)C1CC(C)CCN1C1CCCCC1</smiles>

19a: $\mathrm{R}=\mathrm{Ph}^{37}$

19b: $R=H^{37}$<smiles>[R]C([R])(O)C1CCCCC1N1CCCC1</smiles>

20a: $\mathrm{R}=\mathrm{H}^{38}$ 20b: $R=P h^{38}$<smiles>[X]N1CCN(C(=O)C23CC4CC5C(CC4C5C2)C3)CC1</smiles>

23a: $\mathrm{X}=\mathrm{Ac}, \mathrm{Boc}, \mathrm{Ms}^{43}$ 23b: $X=\mathrm{CH}_{2} \mathrm{CH}_{2} \mathrm{OH}^{43}$<smiles>CNCC1CCCCC1NC</smiles>

$21 a^{39}$<smiles>[R17][NH2+]C[C@H]1CCCC[C@@H]1N1CCCC1</smiles>

$\mathbf{2 1 b}^{39}$<smiles>[Z14]C(O)CC(C)(C)N1CN(n2c(C(C)O)nc3ccccc3c2=O)C(C)(C)C1</smiles><smiles>[R20]C(C(=O)N1CCC[C@H]1C(C)=O)c1ccccc1</smiles>

22a: $\mathrm{R}-\mathrm{R}=\left(\mathrm{CH}_{2}\right)_{5}{ }^{42}$

22b: $\mathrm{R}=\mathrm{Bn}^{42}$<smiles></smiles>

1,3-Amino alcohols 20a and 20b (Figure 1) were able to provide both enantiomeric secondary alcohols by addition of diarylzinc to aromatic aldehydes. ${ }^{38}(S)$ Diarylcarbinols were obtained with ee up to 99\% employing 20a and $(R)$-alcohols with ee up to $75 \%$ with ligand $20 \mathbf{b}$. Better results were obtained by the same group using chiral 1,3-amino sulfonamides 21a and 21b (10 mol\%) for the general enantiodivergent addition of diethylzinc to aliphatic and aromatic aldehydes, generally in high yields. ${ }^{39}(S)$-Alcohols were formed with ligand 21a in toluene at $0{ }^{\circ} \mathrm{C}$ with ee up to $94 \%$ and the enantiomers by means of $\mathbf{2 1 b}$ in hexane at $0{ }^{\circ} \mathrm{C}$ with ee up to $98 \%$ (Scheme 7). According to Yamakawa and Noyori mechanistic studies for 2-aminoalcohols as ligands 
through a 5/4/4 tricyclic transition state, ${ }^{40,41}$ the authors proposed the anti-6/4/4 tricyclic transition state for 1,3-aminoalcohols 21. ${ }^{38}$ Similarly, for ligands $\mathbf{2 1}$, transition state anti-21A gave (S)-products, likewise anti-21B provided the $(R)$-alcohols. ${ }^{39}$

Scheme 7. Enantiodivergent Addition of Diethylzinc to Aldehydes Catalyzed by 1,3-Diamine Sulfonamides 21

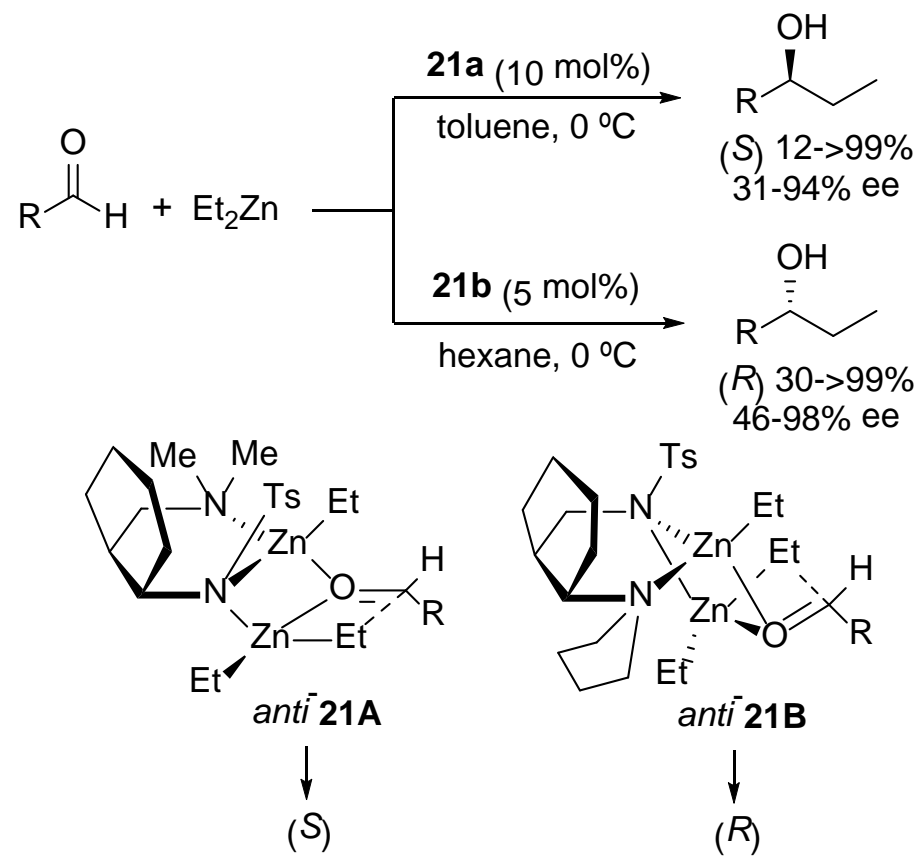

Further examples have been described such as the addition of dimethylzinc to aldehydes in the presence of proline derived dipeptides 22 (Figure 1) bearing different substituents in the N-terminus with low values of enantioselectivity. ${ }^{42}$ The addition of diethylzinc to benzaldehyde catalyzed by (1S)-ketopinic acid derived amides 23a and 23b (Figure 1) gave (R)-1-phenyl-1-propanol with ee up to $78 \%$ and the $(S)$-alcohol with ee up to $66 \%$, respectively. ${ }^{43}$ Another case of enantiodivergence in the addition of diorganozinc compounds to aldehydes has been described using diastereomeric aziridine diols as chiral ligands $\mathbf{2 4 a}$ and $\mathbf{2 4} \mathbf{b}^{\mathbf{4 4}}$ (Figure 1). The addition of diethylzinc to aromatic aldehydes in the presence of $\mathbf{2 4 a}(10 \mathrm{~mol} \%)$ at $-15{ }^{\circ} \mathrm{C}$ gave $(R)$-alcohols in high yields and with ee up to $92 \%$. On the contrary, ligand $24 \mathbf{b}(10 \mathrm{~mol} \%)$ at $-30{ }^{\circ} \mathrm{C}$ afforded $(S)$-alcohols with ee up to $86 \%$. All these examples are based on changes in the structure of catalysts.

In the addition of diethylzinc to trifluoromethyl ketones catalyzed by bis(oxazolines) (BOX) 25-27, the reversed enantioselectivity was observed by changing the substitution on these ligands (Scheme 8). ${ }^{45}$ In the case of BOX ligands 25 and 26, with different substituents in the oxazoline ring, the corresponding tertiary alcohols $(S)$-28 and $(R)-\mathbf{2 8}$ were obtained, respectively. Similar inversion of the enantioselectivity was observed with catalysts $\mathbf{2 7}$ and $\mathbf{2 6}$ giving $(S)$ - and $(R)$-28 alcohols, respectively.

Scheme 8. Enantiodivergent Addition of Diethylzinc to Trifluoromethyl Ketones Catalyzed by Bis(oxazolines) 25-27 


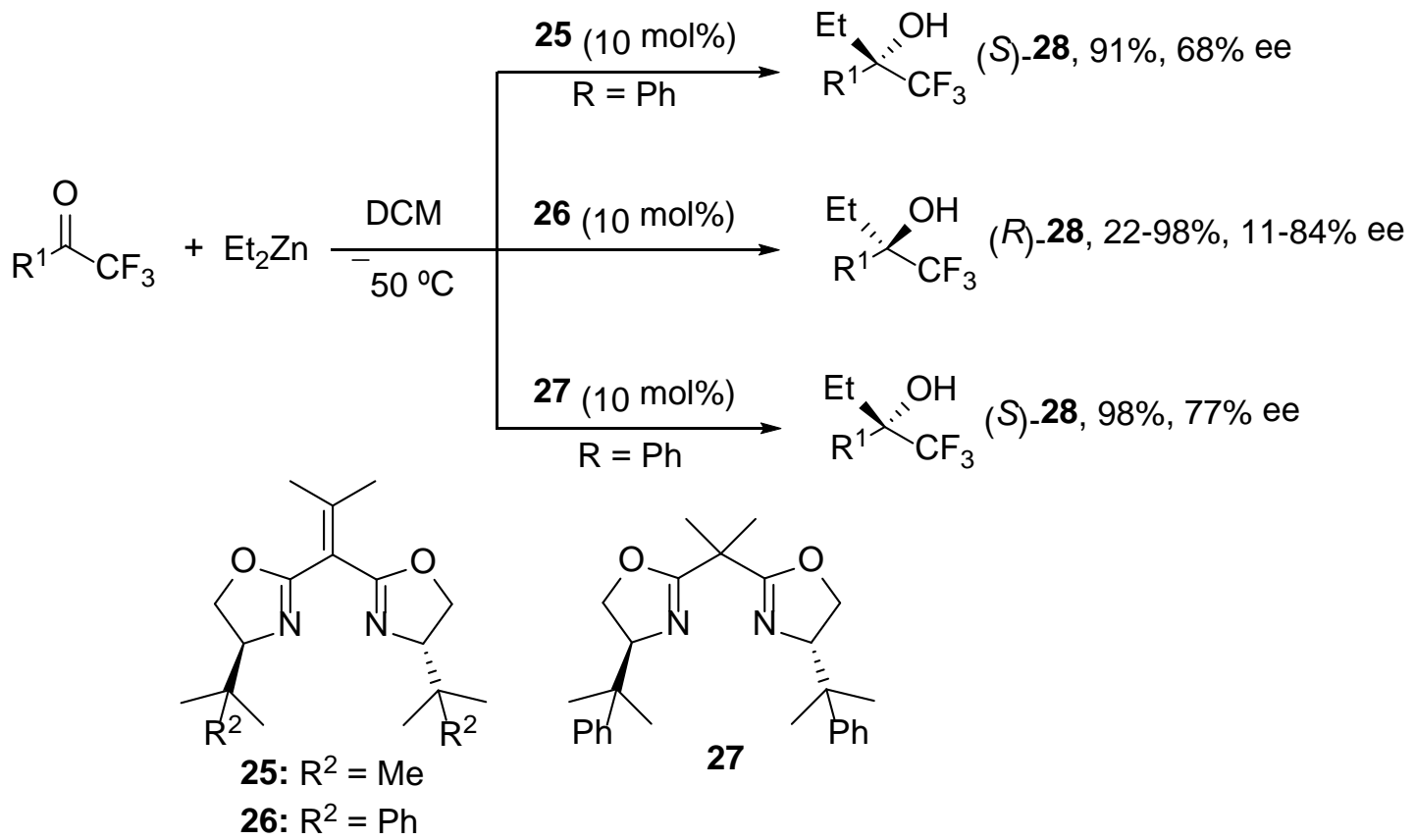

The presence and absence of titanium tetraisopropoxide caused significant changes in the enantioselective addition of diorganozinc reagents to carbonyl compounds. Lake and Moberg ${ }^{46}$ used the tridentate ligand 29 derived from L-valine yielding mainly the $(R)$-enantiomer in $26 \%$ ee in the absence of $\mathrm{Ti}(\mathrm{OiPr})_{4}$ and the $(S)$-isomer in $72 \%$ ee by adding $\mathrm{Ti}(\mathrm{OiPr})_{4}$ (Figure 2). Inversion of enantioselectivity has been observed in the case of (-)-ephedrine-derived sulfonamide ligand $30^{47}$ (10 mol\%) in the addition of diethylzinc to aldehydes (Figure 2). Without $\mathrm{Ti}(\mathrm{OiPr})_{4},(S)$-alcohols were obtained in $77-83 \%$ ee, whereas in the presence of 1.2 eq of $\mathrm{Ti}(\mathrm{OiPr})_{4}$ the enantiomeric $(R)$ alcohols were formed in modest $17-47 \%$ ee. Trivalent binding modes of these ligands to $\mathrm{Zn}$ and multivalent binding to $\mathrm{Ti}$ have been proposed. $(R, R)$-Hydroxybenzoin (31) catalyzed the addition of diethylzinc to aldehydes affording ( $S)$-alcohols with ee up to $85 \% .{ }^{48}$ However, in the presence of 1 eq of $\mathrm{Ti}(\mathrm{OiPr})_{4},(R)$-enantiomers were obtained with ee up to $68 \%$. Enantiodivergent pathways have been proposed tentatively by the transition states 31A and 31B (Figure 2).

Figure 2. Ligands Used for the Enantiodivergent Addition of Diorganozinc Reagents to Aldehydes with or without $\mathrm{Ti}(\mathrm{OiPr})_{4}$ 
<smiles>CC(C)[C@H](N)CNC[C@H]([NH])C(C)C</smiles>

$29^{46}$<smiles>C[C@H](C(O)c1ccccc1)N(C)C[C@H](N)c1ccccc1</smiles>

$30^{47}$<smiles>OC(c1ccccc1)C(O)[Pb](O)c1ccccc1</smiles>

$31^{48}$

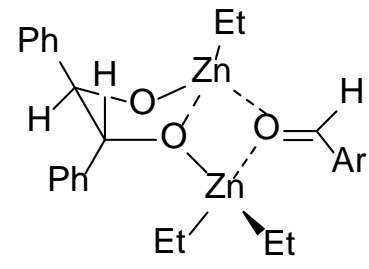

31A

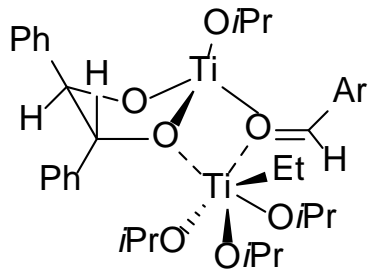

31B

Soai and co-workers, during outstanding studies on the amplification of chirality, discovered that small amounts of achiral $\beta$-amino alcohols also reversed the enantioselectivity in the model reaction, the addition of diisopropylzinc to 2-(tertbutylethynyl)-pyrimidine-5-carbaldehyde $\quad$ (32). ${ }^{49}$ By using (1S,2R)- N,N-dimethylnorephedrine (33, $20 \mathrm{~mol} \%$ ) the corresponding $(R)$-alcohol 34 was formed in $98.7 \%$ ee. On the other hand, by adding $N, N$-dibutylaminoethanol, the (S)-alcohol 34 was obtained in $95.2 \%$ ee (Scheme 9). Based on kinetic studies the authors later proposed a mechanistic pathway for this inversion of enantioselectivity by the formation of a heterodimer complex with $\mathbf{3 3}$ and the achiral $\beta$-amino alcohol. ${ }^{50}$ The same reaction carried out with chiral diols such as (2S,3S)-butane-2,3-diol (20 mol\%) gave the $(R)$ alcohol 34 in 99\% ee, whereas in the presence of an achiral additive such as phenol $(S)$ alcohol 34 was formed in $97 \%$ ee. ${ }^{51}$ All these examples have in common that the stereocontrol is governed by means of additives.

\section{Scheme 9. Enantiodivergent Addition of Diisopropylzinc to Pyrimidine-5-} carbaldehyde 32 in the Absence or Presence of an Amino Alcohol

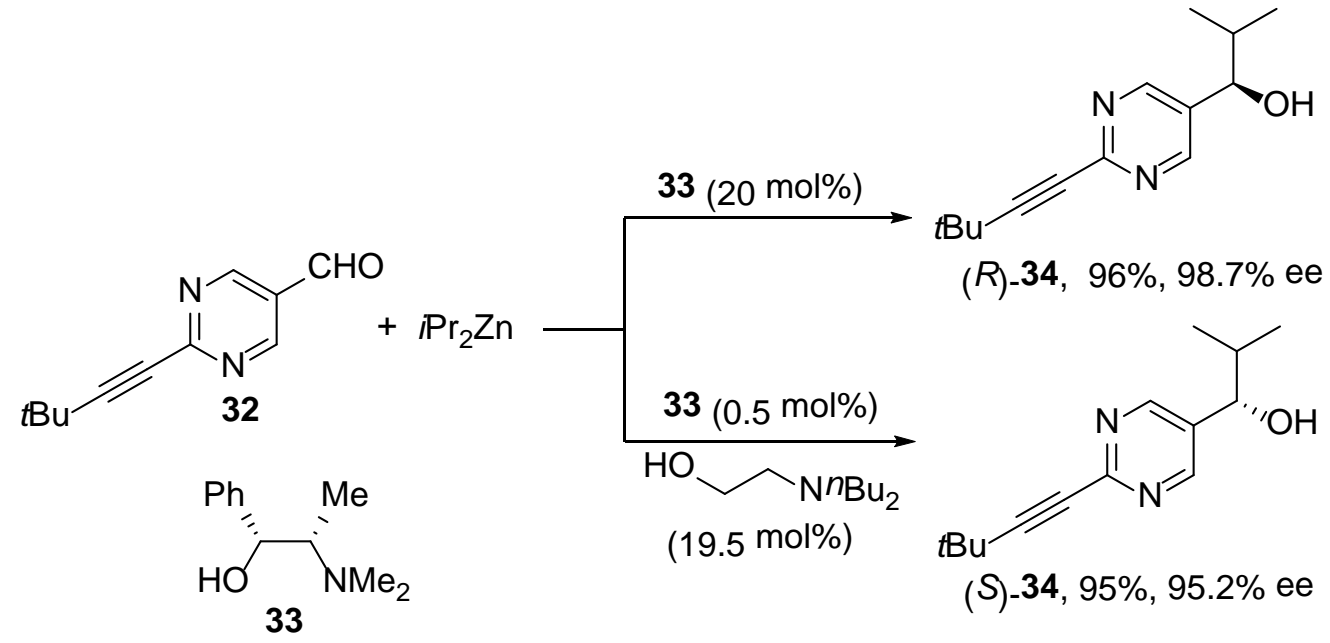


Temperature-dependent inversion of the enantioselectivity has been observed by the Soai group in the asymmetric autocatalysis reaction of $\mathbf{3 2}$ with diisopropylzinc with enantioenriched alcohols. Different $(S)$-1-arylethanols gave $(S)-34$ at $0{ }^{\circ} \mathrm{C}$ in $>99 \%$ yields and $75-90 \%$ ee, whereas at $-44{ }^{\circ} \mathrm{C}(R)$-34 was obtained in $64-88 \%$ yields and 21 $59 \%$ ee. Surprisingly, $(R)$-1-arylethanamines behave the same giving at $0{ }^{\circ} \mathrm{C}(S)$-34 with ee up to $89 \%$ and at $-44{ }^{\circ} \mathrm{C}(R)-34$ with ee up to $74 \% .{ }^{52}$ In this case, the mechanism of this effect has not been clarified. Recently, another example of excellent switch of enantioselectivity has been observed for the same reaction using 1- and 2aza[6] helicenes as promoters. ${ }^{53}(P)-(+)-1$-Aza[6] helicene (35) gave $(S)$-34 in 89\% yield and 99\% ee (Figure 3). Surprisingly, $(P)-(+)-2-a z a[6]$ helicene (36) afforded $(R)-34$ in $88 \%$ yield and $93 \%$ ee. The sense of enantioselectivity is controlled not only by the helicity of the azahelicene but also by the position of the nitrogen atom.

Figure 3. Aza[6]helicenes 35 and 36 Used for the Enantiodivergent Addition of Diisopropylzinc to Pyrimidine-5-carbaldehyde 32

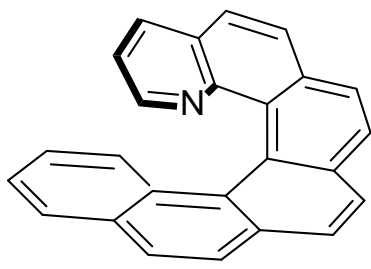

35

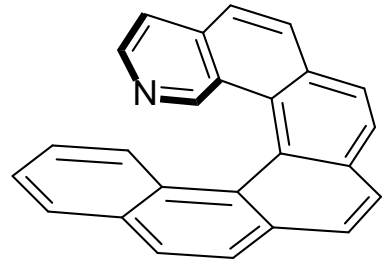

36

The group of Burguete and Luis described an interesting example about the addition of diethylzinc to aldehydes catalyzed by low loading of nickel complexes. ${ }^{54,55}$ In the case of a 1:1 ligand/nickel complex 37a, derived from chiral $\alpha$-amino amides, the formation of (S)-1-phenyl-1-propanol was mainly favored, whereas the 1:2 complex 37b provided the $(R)$-enantiomer (Scheme 10$){ }^{55}$

Scheme 10. Enantiodivergent Addition of Diethylzinc to Benzaldehyde Catalyzed by $\alpha$-Amino Amide Ni Complexes 37

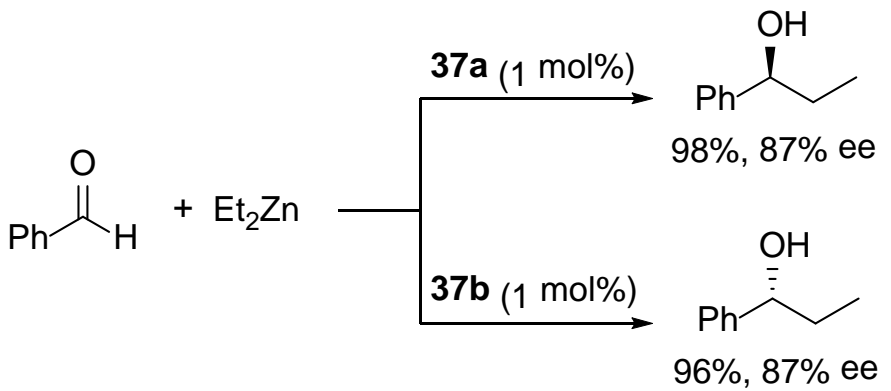<smiles>CO[N+]1(O)NC(Br)C(=O)N1Cc1ccccc1</smiles>

$37 a$<smiles>NN(C(=O)C(Br)Br)N(Cc1ccccc1)C(=O)N(Cc1ccccc1)Cc1ccccc1</smiles>

37b 
Catalytic enantiodivergent addition of vinylaluminum reagents to aldehydes in the presence of chiral $\mathrm{Ti}$ or $\mathrm{Al}$ catalysts has been recently described. By using the Zrcatalyzed carboalumination of terminal alkynes with $\mathrm{Me}_{3} \mathrm{Al}$, it was possible to prepare in situ the corresponding $(E)$-alkenylaluminium compounds $\mathbf{3 8}$, which in the presence of $(R)-\mathrm{DPP}-\mathrm{H}_{8}$-BINOL (39)-derived Ti catalyst reacted with aldehydes to afford $(R)$ alcohols 40. ${ }^{56}$ However, in the absence of $\mathrm{Ti}(\mathrm{OiPr})_{4}$ the reversal of enantioselectivity was observed, just by changing $\mathrm{Ti}$ by $\mathrm{Al}$ as a metal source, giving allylic alcohols $(S)$ $\mathbf{4 0}^{57}$ (Scheme 11).

Scheme 11. Enantiodivergent Addition of Vinylaluminum Reagents to Aldehydes Catalyzed by (R)-DPP-H $-H_{8}$-BINOL 39 Ti and Al Complexes

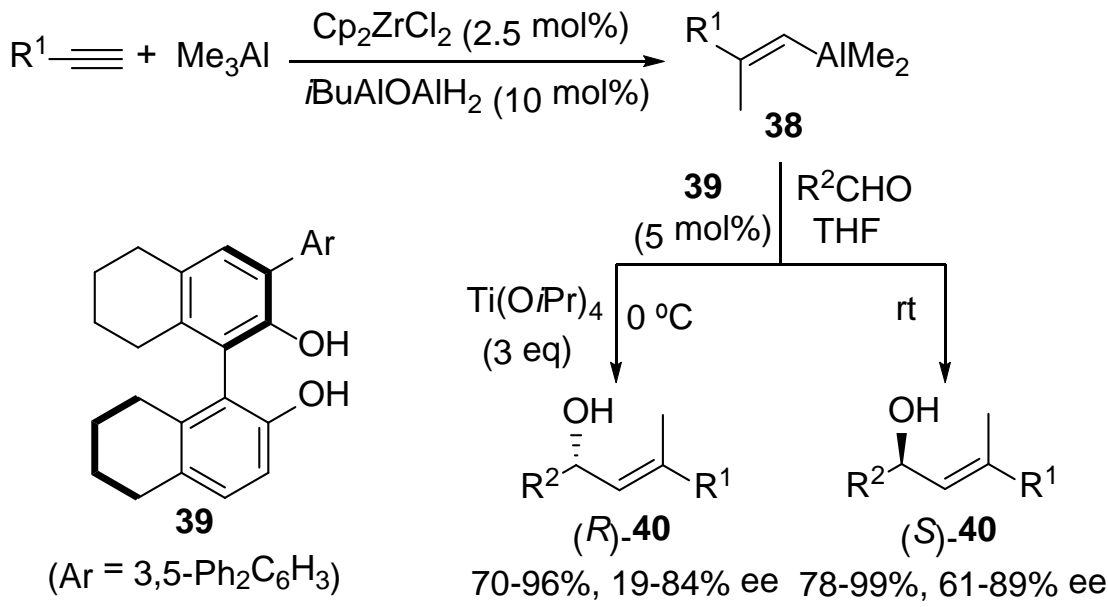

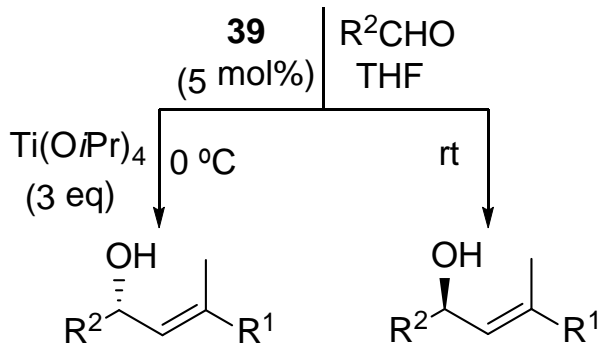

Asymmetric alkynylation of carbonyl compounds provided enantioenriched propargylic alcohols. Diastereodivergent addition of alkynylmetals to Garner's aldehyde 9 was observed in the pioneering work of Herold ${ }^{58}$ during the synthesis of erythro- and threo-sphingoshine derivatives. Addition of 1-pentadecynyllithium in THF led to the formation of anti-41 in 95\% de. On the other hand, the predominant formation of syn-41 was achieved by addition of $\mathrm{ZnBr}_{2}$ at $-78{ }^{\circ} \mathrm{C}$ in $95 \%$ de, based on a chelation-controlled mechanism (Scheme 12). Similar diastereodivergence was observed by Fujisawa and co-workers ${ }^{59}$ in the synthesis of (+)-deoxybiotin, a precursor of (+)-biotin, starting from the thio derivative of $\mathbf{9}$. The addition of 1-hexynyllithium in the presence of HMPA gave the anti-adduct in $66 \%$ de, whereas the chlorozincacetylide exclusively gave the syn-adduct in $86 \%$ yield.

Scheme 12. Diastereodivergent Addition of 1-Pentadecynyllithium to Garner's Aldehyde 9 


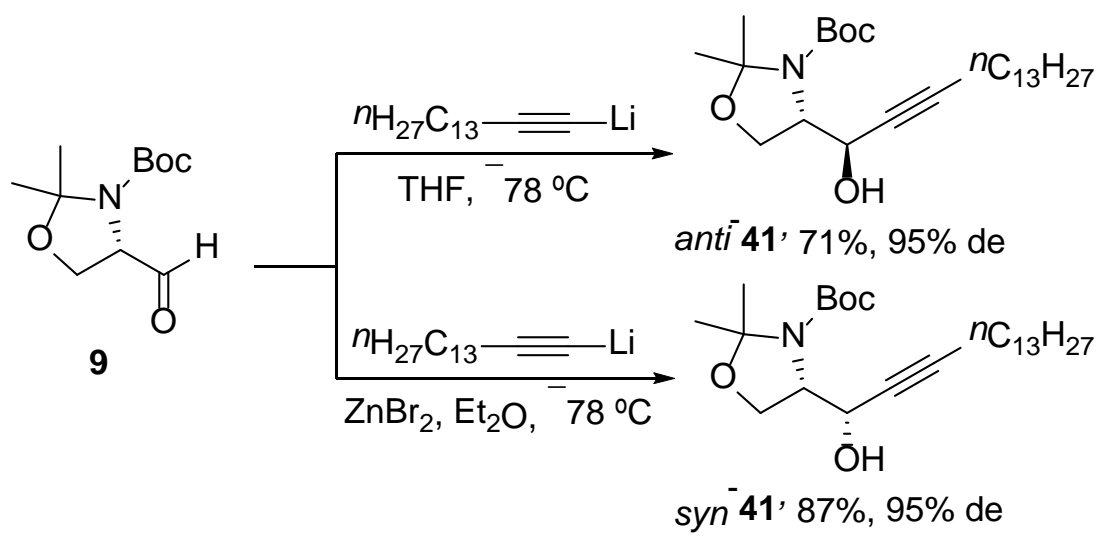

Diastereodivergent additions of alkynyllithium and magnesium reagents to chiral 2acyl-1,3-oxathianes $\mathbf{4 2}$ were performed by Utimoto and co-workers. ${ }^{60}$ In the absence and presence of ytterbium trichloride, compounds $\mathbf{4 3}$ and $\mathbf{4 4}$ were formed, respectively (Scheme 13). Pentynylmagnesium bromide showed the same behavior as pentynyllithium but in lower yields. These products, 43 and 44, can be further transformed into optically active $\alpha$-hydroxy aldehydes following Eliel and co-workers methodology. ${ }^{61,62}$

Scheme 13. Diastereodivergent Addition of 1-Pentynylmetals to 2-Acyl-1,3oxathianes 42

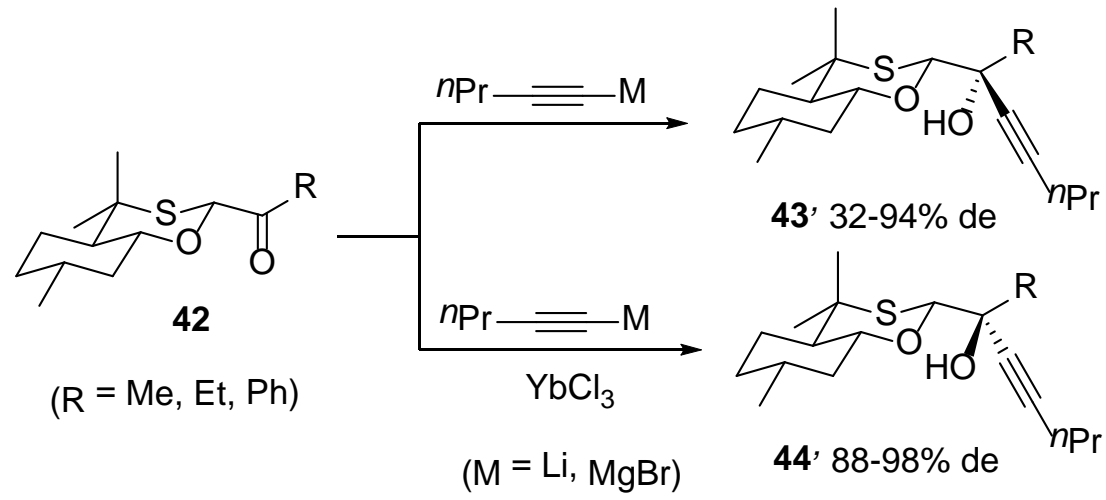

Synthetic studies of Annonaceous acetogenins performed by Tanaka and coworkers, ${ }^{63-66}$ used the Carreira protocol $^{67}$ for the alkynylation of aldehydes with $\mathrm{Zn}(\mathrm{OTf})_{2}$ in the presence of an amine. They found out the diastereodivergent addition of acetylene $\mathbf{4 6}$ to aldehyde $\mathbf{4 5}$ depending on the amine ligand. Diastereomeric adducts 47 and 48 were obtained in $>94 \%$ de, depending on the use of 2.4 eq of $(1 R, 2 S)$ - and $(1 S, 2 R)-N$-methylephedrine (NME), respectively (Scheme 14). ${ }^{66}$ The resulting propargylic alcohols have been transformed into the THF-core of acetogenins.

Scheme 14. Diastereodivergent Alkynylation of Aldehyde 45 by Means of $\mathrm{Zn}(\mathrm{OTf})_{2}$ and $N$-Methylephedrines 


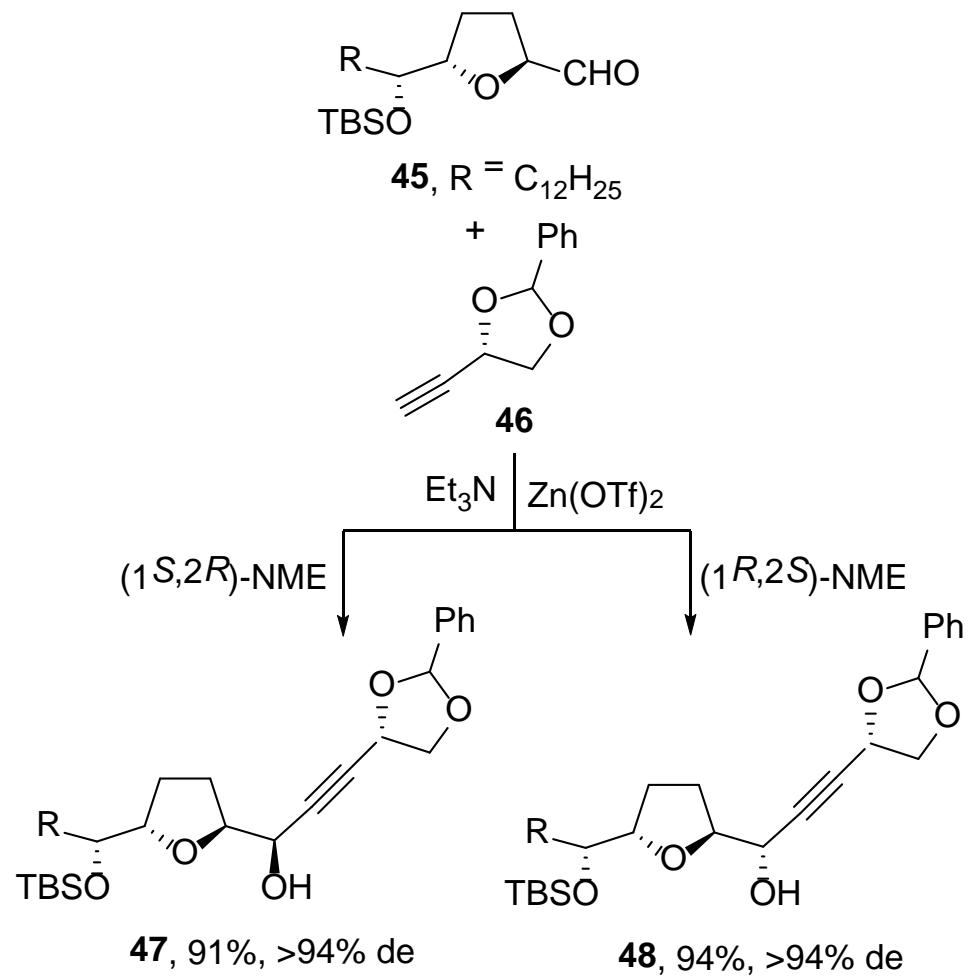

Reversal of enantioselectivity was observed also in the addition of phenylacetylene to aldehydes by adding $\mathrm{Ti}(\mathrm{OiPr})_{4}$ and using $(S)$-prolinol 49 as chiral ligand. ${ }^{68}$ In the presence of $\mathrm{Ti}$, $(S)$-propargylic alcohols (24-56\% ee) were obtained and in the absence of $\mathrm{Ti},(R)$-enantiomers were formed (37-61\% ee). The same group described similar effect just with different prolinols $\mathbf{5 0}$ and $\mathbf{5 1}$ for the addition of terminal alkynes to aromatic aldehydes. Propargylic alcohols with $(R)$-configuration were obtained using ligand 50 and the corresponding (S)-isomer in the case of ligand $\mathbf{5 1}$ in moderate enantioselectivities (Figure 4). ${ }^{69}$ The addition of phenylacetylene to aromatic aldehydes in the presence of dimethylzinc and ligand 52 afforded the corresponding (S)propargylic alcohols (54-94\% ee), while in the presence of $\mathrm{CaH}_{2}$ and $n \mathrm{BuLi}$ gave $(R)$ alcohols (65-99\% ee) (Figure 4). ${ }^{70}$

\section{Figure 4. Ligands Used for the Enantiodivergent Alkynylation of Aldehydes}<smiles>CCOC(=O)N1CCCC1C(O)(c1ccccc1)c1ccccc1</smiles><smiles>[NH3+]C(CN1CCCC1CO)c1ccccc1</smiles>

$51^{69}$<smiles>[NH3+]C(CN1CCCC1C(O)(c1ccccc1)c1ccccc1)[PH2]c1ccccc1</smiles>

$50^{69}$<smiles>Oc1c(-c2ccccc2)cc2ccccc2c1-c1c(C(O)c2ccccc2)ccc2ccccc12</smiles>

$52^{70}$ 
A diastereodivergent addition of allenylzincs to aryl glyoxylates $\mathbf{5 3}$ has been observed depending on the structure of these zinc reagents. ${ }^{71}$ The allenylzinc generated by the treatment of propargyl benzoate 54 with $\mathrm{Pd}(\mathrm{OAc})_{2} / \mathrm{PPh}_{3}(10 \mathrm{~mol} \%)$ and diethylzinc, reacted with glyoxylates $\mathbf{5 3}$ affording products $\mathbf{5 6}$ with de up to $78 \%$ (Scheme 15). However, the allenylzinc formed by the lithiation of silylated alkyne 55 followed by transmetallation with $\mathrm{ZnBr}_{2}$ led to the formation of products $\mathbf{5 7}$ with de up to $92 \%$. These divergent results have been explained by the proposed transition states 56A and 57A, respectively.

\section{Scheme 15. Diastereodivergent Addition of Allenylzincs to Aryl Glyoxylates 53}
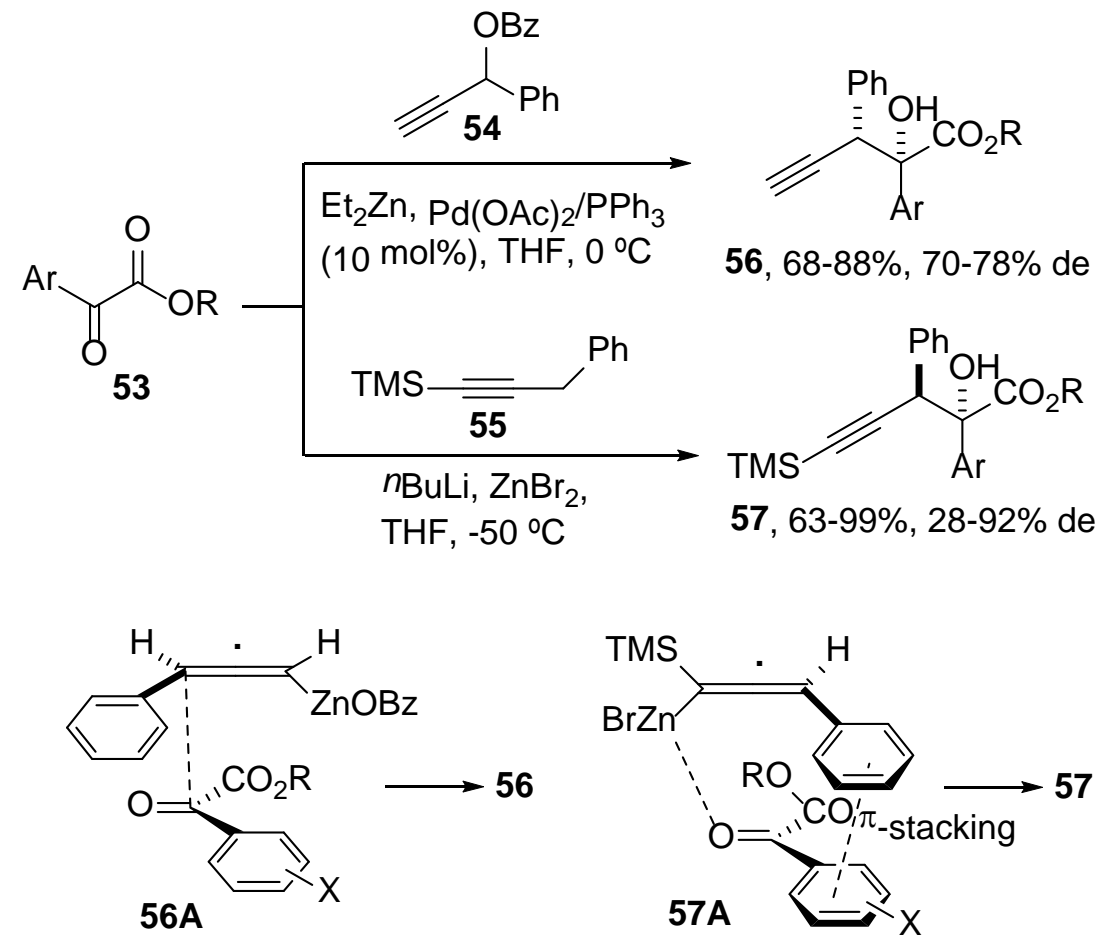

The addition of pinacol derived allylboronates $\mathbf{5 9}$ to aldehyde $\mathbf{5 8}$ in the presence of $\mathrm{SnCl}_{4}$ (10 mol\%) afforded predominantly syn-alcohol $\mathbf{6 1}$. On the other hand, in the presence of chiral diol $\mathbf{6 0}$ (11 mol\%) the anti-isomer $\mathbf{6 1}$ was mainly obtained (Scheme 16). ${ }^{72}$ In the last case, achiral Brønsted acid formed by diol $\mathbf{6 0}$ and $\mathrm{SnCl}_{4}$ catalyzed the enantioselective allylboration.

Scheme 16. Diastereodivergent Addition of Pinacol Allylboronate (59) to Aldehyde 58 Catalyzed by $\mathrm{SnCl}_{4}$ 


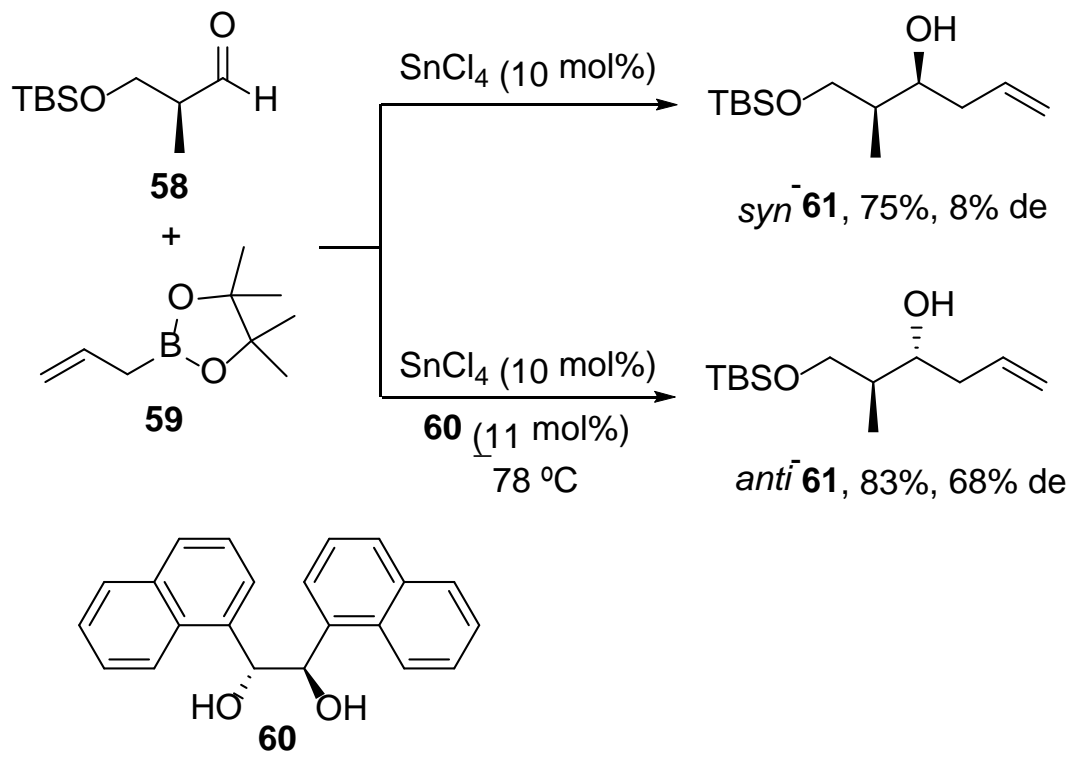

Crotylation of aldehydes allows the synthesis of $\alpha$-methyl homoallylic alcohols using generally premetalated reagents. ${ }^{14,15,73-75}$ Modest values of diastereoselectivity were obtained in the diastereo- and enantiodivergent addition of crotyl bromide to aromatic aldehydes catalyzed by $\mathrm{Cr}^{\mathrm{II}}$ (salen) complex 62 (Scheme 17). ${ }^{76}$ When this enantioselective version of the Nozaki-Hiyama-Kishi reaction of benzaldehyde with crotylbromide was performed in the presence of $10 \mathrm{~mol} \%$ of complex 62, product anti63 was obtained in 50\% yield and 34\% de but only in 5\% ee. On the other hand, when a 2:1 mixture of salen/ $\mathrm{CrCl}_{3}$ was used as catalyst, syn-63 was preferentially formed in $56 \%$ yield, 66\% de, and $89 \%$ ee. This syn-diastereoselectivity was explained by formation of an acyclic transition state in which the aldehyde was coordinated either by the manganese salt or by the $\mathrm{Cr}^{\mathrm{II}}$ (salen) complex. However, the anti-diastereoselection occurred probably via a cyclic Zimmermann-Traxler transition state.

Scheme 17. Diastereo- and Enantiodivergent Addition of Crotyl Bromide to Benzaldehyde Catalyzed by the $\mathrm{Cr}^{\mathrm{II}} /$ Salen Complex 62 


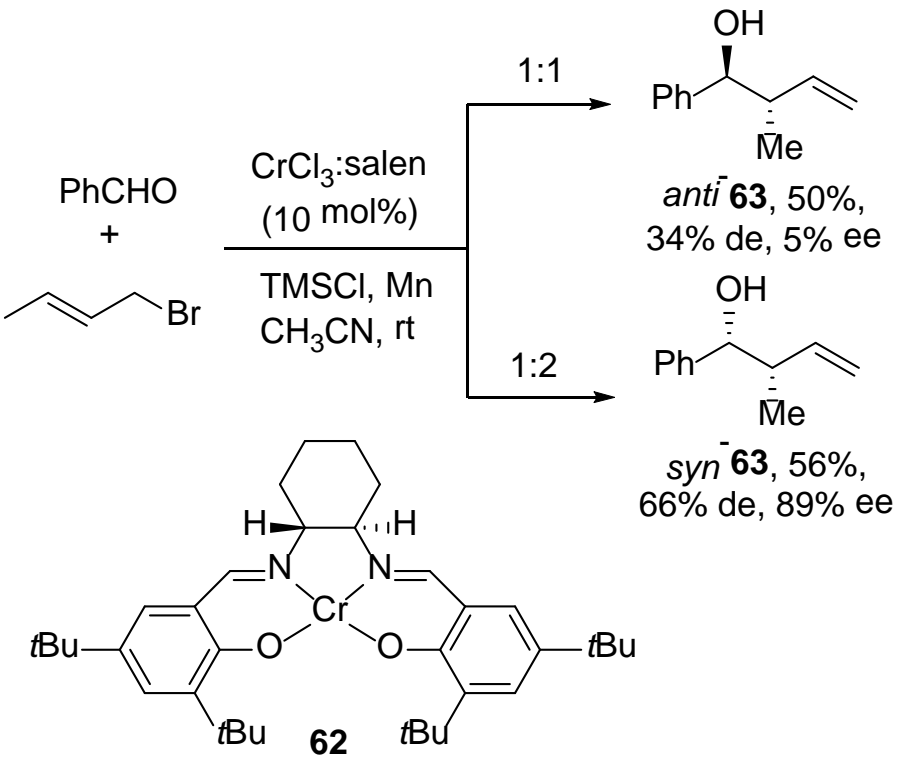

Krische and co-workers have implemented a ruthenium-catalyzed asymmetric hydrohydroxyalkylation of butadiene as an alternative for crotylation of carbonyl compounds. ${ }^{77}$ They have demonstrated a chiral-anion-dependent diastereodivergence in this carbonyl crotylation catalyzed by $\mathrm{H}_{2} \mathrm{Ru}(\mathrm{CO})\left(\mathrm{PPh}_{3}\right)_{3}$ and (S)-SEGPHOS or DPPF complexes (Scheme 18). ${ }^{78}$ Butadiene hydrohydroxyalkylation with primary alcohols took place with these complexes and chiral phosphoric acids. When BINOL-derived phosphoric acid $\mathbf{6 4}$ worked as counteranion, the corresponding anti-alcohols $\mathbf{6 6}$ were mainly formed. However, syn-crotylation occurred by using TADDOL-phosphoric acid 65 (Scheme 18). In addition to diastereodivergent crotylation, inversion of the enantioselectivity was observed in the alcohol stereocenter. The observed stereochemical outcomes were controlled by the phosphine and the phosphate ligands. The DFT calculations supported the formation of TS anti-66A and syn-66A. ${ }^{79}$ The phosphate-dependent stereoselectivity arose from TS syn-66A through a hydrogen bond between the phosphonyl oxygen and the formyl hydrogen present in the TADDOLderived catalyst. The aldehyde was formed by hydrogen-transfer between the primary alcohol and the $\pi$-unsaturated reactant. This hydrogen bond is absent in the BINOLderived systems inducing the reversal of enantioselectivity for the anti-product.

Scheme 18. Diastereodivergent Asymmetric Crotylation of Aldehydes via RuCatalyzed Butadiene Hydrohydroxyalkylation 


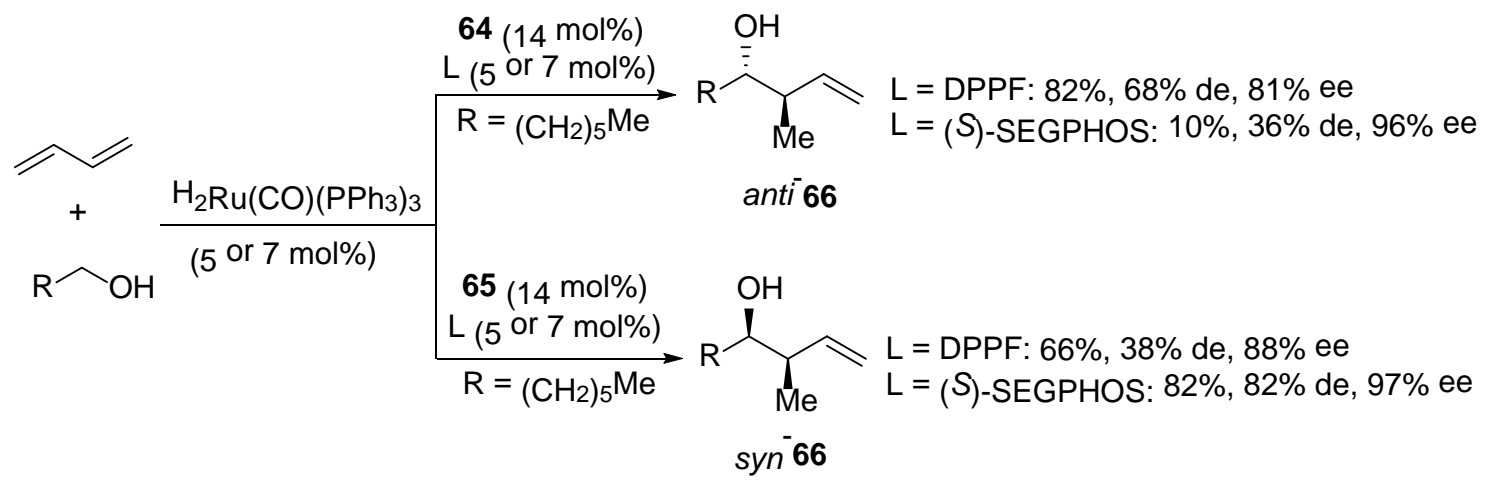<smiles>CCc1cc2c(c(-c3c(OP(=O)(O)O)cc4c(c3OP(=O)(O)O)CCCC4)c1)CCCC2</smiles>

64

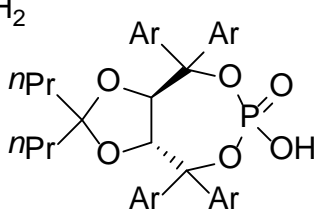

$65\left(\mathrm{Ar}=m_{-\mathrm{xylyl})}\right.$<smiles>Pc1cccc(-c2ccccc2)c1-c1c(P)ccc2c1OCO2</smiles>

(S)-SEGPHOS

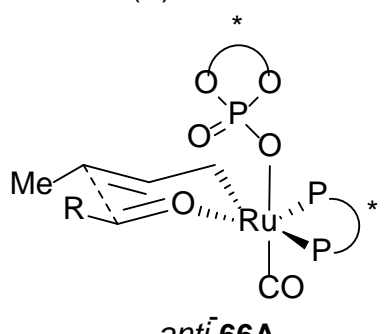

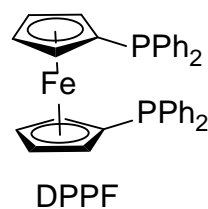

DPPF

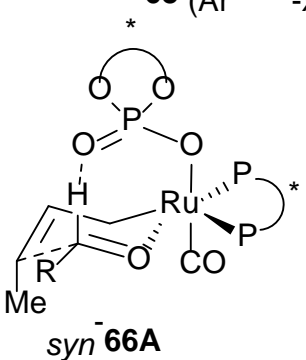

syn ${ }^{\top} 66 \mathrm{~A}$

anti 66A

It can be concluded that diastereodivergent addition of organometallics to acyclic carbonyl compounds can be modulated by different Lewis acids either by intermolecular chelation or by nonchelation control. In addition, the presence or the absence of a Lewis acid and the polarity of the solvent can be also crucial. In the case of enantiodivergent additions, substituent modifications on the chiral ligand or the presence or absence of a Lewis acid, as well as the presence of additives and temperature changes can be crucial.

2.1.2. Aldol Reactions. Aldol reactions are one of the most important transformations not only in organic synthesis but also in biological systems. It has been widely applied to build up polyketide units of bioactive and natural products. Significant advances have been made in the last 40 years to control the diastereo- and also the enantioselectivity of this reaction. ${ }^{80,81}$ In the case of acyclic aldols with two stereocenters at the $\alpha$ and $\beta$-position, the syn and anti-relationship depends mainly from the $Z$ and $E$-configuration of the precursor enolate, respectively, in cyclic TS. Stereodivergent methodologies have been developed over the years controlling structural features of the substrates as well as the enolate counteranion, Lewis acids and bases being used as catalysts. Catalytic stereodivergent processes have been considered by using metal complexes or organocatalysts.

2.1.2.1. Aldol Reactions Involving Metal Enolates. Silicon enolates are less reactive than other metal enolates in aldol reactions. However, using a Lewis acid the carbonyl acceptor can be activated and the nucleophilic character of enol silanes can be 
increased by binding to a Lewis base such as a phosphoramide. ${ }^{81}$ This Mukaiyama aldol reaction proceeds through an open TS antiperiplanar approaches, favored by the minimization of dipolar interactions (Figure 5). ${ }^{82,83}$

Figure 5. Open Transition States for Mukaiyama Aldol Reactions
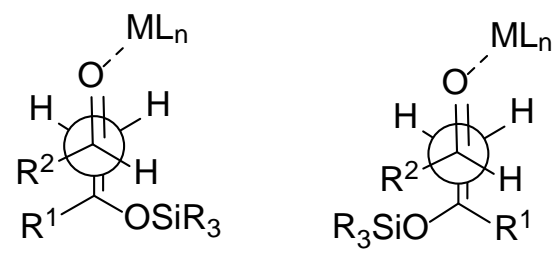

In the typical Mukaiyama aldol reaction of a protected $\alpha$-methyl- $\beta$-alkoxy aldehyde 67 with the acetone silyl enol ether, a diastereodivergent addition was found to be dependent on the Lewis acids, which are used in stoichiometric amounts. ${ }^{84}$ In the case of $\mathrm{BF}_{3} \cdot \mathrm{OEt}_{2}$ the 1,3-anti-68 isomer was mainly formed according to anti-Felkin-Anh preference. However, for the sterically demanding Lewis acid trityl perchlorate, reversal of the facial selectivity was observed providing aldol 1,3-syn-68 by a Felkin-Anh preference (Scheme 19).

Scheme 19. Diastereodivergent Aldol Reaction of Acetone Silyl Enol Ether with $\alpha$ Methyl- $\beta$-alkoxy Aldehyde 67

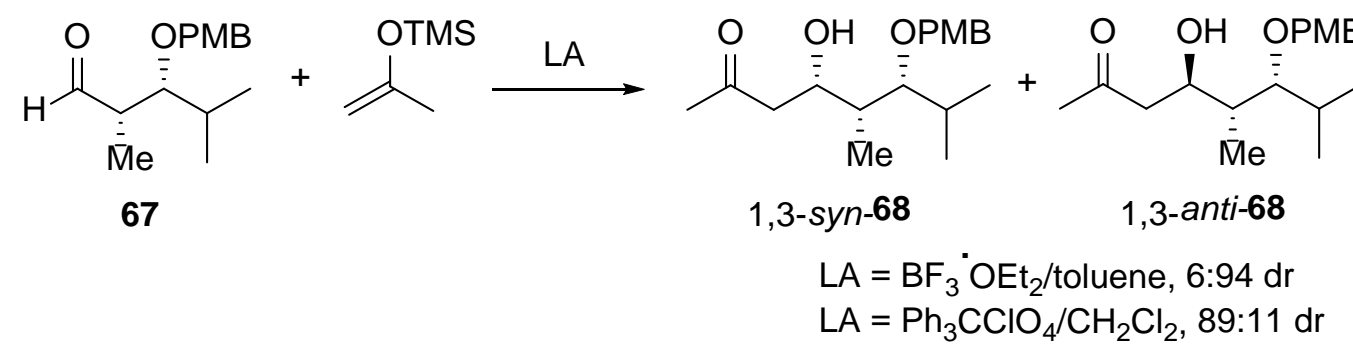

Previous work from Evans and co-workers about Mukaiyama aldol reaction with enol silanes of pinacolone and $\alpha$-methyl- $\beta$-alkoxy aldehydes in the presence of different Lewis acids has shown the diastereodivergent formation of aldols. ${ }^{85}$ In the case of $\alpha$ methyl- $\beta$-silyloxy aldehyde 69, $\mathrm{BF}_{3} \cdot \mathrm{OEt}_{2}, \mathrm{SnCl}_{4}$ and $\mathrm{TiCl}_{4}$ gave mainly syn-70 resulting from a Felkin-Anh controlled reaction model. However, $\mathrm{Me}_{2} \mathrm{AlCl}$ and $\mathrm{MeAlCl}_{2}$ favored the formation of the anti-aldol $\mathbf{7 0}$ according to a chelation mechanism (Scheme 20).

Scheme 20. Diastereodivergent Addition of Pinacolone Silylenolate to $\alpha$-Methyl- $\beta$ silyloxy Aldehyde 69 in the Presence of Lewis Acids 


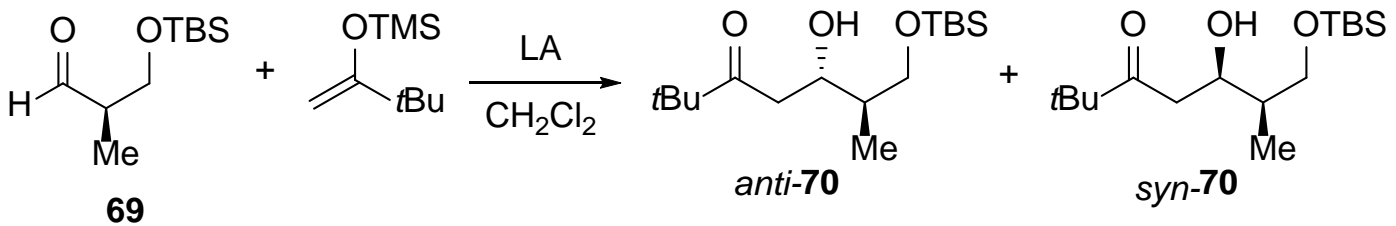
LA : $\mathrm{BF}_{3}{ }^{\circ} \mathrm{OEt}_{2}, 55 \%, 9: 91 \mathrm{dr}$
LA : $\mathrm{SnCl}_{4}, 41 \%, 7: 93 \mathrm{dr}$
LA : $\mathrm{TiCl}_{4}, 55 \%, 7: 93 \mathrm{dr}$
LA : $\mathrm{Me}_{2} \mathrm{AlCl}, 62 \%, 97: 3 \mathrm{dr}$
LA : $\mathrm{MeAICl}_{2}, 55 \%, 77: 23 \mathrm{dr}$

Diastereodivergent aldol reactions have been carried out with $\alpha$-methyl- $\beta$-alkoxy aldehydes such as $\mathbf{7 1}$ by adding a mixture of $E$ and Z-enolsilanes $\mathbf{7 2}$ derived from 2bromopropionate in the presence of different Lewis acids. ${ }^{86}$ A high 3,4-syn diastereoselectivity was observed when the monodentate Lewis acid $\mathrm{BF}_{3} \cdot \mathrm{OEt}_{2}$ was used giving esters 73. On the contrary, when a bidentate Lewis acid such as $\mathrm{Ti}(\mathrm{OiPr}) \mathrm{Cl}_{3}$ was employed the Cram's chelated Mukaiyama aldol product 3,4-anti-73 was predominantly formed (Scheme 21). Similar results have been observed with aldehydes 74-76. After reductive debromination by means of a hydrogen transfer reaction, up to 16 diastereomeric stereopentads have been prepared. Following this methodology, the synthesis of the C1-C11 fragment of the natural product zincophorin with antibiotic properties has been achieved.

Scheme 21. Diastereodivergent Addition of Enolsilanes 72 to $\alpha$-Methyl- $\beta$-alkoxy Aldehyde 71

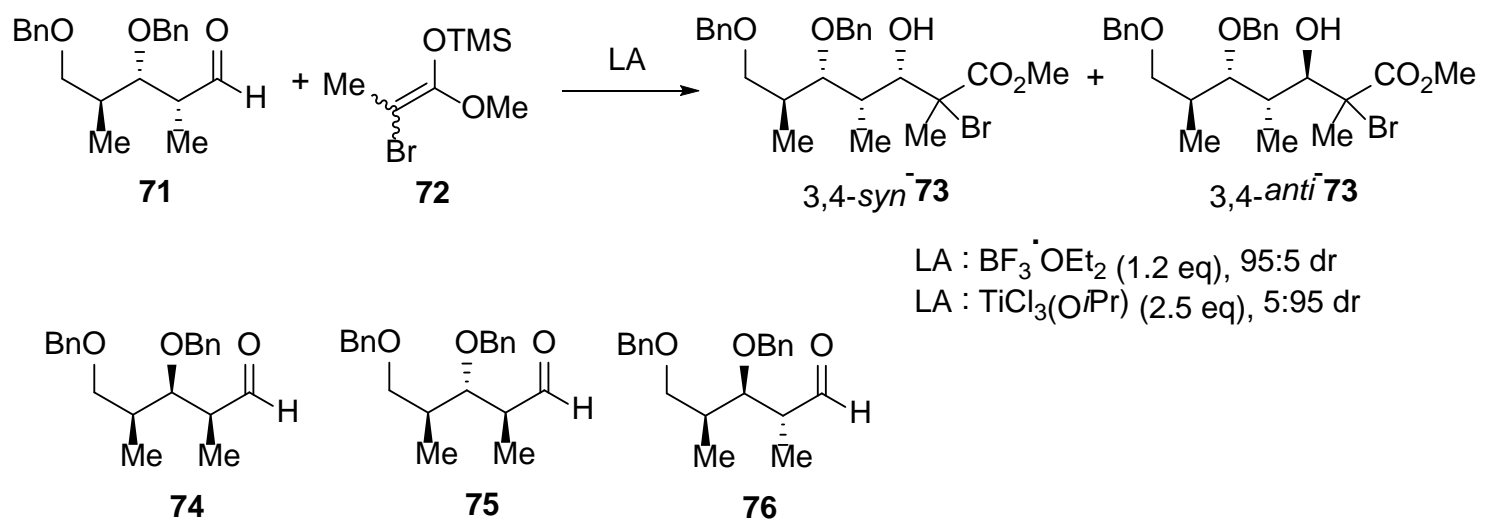

In 1994 Kobayashi and Horibe discovered an enantiodivergent Mukaiyama aldol reaction using stoichiometric amounts of $\operatorname{Sn}(\mathrm{OTf})_{2}$ as Lewis acid and chiral diamines $\mathbf{7 8}$ derived from L-Pro. ${ }^{87-91}$ In the aldol reaction of silylketene thioacetal $77 \mathbf{a}$ with aldehydes, small changes in the structure of these homochiral diamine ligands produced both enantiomeric aldols 79 (Scheme 22). The resulting 2,3-dihydroxy thioesters were obtained in high syn-selectivity (syn/anti: 99:1 dr) and high enantioselectivities. These unique selectivities have been explained assuming the participation of transition states shown in Scheme 22. In the case of chiral diamine 78a-coordinated tin(II) complex, the aldehyde will approach from the bottom side and its $R e$ face is shielded by the amine 
unit and therefore the silylketene thioacetal attacks the aldehyde by its $S i$ face via an acyclic TS (see, Figure 5) forming the syn-(2S,3R)-aldol 79. In the case of chiral diamine $\mathbf{7 8 b}$, the aldehyde coordination occurred in the opposite side of the tin complex being the $S i$ face shielded and the silylketene thioacetal attacks the aldehyde from the $R e$ face. $^{91}$

Scheme 22. Enantiodivergent Aldol Reaction of Silylketene Thioacetal 77a with Aldehydes Catalyzed by Sn(OTf $)_{2}$ and Homochiral Diamines 78
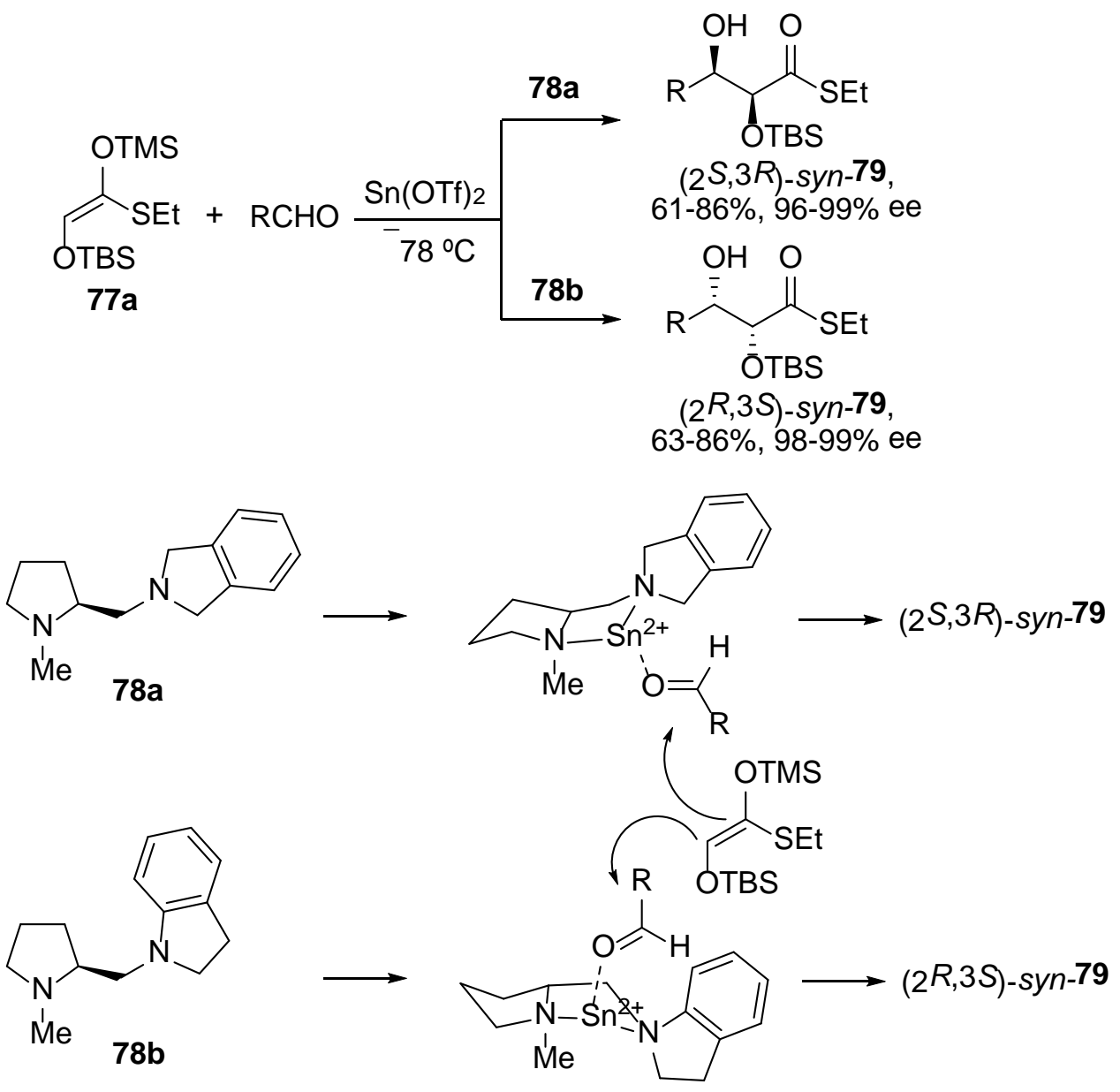

The group of Evans also found that copper complexes with the same ligand, $(S, S)$ tert-BuBox 80, can give enantiomeric aldols depending on the copper salt used. ${ }^{92}$ When $\mathrm{Cu}(\mathrm{OTf})_{2}$ was used as copper source in the Mukaiyama aldol reaction between (benzyloxy)acetaldehyde and tert-butylthioacetate trimetylsilylketene acetal (77b), $(R)$ $\beta$-hydroxy thioester $\mathbf{8 2}$ was formed in 91\% ee. Reversal of the enantioselectivity took place when $\mathrm{Cu}\left(\mathrm{SbF}_{6}\right)_{2}$ was employed affording $(S)-82$ in a lower $64 \%$ ee. The copper complex 81 (X = OTf) formed with (benzyloxy)acetaldehyde a square pyramidal intermediate 81A and the approaching of the silylketene acetal $\mathbf{7 7 b}$ is by the $R e$ face. However, in the case of $\mathbf{8 1}\left(\mathrm{X}=\mathrm{SbF}_{6}\right)$, a square plane intermediate $\mathbf{8 1 B}$ is formed and the attack of the nucleophile is by the Si face forming the enantiomer $(S$ )-82 (Scheme 
23). This example can be considered as a case of counteranion-dependent enantiodivergence.

Scheme 23. Enantiodivergent Aldol Reaction of Silylketene Thioacetal 77b with (Benzyloxy)acetaldehyde Catalyzed by Cu(II) Salts and $(S, S)$-tBuBox 80

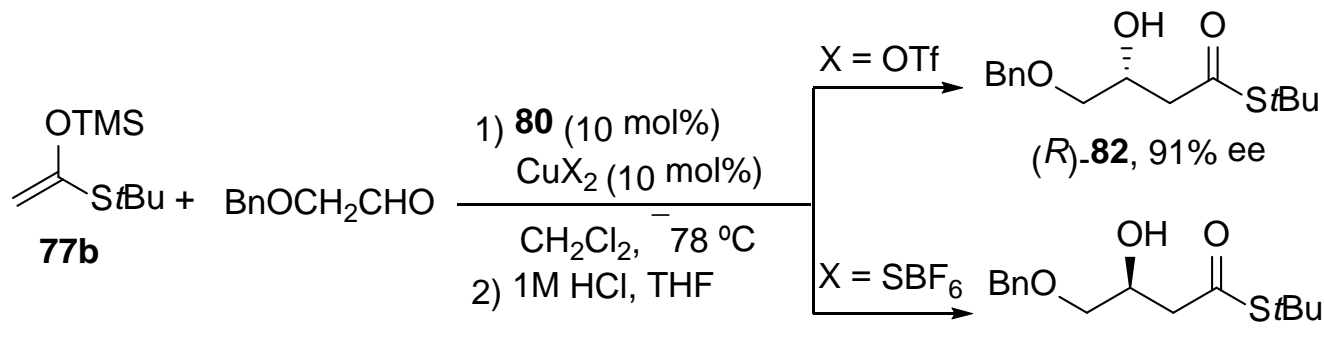<smiles>CC(C)(C1=N[C@@H](C(C)(C)C)CO1)C1=N[13CH](C(C)(C)C)CO1</smiles>

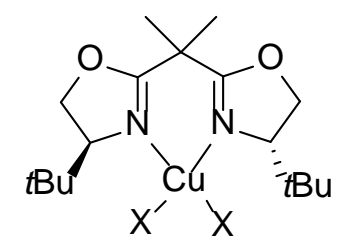

(S)-82, 64\% ee

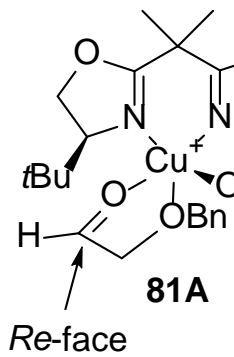

81

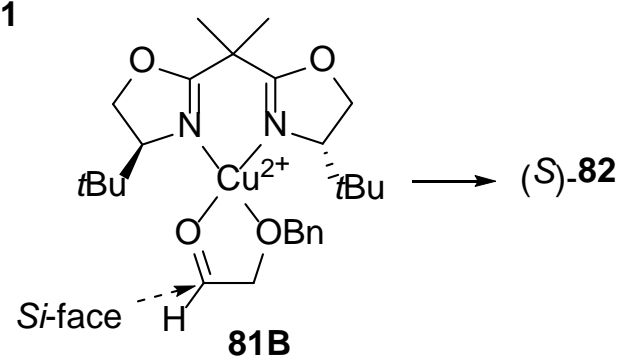

Inversion of the enantioselectivity promoted by the solvent has been also observed in the Mukaiyama aldol reaction of tert-butylthioacetate trimetylsilylketene acetal (77b) with pyruvate methyl ester. ${ }^{93,94}$ Using complex 81 (X = OTf) in acetonitrile the $(R)$ enantiomer $\mathbf{8 3}$ was formed although in low ee, whereas in other solvents aldol (S)-83 was enantioselectively obtained (Scheme 24). Apparently, acetonitrile is acting as ligand giving a square pyramidal $\mathrm{Cu}(\mathrm{II})$ geometry, like in $\mathbf{8 1 A}$, instead of the square planar geometry as in $\mathbf{8 1 B}$.

Scheme 24. Enantiodivergent Aldol Reaction of Silylketene Thioacetal 77b with Methyl Pyruvate Catalyzed by $\mathrm{Cu}(\mathrm{OTf})_{2}$ and $(S, S)$-tBuBox 80

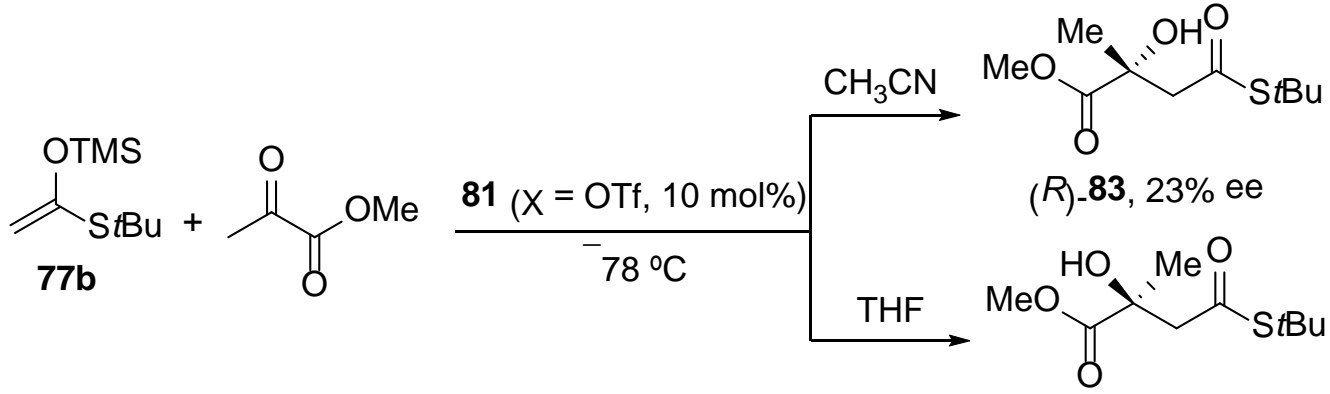

(S)-83, 99\% ee 
One example of diastereodivergent vinylogous aldol reaction of 2trimethylsilyloxyfuran (84) with aldehydes gave racemic threo- and erythro- $\delta$-hydroxy$\gamma$-butenolides 85 just varying the reaction conditions. ${ }^{95}$ Lewis acids such as $\mathrm{BF}_{3} \cdot \mathrm{Et}_{2} \mathrm{O}$, trityl perchlorate and triethylsilyl triflate (TESOTf) catalyzed this condensation to afford products threo-85, the later Lewis acid giving the highest de (up to 64\%). On the contrary, using tetra-n-butylammonium fluoride (TBAF), erythro-isomers 85 were obtained in moderate de (Scheme 25). These butenolides were further transformed, by reduction with $\mathrm{NaBH}_{4} / \mathrm{NiCl}_{2}$, into the corresponding lactones.

\section{Scheme 25. Diastereodivergent Catalyzed Vinylogous Aldol Reaction of 2- Trimethylsilyloxyfuran (84) with Aldehydes}

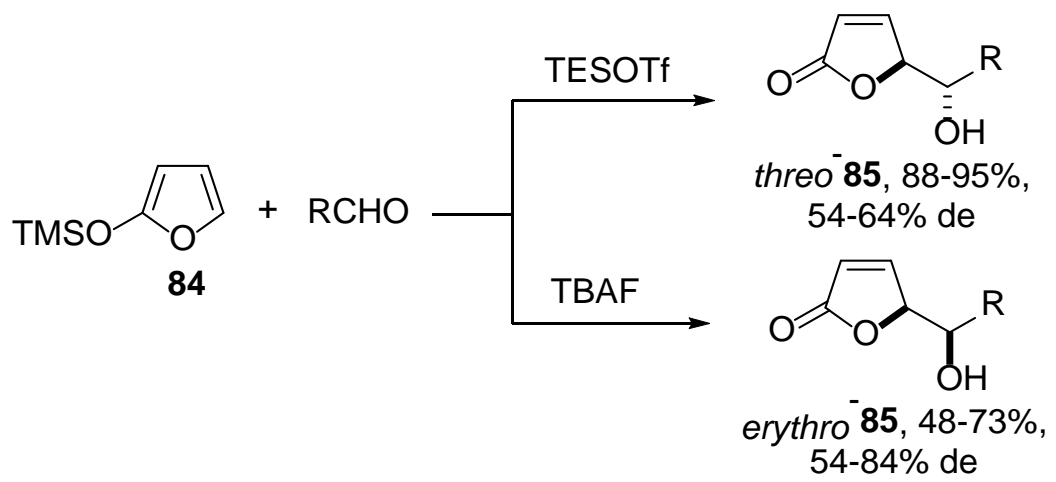

A diastereodivergent vinylogous Mukaiyama aldol reaction has been found by changing the Lewis acid for the reaction of (S)-Roche aldehyde 86 with 3,4-(Z)-ketene acetal 87 (Scheme 26). ${ }^{96}$ In the case of $\mathrm{MgBr}_{2} \cdot \mathrm{OEt}_{2}$, the corresponding 4,5-syn-5,6-anti isomer 88, a fragment of the natural product soraphen, was preferentially formed according to a chelation-controlled transition state. However, in the presence of dicyclohexylboron chloride, the polyketide all-syn-88, precursor of the natural product oleandolide, was obtained by a Felkin-Anh transition state.

\section{Scheme 26. Diastereodivergent Catalyzed Vinylogous Mukaiyama Aldol Reaction} of Vinyl Ketene Acetal 87 with (S)-Roche Aldehyde 86

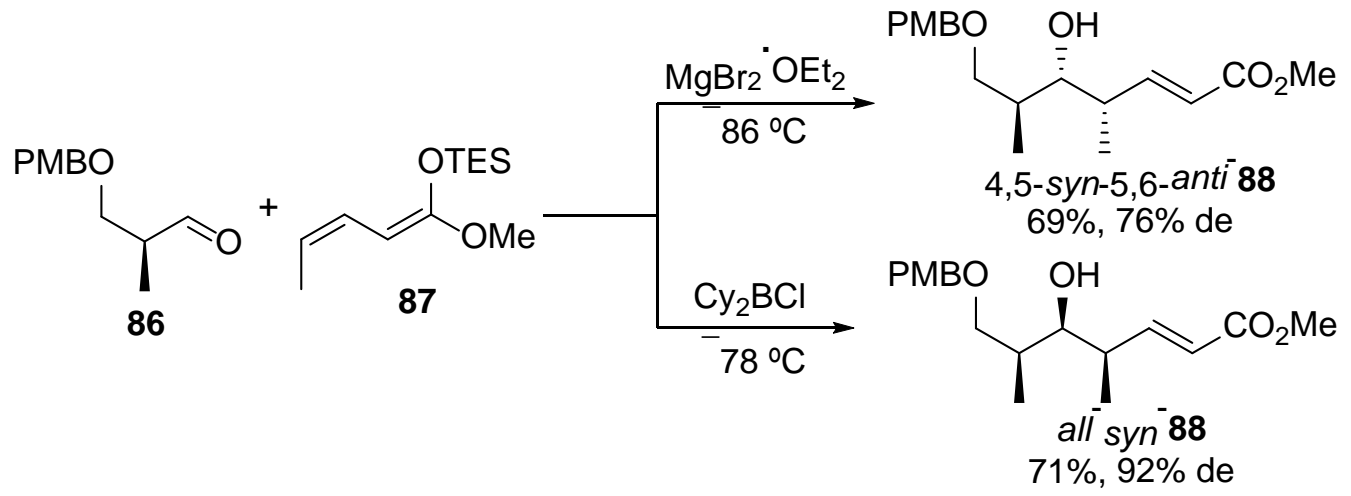

A direct metal-catalyzed temperature-dependent enantiodivergent aldol reaction has been reported by Trost and co-workers. ${ }^{97}$ During the screening at different temperatures for the reaction of methyl ynone $\mathbf{8 9}$ with the aldehyde $\mathbf{9 0}$ using a chiral zinc complex of 
ligand 91 and $\mathrm{Et}_{2} \mathrm{Zn}$, they found out that at $-25{ }^{\circ} \mathrm{C}$ the $(S)$-aldol 92 was formed in $44 \%$ ee. However, at $0{ }^{\circ} \mathrm{C}$ the corresponding enantiomer $(R)$-26 was obtained in $72 \%$ ee (Scheme 27). At the early stage of the aldol reaction at $0{ }^{\circ} \mathrm{C}$, the main compound is the $S$ enantiomer, which changed to the $R$ along the process. Further optimization studies demonstrated that the (R)-92 (with $\mathrm{SiEt}_{3}$ as substituent) could be obtained in 75\% yield and $99 \%$ ee working at $\mathrm{rt}$ in THF.

Scheme 27. Enantiodivergent Aldol Reaction of Methyl Ynone 89 with the Aldehyde 90 Catalyzed by a Chiral Zn Complex at Different Temperatures

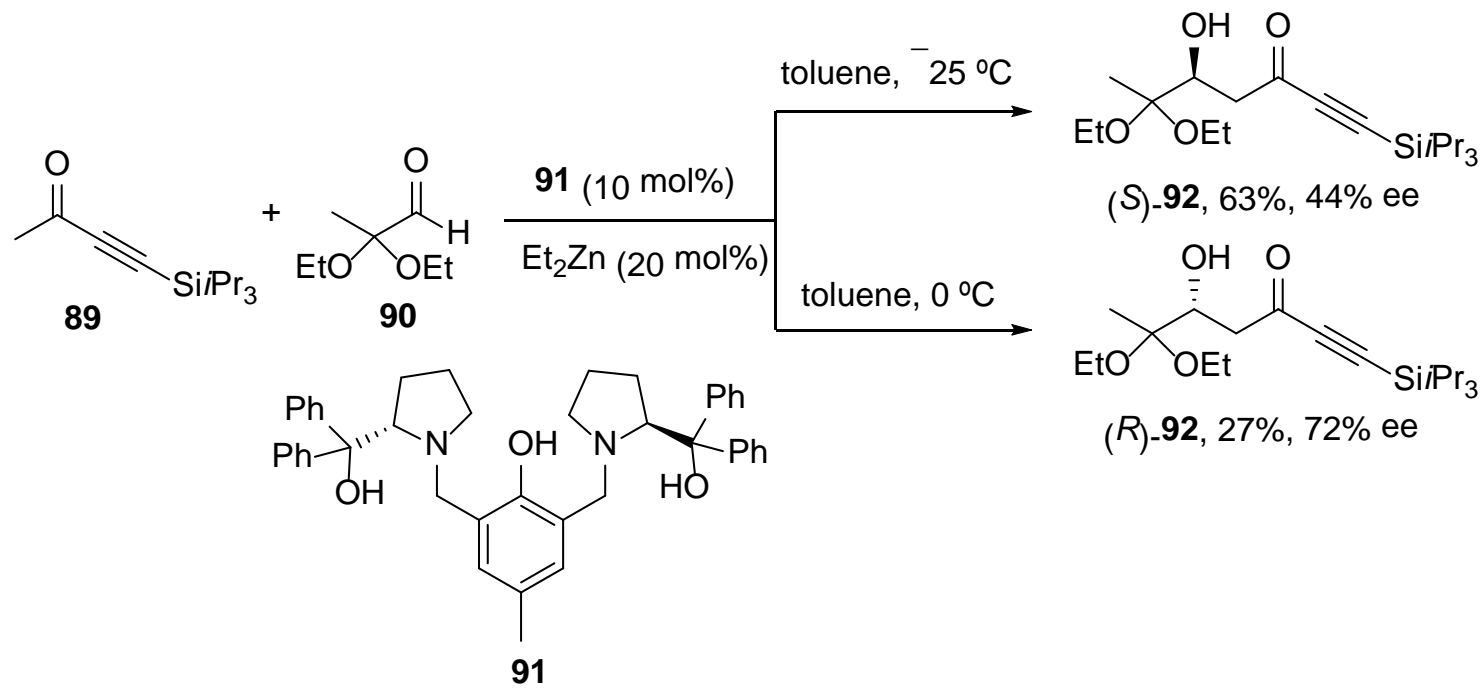

2.1.2.2. Organocatalyzed Aldol Reactions. After the breakthrough on asymmetric organocatalysis, the direct intermolecular aldol reactions could be performed without the presence of any metal just using small organic molecules namely L-Pro. ${ }^{80,81}$ Prolinamides have shown excellent catalytic properties than L-Pro due to a higher solubility in organic solvents. However, few examples have been found about stereodivergent organocatalyzed aldol reactions. The influence of additives is an important factor for increasing the diastereo- and the enantioselectivity in the direct aldol reaction. In the case of using binaphthyl-based axially chiral amine-derived prolinamides 93a and 93b, the structure of these organocatalysts determined the observed reversal of enantioselectivity. This was the first example in which an enantiodivergent effect has been observed when the benchmark reaction between cyclohexanone and 4-nitrobenzaldehyde was performed in THF at $-20{ }^{\circ} \mathrm{C}$ (Scheme 28). ${ }^{98}$ In the case of $93 \mathbf{a}$, anti-(2S,1'R)-aldol 94 was diastereo- and enantioselectively prepared, whereas organocatalysts $93 \mathbf{b}$, also derived from L-Pro, gave mainly its enantiomer anti-(2R,1'S)-aldol 94, in both cases with modest results.

Scheme 28. Enantiodivergent Aldol Reaction Organocatalyzed by Diastereomeric Prolinamides 93 


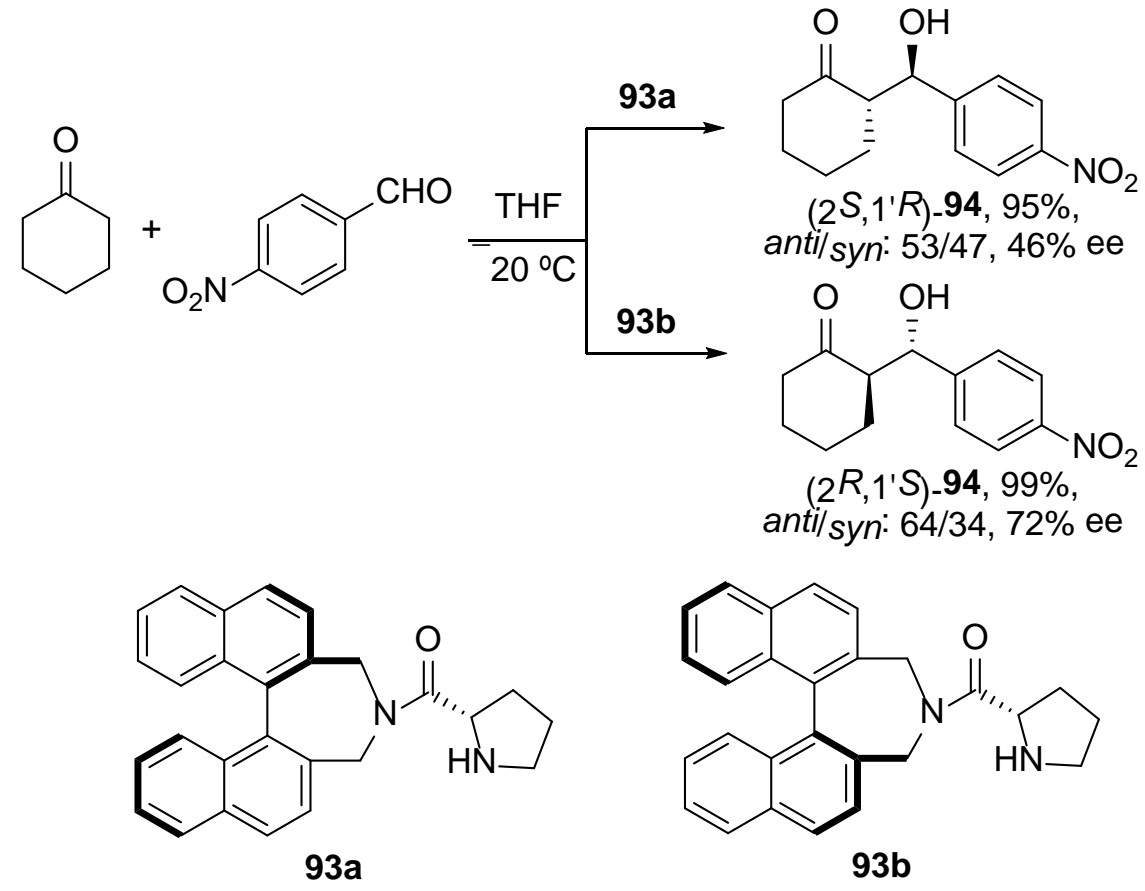

Recently, Kaskel and co-workers reported a reversal of diastereoselectivity using proline functionalized metal-organic frameworks (MOFs) UiO-67 and 68. ${ }^{99}$ These Zrbased MOFs were used as host for Boc-protected L-prolinamides 95 and 96, which were deprotected in situ (Figure 6). The reaction of cyclohexanone with 4-nitrobenzaldehyde catalyzed by deprotected 95 afforded mainly aldol (2R,1'S)-anti-94 in 60\% yield and 40\% de. However, the corresponding chiral MOF UiO-67-NHPro prepared from 95 gave preferentially aldol (2R,1'R)-syn-94 in 39\% yield and 58\% de and UiO-68-NHPro prepared from 96 provided the same aldol in better $97 \%$ yield and 76\% ee. The switch of diastereoselectivity anti to syn was attributed to the catalytic sites in the confined space of the MOF.

Figure 6. Prolinamides Used for the Preparation of Chiral MOFs UiO-67-NHPro and UiO-68-NHPro<smiles>CC(C)(C)OC(=O)N1CCCC1C(=O)Nc1cc(C(=O)O)ccc1-c1ccc(C(=O)O)cc1</smiles><smiles>CC(C)(C)OC(=O)N1CCCC1C(=O)Nc1cc(-c2ccc(C(=O)O)cc2)ccc1-c1ccc(C(=O)O)cc1</smiles>

Several examples of enantiodivergent aldol reactions catalyzed by L-Pro were further described. (S)-Proline adsorbed on $\gamma-\mathrm{Al}_{2} \mathrm{O}_{3}$ promoted an inversion of the 
enantioselectivity in aldol reactions of acetone with different aldehydes. $(R)$-Aldols were obtained with ee up to $88 \%$ using L-Pro, while L-Pro $/ \gamma-\mathrm{Al}_{2} \mathrm{O}_{3}$ provided (S)-aldols with modest enantioselectivities (up to $21 \%$ ee). ${ }^{100}$ A similar effect was observed with other $\alpha$-amino acids. More recently, Bartók and co-workers studied the aldol reaction of acetone and cycloalkanones with aliphatic and aromatic aldehydes catalyzed by L-Pro $/ \gamma$ $\mathrm{Al}_{2} \mathrm{O}_{3}$ either in homogeneous liquid phase or under heterogeneous organic-inorganic hybrid catalyst. Modest reversal of the enantioselectivity was observed in the case of acetone (up to $40 \%$ ee). ${ }^{101}$

The same group has found that the hydroxyproline-derived organocatalyst $\mathbf{9 7}$ gave reversal of enantioselectivity in the aldol reaction of 2-nitrobenzaldehyde with aqueous acetone under acidic or basic conditions. In the presence of ammonium chloride $(R)-\mathbf{9 8}$ was obtained in $58 \%$ ee, whereas in the presence of $\mathrm{NaOAc}$ its enantiomer was formed in only $20 \%$ ee (Scheme 29). ${ }^{102}$ This methodology was further studied with different aromatic aldehydes. ${ }^{103}$ The formation of a biphasic micellar system in the aqueous media seems to be crucial for the observed enantioselectivities.

Scheme 29. Enantiodivergent Aldol Reaction Organocatalyzed by the Hydroxyproline Derivative 97

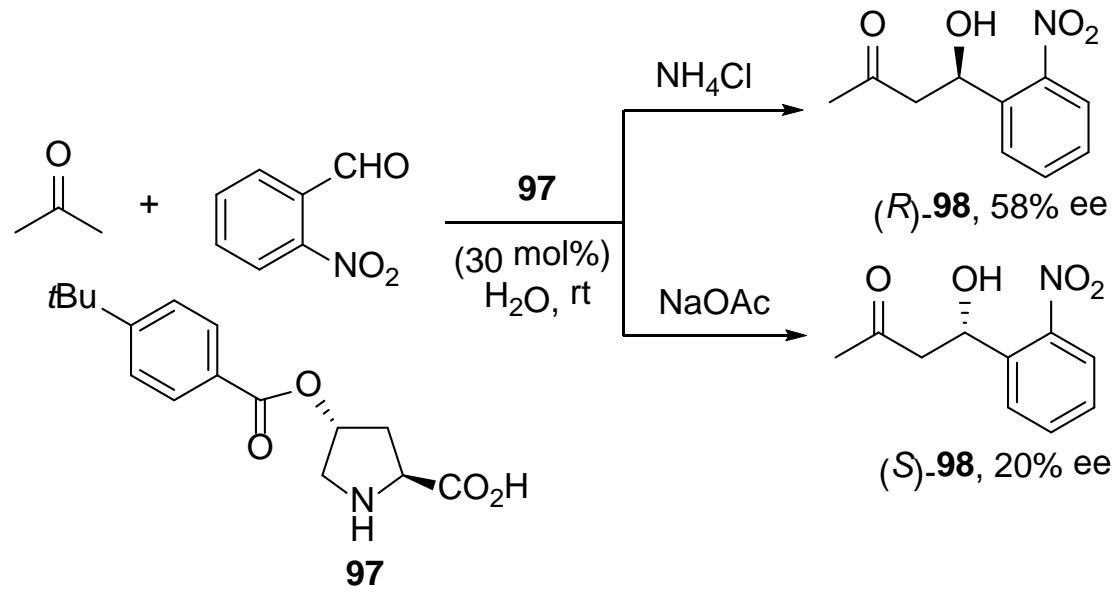

Substrate-dependent reversal of the enantioselectivity has been found in the aldol reaction of acetone with isatins 99 catalyzed by L-Pro. When isatin (99a) was used as acceptor, (S)-100a was obtained with ee up to 37\%, while 4,6-dibromoisatin (99b), gave $(R)$-convolutamydine A $[(R)-\mathbf{1 0 0 b}]$ in modest 35-55\% ee (Scheme 30). ${ }^{104-106}$ DFT and AIM calculations of the transition state supported that the product formation followed different pathways. In the case of isatin (99a), the $S$ enantiomer 100a was favored due to stereoelectronic effects. In contrast, 4,6-dibromoisatin (99b) furnished the expected product $(R)$-100b owing to a steric effect of the 4-bromo substituent. ${ }^{106}$

Scheme 30. Enantiodivergent Aldol Reaction of Acetone with Isatins 99 Catalyzed by L-Proline 


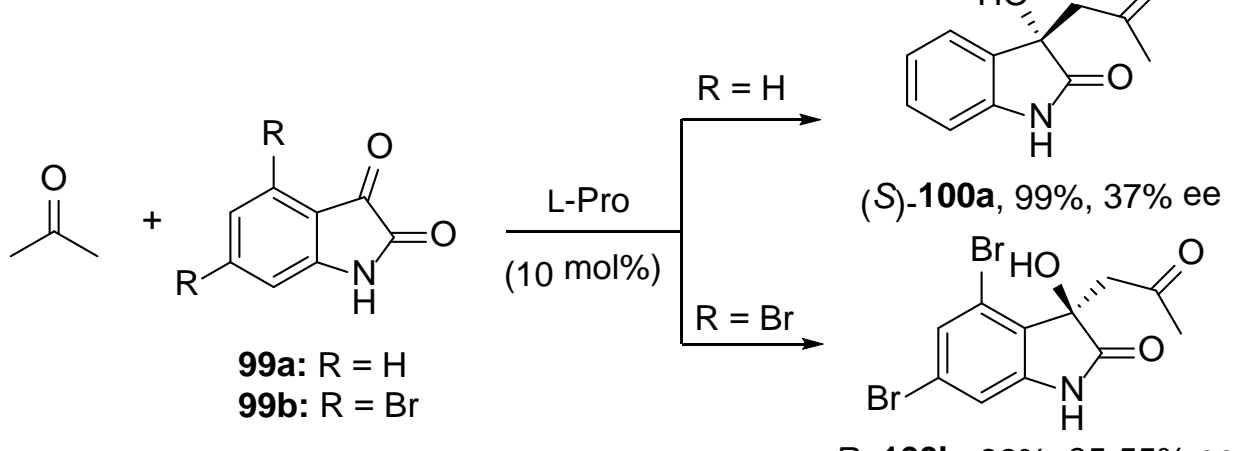

(R)-100b, 86\%, 35-55\% ee

Diastereodivergent aldol reaction of cyclohexanone with 4-nitrobenzaldehyde catalyzed by chiral diamine $\mathbf{1 0 1}$ has been observed when a carboxylic acid was used as additive. In the case of trifluoroacetic acid (TFA) a 86/14 anti/syn ratio was observed having the major anti-94 isomer a $\left(2 S, 1^{\prime} R\right)$ absolute configuration in $97 \%$ ee. In the presence of different dicarboxylic acids, eg succinic acid, the syn-isomer 94 with $\left(2 S, 1^{\prime} S\right)$ configuration was mainly isolated in $84 \%$ ee (Scheme 31). ${ }^{107}$

Scheme 31. Diastereodivergent Aldol Reaction of Cyclohexanone with 4Nitrobenzaldehyde Catalyzed by Diamine 101 in the Presence of Different Carboxylic Acids

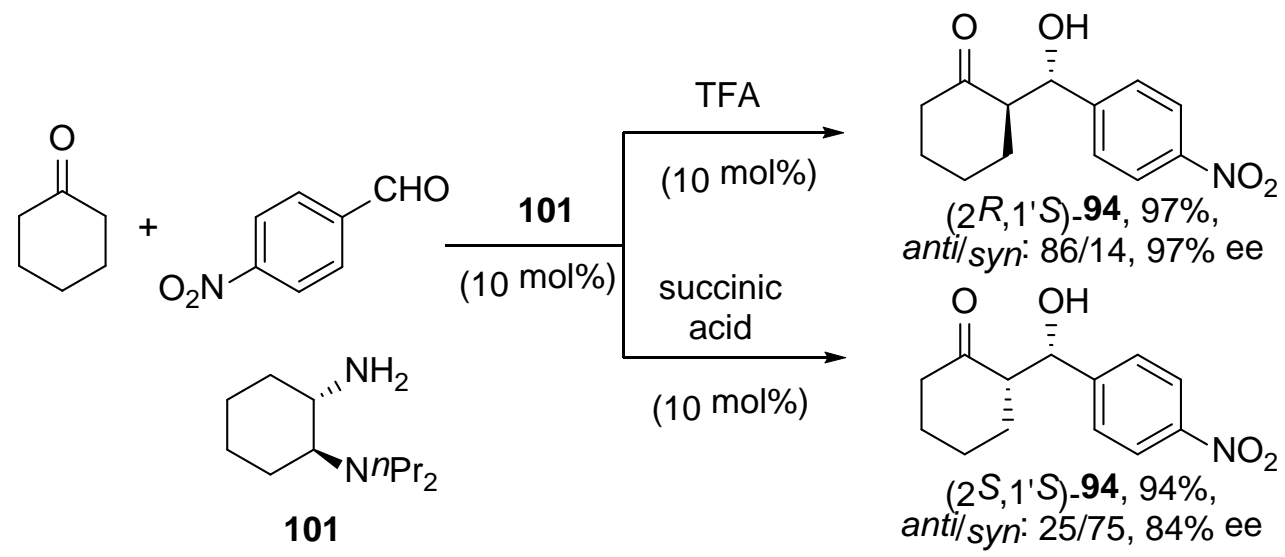

The influence of acids as additives in enantiodivergent organocatalyzed aldol reactions has been observed in the addition of ketones to $\alpha$-keto esters 102 catalyzed by 103. ${ }^{108}$ For instance, when this reaction was carried out in methanol with 102a, aldol syn-104a was formed with configuration $\left(2 R, 1^{\prime} R\right)$ in $94 \%$ ee. On the other hand, its enantiomer (2S,1'S)-104a was isolated in $88 \%$ ee using pentafluorobenzoic acid as additive in aqueous acetonitrile (Scheme 32). High enantioselectivities were achieved in both cases with other substrates, the first case being a synthetically useful enantiodivergent organocatalyzed aldol reaction. The resulting syn-aldols $\left(2 R, 1^{\prime} R\right)-104$ were obtained in high diastereoselectivities (20:1-23:1 syn:anti $\mathrm{dr})$ and enantioselectivities (87-95\% ee) and isomers (2S,1'S)-104 with dr up to 8:1 and 87-96\% ee. Based on the DFT calculations two transition states, 103A and 103B, have been proposed. In the absence of acid, the keto group of $\mathbf{1 0 2}$ is activated by hydrogen 
bonding with both $\mathrm{NH}$ groups according to transition state 103A. The enamine will attack from the back side to give $\left(2 R, 1^{\prime} R\right)$-syn-104a. In the presence of an acid as additive and water both carbonyl groups in the keto ester $\mathbf{1 0 2}$ are chelated by three hydrogen bonds. In the case of the keto group, the $\mathrm{NH}$ of the triflamide and the water molecule can be coordinated with the carboxy group. The carbonyl group of the ester will form a third hydrogen bond with the water molecule in the TS 103B.

Scheme 32. Enantiodivergent Aldol Reaction of Cyclohexanone with $\alpha$-Keto Ester 102a Organocatalyzed by 103 and Induced by an Achiral Acid

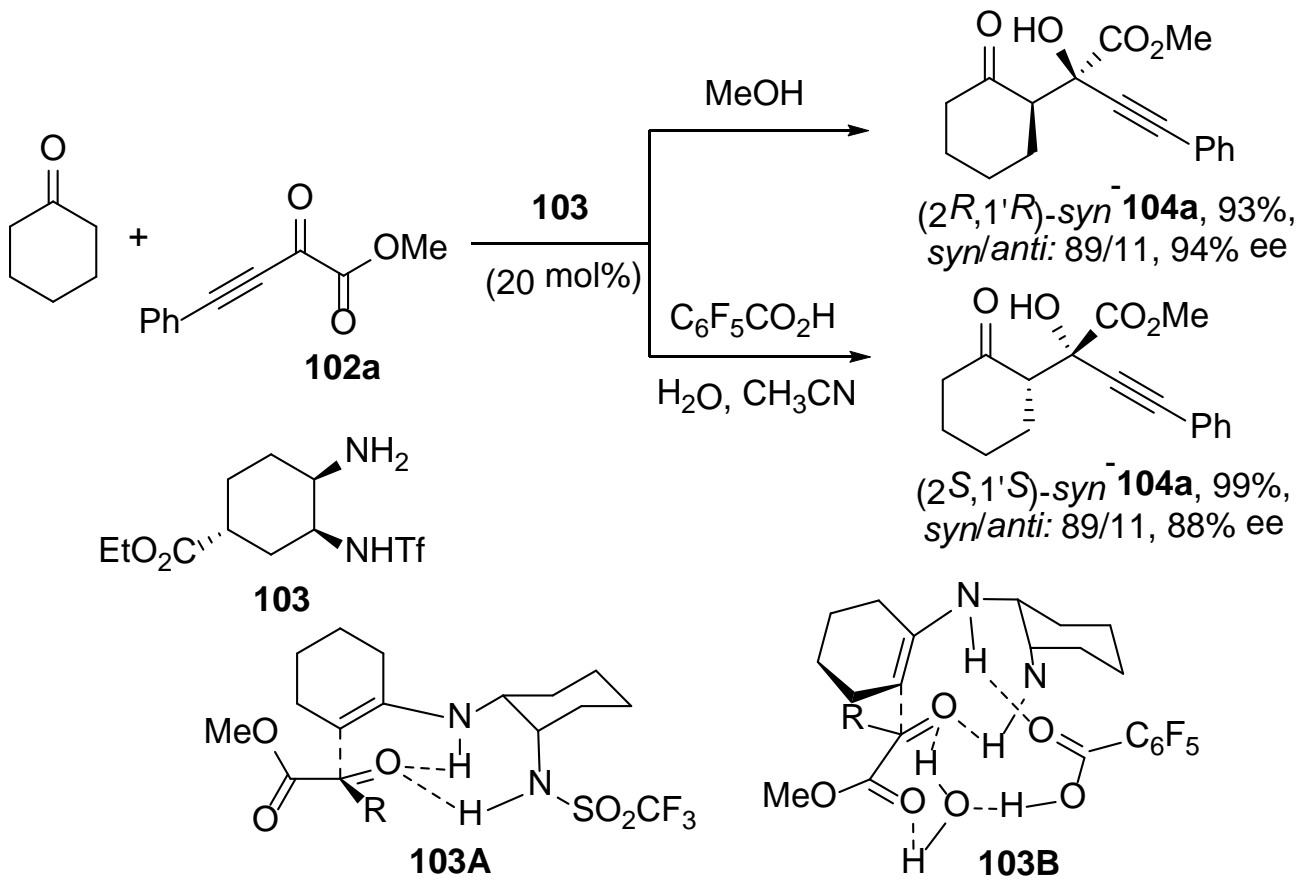

An interesting reversal of the enantioselectivity promoted by the solvent has been observed in the reaction of acetone with 4-nitrobenzaldehyde catalyzed by the $\beta$-turn tetrapeptide $105 .{ }^{109}$ Formation of the $(R)$-aldol 98 with ee up to $66 \%$ was observed in $\mathrm{MeOH}$, whereas in 1,2-dichloroethane (DCE) product (S)-98 was obtained in low 40\% ee (Scheme 33).

Scheme 33. Enantiodivergent Aldol Reaction for the Addition of Acetone to 4Nitrobenzaldehyde Catalyzed by Tetrapeptide 105 in Different Solvents 


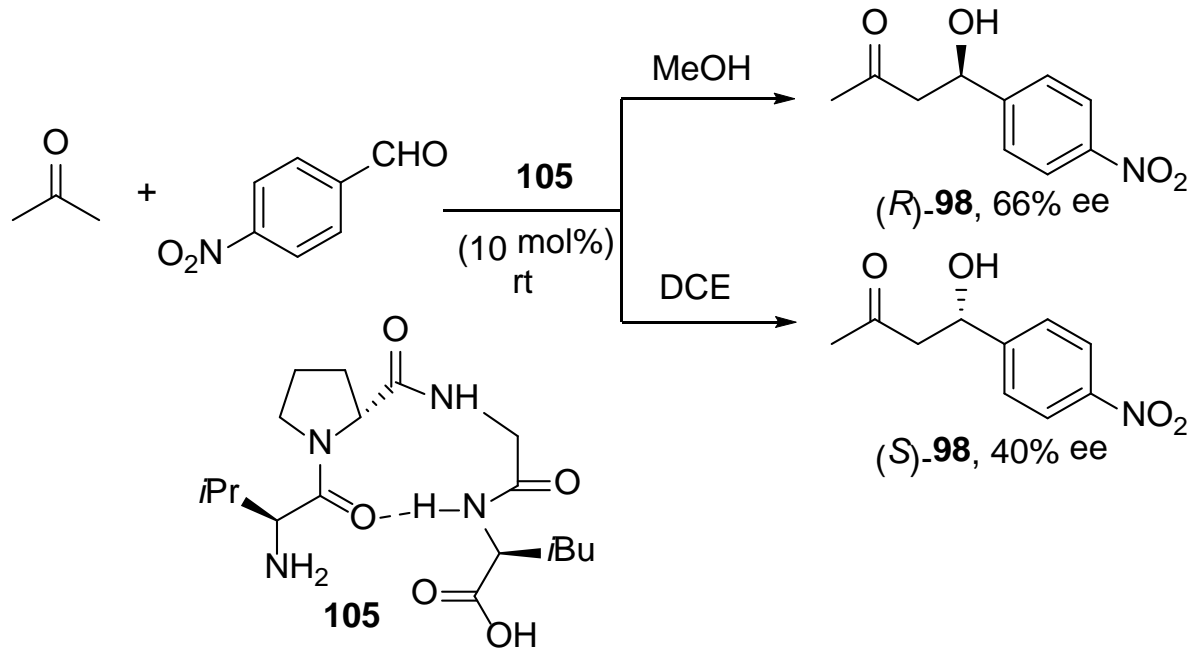

Modest values of reversal of enantioselectivities have been observed when either LPro or L-Pro-functionalized with the MOF $\mathrm{H}_{2} \mathrm{~N}-\mathrm{MIL}-101(\mathrm{Al})$ were used as catalysts for the aldol reaction of acetone with 4-nitrobenzaldehyde. ${ }^{110}$ Aldol (S)-98 was obtained with ee up to $28 \%$ in $63 \%$ yield, whereas L-Pro gave $(R)-98$ in $97 \%$ yield and 60\% ee.

Solvent-dependent enantiodivergent effect was observed in the proline-based peptide-catalyzed aldol reaction of cyclohexanone with 4-nitrobenzaldehyde. ${ }^{111}$ Thus, the peptide H-Pro-Pro-Asp- $\mathrm{NH}\left(\mathrm{CH}_{2}\right)_{11} \mathrm{CH}_{3}$ (106) catalyzed the formation of (2S,1'R)anti-94 in 55\% ee in aqueous DMSO or $\mathrm{MeOH}$, whereas (2R,1'S)-anti-94 was formed in pure DMSO or MeOH (Scheme 34). This stereochemical outcome has been attributed to the conformational differences of the organocatalyst in different solvents.

Scheme 34. Enantiodivergent Aldol Reaction for the Addition of Cyclohexanone to 4-Nitrobenzaldehyde Catalyzed by Tripeptide 106 in Different Solvents

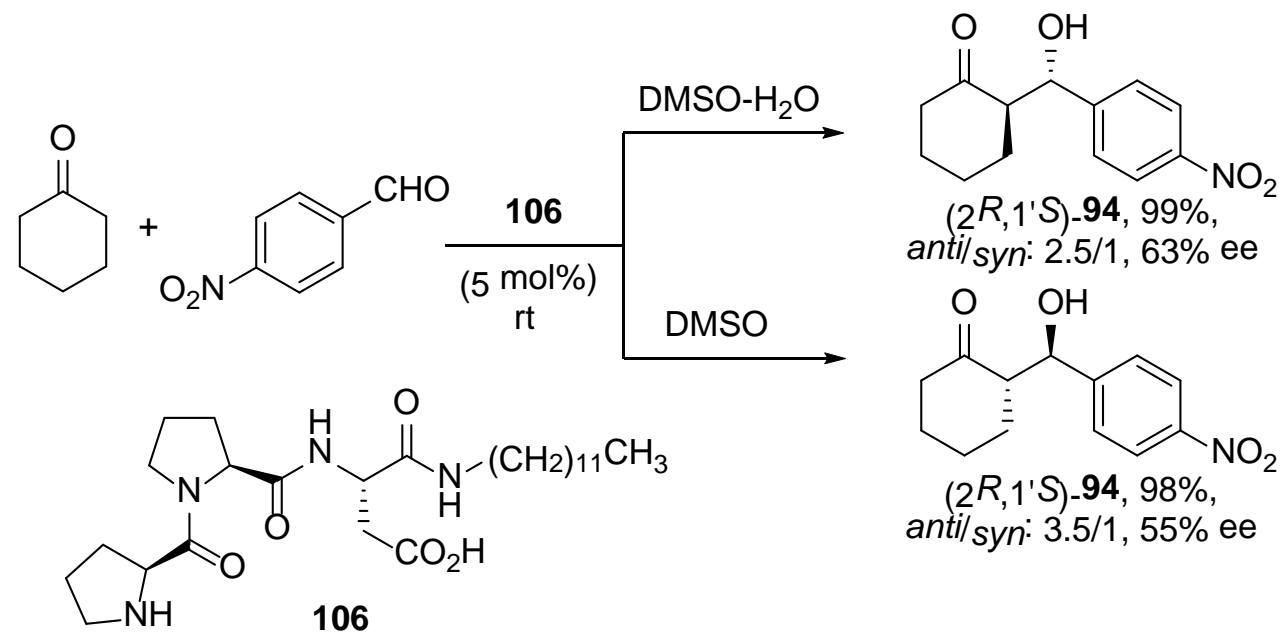

A diastereodivergent effect based on the counteranion of an achiral 1,5,7triazabicyclo[4.4.0]dec-5-ene (TBD) guanidinium salt 107 has been found in the L-Procatalyzed aldol reaction of cycloalkanones with aldehydes under solvent-free conditions. ${ }^{112,113}$ For instance, the tetraphenylborate salt 107a used as co-catalyst in the addition of cyclohexanone to 4-nitrobenzaldehyde gave the $\left(2 R, 1^{\prime} R\right)$-syn-aldol 94 and 
the tetrafluoroborate salt $\mathbf{1 0 7} \mathbf{b}$ afforded the (2R,1'S)-anti-94 in high ee (Scheme 35). ${ }^{113}$ The origin of this syn diasteroselectivity has been attributed to an equilibrium between the anti and the syn-diastereomers, which are the thermodynamically more stable compounds. The larger tetraphenylborate anion allows the syn-94 product to form hydrogen bonding with the guanidinium cation displacing the equilibrium to its formation.

Scheme 35. Diastereodivergent Aldol Reaction for the Addition of Cyclohexanone to Aldehydes Catalyzed by L-Pro and TBD Guanidinium Salts 107 as Additives

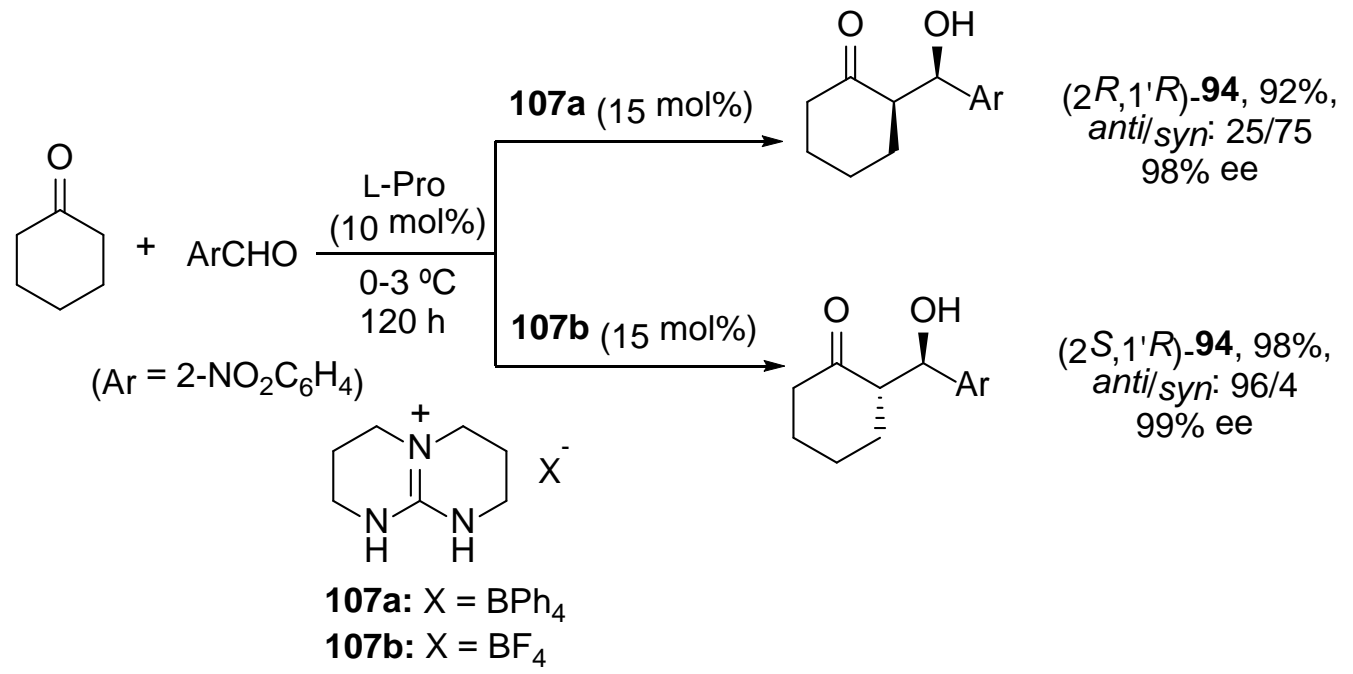

Enantiodivergent organocatalyzed aldol reactions between acetone and aldehydes have been recently performed by means of di- and tripeptides supported on a polystyrene resin (PS). The polystyrene-supported tripeptide H-Pro-Pro-Glu(OH)-PS (108) provided $(S)$-aldols 99 in 39-50\% ee and polystyrene-supported dipeptide H-ProGlu(OH)-PS (109), the corresponding enantiomers in 52-95\% ee. ${ }^{114}$ The conformation of intermediate adduct-models 108A and 109A are responsible for the observed asymmetric induction (Scheme 36). This methodology has been also implemented under continuous flow conditions. ${ }^{115}$

Scheme 36. Enantiodivergent Aldol Reaction Models for the Addition of Acetone to Aldehydes Catalyzed by Di- and Tri-peptides 108 and 109 


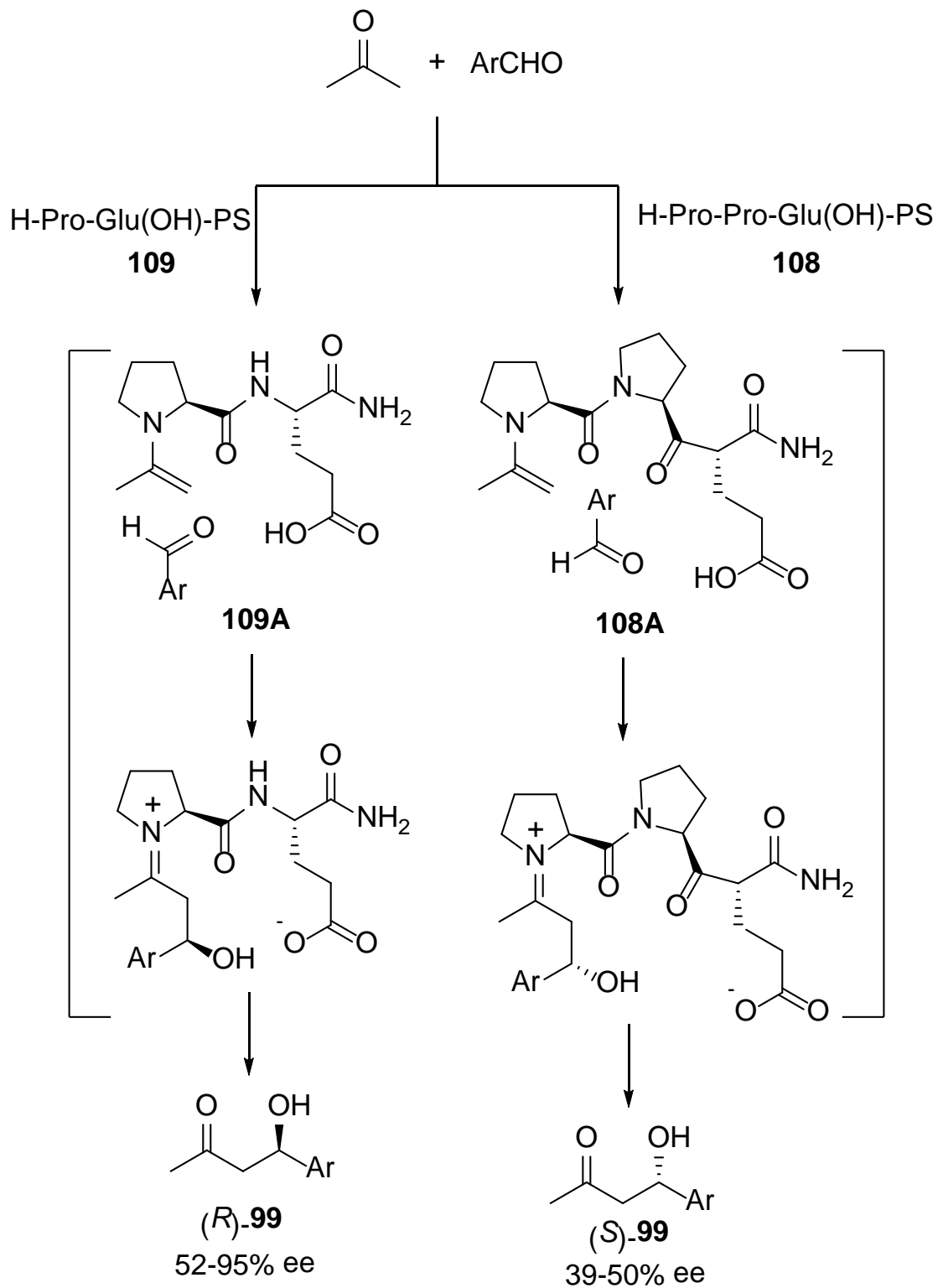

Denmark and co-workers described the diastereodivergent asymmetric aldol reaction of ketone trichlorosilyl enolates with aldehydes catalyzed by chiral phosphoramides 110. ${ }^{116-118}$ For example, the reaction of cyclohexanone trichlorosilyl enolate with benzaldehyde and $(S, S)$-110a as catalyst, gave the anti-aldol (2R,1'S)-94 in 97\% de and 92\% ee (Scheme 37). By using phosphoramide $(S, S)-\mathbf{1 1 0 b}$ as organocatalyst, aldol syn94 was isolated in $98 \%$ de and 51\% ee. The less demanding phosphoramide 110a binds in a 2:1 fashion in a chair-like approach $110 \mathrm{~A}$, whereas the sterically demanding $\mathbf{1 1 0 b}$ binds to the enolate in a boat-like disposition $\mathbf{1 1 0 B} .{ }^{117}$ The reaction of acetone trichlorosilyl enolate with benzaldehyde afforded excellent enantiodivergent results using the same catalyst 110a in different solvents. ${ }^{118}$ Aldol (S)-98 was obtained in dichloromethane in 92\% yield and 92\% ee, whereas in propionitrile $(R)-\mathbf{9 8}$ was formed in $88 \%$ yield and $90 \%$ ee.

Scheme 37. Diastereodivergent Aldol Reaction of Cyclohexanone Trichlorosilyl Enolate with Benzaldehyde Organocatalyzed by Chiral Phosphoramides 110 


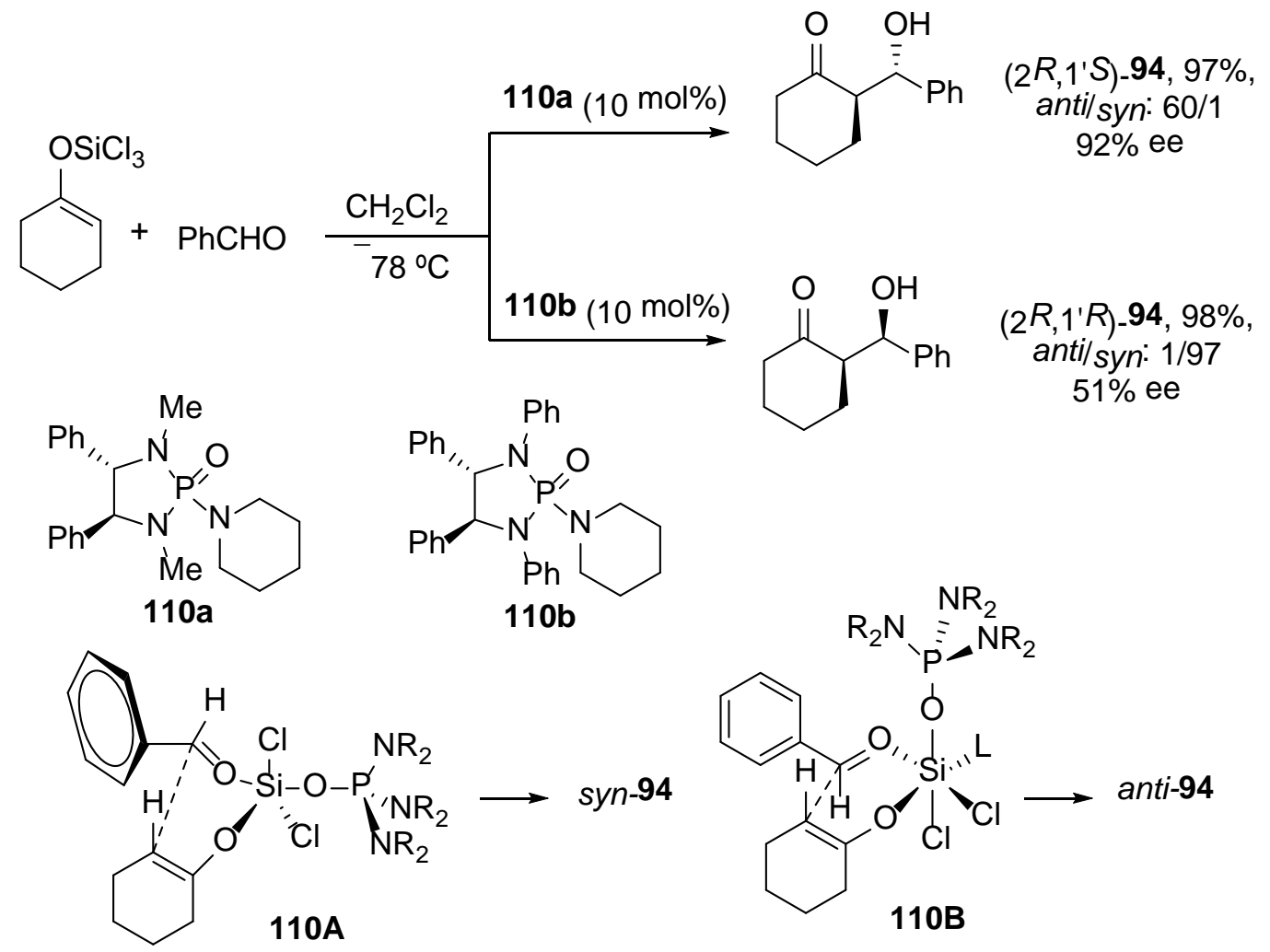

In conclusion, diastereodivergent Mukaiyama aldol reactions show syn- or antipreference by using different Lewis acids according to their non-chelation or chelation abilities, respectively. On the other hand, enantiodivergent Mukaiyama reactions can be performed using chiral complexes with different substituents in the ligand or with different metal salts, or even by changing the solvents. The enantiodivergent reaction of silylketene thioacetal derived from $\alpha$-hydroxythioacetate catalyzed by $\operatorname{Sn}(\mathrm{OTf})_{2}$ and diamines gave the best results. Direct enantiodivergent intermolecular aldol reactions have been mainly performed with chiral organocatalysts using different additives or solvents. For the diastereodivergent anti/syn processes of cycloalkanones-derived aldols, L-Pro and the presence of different guanidinium salts as additives gave the best results. For the enantiodivergent aldol reactions of cyclohexanone, its trichlorosilyl enolate organocatalyzed by different homochiral phosphoramides is the best methodology by far.

2.1.3. Nitroaldol Reactions. The so-called Henry reaction is a versatile carboncarbon bond forming reaction which can be metal- or organocatalyzed providing the corresponding synthetically useful nitroaldols. ${ }^{119-121}$ Stereodivergent nitroaldol reactions changing the metal center with the same ligand have been reported using chiral metal complexes. The addition of nitromethane to $\alpha$-keto esters catalyzed by $\mathrm{Zn}$ and $\mathrm{Cu}$ complexes and $C_{2}$-symmetric tridentate bis(oxazoline) and bis(thiazoline) as chiral ligands took place with reversal of enantioselectivity just by changing the metal salt. ${ }^{122,123}$ In the case of $\mathrm{Cu}(\mathrm{OTf})_{2}$ and ligand 111, products $(R)-\mathbf{1 1 2}$ were isolated with ee up to $60 \%$ and by changing $\mathrm{Cu}$ by $\mathrm{Zn}$, products $(S)-\mathbf{1 1 2}$ were obtained with ee up to 85\% (Scheme 38). 


\section{Scheme 38. Enantiodivergent Nitroaldol Reaction of Nitromethane with $\alpha$-Keto Esters Catalyzed by $\mathrm{Cu}(\mathrm{II})$ and Zn/111 Complexes}

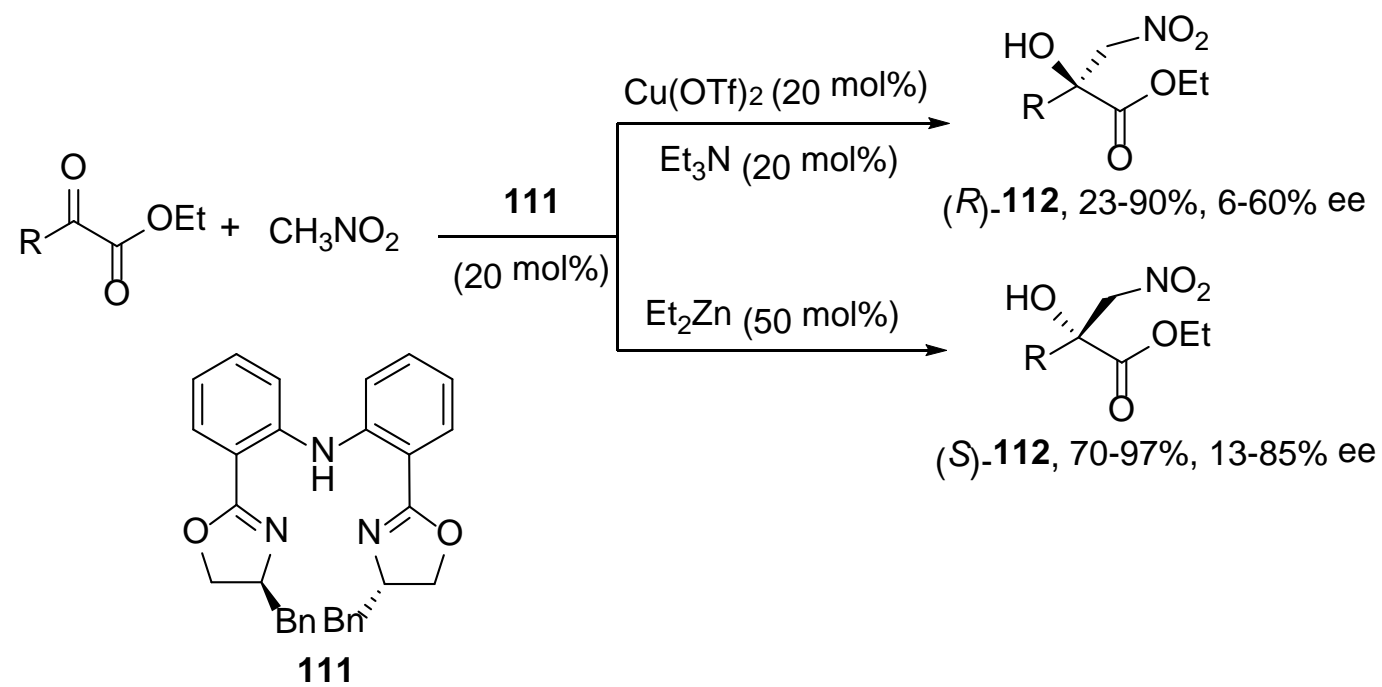

An aminoindanol-derived bis(oxazolidine) $\mathbf{1 1 3}$ is also an efficient chiral ligand for the metal-catalyzed enantiodivergent Henry reaction of nitromethane with aliphatic and aromatic aldehydes. Using $10 \mathrm{~mol} \%$ of CuOAc, (S)-nitroaldols 114 were obtained in 74-97\% ee, whereas with $\mathrm{Me}_{2} \mathrm{Zn}(R)$-enantiomers were mainly formed in 82-95\% ee (Scheme 39). ${ }^{124}$ When the brucine-derived amino alcohol 115 (Figure 7) was used as ligand of CuOAc, $(S)$-nitroaldols 114 were isolated in $90-97 \%$ ee. Similarly to the former case with $\mathrm{Zn}(\mathrm{OTf})_{2}$ as metal salt, compounds $(R)-\mathbf{1 1 4}$ were formed in $42-90 \%$ ee. ${ }^{125}$ When nitroethane was the nucleophile, almost equimolecular mixtures of antiand syn-nitroaldols were formed. Again, the absolute configuration of both diastereomers was opposite depending on the metal salt used.

Scheme 39. Enantiodivergent Nitroaldol Reaction of Nitromethane with Aldehydes Catalyzed by $\mathrm{Cu}(\mathrm{I})$ and $\mathrm{Zn} / 113$ Complexes

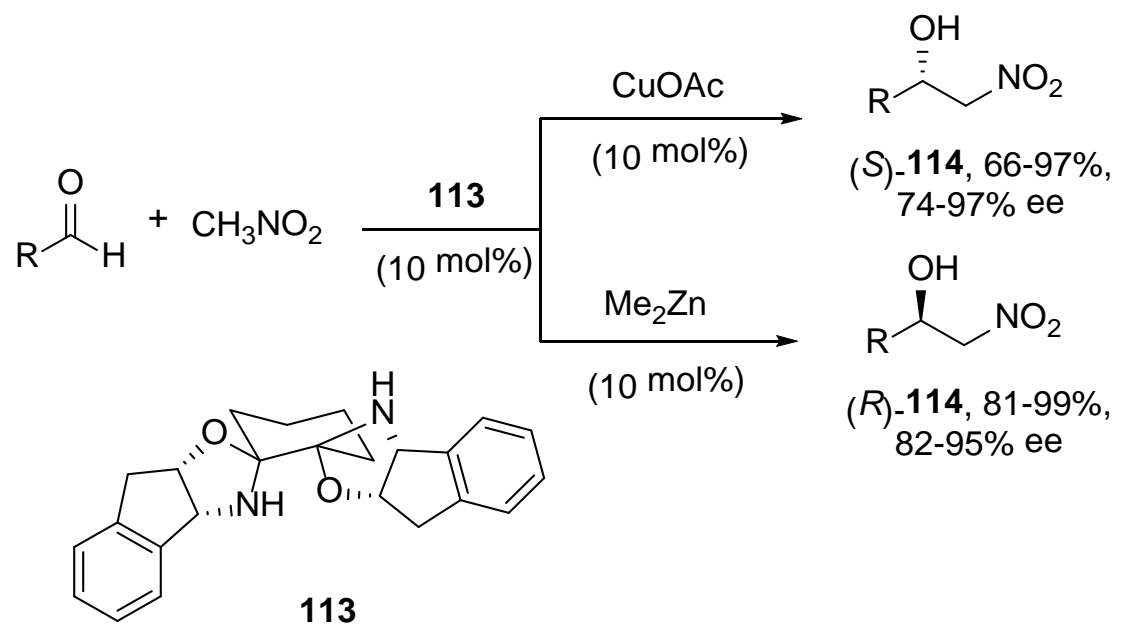

By means of $C_{2}$-symmetric salen ligands 116 (Figure 7) different metal salts have been screened in the enantiodivergent nitroaldol addition of nitromethane to 
aldehydes. ${ }^{126} \mathrm{Co}(\mathrm{OAc})_{2}$ complex gave $(S)$-nitroaldols 114 in $65-90 \%$ yields and $81-79 \%$ ee, and reversal of enantioselectivity was achieved with $\mathrm{Yb}(\mathrm{OiPr})_{3}$ derived complexes affording (R)-114 in 77-98\% yields and lower 10-87\% ee. Enantiodivergent Henry reaction has been described using the Co-salen derived from ligand $\mathbf{1 1 6}$ as catalyst and Co-tetrahydro-salen 117a or Co-salan 118 complexes (Figure 7), both bearing the same $(R, R)$-trans-cyclohexane-1,2-diamine chiral unit. ${ }^{127}$

Figure 7. Chiral Ligands Used in Enantiodivergent Nitroaldol Reactions of Nitromethane with Aldehydes Catalyzed by Metal Complexes

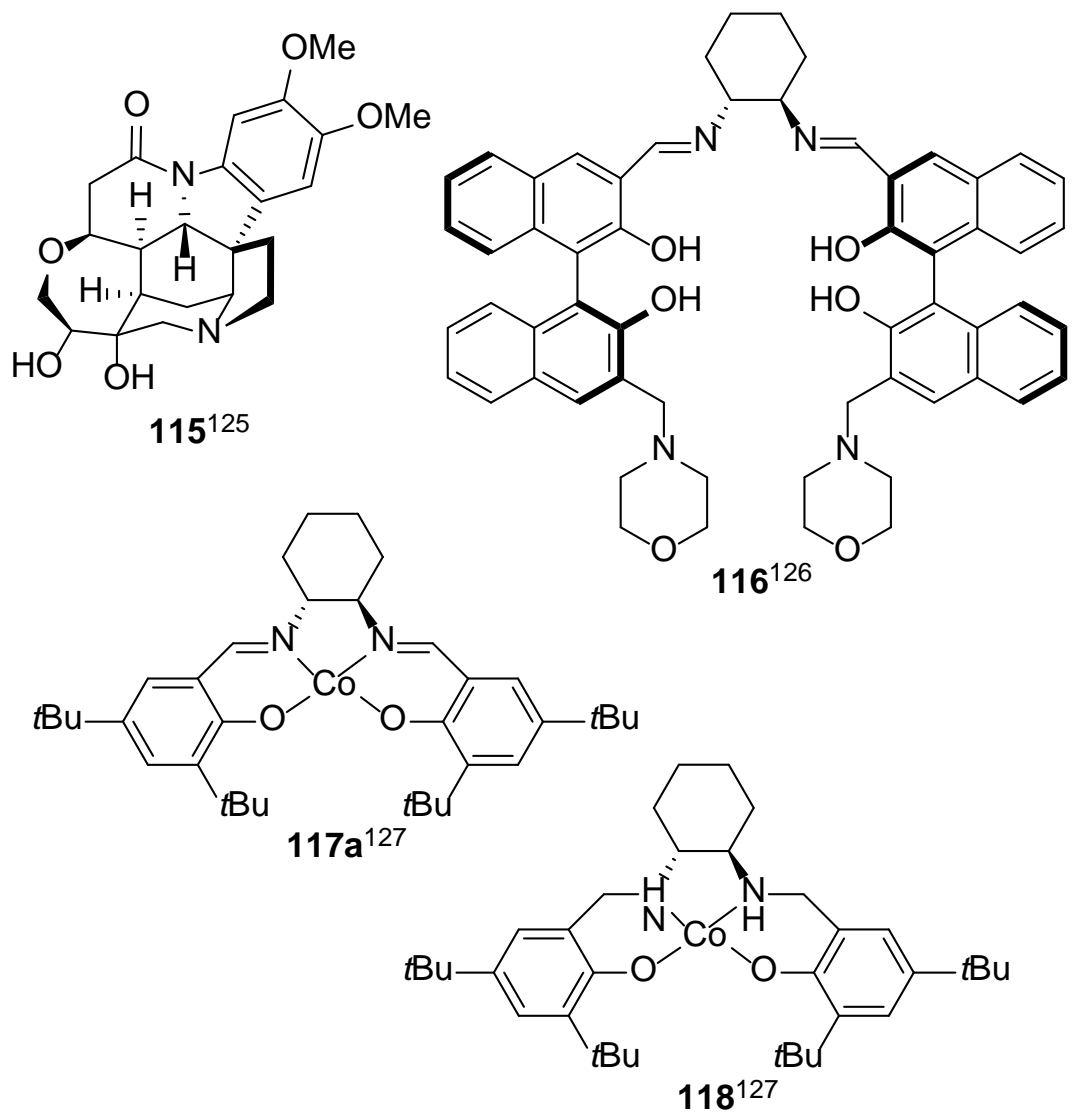

Diastereodivergent Henry reactions between $\alpha$-chiral aldehydes (X = OBn, NHPG), 2,3-O-isopropylidene-D-glyceraldehyde and Garner's aldehyde 9 (Section 2.1.1) with nitroacetaldehyde dimethylacetal can be carried out using two types of heterobimetallic complexes. The $\mathrm{LaLi}_{3}$ tris(binaphthoxide) complex 119 is acting as chiral Lewis acidBrønsted base bifunctional catalyst providing nitroaldol anti,syn-121 products. On the contrary, the bimetallic Pd-La-salen-based complex 120 gave syn,syn-121 nitroaldols (Scheme 40). ${ }^{128}$

Scheme 40. Diastereodivergent Nitroaldol Reaction of Nitroacetaldehyde Dimethyl Acetal with $\alpha$-Chiral Aldehydes Using Heterobimetallic Catalysts 119 and 120 


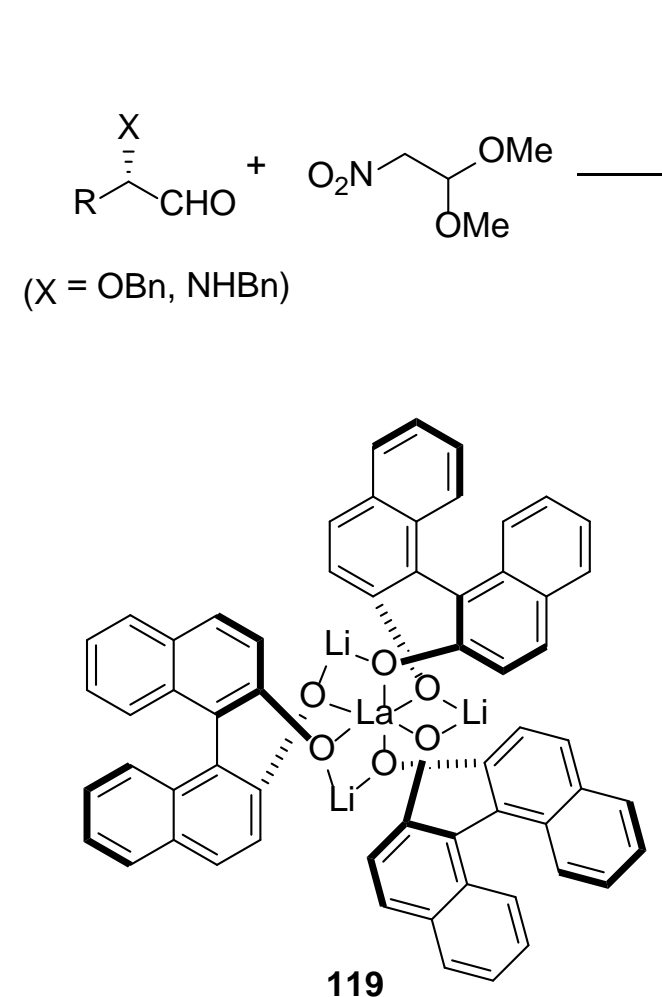

119

(5 mol\%)

120

(10 mol\%)<smiles>[R]C([X])C(O)C(C(OC)OC)[N+](=O)[O-]</smiles>

anti,syn ${ }^{-121}, 55-98 \%$, $86-90 \%$ ee<smiles>[R]C([X])C(O)[C@H](OC)C(OC)O[Na]</smiles>

syn, syn ${ }^{-121}, 70-87 \%$, $66-82 \%$ ee

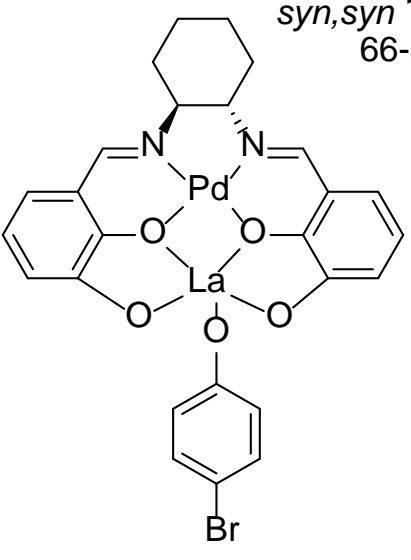

120

In conclusion, diastereo- and enantiodivergent nitroaldol reactions have been modulated by changing the metal in the chiral metal complex.

2.1.4. Addition of Other Nucleophiles. Cyanosilylation of aldehydes by means of trimethylsilyl cyanide under metal catalysis gave the reversal of enantioselectivity in the case of using Schiff bases 122 and 123 as chiral ligands. Both ligands derived from (S)valinol, and their corresponding $\mathrm{Ti}(\mathrm{OiPr})_{4}$ complexes $^{129-131}$ afforded enantiodivergent cyanosilylation in the case of benzaldehyde. (S)-Cyanohydrin 124 was obtained by using ligand 122 only in 22\% ee, whereas $(R)-\mathbf{1 2 4}$ was isolated by means of the $\mathrm{Ti}$ complex derived from ligand 123 in higher 85\% ee (Scheme 41). ${ }^{131}$

Scheme 41. Enantiodivergent Cyanosilylation of Benzaldehyde Catalyzed by Ti Schiff Bases 122 and 123 Complexes 


$$
\mathrm{PhCHO}+\mathrm{Me}_{3} \mathrm{SiCN} \frac{\mathrm{Ti(OiPr})_{4}(20 \mathrm{~mol} \%)}{-80^{\circ} \mathrm{C}} \stackrel{\text { (20 mol\%) }}{\longrightarrow} \stackrel{\mathrm{HCl}}{\longrightarrow}
$$

Shibasaki and co-workers discovered a reversal of the enantioselectivity during the cyanosilylation of ketones devoted to the synthesis of an intermediate for the synthesis of the (20S)-camptothecin family. ${ }^{132}$ In the cyanosilylation of ketones using chiral ligand 125a or 125b, by changing the central atom from $\mathrm{Ti}^{133,134}$ to Gd or Sm, reversal of enantioselectivity was observed (Scheme 42). Titanium complexes with ligands 125 provided $(R)$-cyanohydrins 126 by the mechanism proposed through a TS 125A and lanthanides complex gave (S)-cyanohydrins 126 by an intermediacy of TS 125B. According to ${ }^{1} \mathrm{H}$ NMR and EIS-MS analyses, the Ln complexes are binuclear systems in which the more electron-rich lanthanides activate the cyanide and the other atom is acting as Lewis acid coordinating to the aldehyde. For the synthesis of the intermediate 127 of (20S)-camptothecin a screening of the appropriate catalyst, was needed, the best being $\mathrm{Gd}(\mathrm{OiPr})_{3}$ and ligand 125a in a 2:1 molar ratio.

Scheme 42. Enantiodivergent Cyanosilylation of Ketones Catalyzed by Phosphine Oxide 125/Metal Complexes 


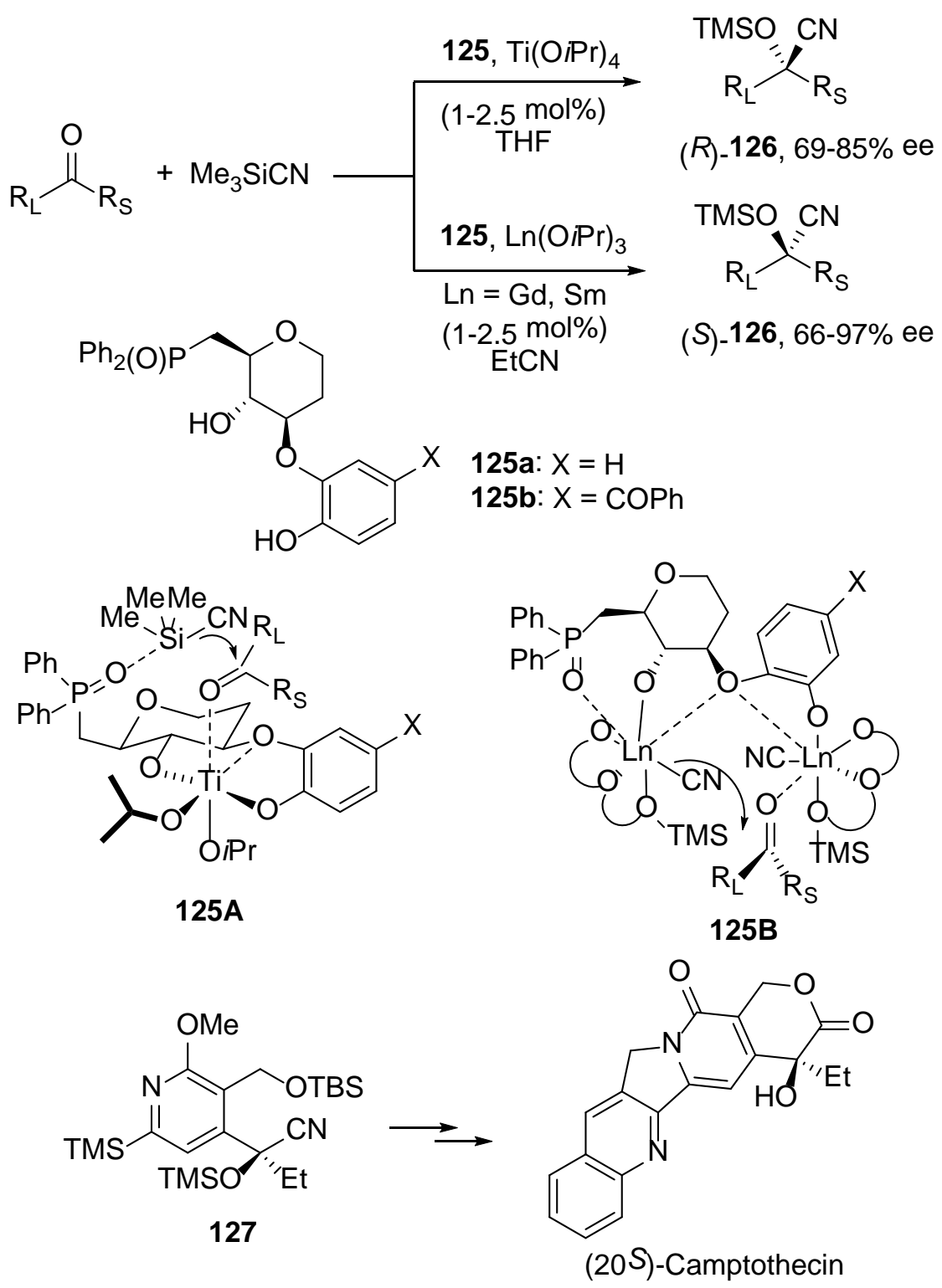

In the hydrophosphonylation of aldehydes mediated by Lewis acids, diastereodivergent results have been found to be dependent on the hydrophosphonylating reagents. The reaction of $\alpha$-dibenzylamino- $\beta$-phenylpropanal 128 with diethyl dimethyl (tert-butyl)silyl phosphite under Ti-mediated conditions gave anti-129 in more than $96 \%$ de according to a non-chelated model. On the other hand, the addition of a less reactive nucleophile such as diethyl phosphite to $\mathbf{1 2 8}$ provided the diastereoselective formation of syn-129 through a chelated model (Scheme 43). ${ }^{135}$

Scheme 43. Diastereodivergent Hydrophosphonylation of $\alpha$-Dibenzylamino- $\beta$ phenylpropanal 128 Mediated by $\mathrm{TiCl}_{4}$ 


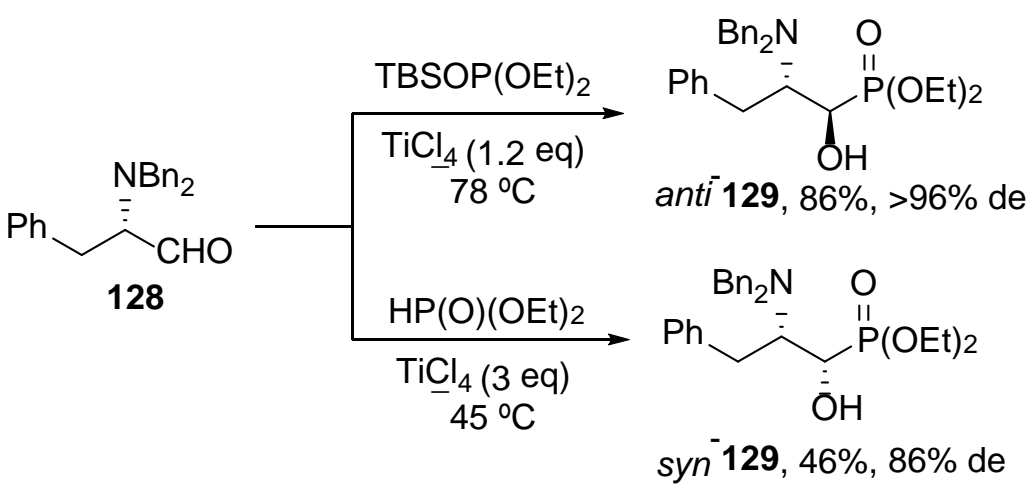

Diastereodivergent hydrophosphinylation of $\alpha$-amino aldehydes 128 has been performed by the addition of ethyl phosphinate using enantiomeric aluminum lithium bis(binaphthoxide) (ALB) 130. When (S)-ALB was employed as catalyst anti-131 products were diastereoselectively formed, whereas (R)-ALB $\mathbf{1 3 0}$ gave the corresponding syn- $\beta$-amino- $\alpha$-hydroxy- $H$-phosphinates 131 (Scheme 44$){ }^{136}$

Scheme 44. Diastereodivergent Hydrophosphinylation of $\alpha$-Amino Aldehydes 128 with Ethyl Phosphinate Catalyzed by Chiral AlLiBis(binaphthoxide) 130

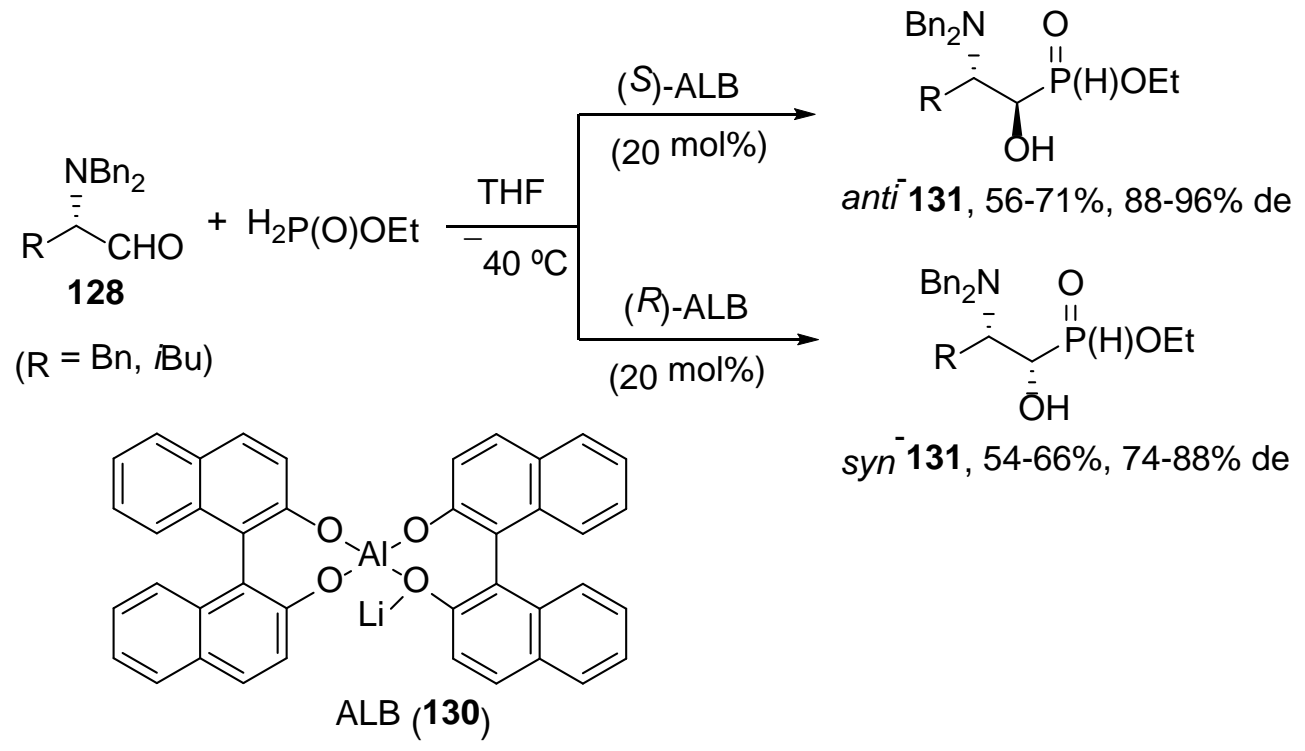

One example on a moderate enantiodivergent hydrophosphonylation has been described using $10 \mathrm{~mol} \%$ of ligand 122 (Scheme 41) and different aluminum Lewis acids. ${ }^{137}$ The addition of diethyl phosphite to benzaldehyde catalyzed by $\mathbf{1 2 2}$ and $\mathrm{Et}_{2} \mathrm{AlCl}$ gave adduct $(S)-\mathbf{1 3 2}$ in $\mathbf{8 6 \%}$ ee, whereas in the presence of $\mathrm{Al}(\mathrm{OiPr})_{3}$ the enantiomeric ( $R$ )-132 was obtained in low $30 \%$ ee (Scheme 45). This type of stereocontrol can be classified using changes in the metallic precursor for the catalytic complex.

Scheme 45. Enantiodivergent Hydrophosphonylation of Benzaldehyde with Diethyl Phosphite Catalyzed by Al/122 Lewis Acids 


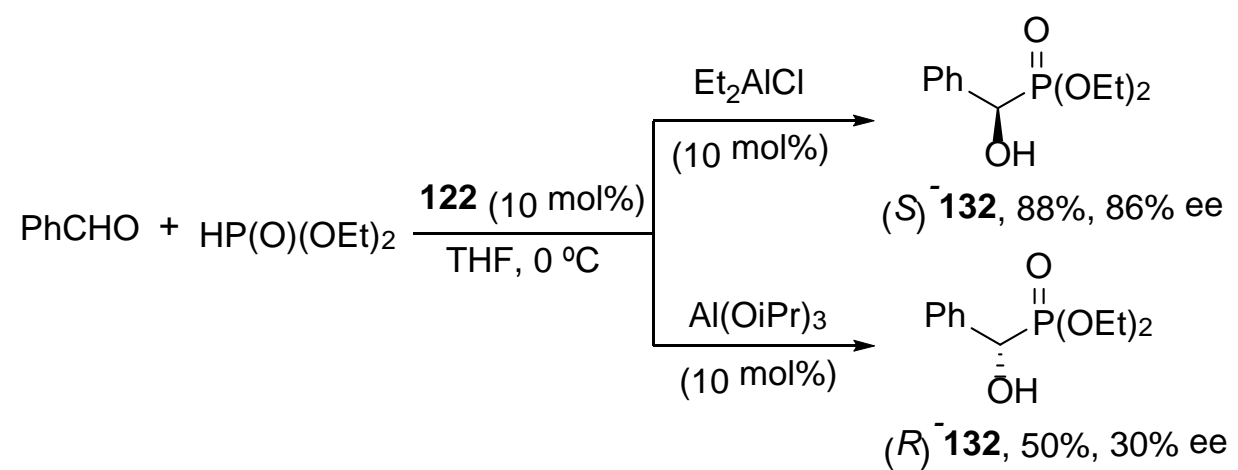

Diastereodivergent Morita-Baylis-Hillman (MBH) reactions have been observed in the case of chiral acryloylhydrazide 133 and aldehydes catalyzed by 1,8diazabicyclo[5.4.0] undec-7-ene (DABCO) depending on the solvent employed. ${ }^{138}$ When the reaction was carried out in DMSO, adducts $\mathbf{1 3 4}$ were formed and in aqueous THF diastereomeric products 135 were obtained, both with high diastereoselectivities (Scheme 46).

Scheme 46. Diastereodivergent Morita-Baylis-Hillman Reaction of Acryloylhydrazide 133 with Aldehydes

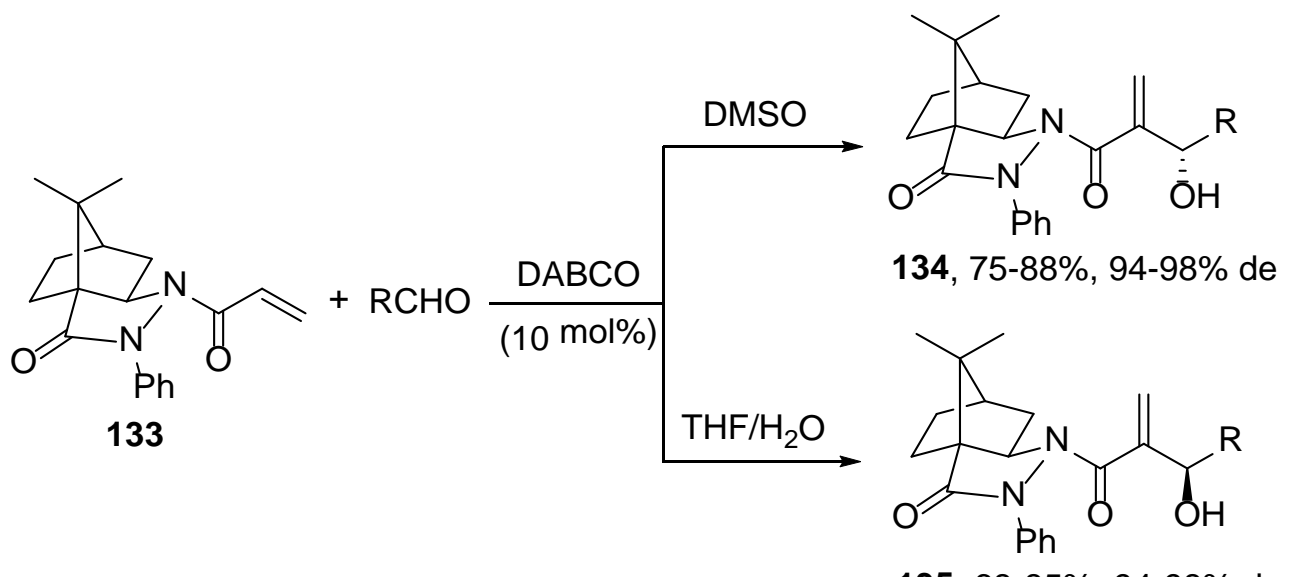

Recently, a switch of diastereoselectivity has been reported by Gravel and coworkers in the cross-benzoin or cross-acyloin reaction catalyzed by an N-heterocyclic carbene (NHC) derived from 137. ${ }^{139,140}$ Substrate-controlled diastereodivergent results were observed for the reaction of aldehydes with $\alpha$-amino aldehydes 136 . In the case of 136a (NHBoc), anti-selective cross-benzoin reaction took place giving $\alpha$ hydroxyketones 138 with de up to $90 \%$ (Scheme 47). ${ }^{139}$ On the other hand, when $\alpha$ amino aldehydes 136b (NBnBoc) were used, syn-selectivity was observed affording products 139 with de up to $82 \% .{ }^{140}$ The observed diastereoselectivity was rationalized using a Cram-chelate model $\mathbf{1 3 8} \mathbf{A}^{139}$ in the first case and a polar Felkin-Anh TS model 139A ${ }^{140}$ for the last case.

Scheme 47. Diastereodivergent Cross-Benzoin Reaction of Different $N$-Substituted $\alpha$-Amino Aldehydes 136 Catalyzed by the NHC Derived from 137 


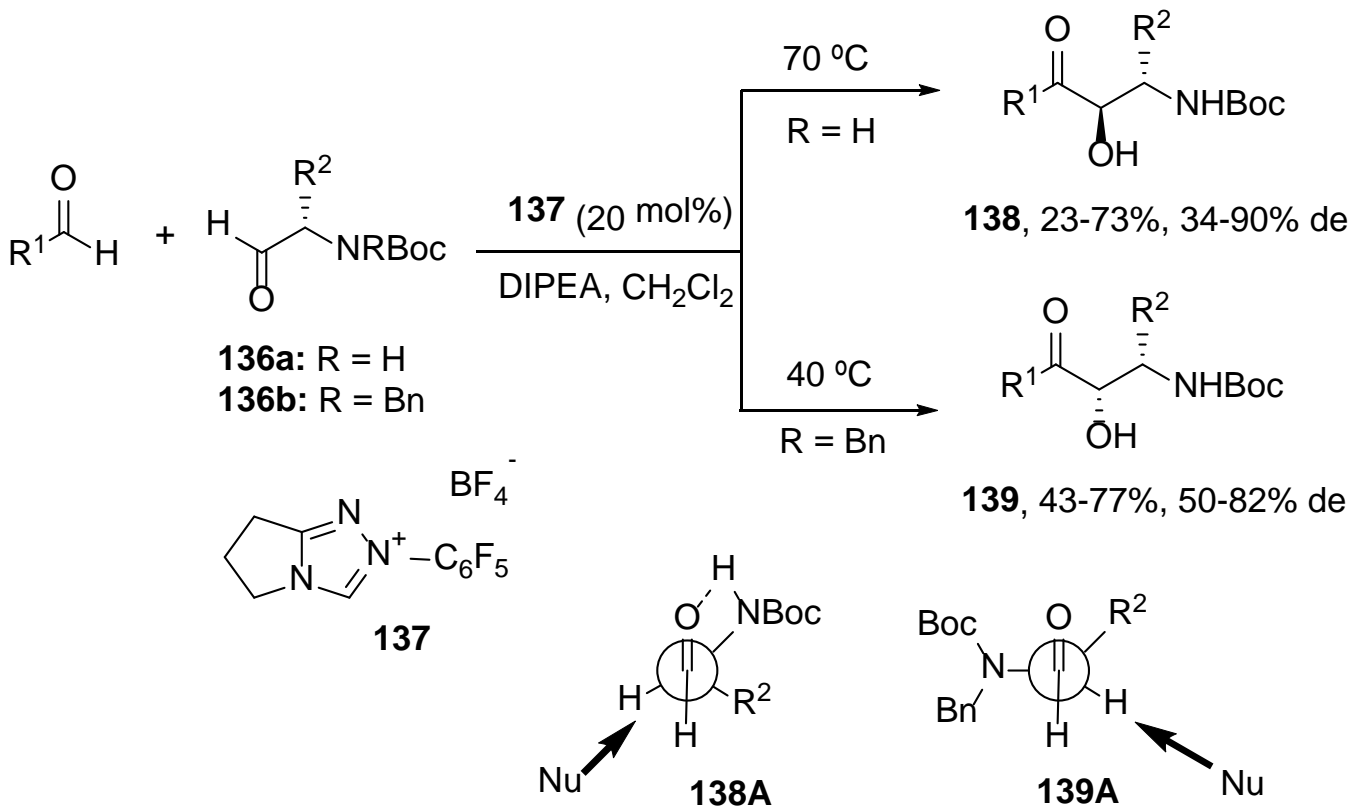

In conclusion, from the few examples described in this section, the addition of different nucleophiles to carbonyl compounds, can be carried out under enantiodivergent conditions changing the ligand (for aldehydes) or the metal of the chiral metal complexes (for ketones) in the case of the cyanosilylation. Hydrophosphonylation and hydrophosphinylation of aldehydes occurred with the inversion of enantioselectivity by changing the nature of the aluminum Lewis acid. In the case of the cross-benzoin reaction substrate-dependent diastereodivergent effects were observed.

2.1.5. Reduction of Ketones. The asymmetric reduction of ketones is one of the most important strategies for the synthesis of enantioenriched secondary alcohols. ${ }^{141}$ In this section enantiodivergent methodologies such as hydride addition, catalytic hydrogenation, transition metal-catalyzed hydrogen transfer and enzyme-catalyzed reductions are considered.

2.1.5.1. Addition of Hydrides. The reduction of ketones with hydrides can be stereocontrolled by chelation and non-chelation modes. This methodology has been widely used in diastereodivergent processes based on the use of different types of reagents and has been applied to the synthesis of numerous biologically active molecules and natural products. Catalytic sterodivergent reductions by hydride addition have been performed with borane and substoichiometric amounts of chiral ligands and also by metal-catalyzed hydrosilylation.

Control of the enantiodivergence by different substitution in the catalyst has been achieved in the addition of borane to ketones using chiral ligands 140 and 141, prepared from the same chiral source, the $(S)$-indoline-2-carboxylic acid. ${ }^{142}$ Secondary alcohols with $(R)$-configuration were enantioselectively obtained when $5 \mathrm{~mol} \%$ of amino alcohol 140 was used as ligand for borane (Scheme 48). On the contrary, a reversal of enantioselectivity resulted when ligand 141 was employed. These amino alcohols 
formed in situ the corresponding oxazaborolidines by reaction with borane. The presence of two phenyl groups on the alcohol 140 promotes the formation of TS 140A by steric effects. On the other hand, the steric effects of the cyclohexyl group cause the formation of TS 141A explaining the observed enantiofacial discrimination.

Scheme 48. Enantiodivergent Reduction of Ketones with Borane Catalyzed by Oxazaborolidines from Amino Alcohols 140 and 141

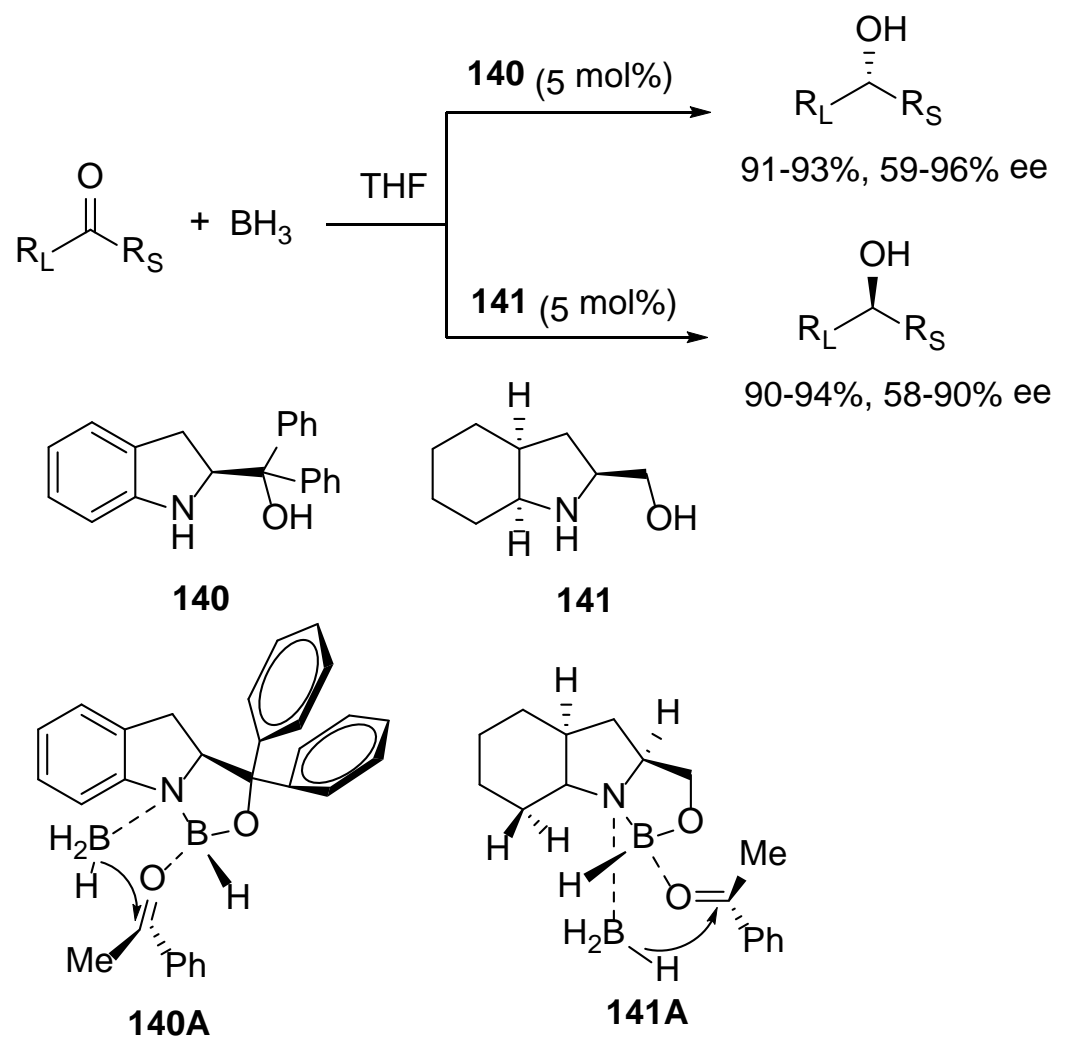

Asymmetric hydrosilylation of prochiral ketones catalyzed by chiral metal complexes has been found to be an enantiodivergent strategy controlled by the metal center for the synthesis of enantiomeric alcohols. ${ }^{143-145}$ In 1989, Kreuzfeld and coworkers reported the first enantiodivergent hydrosilylation of acetophenone using $\mathrm{Pt}(\mathrm{COD})_{2}$ or $[\mathrm{Rh}(\mathrm{COD}) \mathrm{Cl}]_{2}$ in the presence of a chiral N,P-type ligand with moderate $27 \%$ ee for $R$ alcohol and $51 \%$ ee for the $S$ enantiomer. ${ }^{146}$ Another enantiodivergent hydrosilylation was later described by Faller and Chase using a tridentate ligand and either $[\mathrm{Rh}(\mathrm{COD}) \mathrm{Cl}]_{2}$ or $[\mathrm{Ir}(\mathrm{COD}) \mathrm{Cl}]_{2}$ as metal complexes achieving ee up to $62 \%{ }^{147}$ Efficient enantiodivergent hydrosilylation was described by Uemura and co-workers with the above mentioned metal complexes and the chiral ligand $[(S, S, S)$-DIPOF] 142 with ee up to $91 \%$ for the $(R)$ and $96 \%$ for the $(S)$-1-phenylethanol (Scheme 49$).{ }^{148}$

Scheme 49. Enantiodivergent Hydrosilylation of Acetophenone Catalyzed by Rh and Ir Complexes Using Chiral Ligand 142 


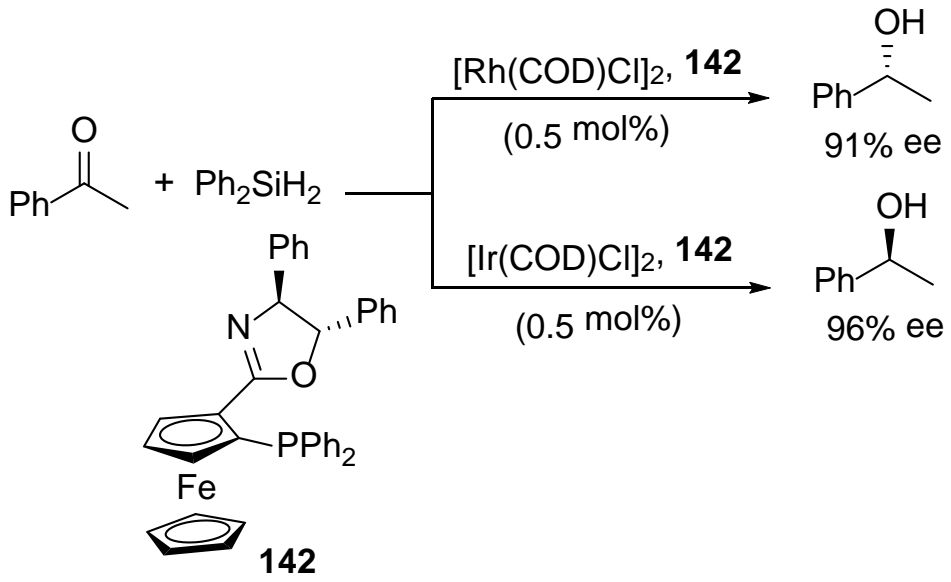

Subsequent studies by Frölander and Moberg in enantiodivergent hydrosilylation reactions, showed that the PHOX ligand 143, with a suitably located hydroxy function, and a Rh complex led to an enhancement of the enantioselectivity. ${ }^{149}$ The presence of $\mathrm{AgBF}_{4}$ increases the enantioselectivity, for instance for $(R)-1$-phenylethanol from $75 \%$ to $95 \%$ ee. By changing Rh by Ir (S)-1-phenylethanol was formed in $78 \%$ ee (Scheme $50)$.

Scheme 50. Enantiodivergent Hydrosilylation of Ketones Catalyzed by Rh and Ir Complexes and Chiral Ligand 143

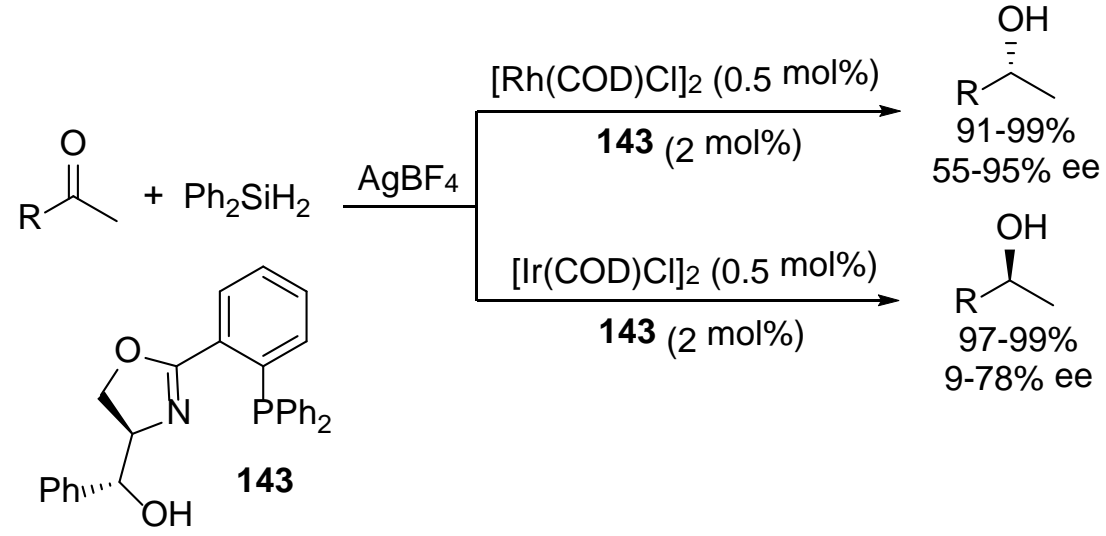

Asymmetric Fe-catalyzed hydrosilylation of ketones by $(\mathrm{EtO})_{2} \mathrm{MeSiH}$ using ligand 144 and $\mathrm{Fe}(\mathrm{OAc})_{2}$ or complex 145 and $(\mathrm{EtO})_{3} \mathrm{SiH}$, bearing the same chiral ligand, in the presence of subtoichiometric amounts of $\mathrm{Zn}$ metal afforded $R$ or $S$ alcohols, respectively (Scheme 51). ${ }^{150}$ In the presence of $\mathrm{Zn}$, reduction of $\mathrm{Fe}$ (III) to $\mathrm{Fe}$ (II) with the formation of a high-spin Fe(II) complex was postulated. However, the reaction mechanism for this enantiodivergent hydrosilylation has not been clarified. In general, excellent yields and moderate to high enantioselectivities were obtained for a wide variety of acetophenones.

Scheme 51. Enantiodivergent Hydrosilylation of Ketones Catalyzed by Fe Complexes with or without Zn 


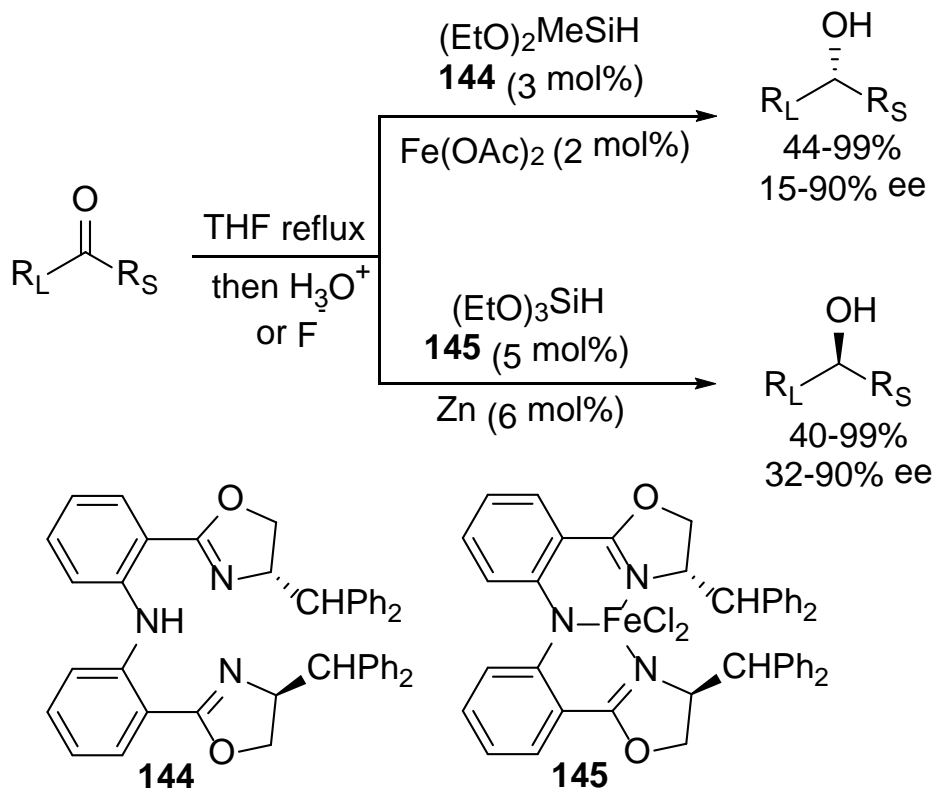

Asymmetric hydrosilylation of aryl ketones with $(\mathrm{EtO})_{3} \mathrm{SiH}$ catalyzed by the iridium complex derived from the N-heterocyclic carbene (NHC) 146 and $[\operatorname{Ir}(\mathrm{COD}) \mathrm{Cl}]_{2}$ at rt in Me-THF gave mainly $S$ alcohols in 68-92\% ee. ${ }^{151}$ Interestingly, an unexpected inversion of the enantioselectivity was observed by the same group in the Ir-catalyzed transfer hydrogenation (see, Section 2.2.5.3) of acetophenone when [ $\operatorname{IrCp} * \mathrm{Cl}_{2} \cdot \mathbf{1 4 6}$ ] was used as catalyst and isopropyl alcohol (IPA) as hydrogen source affording $(R)$-1-phenylethanol in $60 \%$ ee (Scheme 52). ${ }^{152}$

Scheme 52. Enantiodivergent Hydrosilylation of Ketones Catalyzed by Ir Complexes
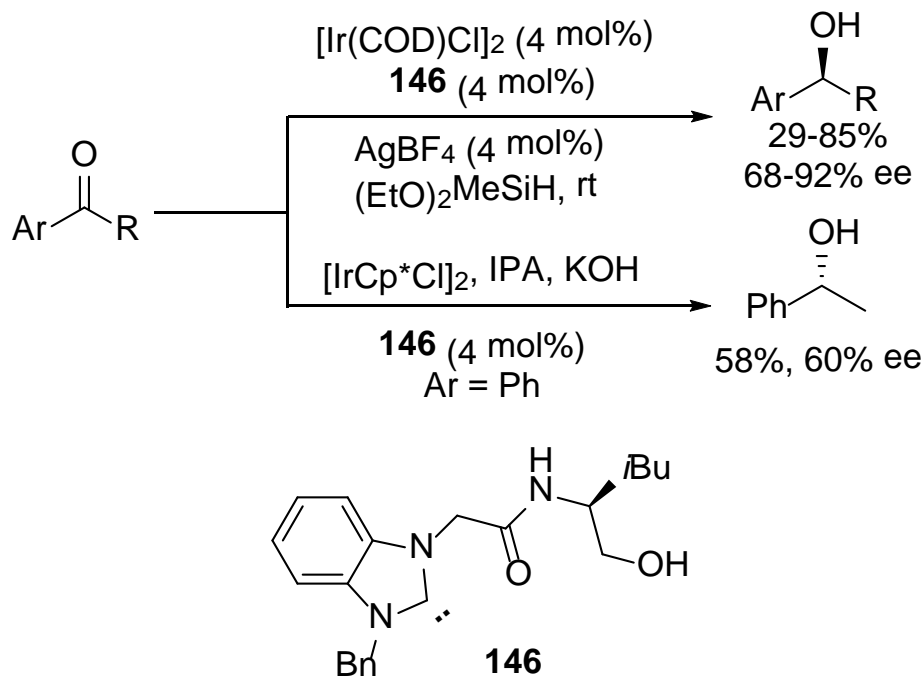

2.1.5.2. Hydrogenation. Homogeneous asymmetric hydrogenation of $\beta$-keto esters and related substrates was first developed by Noyori and co-workers using a Ru(II) complex bearing a chiral diphosphine. ${ }^{7,153,154}$ However, only under heterogeneous conditions enantiodivergent results have been reported. Catalytic asymmetric hydrogenation of ketones under heterogeneous conditions has been performed mainly 
by means of supported Pt and Cinchona alkaloids (Orito reaction). ${ }^{6,153,154}$ In the case of the Orito reaction, Pt-cinchonine (CN) or quinidine (QD) gave ethyl (S)-lactate in the reduction of ethyl pyruvate, and Pt-cinchonidine (CD) or quinine (QN) afforded the corresponding $(R)$-enantiomer according to the pseudoenantiomeric nature of these alkaloids. Therefore, changes of these chiral modifiers should not be considered strictly as enantiodivergent processes.

During the hydrogenation of pyruvate esters in the presence of cinchonidine (CD), (R)-lactate was obtained on $\mathrm{Pt} / \mathrm{SiO}_{2}$ with ee up to $70 \%$. On the other hand, the $S$ isomer was isolated by using oxide-supported palladium catalysis, a solvent and/or substituent effects being observed. Thus, in EtOH, THF or methyl ethyl ketone, methylpyruvate gave the opposite $(S)$-lactate with ee up to $12 \% .{ }^{155}$ It is reasonable to assume that the adsorption of the alkaloid onto a Pt surface provides an adjacent site at which the selective enantiofacial adsorption of pyruvate occurs. However, the mechanistic pathway of the Pd-catalyzed reaction is not well-understood and coadsoption of solvent/substituent molecules on the Pd surface is crucial in determining the stereochemical outcome of the hydrogenation.

Inversion of the enantioselectivity has been observed during the Pt-catalyzed hydrogenation of 4,4,4-trifluoroacetoacetate with O-methylcinchonidine (MeOCD) 147 $\left(\mathrm{R}=\mathrm{Me}\right.$ ) in acetic acid to provide $(S)-\mathbf{1 4 8}$ in 90\% ee (Scheme 53). ${ }^{156}$ In the presence of water a low inversion of the enantioselectivity occurred with ee up to $12 \%$ only.

Scheme 53. Enantiodivergent Hydrogenation of 4,4,4-Trifluoroacetoacetate with $5 \% \mathrm{Pt} / \mathrm{Al}_{2} \mathrm{O}_{3}$ and MeOCD 147 as Catalyst

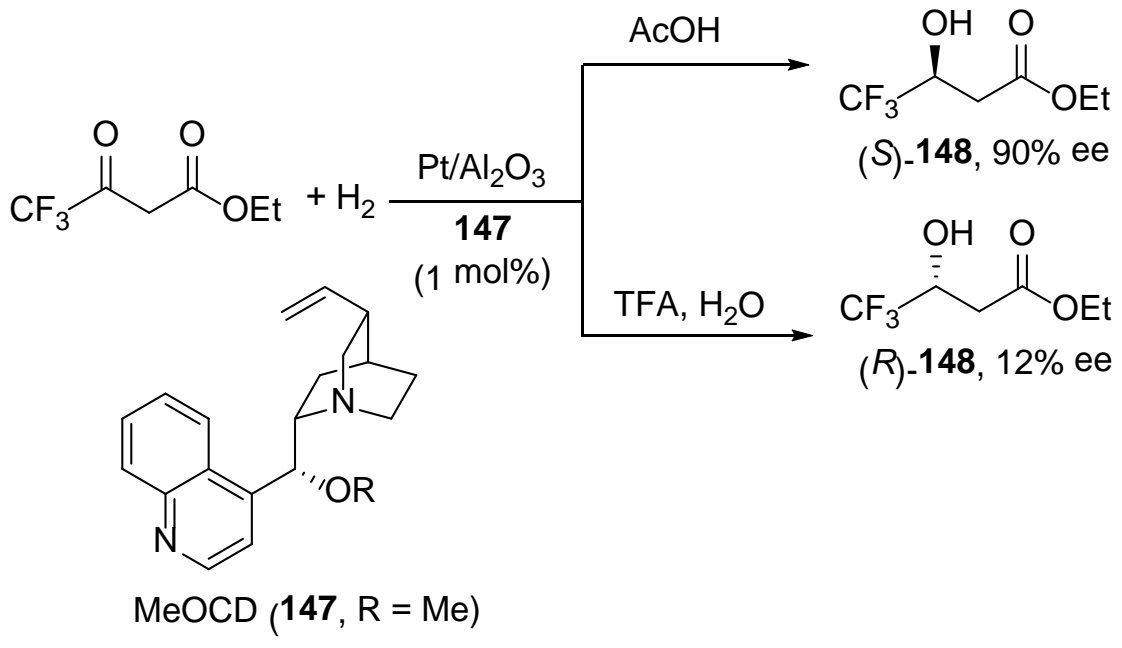

Heterogeneous hydrogenation of ethyl pyruvate (EtPy) by Pt- $\mathrm{Al}_{2} \mathrm{O}_{3}$ containing $\beta$ isocinchonine $(\beta$-ICN) 149a led to the formation of ethyl $(R)$-lactate in $50 \%$ ee in toluene, whereas in $\mathrm{AcOH}$ the corresponding $(S)$-enantiomer was formed in $60 \%$ ee (Scheme 54). ${ }^{157}$ A linear relationship between the composition of the solvent (AcOH+toluene) and the sense of chiral induction was found. The proposed structure for the 1:1 complex of $\beta$-ICN (149a) and ethyl pyruvate (Scheme 54) in toluene showed the binding between the nitrogen of the quinuclidine unit and the carbonyl group. 
However, in $\mathrm{AcOH}$ this nitrogen is protonated and this proton will form a hydrogen bond with the oxygen of the keto group inverting the enantiofacial approach. The same enantiodivergent effect has been detected in the hydrogenation of trifluoromethyl cyclohexyl ketone. ${ }^{158}$ Modest ee has been observed in the enantiodivergent hydrogenation of ethyl pyruvate in the presence of $\beta$-ICN (149a) or $\alpha$-ICN (149b) giving either ethyl $(S)$ - or $(R)$-lactate, respectively, in $27 \%$ and $50 \%$ ee. ${ }^{159}$

\section{Scheme 54. Enantiodivergent Hydrogenation of Ethyl Pyruvate with $\beta$ -} Isocinchonine (149a) Modified $\mathrm{Pt} / \mathrm{Al}_{2} \mathrm{O}_{3}$ Catalyst

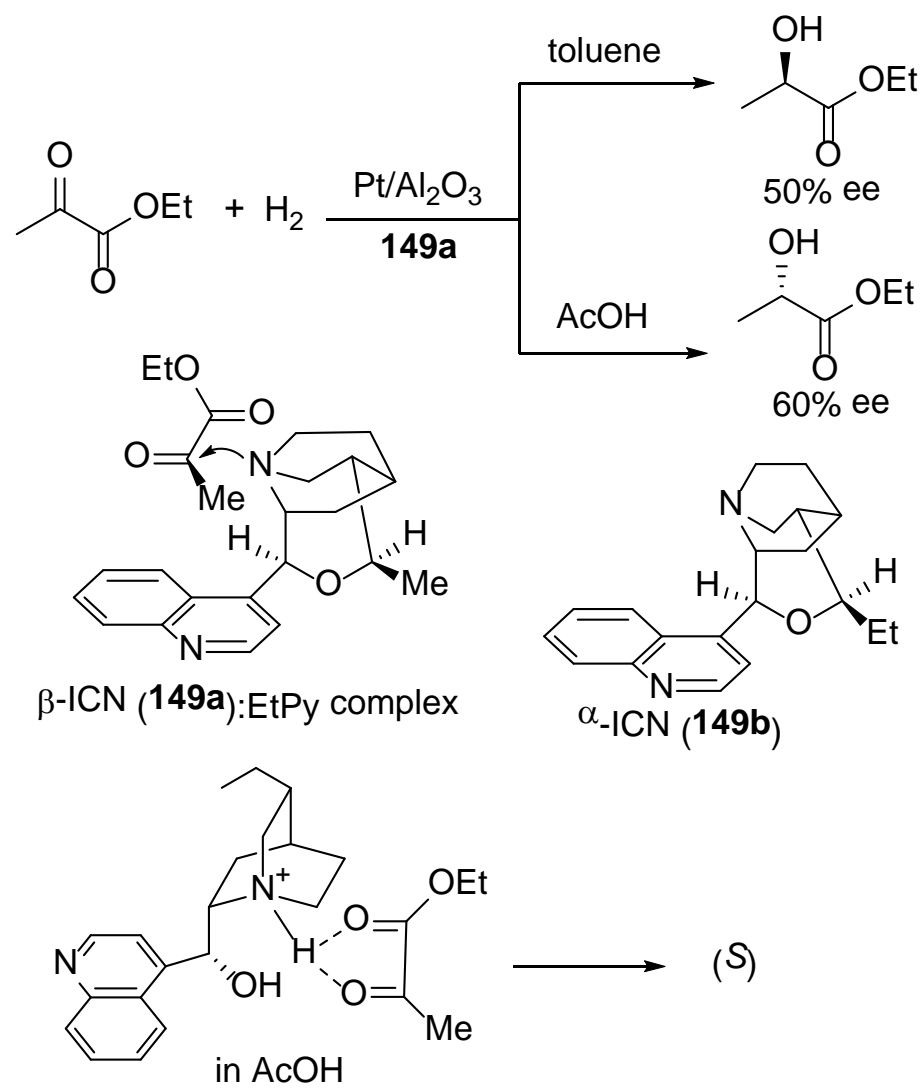

In the case of 2,2,2-trifluoroacetophenone hydrogenation, a modest inversion of the enantioselectivity was observed using $O$-methylcinchonine (MeOCN) and $\beta$-ICN (149a). ${ }^{160}$ Reversal of the enantioselectivity has been observed in the hydrogenation of acetophenones using $\mathrm{Pt} / \mathrm{Al}_{2} \mathrm{O}_{3}$ modified by cinchonidine (CD), for instance, the resulting (S)-1-phenylethanol was obtained in ca. 70\% ee. However, in polar solvents or by adding TFA in weak polar solvents a modest reversal of the enantioselectivity was observed. ${ }^{161}$ The hydrogenation of 1,1,1-trifluoro-2,4-diketones with CD or MeOCD (147) modified $\mathrm{Pt} / \mathrm{Al}_{2} \mathrm{O}_{3}$ gave regioselectively ( $\mathrm{CF}_{3} \mathrm{CO}$ group) the corresponding $\beta$ hydroxy ketones in 28-86\% ee. The inversion of the enantioselectivity increased with the solvent polarity with ee up to $22 \%$ in the case of MeOCD (147). ${ }^{162}$

Inversion of the enantioselectivity has been observed in the hydrogenation of $\alpha$ oxopantolactone (150) to pantolactone (151), an intermediate in the synthesis of pantothenic acid (vitamin B family) and a constituent of coenzyme A. ${ }^{163}$ In this case, 
the presence of bulky groups in the $\mathrm{OH}$ group of $\mathrm{CD}$ caused the inversion of enantioselectivity. The dominant $R$ enantiomer 151 was obtained with CD in toluene (79\% ee) whereas CD derived $O$-trimethylsilyl or $O$-phenyl ethers gave $(S)$-151 with ee up to 52\% (Scheme 55). DFT calculations supported the adsorption of CD and PhOCD (147, $\mathrm{R}=\mathrm{Ph}$ ) on an idealized flat Pt surface showing the steric hindrance by the phenyl group.

\section{Scheme 55. Enantiodivergent Hydrogenation of $\alpha$-Oxopantolactone with Cinchonidine and $O$-Phenylcinchonidine Modified $\mathrm{Pt} / \mathrm{Al}_{2} \mathrm{O}_{3}$ Catalysts}

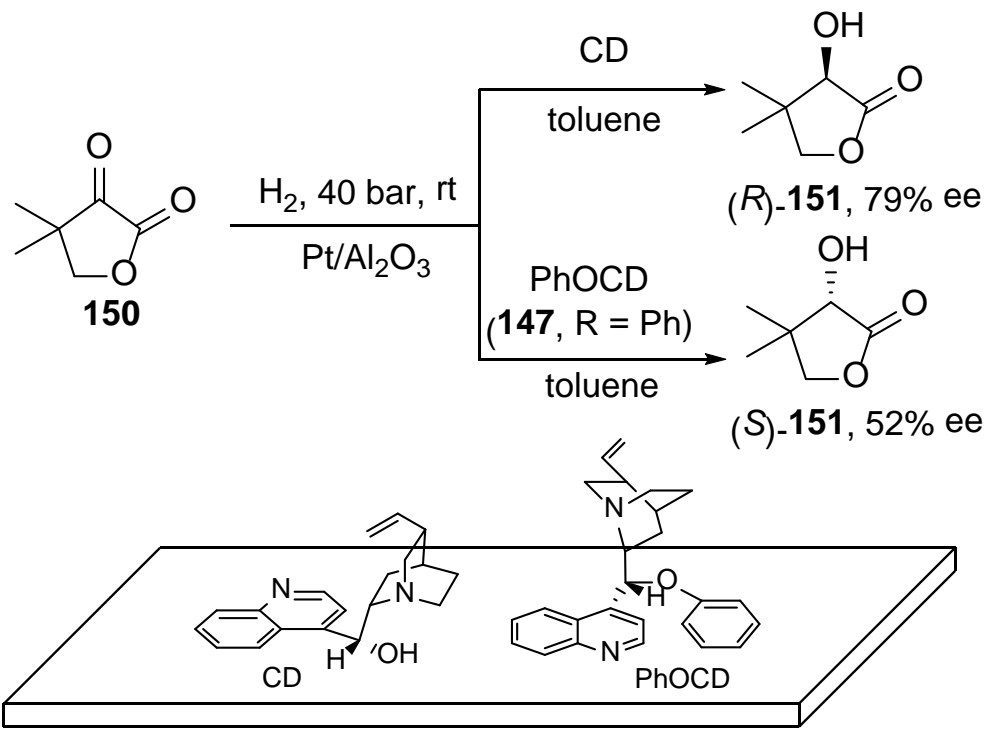

Inversion of the enantioselectivity in the hydrogenation of $\alpha$-oxopantolactone (150) was also described by Bartók and co-workers using $\beta$-ICN (149a) in AcOH and in toluene. Pantolactone $(R)-\mathbf{1 5 1}$ was obtained in $60 \%$ ee in toluene and $(S)-\mathbf{1 5 1}$ in a very low 5\% ee in $\mathrm{AcOH} .{ }^{164}$ The same enantiodivergence has been observed by Baiker and co-workers in the hydrogenation of ethyl pyruvate, 4,4,4-trifluorocetoacetate and 1,1,1trifluoro-2,4-pentadione. ${ }^{165}$ Experimental and theoretical calculations to explain this switch of enantioselectivity have been performed. ${ }^{166}$ The approach of CD and PhOCD (147) to the solid surface corresponds to the models in Scheme 55 showing that CD is absorbed more strongly than PhOCD. Attempts to substitute Pt by Rh on alumina using $\mathrm{CD}$ and $\mathrm{O}$-substituted CDs 147 as chiral modifiers in the hydrogenation of 3,5di(trifluoromethyl)acetophenone gave lower enantioselectivities. ${ }^{167}$

Murzin and co-workers ${ }^{168,169}$ also studied the influence of $\mathrm{AcOH}$ using $\mathrm{Pt} / \mathrm{Al}_{2} \mathrm{O}_{3}$ as catalyst in the hydrogenation of 1-phenylpropane-1,2-dione with CD and 9-methoxy10,11-dihydrocinchonidine as chiral modifiers. The corresponding (1S,2R)-1-phenyl1,2-propanediol was isolated in both cases using $\mathrm{AcOH}$ in $67 \%$ and $78 \%$ ee, respectively. Changing $\mathrm{AcOH}$ by toluene a modest inversion of the enantioselectivity was observed and $(1 R, 2 S)$-diol was formed as a main product in $38 \%$ ee. Also modest enantioselectivities have been observed in the screening of other experimental 
parameters, such as the chiral modifier concentration, in the enantiodivergent hydrogenation of ethyl pyruvate in the gas phase over $\mathrm{Pt} / \mathrm{Al}_{2} \mathrm{O}_{3} \cdot{ }^{170,171}$ Low concentrations of hydroquinidine 4-chlorobenzoate gave ethyl (S)-lactate in $15 \%$ ee and in higher concentrations the $(R)$-enantiomer was obtained in $17 \%$ ee.

It can be concluded that in spite of the big effort carried out in this type of heterogeneous enantiodivergent hydrogenation of ketones modest results have been obtained.

2.1.5.3. Transfer Hydrogenation. Isopropanol (IPA) and formic acid and its salts are the most common hydrogen donors for the metal-catalyzed reduction of carbonyl compounds. ${ }^{7,141,172-174}$ Generally, isopropanol is used in asymmetric metal-catalyzed hydrogen-transfer reactions. Hantzsch esters acted also as hydrogen donors mimicking $\mathrm{NADH}$ in organocatalyzed processes.

Ru-based asymmetric catalysis for hydrogen transfer was initially reported by Noyori and co-workers. ${ }^{175} \mathrm{Ru}$, Ir and Rh complexes have been mainly used as chiral catalysts for the hydrogen transfer of ketones to give chiral alcohols. Enantiodivergent transfer hydrogenation of acetophenone using axially chiral Ir(III) catalysts has been observed. Bidentate monosulfonated diamines with a chiral biaryl backbone 152 (Figure 8) in combination with $\operatorname{Ir}(\mathrm{III})$ complexes acted as catalysts in the reduction of acetophenone with isopropanol and potassium isopropoxide. ${ }^{176}$ In the presence of 1 eq of KOiPr, $(R)$ 1-phenylethanol was obtained (42-92\% ee), whereas in the presence of an excess of base (10 eq) the $S$ alcohol was mainly formed in only 5-20\% ee. Similar enantioselectivities were obtained in the transfer hydrogenation with NaiOPr/IPA and $\left[\mathrm{Ru}(p \text {-cymene }) \mathrm{Cl}_{2}\right]_{2}$ as catalysts using amide and thioamide ligands 153 (Figure 8). ${ }^{177}$ Thus, 153a gave $(S)$-1-phenylethanol in 92\% ee, whereas using ligand 153b the $(R)$ alcohol was isolated in low $20 \%$ ee.

Figure 8. Chiral Ligand for the Enantiodivergent Transfer Hydrogenation of Acetophenone to 1-Phenylethanol

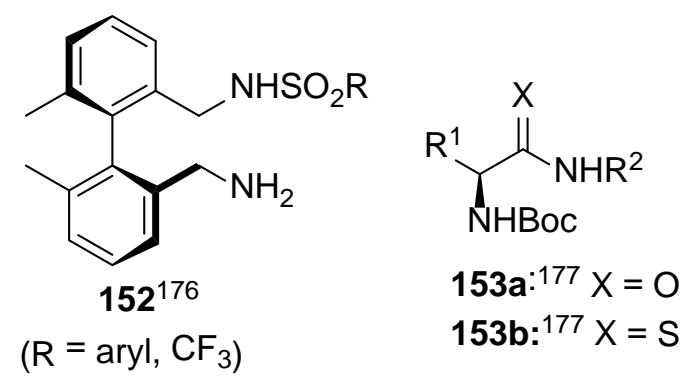

During optimization reaction studies on the reduction of acetophenone to 1phenylethanol by $\mathrm{Ru}(\mathrm{II})$ complexes $\mathbf{1 5 4}$ bearing tridentate NNN ligands, enantiodivergent hydrogen transfer was also observed. ${ }^{178}$ Complex 154a gave the $(S)$-1phenylethanol in 56\% ee, whereas $\mathbf{1 5 4 b}$ afforded the $(R)$-alcohol in $79 \%$ ee (Scheme 56). These results were explained by the steric effects of the chiral oxazolinyl moiety. 
Scheme 56. Enantiodivergent Transfer Hydrogenation of Acetophenone Catalyzed by Ru(II) Complexes 154

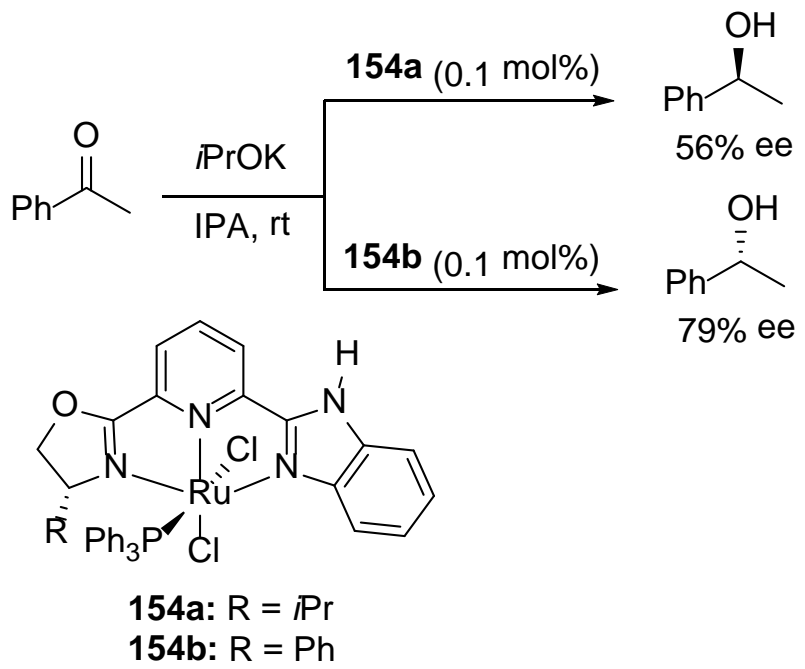

1,4-Dihydropyridines are the active part of the reduced form of the nicotinamide adenine dinucleotide (NADH), which is able to perform many biological reductions by a hydride or two electrons plus one proton transfer to a substrate bound to an enzyme in living organisms. ${ }^{141}$ The first reported example of an enantiodivergent H-transfer reduction is the case of ethyl phenylpyruvate, which was carried out with 1,4dihydronicotinamides 155 bearing a chiral amino acid unit giving ethyl mandelate (156) in modest enantioselectivities (Scheme 57). ${ }^{179}$

\section{Scheme 57. Enantiodivergent Chiral NADH-Type Reduction of Ethyl} Phenylpyruvate

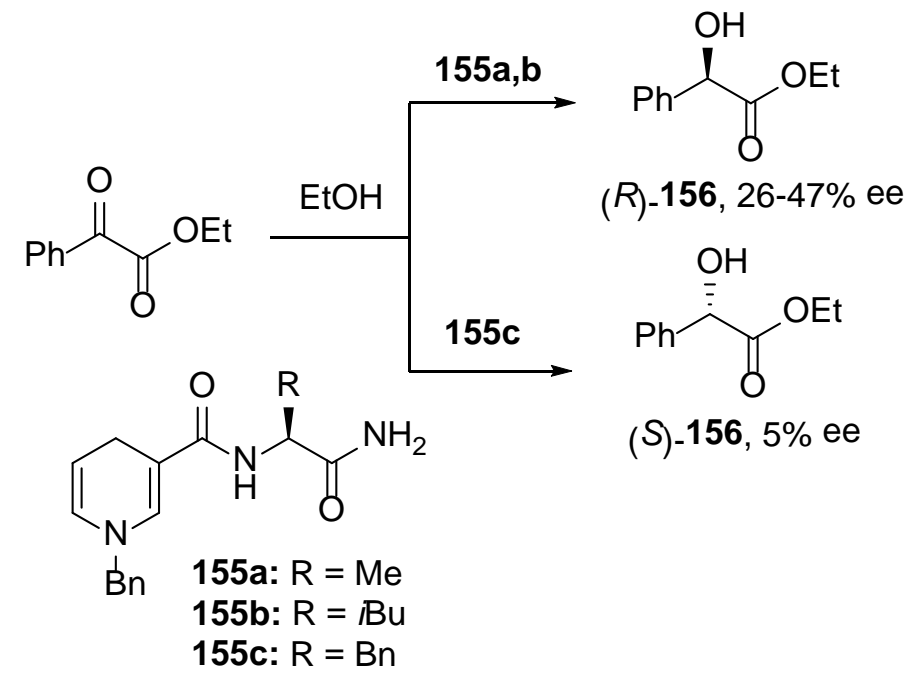

A similar effect has been observed using 2-amino ethanols as chiral source. Thus, in the case of compound 157, methyl $(R)$-mandelate was obtained with $49 \%$ ee, while NADH 158 gave methyl (S)-mandelate in $52 \%$ ee ${ }^{180}$ (Figure 9). Higher enantioselectivities were obtained using the seven-membered fused ring NADHs, which are conformational diastereomers, $(\mathrm{a} S, 1 S, 4 R)-\mathbf{1 5 9}$ and $(\mathrm{a} R, 1 S, 4 S)-\mathbf{1 6 0}$, affording in the 
presence of $\mathrm{Mg}\left(\mathrm{ClO}_{4}\right)_{2}$ methyl $(R)$-mandelate in $88 \%$ ee and the $(S)$-enantiomer in $78 \%$ ee, respectively. ${ }^{181}$ Under similar reaction conditions NADH 161 gave the reversal of enantioselectivity just changing the amino acid methyl ester unit. (S)-Alanine and proline derivatives 161 provided (S)-mandelate, whereas valine, phenylalanine and phenylglycine gave $(R)$-mandelate but in low enantioselectivities, which are dependent upon the temperature. ${ }^{182}$

Figure 9. NADH Models for the Enantiodivergent Reduction of Phenylpyruvates<smiles>CCC(CO)NC(=O)C1=CN(C)C=CC1</smiles>

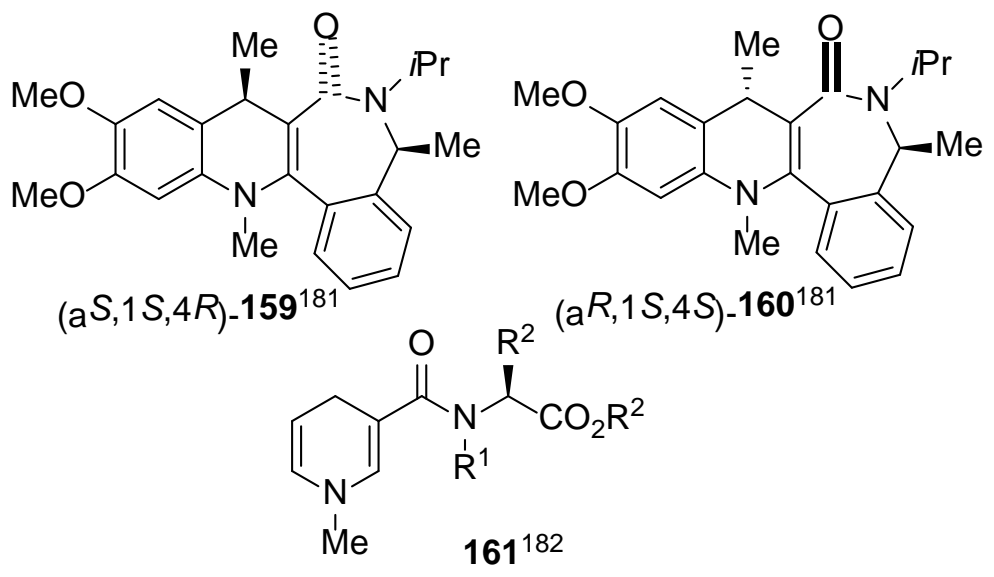

In general, it is very difficult to explain the observed inversion of enantioselectivity in these transfer hydrogenations reactions.

2.1.5.4. Enzymatic Reduction. Biological reduction of carbonyl compounds has some advantages over chemical processes with respect to selectivity, safety and sustainability. ${ }^{183}$ Enantiodivergent enzymatic reductions of $\alpha$-chloro- $\beta$-keto esters $\mathbf{1 6 2}$ have been performed with baker's yeast for the preparation of taxol side chains. ${ }^{184}$ NADPH-mediated reductions were carried out with whole Escherichia coli cells that overexpressed one of the two key Saccharomyces cerevisae reductases with $\mathrm{pH}$ maintained at 5-6. The corresponding ethyl (2S,3R)-2-chloro-3-hydroxy-3phenylpropanoate (163) was obtained in $>98 \%$ de and $>98 \%$ ee. For the synthesis of the (2R,3S)-enantiomer with the same stereoselective values, Escherichia coli strain overexpressing the yeast YGL039w short chain dehydrogenase was used (Scheme 58).

Scheme 58. Enantiodivergent Bioreduction of Ethyl $\alpha$-Chloro- $\beta$-Keto Ester 162 


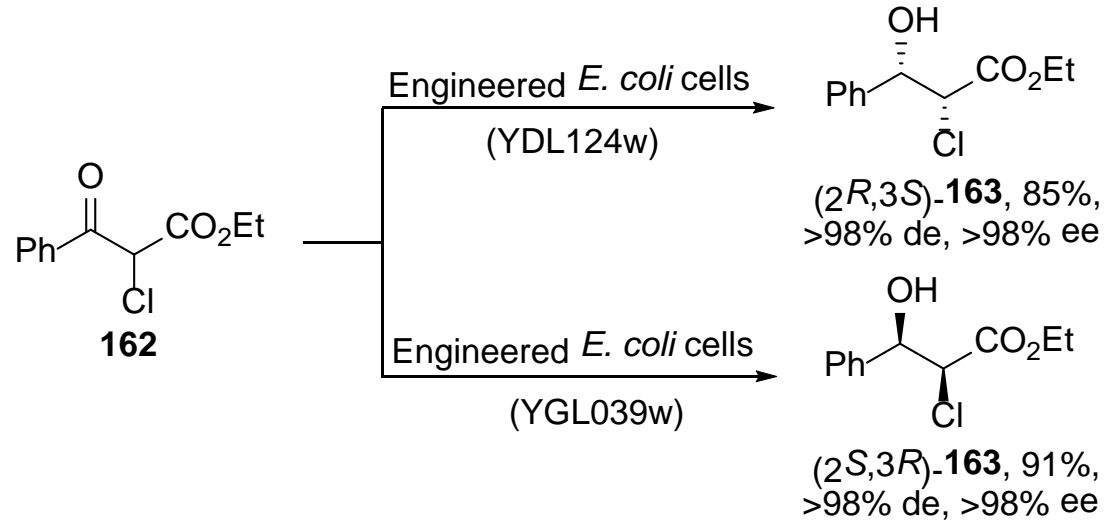

By using alcohol dehydrogenases (ADHs), ketones 164 could be enantiodivergently reduced to the corresponding axially chiral alcohols 165. Various ADHs provide $R$ alcohols, while the direct evolution of Thermoethanolicus brockii (TbSADH) provided (S)-165 with ee up to $98 \%$ (Scheme 59). ${ }^{185}$

Scheme 59. Enantiodivergent Bioreduction of Ketones 164 with Alcohol Dehydrogenases

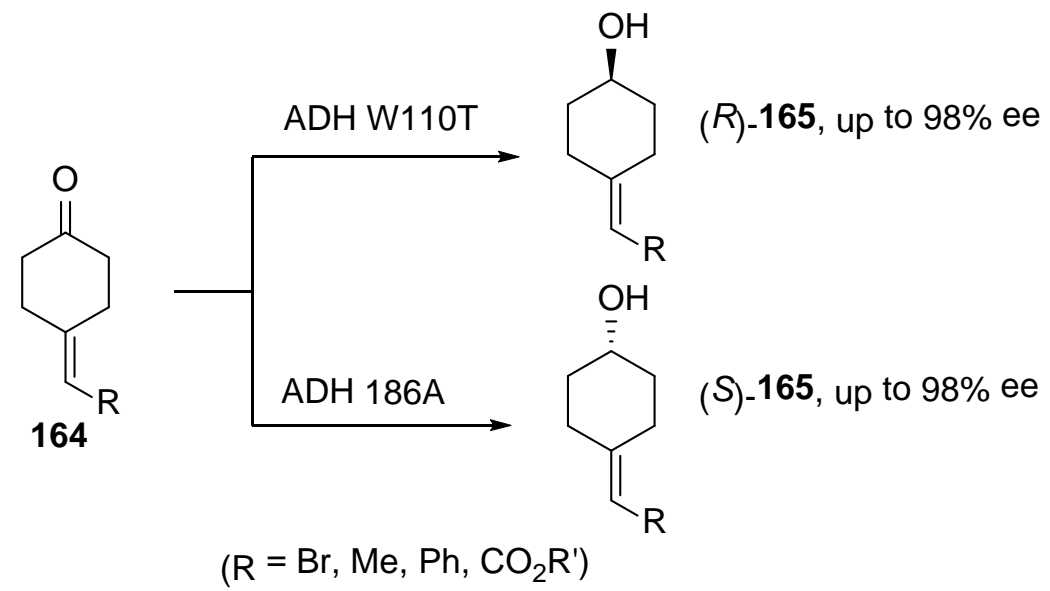

In the reduction of keto esters 166, Gotor and co-workers used ADH from Rodococcus Ruber (ADH-A) giving (S)-lactones 167 derived from a subsequent lactonization of the hydroxy esters. By means of Lactobacillus brevis ADH (LBADH) the enantiomeric lactones $(R)-\mathbf{1 6 7}$ were obtained (Scheme 60$){ }^{186}$

Scheme 60. Enantiodivergent Bioreduction of Keto Esters 166 with Alcohol Dehydrogenases

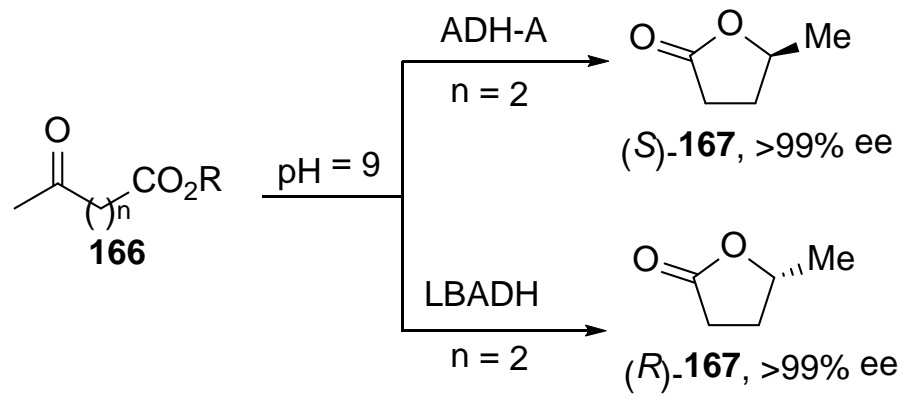


The mutant M242F/Q245T of a carbonyl reductase (SSCR) containing a coenzyme NADPH from Sporobolomyces salmonicolor AKU4429, catalyzed the reduction of 3(dimethylamino)-1-phenylpropan-1-one

(168) to

(S)-3-(dimethylamino)-1phenylpropan-1-ol (170) in 28\% ee. The combinatorial active-site saturation of this enzyme resulted in two mutants P170R/L174Y and P170H/L174Y, which catalyzed the enantiodivergent reduction of $\mathbf{1 6 8}$ and 169 to $(R)-\mathbf{1 7 0}$ and $(R)-\mathbf{1 7 1}$ in 93\% ee and 95\% ee, respectively. On the other hand, mutant L174W gave (S)-170 and (S)-171 in 96\% and $67 \%$ ee, respectively (Scheme 61). ${ }^{187}$

\section{Scheme 61. Enantiodivergent Bioreduction of $\beta$-Amino Ketones 168 and 169 with Carbonyl Reductases}<smiles>CN(C)CCC(=O)[Al]</smiles>

168: $\mathrm{Ar}=\mathrm{Ph}$

169: Ar = Thienyl

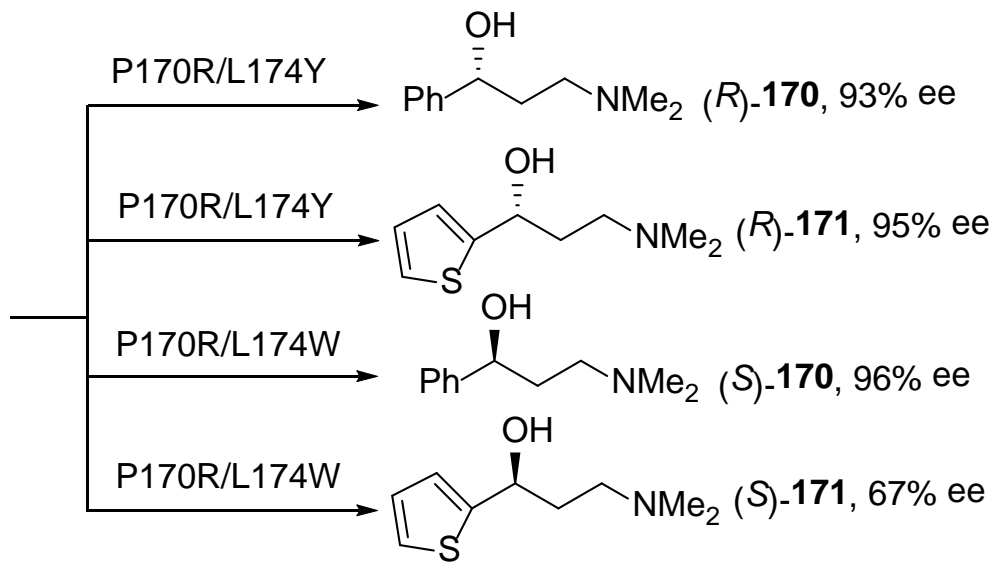

In conclusion, the enantiodivergent reduction of ketones by borane can be controlled taking in account the substitution of the chiral amino alcohol. In the case of metalcatalyzed hydrosilylation the reversal of enantioselectivity has been achieved by changing the metal of the chiral catalyst from Rh to Ir. Asymmetric heterogeneous hydrogenation afforded enantiodivergent reductions over Pt catalyst on alumina with Cinchona alkaloids as chiral modifiers mainly due to their different conformations on the surface complex. Enantiodivergent transfer hydrogenation catalyzed by transition metal complexes can be modulated by changing the substituents on the chiral ligand or on the chiral NADH-models in the organocatalyzed processes. For biological enantiodivergent reductions of ketones the elaboration of engineered enzymes is the most successful strategy.

\subsection{Nucleophilic Addition to $\mathrm{C}=\mathrm{N}$ Bonds}

In this section, diastereodivergent and enantiodivergent additions of organometallic compounds to imines and nitrones catalyzed by chiral complexes or Lewis acids will be described. In the case of diastereodivergent Mannich reactions, metal enolate additions to acyclic imines and nitrones with stereocenters in the skeleton or at the nitrogen will be considered. Enantiodivergent Mannich reactions can be catalyzed either by chiral Lewis acids or by organocatalysts. Diastereo- and enantiodivergent additions of other 
nucleophiles such as nitroalkanes (aza-Henry) and cyanides (Strecker reaction) to imines will be also considered. Enantiodivergent hydrogenations catalyzed by chiral metal complexes or by organocatalysts will allow the synthesis of enantioenriched amines.

2.2.1. Addition of Organometallic Reagents. The $\mathrm{C}=\mathrm{N}$ bond has lower electrophilicity than the $\mathrm{C}=\mathrm{O}$ group; for this reason, $N$-substituted imines, nitrones, and hydrazones are mainly used. ${ }^{188-192}$ Very reactive organometallic reagents react efficiently with these $\mathrm{C}=\mathrm{N}$ bonds containing substrates allowing the diastereodivergent synthesis of amines by changing the metal, additives and reaction conditions, such as the temperature, as in the case of carbonyl compounds. Moreover, for imines it is possible to have not only stereocenters in the acyclic backbone but also a chiral auxiliary at the nitrogen, or both. In addition, changes in the substituent of the nitrogen can afford sterodivergent results. The presence or absence of a Lewis acid and also changes in the metal center can control the diastereodivergence in the addition reaction. Enantiodivergent additions of organometallic compounds to imines have been performed by changing the metal salt. Nitrones are generally derived from chiral aldehydes and therefore have been mainly used in diastereodivergent addition reactions in the presence or absence of a Lewis acid.

2.2.1.1. Imines. Chiral sulfinimines have been extensively used over the years for the synthesis of enantioenriched amines by addition of different organometallics and nucleophilic reagents followed by simple deprotection methods specially in the case of Ellman's $N$-tert-butylsulfinylimines. ${ }^{193-197}$ For the addition of organometallic reagents to sulfinimines 172 affording chiral diastereomeric amines 173 and 174, two TS models have been proposed to explain the observed diastereoselectivity (Scheme 62). Depending on the reaction conditions a chelated TS 173A for the addition of Grignard reagents in non-coordinating solvents, such as $\mathrm{CH}_{2} \mathrm{Cl}_{2}$ or toluene, has been postulated for the formation of products 173. On the other hand, the addition of organolithium compounds gave products 174 by intermediacy of an open TS 174A in coordinating solvents such as THF. ${ }^{196,198,199}$

Scheme 62. Diastereoselective Addition of Organometallics to $\mathrm{N}$-tert-Butylsulfinyl Aldimines 172

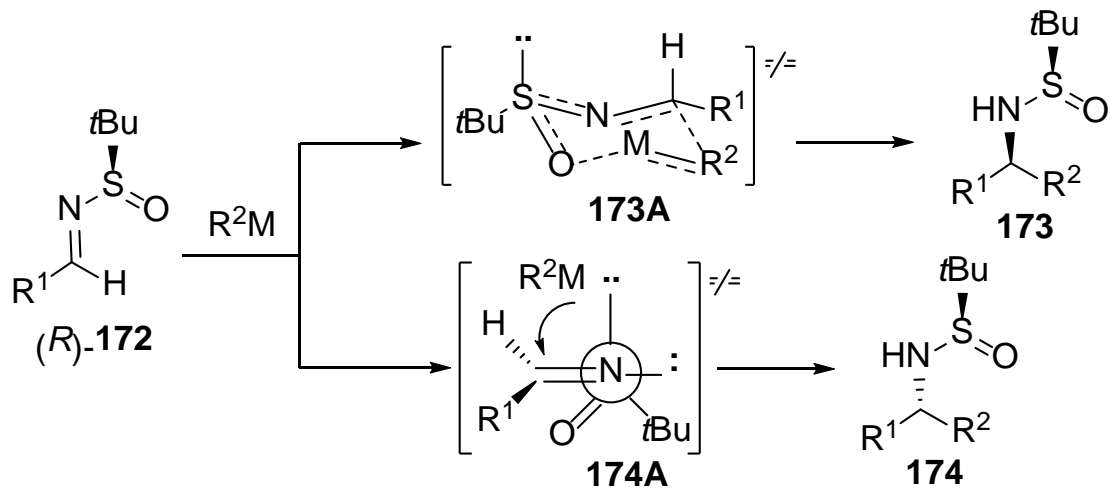


A diastereodivergent addition of isobutyllithium to sulfinyl imines $\mathbf{1 7 5}$ depending on the solvent and the Lewis acid employed has been found. ${ }^{200}$ Either in toluene or THF and $\mathrm{BF}_{3} \cdot \mathrm{OEt}_{2}$ as additive, the sulfinyl imine 175a gave $(R)$-amine $\mathbf{1 7 6}$ after hydrolysis. On the contrary, in the presence of trioctylaluminum in toluene the enantiomer (S)-176 was isolated. Amine (R)-176 is an active metabolite of sibutramine, a potent serotonin, norepinephrine, and dopamine re-uptake inhibitor. (R)-(Triethyl)methylsulfinyl aldimine [(R)-TESA] 175b $\left(\mathrm{R}=\mathrm{Et}_{3} \mathrm{C}\right)$ was selected instead of 175a as starting sulfinimine, giving the amine $(R)-\mathbf{1 7 6}$ in $99 \%$ ee, whereas $(S)-\mathbf{1 7 6}$ was obtained in 76\% ee (Scheme 63).

Scheme 63. Diastereodivergent Addition of Isobutyllithium to $N$ (Triethylmethyl)sulfinyl Aldimine 175b in the Presence of Lewis Acids

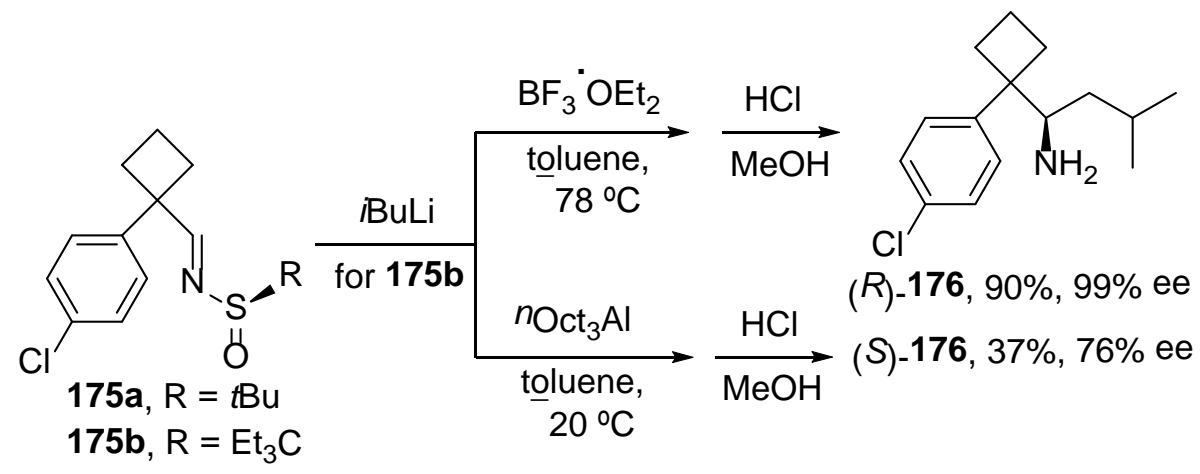

The same group has found out a strong solvent effect in the diastereodivergent addition of chiral Grignard reagents 177 to imine 175a. ${ }^{201}$ For instance, $(R)$-177 gave adduct 178a in $74 \%$ yield and $94 \%$ ee working in $\mathrm{CH}_{2} \mathrm{Cl}_{2}$ according to the formation of a chelated TS. Under other reaction conditions, $\left(n-\mathrm{C}_{8} \mathrm{H}_{17}\right)_{3} \mathrm{Al}$ in THF, the corresponding diastereomer 179a was obtained in $72 \%$ ee (yield not provided) (Scheme 64). In this case, by addition of the organolithium compound only one diastereomer was formed under both reaction conditions showed in Scheme 63.

Scheme 64. Diastereodivergent Addition of Chiral Grignard Reagent (R)-177 to $N$ tert-Butylsulfinyl Aldimine 175a 


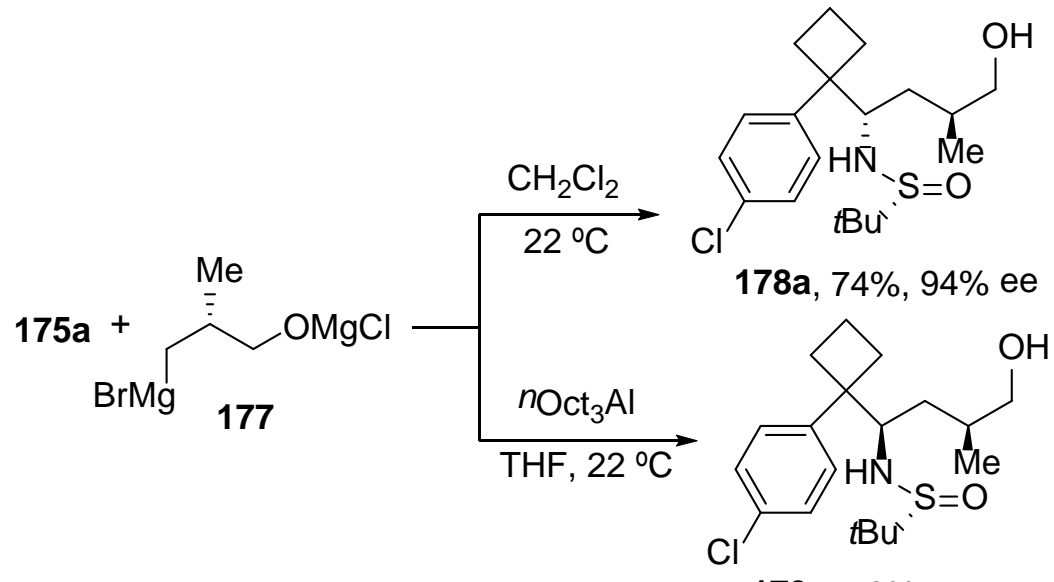

$179 a, 72 \%$ ee

The first enantiodivergent addition of organolithium compounds to $N$-aryl aromatic aldimines catalyzed by the amino ethers 180 and 181, both with $(S)$-configuration, gave very low ee of the corresponding $(S)$ - and $(R)$-amines, respectively (Figure 10$){ }^{202}$ In the case of similar ligands 182a and 182b, a reversal of enantioselectivity was observed in the addition of $\mathrm{PhLi}$ to $\mathrm{N}$-(4-methoxyphenyl)imine derived from thiophenecarbaldehyde but in very low ee. ${ }^{203}$

Figure 10. Chiral Ligands for the Enantiodivergent Addition of Organolithium Compounds to $N$-Aryl Aromatic Imines<smiles>COc1ccccc1OCC(Cc1ccccc1)N(C)C</smiles><smiles>COc1ccccc1OCC1CCCN1C(C)(C)C</smiles><smiles>[R]CN(C)C1CCCCC1N(C)C[R]</smiles>

$\left.182 a_{(R}=H\right)^{203}$

$182 b(R=B n)^{203}$

An efficient enantiodivergent addition of arylacetylenes to $\alpha$-imino esters was carried out with a chiral copper(I) complex CuOTf·0.5PhH with PyBOX 184 as a common chiral ligand. ${ }^{204}$ In the addition of ethyl glyoxylate 183 to the $N$ - $(p$ methoxyphenyl)imine just adjusting the ligand-to-metal ratio from 1:1 to 2.5:1 switched the enantioselectivity of the product $\mathbf{1 8 5}$ to the opposite sense (Scheme 65).

Scheme 65. Enantiodivergent Addition of Phenylacetylene to $N$-4-Methoxyphenyl Imine of Ethyl Glyoxylate 183 Catalyzed by Cu(I)/PyBOX 184 Complex 


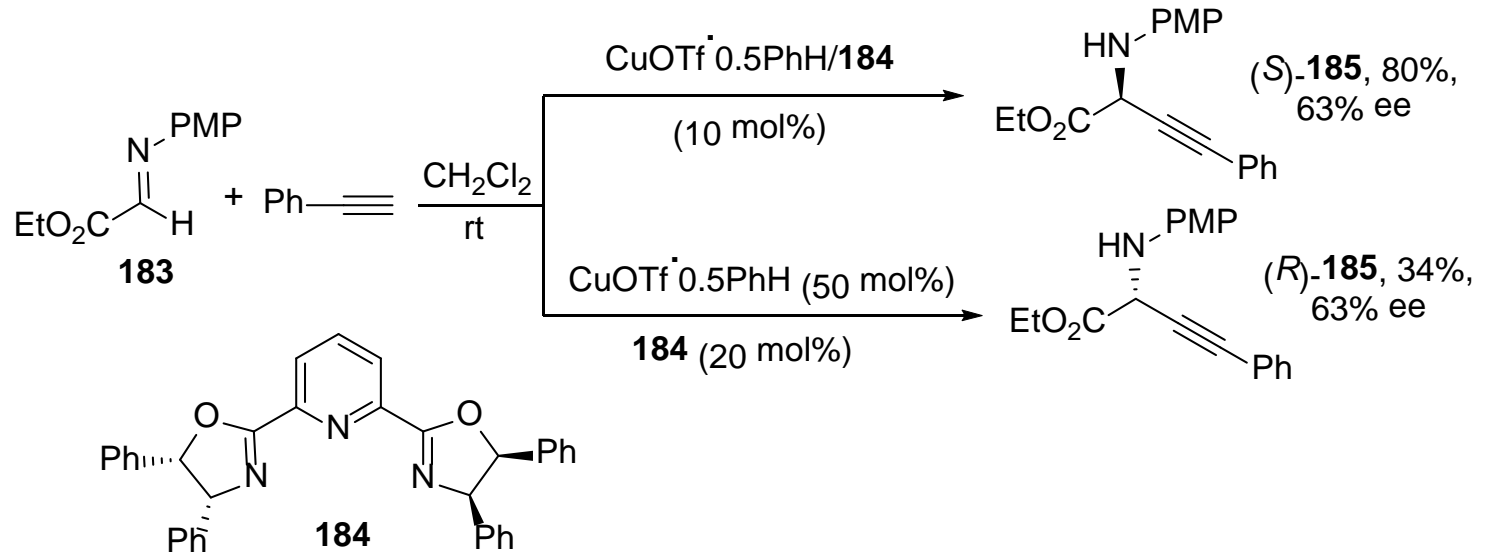

The group of Feringa and Minnaard reported an efficient enantiodivergent addition of different organometallic reagents to $N$-formyl imines generated in situ by elimination of 4-toluenesulfinate from aliphatic and aromatic $\alpha$-amido sulfones $\mathbf{1 8 6}^{205}$ Monodentate phosphoramidite ligand 187 (4 mol\%) was the only source of chirality in the addition of dialkylzinc compounds catalyzed by the $\mathrm{Cu}(\mathrm{OTf})_{2}$ (2 mol\%) complex providing $(R)-N$-formyl protected amines 188. The same ligand (10 mol\%), but changing the metallic precursor to $\mathrm{Cu}(\mathrm{acac})_{2}$ (5 mol\%) catalyzed the addition of trimethylaluminum to $186\left(\mathrm{R}^{1}=\mathrm{Ph}\right)$ giving $(S)$-adducts 188 (Scheme 66). This reversal of enantioselectivity was attributed to the formation of different catalysts.

Scheme 66. Enantiodivergent Addition of Dialkylzinc and Trimethylaluminum Compounds to $\alpha$-Amido Sulfones Catalyzed by Phosphoramidite 187 and Cu(II) Salts

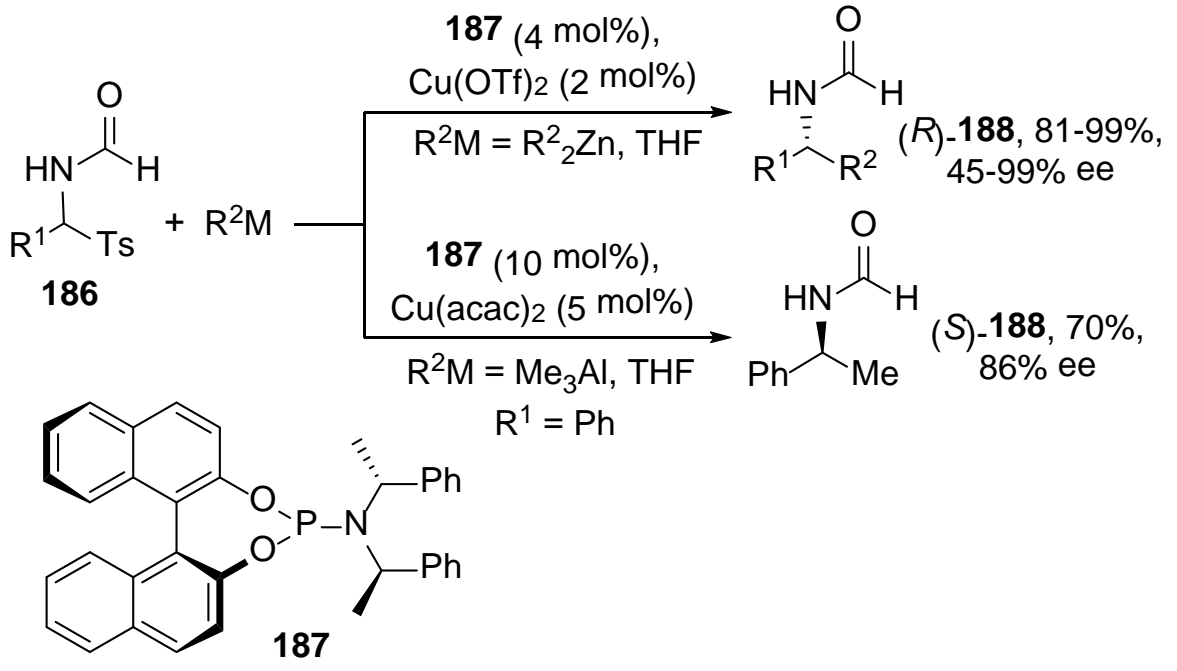

2.2.1.2. Nitrones. According to the nature of the functional group, nitrones have the most highly polarized $\mathrm{C}=\mathrm{N}$ bond and the oxygen atom can chelate organometallic reagents controlling the stereoselectivity of the addition reaction giving $N, N$ disubstituted hydroxylamines, which can be further reduced to secondary amines. Nitrones derived from chiral aldehydes are able to exert diastereodivergent additions of organometallic reagents. In the first example described by Dondoni, Merino and co- 
workers, the addition of 2-lithiothiazole, a masked formyl anion, to D-glyceraldehyde acetonide-derived nitrone 189 provided syn-adducts 190 diastereoselectively. ${ }^{206}$ However, in the presence of a Lewis acid such as $\mathrm{Et}_{2} \mathrm{AlCl}$ or $\mathrm{TiCl}_{4}$ anti-products were mainly obtained, the former giving the highest diastereoselectivity (Scheme 67). TS model 189A, developed by DFT calculations by Houk and co-workers ${ }^{207}$ for the nucleophilic addition to alkenes, explain the syn-selectivity. In the presence of a Lewis acid, a precomplexation takes place before addition of the organolithium reagent giving a TS 189B precursor for the anti-isomer. Similar diastereodivergent effect has been observed for the addition of 2-lithiofuran and $N$-methyl-2-lithioimidazole to $\alpha$-alkoxy nitrones in the absence and in the presence of $\mathrm{Et}_{2} \mathrm{AlCl}^{208-210}$ In the first case, the addition to $\mathbf{1 8 9}$ gave the syn adduct in $92 \%$ de and $90 \%$ for the anti one. On the other hand, $N$-methyl-2-lithioimidazole gave lower diastereoselectivity, $76 \%$ and $58 \%$, respectively. Further transformation of the obtained hydroxylamines can be performed with $\mathrm{TiCl}_{3}$ in aqueous $\mathrm{MeOH}^{206}$ or by hydrogen transfer with ammonium formate in refluxing $\mathrm{MeOH}$ catalyzed by $\mathrm{Pd} / \mathrm{C}^{208-210}$ The heterocyclic fragment can be transformed into a carboxylic function by oxidation with $\mathrm{RuO}_{2} / \mathrm{NaIO}_{4}$.

\section{Scheme 67. Diastereodivergent Addition of 2-Lithiothiazole to D-Glyceraldehyde $N$-Benzyl Nitrone 189}

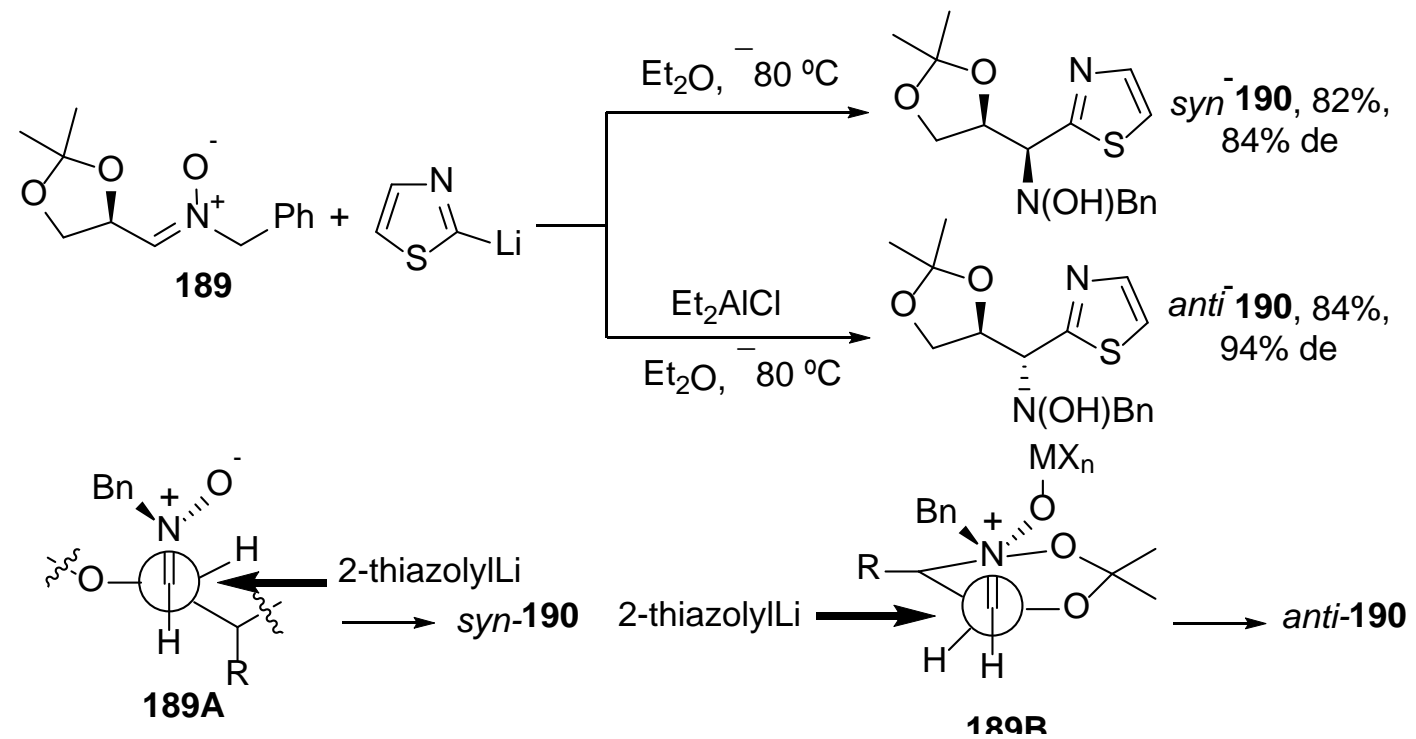

The addition of 2-lithiothiazole to nitrone 191, derived from 1,2,3,4-di-Oisopropylidene- $\alpha$-D-galactohexodialdo-1,5-pyranose, gave the syn-adduct 192 in 34\% or $58 \%$ de, by complexation with $\mathrm{MgBr}_{2}$ or $\mathrm{ZnBr}_{2}$, respectively. A reversal of diastereoselectivity could be achieved using $\mathrm{Et}_{2} \mathrm{AlCl}$ in $82 \%$ de. However, the Grignard or the diethylaluminum organometallics gave lower de (Scheme 68). ${ }^{211}$ The thiazole unit can be transformed into an aldehyde and this methodology has been applied to the synthesis of advanced intermediates of destomic acid and lincosamine.

Scheme 68. Diastereodivergent Addition of 2-Lithiothiazole to Nitrone 191 Mediated by Lewis Acids 


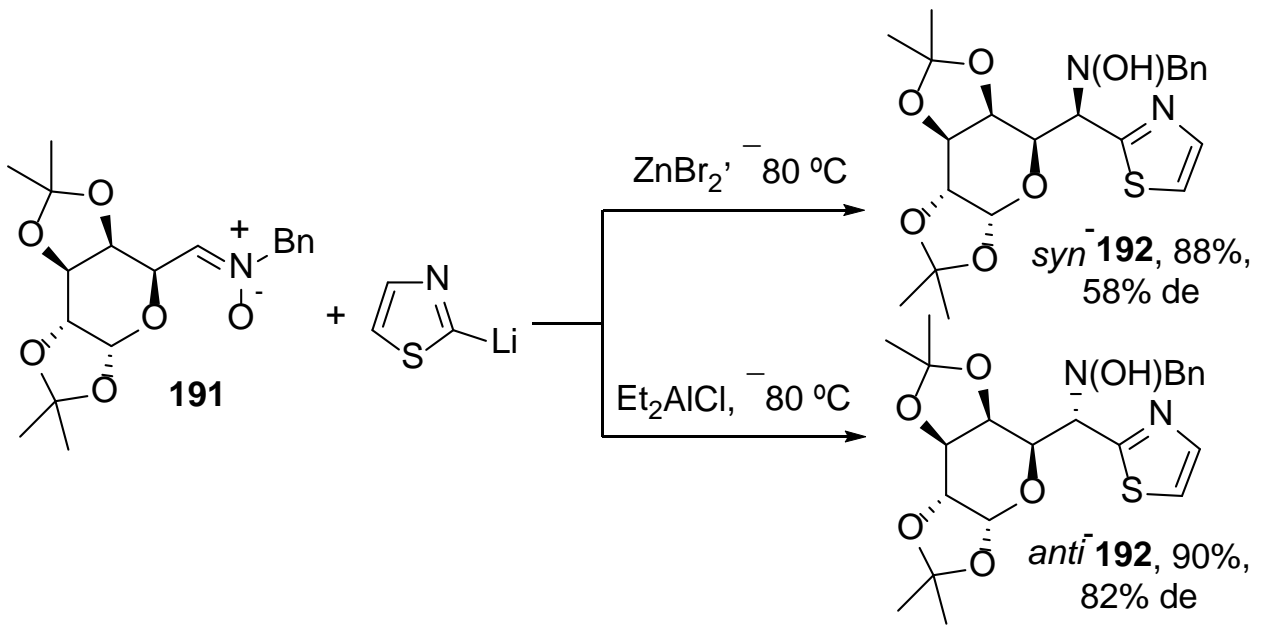

Diastereodivergent addition of vinylmetal reagents to D-glyceraldehyde nitrone $\mathbf{1 8 9}$ gave syn and anti-isomers in the absence or presence of $\mathrm{Et}_{2} \mathrm{AlCl}$. The best results for the preparation of syn-allylamine were obtained with vinylmagnesium bromide at $0{ }^{\circ} \mathrm{C}$ in THF (86\% yield and 52\% de). Vinyllithium in the presence of $\mathrm{Et}_{2} \mathrm{AlCl}$ at $-80{ }^{\circ} \mathrm{C}$ in ether afforded anti-193 in 92\% yield and 92\% de, whereas vinylmagnesium bromide in ether at $0{ }^{\circ} \mathrm{C}$ gave the same isomer in $86 \%$ yield and $84 \%$ de. Propargylamine derivatives were synthesized by the addition of trimethylsilylethynyllithium in THF giving syn-194 and anti-194 in the absence and presence of $\mathrm{Et}_{2} \mathrm{AlCl}$, respectively (Scheme 69). ${ }^{212,213}$ The last methodology has been applied to the synthesis of epimeric $N$-hydroxy $\alpha$-amino esters. ${ }^{213}$

Scheme 69. Diastereodivergent Addition of Vinyl- and Trimethylsilylethynyl Organometallic Reagents to D-Glyceraldehyde Nitrone 189

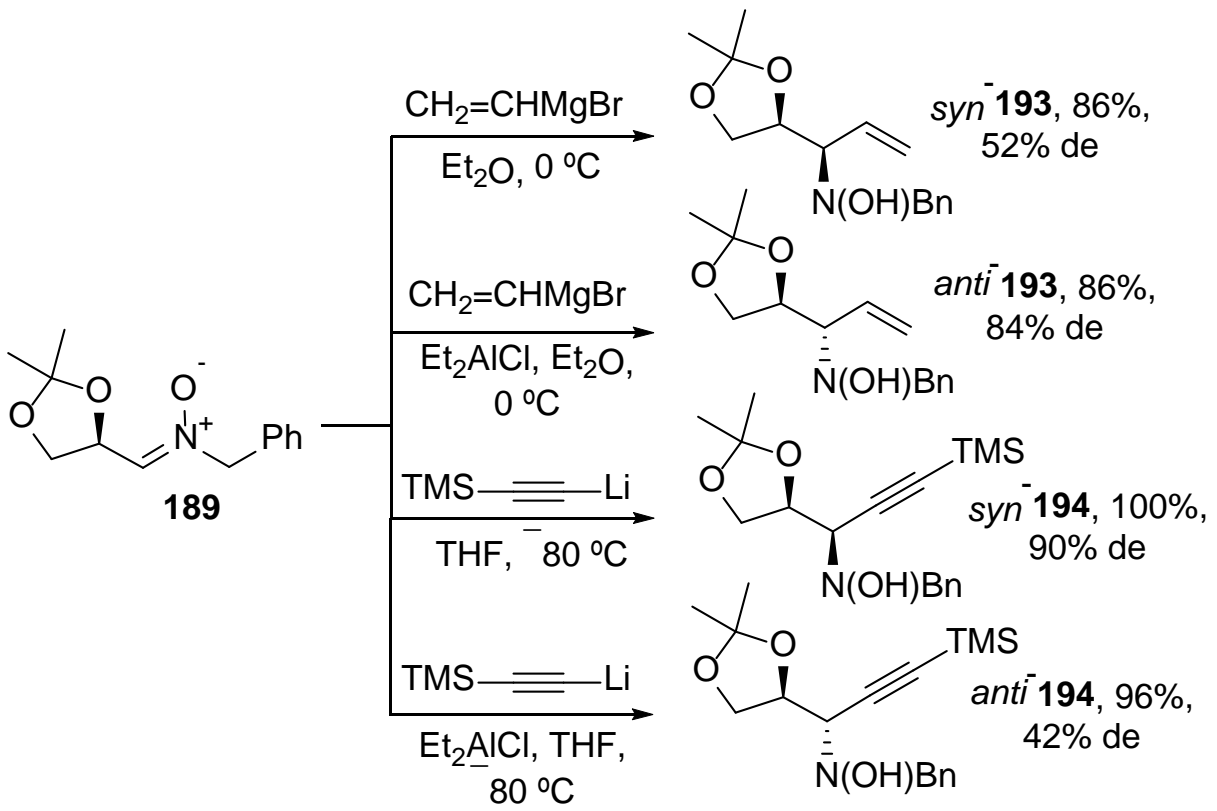


Diastereodivergent addition of Grignard reagents to nitrone 189 has been applied to the synthesis of $N$-hydroxy $(R)$ - and $(S)$-amino acids where the nitrone moiety was considered as an $N$-hydroxyglycine cation equivalent. ${ }^{214}$ In this case, the dioxolane ring from the starting isopropylidene-D-glyceraldehyde was oxidized with periodic acid. The diastereodivergent addition of Grignard reagents to $\alpha$-alkoxy nitrones gave syn-adducts in the presence of $\mathrm{ZnBr}_{2}$ and anti isomers using $\mathrm{Et}_{2} \mathrm{AlCl}$ as Lewis acids. ${ }^{215}$ The stereochemical outcome of these additions has been explained with models 189C and 189D, respectively (Figure 11). The obtained diastereomeric hydroxylamines have been further transformed into 3-amino-1,2-diols.

Figure 11. Proposed Models for the Diastereodivergent Addition of Grignard Reagents to D-Glyceraldehyde Nitrone 189
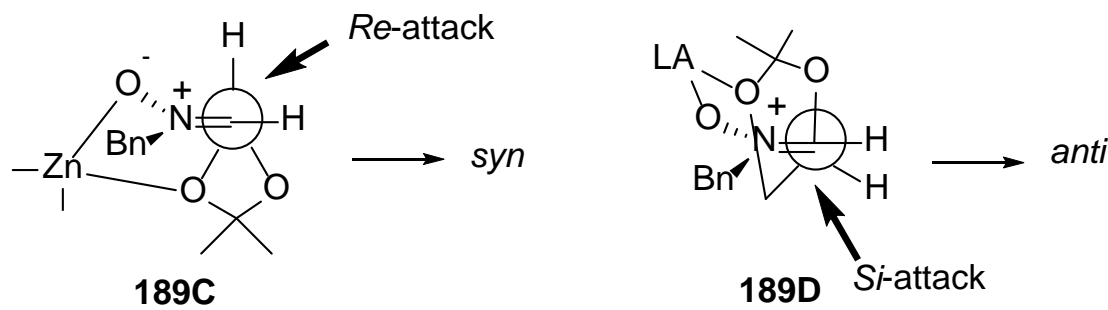

d

The allylation of nitrones derived from differently protected D-glyceraldehyde derivatives has been performed with different allylmetals ( $\mathrm{Li}, \mathrm{MgBr}, \mathrm{SnnBu} \mathrm{B}_{3}$ ) in the absence or presence of Lewis acids. In general, low diastereoselectivities were observed except in the case of allylmagnesium bromide which gave enantiopure syn-adducts in the presence of $\mathrm{ZnBr}_{2}$ and the anti-products in the presence of $\mathrm{BF}_{3} \cdot \mathrm{Et}_{2} \mathrm{O}$ at $-50{ }^{\circ} \mathrm{C} .{ }^{216}$ Grignard reagents have been also added to chiral $\alpha$-amino nitrones ${ }^{217}$ and to protected nitrones derived from L-serine, ${ }^{218}$ allowing the diastereodivergent synthesis of 1,2diamines and 2,3-diaminobutanoic acids, respectively. The addition of $\alpha$ alkoxymethyllithium, $\mathrm{LiCH}_{2} \mathrm{OMe}$ and $\mathrm{LiCH}_{2} \mathrm{OBn}$, to nitrones from D-glyceraldehyde derivatives gave the hydroxymethylation products. The obtained syn and anti products were further transformed into $(2 R, 3 S)$ - and $(2 S, 3 S)$-2-amino-1,3,4-butanetriols. ${ }^{219}$

Diastereodivergent addition of benzylmagnesium bromide to nitrones 195 gave 196 precursors of pseudo $C_{2}$-symmetric 1,3-diamino-2-propanol $(S, S)-197$ and its meso stereoisomer. ${ }^{220}$ The addition was carried out in the absence and presence of $\mathrm{Et}_{2} \mathrm{AlCl}$ in high yields and de (Scheme 70). Further functional group transformations provided dibenzyl-1,3-diamino-2-propanols, the core units of potent and selective HIV-1 protease inhibitors.

Scheme 70. Diastereodivergent Addition of Benzylmagnesium Bromide to Nitrone 195 

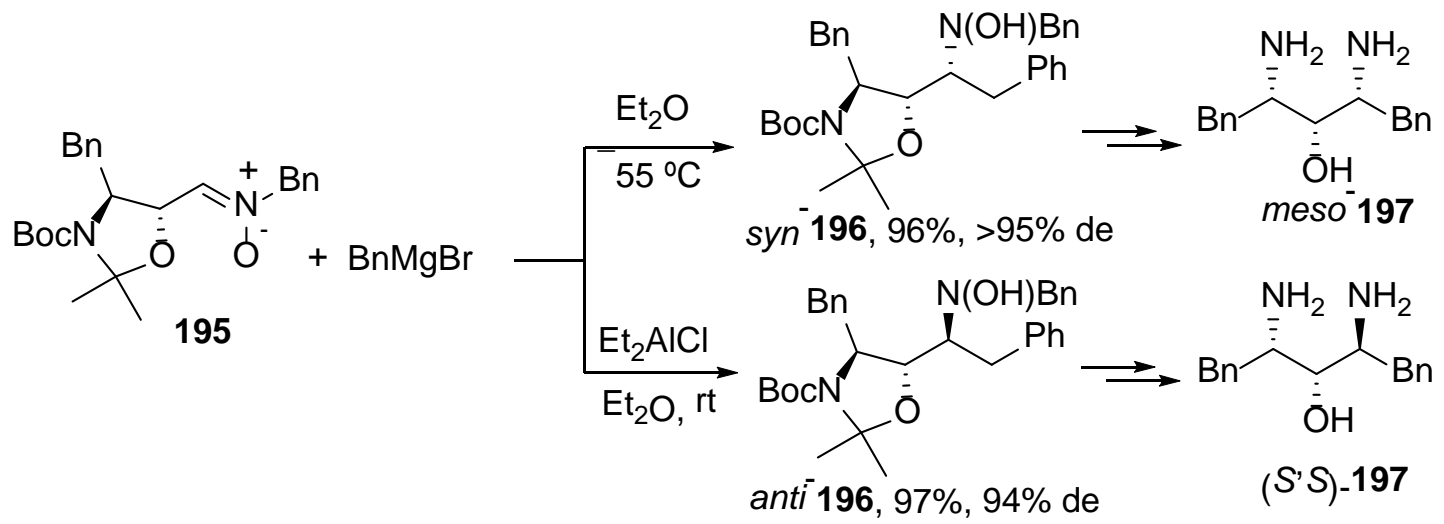

Reissig and co-workers have described the diastereodivergent addition of lithiated alkoxyallenes to carbohydrate-derived aldonitrones in the absence or presence of $\mathrm{Et}_{2} \mathrm{AlCl}$ giving syn or anti adducts, respectively. ${ }^{221,222}$ In both cases high de were obtained using D-glyceraldehyde $N$-benzyl and $N$-methyl nitrones 189. Instead of the expected formation of hydroxylamines a novel [3+3] cyclization takes place giving 4alkoxy-3,6-dihydro-2H-1,2-oxazines 198 (Scheme 71). ${ }^{21,222}$ This diastereodivergent methodology has been applied to the synthesis of differently configured C2-branched 4amino sugar derivatives. ${ }^{223}$

Scheme 71. Diastereodivergent Addition of Lithiated Alkoxyallenes to DGlyceraldehyde Nitrones 189

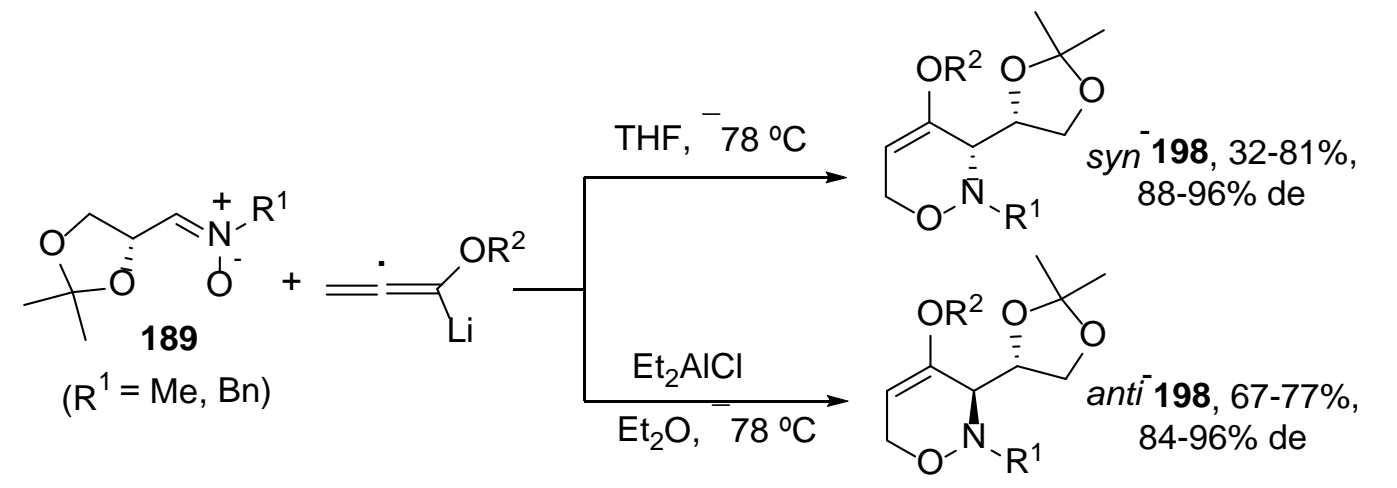

Employing $\alpha$-ethoxyvinyllithium as nucleophile and subsequent $O$-silylation, the corresponding open chain adducts 199 were isolated. Under similar reaction conditions syn or anti products were obtained. Further treatment of compounds 199 with TMSOTf gave diastereomeric 3,6-dihydro-2H-1,2-pyrans 200 (Scheme 72). ${ }^{224}$

Scheme 72. Diastereodivergent Addition of $\alpha$-Ethoxyvinyllithium to DGlyceraldehyde Nitrone 189 


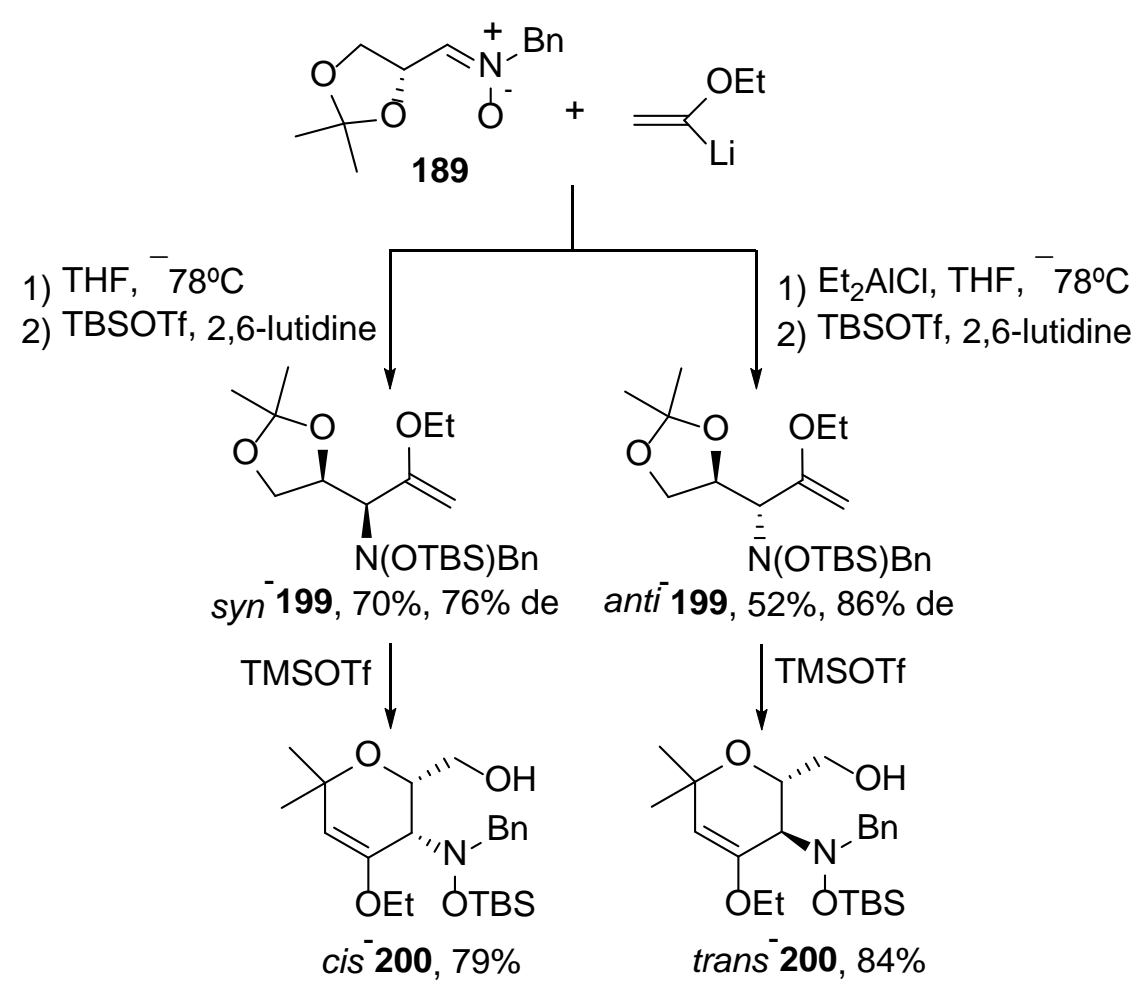

In conclusion, diastereodivergent addition of organometallics reagents to $\mathrm{N}$-tertbutylsulfinyl imines can be controlled either by the presence of Lewis acids, able to chelate the sulfinylimine unit, or without coordination. Efficient enantiodivergent additions of organometallic compounds have been achieved by changing the metal salt. In the case of nitrones, diastereodivergent processes can be achieved to the presence or absence of a Lewis acid under chelation or non-chelation conditions. The same mechanism can take place by using different types of Lewis acids.

2.2.2. Mannich Reactions. The Mannich reaction is a very important $C-C$ bond forming process, which allows the synthesis of $\beta$-amino carbonyl compounds. Asymmetric diastereoselective Mannich reaction with chiral imines has been widely investigated. ${ }^{225,226}$ Metal enolates can be diastereodivergently added depending on the substitution in the chiral skeleton of the imine, the base, the metal center, and the Lewis acid used. Recently, catalytic enantioselective Mannich-type reactions ${ }^{191,227-231}$ by means of chiral metal complexes and organocatalysts have been reported. In the first case, enantiodivergent processes have been carried out by changing the protecting group in the imine, also in the silyl enol ether, substituents in the imine backbone, substituents in homochiral ligands and the metal center. For enantiodivergent organocatalyzed reactions, substituents in the carbon nucleophile or in the organocatalysts and in the solvent used have been studied.

2.2.2.1. Metal-Catalyzed Mannich Reactions. The influence of the base as catalyst in the diastereodivergent addition of malonic acid derivatives to (S)- $N$-tertbutanesulfinyl-3,3,3-trifluoromethylacetaldimine (201) has been evaluated. ${ }^{232,233}$ In the presence of substoichiometric amounts of bases, $n \mathrm{BuLi}$ or DMAP, the reaction provided 
$\beta$-aminomalonates $\left(R, S_{\mathrm{S}}\right)$-202 with de up to $90 \%$ (Scheme 73 ). When phosphazene bases were employed, such as $\mathrm{P}_{2}$-Et, $\left(S, S_{S}\right)$-202 diastereomers, with de up to $98 \%$, were mainly obtained. The obtained stereochemical outcomes have been explained by a chelated TS 202A for the $\left(R, S_{\mathrm{S}}\right)$-202 and in the presence of phosphazene a 'naked' enolate is generated and a non-chelated TS 202B should operate.

Scheme 73. Diastereodivergent Mannich Reaction of Malonates with Trifluoroacetaldehyde-Derived $\mathbf{N}$-tert-Butylsulfinyl Imine 201

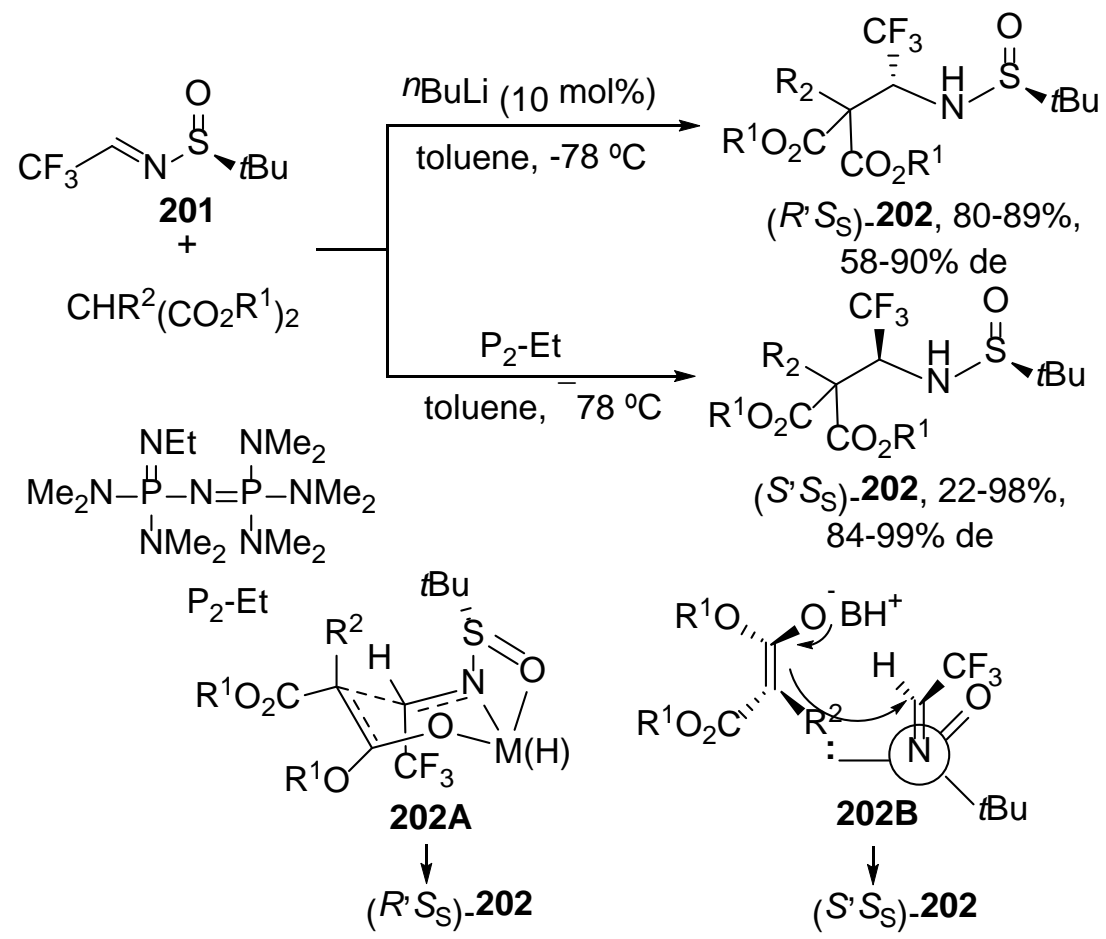

A vinylogous Mannich-type reaction catalyzed by Lewis acids has been performed between $N$-tert-butylsulfinylimino acetates 203 and dioxinone-derived silyl dienolate 204. ${ }^{234}$ This process resulted by a regioselective and diastereodivergent reaction pathway depending on the appropriate choice of the Lewis acid. The $\gamma$-regioselectivity can be controlled by AgOTf, $\mathrm{AgClO}_{4}$, CuOTf, $\mathrm{Cu}(\mathrm{OTf})_{2}$ and $\mathrm{Zn}(\mathrm{OTf})_{2}$, whereas $\alpha$ regioisomers are mainly obtained with $\mathrm{AgOAc}, \mathrm{AgNO}_{3}$ and AgTFA. Concerning the diastereoselectivity, in the case of the $\gamma$-products, AgOTf afforded $\left(S, R_{\mathrm{S}}\right)$-205 isomers and $\mathrm{Zn}(\mathrm{OTf})_{2}$ gave mainly $\left(R, R_{\mathrm{S}}\right)$-205 (Scheme 74$)$. Under the silver-catalysis larger anions such as $\mathrm{TfO}^{-}$and $\mathrm{ClO}_{4}{ }^{-}$inhibit the approach of OTMS to silver and the sulfinyl nitrogen, allowing the oxygen atoms from the sulfinyl and ester groups to afford the $\gamma$ isomer due to a Si-attack (TS 205A). For the later catalyst the TS 205B with Zn coordinating the oxygen of the ester and the imine nitrogen atom, has been proposed.

Scheme 74. Diastereodivergent Mannich Reaction of Dioxinone-Derived Silyl Dienolate 204 with $N$-tert-Butanesulfinyl Imines 203 Catalyzed by Lewis Acids 


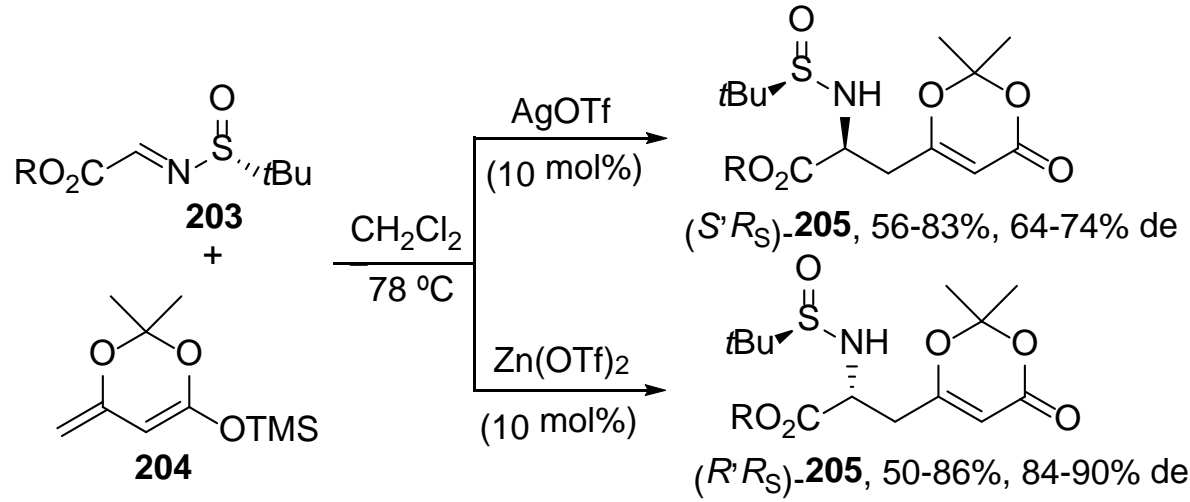

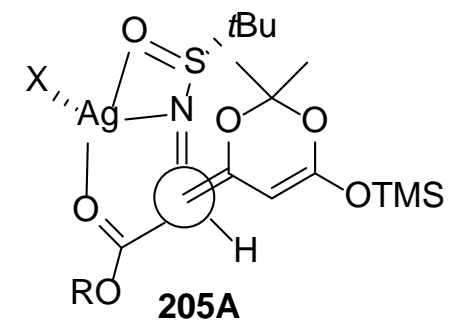

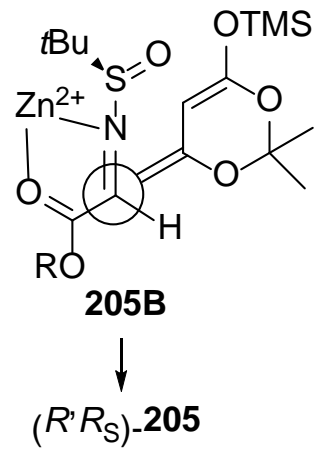

In a three-component phenolic Mannich-type reaction a dramatic temperaturedependent diastereodivergence has been observed. ${ }^{235}$ Electron-rich phenols, a primary amine and chiral $\alpha$-dibenzylamino aldehydes gave o-1,2-diaminoalkyl phenols (Scheme 75). The process takes place under $\mathrm{Yb}(\mathrm{OTf})_{3}$ catalysis giving products anti-206 with de up to $>99 \%$ at $-20^{\circ} \mathrm{C}$. In the reaction between $20^{\circ} \mathrm{C}$ and $60{ }^{\circ} \mathrm{C}$, syn-206 were obtained with de up to $99 \%$.

Scheme 75. Diastereodivergent Three Components Mannich Reaction of Phenols with Aldimines Generated in situ from $\alpha$-(N,N-Dibenzylamino) Aldehydes 129 Catalyzed by $\mathbf{Y b}(\mathrm{OTf})_{3}$

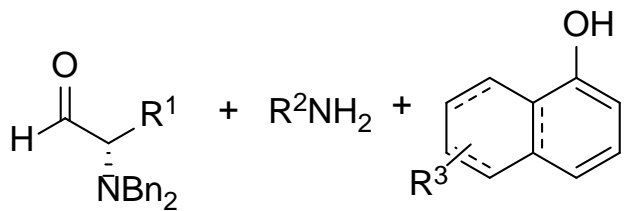

129

$\mathrm{Yb}(\mathrm{OTf}) 3(10 \mathrm{~mol} \%)$

$\mathrm{Na}_{2} \mathrm{SO}_{4}, \mathrm{DCM}$ or DCE

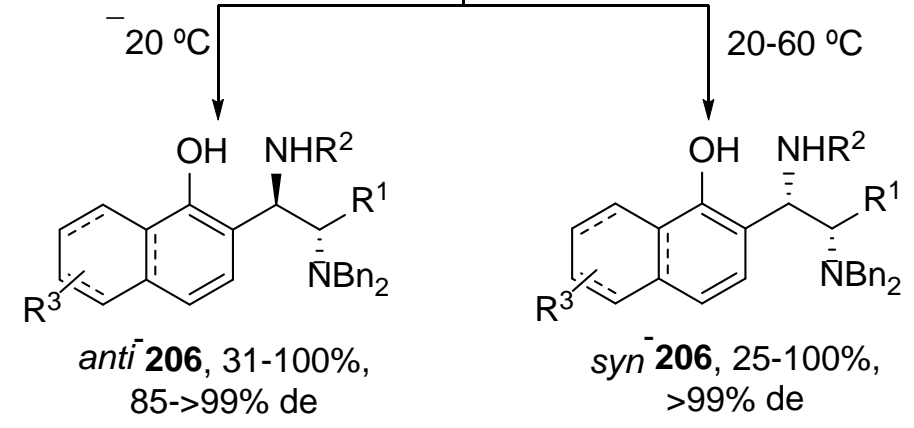


Chiral nitrones such as $\mathbf{1 8 9}$, have been used as acceptors of $\alpha$-silylketene acetal 207, a diastereodivergent Mannich-type reaction took place depending on the Lewis acid employed. ${ }^{236}$ Using Zn(OTf) 2 catalysis diastereomer (3S,4S)-208 was obtained in 94\% de after desilylation with citric acid. When $\mathrm{SnCl}_{4}$ was used as Lewis acid, the reaction gave (3R,4S)-208 in lower diastereoselectivity (Scheme 76). The major adducts were used for the preparation of polyhydroxy $\beta$-amino esters, precursors of $\mathrm{D}$ - and L-erythrosphingosines.

Scheme 76. Diastereodivergent Mannich Reaction of 2-Silyloxy Silyl Ketene Acetal 207 with D-Glyceraldehyde Nitrone 189 Catalyzed by Lewis Acids

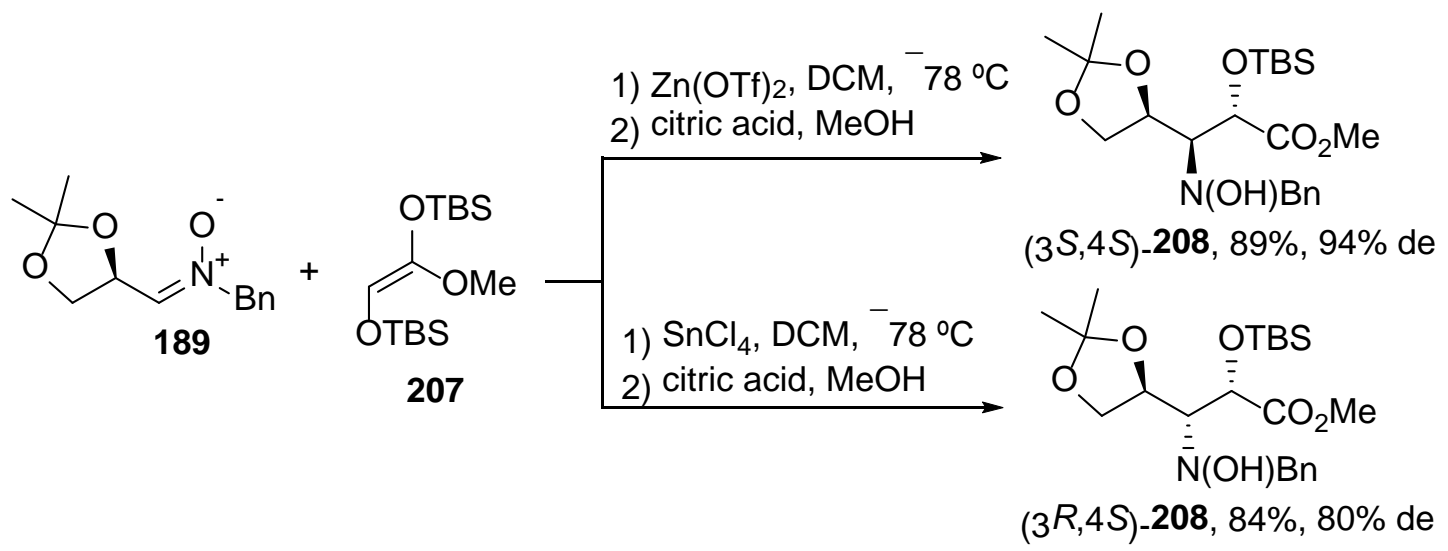

The first direct metal-catalyzed asymmetric Mannich reaction was described by Shibasaki and co-workers using propiophenone, formaldehyde and pyrrolidine in a three- component process catalyzed by $(R)$-LaLi 3 tris(binaphthoxide) complex 119 (Scheme 40). ${ }^{237}$ Low chemical yield and enantioselectivity were obtained. However, diastereodivergent results were observed depending on the protection of the imine. Thus, working with $\alpha$-hydroxy aromatic ketone 211 as a nucleophile and $N$ diphenylphosphinoyl (Dpp) imines 209 or $N$-Boc imines 210, in the presence of catalyst formed by $(S, S)$-linked BINOL ligand 212 and $\mathrm{Et}_{2} \mathrm{Zn}$, anti-213 or syn-214 adducts were obtained, respectively (Scheme 77). ${ }^{238,239}$ The substituent effects in the stereocontrol of this diastereodivergent Mannich reaction have been explained by the participation of a zinc enolate 215. In the case of the $N$-Dpp imines, TS 213A with less interactions between the aromatic ring of the ketone and the protecting group controls the formation of anti-213. For $N$-Boc imines, the bulky tert-butyl group and the R group in the imine forces the formation of syn-214 through TS 214A.

Scheme 77. Diastereodivergent Mannich Reaction of $\alpha$-Hydroxy o-Methoxy Acetophenone with Different $N$-Protected Imines Catalyzed by Chiral $\mathrm{Zn} /(S, S)$ Linked BINOL 212 Complex 


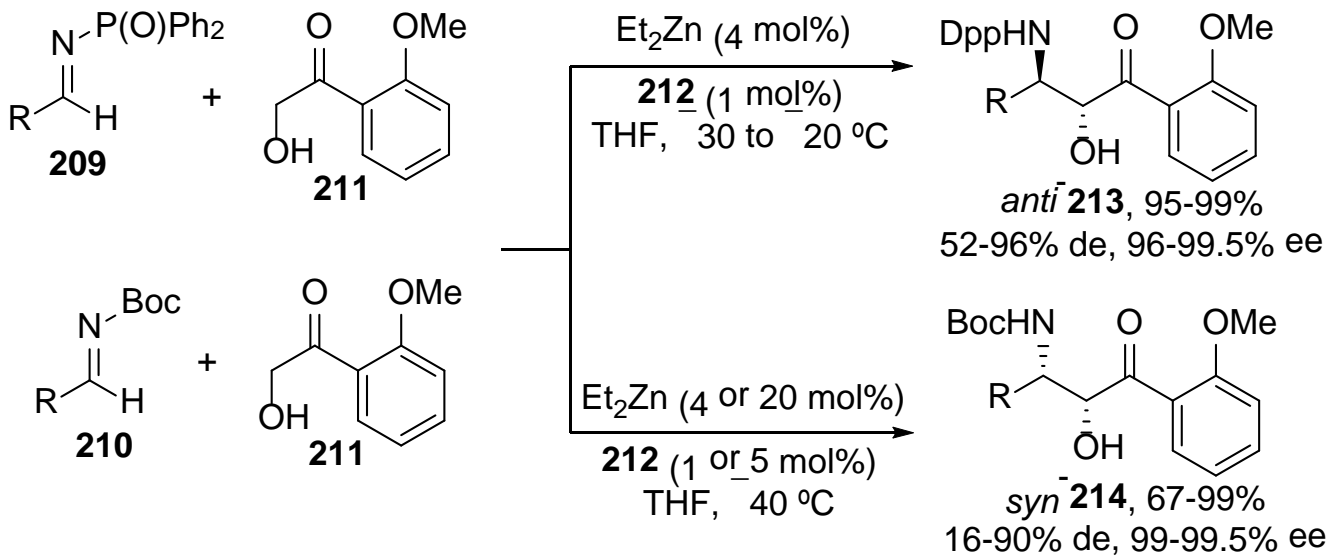<smiles>Oc1ccc2ccccc2c1-c1c(O)c(O)c(COCc2ccc3ccccc3c2)c(-c2c(O)ccc3ccccc23)c1O</smiles><smiles>COc1ccccc1C1=CO[Ge]O1</smiles>

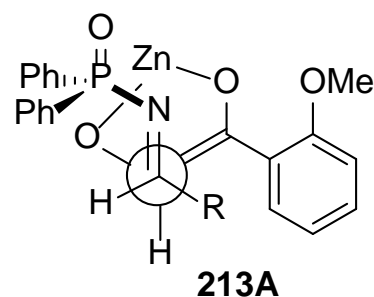

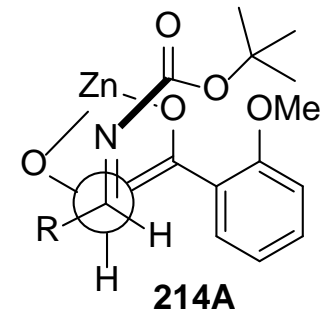

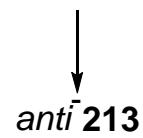<smiles>C[14C](C)S</smiles>

The influence of protecting groups in the diastereoselectivity of metal-catalyzed asymmetric Mannich reactions was previously described by Kobayashi and co-workers for the addition of $\alpha$-oxygenated silyl ketene acetals 217 to aldimines $216 .{ }^{240}$ For example, in the reaction of 216 with Z-ketene acetal 217 (PG = TBS) the syn-219 product was exclusively obtained using Zr-complex 218 as catalyst. On the other hand, for $217(\mathrm{PG}=\mathrm{Bn})$ the anti-isomer 219 was mainly formed (Scheme 78).

Scheme 78. Diastereodivergent Mannich Reaction of $\alpha$-Oxygenated Silyl Ketene Acetals 217 with Aldimine 216 Catalyzed by Chiral Zr Chiral Complex 218 


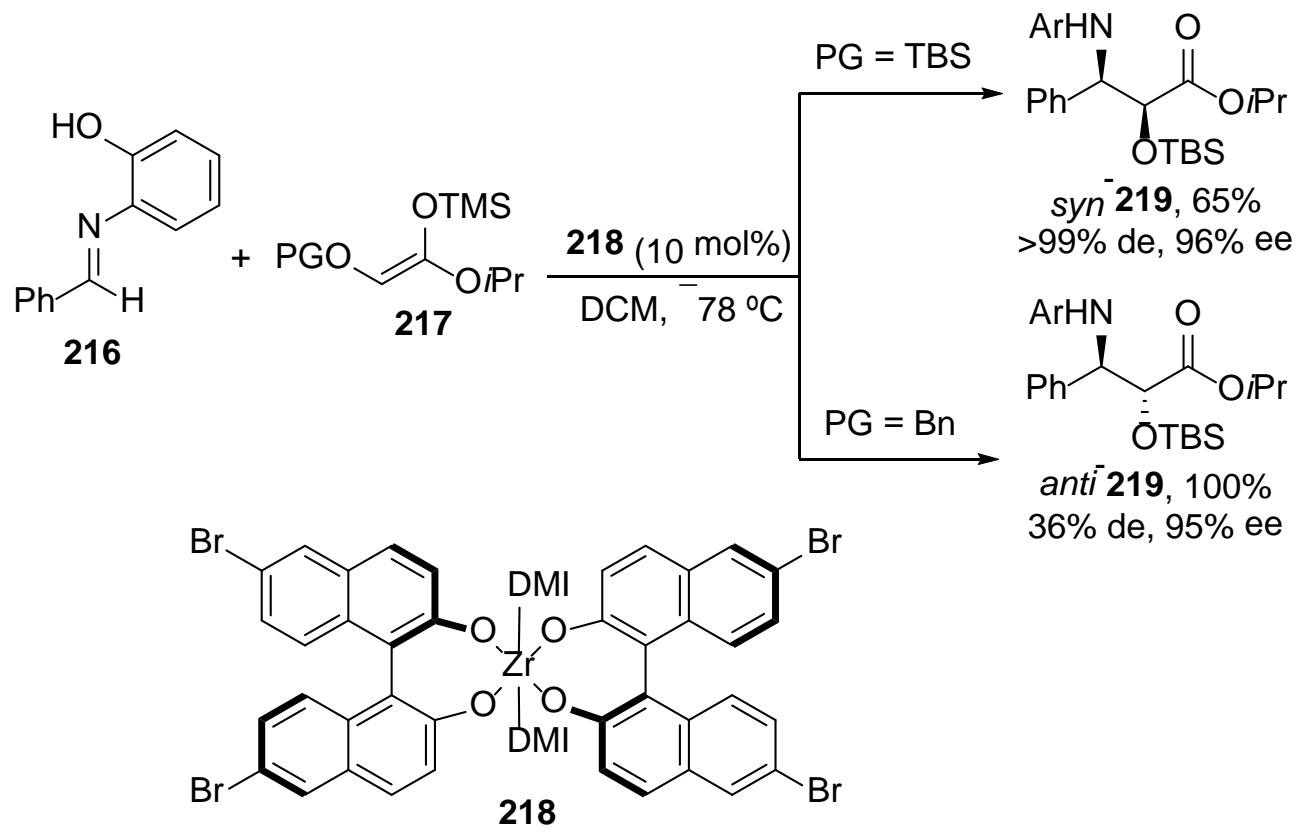

Trost and co-workers have used the Zn-complex 220 of ligand 91 (Scheme 27), for the Mannich reaction of $N$-Dpp-209 and $N$-Boc-imines 210. ${ }^{241}$ Diastereoselective addition of $\alpha$-hydroxy acetophenone to 209, even derived from aliphatic aldehydes, provided anti-221 in high 57-99\% ee. Diastereodivergently, imines $\mathbf{2 1 0}$ gave syn-amino alcohols 221 in moderate diastereoselectivity and high enantioselectivity (90-95\%) (Scheme 79).

Scheme 79. Diastereo- and Enantiodivergent Mannich Reaction of $\alpha$-Hydroxy Acetophenone with $N$-Dpp and $N$-Boc Aldimines Catalyzed by Zn Chiral Complex 220

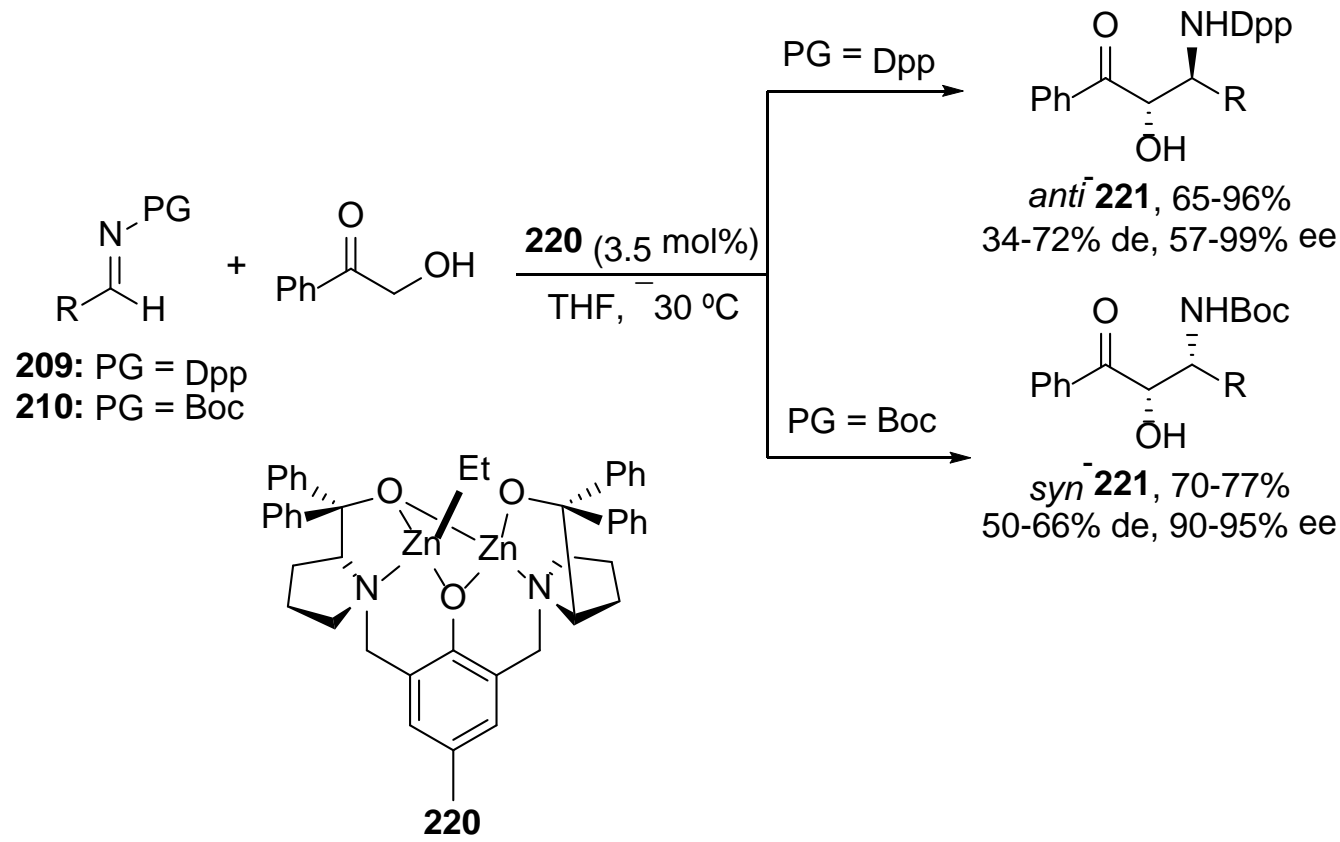


Shibasaki and co-workers observed diastereodivergent results depending on the substitution pattern of $\mathrm{N}$-o-tosyl aldimines 222. ${ }^{242}$ Mannich condensation of $\mathrm{N}$-(2hydroxyacetyl)pyrrole 223, catalyzed by the In complex of $(S, S)$-linked BINOL 212 (Scheme 77), with imines bearing styryl, phenyl and 4-chlorophenyl groups, gave mainly syn-adducts 224 (with de up to $82 \%$ and ee up to 97\%). On the other hand, naphthyl and $o$-substituted aryl groups as well as cyclopropyl, favored the formation of anti-224 (with de up to $72 \%$ and ee up to 98\%) (Scheme 80 ). Transition state 224A and 224B have been proposed by the authors to explain the observed diastereoselectivity and the same enantiofacial control in the imine moiety. The utility of the $N$-acylpyrrole unit as ester surrogate has been demonstrated through several functional group interconversions.

\section{Scheme 80. Diastereodivergent Mannich Reaction of $\alpha$-(Hydroxyacetyl)pyrrole with $N$-o-Tosyl Aldimines Catalyzed by Chiral In Complex from $(S, S)$-Linked BINOL 212 Complex}

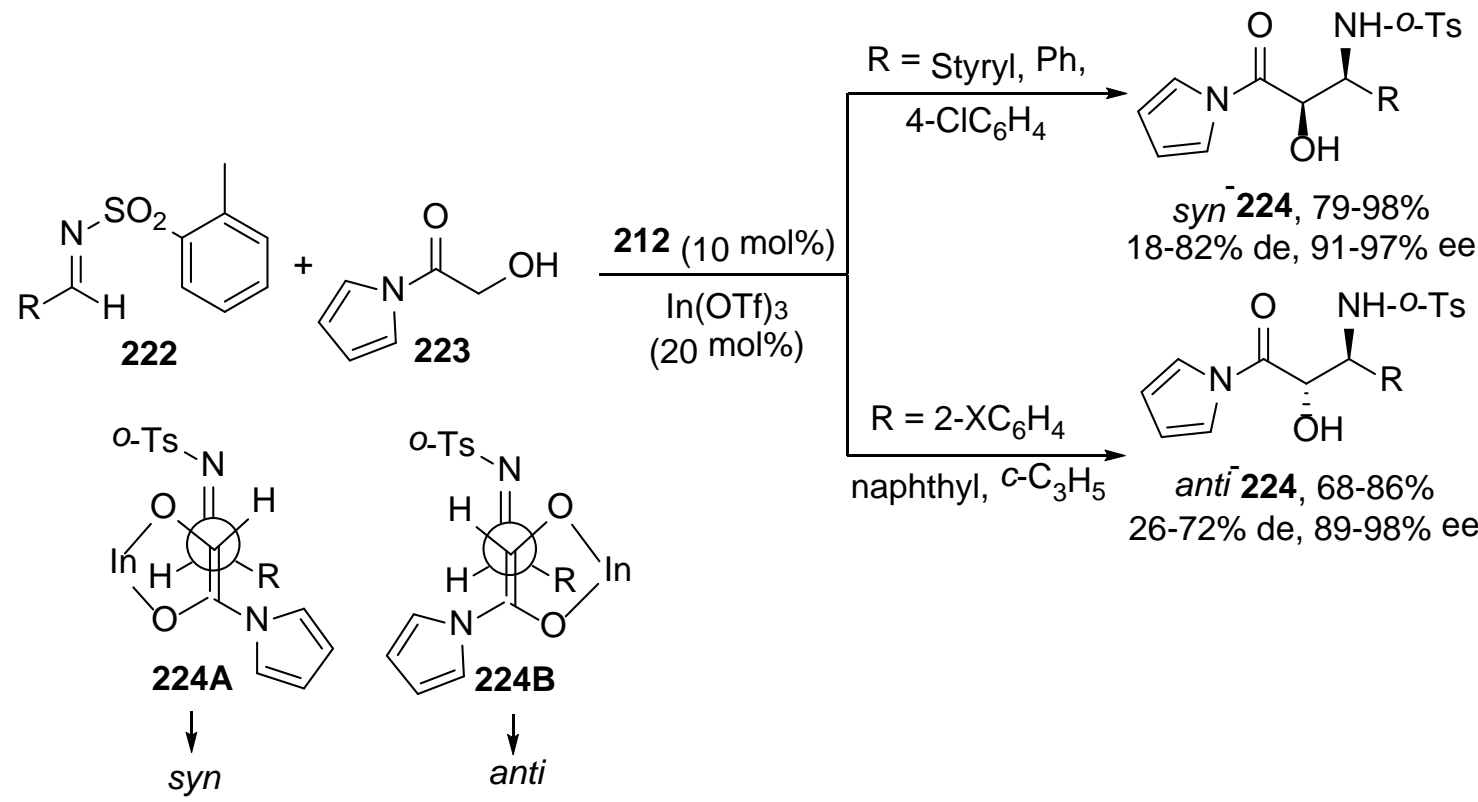

$N$-Tosyl imines 225, derived from aliphatic and aromatic aldehydes, have been used as Mannich acceptors in the diastereo- and enantiodivergent reaction with benzophenone-derived glycine methyl ester 226a catalyzed by chiral $\mathrm{Cu}(\mathrm{I})$ complexes. $^{243}$ In this case, the electronic properties of the chiral ligands 227 was crucial for the switching of the diastereoselectivity (Scheme 81). Using ligand 227a $(\mathrm{Ar}=4-$ $\mathrm{MeOC}_{6} \mathrm{H}_{4}$ ), with an electron-donating substituent at the 4-position, anti-adducts 228 were mainly formed. However, in the case of the ligand with a 3,5-difluorophenyl group 227b, syn selectivity was observed. Both diastereomeric adducts were obtained with the same (S)-configuration at the $\alpha$-position of the $\alpha, \beta$-diamino acid derivatives in excellent enantioselectivities. In addition, a reversal of the diastereoselectivity took place when the substituent in the imine 225 is $o-\mathrm{BrC}_{6} \mathrm{H}_{4}$ and using ligand 227a, affording the synadduct 228. 
Scheme 81. Diastereodivergent Mannich Reaction of Benzophenone Imine Glycine Methyl Ester with $\mathrm{N}$-Tosyl Aldimines Catalyzed by Chiral $\mathrm{CuClO}_{4} \mathrm{Complexes} \mathrm{of}$ Ligands 227

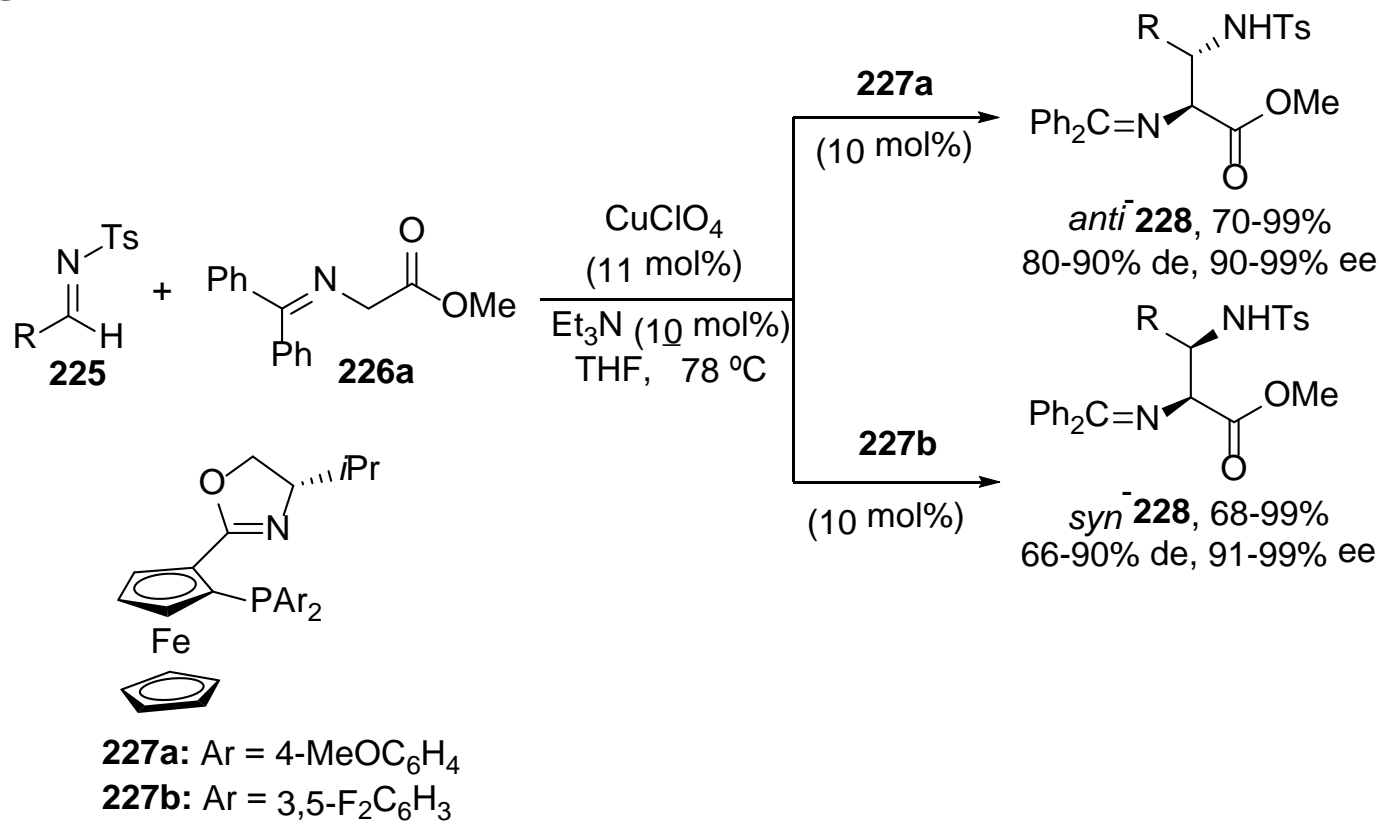

Diastereodivergent addition of $\alpha$-cyanocyclopentanone 229 to $N$-Boc-benzaldimine 210a takes place using rare earth metal salts as Lewis acids with the amide-based ligand 230 (Scheme 82). ${ }^{244,245}$ A 2:1 mixture of 230 and the $\mathrm{Sc}(\mathrm{OiPr})_{3}$ gave the anti-product 231a, whereas $\operatorname{Er}(\mathrm{OiPr})_{3}$ provided syn-231a under identical reaction conditions (ee not provided). The scope of this diastereodivergent process was not further studied.

Scheme 82. Diastereodivergent Mannich Reaction of $\alpha$-Cyanocyclopentanone 229 with $N$-Boc-Benzaldimine Catalyzed by Sc and Er Alkoxides Complexes and Chiral Ligand 230 

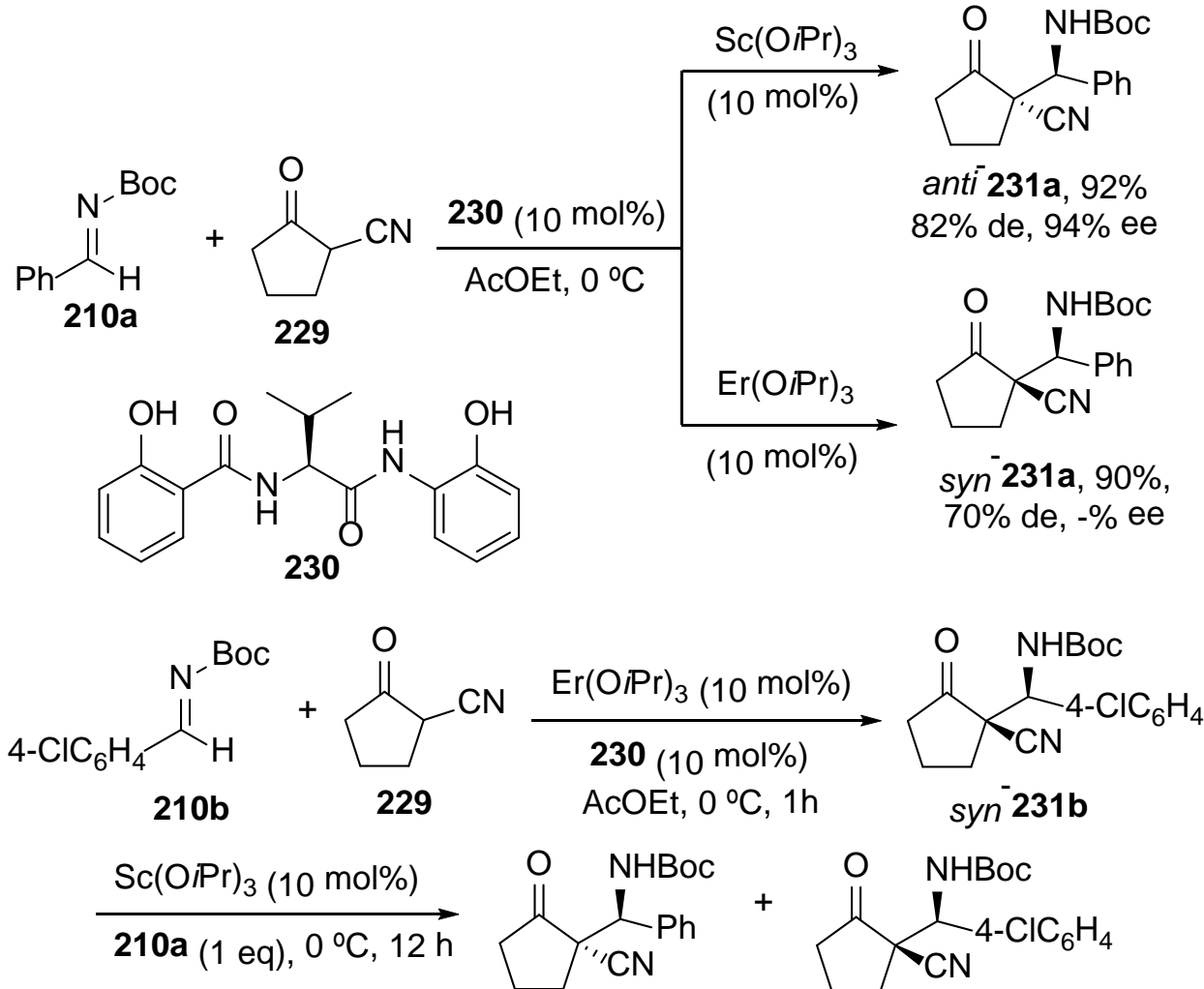<smiles>CC(C)(C)OC(=O)N[C@@H](N)C1(C#N)CCCC1=O</smiles>

anti $231 \mathrm{a}, 88 \%$, $80 \%$ de, $91 \%$ ee

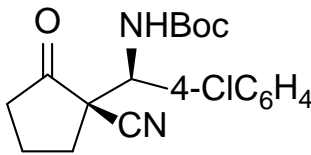

syn ${ }^{-231 b}, 85 \%$, $82 \%$ de, $98 \%$ ee

Sequential diastereodivergent catalysis ${ }^{246}$ was observed in the Mannich reaction of imine 210b with 229 using $\mathrm{Er}(\mathrm{OiPr})_{3} / 230$ as catalysts giving syn-231b. When $\mathrm{Sc}(\mathrm{OiPr})_{3}$ and imine 210a were added to this reaction medium, a mixture of syn-adduct $\mathbf{2 3 1 \mathbf { b }}$ in 91\% ee and anti-231a in 98\% ee were obtained (Scheme 82).

The Matsunaga and Shibasaki group has described a metal-induced diastereodivergent Mannich-type reaction of $N$-Dpp-ketimines 232 with $\alpha$-methyl- $\alpha$ isothiocyanate methyl ester (233) catalyzed by a salen ligand 234 and $\mathrm{Sr}$ or $\mathrm{Mg}$ as metal centers. ${ }^{247-249}$ By using $\mathrm{Sr}(\mathrm{OiPr})_{3}$ anti-products 235 were formed with de up to $92 \%$ and ee up to $97 \%$, while $n$ - $\mathrm{Bu}_{2} \mathrm{Mg}$ gave syn-235 with de up to $86 \%$ and ee up to $95 \%$ (Scheme 83). These cyclic thioureas have been further transformed into thioimidazolines.

Scheme 83. Diastereodivergent Mannich-Type Reaction of $\alpha$-Methyl- $\alpha$ isothiocyanate Methyl Ester with $N$-Dpp Ketimines Catalyzed by $\mathrm{Sr}$ and $\mathrm{Mg}$ Complexes with Chiral Ligand 234 


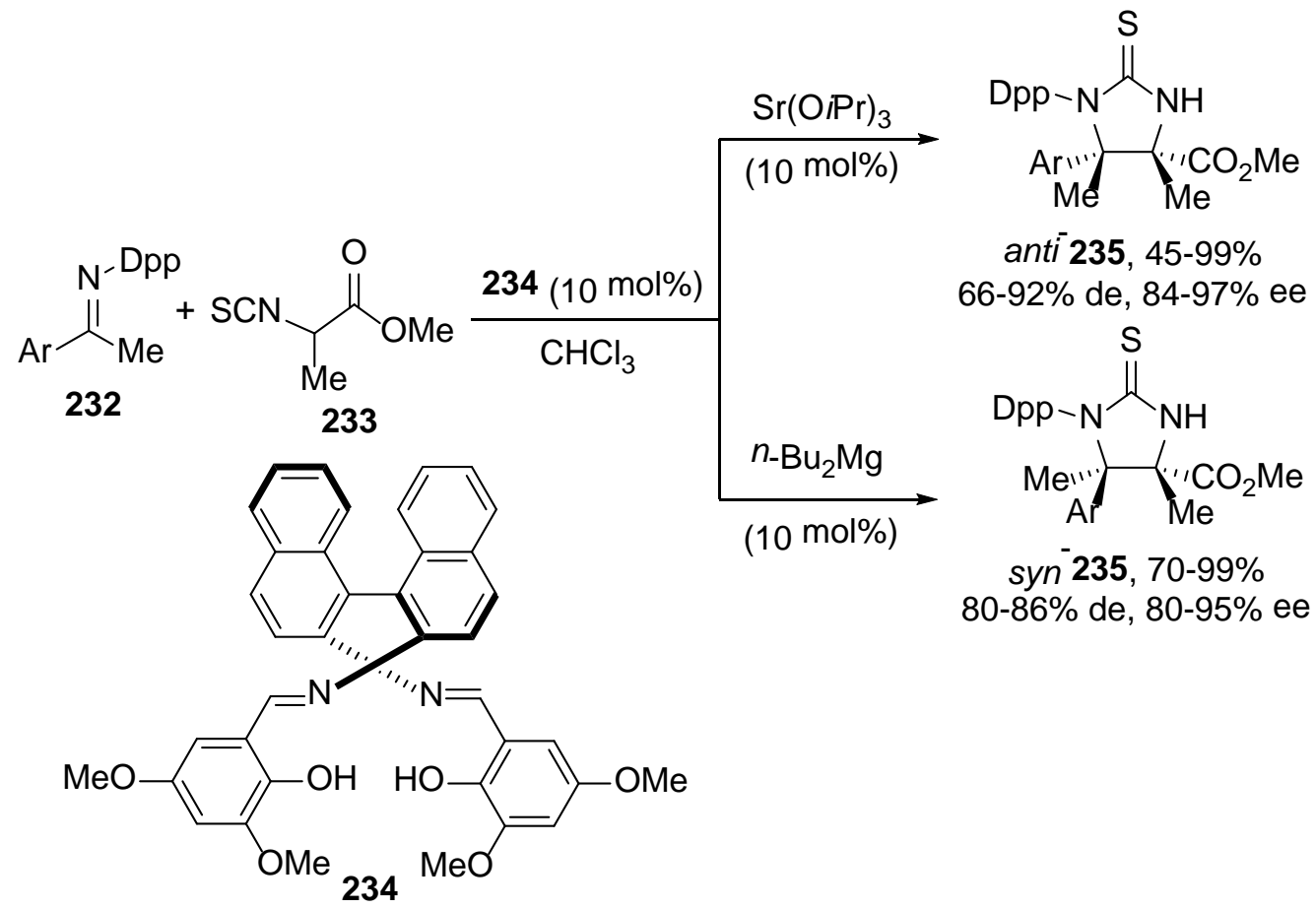

Reversal of enantioselectivity has been achieved in the addition of indole to $\mathrm{N}$ tosylarylimines 225 catalyzed by N-heterocyclic carbene (NHC) Pd(II) complexes 236 by changing the R substituent in the NHC ligand (Scheme 84 ). ${ }^{250}$ In the case of complex 236a $(\mathrm{R}=\mathrm{H})$ adducts 237 with $R$-configuration were obtained with ee up to $66 \%$ and using complex 236b $\left(\mathrm{R}=\mathrm{CF}_{3}\right)$, $(S)$-237 were produced with ee up to $74 \%$.

Scheme 84. Enantiodivergent Mannich-Type Reaction of $\mathbf{N}$-Tosyl Aldimines with Indole Catalyzed by Chiral NHC Pd(II) Complexes 236

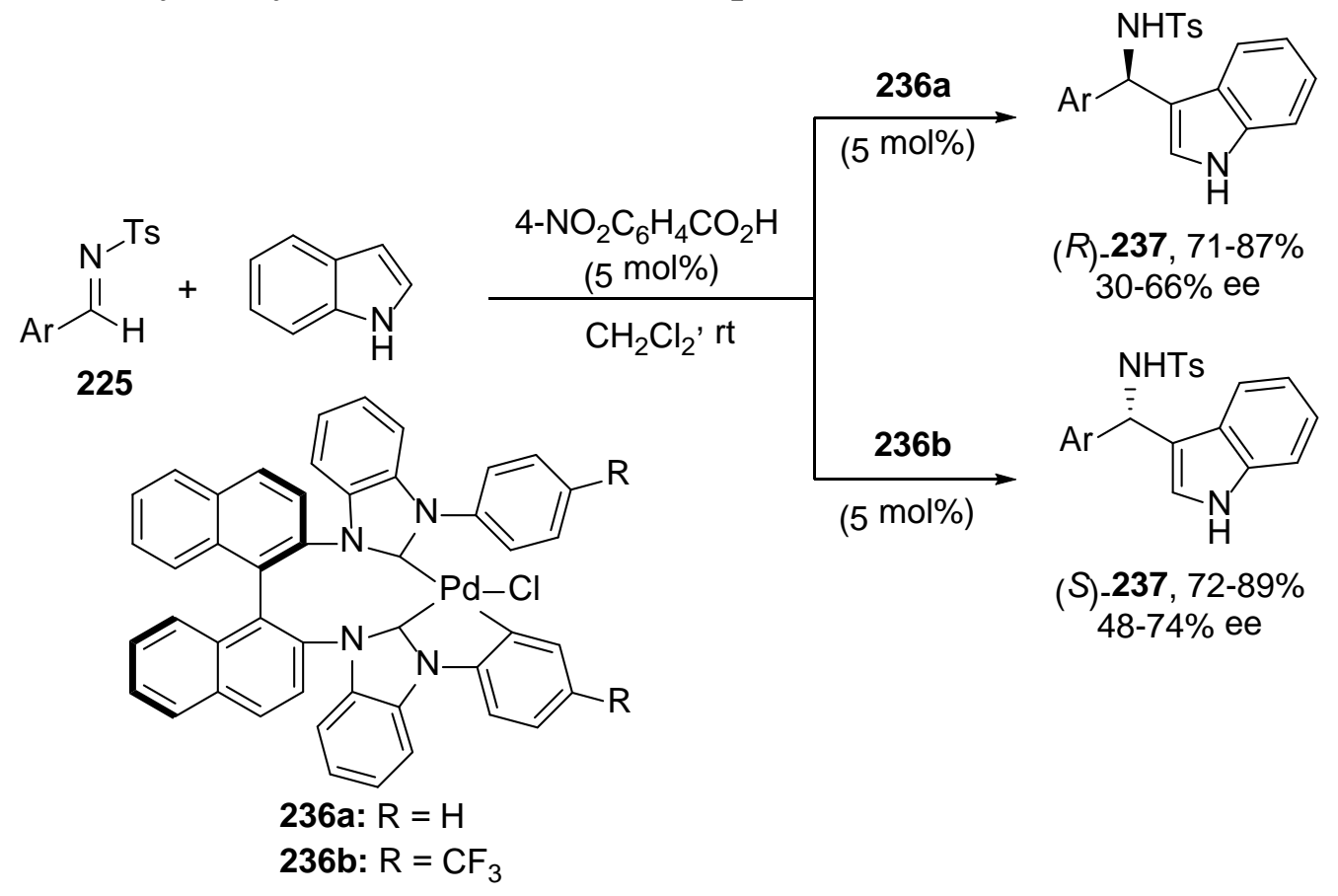


Sulfonylimidate 238 has been used as a new nucleophile for the Mannich reaction with $N$-Boc imines 210 catalyzed by DBU giving adducts 240 in high yields and antidiastereoselectivity. Diastereodivergent results were observed in the presence of ligand 239 and by using as alkali earth metal alkoxide $\mathrm{Mg}(\mathrm{O} t \mathrm{Bu})_{2}$ in DMF, providing antiproducts $\mathbf{2 4 0}$, while $\left[\mathrm{Sr}(\mathrm{HMDS})_{2}\right]_{2}$ in THF gave syn selectivity (Scheme 85$){ }^{251}$

Scheme 85. Diastereodivergent Mannich-Type Reaction of Sulfonylimidate 238 with $N$-Boc Aldimines Catalyzed by Alkali Earth Metal Salts

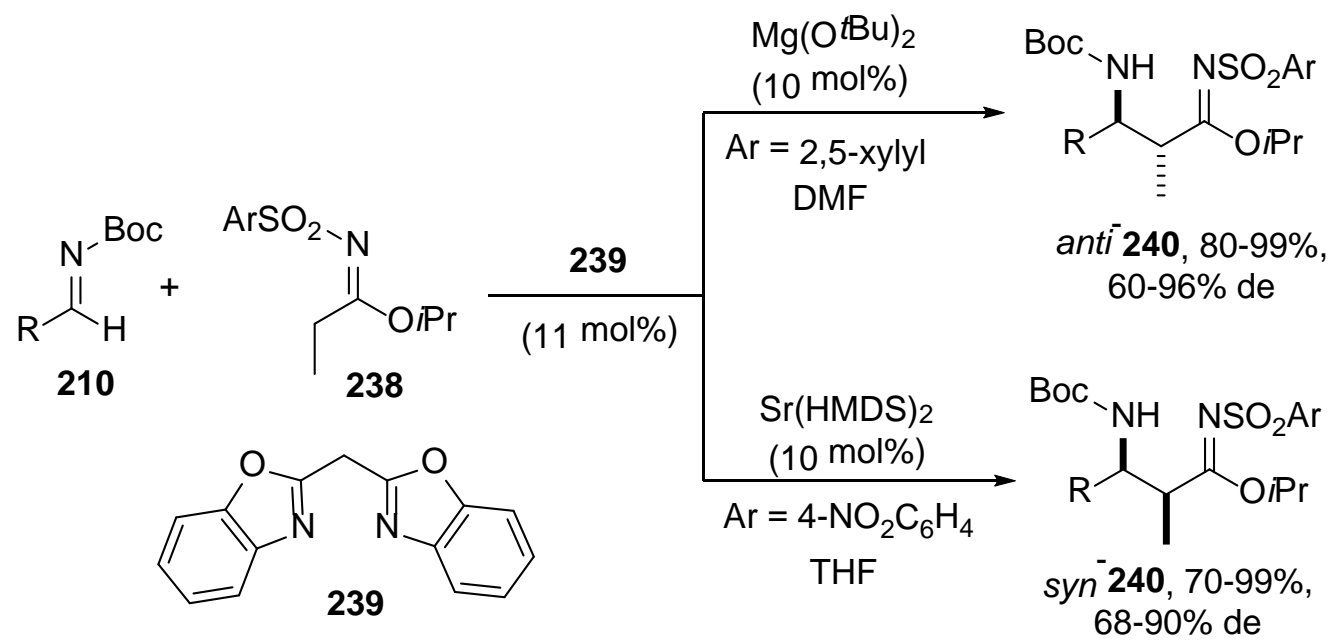

2.2.2.2. Organocatalyzed Mannich Reactions. Enantioselective organocatalyzed Mannich reactions were initially studied using L-Pro ${ }^{252,253}$ as catalyst in a proper threecomponent process between acetone, 4-nitrobenzaldehyde and 4-anisidine to give the corresponding Mannich product in 94\% ee. In the case of $\alpha$-hydroxyacetone, isobutanal and 4 -anisidine mainly the syn-adduct was obtained in $89 \%$ de and $65 \%$ ee. Since then, a high number of publications about organocatalyzed Mannich reaction were described. ${ }^{228}$ However, few cases of stereodivergent transformations have been found. The first example was the diastereodivergent addition of benzyl $\alpha$-aryl cyanoacetates 242 to $N$-Boc $\alpha$-imino esters 241 organocatalyzed by (DHQD) ${ }_{2}$ PYR 243 (Scheme 86). ${ }^{254}$ Only in the case of 2-bromophenyl cyanoacetate an anomalous inversion of the diastereoselectivity was observed for products 244 (ee was not reported for syn-244).

Scheme 86. Diastereodivergent Mannich-Type Reaction of Benzyl $\alpha$-Cyanoacetate with $N$-Boc $\alpha$-Imino Ester Organocatalyzed by Chiral (DHQD) ${ }_{2}$ PYR 243 


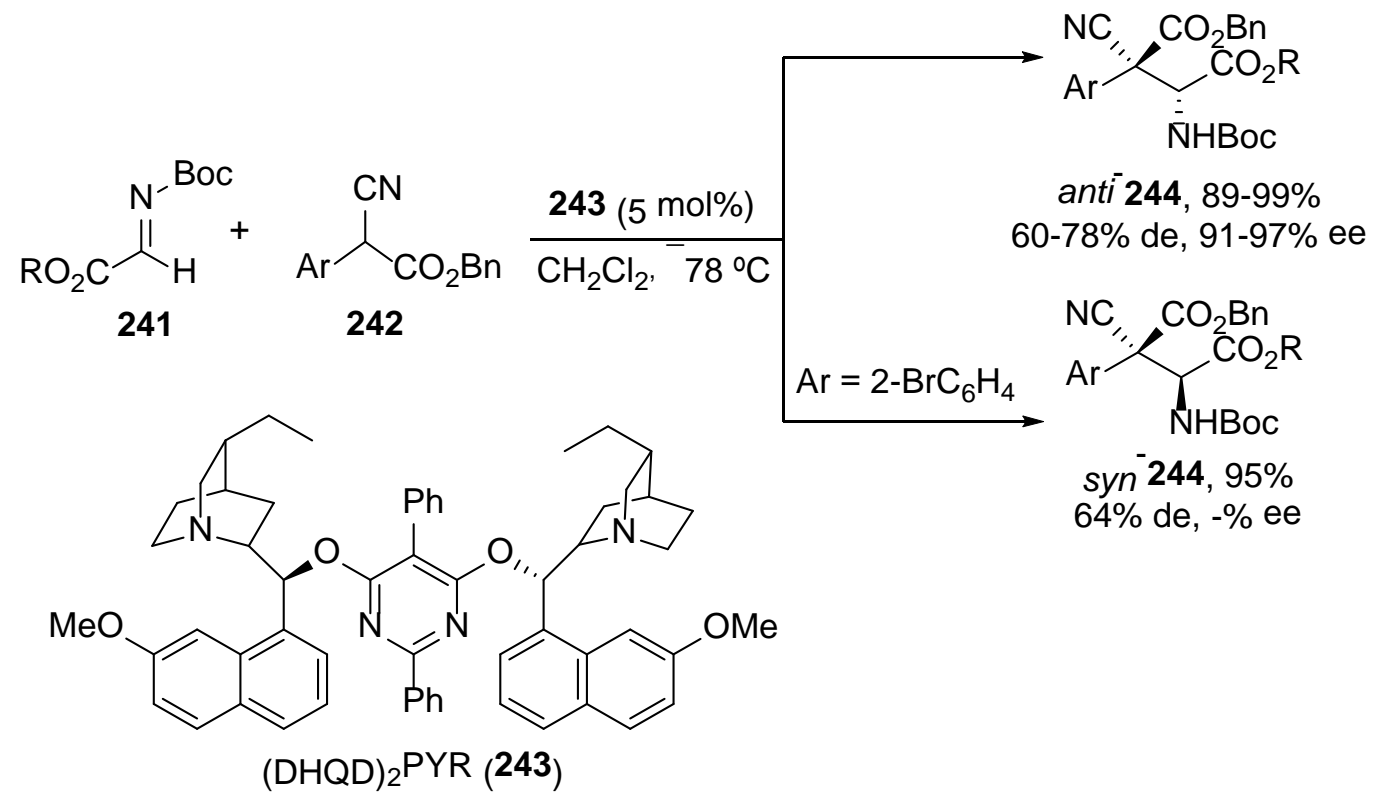

Ishihara and co-workers have reported the use of chiral phosphoric acids and their calcium salts as catalysts for the enantiodivergent Mannich reaction between $N$-Boc benzaldimine 210a and 1,3-dicarbonyl compounds. ${ }^{255}$ The best reversal of enantioselectivities for compounds $(S)$ - and $(R)$-247 derived from acetylacetone were obtained with free phosphoric acid 245 (93\% ee) and its Ca salt 246 (92\% ee), respectively (Scheme 87 ). The scope of this enantiodivergent reaction was studied with the organocatalyst 245 and acyclic 1,3-diketones giving compounds such as (S)-247 in 92-95\% ee. When cyclic $\beta$-diketones and $\beta$-keto esters were employed good diastereoselectivities (syn/anti: >10/90) and enantioselectivities for the anti-adduct (95$98 \%$ ee) were achieved. In the case of catalyst 246, products $(R)-247$ were mainly formed, the best results being obtained with $\beta$-keto thioesters (90-98\% ee). These adducts were further transformed into different functionalities. This is a good example of the use of chiral Brønsted acid and its salt as catalysts. ${ }^{256}$

Scheme 87. Enantiodivergent Mannich Reaction of Acetylacetone with $N$-BocBenzaldimine Catalyzed by Chiral Phosphoric Acid 245 and its Calcium Salt 246 


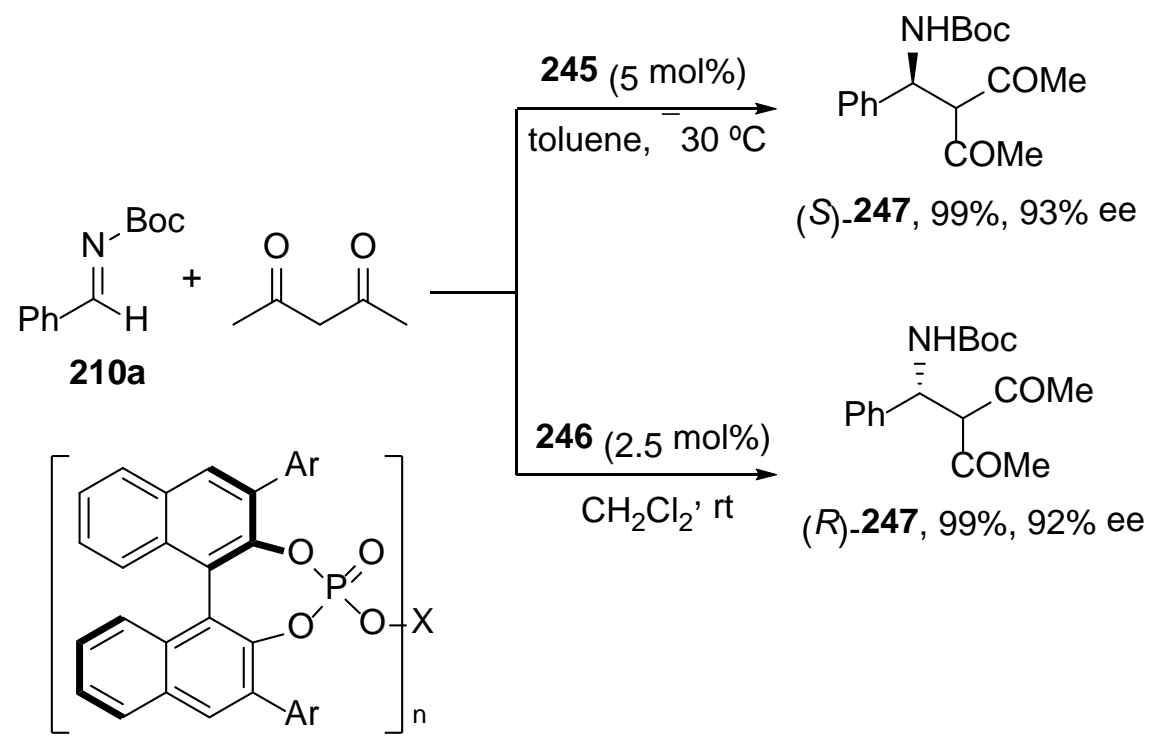

245: $X=H, A r=9$-anthryl, $n=1$

246: $X=C a, A r=4-(2-$ naphthyl), $n=2$

An interesting example of enantiodivergent Mannich reaction was observed by using a single organocatalysts $\mathbf{2 4 9}$ derived from the ester $\mathbf{1 0 3}$ (Scheme 32) in the presence or absence of achiral acids as additives. ${ }^{257}$ The addition of ketones to cyclic imino ester 248 in DMF in the absence of acid gave mainly the corresponding adducts anti-250 with de up to $90 \%$ and in high enantioselectivities (Scheme 88). On the other hand, in the presence of 2,6-dinitrobenzoic acid (10 mol\%) a reversal of enantioselectivity was achieved. Products $\mathbf{2 5 0}$ can be deprotected to give enantioenriched free $\alpha$-amino acids by hydrogenation.

Scheme 88. Enantiodivergent Mannich Reaction of Ketones with Cyclic Imino Ester 248 by Means of a cis-Diamine-Based Chiral Organocatalyst 249 
<smiles>O=C1C=NC(c2ccccc2)(c2ccccc2)CO1</smiles>

248

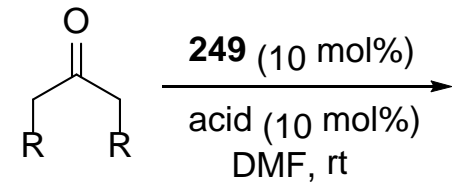

$\mathrm{H}_{2} \mathrm{~N}$<smiles>N[C@H]1CCC(C(=O)O)C[C@H]1N</smiles><smiles>[R]CC(=O)C([R])C1NC(c2ccccc2)(c2ccccc2)COC1=O</smiles>

anti 250

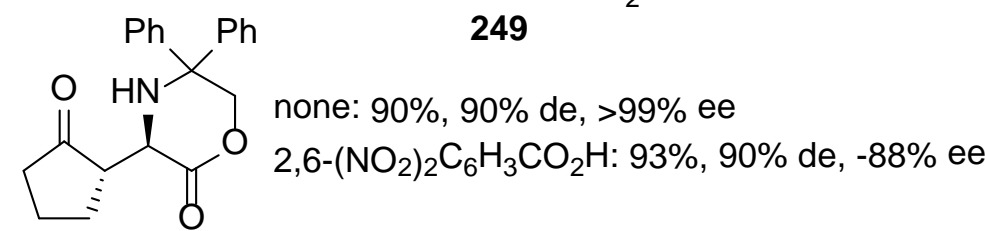

$\mathrm{Ph} \mathrm{Ph}$<smiles>O=C1CCCC[C@H]1[C@@H]1NC(c2ccccc2)(c2ccccc2)COC1=O</smiles>

none: $98 \%, 90 \%$ de, $>99 \%$ ee 2,6- $\left(\mathrm{NO}_{2}\right)_{2} \mathrm{C}_{6} \mathrm{H}_{3} \mathrm{CO}_{2} \mathrm{H}: 93 \%, 90 \%$ de, $-83 \%$ ee<smiles>CCC(=O)C(C)C1NC([In])(c2ccccc2)COC1=O</smiles>

none: $70 \%, 88 \%$ de, $98 \%$ ee

$2,6-\left(\mathrm{NO}_{2}\right)_{2} \mathrm{C}_{6} \mathrm{H}_{3} \mathrm{CO}_{2} \mathrm{H}: 74 \%, 89 \%$ de, $-87 \%$ ee

Sohtome and Nagasawa have developed a strategy for the enantiodivergent Mannich reaction of $\mathrm{N}$-Boc aldimines $\mathbf{2 1 0}$ with malonates using a conformationally flexible guanidine/bisthiourea organocatalyst 251. In this case, enormous solvent-dependent reversal of the enantioselectivity was observed: in toluene or $m$-xylene afforded products 252 with $(S)$-configuration (87-97\% ee), whereas in acetonitrile $(R)$-252 were isolated in slightly lower $80-89 \%$ ee (Scheme 89). This solvent-dependent enantiodivergence is due to the different organocatalyst conformations and the different enthalpies and entropies of activation in these solvents. ${ }^{246,258-260}$

Scheme 89. Enantiodivergent Mannich Reaction of Malonates with $N$-Boc Aldimines Organocatalyzed by a Chiral Guanidine/Bisthiourea 251 in Different Solvents 


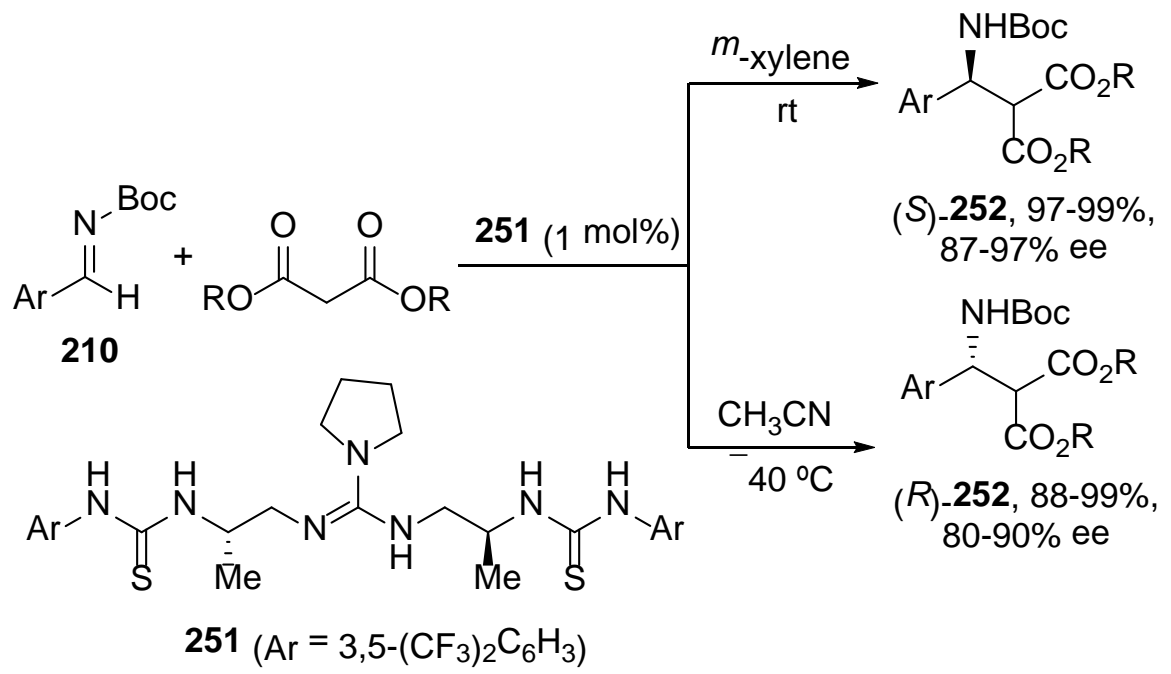

In the addition of indole to $N$-phenylsulfonyl arylimines 225 catalyzed by a chiral metal-organic frameworks (CMOFs), formed from $\mathrm{Cu}_{2}$ (carboxylate) ${ }_{4}$ and chiral carboxylate anions derived from chiral phosphoric acid 253, a reversal of the enantioselectivity under homogeneous catalysis was induced (Scheme 90). ${ }^{261}$ Under homogeneous conditions the so-called Friedel-Crafts adduct $(S)-\mathbf{2 5 4}$ was obtained in $72 \%$ ee, whereas under heterogeneous conditions $(R)-254$ was formed in $42 \%$ ee. This switch of enantioselectivity has been explained from the structural analysis and $\mathrm{QM} / \mathrm{MM}$ calculations as a result of the flip of handedness in the MOF cavity.

Scheme 90. Enantiodivergent Mannich Reaction of Indole with $N$-Phenylsulfonyl Aldimine 225 Organocatalyzed by a Chiral Phosphoric Acid 253 and its Derived MOF

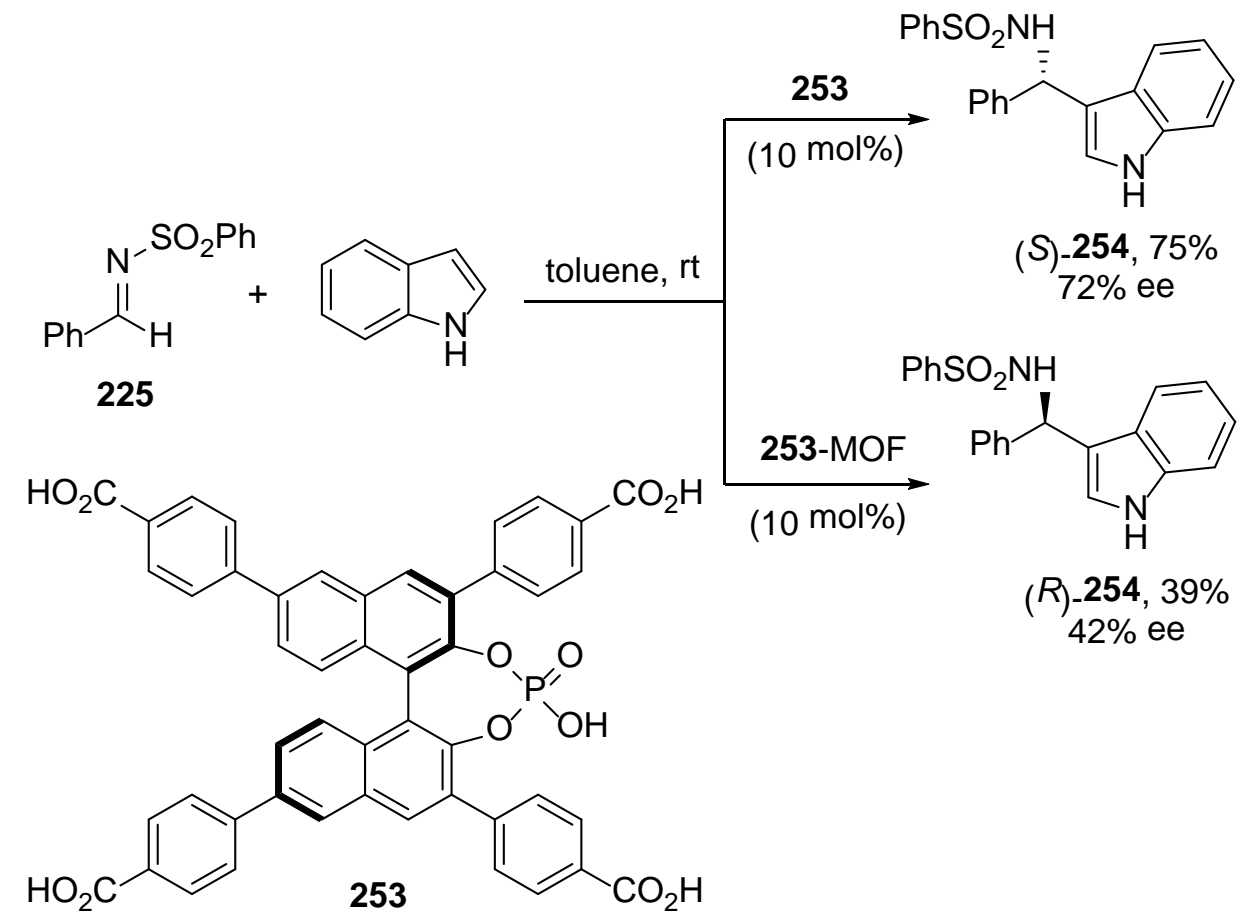


In conclusion, metal-catalyzed Mannich reactions have been performed diastereodivergently with chiral $N$-tert-butylsulfinyl imines and nitrones by controlling the chelation mode with an appropriate Lewis acid. The substituent in the aldimine (at carbon or nitrogen atoms) was crucial to control the reversal of diastereoselectivity in the metal-catalyzed reactions with chiral ligands. In the case of enantiodivergent organocatalyzed Mannich reactions Brønsted acids are the most appropriate catalysts.

2.2.3. Addition of Other Nucleophiles. Aza-Henry reaction or nitro-Mannich reaction involves the addition of nitroalkanes to imines to afford $\beta$-nitroamines, which can be further transformed into $\alpha$-amino acids or diamines. Stereodivergent aza-Henry reactions have been seldom described. Johnston and co-workers found out that the addition of nitroacetates 255 to $N$-Boc aldimines 210 (Section 2.2) in the presence of bis(amidine) triflate salts 256a gave anti-adducts $257 .{ }^{262,263}$ When $\alpha$-substituted nitroacetates 255 were used, diastereodivergent results were observed depending on the substitution in the chiral organocatalysts. syn-Diastereoselectivity occurred with catalyst 256b, whereas 256a provided adducts anti-257 (Scheme 91). The resulting $\alpha$-nitro- $\beta$ amino esters 257 were obtained in good yields, $66 \%$ to $88 \%$ de and high enantioselectivities with ee up to $97 \%$.

\section{Scheme 91. Diastereodivergent Addition of Nitroacetates to $N$-Boc Aldimines Organocatalyzed by Chiral Bis(amidine) Triflate Salts 256}

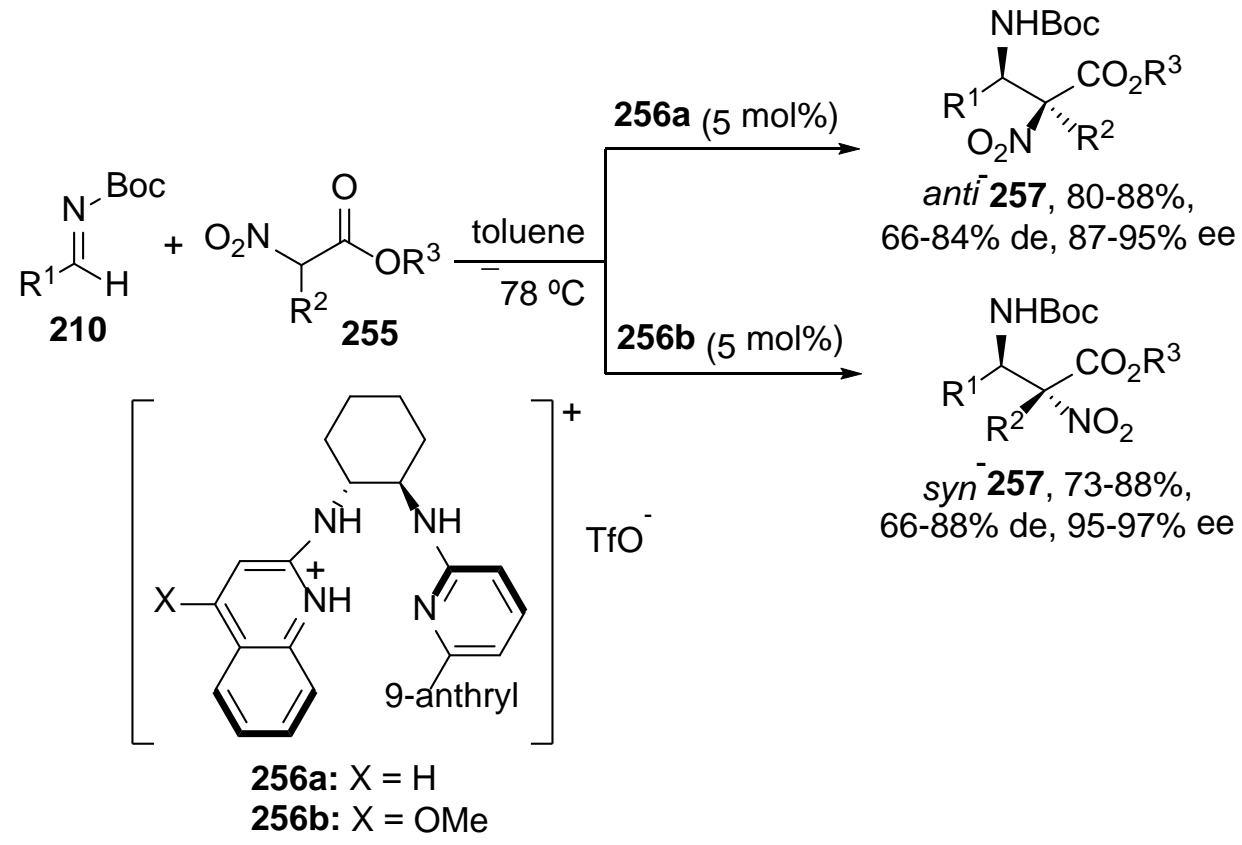

An example of an enantiodivergent aza-Henry reaction was achieved in the addition of nitroalkanes to $\mathrm{N}$-Boc aldimines 210 (Scheme 77) derived from aromatic aldehydes. By using guanidine 258a as chiral organocatalyst, the corresponding $\beta$-nitroamines 259 were isolated with $(R)$-configuration, and homochiral bisguanidine $\mathbf{2 5 8 b}$ led to the formation of (S)-259, although with moderate enantioselectivities (Scheme 92). ${ }^{264}$ 
Scheme 92. Enantiodivergent Aza-Henry Reaction of Nitroalkanes to $N$-Boc Aldimines Organocatalyzed by Chiral Guanidines 258

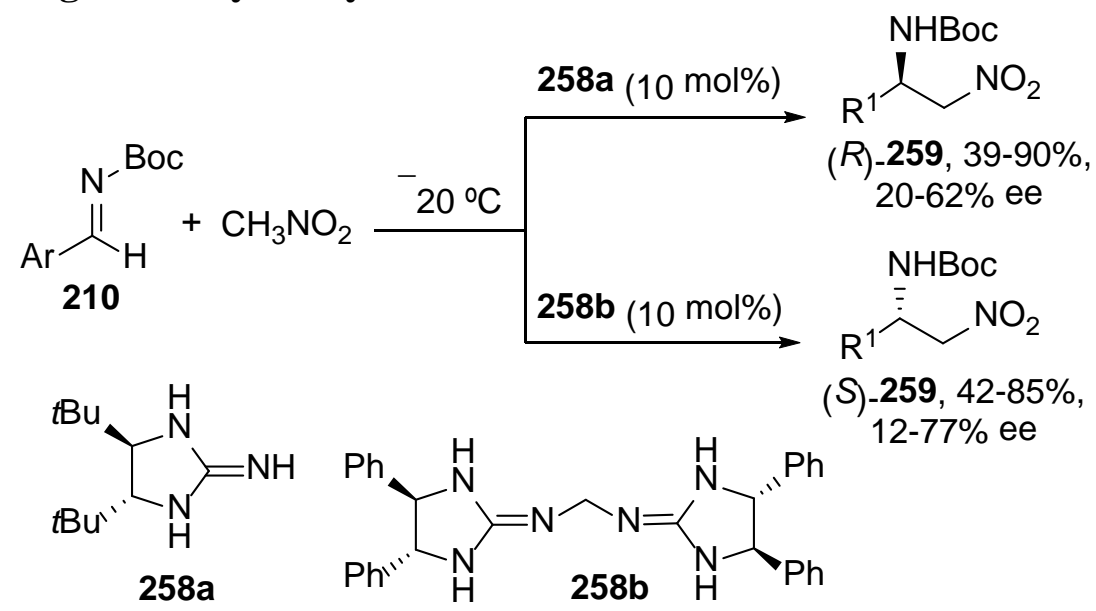

Aza-Morita-Baylis-Hillman (AMBH) reaction ${ }^{265}$ of $\alpha, \beta$-unsaturated carbonyl compounds with imines allows the synthesis of $\alpha$-methylene- $\beta$-amino carbonyl compounds. Enantiocatalyzed AMBH reactions are mainly carried out with chiral phosphines and amines. Shi and co-workers used $\beta$-isocupreidine $(\beta$-ICPD, 149a, Scheme 54) as an efficient organocatalyst for the asymmetric AMBH reaction. ${ }^{266}$ The corresponding adducts of methyl and ethyl vinyl ketones had an opposite absolute configuration to those from acrolein and acrylates. In the case of the reaction of methyl vinyl ketone with $N$-tosyl salicylaldimines 222, ${ }^{267}$ adducts 260 resulted with an opposite configuration than for $\mathbf{2 6 1}$ obtained with $N$-tosyl benzaldimines ${ }^{268}$ (Scheme 93).

Scheme 93. Enantiodivergent AMBH Reaction of Methyl Vinyl Ketone with $\mathrm{N}$ Tosyl Aldimines Organocatalyzed by $\beta$-Isocupreidine 149a

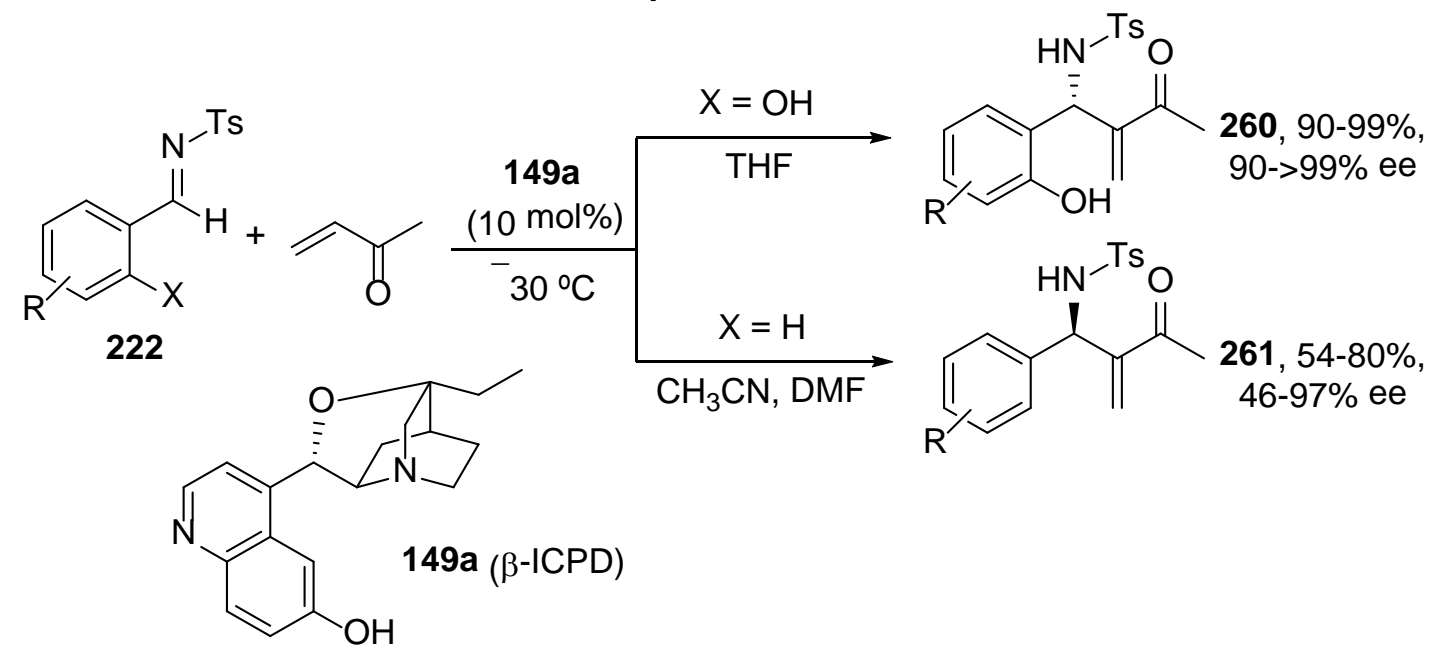

Further studies about AMBH reaction between aromatic and aliphatic $N$-sulfonyl aldimines 222 and $\alpha, \beta$-unsaturated ketones catalyzed by $\beta$-isocupreidine 149a or its amide 149c ( $\beta$-ICPD-amide) produced $(R)$-adducts 262. On the contrary, using 149a 
and $\beta$-naphthol as additive, a reversal of the enantioselectivity was observed giving adducts $(S)$-262 (Scheme 94). ${ }^{269}$

Scheme 94. Enantiodivergent AMBH Reaction of $\alpha, \beta$-Unsaturated Ketones with $N$ Sulfonyl Aldimines 222 Organocatalyzed by $\beta$-Isocupreidines 149

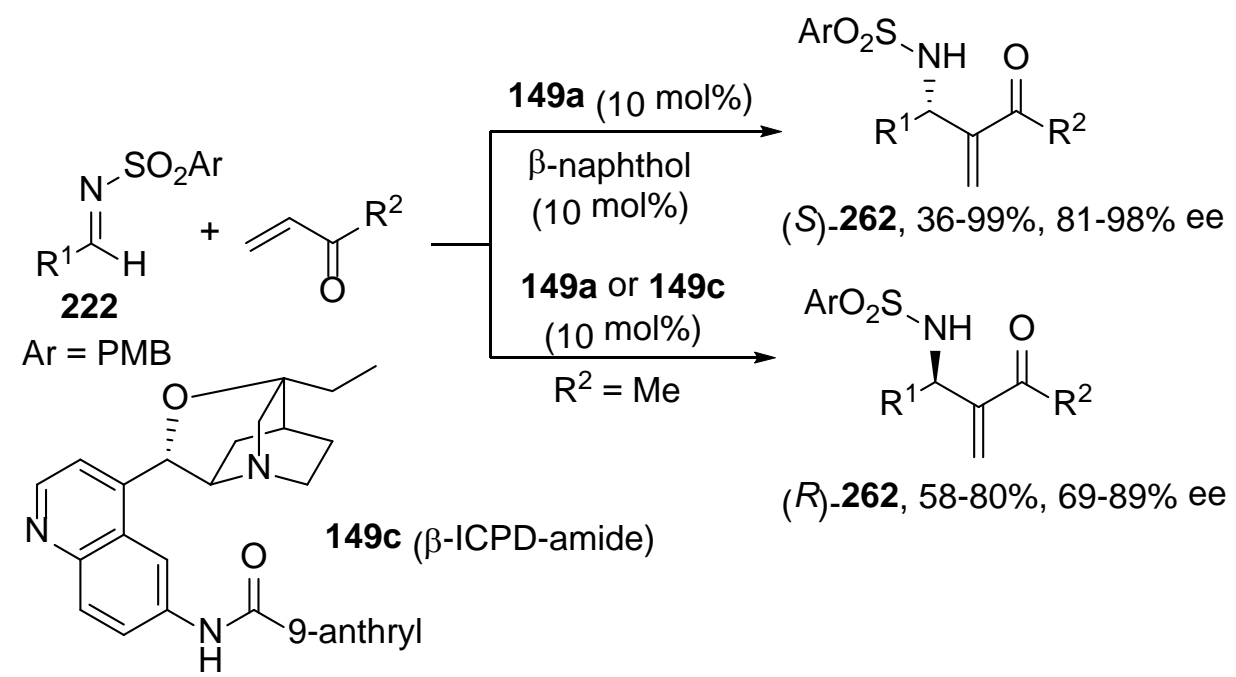

The Strecker reaction involves the hydrocyanation of imines to provide $\alpha$ aminonitriles, which can be further transformed into $\alpha$-amino acids. This fact has attracted the attention of asymmetric protocols using chiral metal complexes and organocatalysts. ${ }^{230,270}$ Enantiodivergent addition of HCN to $N$-benzyl aldimines has been observed using either oxazaborolidine $\mathbf{2 6 3}$ or the protonated one $\mathbf{2 6 4}$ affording (S) or $(R)$-isomers 265 in modest enantioselectivities (Scheme 95). ${ }^{271}$ Looking for an increase in the enantioselectivity the protonated derivative $\mathbf{2 6 4}$ was assayed giving an unexpected reversal of the configuration.

\section{Scheme 95. Enantiodivergent Hydrocyanation of $N$-Benzyl Aldimines Organocatalyzed by Oxazaborolidines 263 and 264}

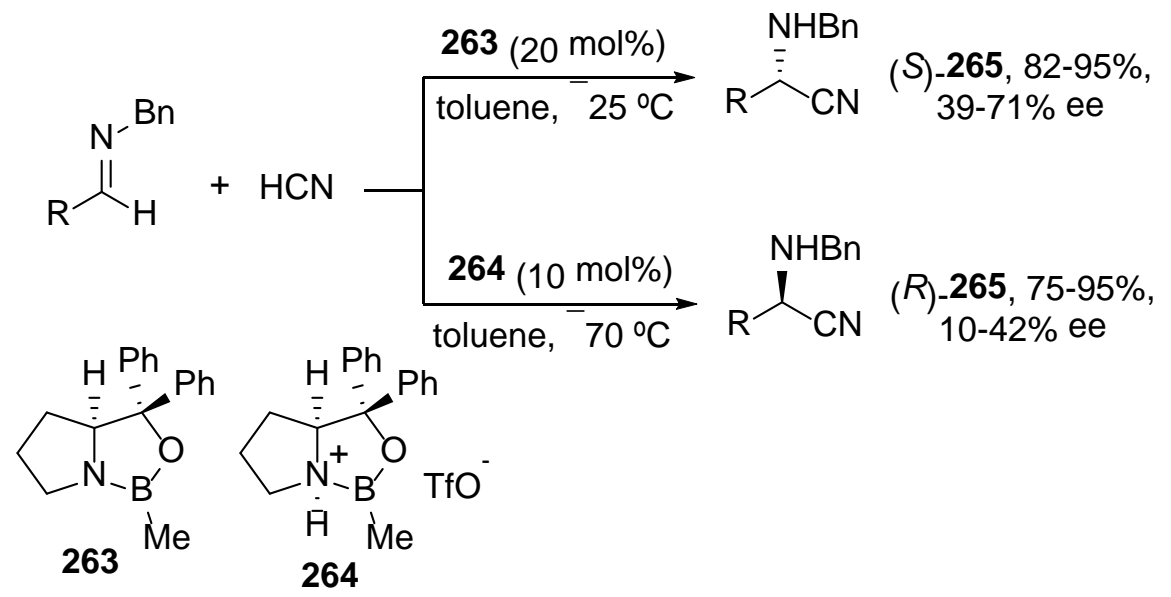


Solvent-controlled diastereodivergent asymmetric Strecker reaction has been described during the cyanosilylation of trifluoromethyl ketone-derived sulfinylimines 201 (Scheme 96). ${ }^{272}$ Working in DMF $\left(R, R_{\mathrm{S}}\right)$-isomers 266 were obtained as major products with de up to $90 \%$. On the contrary, in hexane as solvent $\left(S, R_{\mathrm{S}}\right)$-diastereomers 266 were mainly formed with de up to 99\%. This method allows the synthesis of enantiomerically enriched $\alpha$-trifluoromethyl $\alpha$-amino acids.

Scheme 96. Diastereodivergent Cyanosilylation of Trifluoromethyl Substituted $(R)$ tert-Butanesulfinyl Ketimines 201

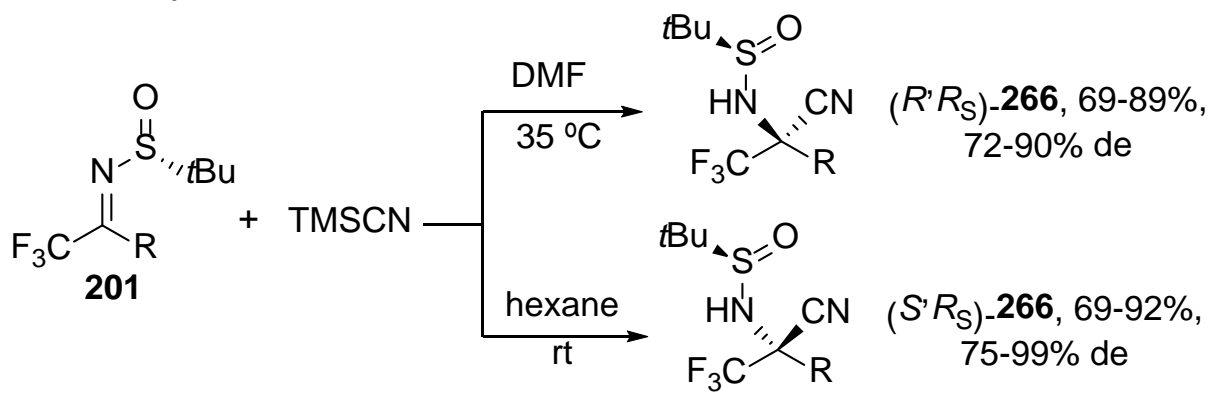

Shibasaki and co-workers realized that the in situ prepared gadolinium complex formed by ligand 267 and $\mathrm{Gd}(\mathrm{OiPr})_{3}$ catalyzed the enantioselective cyanosilylation of $N$-diphenylphosphinoyl (Dpp) ketimines 232 giving selectively the corresponding $(S)$ amino nitriles 268. ${ }^{273}$ This methodology was later successfully used for the synthesis of lactacystin. ${ }^{274}$ On the other hand, using the crystalline 4:5 complex of $\mathrm{Gd}(\mathrm{OiPr})_{3}$ and 267, the opposite enantiomer $(R)$-268 was formed with the same or improved level of enantioselectivity (Scheme 97). ${ }^{275}$

\section{Scheme 97. Enantiodivergent Cyanosilylation of $N$-Dpp Ketimines 232 Catalyzed by $\mathrm{Gd}(\mathrm{OiPr})_{3}$ and Ligand 267}

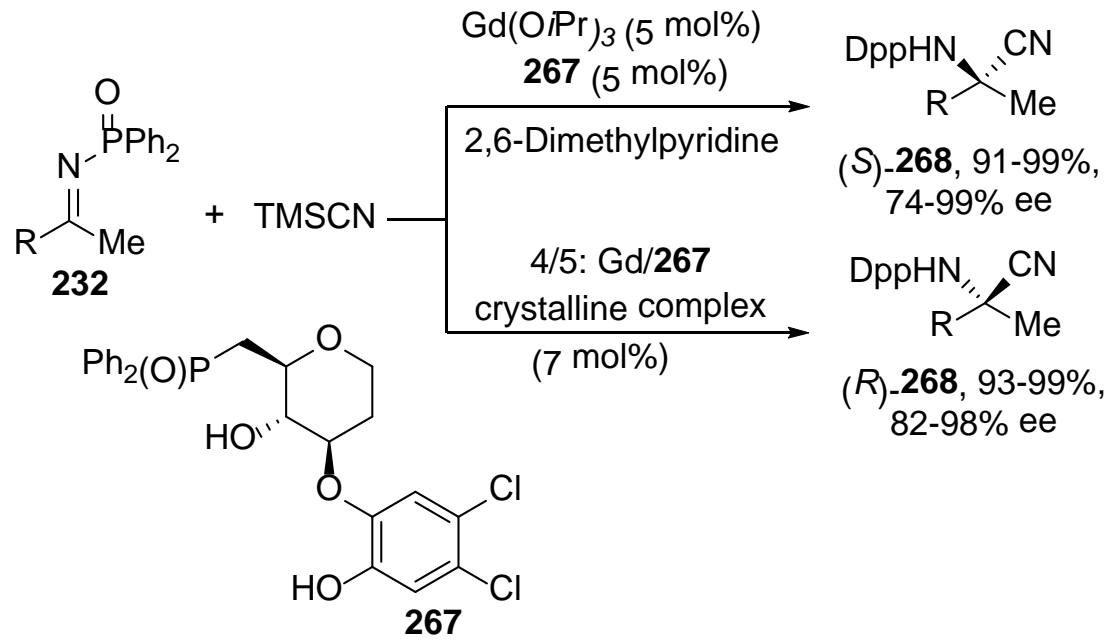

In conclusion, diastereo- and enantiodivergent aza-Henry reactions organocatalyzed by chiral bases can be carried out by changing the substituents on the structure of these 
organocatalysts. In the case of the aza-Morita-Baylis-Hillman reaction (AMBH) it can be enantiodivergently performed by $\beta$-isocupreidines using different additives. With respect to the enantiodivergent Strecker reaction of ketimines the best results have been achieved under metal-catalyzed conditions according to the metal-to-ligand stoichiometry. On the other hand, in the case of diastereodivergent cyanosilylation of $\mathrm{N}$ tert-butylsulfinyl ketimines the polarity of the solvent controls this process.

2.2.4. Reduction of Ketimines. In spite of the importance of the enantioselective reduction of ketimines for the synthesis of chiral amines as pharmaceutical and agrochemical compounds, ${ }^{141,276}$ very few enantiodivergent processes have been described.

Diastereodivergent reduction of chiral $O$-benzyl oximes 269 bearing an stereocenter in the aliphatic backbone has been studied using aluminum hydrides and Pd-catalyzed hydrogenation methodologies. ${ }^{277}$ The reduction with $\mathrm{LiAlH}_{4}$ or $\mathrm{Na}\left[\mathrm{AlH}_{2}\left(\mathrm{OCH}_{2} \mathrm{CH}_{2} \mathrm{OCH}_{3}\right)_{2}\right]$ afforded syn-products 270 in high diastereoselectivities. However, under Pd catalyzed hydrogenation conditions the reduction of the oximes 269 gave anti-amino alcohols $\mathbf{2 7 0}$ (Scheme 98). In all cases, cleavage of the $\mathrm{N}-\mathrm{O}$ bond takes place providing primary amines.

\section{Scheme 98. Diastereodivergent Reduction of Chiral $\alpha$-Hydroxy $O$-BenzylOximes} 269

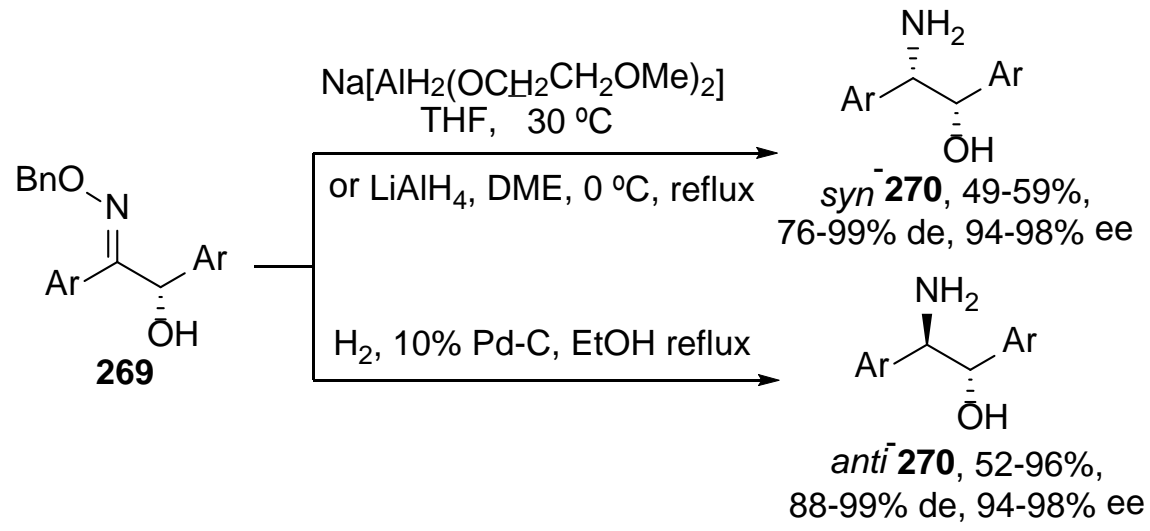

Asymmetric hydrogenation of $N$-benzyl ketimines catalyzed by chiral Rh complexes 271 was enhanced by the presence of reverse micellar systems of sodium bis(2ethylhexyl)sulfosuccinate (AOT, 272), giving the corresponding amines $(R)$-273 with ee up to $87 \%$ in high yields. ${ }^{278}$ Reversal of the enantioselectivity was observed for the $N$ benzyl ketimine derived from acetophenone when AOT was replaced by ammonium halides (iodide or chloride) giving (S)-273 with ee up to $80 \%$ (Scheme 99). This enantiodivergent process induced by the presence of different additives has been explained by the participation of a dihydride in the case of iodide, whereas a monohydride is operating in the presence of AOT.

Scheme 99. Enantiodivergent Hydrogenation of a $N$-Benzyl Ketimine Catalyzed by a Rh(I) Chiral Complex 271 in the Presence of Anionic Additives 


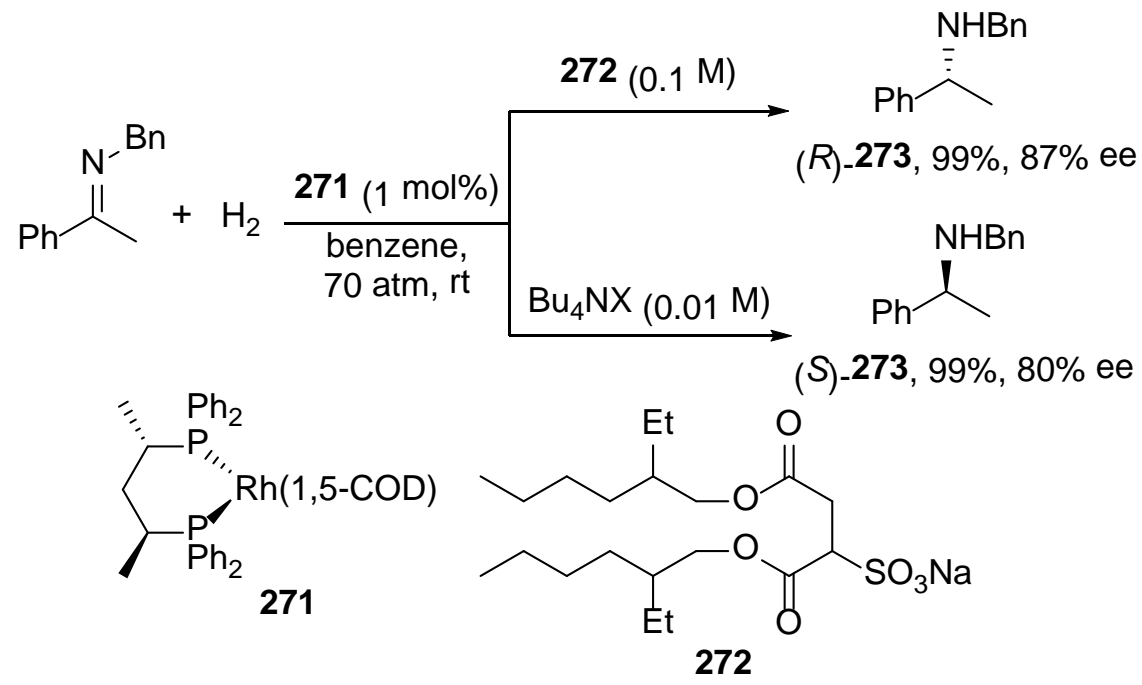

Recently, an enantiodivergent atroposelective synthesis of chiral biaryls by transfer hydrogenation under a chiral phosphoric acid catalysis has been described by Akiyama and co-workers. Biaryl lactols 274 reacted with anilines and a Hantzch ester 275 in the presence of chiral phosphoric acids 276 or $\mathbf{2 4 5}$ forming $(R)$-277 or $(S)$-278, respectively (Scheme 100). ${ }^{279} \mathrm{~A}$ dynamic kinetic resolution (DKR), involving a reductive amination, took place with high enantioselectivity. The ring-opening/ring-closing equilibrium between the biaryls acetal and the formed intermediate biaryl imine (racemization), and the final kinetic-resolution-type asymmetric transfer hydrogenation, afforded the chiral biaryls 277 and 278. (S)-Products 277 were obtained in the presence of 3-hydroxy-5trifluoromethylaniline, whereas $(R)$-278 were formed in the presence of 2-hydroxy-4trifluoromethylaniline.

Scheme 100. Enantiodivergent Reductive Amination of Biaryl Lactols 274 with Hantzch Ester 275 Catalyzed by Chiral Phosphoric Acids and Anilines as Additives 


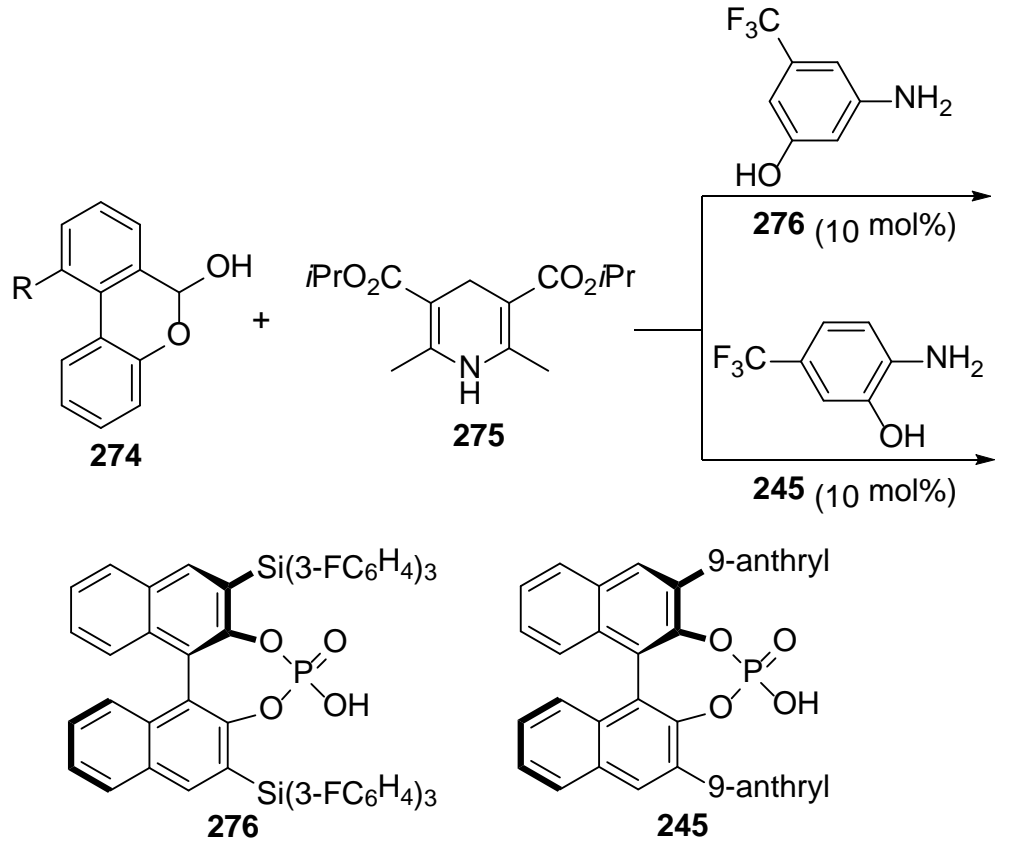<smiles>[R]c1cccc(CNc2cc(O)cc(C(F)(F)F)c2)c1-c1ccccc1O</smiles>

(S)-277, 75->99\%, 84-99\% ee<smiles>[R]c1cccc(CNc2ccc(C(F)(F)F)cc2O)c1-c1ccccc1O</smiles>

(R)-278, 82->99\%, 77-94\% ee

In conclusion for this section, diastereodivergent reduction of $\alpha$-hydroxy ketimines can be controlled either by the addition of hydrides or by catalytic hydrogenation. In the case of enantiodivergent reduction of ketimines, the homogeneous hydrogenation can be modulated by the presence of anionic additives.

\subsection{Addition to Alkenes}

According to the electronic feature of alkenes several types of nucleophilic or electrophilic addition reactions can be performed. One of the most important C-C and also C-heteroatom bond forming reactions are the conjugate additions to electrophilic olefins. The asymmetric version of this Michael-type reaction can be catalyzed by metal complexes and organocatalysts. Catalytic hydrogenation of all kinds of alkenes is a very useful strategy for the asymmetric generation of stereocenters using chiral metal complexes. Another important transformation is the enantioselective metal-catalyzed hydroformylation for the preparation of branched aldehydes. Tandem electrophilic hydroboration-oxidation of alkenes is an important asymmetric oxidation processes for the functionalization of $\mathrm{C}=\mathrm{C}$ bonds to afford the corresponding alcohols. Asymmetric metal-catalyzed epoxidation and cyclopropanation of alkenes allow the diastereo- and enantioselective preparation of oxiranes and cyclopropanes. Many of these addition reactions have been applied to industrial scale processes.

2.3.1. Conjugate Additions. Stereodivergent metal- and organocatalyzed conjugate addition (CA) to electrophilic alkenes ${ }^{280-288}$ can be performed with carbon and heteroatom nucleophilic reagents but also under radical conditions which will be considered in different sections. 
2.3.1.1. Metal-Catalyzed Conjugate Additions. Among carbon nucleophiles, the conjugate addition of organometallic reagents to $\alpha, \beta$-unsaturated carbonyl compounds in the presence a chiral ligand or a chiral metal complex have been extensively studied mainly with cuprates or organomagnesium, organozinc, organoaluminum and organoboron reagents. ${ }^{280}$ According to the nature of the organometallic compounds stereodivergent processes will be initially considered.

The enantioselective CA of lithium cuprates to acyclic enones was initially studied by Tomioka and co-workers using chiral ligands. ${ }^{289-292}$ They used a stoichiometric amount of chiral amidophosphines and found out that the carbonyl group of the ligand c oordinates the lithium atom and the phosphorous atom to the copper. Solvent-dependent enantiodivergence was observed in the addition of lithium methylcuprate to chalcone in the presence of ligands 279 (Scheme 101). ${ }^{289}$ Working with 279a, (S)-adduct 280 was formed in ether in $\mathbf{8 8 \%}$ ee, whereas in THF $(R)-\mathbf{2 8 0}$ was formed in $\mathbf{7 2 \%}$ ee. Similar behavior was observed with phosphines $\mathbf{2 7 9 b}$ and 279c. Although, the best results were achieved with ligand 279d in ether, which gave (S)-280 in 90\% ee and 99\% yield. ${ }^{290}$

\section{Scheme 101. Enantiodivergent Conjugate Addition of Lithium Cuprates to Chalcone in the Presence of Amidophosphines 279}

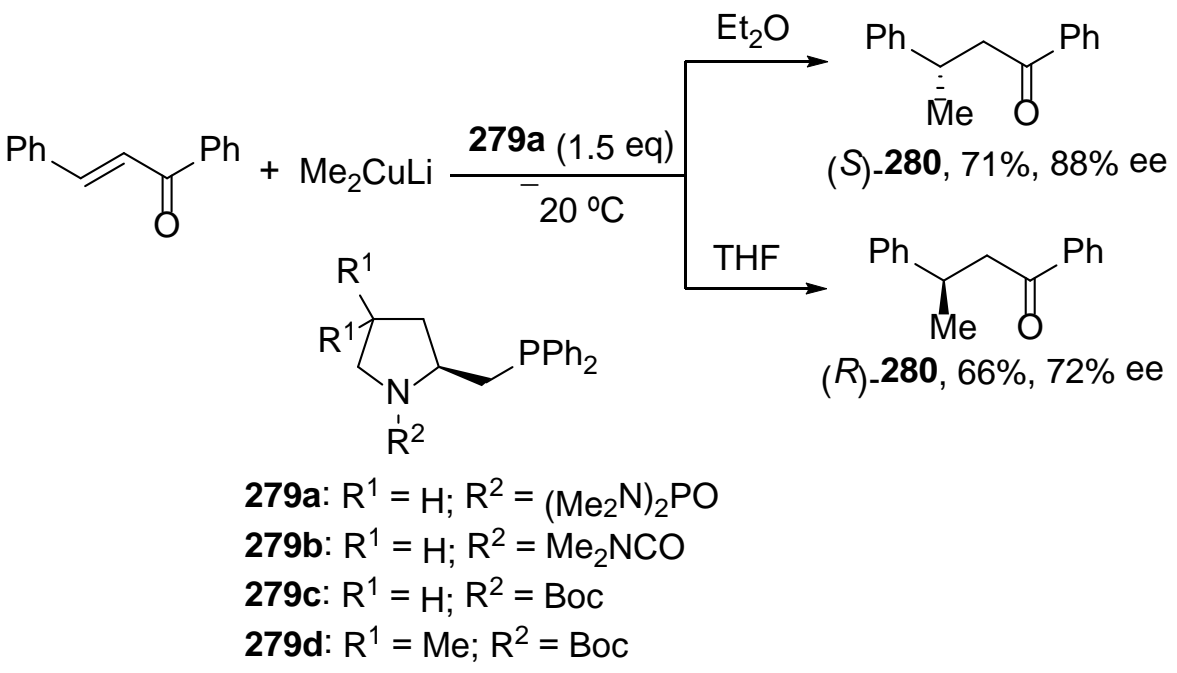

The same group performed the enantioselective CA of Grignard reagents to cyclic enones under substoichiometric amounts of $\mathrm{Cu}(\mathrm{I})$ salts (see, Section 3) using ligand 279b (32 mol\%). ${ }^{291}$ Enantioselective CA of methylmagnesium bromide to $\alpha, \beta$ unsaturated esters catalyzed by CuI and Tol-BINAP has been performed by Loh and coworkers. ${ }^{293}$ Methylation of ester $\mathbf{2 8 1}$ in the presence of $(R)$ - or $(S)$-Tol-BINAP allows the diastereodivergent synthesis of esters anti- or syn-282, respectively (Scheme 102). This methodology has been applied to the synthesis of the C14-C20 fragment of the antibiotic TMC-151A and of the two products from marine organisms siphonarienal and siphonarienone. 
Scheme 102. Diastereodivergent Asymmetric Conjugate Addition of Methylmagnesium Bromide to $\alpha$, $\beta$-Unsaturated Ester 281 Catalyzed by CuI/TolBINAP

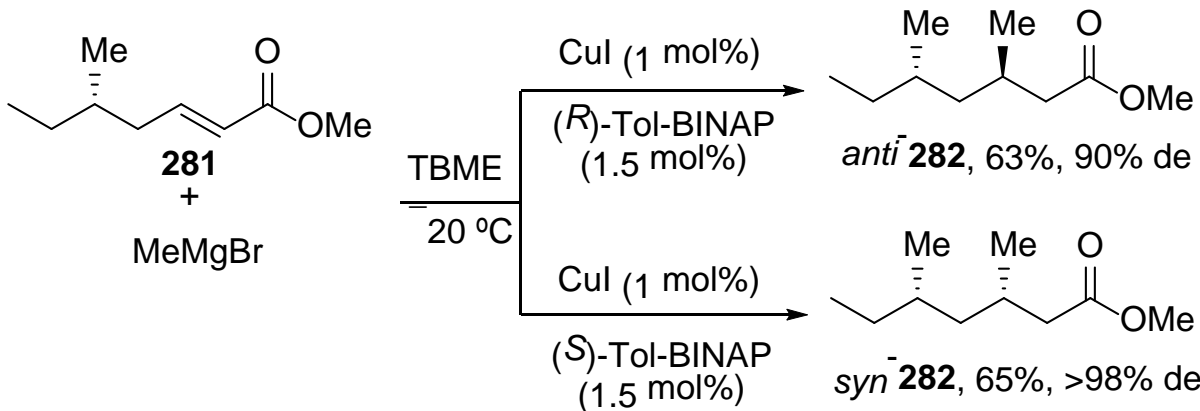

This copper-catalyzed CA protocol was applied to the total synthesis of the different diastereomers of stylopsal, a sex pheromone of Strepsiptera, a twisted-wing parasites of various taxa insects. ${ }^{294}$ One application of this CA methodology is illustrated with ester 283, which give products $\mathbf{2 8 4}$ and $\mathbf{2 8 5}$ (Scheme 103). The compound $\mathbf{2 8 4}$ is a precursor for the corresponding aldehyde stylopsal.

Scheme 103. Diastereodivergent Asymmetric Conjugate Addition of Methylmagnesium Bromide to $\alpha, \beta$-Unsaturated Ester 283 Catalyzed by CuBr/TolBINAP

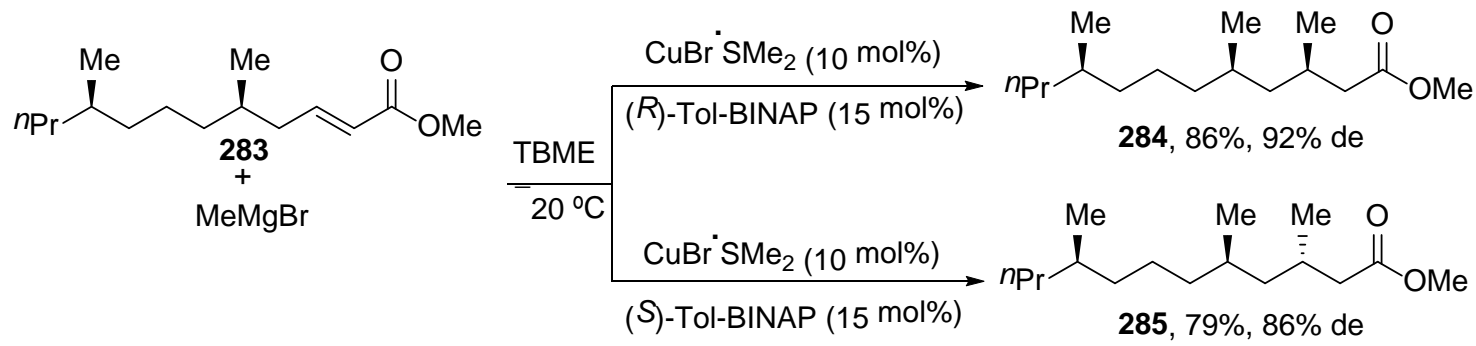

The CA of organozinc reagents to enones is very slow but can be accelerated either by a chiral ligand or by metal complexes. Diastereodivergent asymmetric addition of dimethylzinc to alkylidenemalonate $\mathbf{2 8 6}$ has been carried out in the presence of copper(II) complexes with phosphoramidites $\mathbf{2 8 7}$ and $\mathbf{2 8 8}$ as ligands giving syn- and anti-289, respectively (Scheme 104). ${ }^{295}$

Scheme 104. Diastereodivergent Enantioselective Conjugate Addition of Dimethylzinc to $\alpha$, $\beta$-Unsaturated Malonate 286 Catalyzed by $\mathrm{Cu}(\mathrm{OTf})_{2}$ /Phosphoramidites 287 and 288 


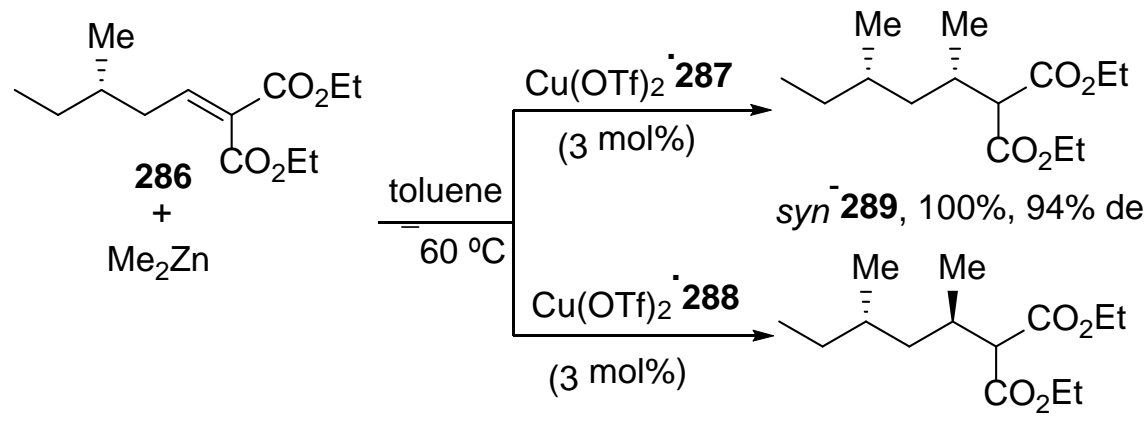

anti $\mathbf{2} 89,100 \%, 94 \%$ de<smiles></smiles><smiles>C[C@H](c1ccccc1)N([C@H](C)c1ccccc1)P(Oc1ccc2ccccc2c1)c1c(OP(c2ccccc2)c2ccccc2)ccc2ccccc12</smiles>

Solvent-dependent enantiodivergence was observed in the copper-catalyzed CA of diethylzinc to $\alpha, \beta$-unsaturated ketones with $D_{2}$-symmetric biphenyl-derived phosphoramidites 290 as chiral ligands (Scheme 205). ${ }^{296}$ In toluene, products (R)-291 were obtained in high yields with ee up to 99\%, whereas THF promoted the formation of (S)-291 with ee up to 92\%. These results were explained by the stronger coordination ability of THF than toluene to the Zn ion, consequently modifying the structure of the complex. Moreover, by changing ligand cis-290a to trans-290, and also the solvent, reversal of enantioselectivity was also observed.

Scheme 105. Enantiodivergent Conjugate Addition of Diethylzinc to $\alpha, \beta$ Unsaturated Ketones Catalyzed by $\mathrm{Cu}(\mathrm{OAc})_{2} / \mathrm{Phosphoramidites} 290$ in Different Solvents

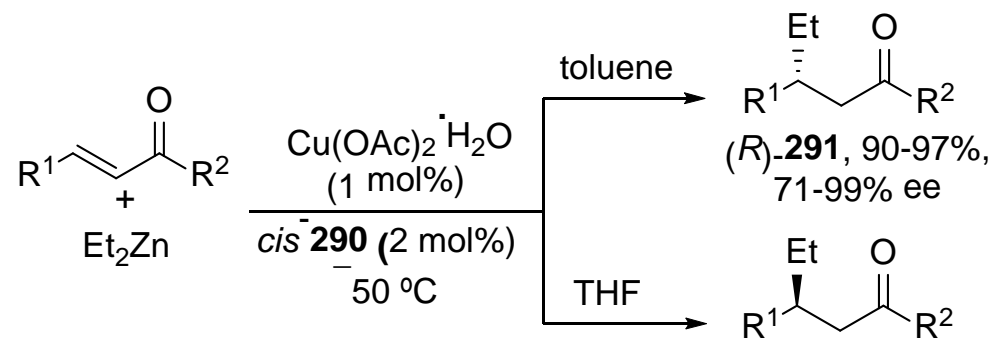

(S)-291, 90-94\%, $60-92 \%$ ee

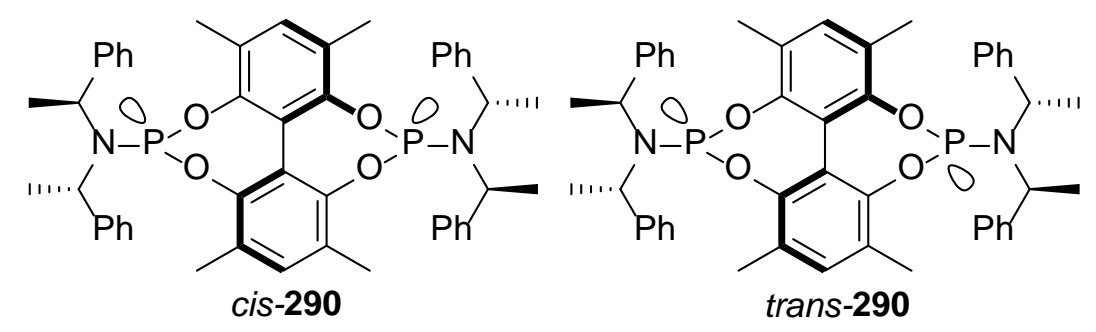


In the case of the Cu-catalyzed CA of dialkylzincs to nitrostyrene followed by a Mannich reaction a similar solvent effect has been observed. ${ }^{297}$ In this one-pot process high diastereo- and enantiocontrol were achieved using Charette's procedure. ${ }^{298}$ BozPHOS 292 as ligand for $\mathrm{Cu}(\mathrm{OTf})_{2}$ in ether or THF afforded products syn,anti-294 as well as following the Hoveyda's protocol ${ }^{299}$ with ligand 293. However, in toluene or ether $\beta$-nitroamines syn,syn-294 were mainly obtained (Scheme 106). The observed diastereoselectivity was controlled by the presence or absence of $\mathrm{Zn}(\mathrm{OTf})_{2}$. According to a Felkin-Anh model transition state 294A the syn-isomers are formed, whereas in the nitro-Michael process cyclic 294B transition state should provide the syn,anti-products working under kinetic control $\left(-70{ }^{\circ} \mathrm{C}\right)$. However, in toluene, an open structure 294C can be the transition state without the participation of $\mathrm{Zn}(\mathrm{OTf})_{2}$, which can precipitate in the less polar solvent, minimizing dipole-dipole interactions forming the major syn,syn-294 diastereomer.

\section{Scheme 106. Diastereodivergent Enantioselective Conjugate Addition-Nitro Michael Reactions of Dialkylzincs to $\beta$-Nitrostyrenes and Imines Catalyzed by $\mathrm{Cu}(\mathrm{OTf})_{2}$ and Ligands 292 and 293}

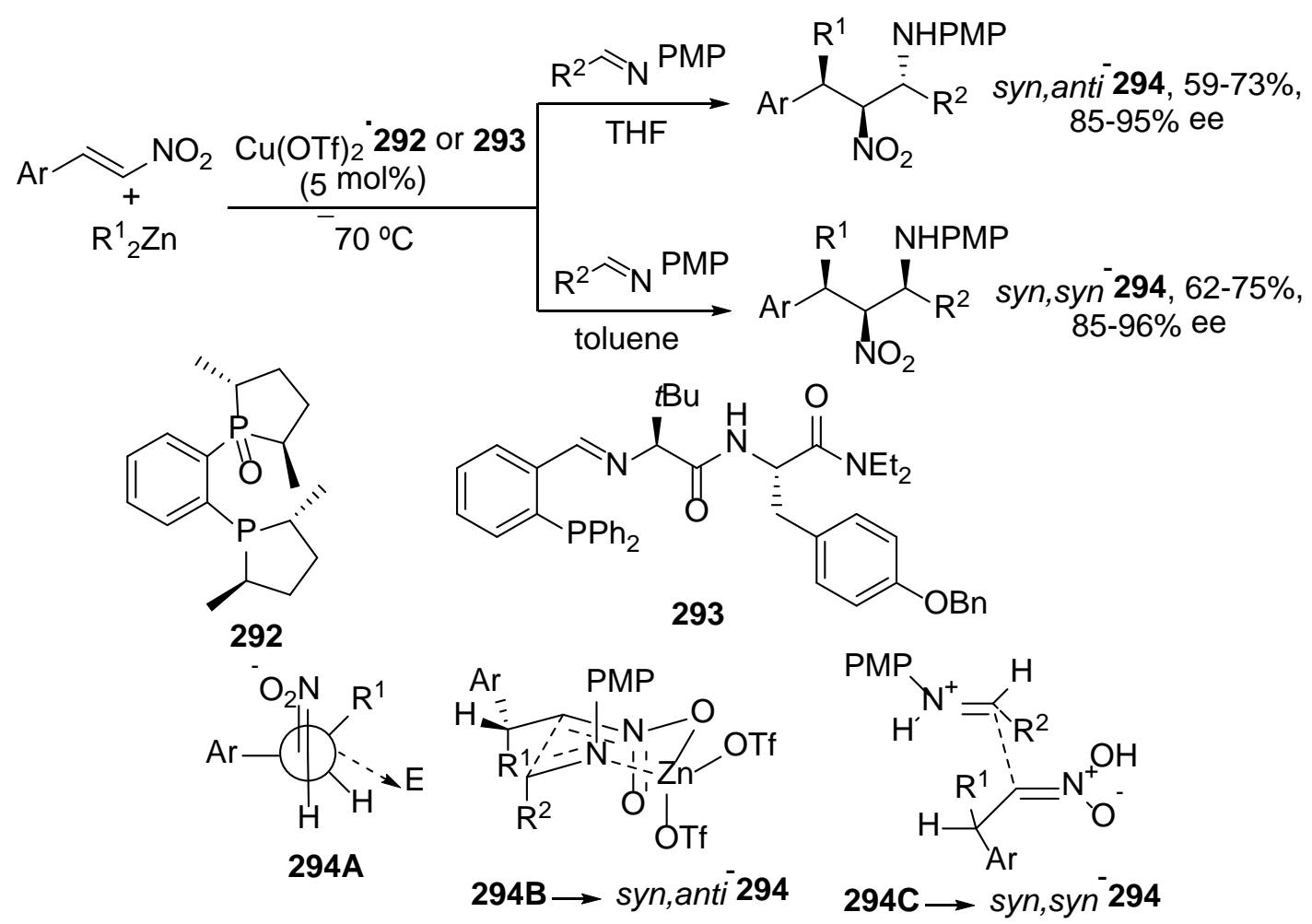

Nickel(II) acetylacetonate catalyzes the enantiodivergent addition of diethylzinc to chalcone using chiral amines $\mathbf{2 9 5}$ and 296 as ligands providing Michael adducts $(R)-\mathbf{2 9 1}$ and $(S)-291\left(\mathrm{R}^{1}=\mathrm{R}^{2}=\mathrm{Ph}\right.$ ), respectively, in low enantioselectivity (Figure 12). ${ }^{33}$ Zhang and co-workers have found, in the copper-catalyzed enantioselective CA of triethylaluminium to $\alpha, \beta$-unsaturated ketones, that the substituents at the 3,3',5,5'positions of the biphenyl backbone of the phosphoramidite ligands $\mathbf{2 9 0}$ played a crucial role in the switching of enantioselectivity. ${ }^{300}$ This effect was not observed in the CA of 
diethylzinc (Scheme 105). ${ }^{295}$ Ligands trans-290 with different substituents ( $\mathrm{R}=\mathrm{Me}, \mathrm{Et}$, $\mathrm{Ph}$ ) gave products $(R)$-291 with ee up to 93\%, whereas phosphoramidite cis-290 $(\mathrm{R}=\mathrm{H})$ afforded (S)-291 with ee up to 81\% (Figure 12).

Figure 12. Ligands Used for the CA of $\mathrm{Et}_{2} \mathrm{Zn}$ and $\mathrm{Et}_{3} \mathrm{Al}$ to Chalcones Catalyzed by $\mathrm{Ni} / 295$ or 296 and $\mathrm{Cu} / 290$ Complexes<smiles>CN(CC(O)c1ccccc1)[C@H]1CCCC[C@H]1CC(O)c1ccccc1</smiles><smiles>CN(CC(O)c1ccccc1)[C@H]1CCCC[C@H]1NCC(O)c1ccccc1</smiles>

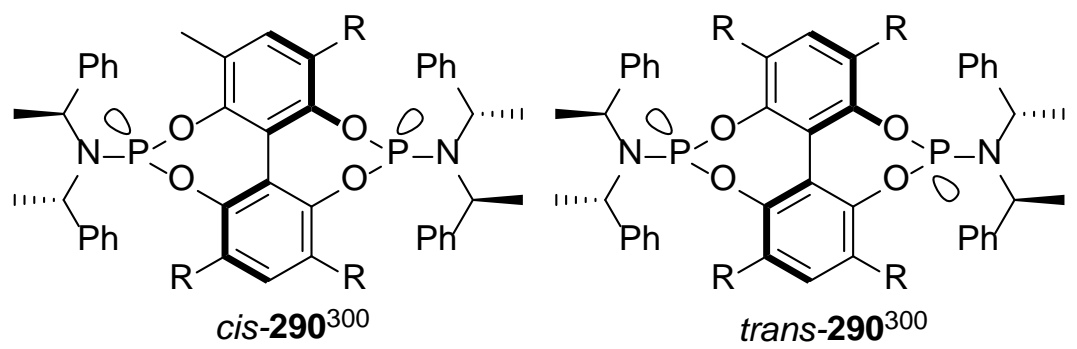

Silyl enol ethers are the carbon nucleophiles in stereodivergent Mukaiyama-Michael reactions catalyzed by $\mathrm{Cu}$ (II) bis(oxazolines) (BOX) complexes. These neutral nucleophiles are less reactive than the corresponding organometallic compounds and, as in the case of the Mukaiyama-aldol reaction, a Lewis acid is commonly used as well as strong Michael acceptors. Evans and co-workers have explored the CA of silylketene acetals to alkylidene malonates under $\mathrm{Cu}\left(\mathrm{SbF}_{6}\right)_{2}$ and chiral BOX ligand $\mathbf{8 0}$ (Scheme 23) in the presence of hexafluoroisopropanol (HFIP) as Brønsted acid. The substituents in the alkylidene malonate $(\mathrm{R}=\mathrm{Ph}, 2$-furyl, $t \mathrm{Bu})$ gave the opposite configuration for products 297 than when $\mathrm{R}=$ Me was used in the substrate (Scheme 107). ${ }^{301-303}$ From the X-ray diffraction analyses of the phenyl substituted and the methyl substituted alkylidene malonates with the $\mathrm{Cu} / \mathrm{BOX}$ complex, it can be deduced that the phenyl derivative forms a six-membered chelate in a half-open envelope conformation favoring the approach of the nucleophile from the Si-face. However, the methyl derivative forms an almost flat six-membered chelate ring and the $R e$-face attack is favored giving the enantiomeric adduct.

Scheme 107. Enantiodivergent Mukaiyama-Michael Reaction of Silylketene Acetals to Alkylidene Malonates Catalyzed by $\mathrm{Cu}(\mathrm{II}) / \mathrm{Box} 80$ Complex 


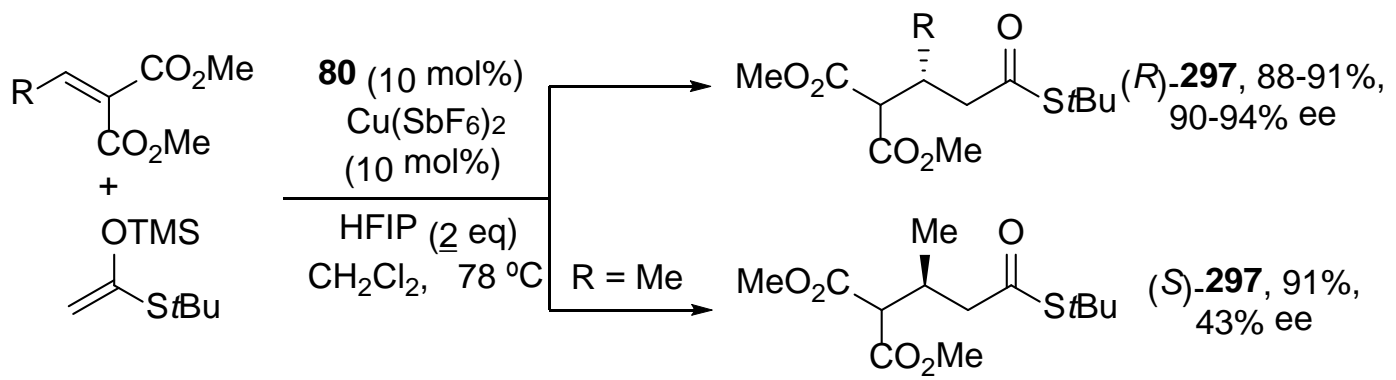

Vinylogous Mukaiyama-Michael reaction has been carried out with 2trimethylsilyloxyfuran 84 (Section 2.1.2.1) and $N$-crotonoyloxazolidinone (298) catalyzed by chiral pyridine 2,6-bis(oxazoline) (PyBOX) 299-lanthanide derived complexes (Scheme 108). ${ }^{304}$ In the case of $\mathrm{Sc}(\mathrm{OTf})_{3}$ compound $(S, S)-\mathbf{3 0 0}$ was prepared in high diastereoselectivity (anti/syn: 98/2) and enantioselectivity (94\%). Reversal of enantioselectivity was observed using a medium-size lanthanide, Eu(OTf $)_{3}$, giving compound $(R, R)-300$ in $74 \%$ de and $70 \%$ ee.

Scheme 108. Enantiodivergent Vinylogous Mukaiyama-Michael Reaction of 2Trimetylsilyloxyfuran 84 with $N$-Crotonoyloxazolidinone 298 Catalyzed by PyBOX 299 and Lanthanide Triflates

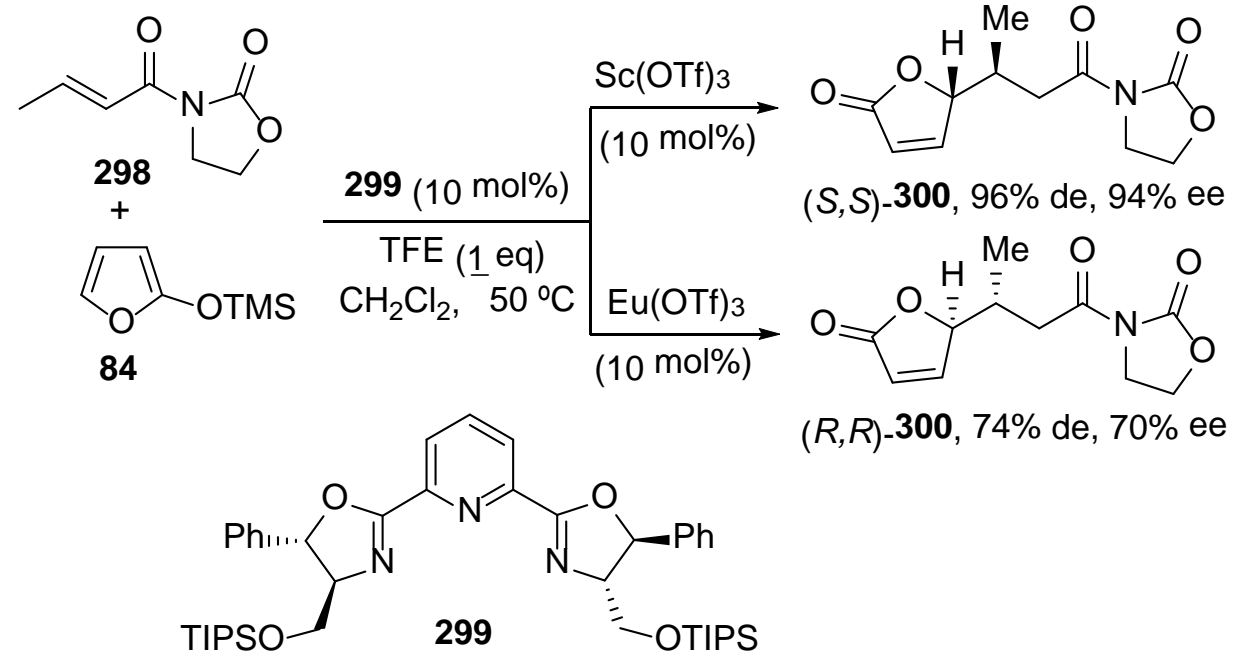

Modest levels of enantioselectivity have been obtained in the enantiodivergent rhodium-catalyzed CA of triphenylboroxin to 4-methyl- $\beta$-nitrostyrene using a combination of achiral and chiral ligands. ${ }^{305}$ Ligands $\mathbf{3 0 1}$ and $\mathbf{3 0 2}$ gave (R)-adduct $\mathbf{3 0 3}$ in 7\% ee, whereas ligands $\mathbf{3 0 1}$ and $\mathbf{2 8 8}$ gave $(S)$-303g in 30\% ee (Scheme 109).

Scheme 109. Enantiodivergent Conjugate Addition of Triphenylboroxin to 4Methyl- $\beta$-nitrostyrene Catalyzed by Rh(acac) and Phosphoramidites 


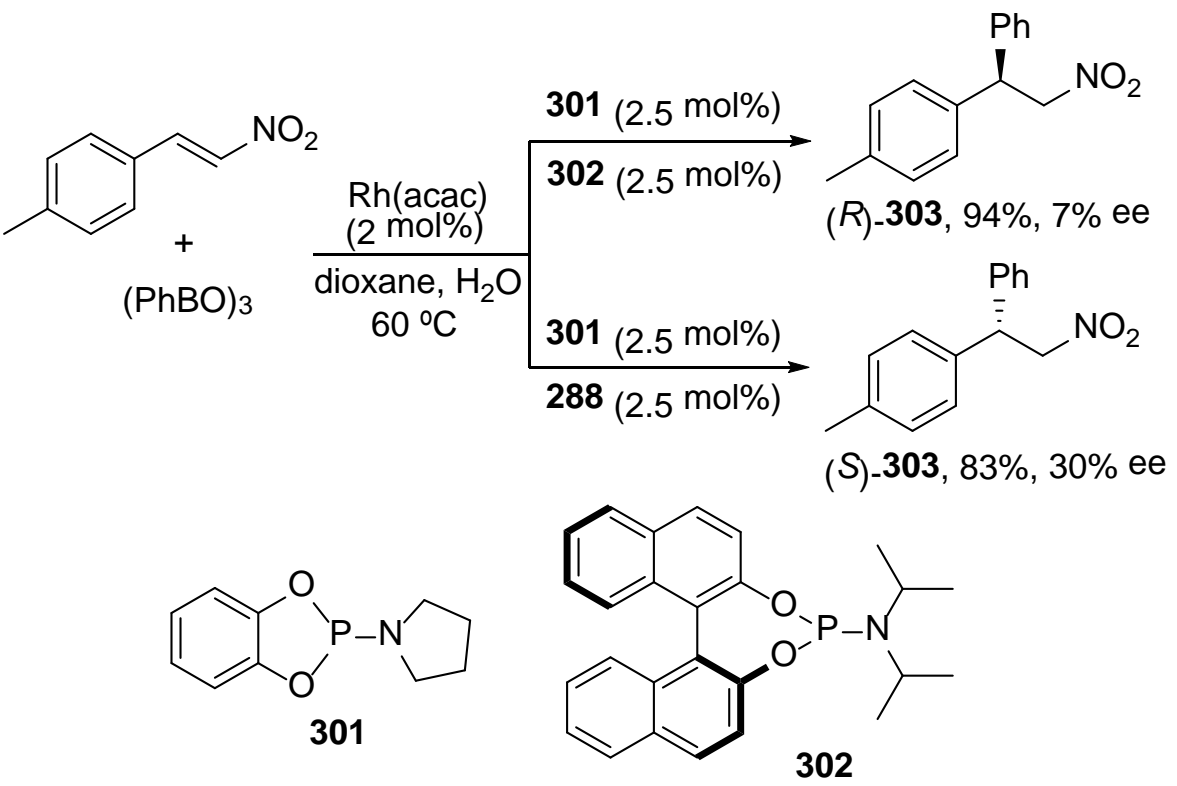

Diastereodivergent asymmetric conjugate addition of oxindoles 304 to nitrostyrenes has been carried out under Ni(II)-catalyzed conditions. The catalyst used $\mathbf{3 0 5}$ contains a $\mathrm{Ni}$ (II)-bis(phenoxyimine) unit and an axially chiral bisimidazolium salt (Scheme 110). ${ }^{306}$ By changing the substitution in the nitrogen of the bis(phenoxymine), a diastereodivergent CA was observed. Complex 305a gave mainly adducts 306a, whereas 305b gave diastereomers 306b with moderate de and high ee. To explain the observed diastereodivergence, a coordination of the $N$-Boc-oxindole 304 to the $\mathrm{Ni}$ center is directed by the imino alcohol unit determining the $\mathrm{R}$ and $\mathrm{S}$ configuration of the 3-position in the product 306 by catalysts 305a and 305b, respectively. In model 306A and 306B the nitroalkene is coordinated by the imidazolium linker in the same manner.

Scheme 110. Diastereodivergent Enantioselective Conjugate Addition of Oxindoles 304 to Nitrostyrenes Catalyzed by Ni(II) Complexes 305a and 305b 

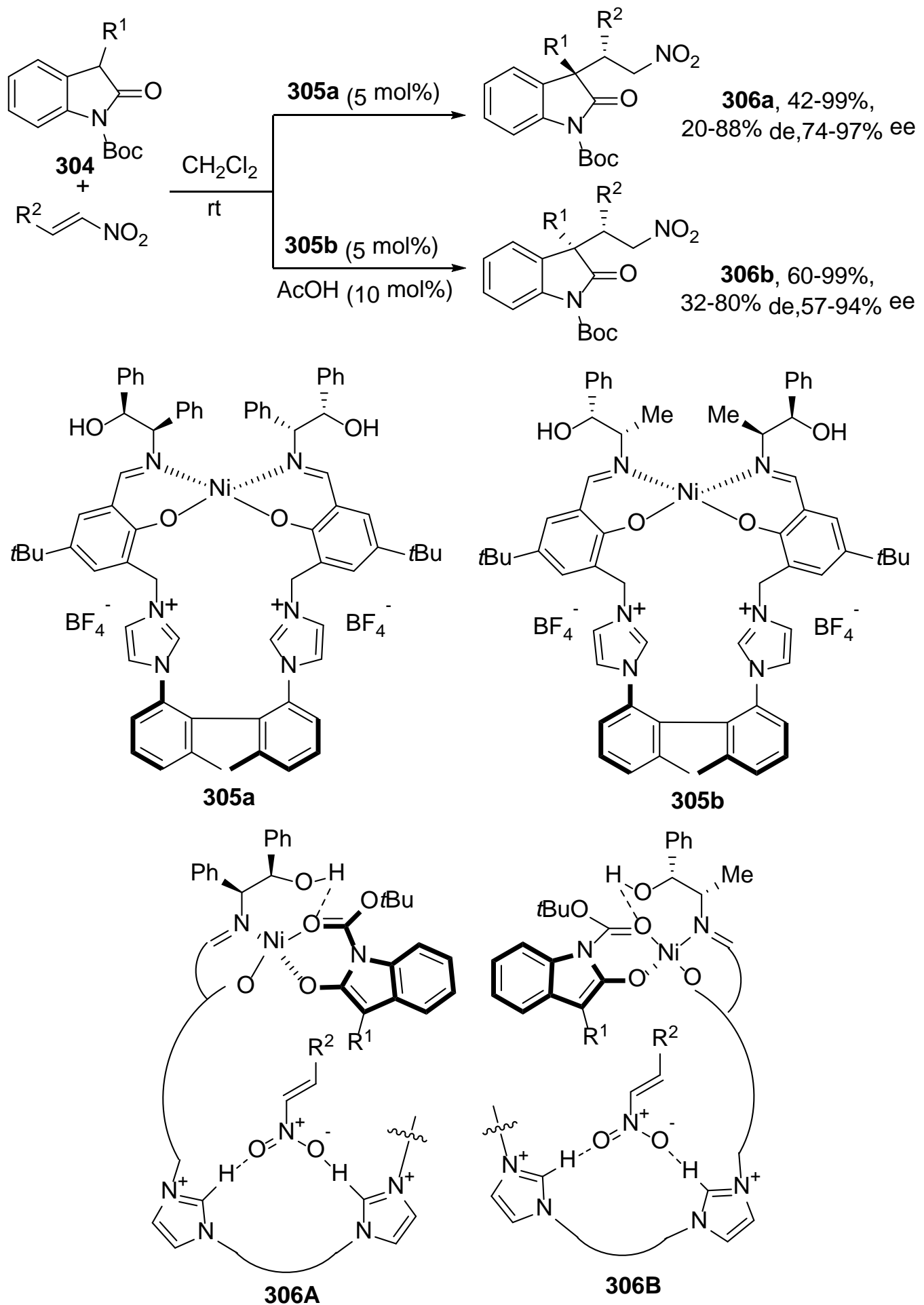

Fukuzawa and co-workers reported the diastereodivergent CA of 1-pyrroline esters 307 to nitroalkenes catalyzed by $\mathrm{Cu}$ and Ag complexes using different chiral ligands 308 and 309, respectively (Scheme 111$){ }^{307}$ In the case of CuOAc and $\left(S, S_{P}\right)$-FcPHOX 308 syn-310 adducts were obtained in high de and excellent ee, whereas using AgOAc and $(R, S p)$-ThioClickFerrophos 309 as catalyst, products anti-310 were formed in excellent de and high ee. From the DFT calculations the TS 310A and 310B have been proposed to explain the observed diastereo- and enantioselectivity. In the model 310A, 
the nitroalkene will approach the $S i$ face of the pyrroline ester from its $R e$ face, avoiding the sterically hindered oxazoline unit of the ligand. The anti-selective CA catalyzed by the Ag complex took place from the $R e$ face of the nitroalkene.

Scheme 111. Diastereodivergent Enantioselective Conjugate Addition of 1Pyrroline Esters 307 to Nitrostyrenes Catalyzed by CuOAc/308 and AgOAc/309 Complexes

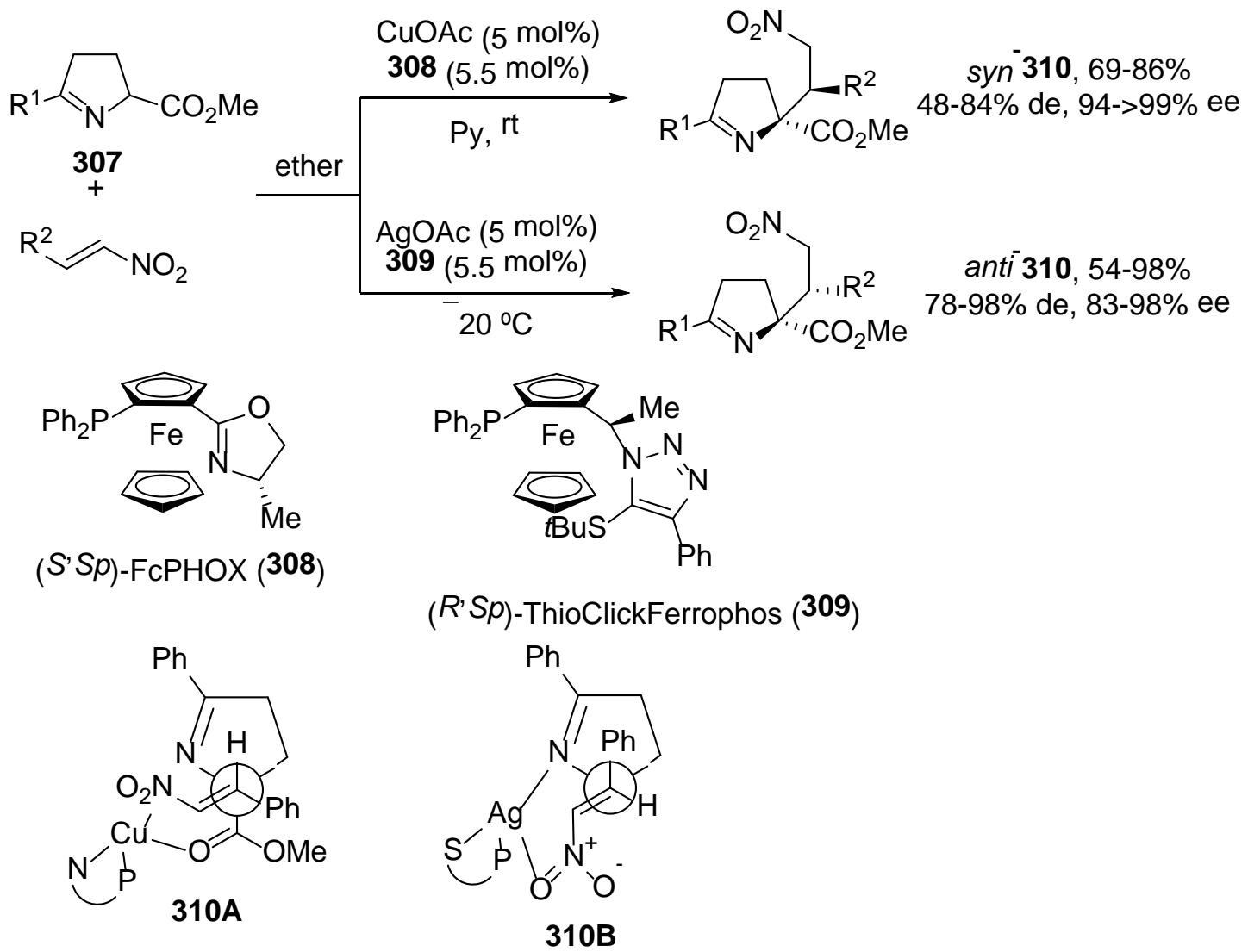

An ambidextrous redox-configurable copper catalyst 311, derived from Lmethionine, has been used for the enantiodivergent CA of diethyl malonate to $\beta$ nitrostyrene. Utilising the different oxidation states of the copper the reversal of enantioselectivity can be achieved. Thus, the corresponding (S)-adduct can be obtained in $72 \%$ ee when the $\mathrm{Cu}(\mathrm{II})$ complex 311a was used (Figure 13). ${ }^{308}$ Upon one-electron reduction of the $\mathrm{Cu}(\mathrm{II})$ to the $\mathrm{Cu}(\mathrm{I})$ complex $\mathbf{3 1 1 b}$, the helical inversion takes place and the corresponding enantiomeric $(R)$-adduct was obtained in $70 \%$ ee.

Figure 13. Helical $\mathrm{Cu}(\mathrm{II})$ and $\mathrm{Cu}(\mathrm{I})$ Complexes 311 for the Enantiodivergent Conjugate Addition of Diethyl Malonate to $\beta$-Nitrostyrene 

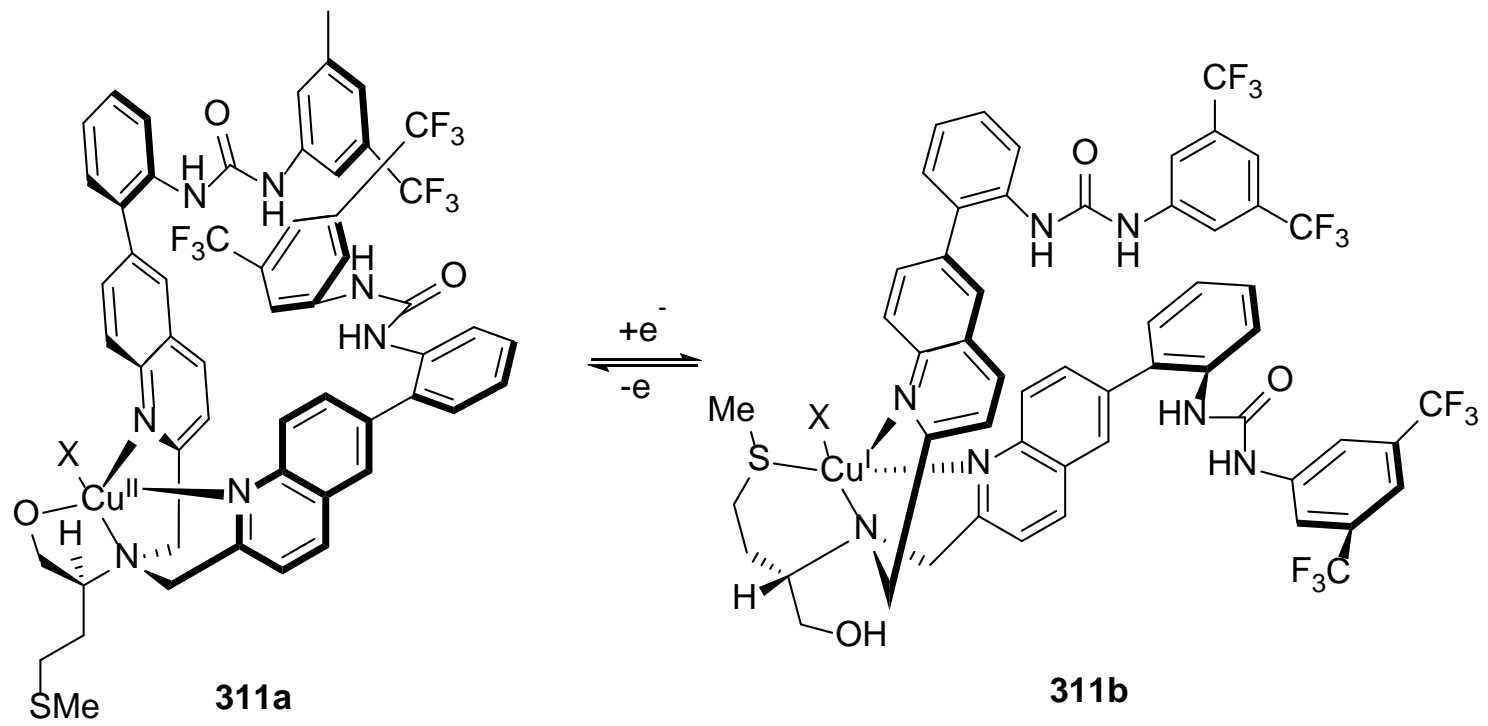

Enantiodivergent CA has been observed using the same ligand and different metal centers in the reaction of pyrazolin-5-ones $\mathbf{3 1 2}$ with 4-oxo-4-arylbutenoates $\mathbf{3 1 3}$ (Scheme 112). ${ }^{309}$ In the presence of Sc(OTf) 3 and ligand 314, the corresponding adducts $(2 S, 4$ 'S)-315 were preferentially formed with good diastereoselectivity and high enantioselectivity. On the other hand, $\mathrm{Y}(\mathrm{OTf})_{3}$ and the same ligand 314 gave the corresponding enantiomers $\left(2 R, 4^{\prime} R\right)-316$ with high diastereoselectivity and high enantioselectivity. This switch in enantioselectivity has been attributed to the smaller ionic radii of scandium(III) than yttrium(III) and therefore the coordination of both reagents can take place in the yttrium complex only.

Scheme 112. Enantiodivergent Conjugate Addition of 4-Substituted Pyrazolones 312 to 4-Oxo-4-arylbutenoates 313 Catalyzed by $\operatorname{Sc}(\mathrm{OTf})_{3}$ or $\mathrm{Y}(\mathrm{OTf})_{3}$ and 314 Complexes

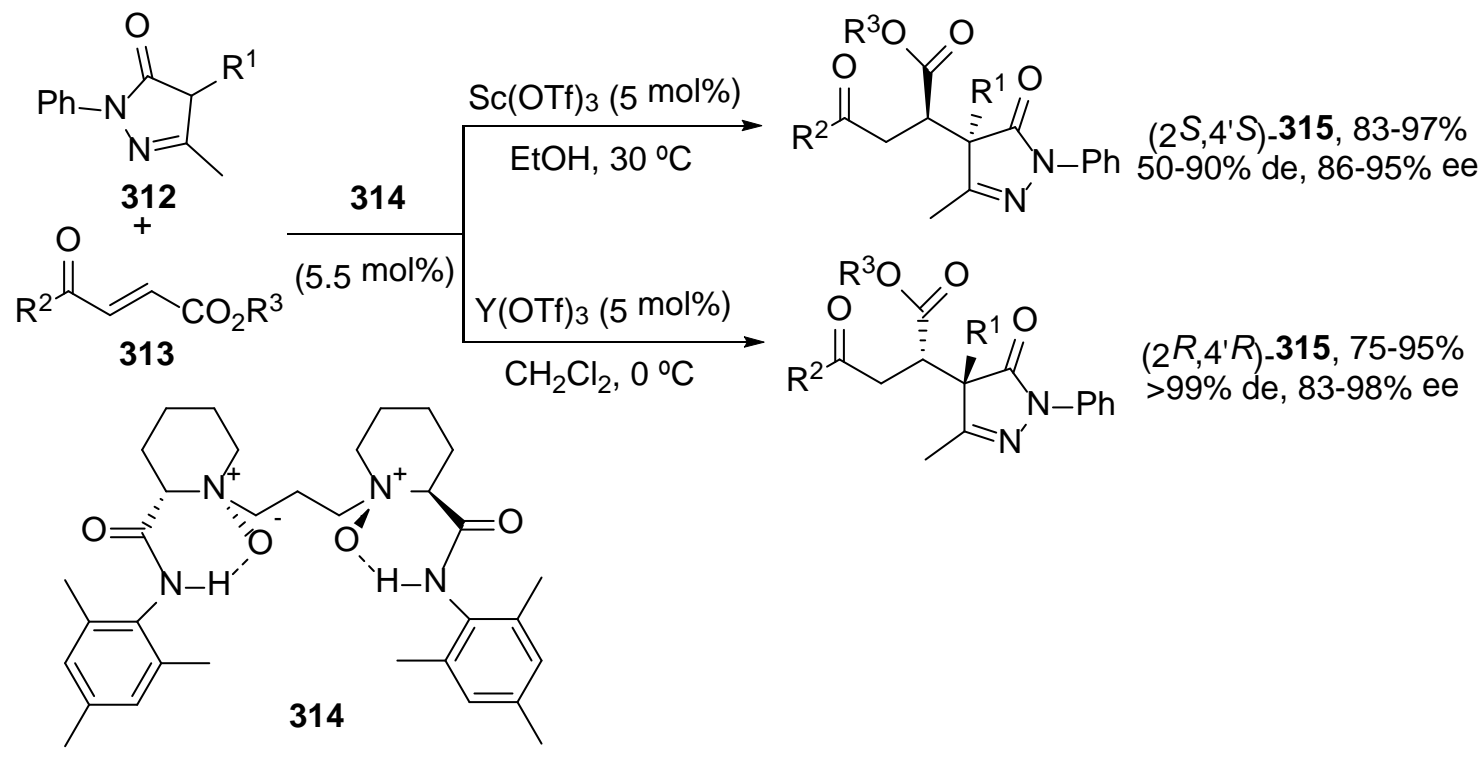


The catalytic asymmetric addition of $\alpha$-cyanoketones 229 (Scheme 82) to vinyl ketones gave the corresponding adducts with lower enantiodivergence than for the Mannich reaction ${ }^{244,245}$ described by the same group. In this case, the use of $\mathrm{Y}(\mathrm{OiPr})_{3}$ and the ligand 230 (10 mol\%) gave the corresponding $(R)$-products with ee up to $98 \%$, whereas $\mathrm{La}(\mathrm{OiPr})_{3}$ provided the $(S)$-products with ee up to $28 \%{ }^{310}$

Conjugate addition of thiols to $N$-alkenoyloxazolidinones 298 catalyzed by $\mathrm{Ni}\left(\mathrm{ClO}_{4}\right)_{2} \cdot 6 \mathrm{H}_{2} \mathrm{O}$ and the bisoxazoline DBFOX 316 has shown a modest enantiodivergent effect caused by the presence of different additives (Scheme 113). ${ }^{311}$ In the presence of $\mathrm{MeOH}$, thiophenol added quantitatively to $N$-crotonoyloxazolidinone 298 giving $(S)$-317 in 82\% ee, whereas in the presence of aqueous $\mathrm{NH}_{4} \mathrm{Cl},(R)-\mathbf{3 1 7}$ was formed in low $27 \%$ ee.

Scheme 113. Enantiodivergent Conjugate Addition of Thiophenol to $N$ Crotonoyloxazolidinone 298 Catalyzed by $\mathrm{Ni}\left(\mathrm{ClO}_{4}\right)_{2} \cdot 6 \mathrm{H}_{2} \mathrm{O} /(\mathrm{R}, \mathrm{R})-\mathrm{DBFOX} 316$ Complex

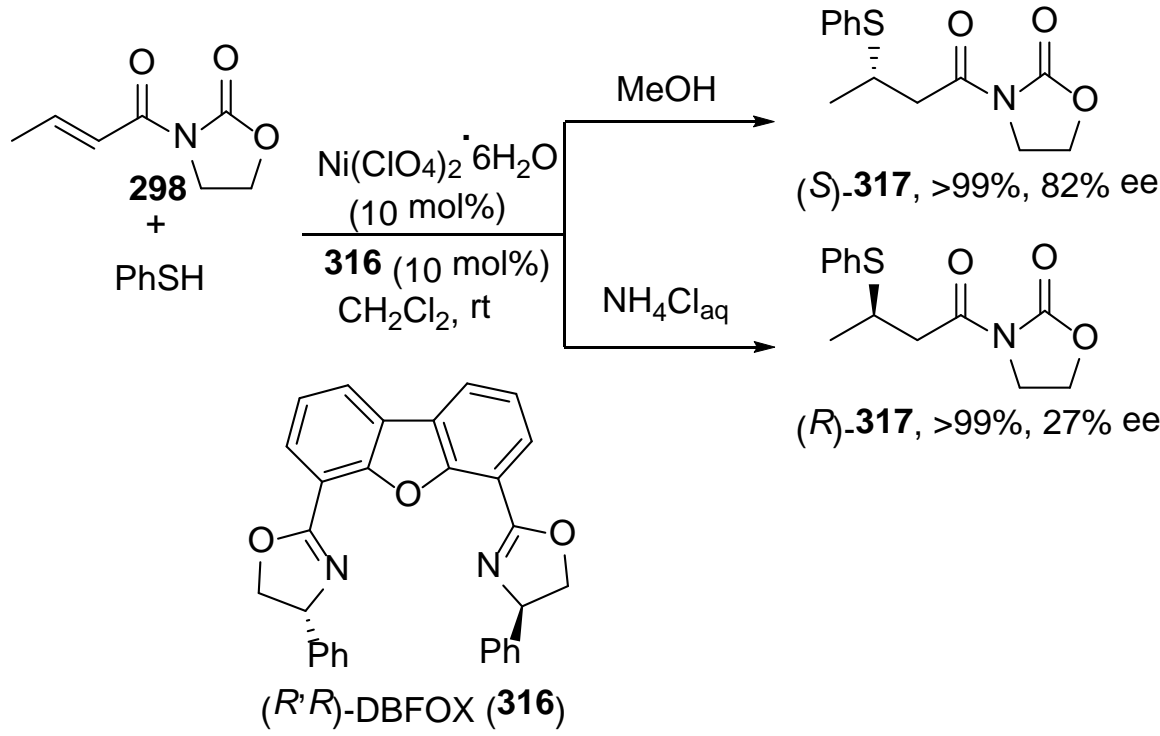

Sibi and co-workers performed the first highly enantioselective conjugate amination using stoichiometric amounts of Lewis acid in the reaction of $O$-benzylhydroxylamine with $\mathrm{N}$-crotonoylpyrazole 318 catalyzed by the complex formed by $\mathrm{MgBr}_{2}$ and chiral BOX 319 (Scheme 114). ${ }^{312}$ They found the reversal of enantioselectivity when $\mathrm{MgBr}_{2}$ was substituted by $\mathrm{Y}(\mathrm{OTf})_{3}$ giving $(R)$-320 or $(S)$-320, respectively. In the case of $\mathrm{Y}(\mathrm{OTf})_{3}(S)$-320 was obtained in a higher yield but in lower enantioselectivity.

Scheme 114. Enantiodivergent Conjugate Addition of $O$-Benzylhydroxylamine to $\mathrm{N}$-Crotonoylpyrazole 318 Catalyzed by $\mathrm{BOX} 319$ and $\mathrm{MgBr}_{2}$ or $\mathrm{Y}(\mathrm{OTf})_{3}$ 


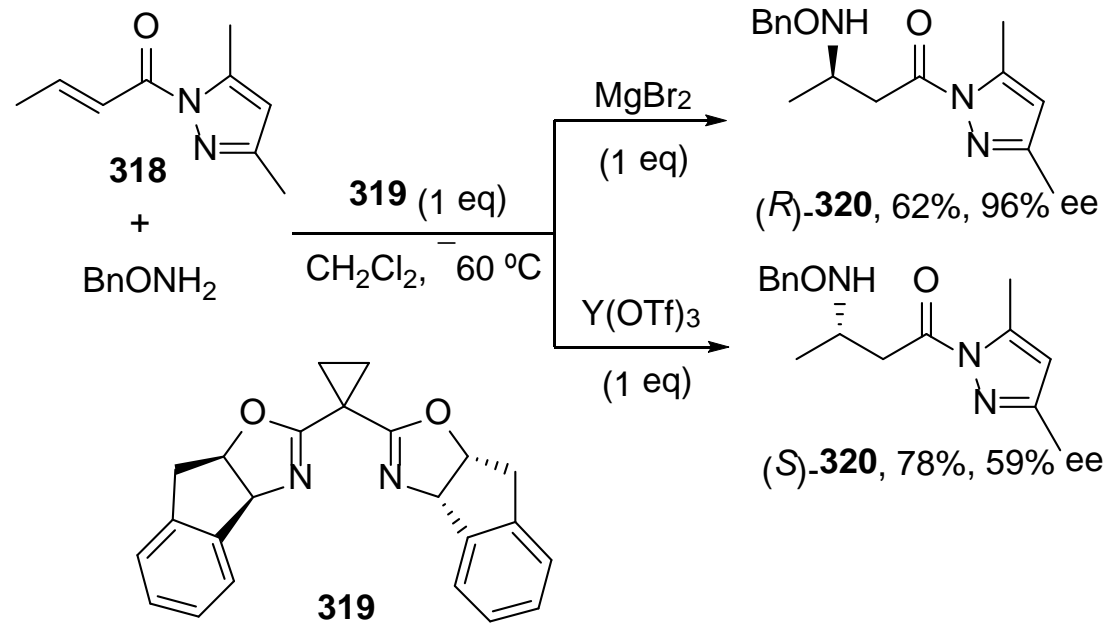

Further studies by the same group revealed a temperature-dependent enantiodivergence for the addition of $O$-benzylhydroxylamine to $\mathrm{N}$ crotonoyloxazolidinone 321 catalyzed by $\mathrm{MgBr}_{2}$ and ligand 319 (Scheme 115). ${ }^{313}$ At room temperature $(R)-322$ was mainly obtained, whereas at $-60{ }^{\circ} \mathrm{C}$ the corresponding enantiomer was predominately formed. The same reversal of enantioselectivity was observed with substrates 321 with substituents at the $\beta$-position by Et, $n \mathrm{Pr}, n \mathrm{C}_{5} \mathrm{H}_{11}$ and $n \mathrm{C}_{6} \mathrm{H}_{13}$ groups. This effect was not observed with an unsubtituted $N$ crotonoyloxazolidinone such as $\mathbf{2 9 8}$.

Scheme 115. Enantiodivergent Conjugate Addition of $O$-Benzylhydroxylamine to the $\mathrm{N}$-Crotonoyloxazolidinone 321 Catalyzed by $\mathrm{MgBr}_{2} / 319$ Complex at Different Temperatures

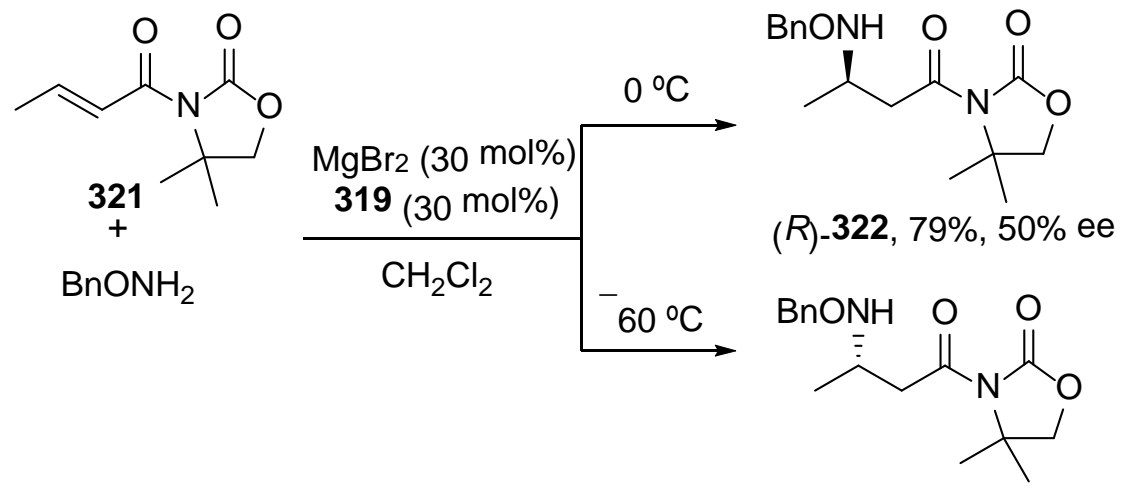

(S)-322, 80\%, 43\% ee

Phospha-Michael addition of diphenylphosphine to 4-oxoenamides 323 has been carried out under Pd-catalysis. ${ }^{314}$ By means of palladacycle 324, compounds 325 were prepared in high yield and ee (Scheme 116). This process is sensitive to the solvent giving a reversal of enantioselectivity in toluene and in a mixture of $\mathrm{CHCl}_{3} / \mathrm{MeOH}$ (10\%). Thus, in toluene $(R)-325$ were obtained, whereas in the mixed solvents the corresponding enantiomers were mainly formed. This behavior was explained just by the different polarity of the solvents. 
Scheme 116. Enantiodivergent Conjugate Addition of Diphenylphosphine to 4Oxoenamides 323 Catalyzed by Palladacycle 324 in Different Solvents
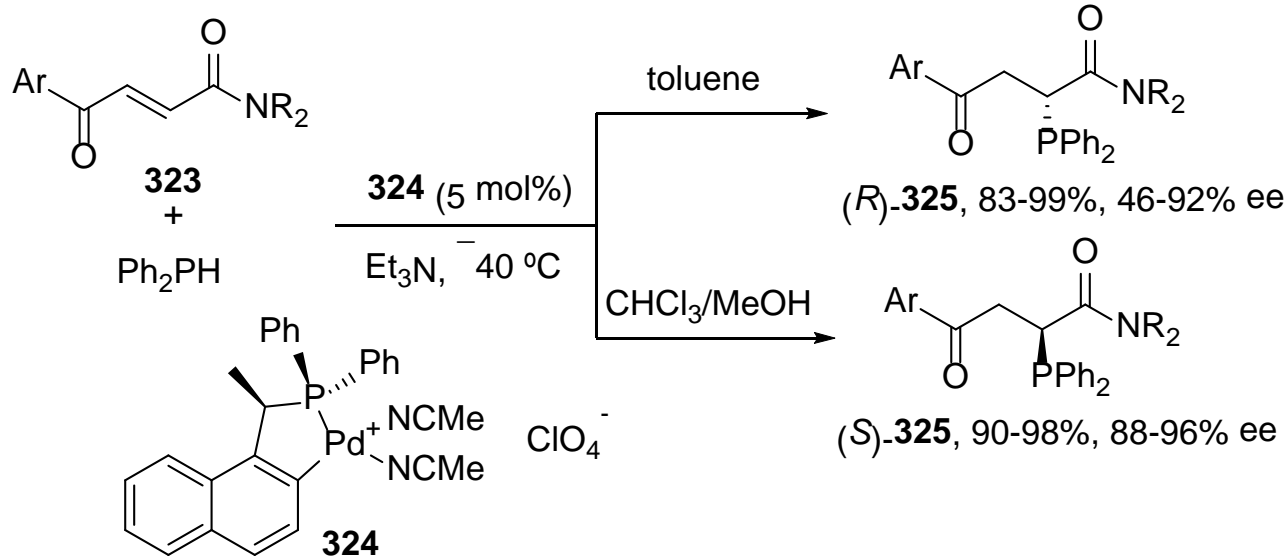

(S)-325, 90-98\%, 88-96\% ee

Metal-catalyzed radical CA reactions have been extensively studied by Sibi and coworkers. ${ }^{11,280}$ They observed the influence of the Michael acceptor in the resulting enantioselectivity of these processes. Thus, the isopropyl radical reacted with $\mathrm{N}$ cinnamoyloxazolidinone 298 catalyzed by Zn(OTf $)_{2}$ and BOX ligand 319 providing (R)-products 326, whereas the $N$-cinnamoyl-3,5-dimethylpyrazole $\mathbf{3 1 8}$ led to the formation of (S)-327 (Scheme 117). ${ }^{315}$ This non-formal enantiodivergency has been explained as a consequence of the size of the intermediate formed by the metal complex and the cinammoyl derivative. In the case of pyrazole 318, a five-membered ring 318A was formed unlike a six-membered one in the case of the oxazolidinone substrate. This methodology can be considered enantiodivergent because both products $\mathbf{3 2 6}$ and $\mathbf{3 2 7}$ afforded, after deprotection of the carboxylate function, the same acid derivative with opposite configuration.

Scheme 117. Enantiodivergent Free-Radical Conjugate Addition to $\mathrm{N}$ Cinnamoyloxazolidinone 298 and $N$-Cinnamoyl-3,5-dimethylpyrazole 318 Catalyzed by Zn(OTf $)_{2}$ and BOX 319

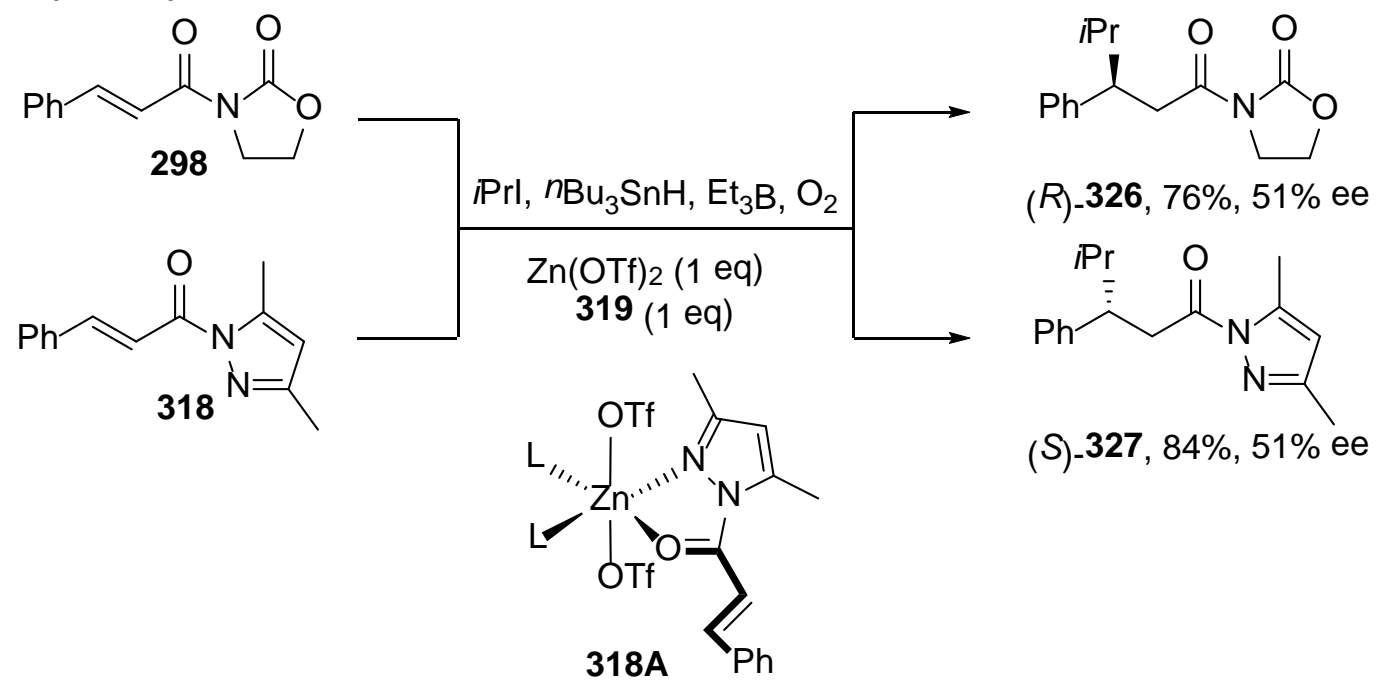


Free-radical CA of the isopropyl radical to $N$-cinnamoyloxazolidinones $\mathbf{3 2 8}$ catalyzed by $\mathrm{Zn}(\mathrm{OTf})_{2}$ and $\mathrm{BOX} 329$ resulted in the reversal of enantioselectivity depending on the substituents of the achiral oxazolidinone $\mathbf{3 3 0}$ used as additive (Scheme 118). ${ }^{316}$ In the case of oxazolidinone 330a (R)-331 was obtained in $82 \%$ ee, whereas in the presence of the $N$-methylated 330b the corresponding enantiomer was formed in low 29\% ee. The role of this additive 330a to achieve high ee can be explained by using its ability as a coordinating ligand for $\mathrm{Zn}$ forming a ternary complex.

Scheme 118. Enantiodivergent Free-Radical Conjugate Addition to $\mathrm{N}$ Cinnamoyloxazolidinone 328 Catalyzed by $\mathrm{Zn}(\mathrm{OTf})_{2} / \mathrm{BOX} 329$ Complex and Additive 330

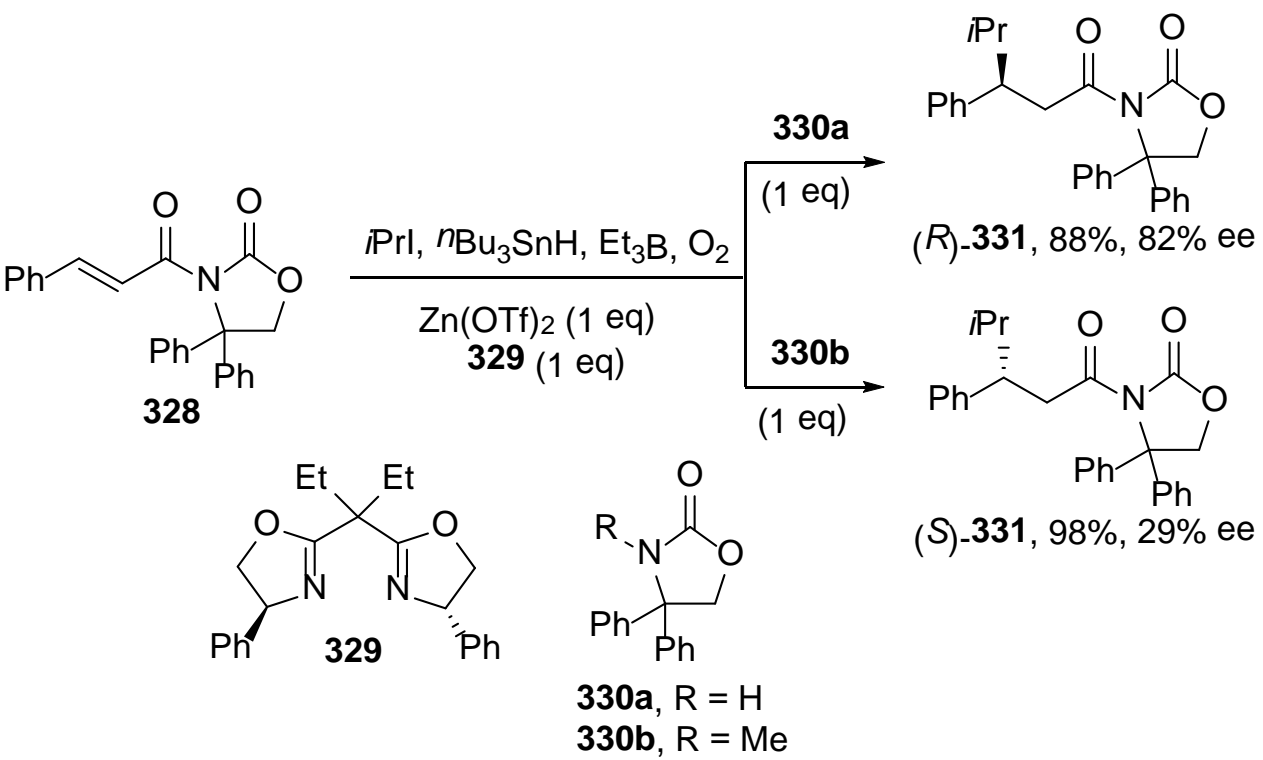

Sibi and Chen have described an enantiodivergent tandem free-radical CA of the isopropyl radical to $N$-cinnamoyloxazolidinone and trapping the intermediate radical with an allylstannane. In this case a metal salt-dependent effect was observed. Thus, working with BOX 319 and $\mathrm{MgI}_{2}$ product anti-332 was obtained in high 94\% de and 93\% ee (Scheme 119). ${ }^{317}$ On the other hand, just changing the metal salt to $\mathrm{Cu}(\mathrm{OTf})_{2}$ ent-332 was obtained in $92 \%$ de and $79 \%$ ee. Similar results were obtained with tertbutyl iodide.

Scheme 119. Enantiodivergent Free-Radical Conjugate Addition to $\mathrm{N}$ Cinnamoyloxazolidinone 298 Catalyzed by Ligand 319 using $\mathrm{MgI}_{2}$ or $\mathrm{Cu}(\mathrm{OTf})_{2}$ and Subsequent $\alpha$-Allylation 


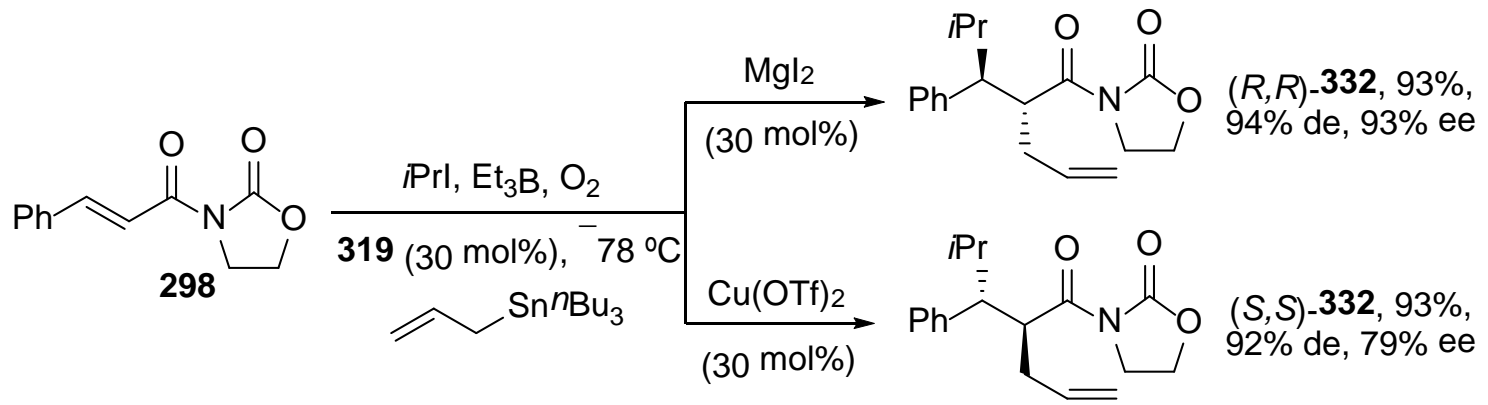

Friedel-Crafts (F-C) reactions between indoles or pyrroles with electrophilic alkenes can be also considered as CA. The first enantiodivergent F-C alkylation of indoles with alkylidene malonates catalyzed by a tris(oxazoline)(TOX)333-copper(II) complex was described by Tang and co-workers. ${ }^{318}$ Solvent-dependent enantioselectivity was observed when the reaction was performed in isobutyl alcohol or 1,1,2,2tetrachloroethane (TCE). In the former solvent products 334 were mainly obtained, whereas in TCE ent-334 were prepared (Scheme 120). This switch of enantioselectivity was explained considering the coordination of the substrates in the metal center. In the model 333A the coordination mode, in which the $\mathrm{Cu}$ atom adopted a square-planar geometry favoring the $R e$-attack by the indole according to Evans studies, is shown in Scheme 120. On the other hand, in the case of working in TCE the copper is in a distorted square-pyramidal geometry and the attack were preferred to the Si-face of malonate in model 333B.

Scheme 120. Enantiodivergent Friedel-Crafts Reaction of Indole with Alkylidene Malonates Catalyzed by $\mathrm{Cu}(\mathrm{OTf})_{2 /}$ Tris(oxazoline) 333 in Different Solvents 


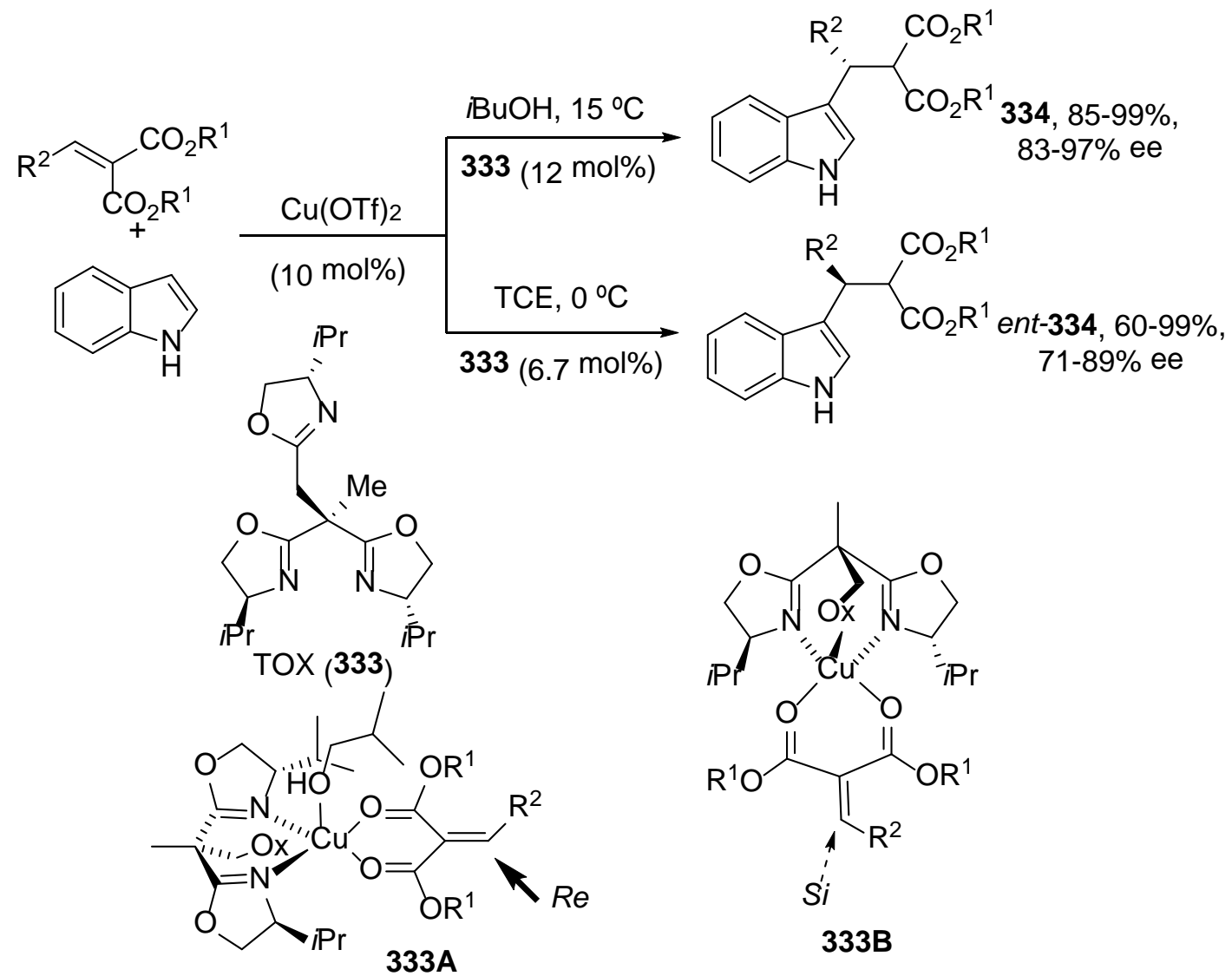

Feng and co-workers have described an example of enantiodivergent F-C alkylation of indoles with $\beta, \gamma$-unsaturated $\alpha$-keto esters 335 using rather similar homochiral ligands 336 and 337a and different metal salts (Scheme 121). ${ }^{319}$ In the case of AgAsF $_{6}$ and ligand 336 in THF, the predominant formation of (S)-338 was achieved. On the contrary, the corresponding enantiomers were the major products when $\operatorname{Sm}(\mathrm{OTf})_{3}$ and ligand 337a in $\mathrm{CH}_{2} \mathrm{Cl}_{2}$ were used. The proposed TS 336A for the Ag-catalyzed process shows the coordination of silver atom with two oxygen atoms of the $\mathrm{N}$-oxide and the attack of the indole to the $\beta$-Si face of the $\alpha$-keto ester 335 affording $(S)$-338. For the Sm-catalyzed reaction TS 337A has been proposed according to the X-ray structure of the complex. ${ }^{320}$ Hexacoordinate $\operatorname{Sm}(\mathrm{OTf})_{3}$ with the ligand and the two oxygen atoms of the keto ester will favor the attack of the indole to the $\beta$-Re face of the $\mathrm{C}$-C double bond providing $(R)-338$.

Scheme 121. Enantiodivergent Friedel-Crafts Reaction of Indoles with $\beta, \gamma$ Unsaturated $\alpha$-Keto Esters 335 Catalyzed by Ligands 336 and AgAsF $_{6}$ or Ligand 337a and $\operatorname{Sm}(\mathrm{OTf})_{3}$ 


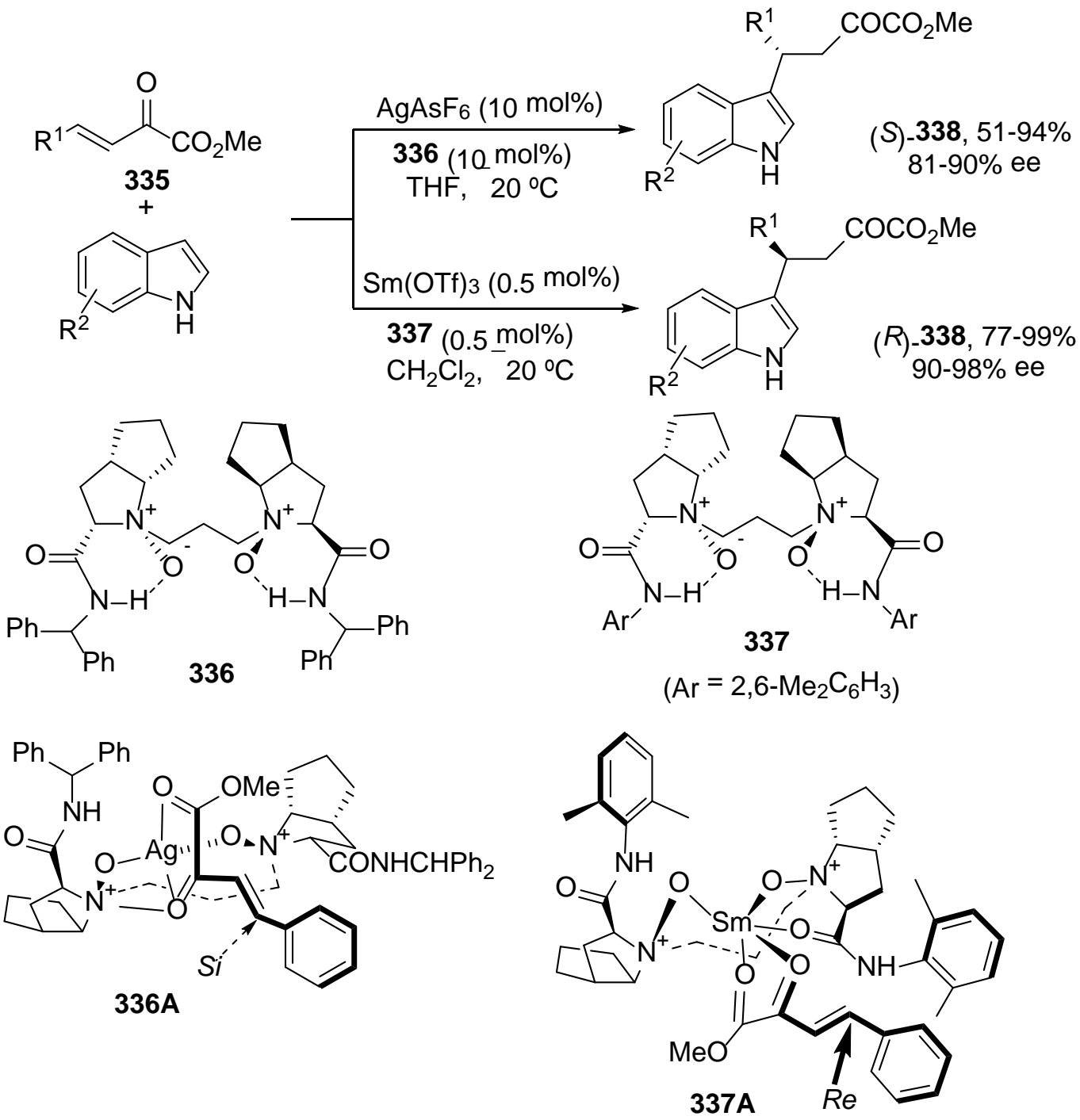

Reversal of the enantioselectivity has been found by Oh and co-workers in the F-C alkylation of indole with nitroalkenes under both homogeneous and heterogeneous conditions. $^{321}$ Adducts derived from this reaction are useful building blocks for the preparation of biologically active indole alkaloids. ${ }^{322}$ Using brucine-derived diol 115 (Figure 7) and $\mathrm{Cu}(\mathrm{OTf})_{2} \cdot \mathrm{PhH}$ under homogeneous conditions, (S)-products 339 were obtained in good ee (Scheme 122). On the contrary, using heterogeneous conditions by adding different solid supports to the reaction media $(R)$-339 were mainly isolated, $4 \AA$ molecular sieves giving the best results. The stereochemical models 339A and 339B have been proposed for both types of catalysts.

Scheme 122. Enantiodivergent Friedel-Crafts Reaction of Indoles with $\beta$ Nitroalkenes Catalyzed by $\mathrm{Cu}(\mathrm{OTf})_{2} \cdot \mathrm{PhH} /$ Ligand 115 under Homogeneous and Heterogeneous Conditions 

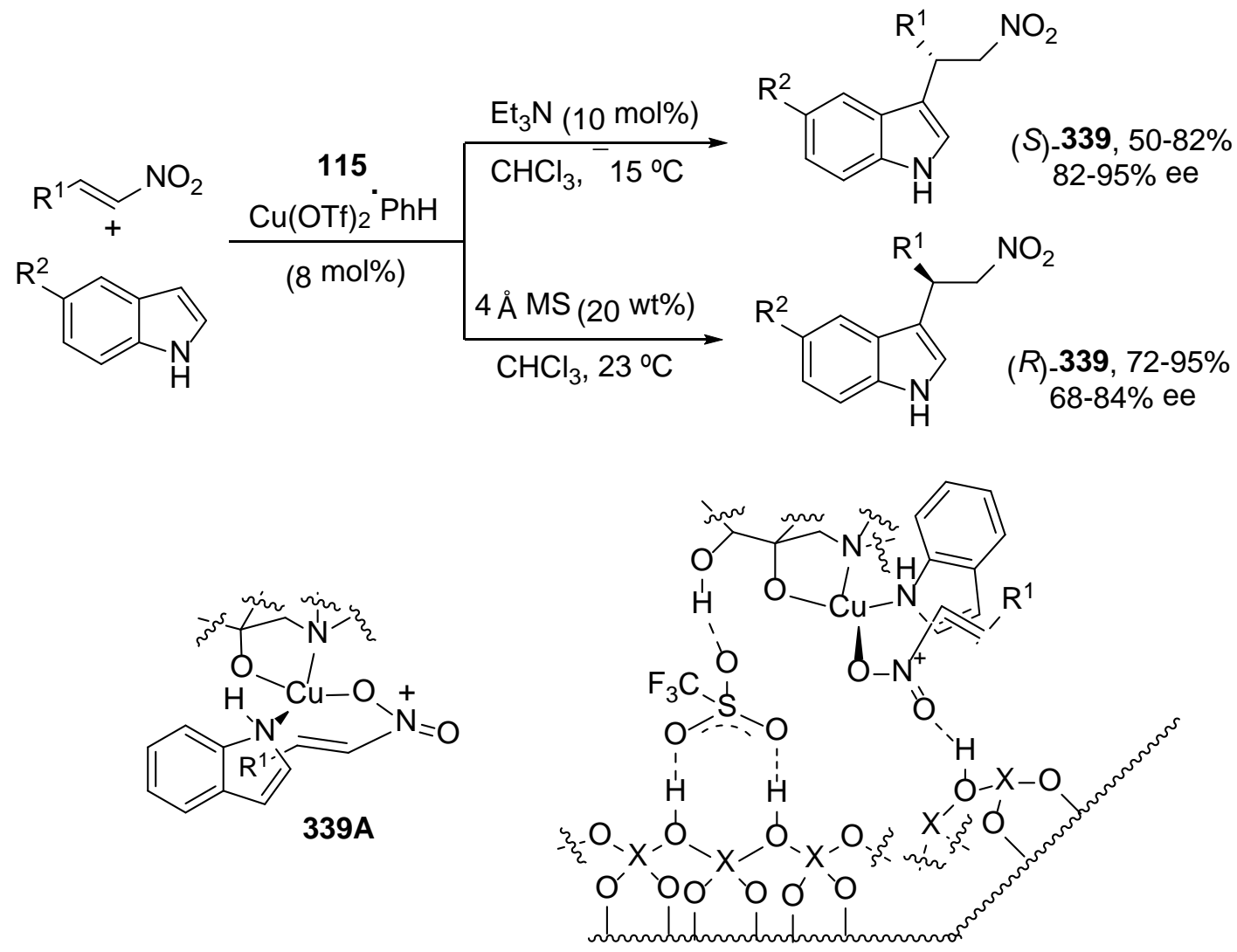

339B

Friedel-Crafts reaction of 2,5-dimethylpyrrole and indole with $\beta, \gamma$-unsaturated $\alpha$-keto esters $\mathbf{3 3 5}$ has been enantiodivergently performed with $N$-oxide derived ligands $\mathbf{3 3 7}$ and $\mathrm{Ni}(\mathrm{OTf})_{2}$. Different substitution in ligands 337b and 337c promoted excellent reversal of ee affording products $\mathbf{3 4 0}$ and ent-340, respectively (Scheme 123). ${ }^{323}$ Similar switch of enantioselectivity was also achieved in the case of indoles giving products 341, in general with high ee. From the X-ray diffraction analysis of the Ni complexes and also from DFT calculations it has been concluded that the steric hindrance of the $O$ isopropyl substituents in ligand 337b favored the alkylation from the Re face giving $(R)$ 340 and 341. In the case of ligand 337c, the tert-butyl substituents shielded the Re face of the substrate giving $(S)$-products.

Scheme 123. Enantiodivergent Friedel-Crafts Reaction of 2,5-Dimethylpyrrole and Indoles with $\beta, \gamma$-Unsaturated $\alpha$-Keto Esters 335 Catalyzed by Ligands 337 and $\mathrm{Ni}(\mathrm{OTf})_{2}$ 


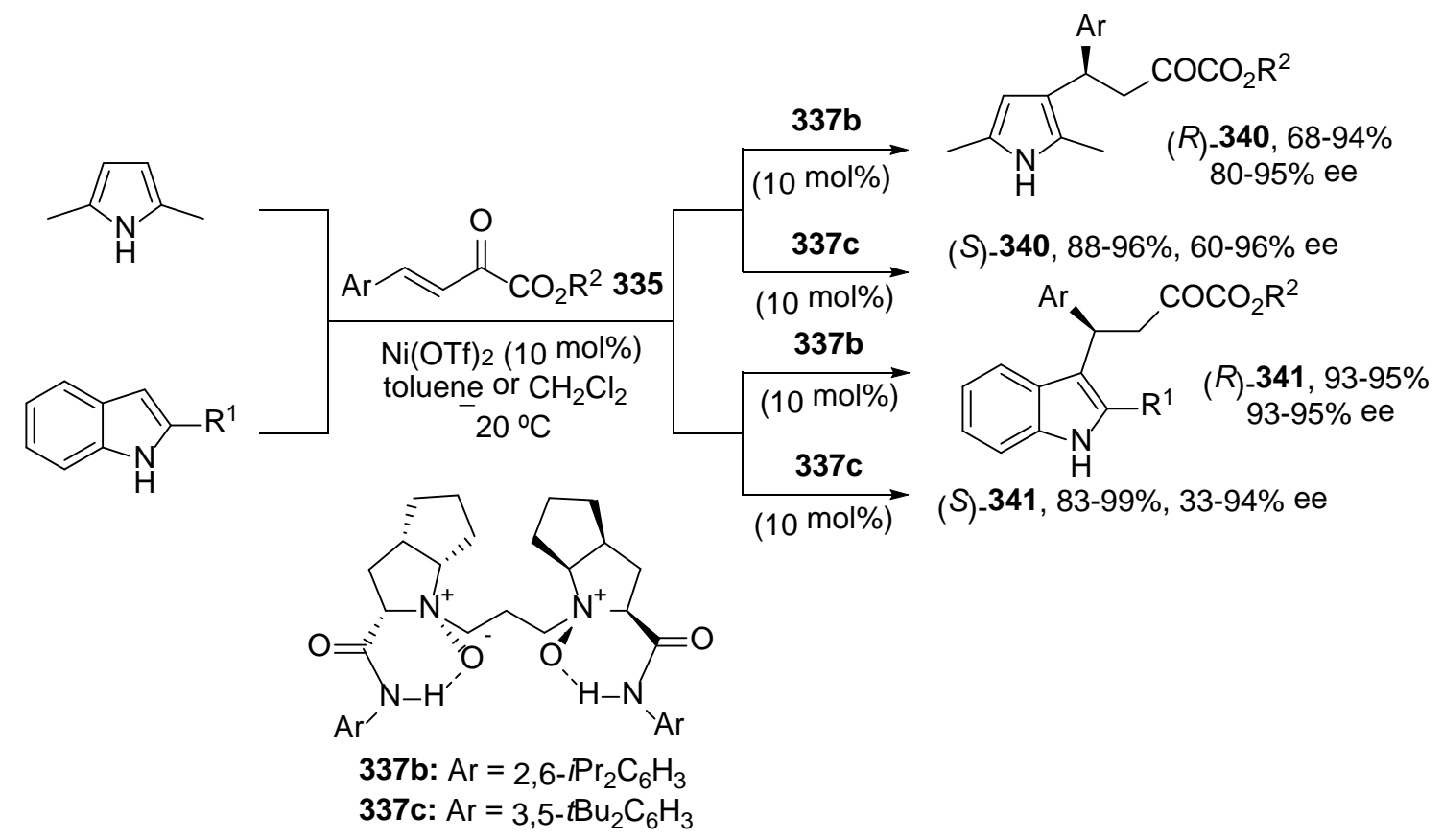

In conclusion, concerning the metal-catalyzed CA of organometallic reagents solvent-dependence plays an important role in the switch of enantioselectivity. When neutral carbon nucleophiles are added to Michael acceptors, in most of the cases changes in the metal complex determine the sense of enantioselectivity either modifying the metal center or the substituents in the ligand. In few cases the presence of additives and the temperature play an important role. An interesting enantiodivergent effect has been observed in the F-C reaction working either under homogeneous or heterogeneous conditions.

2.3.1.2. Organocatalyzed Conjugate Additions. Asymmetric organocatalyzed additions have been extensively studied in the last 15 years. ${ }^{282,284,286-288}$ Activation modes for the organocatalyzed CA can be performed mainly by: (a) covalent bonding either to the nucleophile or to the electrophile, (b) hydrogen bonding to them, and (c) ion paring formation under phase-transfer catalysis (PTC). With respect to the covalent bonding, the nucleophiles can be activated by the transitient formation of a chiral enamine and the electrophilic Michael acceptor by the formation of an ion-pair with the chiral ammonium ion. However, relative few examples deal with stereodivergent processes. In this section only intermolecular organocatalyzed processes will be considered.

Malonates are typical nucleophiles in CA and they have been added to chalcone under PTC. Salunkhe and co-workers observed a moderate solvent effect in the enantioselectivity of this CA working with a quininium salt $\mathbf{3 4 2}$ as catalyst (Scheme 124). ${ }^{324}$ Thus, in organic solvents such as DMSO $(S)-343$ was formed in $96 \%$ yield and $61 \%$ ee, whereas in an ionic liquid the corresponding enantiomer was almost quantitatively formed in $50 \%$ ee in shorter reaction times. 
Scheme 124. Enantiodivergent Conjugate Addition of Dimethyl Malonate to Chalcone Catalyzed by Quininium Salt 342 in Different Solvents

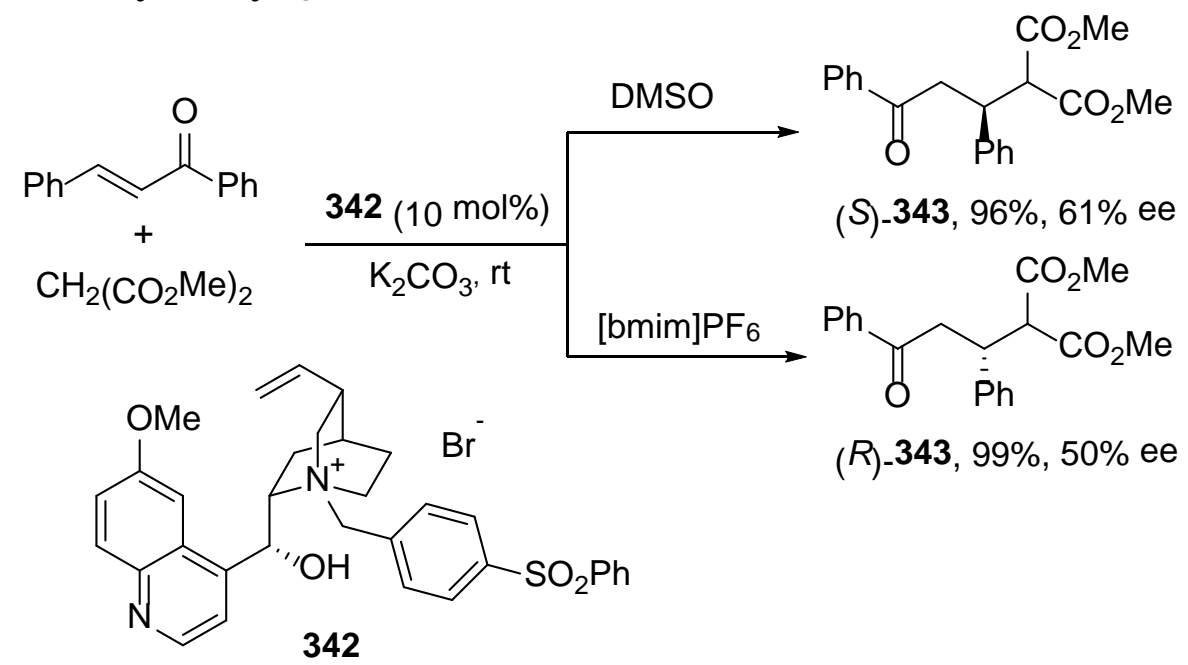

More recently, inversion of the enantioselectivity has been found out using the dimeric cinchonidinium salts $\mathbf{3 4 4}$ in the same type of Michael addition. ${ }^{325}$ Reaction conditions studies revealed that using $\mathbf{3 4 4}$ as catalysts in non polar solvents such as toluene and $\mathrm{NaOH}$ as base, the addition of diethyl malonate to chalcone gave $(R)-345$ $\left(\mathrm{Ar}^{1}=\mathrm{Ar}^{2}=\mathrm{Ph}\right)$ in $99 \%$ ee. However, in polar solvents such as $\mathrm{MeOH}$ the $(S)$-product was obtained in $45 \%$ ee. Besides, a stronger enantiodivergent effect with different substituted chalcones was observed (Scheme 125). Thus, with electron-donating groups in $\mathrm{Ar}^{2}(S)$-345 were formed. However, when $\mathrm{Ar}^{2}$ has an electron-withdrawing substituent, inversion of the configuration took place providing $(R)-345$. This behavior has been attributed to the $\pi-\pi$ interactions of the aromatic rings of the chalcones and the quinoline moiety and to the flexibility of this chiral catalyst.

Scheme 125. Enantiodivergent Conjugate Addition of Diethyl Malonate to Different Substituted Chalcones Catalyzed by Cinchoninium Salts 344 


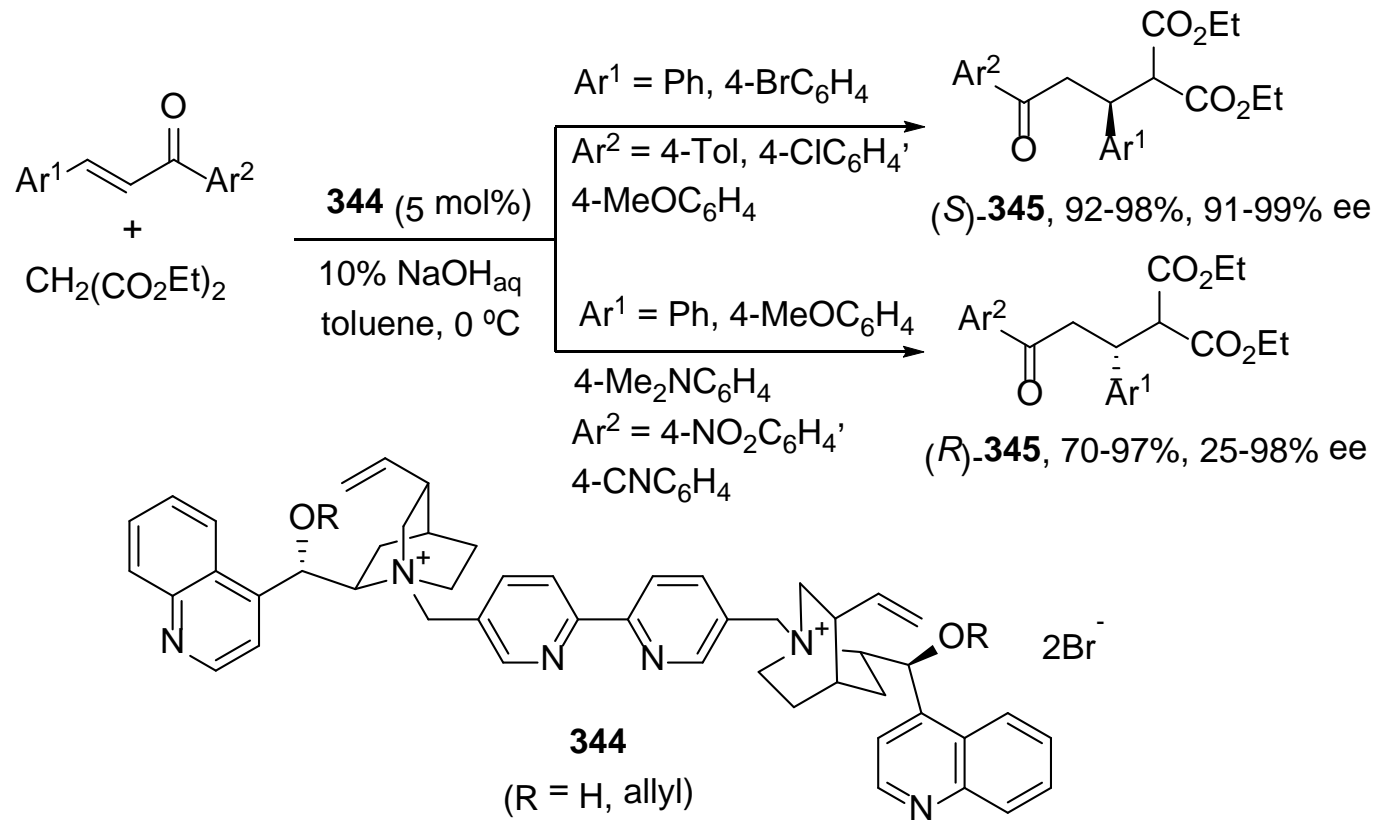

A diastereodivergent tandem CA of $\alpha$-cyano ketones to $\alpha$-chloroacrylonitrile has been reported by Deng and co-workers. ${ }^{326}$ Cinchona bases 346 and 347 acted as bifunctional catalysts bearing hydrogen bond donor and acceptor units in the same molecule. For instance, when 2-cyanoindanone reacted with $\alpha$-chloroacrylonitrile in the presence of 346, syn-adduct 348 was obtained diastereoselectively in 20:1 dr and 93\% ee (Scheme 126). On the other hand, when the thiourea derivative 347 was used as organocatalyst, anti-348 was formed in 10:1 dr and 97\% ee. Transition state models 346A and 347A have been proposed to explain the enantiodivergency observed in these CA.

Scheme 126. Diastereodivergent Enantioselective Conjugate Addition of 2Cyanoindanone to $\alpha$-Chloroacrylonitrile Catalyzed by Cinchona Bases 346 and 347 


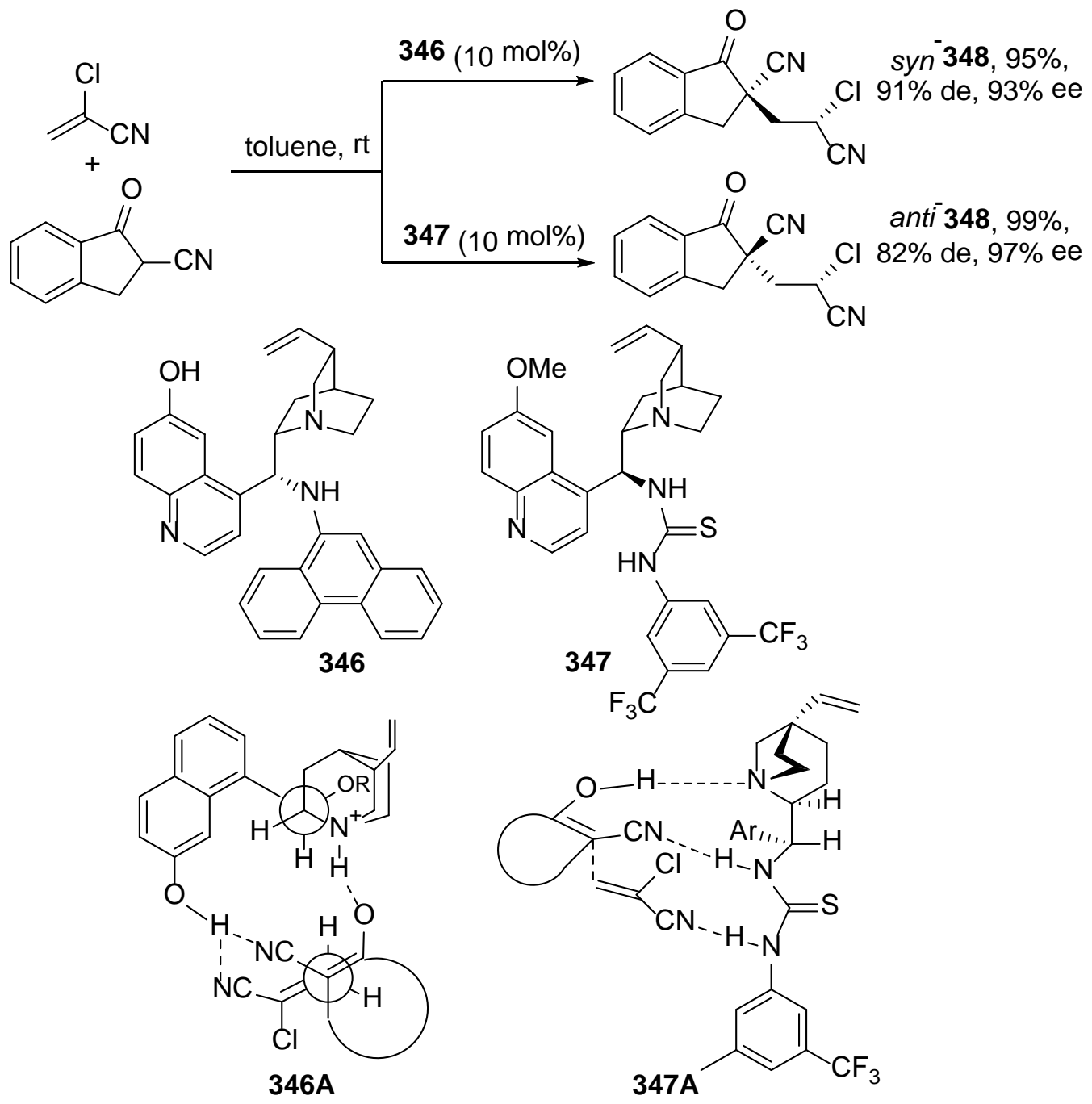

Reversal of enantioselectivity has been observed in the CA of 2-nitropropane to chalcones catalyzed by $N$-spiroammonium salts $349^{327}$ related to Maruoka's catalysts. $^{328}$ Adducts 350a with $(R)$-configuration were mainly obtained using 349a, whereas the more flexible organocatalysts 349b gave products $(S)-350$ (Scheme 127). Excellent results were in general obtained and the catalysts can be recovered and reused.

Scheme 127. Enantiodivergent Conjugate Addition of 2-Nitropropane to Chalcones Catalyzed by Different Spiroammonium Salts 349 


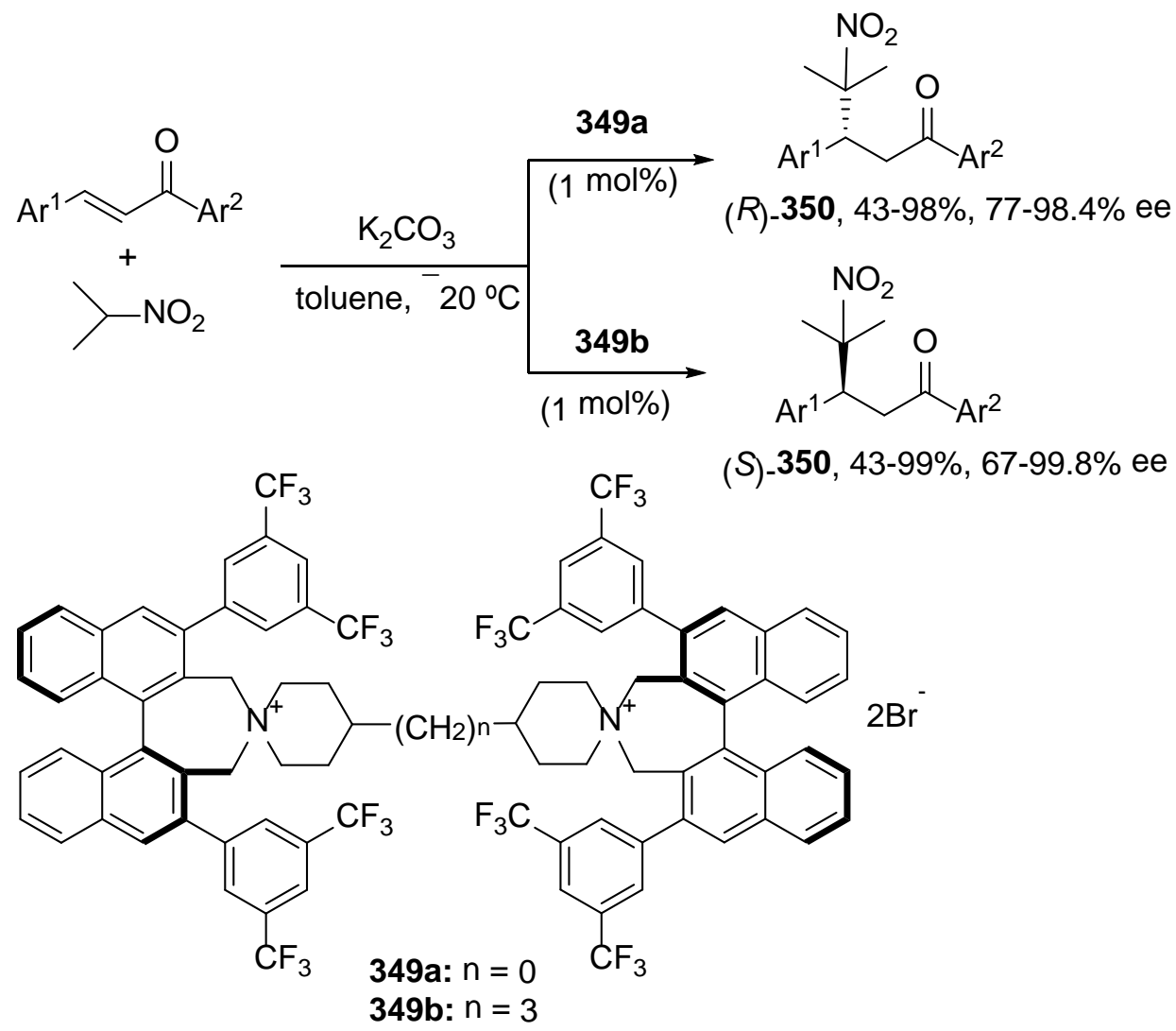

Namboothiri and co-workers have observed a reversal of enantioselectivity in the CA of diethyl benzylphosphonates to nitrostyrene by using chiral bases and in the absence or presence of an achiral additive. ${ }^{329}$ For instance, in the reaction of $p$-chloronitrostyrene with diethyl benzylphosphonate in the absence of additives cinchonine $(\mathrm{CN})$ gave diastereoselectively adduct $(R, R)$-351 in 92\% de and 100\% ee (Scheme 128). By adding $\mathrm{N}$-methylmorpholine, $\mathrm{N}$-dimethylaminopyridine or ether, a switch of the enantioselectivity was observed. Ether turned out superior as additive and as solvent, thus when THF was replaced by ether the corresponding enantiomer $(S, S)$-351 was formed in $88 \%$ de and $100 \%$ ee.

Scheme 128. Enantiodivergent Conjugate Addition of Diethyl Benzylphosphonate to $p$-Chloronitrostyrene Catalyzed by Cinchonine (CN)

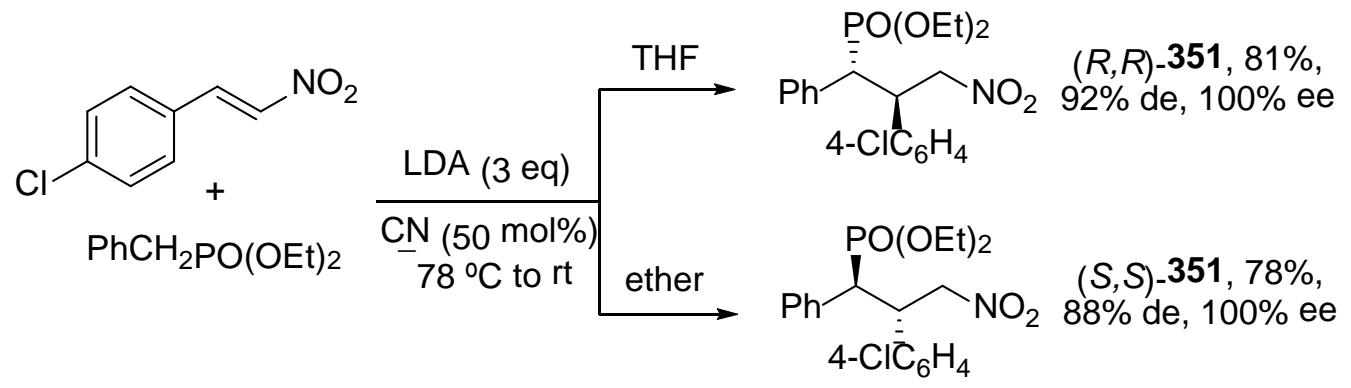

Chiral bicyclic cis-1,2-diamines 352 bearing a thiurea unit catalyzed the enantiodivergent CA of acetylacetone to nitroalkenes. ${ }^{330}$ For example, in the case of the 
addition to $\beta$-nitrostyrene organocatalyzed by 352a the adduct $(R)$-353 was obtained in 89\% ee, whereas 352b gave the enantiomer $(S)$-353 in 95\% ee (Scheme 129). Two plausible TS models 353A and 353B have been proposed to explain this switch of enantioselectivity according to previous DFT calculations performed by Papai. In both cases the thiourea unit coordinates the acetylacetone by dual hydrogen bonding and the protonated dimethylamino group with the oxygen of the nitro group acting as bifunctional organocatalysts.

Scheme 129. Enantiodivergent Conjugate Addition of Acetylacetone to $\beta$ Nitrostyrene Catalyzed by Different Bifunctional Thioureas 352

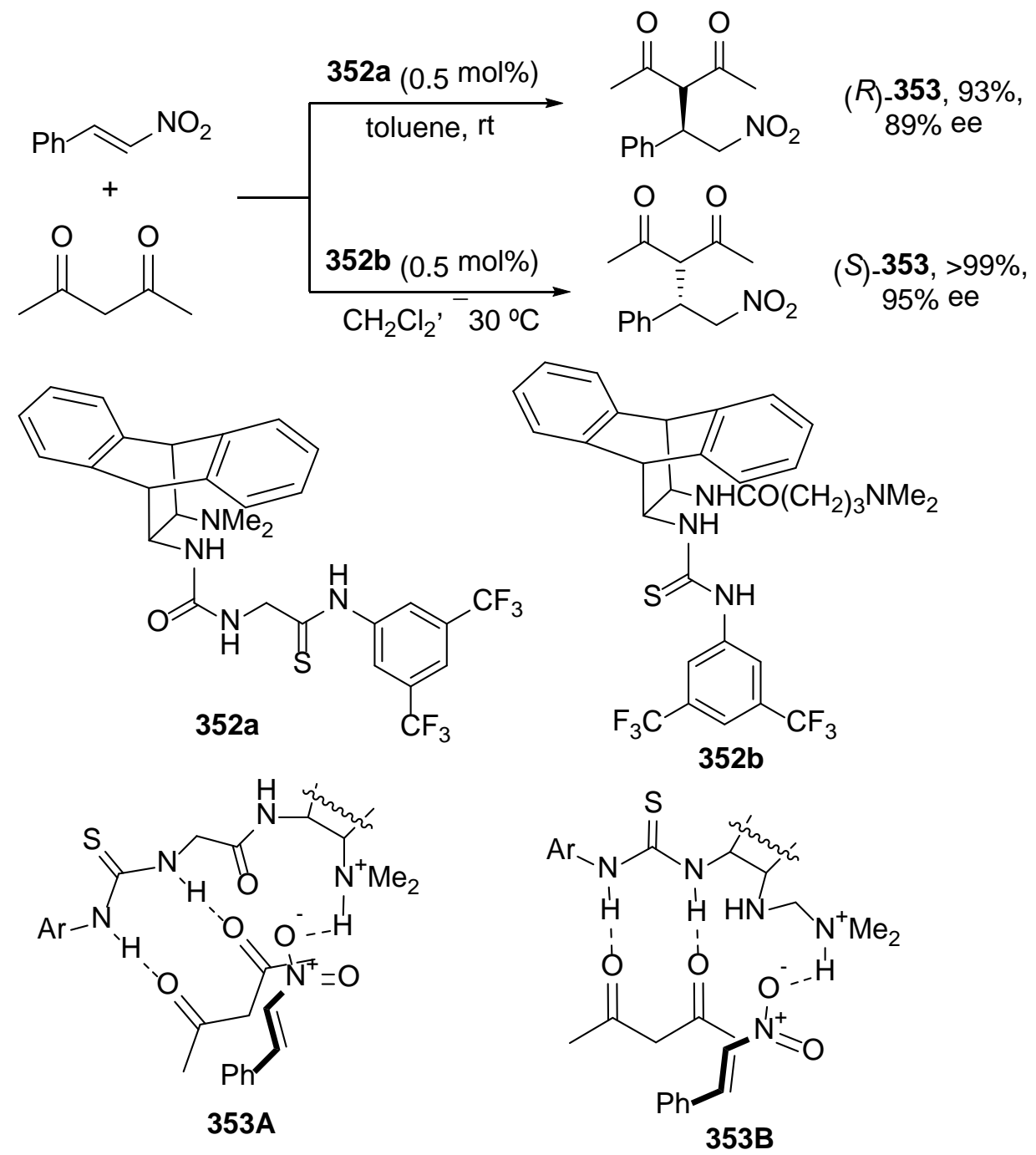

Ye and co-workers have found out, during the reaction condition studies for the CA of 3-substituted oxindoles 304 to $\alpha, \beta$-unsaturated ketones, a diastereodivergent effect working with organocatalysts 354 and $355 .{ }^{331}$ In the presence of diamine 354 (10 mol\%) and $N$-Boc-D-Phg (40 mol\%) as Brønsted acid, products $(R, R)-356$ were mainly obtained in high de and ee (Scheme 130). On the other hand, working with diamine 355 (10 mol\%) and L-tartaric acid (40 mol\%), $(R, S)$ diastereomeric adducts 356 were 
formed in good de and high ee. In the last case, $N$-acetyl-protected oxindole must be used. The observed diastereodivergent results have not been explained.

Scheme 130. Diastereodivergent Enantioselective Conjugate Addition of Oxindoles 304 to $\alpha$, $\beta$-Unsaturated Ketones Catalyzed by Chiral Diamines 354 and 355

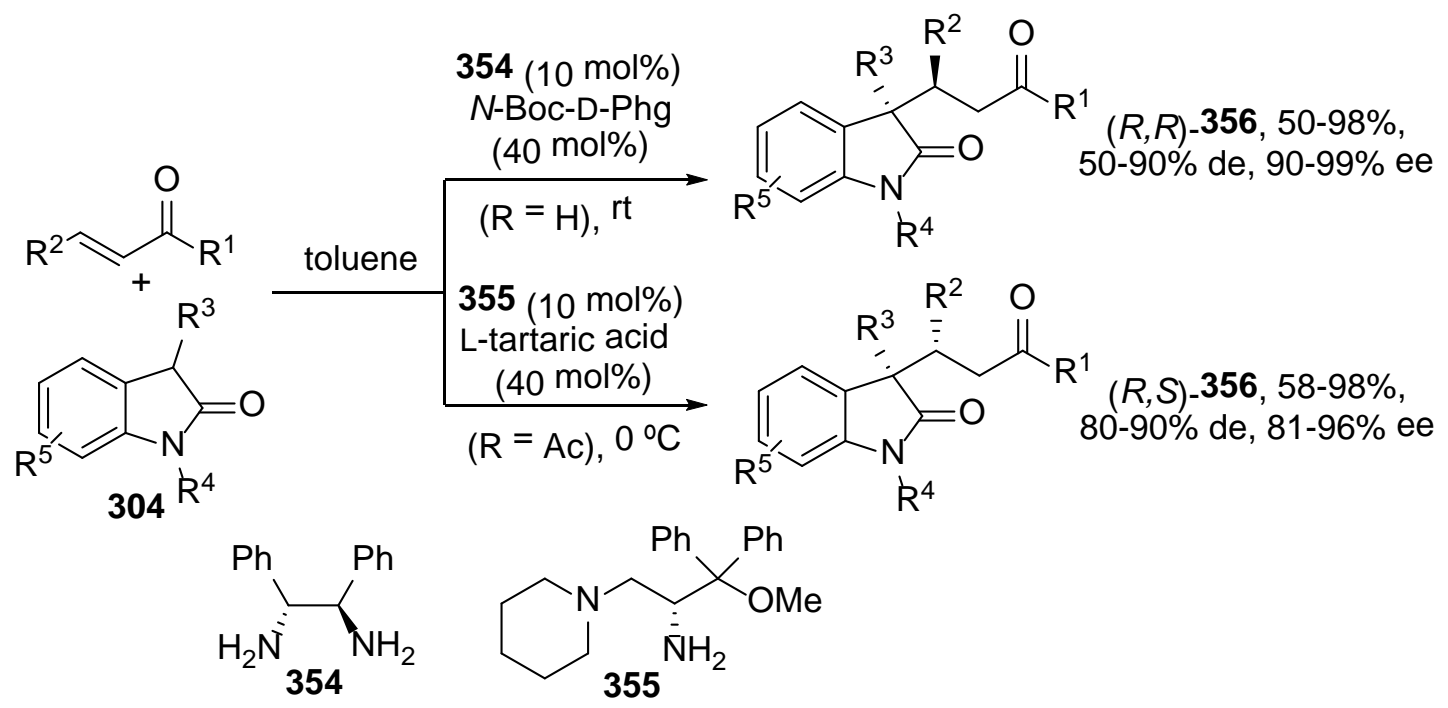

Carbonyl compounds can participate in the asymmetricCA by in situ formation of enamines with primary and secondary amines as chiral organocatalysts. This widely used methodology has been assayed with numerous Michael acceptors. However, very few examples of stereodivergent reactions have been described. A solvent-dependent inversion of the enantioselectivity has been found in the addition of cyclohexanone to chalcones catalyzed by 1-ethyl-3-methylimidazolium-L-prolinate (357) (Scheme 131). ${ }^{332}$ The addition took place at room temperature in $\mathrm{MeOH}$ giving $\left(2 R, 1^{\prime} R\right)$-adducts 358 in high yields and variable ee (23-94\%). On the other hand, when the same process was carried out in DMSO (2R,1'S)-358 were formed in 16-91\% ee.

Scheme 131. Enantiodivergent Conjugate Addition of Cyclohexanone to Chalcones Catalyzed by Prolinate 357 in Different Solvents

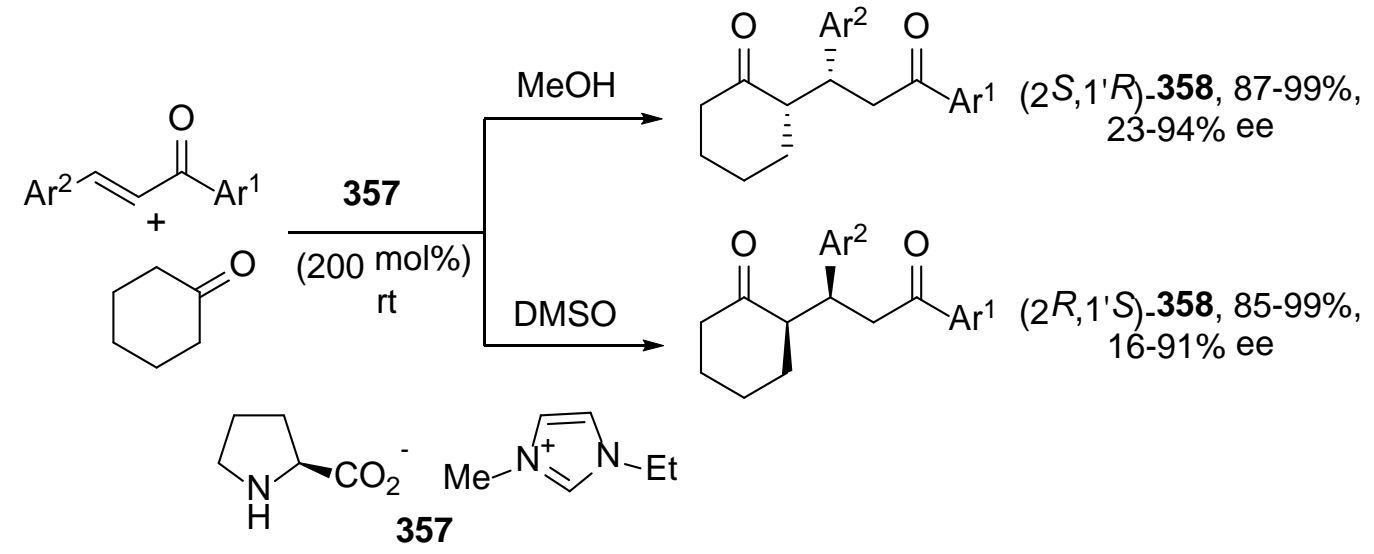


In the CA of aldehydes to nitrostyrenes using a proline-thiourea host-guest complex it has been reported that a different amino acid such as (S)-tert-butyl-Leu promoted an enantiodivergent effect. ${ }^{333}$ By means of L-Pro and thioureas 359 or $\mathbf{3 6 0}$ as additives, $(R, S)$-products 361 were mainly obtained in high ee (Scheme 132). However, $(S)$-tertLeu and thiourea 359 gave $(S, R)$-diastereomers 361 in low syn/anti diastereoselectivity and high ee for the syn-products. According to the Seebach's model for L-Pro catalyzed CA, the authors proposed TS 361A and 361B for both amino acids, respectively, explaining the observed inversion of enantioselectivity.

Scheme 132. Enantiodivergent Enantioselective Conjugate Addition of Aldehydes to Nitrostyrenes Catalyzed by Host-Guest Complexes of L-Pro and (S)-tert-Leu with Thioureas 359 and 360

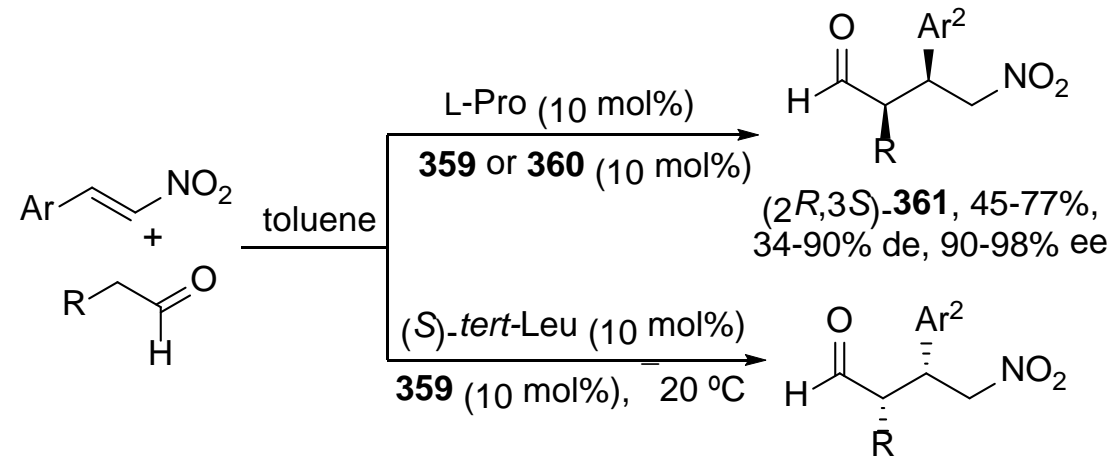

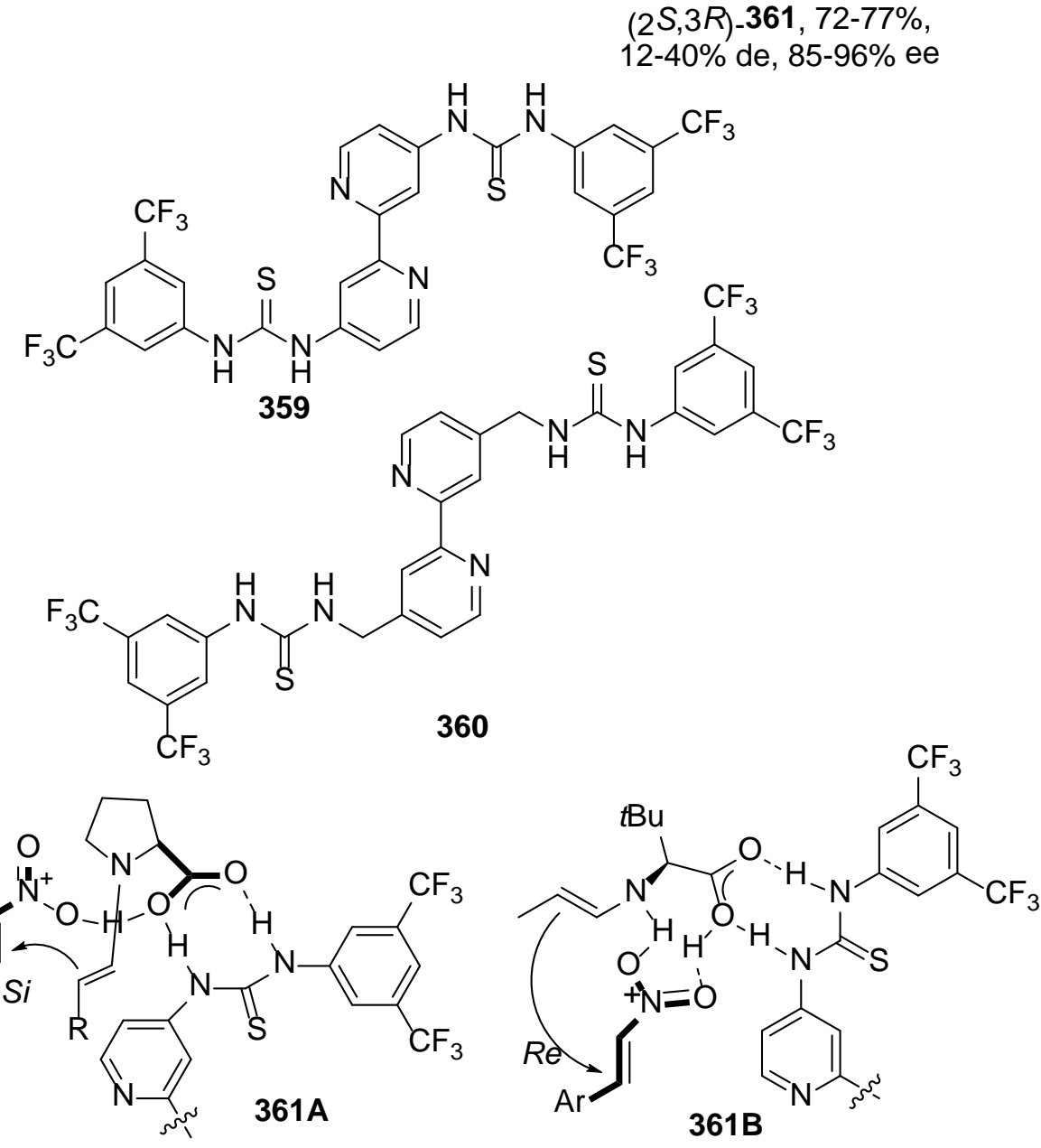


Solvent-dependent enantiodivergent CA of aldehydes to maleimides catalyzed by $\mathrm{N}$ Boc-trans-cyclohexane-1,2-diamine 362 has been observed (Scheme 133). ${ }^{334}$ Working in $\mathrm{CHCl}_{3}$, $(S)$-363 adducts were formed and in aqueous DMF $(R)$-enantiomers were isolated. DFT calculations proposed apolar TS 363A and polarTS 363B according to the different polarity of the media.

\section{Scheme 133. Enantiodivergent Conjugate Addition of Aldehydes to Maleimides Catalyzed by Boc-Diamine 362 in Different Solvents}
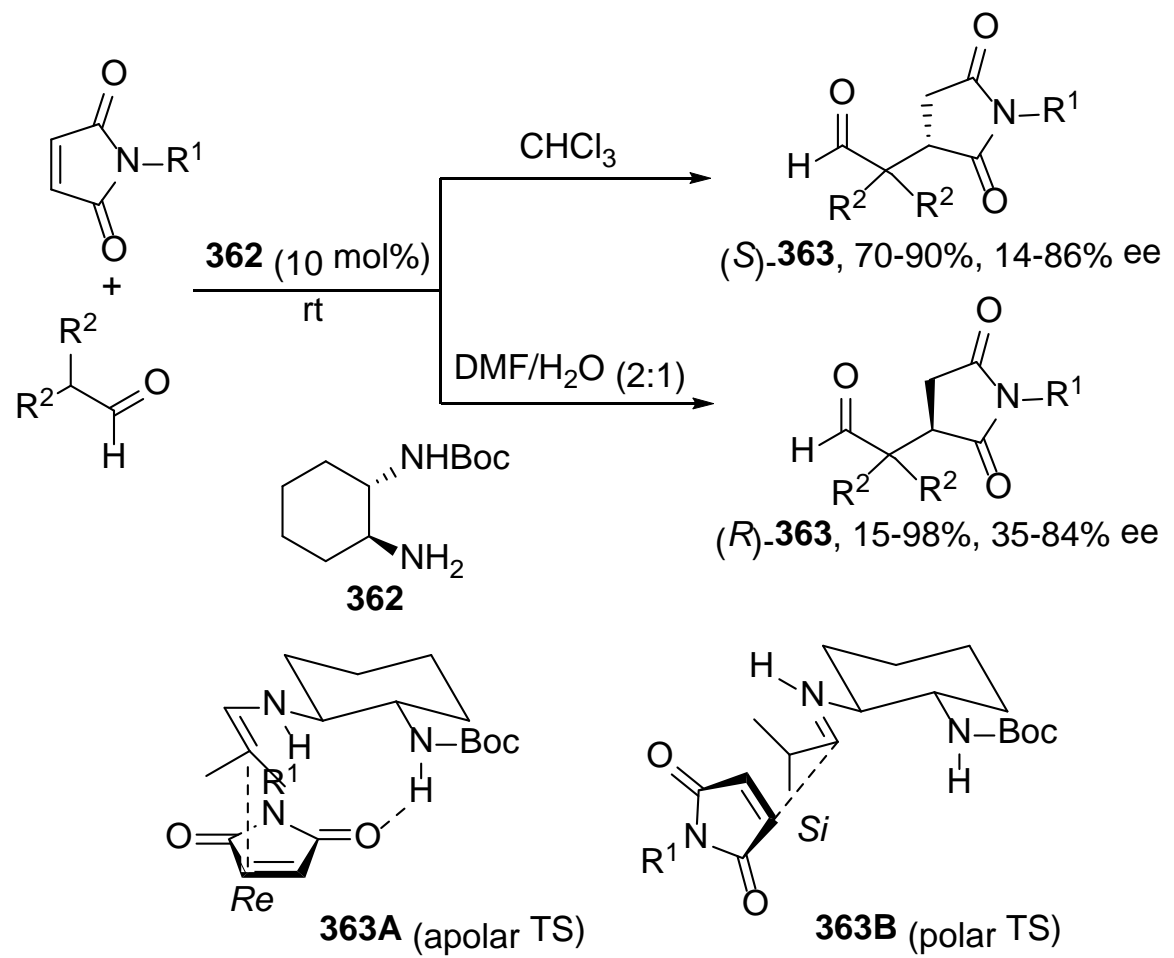

Amine-2-aminopyrimidine chiral organocatalyst $\mathbf{3 6 4}$ has shown moderate solventdependent enantiodivergency in the same CA of aldehydes to maleimides (Scheme 134). ${ }^{335}$ During the optimization studies, isobutyraldehyde reacted with $\mathrm{N}$ phenylmaleimide in the presence of 1,6-hexanedioic acid (HDA) in $\mathrm{CH}_{2} \mathrm{Cl}_{2}$ affording $(S)-363$ adduct in 29\% ee, whereas in aqueous DMF the enantiomeric $(R)$-adduct was obtained in $88 \%$ ee. DFT calculations supported the formation of hydrogen-bonding between the $\mathrm{NH}$ of the aminopyrimidine unit and the carbonyl group of maleimide by the $R e$ face (TS 363A) giving the (S)-enantiomer. However, in protic solvents TS 363B without hydrogen bonding will afford $(R)-363$.

Scheme 134. Enantiodivergent Conjugate Addition of Isobutyraldehyde to $\mathrm{N}$ Phenyl Maleimide Catalyzed by Primary Amine-2-aminopyrimidine 364 in Different Solvents 

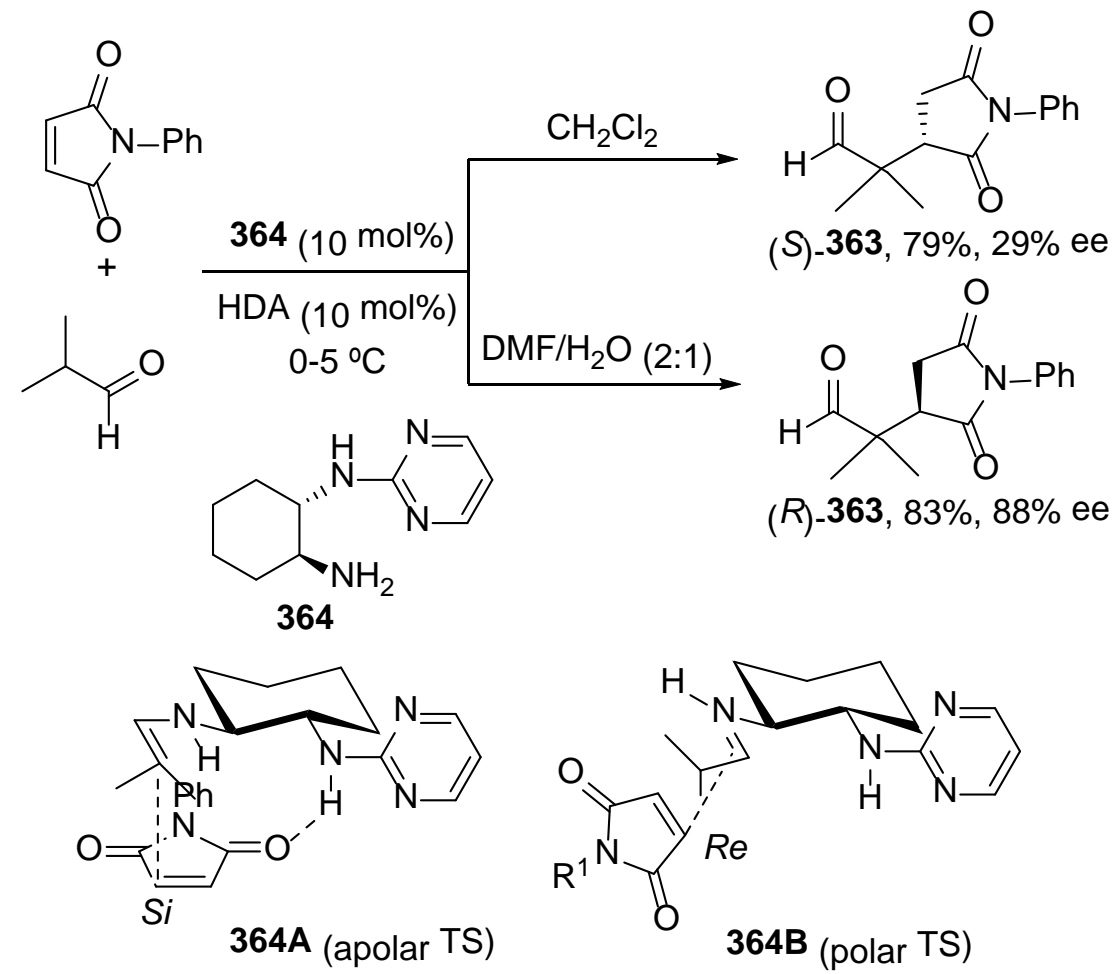

Organocatalyzed asymmetric CA of heteroatom nucleophiles has been performed with nitrogen, oxygen, sulfur, and selenium nucleophilic reagents. However, only a couple of examples can be considered as stereodivergent processes. Diastereodivergent sulfa-Michael addition has been carried out with alkyl thiols and $\alpha$, $\beta$-disubstituted unsaturated ketones. When the quinidine-derived organocatalysts 365 (20 mol\%), forming an iminium intermediate, was used in the presence of 2-fluorobenzoic acid in $\mathrm{CHCl}_{3}$ at $\mathrm{rt}$, the corresponding syn-adducts (2R,3S)-366 were diastereo- and enantioselectively obtained (Scheme 135). ${ }^{336}$ On the other hand, in the presence of Brønsted acids as additives such as phosphoric acid derived from (S)-binaphthol or of an achiral acid such as diphenylhydrogen phosphoric acid (DPP) in acetone at $40{ }^{\circ} \mathrm{C}$, products $(2 R, 3 R)$-anti-363 were diastereo and enantioselectively formed. This is a typical case of the acid controlled diastereoselectivity. In addition, using this methodology the four possible stereoisomers were prepared by changing the chiral amine from quinidine to the quinine-derived pseudoenantiomer.

Scheme 135. Diastereodivergent Enantioselective Conjugate Addition of Alkyl Thiols to $\alpha, \beta$-Disubstituted Enones Catalyzed by Primary Amine 365 and Different Brønsted Acids 


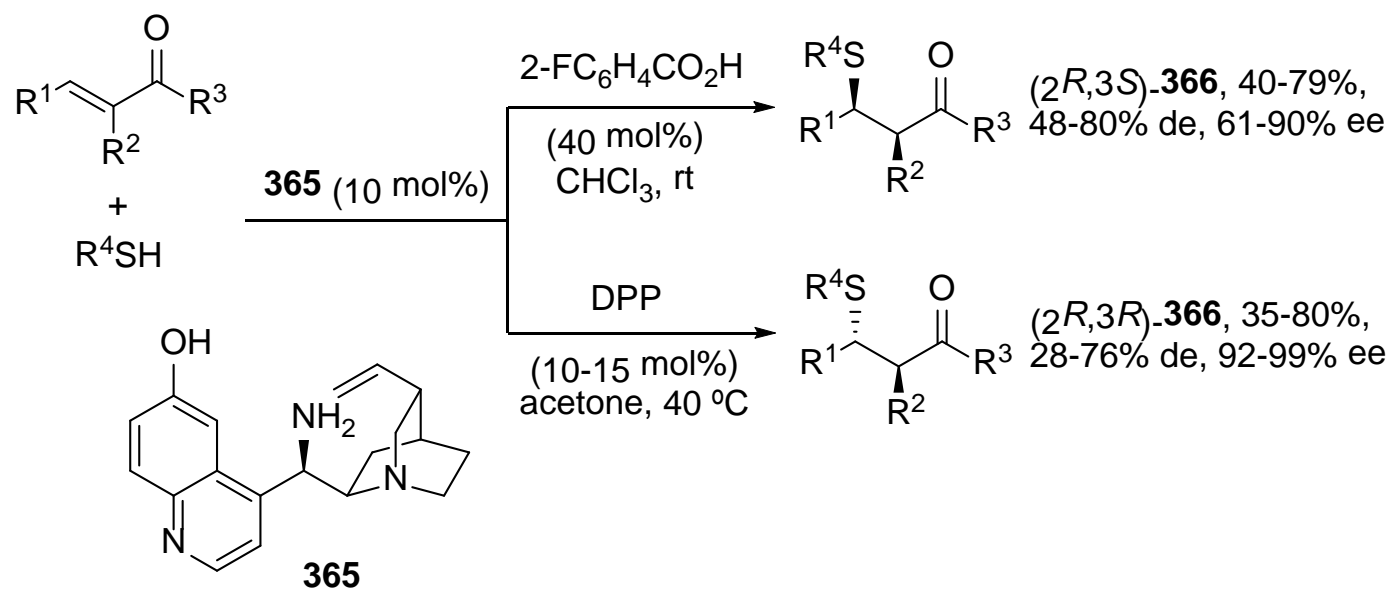

Diphenylphosphite has been used as heteronucleophile in the CA to nitroalkenes catalyzed by bifunctional squaramides 367 and 368 using supercritical carbon dioxide (Scheme 136). ${ }^{337}$ Through the modification of the catalyst structure it was possible to prepare (R)-369 using 367, whereas with organocatalysts 368 (S)-adducts 369 were formed.

Scheme 136. Enantiodivergent Conjugate Addition of Diphenyl Phosphite to $\beta$ Nitroalkenes in Supercritical $\mathrm{CO}_{2}$ Catalyzed by Squaramides 367 and 368

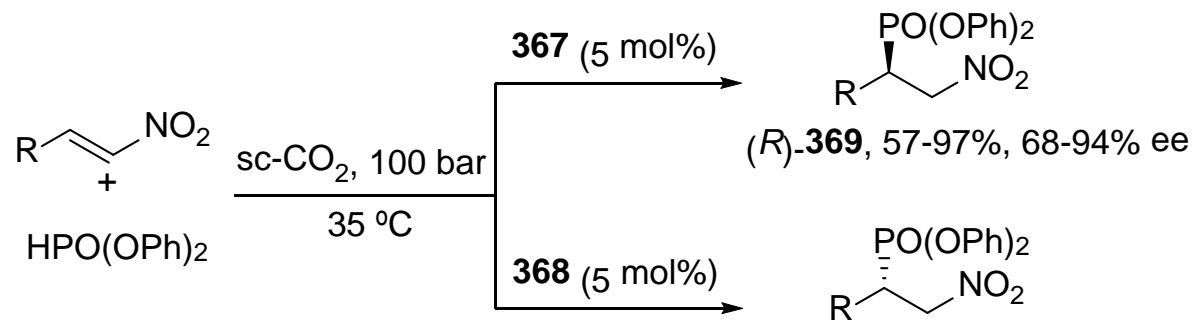<smiles>C=CC1CC2CCN1C(c1ccnc3ccc(OC)cc13)C2Nc1c(Nc2ccc(C(F)(F)F)cc2)c(=O)c1=O</smiles>

Organocatalytic cascade reactions initiated by a Michael addition have been studied by MacMillan and co-workers affording access to different diastereomers starting from the same $\beta$-methylcinnamaldehyde with high de and total ee. ${ }^{338}$ This enal is activated by the imidazolidinone $\mathbf{3 7 0}$ forming a iminium intermediate, which is reduced by the Hantzch ester giving the reduced aldehyde (S)-3-phenylbutanal (371) (Scheme 137). In situ $\alpha$-fluorination with $N$-fluorobenzenesulfonimide (NFSI), catalyzed by enantiomeric imidazolidinones $(S)$ - and $(R)$-372, gave diastereomeric products syn- and anti-373 products, respectively. 
Scheme 137. Diastereodivergent Enantioselective Conjugate Addition of Hydride to $\beta$-Methylcinnamaldehyde Catalyzed by Imidazolidinone 370 Followed by $\alpha$ Fluorination Catalyzed by $(S)$ - and $(R)$-Imidazolidinones 372
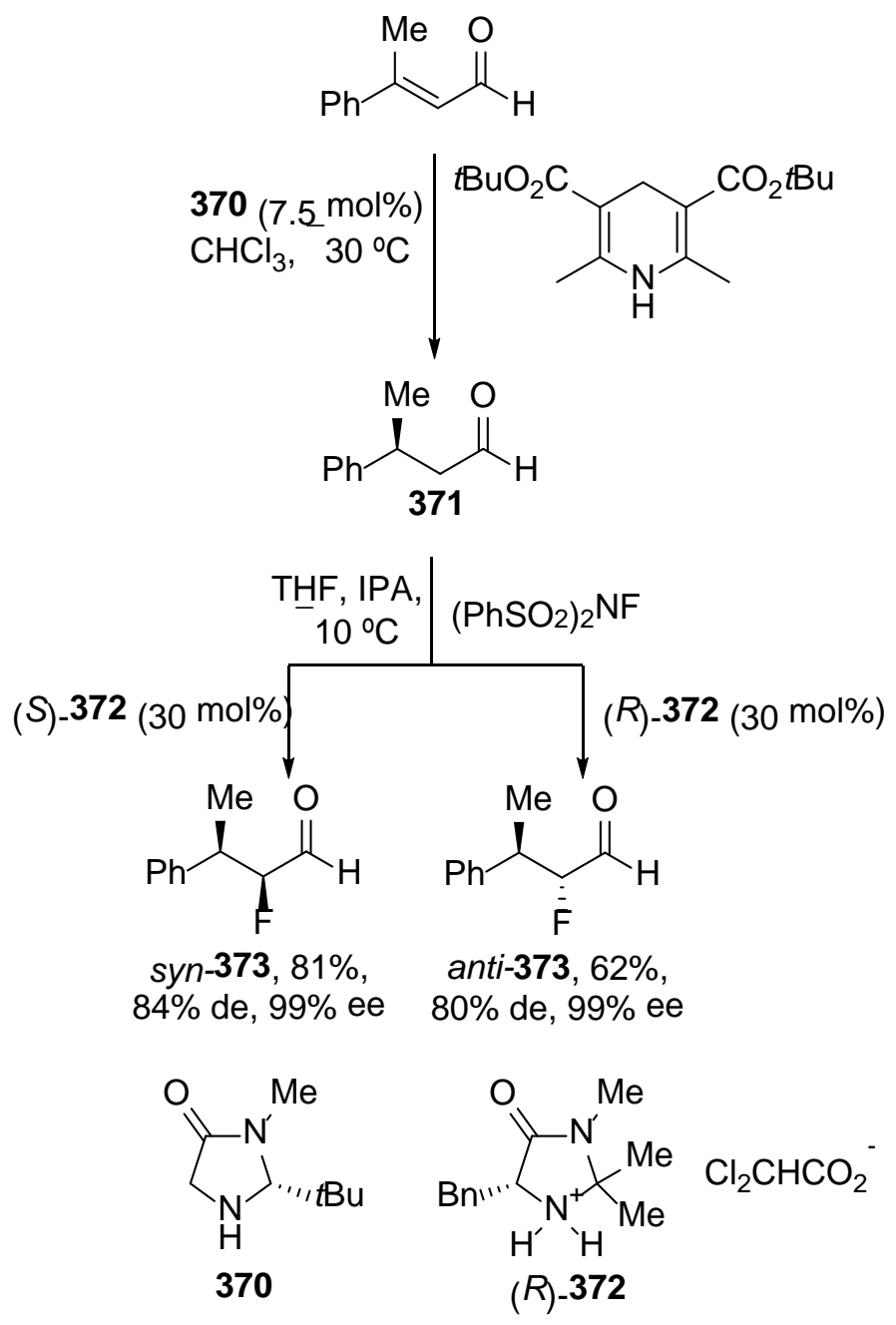

This strategy has been also applied to the corresponding $\alpha$-amination of aldehyde 371 with an azo compound giving products 374 . Working with nitrosobenzene as electrophile the diastereodivergent hydroxylation was achieved affording products $\mathbf{3 7 5}$ after $\mathrm{NaBH}_{4}$ reduction. When the imine derived from ethyl glyoxylic ester was used as electrophile, the corresponding Mannich reaction occurrs providing diasteromers 376 (Scheme 138). ${ }^{338}$

Scheme 138. Diastereodivergent Enantioselective Conjugate Addition of Hydride to $\beta$-Methylcinnamaldehyde Catalyzed by Imidazolidinone 371 Followed by $\alpha$ Functionalization Catalyzed by $(S)$ - and $(R)$-Imidazolidinones 372 


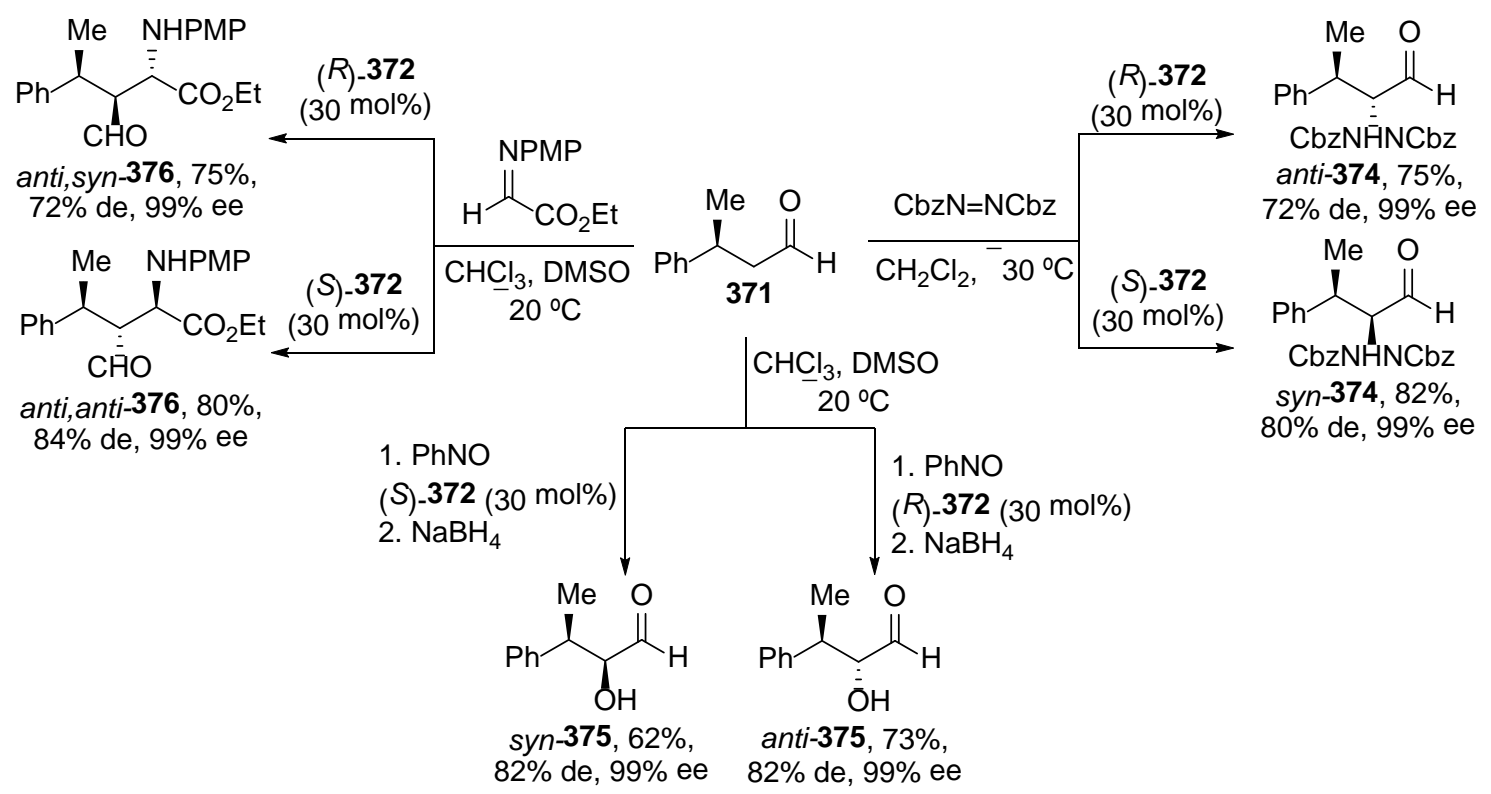

These organocascade organocatalyses have been expanded to crotonaldehyde using different nucleophiles than hydride with imidazolidinone $\mathbf{3 7 7}$ as organocatalysts, for the Michael addition, affording intermediates 378. For the electrophilic $\alpha$-amination reaction, D- and L-Pro were used as organocatalysts (Scheme 139). ${ }^{339}$ In the case of $N$ methylindole syn- and anti-products 379 were obtained after $\alpha$-amination. When azlactone was added to crotonaldehyde followed by $\alpha$-amination, the corresponding diastereomeric products $\mathbf{3 8 0}$ were isolated. Finally, the addition of $O$-silylated $N$-Boc hydroxylamine followed by $\alpha$-amination and reduction with $\mathrm{NaBH}_{4}$ provided products 381.

Scheme 139. Diastereodivergent Enantioselective Conjugate Addition of Different Nucleophiles to Crotonaldehyde Catalyzed by Imidazolidinone 377 Followed by $\alpha$ Amination Catalyzed by ( $S$ )- and (R)-Proline 


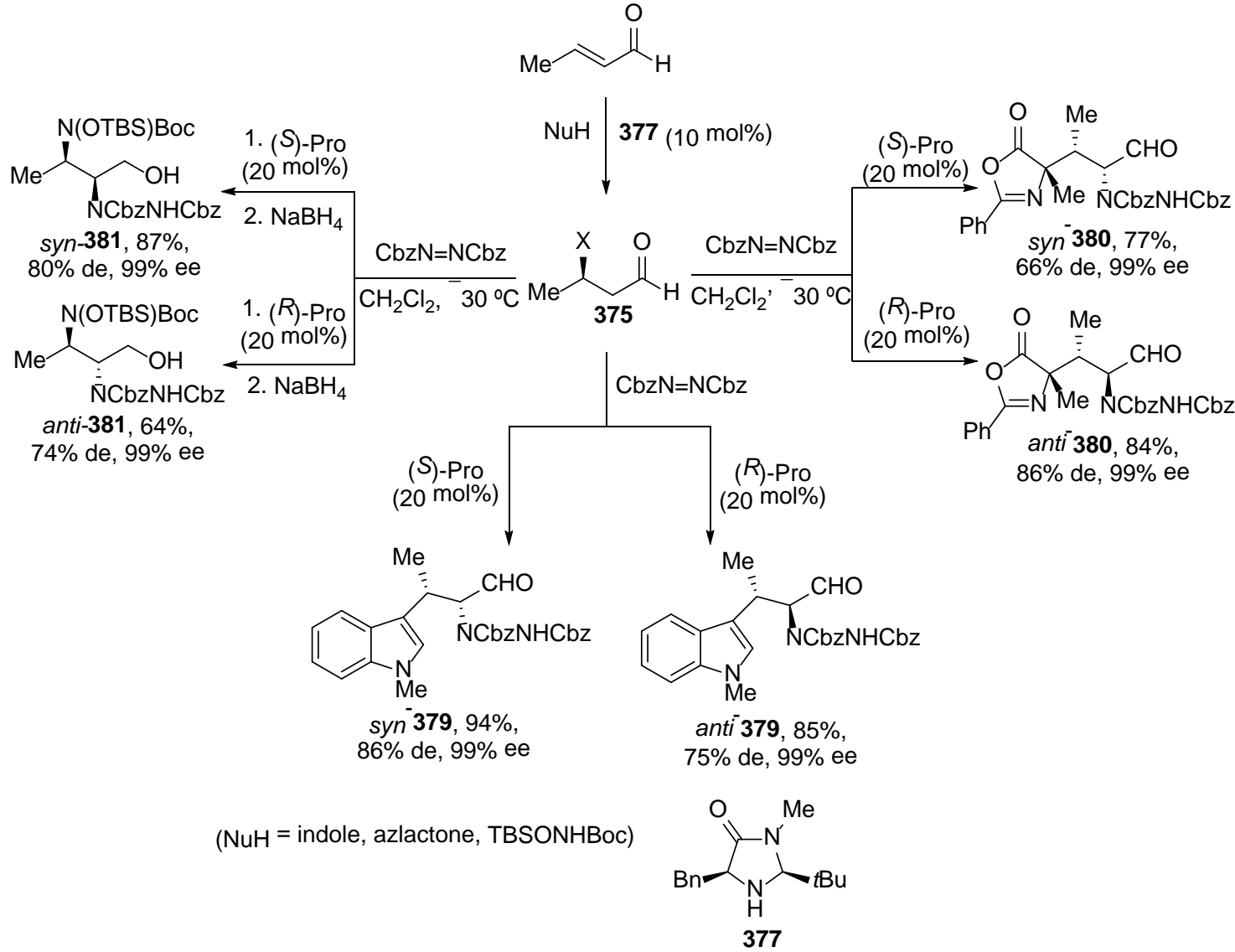

This methodology has been applied to the total synthesis of (-)-aromadendranediol (383), a sesquiterpene isolated from the leaves of the Amazonian tree Xylopia brasiliensis. A key intermediate is the butanolide $\mathbf{3 8 2}$ prepared by a tandem Grubs cross-methatesis/CA/aldol reaction sequence from crotonaldehyde, 5-hexen-2-one and 2-methyl-5-trimethylsilylfuran as starting materials (Figure 14). ${ }^{339}$

Figure 14. Total Synthesis of (-)-Aromadendranediol (383)
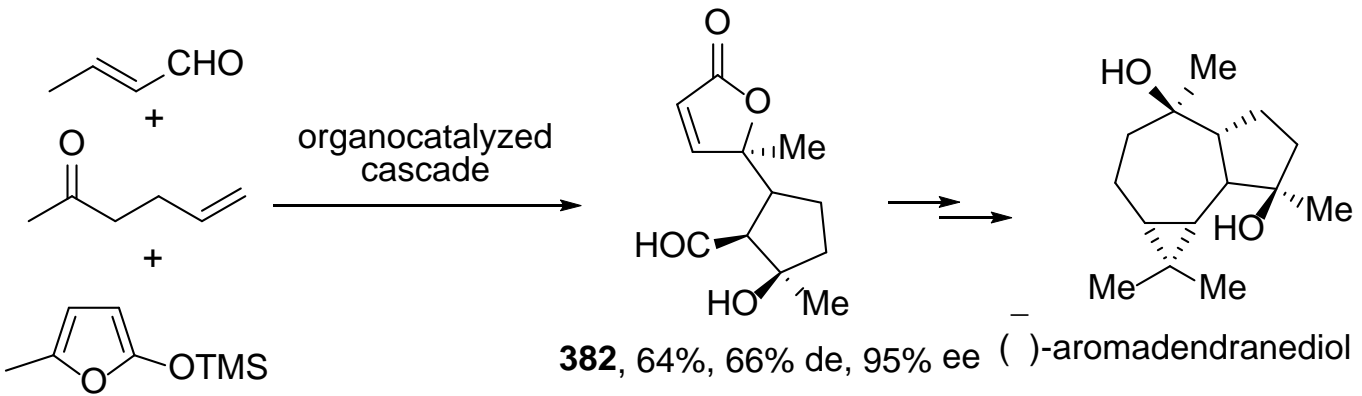

382, 64\%, 66\% de, 95\% ee ( )-aromadendranediol (383)

In conclusion, the enantiodivergent organocatalyzed CA are sensitive to the polarity of the solvent and also to the organocatalyst structure. On the other hand, the diastereodivergent CA is mainly controlled by the catalyst. 
2.3.2. Hydrogenation of Alkenes. Asymmetric hydrogenation of prochiral $C=C$ bonds catalyzed by a transition metal is an useful methodology not only in the academia but also at industrial scale. ${ }^{340,341}$ Based on the models of Knowles, Kagan and Noyori, homogeneous hydrogenation of olefins is performed mainly by $\mathrm{Rh}, \mathrm{Ru}$, and Ir complexes using chiral phosphorous ligands and has been the driving force for the development of asymmetric metal catalysis.

2.3.2.1. Asymmetric Hydrogenation of Functionalized Alkenes. Enantioselective hydrogenation of dehydroamino acids (DAAs) allows the synthesis of $\alpha$-amino acids and it is the benchmark reaction for developing more efficient chiral ligands. A remarkable efficient switching of the enantioselectivity was first achieved by the Sawamura and Ito group during the hydrogenation of methyl 2-( $N$-acetamido)cinnamate using the Rh complex of $(R, R)$ and $(S, S)$-TRAP trans-chelating chiral diphosphines 384 (Scheme 140). ${ }^{342}$ Working at $60{ }^{\circ} \mathrm{C}$ and $0.1 \mathrm{Kg} \cdot \mathrm{cm}^{-2} \mathrm{H}_{2}$ pressure with Et-TRAP 384a as ligand, methyl phenylalaninate $(R)-\mathbf{3 8 5}$, was obtained in $87 \%$ ee, whereas using $\mathbf{3 8 4 b}$ at $15{ }^{\circ} \mathrm{C}$ and atmospheric $\mathrm{H}_{2}$ pressure its enantiomer $(S)-385$ was formed quantitatively in $92 \%$ ee.

\section{Scheme 140. Enantiodivergent Hydrogenation of Methyl 2- $(\mathrm{N}$ - Acetamido)cinnamate Catalyzed by TRAP-Rh Complexes 384 at Different Pressures}

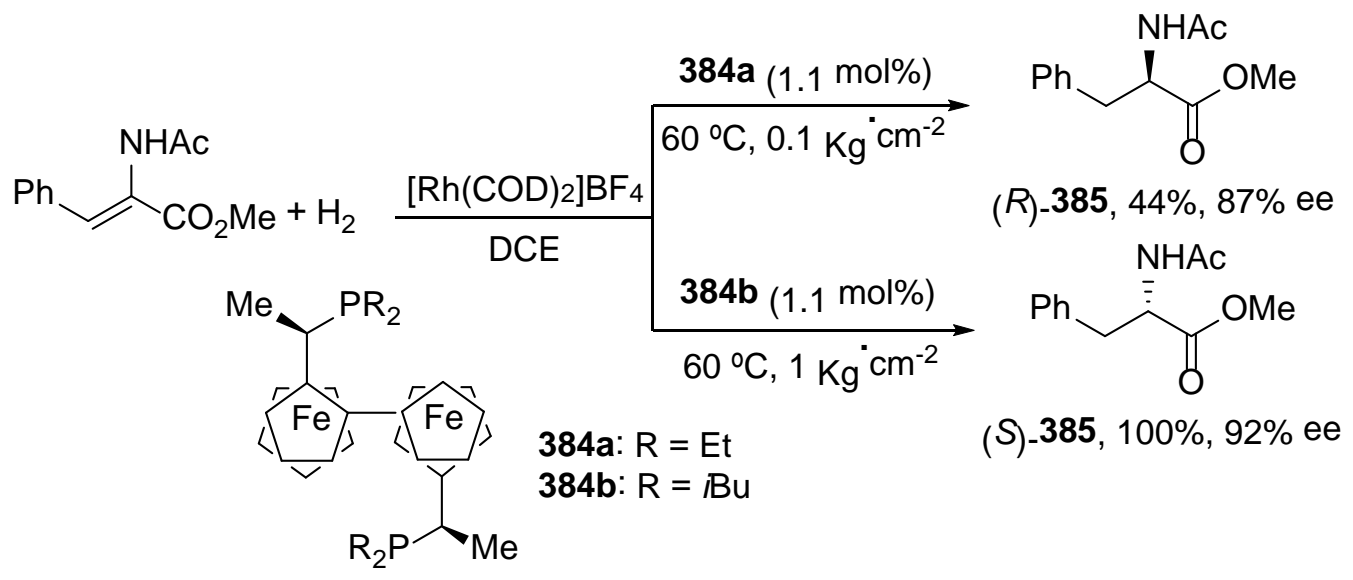

This enantiodivergent effect has been explained by at least two competitive pathways (Scheme 141). In path A, after coordination of the alkene to the Rh complex, oxidative addition of $\mathrm{H}_{2}$ will give $(R)$-385 preferentially. Path $\mathrm{B}$ is in agreement with the Wilkinson's catalyst hydrogenation mechanism, first oxidative addition of $\mathrm{H}_{2}$ and then coordination giving (S)-385. The path $\mathrm{B}$ is suppressed when the $\mathrm{H}_{2}$ pressure decrease and the path $\mathrm{A}$ is favored upon increasing the $\mathrm{H}_{2}$ pressure. Increasing the steric factor in ligand 384b favored the oxidative $\mathrm{H}_{2}$ addition prior to the olefin coordination (path $\mathrm{B}$ ).

Scheme 141. Competitive Pathways in the Enantiodivergent Hydrogenation of Methyl 2-(N-Acetamido)cinnamate Catalyzed by TRAP-Rh Complexes 384 


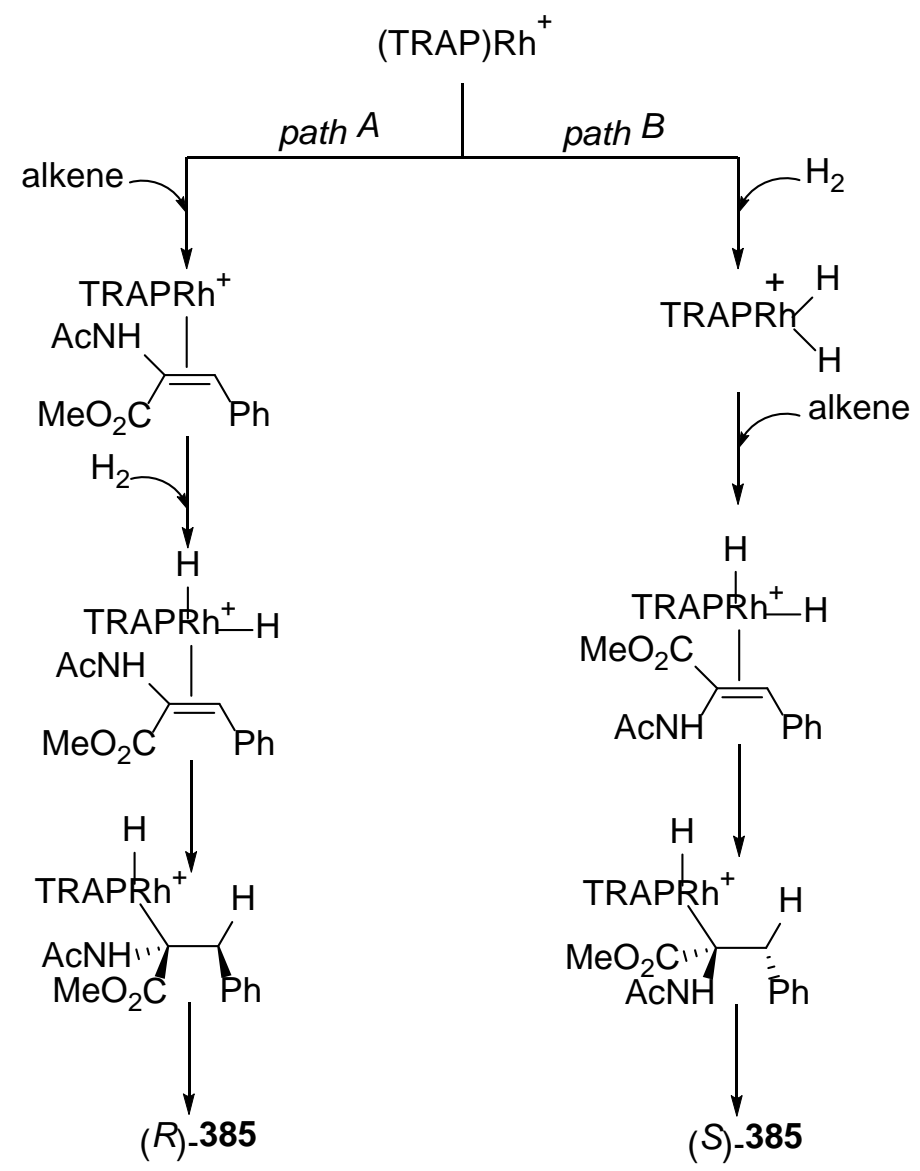

Reetz and Mehler observed a reversal of enantioselectivity in the Rh-catalyzed hydrogenation of methyl acetamidoacrylate using mixtures of chiral and achiral monodentate phosphorous ligands. ${ }^{343}$ This effect was observed in several cases, the most notable example being the combination of phosphinite 386 and phosphine 387, which provided $(R)$-388 in modest conversion with $58.6 \%$ ee, results which are in contrast with the use of phosphinite $\mathbf{3 8 6}$ giving $(S)$-388 in total conversion with $93 \%$ ee (Scheme 142).

Scheme 142. Enantiodivergent Rh-Catalyzed Hydrogenation of Methyl 2-( $N$ Acetamido)acrylate Using Mixtures of Chiral and Achiral Phosphorous Ligands 


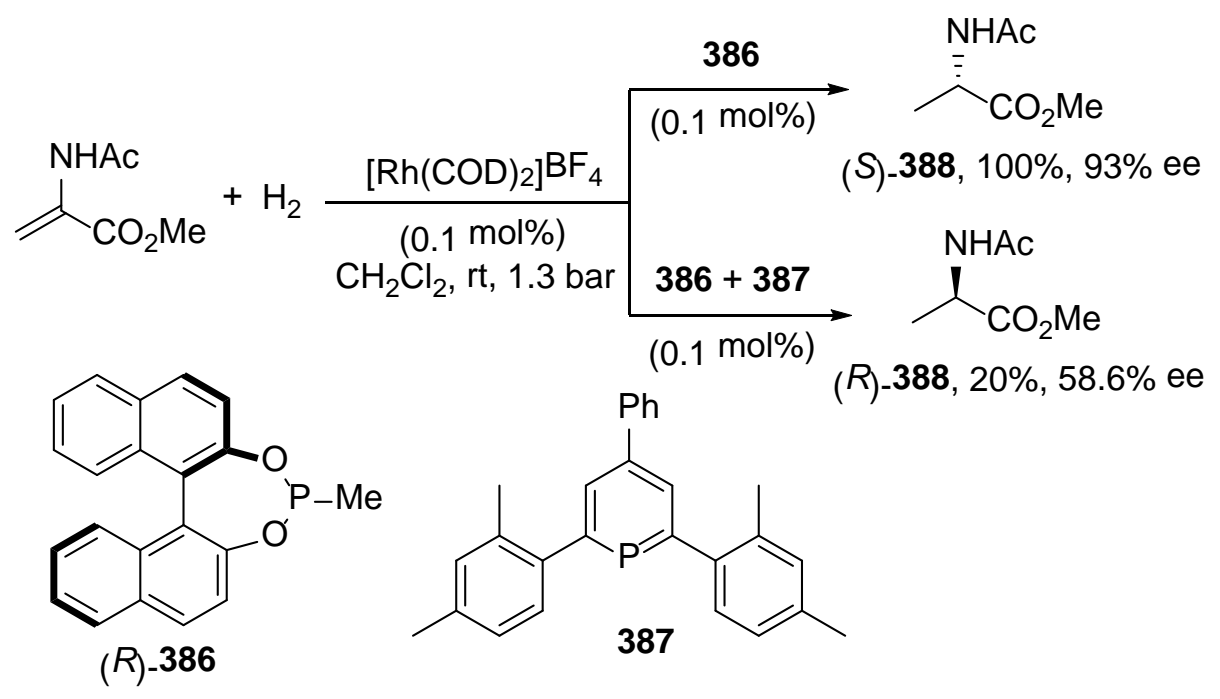

Diastereomeric phosphine-phosphite ligands have been used by Pizzano and coworkers in the Rh-catalyzed asymmetric hydrogenation of methyl $\alpha$ acetamidocinnamate. $^{344}$ Enantiodivergent results were observed depending on the configuration of the ligand. Thus, $(R, S)$-389 gave $(R)$-385 and $(R, R)$-389 the corresponding enantiomer $(S)-385$ in 95\% and 92\% ee, respectively. Similar reversal of enantioselectivity was produced with $(R, S)$-390 and $(R, R)$-390 giving $(R)$-385 and $(S)$ 385 in 93\% and 99\% ee, respectively. Excellent results resulted in the quantitative hydrogenation of dimethyl itaconate (Scheme 143). In the case of employing $(R, S)$-389 or $(R, S)$-390, product $(S)$-391 was isolated in $99 \%$ or $95 \%$ ee, respectively. On the contrary, $(R, R)$-389 and $(R, R)$-390 led to the formation of $(R)-391$ in lower $80 \%$ and $74 \%$ ee, respectively.

Scheme 143. Enantiodivergent Rh-Catalyzed Hydrogenation of Methyl Itaconate with Diastereomeric Phosphine-Phosphite Ligands 389 and 390 


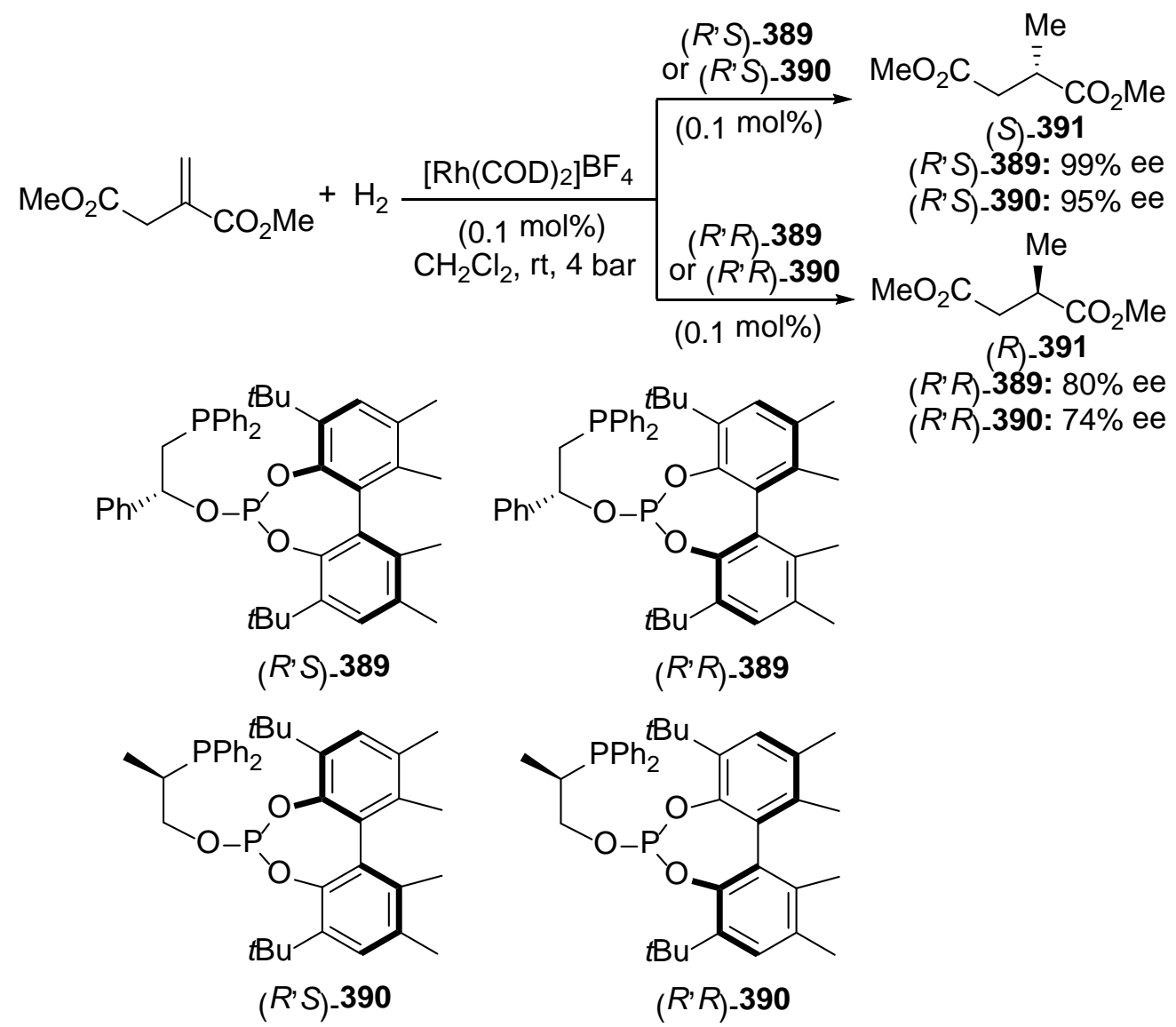

As it has been mentioned in Section 2.1.5.2, the asymmetric hydrogenation of carbonyl compounds is usually performed under heterogeneous conditions with Pt on alumina modified by Cinchona-derived alkaloids. However, in the case of alkenes Pd on alumina is the appropriate heterogeneous catalyst, ${ }^{153}$ although the behavior of Cinchona-derived alkaloids on Pd suffers of weaker absorption than with Pt. During the enantioselective hydrogenation of DAAs such as $\mathrm{N}$-acetyl dehydrophenylalanine methyl ester using Cinchona-modified 5\% $\mathrm{Pd} / \mathrm{TiO}_{2}$ catalysts very low enantioselectivities were obtained. ${ }^{345}$ However, in the case of (E)- $\alpha$-phenylcinnamic acid (392) it was possible to control the enantioselectivity by using different substituents in the Cinchona alkaloid. When, cinchonidine (CD) was used as base in $\mathrm{MeOH}$ as solvent, product (S)-393 was obtained in $49 \%$ ee, whereas $O$-methylcinchonidine (MeOCD, 147) gave the enantiomer (R)-393 in only $12 \%$ ee (Scheme 144). ${ }^{346}$

Scheme 144. Enantiodivergent Hydrogenation of $(E)-\alpha$-Phenylcinnamic 392 Acid Catalyzed by Pd/TiO 2 Modified by CD and MeOCD 147

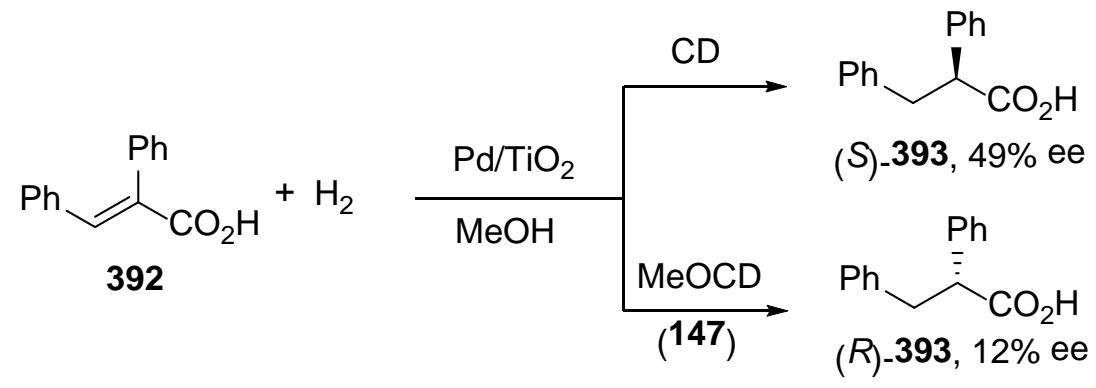


Reversal of the enantioselectivity has been achieved in the hydrogenation of $(E)-2-$ (2-methoxyphenyl)-3-(4-fluorophenyl)propenoic acid (394) over $\mathrm{Pd} / \mathrm{Al}_{2} \mathrm{O}_{3}$ with $\mathrm{CD}$ and with MeOCD as chiral modifiers. In the case of CD, (S)-396 was formed in 86\% ee and with a larger TMS group in the $\mathrm{OH}$ function 395 an inversion of the configuration took place affording (R)-396 in 55\% ee. Decreasing in the interaction strength of the interaction of ether derivatives with the acid and the catalyst surface can explain the switching of enantioselectivity (Scheme 145). ${ }^{347}$ The presence of benzylamine increased in both cases the enantioselectivity and accelerated the desorption of the chiral modifier.

Scheme 145. Enantiodivergent Hydrogenation of (E)-2-(2-Methoxyphenyl)-3-(4fluorophenyl)propenoic Acid 394 Catalyzed by $\mathrm{Pd} / \mathrm{Al}_{2} \mathrm{O}_{3}$ Modified by $\mathrm{CD}$ and TMSOCD 395

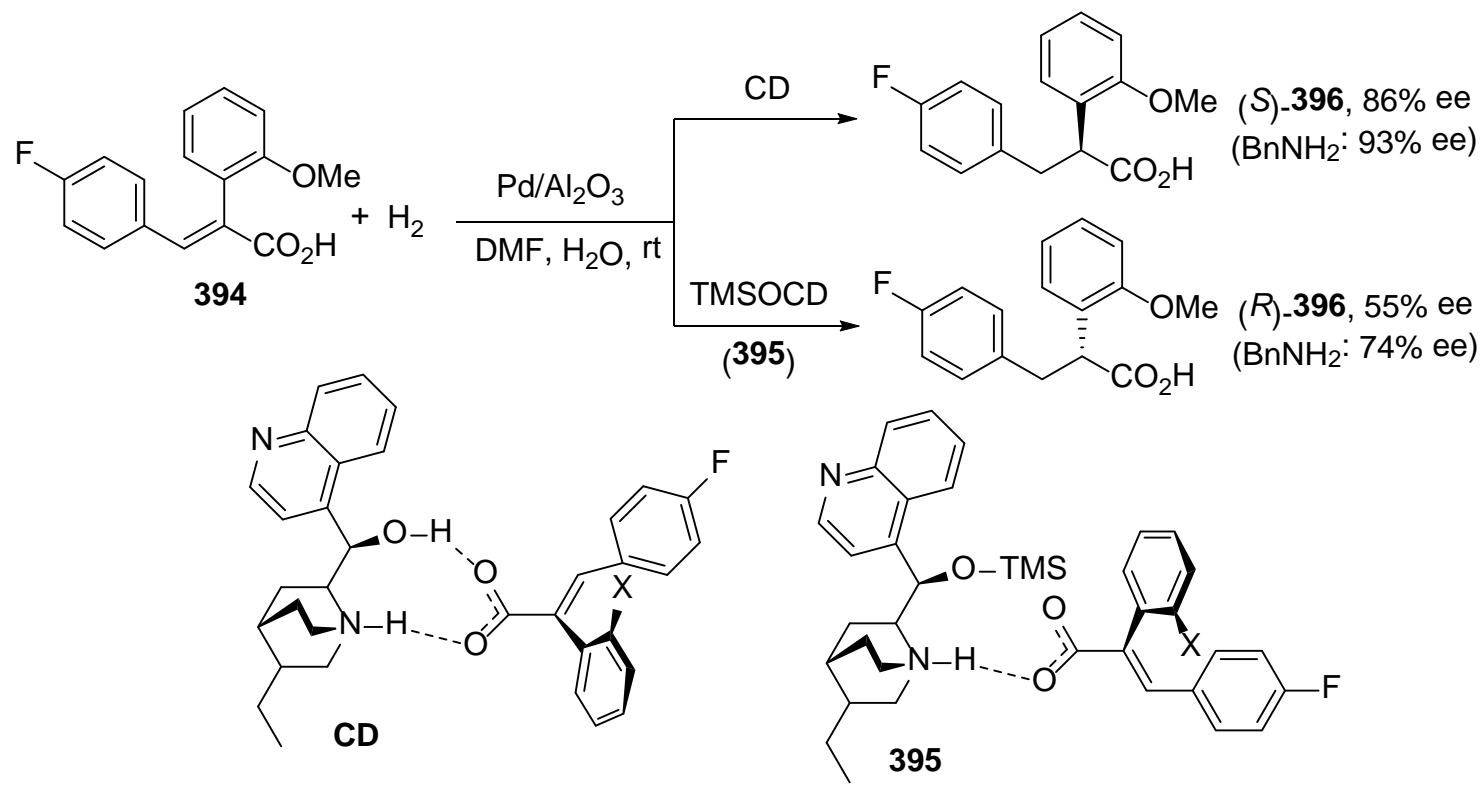

Asymmetric hydrogenation of allylic alcohols has been carried out under $\mathrm{Ru}-$ catalysis. During the hydrogenation of geraniol (397) with $\left[\mathrm{RuCl}_{2}-(S)\right.$ TolBINAP $]_{2} \cdot \mathrm{NEt}_{3}$, Blackmond and co-workers have found an inversion of the configuration depending on the reaction time giving $(R)$ or $(S)$-citronellol (399) (Scheme 146). ${ }^{348}$ Under the ruthenium-catalysis, geraniol (397) isomerized to $\gamma$-geraniol (398) and both substrates underwent asymmetric hydrogenation to $(R)$ and $(S)$ citronellol (399), respectively. Because the hydrogenation of a terminal olefin is faster than the internal one, the preparation of both enantiomers 399 can be controlled according to the reaction time.

Scheme 146. Enantiodivergent Ru-Catalyzed Hydrogenation of Geraniol 397 as a Function of the Reaction Time 


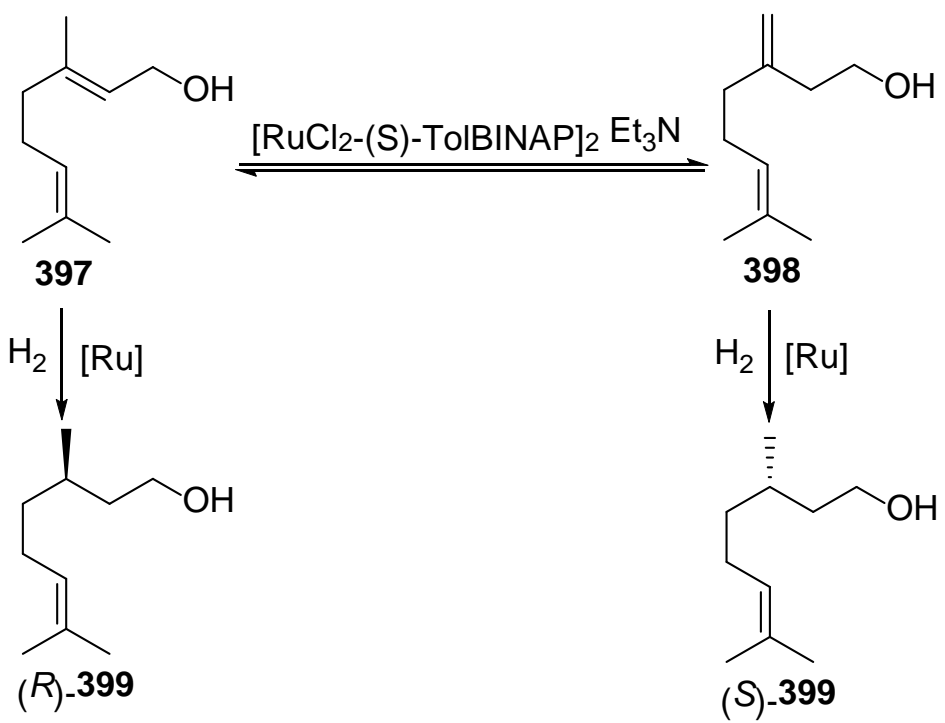

Diastereodivergent hydrogenation of allylic diols 400 and 401 with opposite protection have been performed with the iridium Crabtree's catalyst $\mathbf{4 0 2}$. Thus, diol $\mathbf{4 0 0}$ led to the formation of saturated diol syn-403 in 94\% de, whereas hydrogenation of $\mathbf{4 0 1}$ afforded anti-404 with $98 \%$ de (Scheme 147). ${ }^{349}$

Scheme 147. Diastereodivergent Ir-Catalyzed Hydrogenation of Unsaturated Diols 400 and 401 with Differently Located Protecting Groups
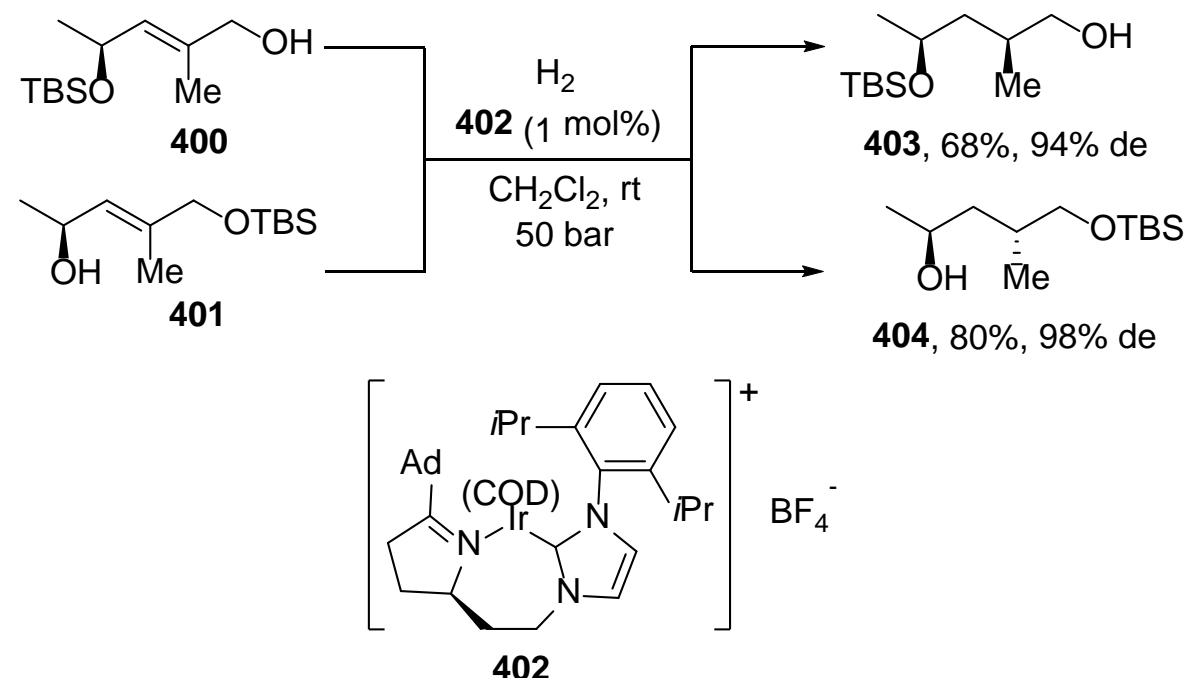

$404,80 \%, 98 \%$ de

In the convergent synthesis of long chain polydeoxypropionates a diastereodivergent hydrogenation of compound $\mathbf{4 0 5}$ has been performed using different Ir catalysts with excellent results. When Ir complex 406 was employed $(8 R, 9 R)-\mathbf{4 0 8}$ was obtained in $96 \%$ de. On the other hand, using complex 407, the corresponding diastereomer $(8 S, 9 R)-\mathbf{4 0 9}$ was isolated also in $96 \%$ de (Scheme 148). ${ }^{350}$

Scheme 148. Diastereodivergent Hydrogenation of Unsaturated Diol 405 Catalyzed by Different Ir Complexes 406 and 407 


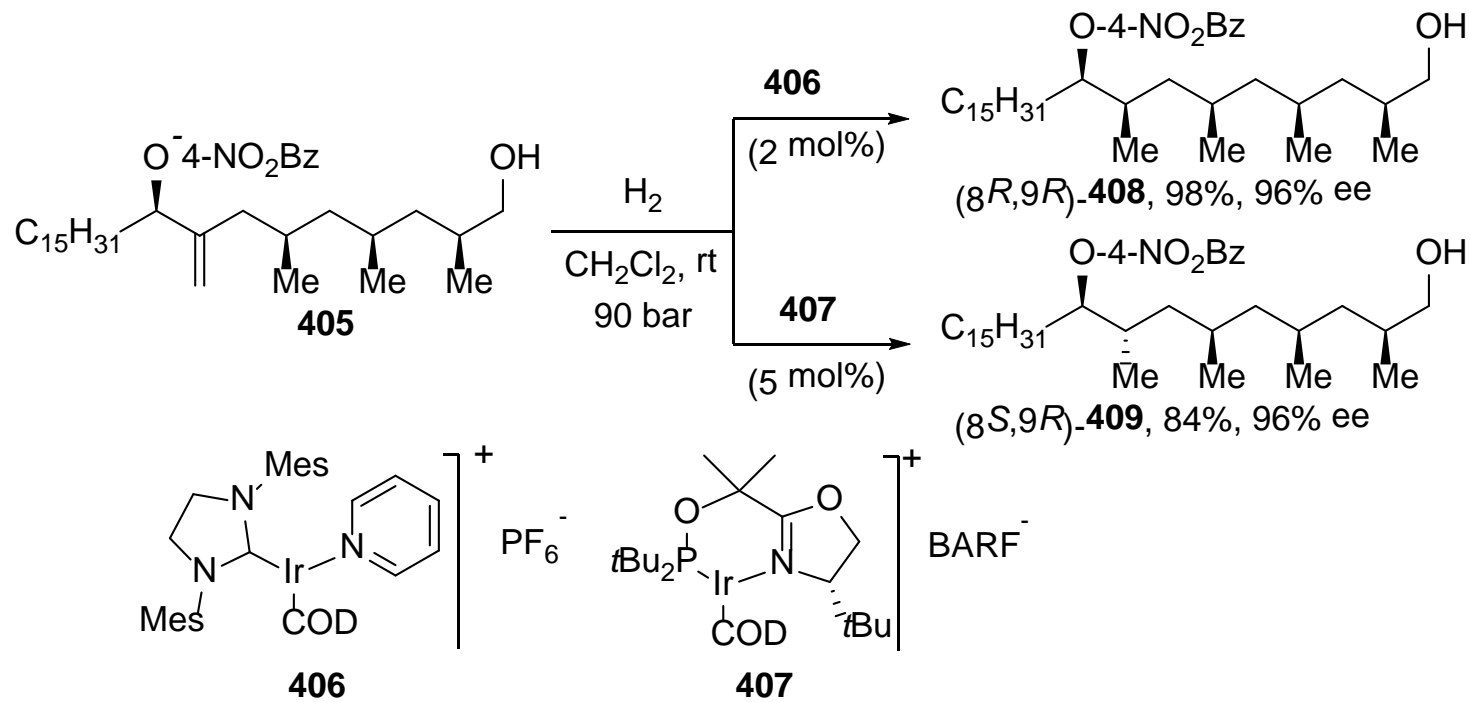

2.3.2.2. Asymmetric Hydrogenation of Unfunctionalized Alkenes. Unfunctionalized alkenes are usually hydrogenated under heterogeneous conditions with Pd on carbon and with $\mathrm{PtO}_{2}$. Under homogeneous conditions Ir catalysts must be used for challenging highly substituted alkenes. Pfaltz and co-workers used chiral Ir complexes derived from phosphinooxazolines (PHOX) as chiral ligands for the asymmetric hydrogenation of $\mathrm{C}=\mathrm{N}$ and $\mathrm{C}=\mathrm{C}$ bonds. ${ }^{351}$ Ligand substitution-dependent enantiodivergent reactions have been reported using Ir complexes of L-serine and L-threonine-derived PHOX ligands 410 and 411. ${ }^{352}$ For instance, during the hydrogenation of $(E)$-1,2-diphenylpropene, $(S)$ 412 was obtained in $89 \%$ ee by the 410-catalyzed hydrogenation (Scheme 149). ${ }^{353}$ On the contrary, catalyst $\mathbf{4 1 1}$ gave enantiomer (R)-412 in 99\% ee. Similar behavior was observed with other substrates such as ethyl (E)- $\beta$-methylcinnamate and with $N$-phenyl acetophenone imine.

Scheme 149. Enantiodivergent Iridium-Catalyzed Hydrogenation of (E)-1,2Diphenylpropene Catalyzed by L-Serine and L-Threonine-Derived PHOX Ligands

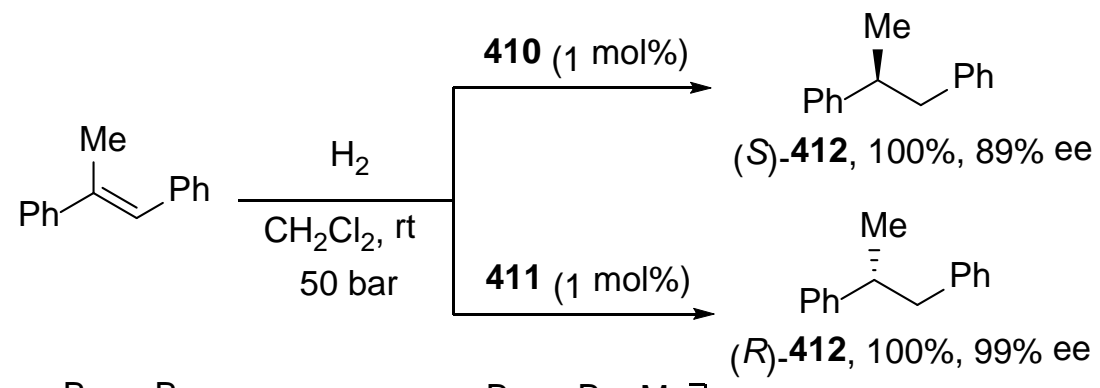

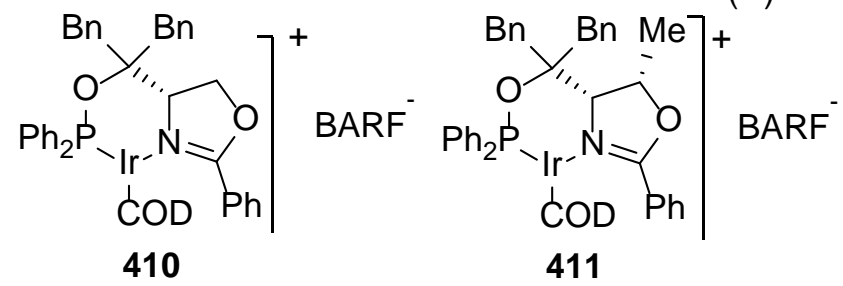


Optically active Ir complexes $\mathbf{4 1 4}$ derived from NHC-oxazoline ligands have been employed in the asymmetric hydrogenation of arylalkenes by Burguess and coworkers. ${ }^{354}$ Enantiodivergent hydrogenation was observed only in one example depending on the reaction conditions such as different pressure and temperature. Thus, 2-(2-methoxyphenyl)but-1-ene (413) gave (R)-415 at rt and 1 bar, whereas at $-15{ }^{\circ} \mathrm{C}$ and 85 bar the enantiomer (S)-415 was formed in 89\% ee (Scheme 150). ${ }^{355}$ This behavior has been attributed to the switch of the prevailing mechanism to an alternative one due to the high hydrogen concentration.

\section{Scheme 150. Enantiodivergent Ir-Catalyzed Hydrogenation of 2-(2- Methoxyphenyl)but-1-ene 413 with Ir complex 414 Under Different Reaction Conditions}

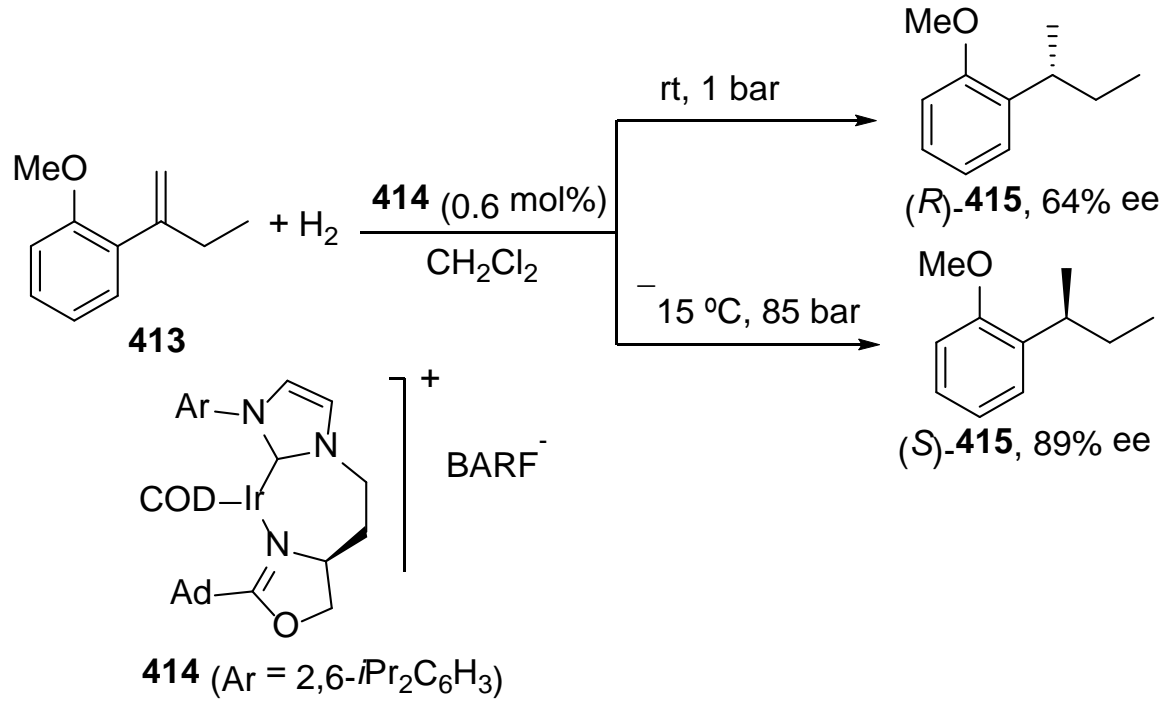

In conclusion, the enantiodivergent hydrogenations of carbon-carbon double bonds are sensitive to the reaction conditions such as pressure and temperature. As in other metal-catalyzed reactions, different substituents in the chiral ligand also render enantiodivergent as well as strong diastereodivergent effects.

2.3.3. Hydroformylation of Alkenes. Regio- and enantioselective hydroformylation of prochiral alkenes by addition of syngas is an important C-C bond forming reaction for the synthesis of branched aldehydes bearing a stereocenter at the $\alpha$-position with important industrial applications, mainly for the synthesis of nonsteroidal antiinflamatory drugs such as 2-arylpropanoic acids and $\alpha$-amino acids. ${ }^{356}$ Enantioselective hydroformylation of propene with Co- and Rh-catalysts and of alkenylaromatics catalyzed by $\mathrm{Rh}$ and Pt ones are the most studied processes. An initial temperature-dependent reversal of the enantioselectivity was found in the hydroformylation of styrenes catalyzed by $[(2 S, 4 S)-\mathrm{BDPP}] \mathrm{Pt}\left(\mathrm{SnCl}_{3}\right) \mathrm{Cl}(\mathbf{4 1 6})$ at $40{ }^{\circ} \mathrm{C}$ giving the branched aldehyde $(S)-\mathbf{4 1 7}$ in $64 \%$ ee. On the other hand, at $100{ }^{\circ} \mathrm{C}(R)-\mathbf{1 4 7}$ was isolated in lower $10 \%$ ee (Scheme 151). ${ }^{357}$ This phenomenon was analyzed by Casey and co-workers performing the deuterioformylation of styrene. ${ }^{358}$ They 
established that the styrene insertion into the Pt-H bond giving the Pt-allyl intermediate is an irreversible process at $40{ }^{\circ} \mathrm{C}$ and became reversible at $100{ }^{\circ} \mathrm{C}$.

\section{Scheme 151. Enantiodivergent Hydroformylation of Styrene Catalyzed by Pt Complex 416 at Different Temperatures}

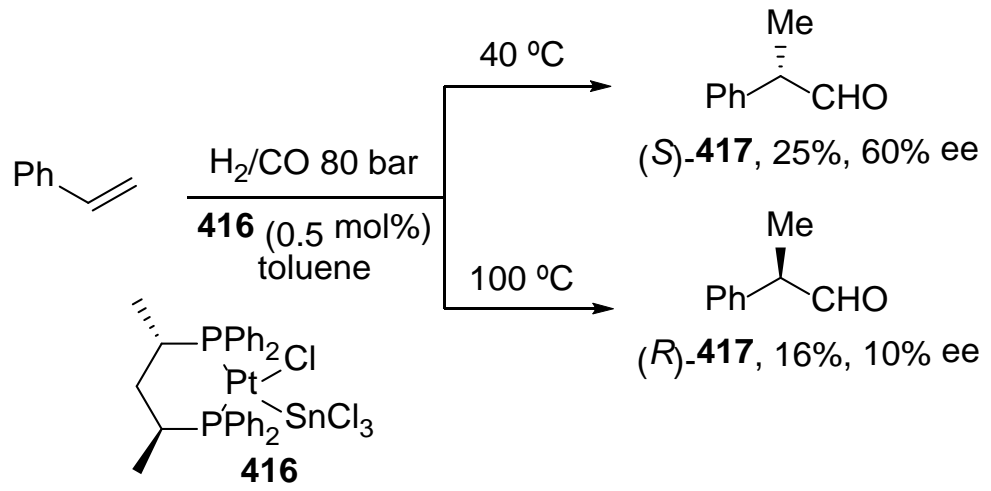

Recent computational studies by Kégl and co-workers about the influence of the electronic properties of the substituents in the phenyl group of $p$-substituted styrenes in the enantioselectivity of these hydroformylations have been performed. They showed an excellent linear correlation of the $p$-substituents Hammet constants and the electrostatic potential at nuclei of the Pt atom as well as in the coordination sphere of Pt. ${ }^{359}$ In the case of electron-donating substituents, a reversal of enantioselectivity was observed at lower temperature than with styrenes containing electron-withdrawing substituents.

In the Rh-catalyzed asymmetric hydroformylation, the electronic nature of the Pligands is related to the relative $\pi$-acidity of the $\mathrm{P}$ atoms. Therefore, phosphites, phosphoramidites, phospholanes and diazaphospholanes are commonly used. In addition, small bite angles of bidentate ligands favored the formation of branched aldehydes. However, few examples have been reported concerning enantiodivergent results. In the case of $\mathrm{Rh}$ complexes derived from phosphine-TADDOL phosphoramidite, IndolPHOS 418, changing substituents in the TADDOL unit promoted a switching on the enantioselectivity. ${ }^{360}$ Several types of substrates have been hydroformylated with moderate reversal of enantioselectivities. Styrene gave with ligand 418a, at different temperatures, $(R)-\mathbf{4 1 7}$ in $40 \%$ conversion and $33 \%$ ee at $40{ }^{\circ} \mathrm{C}$, whereas (S)-417 was obtained at $70{ }^{\circ} \mathrm{C}$ in full conversion and $16 \%$ ee. When vinyl acetate was used as substrate and $\mathbf{4 1 8 b}$ as ligand, product (S)-419 resulted in 97\% conversion and $42 \%$ ee. In addition, $(R)-\mathbf{4 1 9}$ was formed in $99 \%$ conversion and $35 \%$ ee when ligand 418a was used (Scheme 152). In the case of vinyl acetate increasing the syngas pressure gave higher ee with ligand 418a than 418c. On the other hand, allyl cyanide afforded quantitatively $(S)-420$ in $50 \%$ ee with 418 c and $(R)-420$ in $29 \%$ ee with ligand 418a.

Scheme 152. Enantiodivergent Rh-Catalyzed Hydroformylation of Vinyl Acetate and Allyl Cyanide with IndolPHOS Ligands 418 


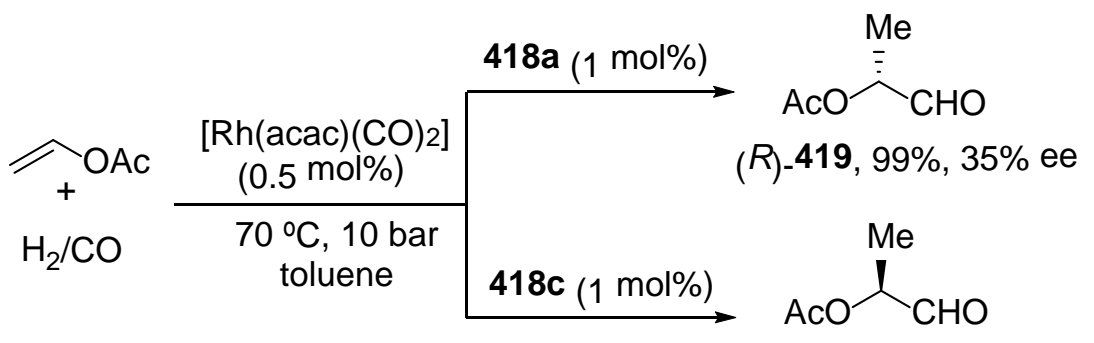

(S)-419, 97\%, 42\% ee

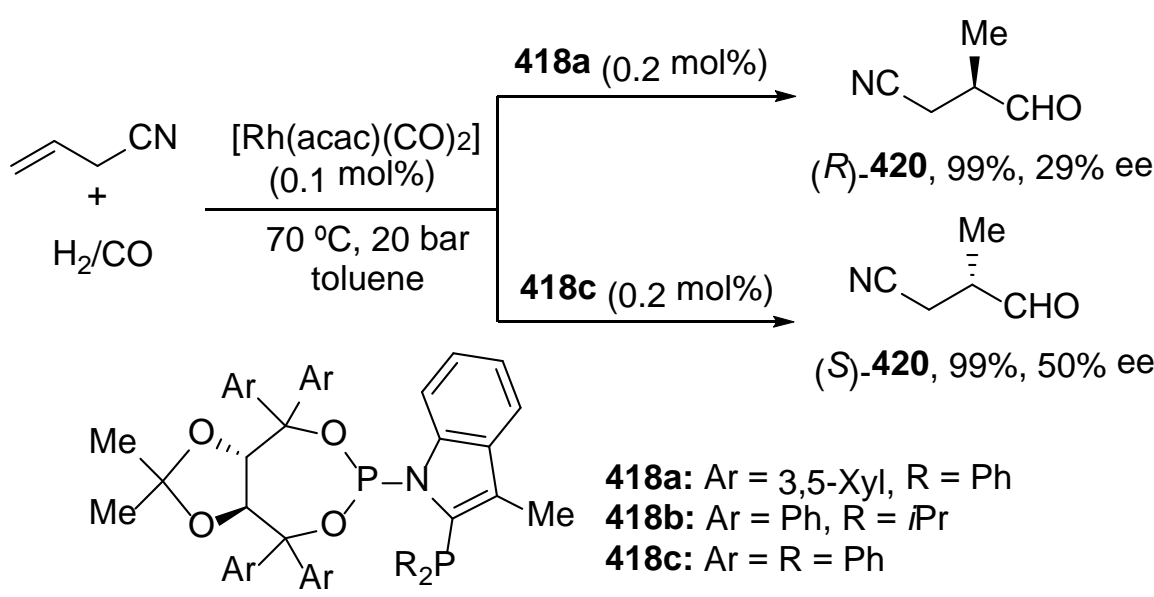

Deuterioformylation of vinyl acetate showed an irreversible insertion of the alkene into the Rh-H bond. According to experimental studies and DFT calculations, an enantiodiscrimination mechanism proposed TS 419A and 419B for ligands 418a and 418b, respectively (Scheme 153). In the TS 419A the xylyl group of TADDOL blocks one coordination site giving $(R)-\mathbf{4 1 9}$. However, in the TS 419B, the phenyl groups of the phosphine blocks the double bond rotation, as proposed by Pizzano and coworkers. $^{361}$

Scheme 153. Proposed Mechanism for the Enantiodivergent Rh-Catalyzed Hydroformylation of Vinyl Acetate with IndolPHOS Ligands 418 


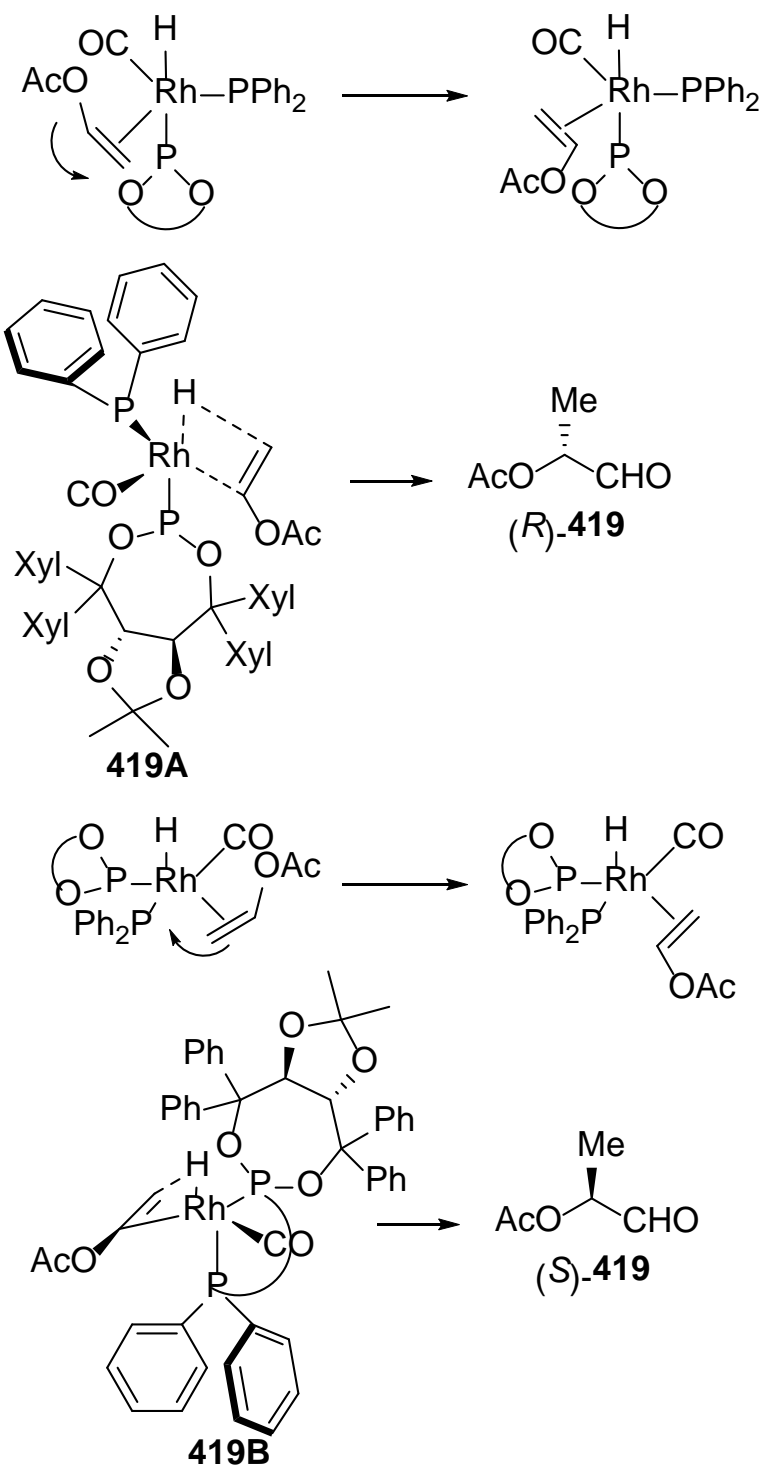

Rhodium complexes bearing chiral phosphine-phosphite ligands $\mathbf{4 2 1}$ with the same absolute configuration and different substituents in the phosphine unit gave enantiodivergent results in the hydroformylation of styrene and allyl cyanide (Figure 15). ${ }^{344,361}$ In the case of ligand 421a, $(S)-\mathbf{4 1 7}$ was formed in $43 \%$ ee, whereas $\mathbf{4 2 1 b}$ gave $(R)-417$ in a modest $25 \%$ ee. Similar reversal of enantioselectivity was observed with other ligands such as 421c and 421d. In the hydroformylation of allyl cyanide 421c gave (S)-420 in 53\% ee, whereas 421d afforded the corresponding enantiomer in only $6 \%$ ee.

Figure 15. Phosphine-Phosphite Ligands 421 for the Enantiodivergent RhCatalyzed Hydroformylation of Styrene and Allyl Cyanide 


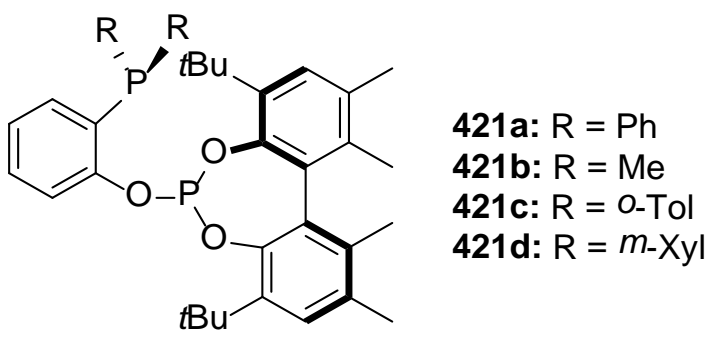

In conclusion, different substituents in the backbone of homochiral ligands play an important role in the Rh-catalyzed enantiodivergent asymmetric hydroformylation of alkenes. For Pt-catalyzed reactions, the temperature-dependent enantiodivergence has been observed. In general, modest effects on the enantiodivergence have been found till now.

2.3.4. Other Addition Reactions. Metal-catalyzed asymmetric additions such as hydroboration-oxidation of prochiral $\mathrm{C}=\mathrm{C}$ bonds as well as epoxidation and cyclopropanation of alkenes are going to be considered in this section.

Tandem enantioselective hydroboration-oxidation of alkenes gave access to enantioenriched alcohols. The first example of an enantioselective metal-catalyzed hydroboration-oxidation was described by Hayashi, Ito and co-workers using $\left[\mathrm{Rh}(\mathrm{COD})_{2}\right] \mathrm{BF}_{4}$ and (+)-BINAP complex as chiral catalyst. ${ }^{362}$ Recently, Smith and Tackas described an enantiodivergent hydroboration of $\beta, \gamma$-unsaturated amides with pinacolborane (pinBH) catalyzed by a cationic Rh-norbornadiene (NBD) complex using TADDOL-derived ligands 423. For instance, in the case of amide $\mathbf{4 2 2}$ and in the presence of phosphoramidite 423a the corresponding product (3S,4R)-424 was obtained in $80 \%$ ee, whereas when the phosphite $\mathbf{4 2 3 b}$ was used as ligand the corresponding enantiomer was obtained in 96\% ee (Scheme 154). ${ }^{363}$ Other substrates showed a similar enantiodivergent effect. This switch of enantioselectivity has been attributed to the catalyst structure as well as to the reactivity and/or reaction mechanism.

Scheme 154. Enantiodivergent Hydroboration-Oxidation of $\beta, \gamma$-Unsaturated Amide 422 Catalyzed by [Rh(NBD) $\left.{ }_{2}\right]_{B F}$ and Different Ligands 423 


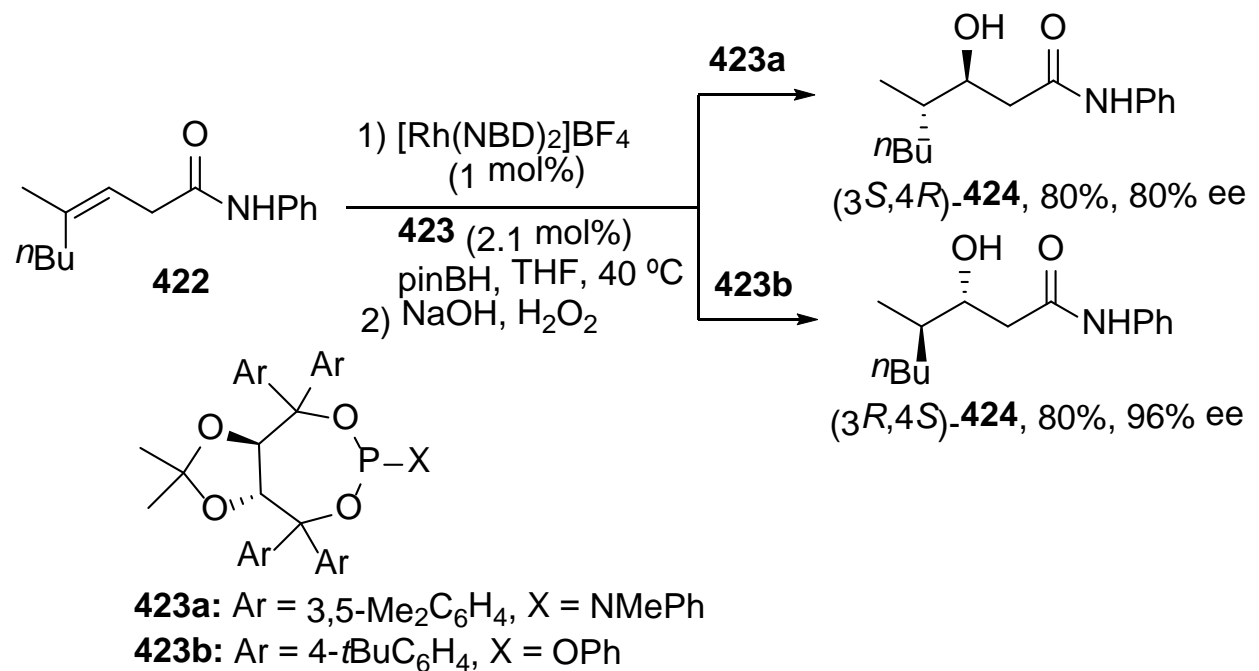

A remarkable influence of the molecular weight of polyethylene glycol (PEG) bonded to L-tartrate in the enantioswitching of the Sharpless asymmetric epoxidation of allylic alcohols has been observed. ${ }^{364-366}$ By using L-tartrate-MPEG 350 (426a), alcohol 425 was transformed into the corresponding epoxide (2S,3S)-427with ee up to $67 \%$ (Scheme 155). ${ }^{366}$ On the other hand, when L-tartrate-MPEG ${ }_{750}$ (426b) was used as ligand of $\mathrm{Ti}(\mathrm{OiPr})_{4}$ enantiomeric (2R,3R)-427 was formed in $75 \%$ ee. This reproducible inversion of enantioselectivity was explained as a consequence of the Ti-ligand complexes, which can be monomeric and dimeric depending on the chain length of the PEG: long chain polymer prevents the formation of dimers. More recently, a similar effect has been observed in the epoxidation of cinnamyl alcohol with L-tartrate derived from $N$-Boc- $N$-methyl-2-aminoethanol, which led to the formation of the $(2 S, 3 S)$ epoxide in $72 \%$ yield and $70 \%$ ee, whereas the ligand supported on silsesquioxane led to the formation of the corresponding enantiomer in $51 \%$ yield and $40 \%$ ee. ${ }^{367}$

\section{Scheme 155. Enantiodivergent Sharpless Epoxidation of (E)-Hex-2-en-1-ol Using MPEG L-Tartrates as Ligands}
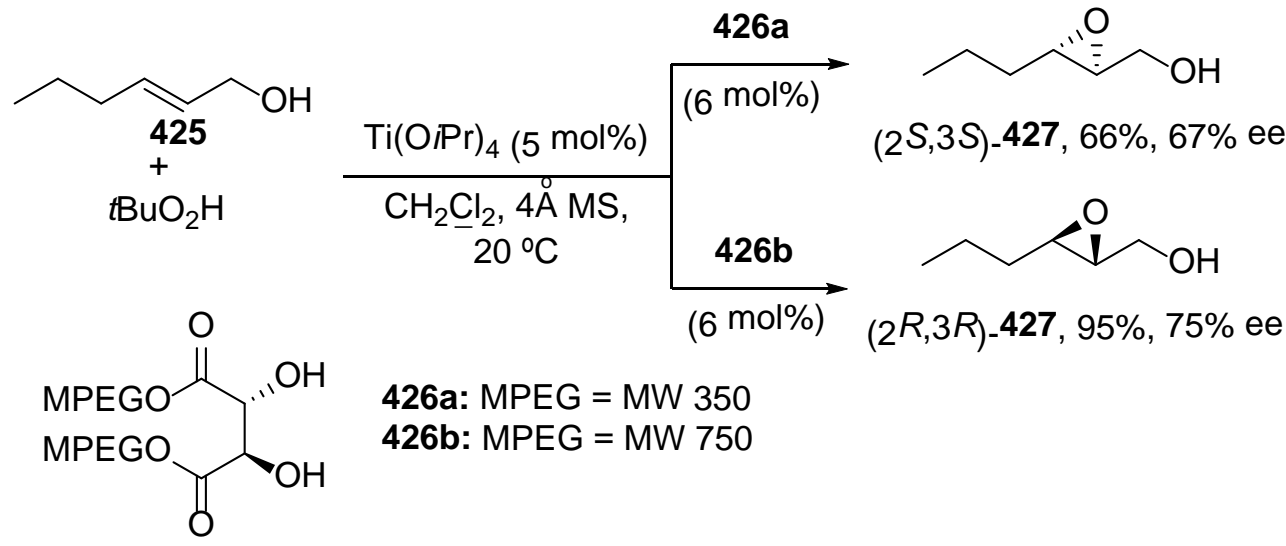

In the case of the asymmetric cyclopropanation of styrene with ethyl diazoacetate catalyzed by clay-immobilized bis(oxazoline)-copper $\mathbf{4 2 8}$ catalysts, a reversal of enantioselectivity has been observed by reduction of the solvent polarity. ${ }^{368,369}$ Just in 
styrene as solvent a 70/30 cis/trans mixture of cyclopropanes $\mathbf{4 2 9}$ was obtained and the major $(1 R, 2 S)$-diastereomer was formed in $34 \%$ ee. However, in $\mathrm{CH}_{2} \mathrm{Cl}_{2}$ the enantiomeric (1S,2R)-product 429 was obtained in $49 \%$ de and $24 \%$ ee (Scheme 156).

Scheme 156. Enantiodivergent Cyclopropanation of Styrene Catalyzed by ClayImmobilized Bis(oxazoline)-Copper Complex 428

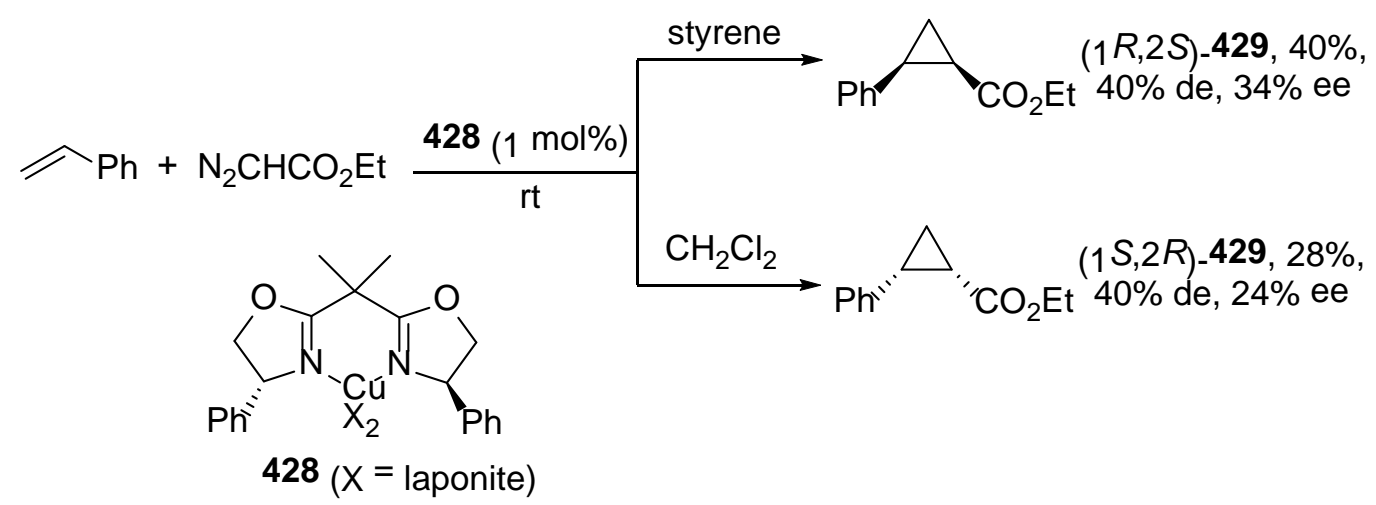

Another example of enantiodivergent cyclopropanation was reported by Mayoral and co-workers using bis(oxazoline)-copper complexes supported in ionic liquid films with different thickness. ${ }^{370}$ This surface effect has been reported for the same cyclopropanation when immobilized pyridinoxazoline-copper complexes were used as catalysts. ${ }^{371}$ Modest values of diastereo- and enantioselectivity were observed.

In conclusion, very few examples of enantiodivergent hydroboration-oxidation, epoxidation and cyclopropanation of carbon-carbon double bonds have been reported. In the first case, different homochiral ligands of the $\mathrm{Rh}$ catalysts determine the enantiodivergence. In the last two cases moderate effects of the polymer or solid supports have been observed.

\section{4. $\alpha$-Functionalization of Carbonyl Compounds}

Asymmetric $\alpha$-functionalization of acyclic carbonyl compounds involves also the formation of $\mathrm{C}-\mathrm{C}$ bonds by the formation of enolates or enamines. Depending on the electrophile used in this process it can take place by nucleophilic substitution or by nucleophilic addition in the presence of organocatalysts. For the asymmetric synthesis of $\alpha$-amino acids, ${ }^{372,373}$ the $\alpha$-alkylation of enolates of imino esters derived from glycine or from other $\alpha$-amino acids is a well-established methodology, which can be performed under phase-transfer catalysis (PTC) with chiral ammonium salts. However, only one example has been described about the enantiodivergent alkylation of $\mathrm{N}$ (diphenylmethylene)glycine isopropyl ester (226b) with benzyl bromide using cinchonidinium salts under metal base-dependent conditions with modest results. ${ }^{374}$ The alkylation in the presence of catalyst $\mathbf{4 3 0 a}$ with $50 \%$ aq $\mathrm{KOH}$ gave (S)-431 in 58\% ee, whereas with $50 \% \mathrm{NaOH}$ the (R)-enantiomer was formed in $40 \%$ ee (Scheme 157). 
Catalysts $\mathbf{4 3 0 b}$ showed the same behavior giving (S)-431 in $98 \%$ yield and $44 \%$ ee with $\mathrm{KOH}$ and $(R)-431$ in $80 \%$ yield and $38 \%$ ee with $\mathrm{NaOH}$. Reasons to explain these unexpected results have not been found.

Scheme 157. Enantiodivergent $\alpha$-Alkylation of Imino Ester 226b under PTC with Cinchonidinium Salts 430

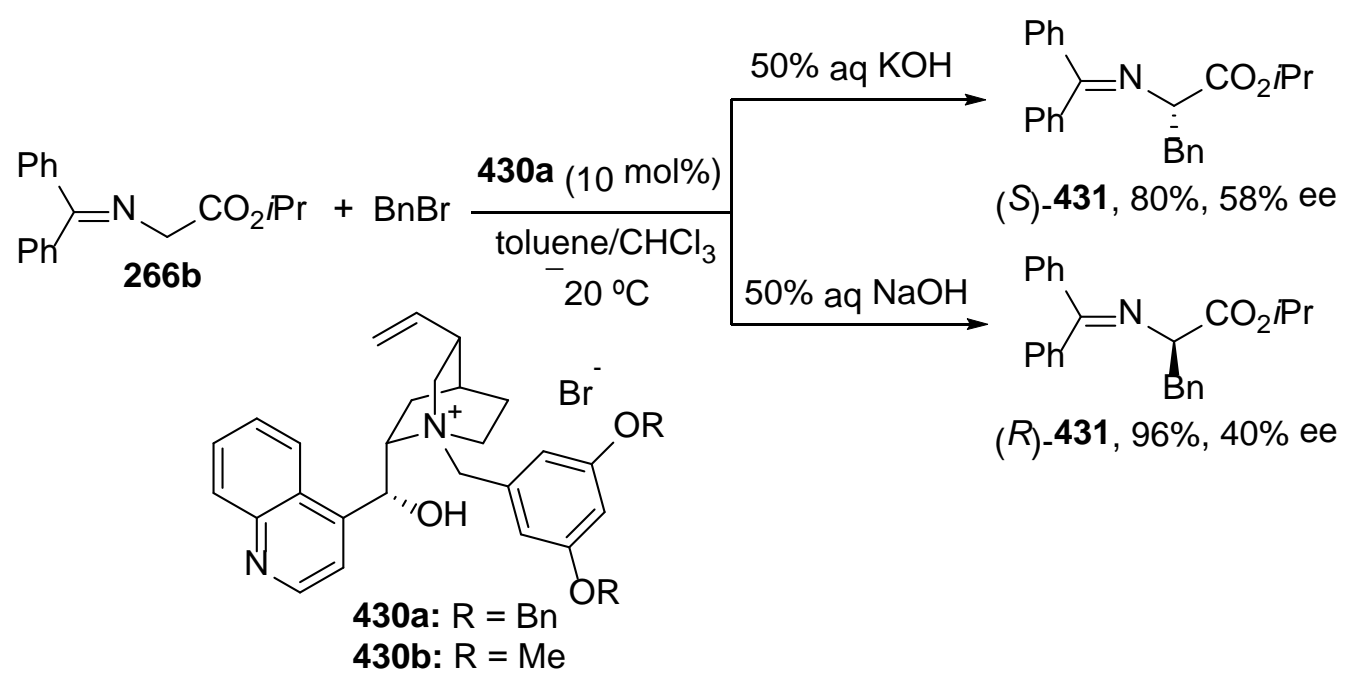

Free radical allylation of $\alpha$-bromo substituted $N$-acyloxazolidines 432 initiated by $\mathrm{Et}_{3} \mathrm{~B}$ has been performed by Sibi and co-workers by using the $(R, R)$-bis(oxazoline) 433 as ligand of Lewis acids. For example, allyltrimethylsilane reacted with the compound 432 in the presence of complexes derived from ligand $\mathbf{4 3 3}$ giving $\alpha$-allylated product 434 with opposite configuration depending on the Lewis acid employed. They observed that in the presence of stoichiometric amounts of $\mathrm{Zn}(\mathrm{OTf})_{2}(R)-\mathbf{4 3 4}$ was obtained in $90 \%$ ee, whereas $\mathrm{MgI}_{2}$ afforded (S)-434 in 68\% ee (Scheme 158). ${ }^{375}$ These are considered excellent results taking in account that it is a free-radical allylation.

Scheme 158. Enantiodivergent Free-Radical Allylation of $\alpha$-Bromo- $N$ acyloxazolines 432 in the Presence of Chiral Ligand 433 and Different Lewis Acids

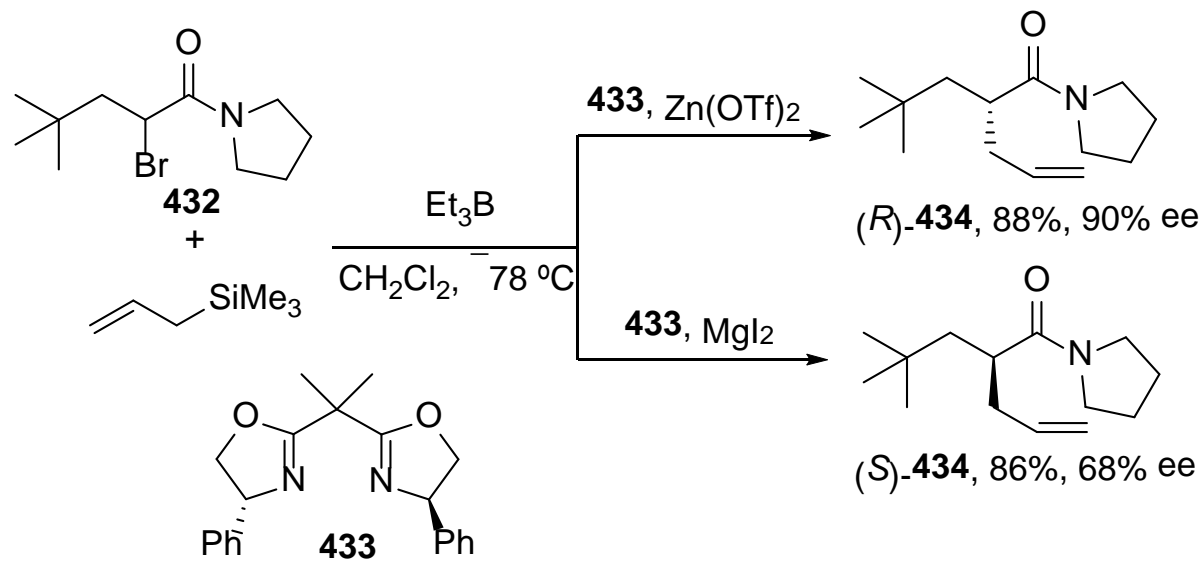

Enamine-based enantiodivergent $\alpha$-oxygenation of aldehydes has been described by Maruoka and co-workers catalyzed by binaphthyl-derived chiral amines 435 and 436 
with excellent results (Scheme 159). ${ }^{376}$ Aminoxylation of aldehydes using 435 took place regioselectively with nitrosobenzene giving, after reduction with $\mathrm{NaBH}_{4}, 2$ aminoxyl alcohols 437 with $R$-configuration and ee up to $88 \%$. When organocatalysts 436, with the same absolute configuration as $\mathbf{4 3 5}$ but different substituents in the binaphthyl unit was used, the enantiomeric products (S)-437 were obtained with ee up to $98 \%$. In the first case, the s-trans-enamine gave TS 435A, according to the activation of nitrosobenzene by the carboxyl group, which will approach by the $R e$ face giving $(R)$-437. On the other hand, the hydrogen of the triflamide unit will favor the approach of nitrosobenzene through hydrogen bonding by the Si-face of the s-cis-enamine in TS 436A.

Scheme 159. Enantiodivergent $\alpha$-Aminoxylation of Aldehydes with Nitrosobenzene Catalyzed by (S)-Binaphthyl Amines 435 and 436

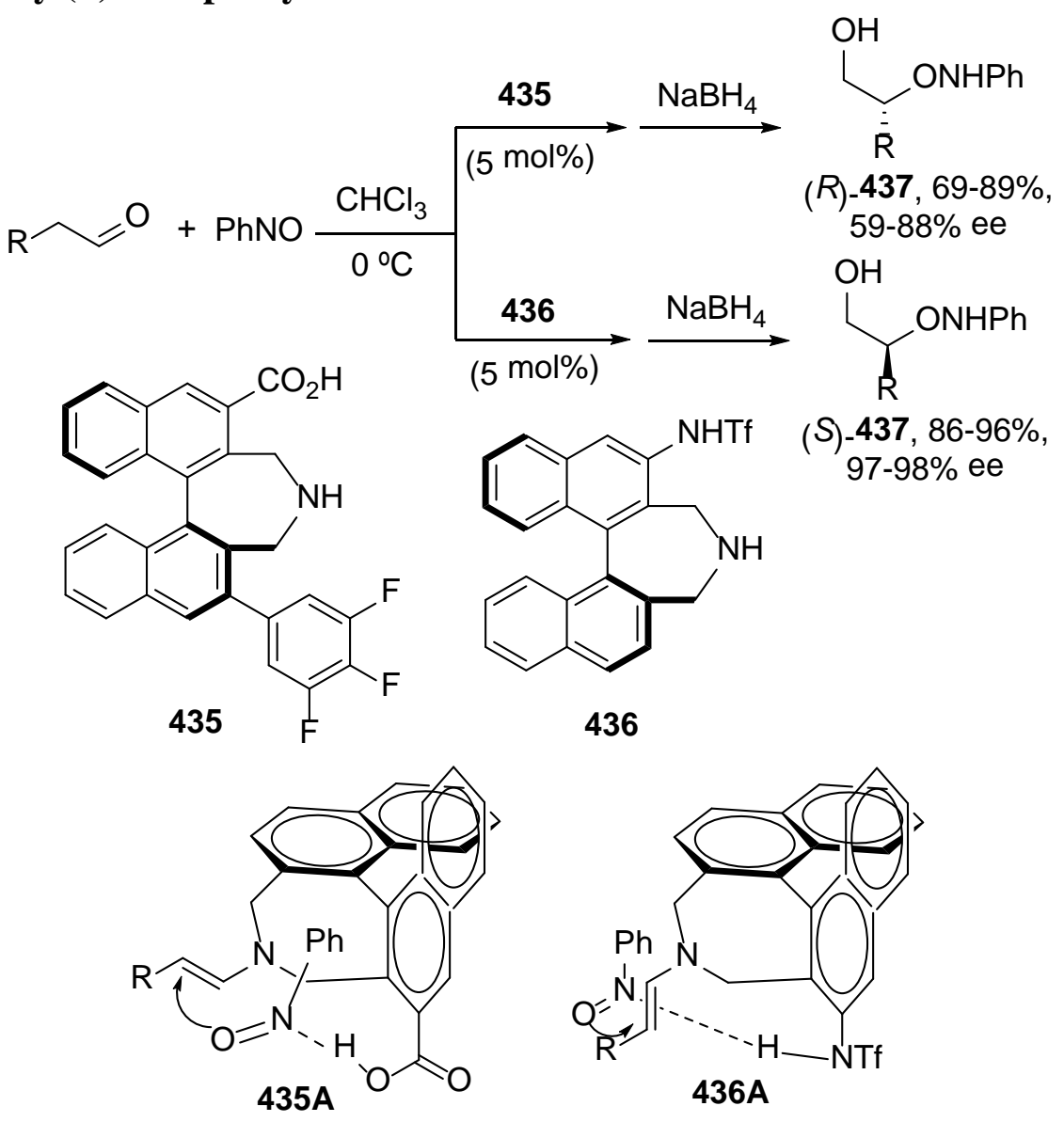

Another example of enantiodivergent enamine-catalyzed nucleophilic addition is the amination of aldehydes with diethyl azodicarboxylate (DEAD). Blackmond, Armstrong and co-workers studied a switch of the enantioselectivity in the $\alpha$-amination of aldehydes under $(S)$-proline catalysis in the absence or presence of DBU as additive. ${ }^{377}$ In the absence or presence of DBU an inversion of the enantioselectivity was observed giving after $\mathrm{NaBH}_{4}$ reduction $(R)-\mathbf{4 3 8}$ or $(S)-\mathbf{4 3 8}$, respectively (Scheme 160). The authors attribute this reversal of enantioselectivity to the formation of prolinate 
salts. ${ }^{378,379}$ Similar results were observed with proline-tetrazole organocatalyst instead of proline.

Scheme 160. Enantiodivergent $\alpha$-Amination of Aldehydes with Diethyl Diazodicarboxylate Catalyzed by L-Pro

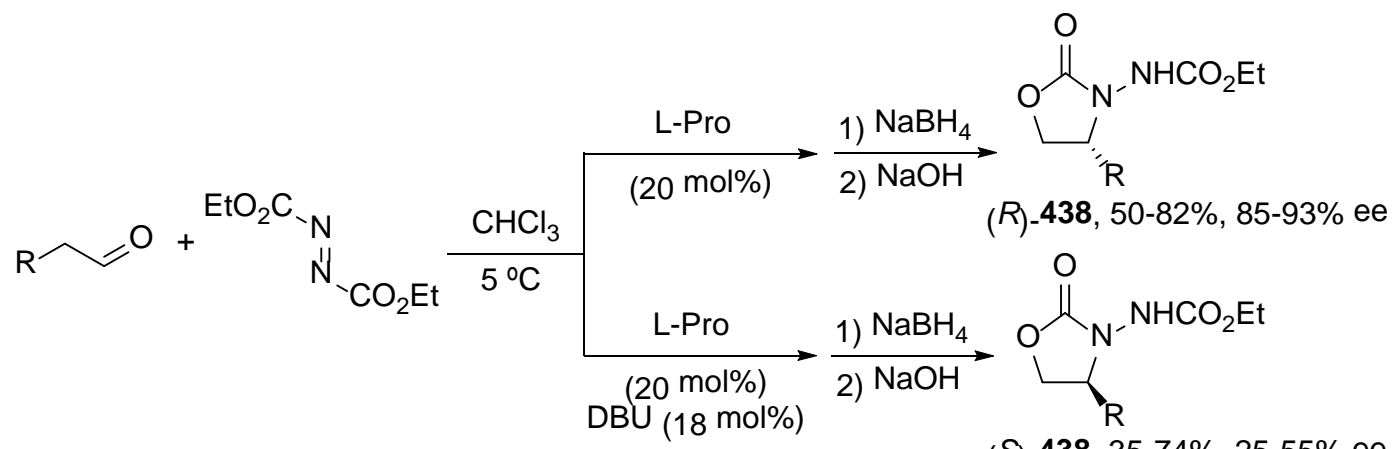

(S)-438, 35-74\%, 25-55\% ee

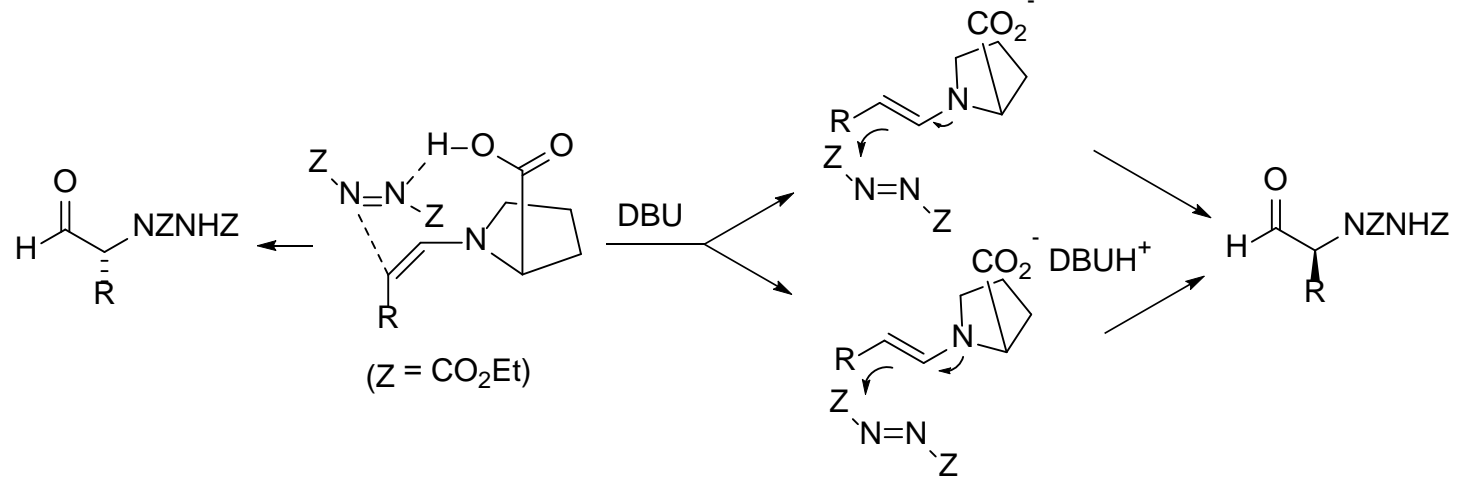

DFT calculations supported the role of the enamine carboxylate in the process. In the absence of DBU, DEAD attacks the syn-face of the s-trans-enamine with a concomitant hydrogen-bonding stabilization. In the presence of DBU anti-addition to the electrophile of the s-trans-enamine carboxylate or the ion pair generates the $(S)$-product. ${ }^{380,381}$

Recently, Vetticatt and co-workers reported excellent enantiodivergent results in the amination reaction of aldehydes with diazodicarboxylates catalyzed by 2-methyl-L-Pro 439a or its tetramethylammonium salt 439b (Scheme 161). ${ }^{382}$ In the case of 439a products $(R)-\mathbf{4 4 0}$ were obtained, whereas the corresponding ammonium salt $\mathbf{4 3 9 b}$ gave the $(S)$ enantiomers. These results were achieved according to the DFT calculations, which predicted the formation of the anti-enamine 439A and the anti-enamine 439B due to the steric interactions, respectively. In the case of propanal the enantiodivergent reaction with nitrosobenzene, using the same catalysts 439a and 439b, provided the hydroxyamination products of type $(R)-\mathbf{4 4 0}$ and $(S)-\mathbf{4 4 0}$ in 66\% yield and $99 \%$ ee and in $64 \%$ yield and $98 \%$ ee, respectively.

Scheme 161. Enantiodivergent $\alpha$-Amination of Aldehydes with Diethyl Diazodicarboxylate Catalyzed by 2-Methyl-L-Pro and Its Ammonium Salt 439 


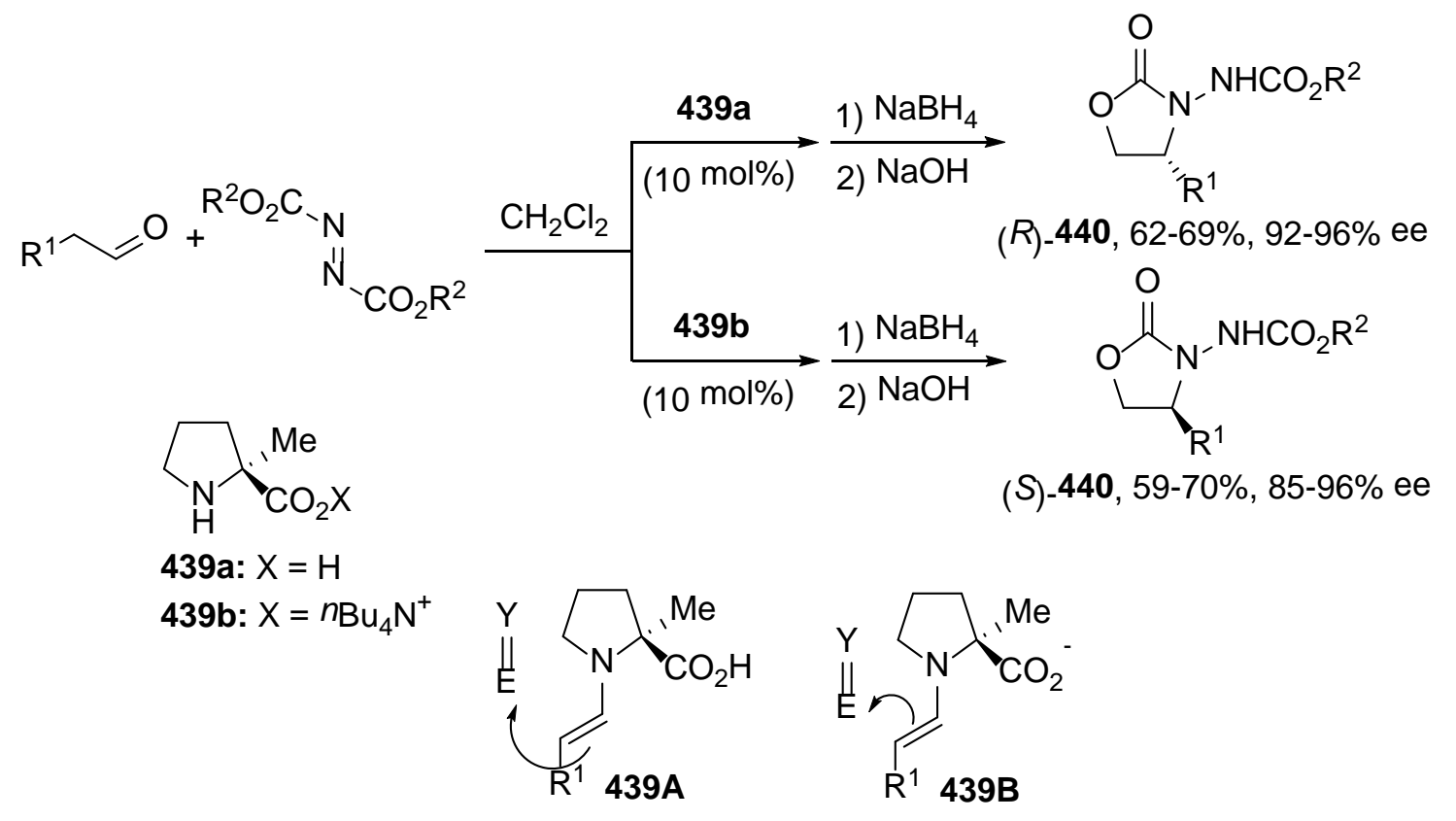

Reversal of enantioselectivity has been achieved by You and co-workers in the asymmetric dearomative amination of $\beta$-naphthols with DEAD catalyzed by different 3,3'-disubstituted BINOL-derived phosphoric acids. ${ }^{383}$ When acid 245 was used as catalyst, product $(R)-\mathbf{4 4 2}$ was obtained with ee up to $81 \%$ ee, whereas the corresponding enantiomer (S)-442, with ee up to 80\%, was isolated using acid 441 (Scheme 162). These enantiodivergent results have been explained by Sunoj and co-workers in base to DFT calculations. ${ }^{384}$ They have explained this enantiodivergent effect by their $\mathrm{C}-\mathrm{H} \cdots \pi$ interactions with the catalyst $\mathbf{2 4 5}$ and the covalent C-H $\cdots$ F interactions for catalyst $\mathbf{4 4 1}$.

Scheme 162. Enantiodivergent Amination of a $\beta$-Naphthol with Diethyl Diazodicarboxylate Catalyzed by BINOL Phosphoric Acids 245 and 441

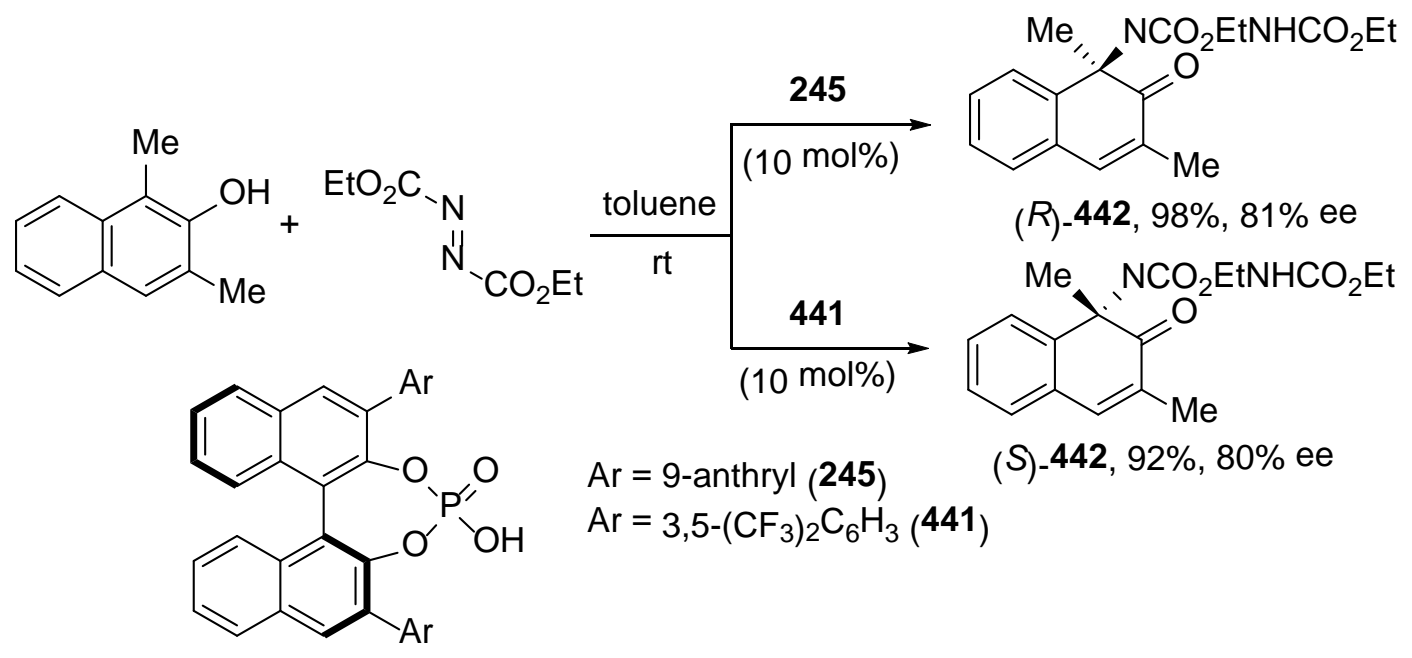

Recently, the enantiodivergent $\alpha$-fluorination of $\beta$-keto ester derivatives organocatalyzed by a chiral primary amine $\mathbf{4 4 3}$ gave enantiodivergent results depending 
on the fluorinating reagent (Scheme 163). ${ }^{385}$ When $N$-fluorobenzenesulfonimide (NFSI) was used, the corresponding products $(R)-445$ were obtained in high yields and ee. On the other hand, $N$-fluorinated pyridinium salt $\mathbf{4 4 4}$ afforded products (S)-445 with excellent results as well. The mechanistic studies revealed a dual hydrogen bonding between the substituents and NFSI in the TS 445A, whereas in the case of the reagent 444 a TS 445B with an attractive $\mathrm{C}-\mathrm{H} \cdots \mathrm{F}$ interaction between the tert-butyl group of the catalyst and the reagent may contribute to the Si-facial attack.

\section{Scheme 163. Enantiodivergent $\alpha$-Fluorination of $\beta$-Keto Ester Derivative Organocatalyzed by Diamine 443 and Different Fluorinating Reagents}

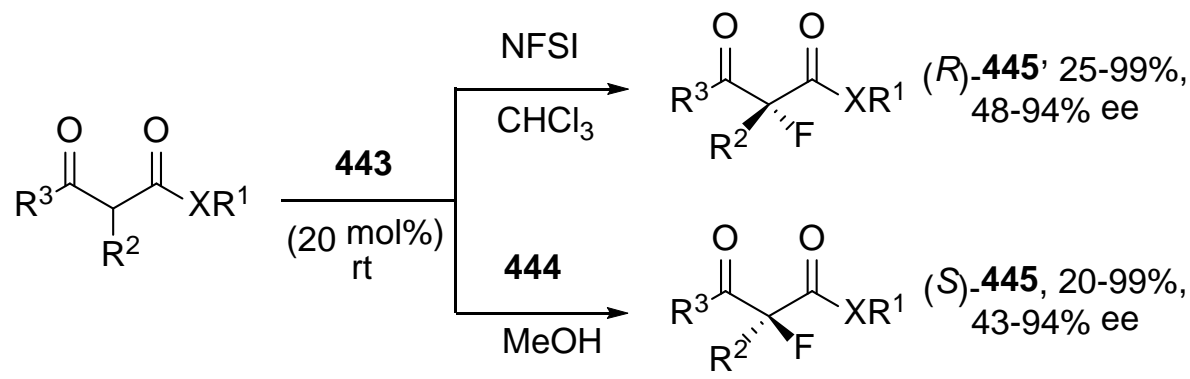<smiles>CCCCNC[C@H](N)CC(C)C</smiles>

443

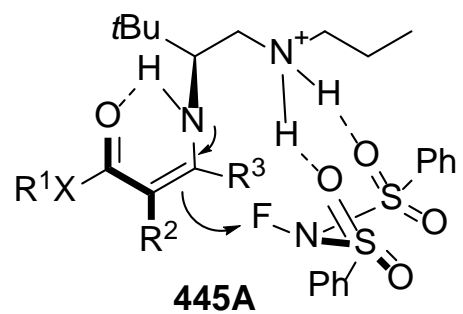<smiles>[N-]=[SiH2]N(S(=O)(=O)c1ccccc1)S(=O)(=O)c1ccccc1</smiles><smiles>Cc1cc(C)[n+](F)c(C=O)c1</smiles>

444

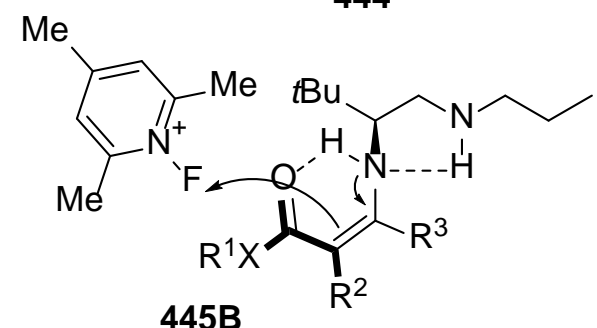

In conclusion, different types of enantiodivergent $\alpha$-alkylations of carbonyl compounds have been described but with only particular examples. In the case of glycine imino esters the alkylation under PTC conditions can be modulated by different metal hydroxides. Only one example about enantiodivergent radical allylation using different Lewis acids and the same chiral BOX ligand has been successfully performed. In the case of the $\alpha$-oxyamination of aldehydes, the enantiodivergence can be controlled by different substitutions in the organocatalysts. In $\alpha$-amination reactions of aldehydes L-Pro derivatives or their ammonium salts can give enantiodivergent results and in the case of $\beta$-naphthol differently 3,3'-substituted BINOL phosphoric acids induced a reversal of enantioselectivity. In one case about $\alpha$-fluorination of $\beta$-keto ester derivatives organocatalyzed by a diamine the enantiodivergence was directed by the use of different fluorinating reagents. 


\subsection{Oxyranyl and Aziridinyl Ring Opening}

Asymmetric ring opening of meso-epoxides and aziridines are usually performed under metal-catalysis, acting as chiral Lewis acids, giving enantioenriched $\beta$-bifunctionalized alcohols and amines, respectively. This nucleophilic substitution can be carried out with different nucleophiles, however, only few enantiodivergent examples have been reported so far.

The ring opening of meso-epoxides in water takes place using chiral Sc/bipyridine 446 complex giving compounds threo-447 in good yields and enantioselectivities (up to $96 \%){ }^{386}$ Reversal of metal-controlled enantioselectivity was achieved when $\mathrm{Cu}(\mathrm{II})$ or $\mathrm{Zn}(\mathrm{II})^{387}$ complexes with ligand 446 were employed in this process. ${ }^{386,387}$ Indoles reacted with cis-stilbene oxide derivatives giving adducts ent-447 with ee up to $96 \%$ using the corresponding $\mathrm{Cu}(\mathrm{II})$ complex (Scheme 164). The same inversion of enantioselectivity was observed when $N$-methylaniline was used as nucleophile giving the corresponding threo-448 amino alcohols in high yields and with ee up to $97 \%$ ee using the $\mathrm{Sc}(\mathrm{III})$ complex as catalyst. ${ }^{386,387}$ In the case of the $\mathrm{Cu}(\mathrm{II})$ complex, the corresponding enantiomeric amino alcohols $\mathbf{4 4 8}$ were isolated with ee up to 91\%. XRay diffraction analysis of the complex of $\mathrm{Cu}(\mathrm{II})$ with ligand 449 showed a square pyramidal structure and a pentagonal bipyramidal structure for the Sc(III) complex, which could be the reason of the enantiodivergence. Further studies by Kobayashi and co-workers about these reactions showed that the $\mathrm{Zn}$ (II) complex gave the same results as $\mathrm{Cu}(\mathrm{II})$ complex. ${ }^{388}$

Scheme 164. Enantiodivergent Ring Opening of meso-Epoxides with Indole and $\mathrm{N}$ Methylaniline Catalyzed by Cu(II) and Sc(III) Bipyridine 446 Complexes

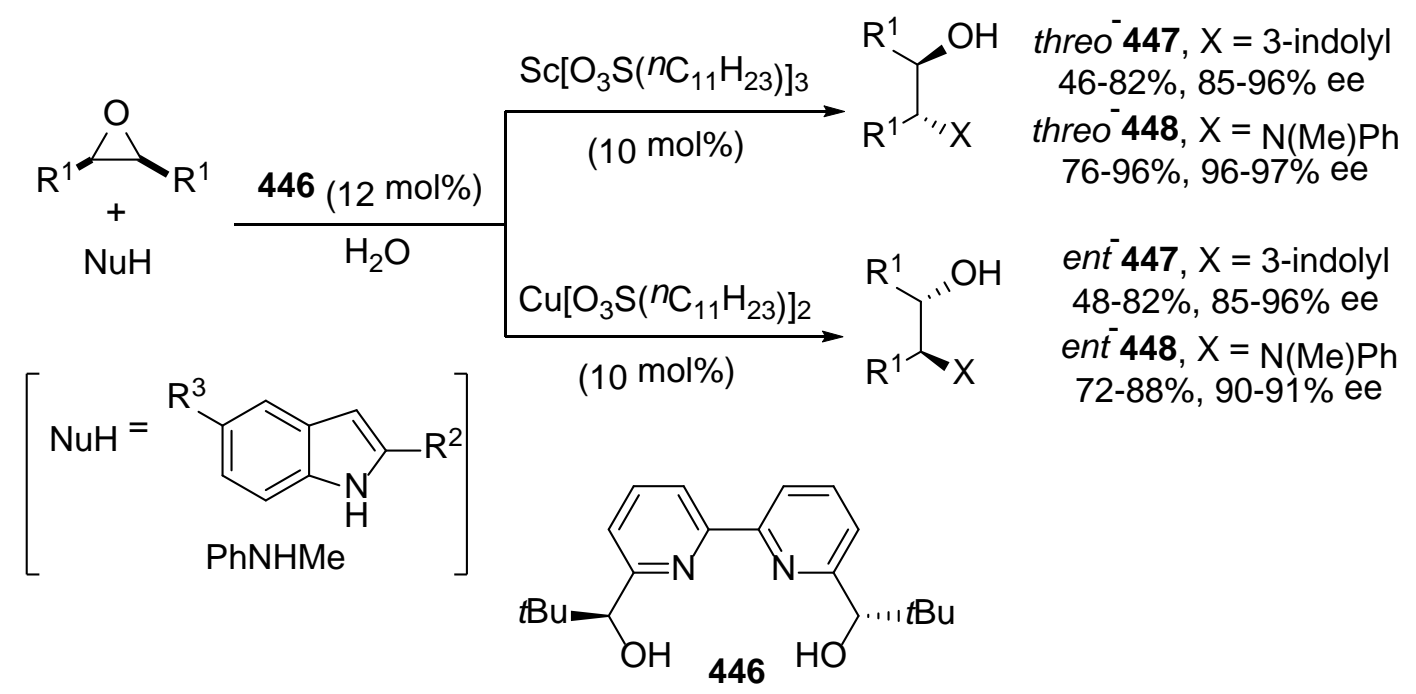

The structural modification of salen-Co(II) complexes gave a reversal of enantioselectivity in the ring opening of epichlorohydrin with 2,3dihydroxybenzaldehyde (Scheme 165). ${ }^{389}$ Addition of $\mathrm{AlCl}_{3}$ increased the reaction rate 
giving under the presence of complex 117a product $(R)-\mathbf{4 4 9}$ with ee up to 50\%, whereas complex 117b afforded enantiomeric compound (S)-449 with ee up to $70 \%$.

Scheme 165. Enantiodivergent Ring Opening of Epichlorohydrin with 2,3Dihydroxybenzaldehyde Catalyzed by Salen-Co(III) Complexes 117

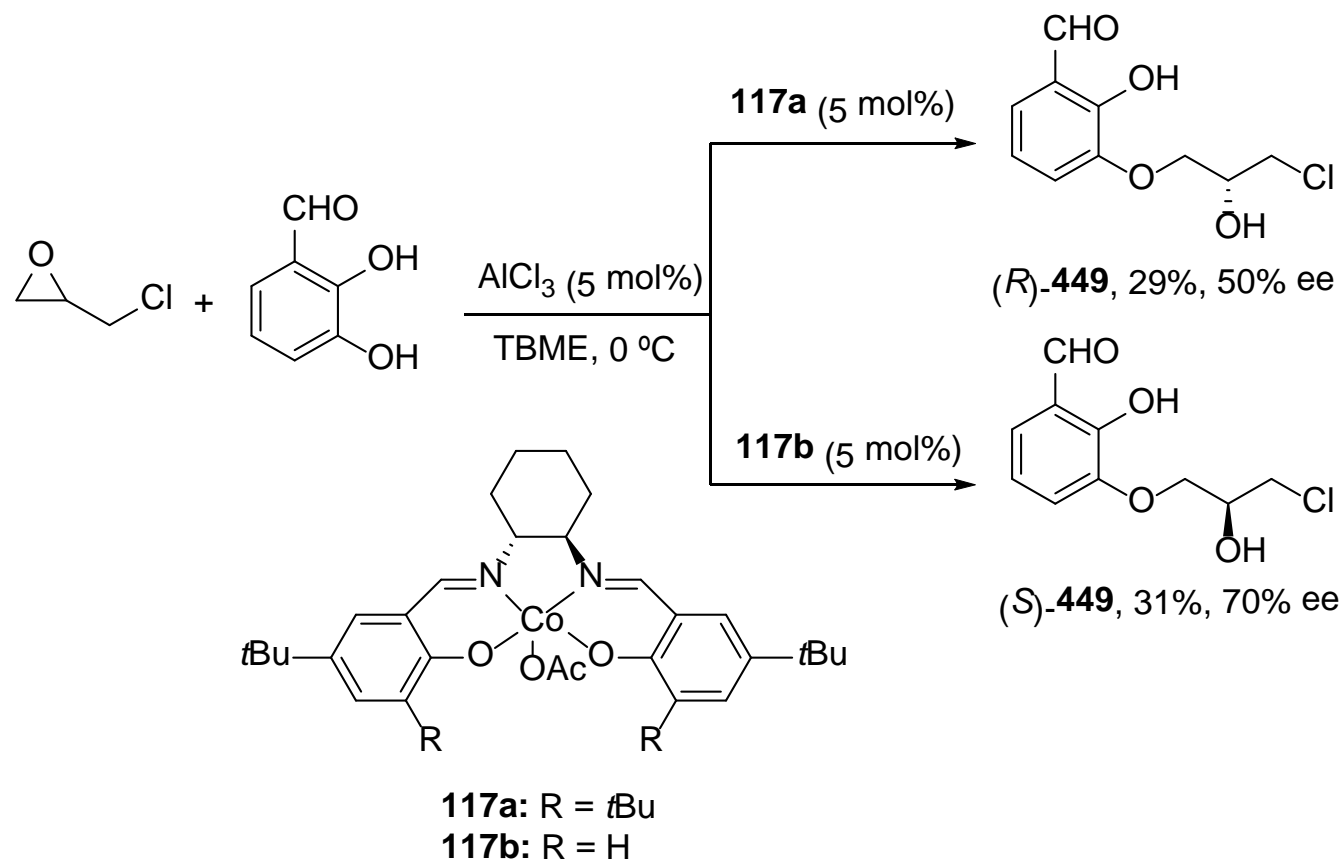

In the case of the ring opening of meso-aziridines 450, the Kanai and Shibasaki group used polymetallic catalysts for the enantiodivergent nucleophilic substitution by means of trimethylsilyl cyanide. When $\mathrm{Gd}(\mathrm{OiPr})_{3}$ and chiral ligand $\mathbf{4 5 1}^{390}$ was employed as catalyst in the presence of TFA (5 mol\%) and 1 eq of 2,6-dimethylphenol (DMP), products $(S, S)-\mathbf{4 5 3}$ were obtained with ee up to 93\% (Scheme 166). Further studies by the same group using ligand $\mathbf{4 5 2}^{391}$ provided an opposite enantioselectivity giving $\beta$-amidonitriles ( $R, R)-\mathbf{4 5 3}$ with ee up to $99 \%$ (Scheme 3 ). Reasons for the excellent reversal of enantioselectivity were not clear and it was attributed to the different structures of the polymetallic complexes.

Scheme 166. Enantiodivergent Ring Opening of meso-Aziridines 450 with Trimethylsilyl Cyanide Catalyzed by Different Gd(III) Complexes 451 and 452 


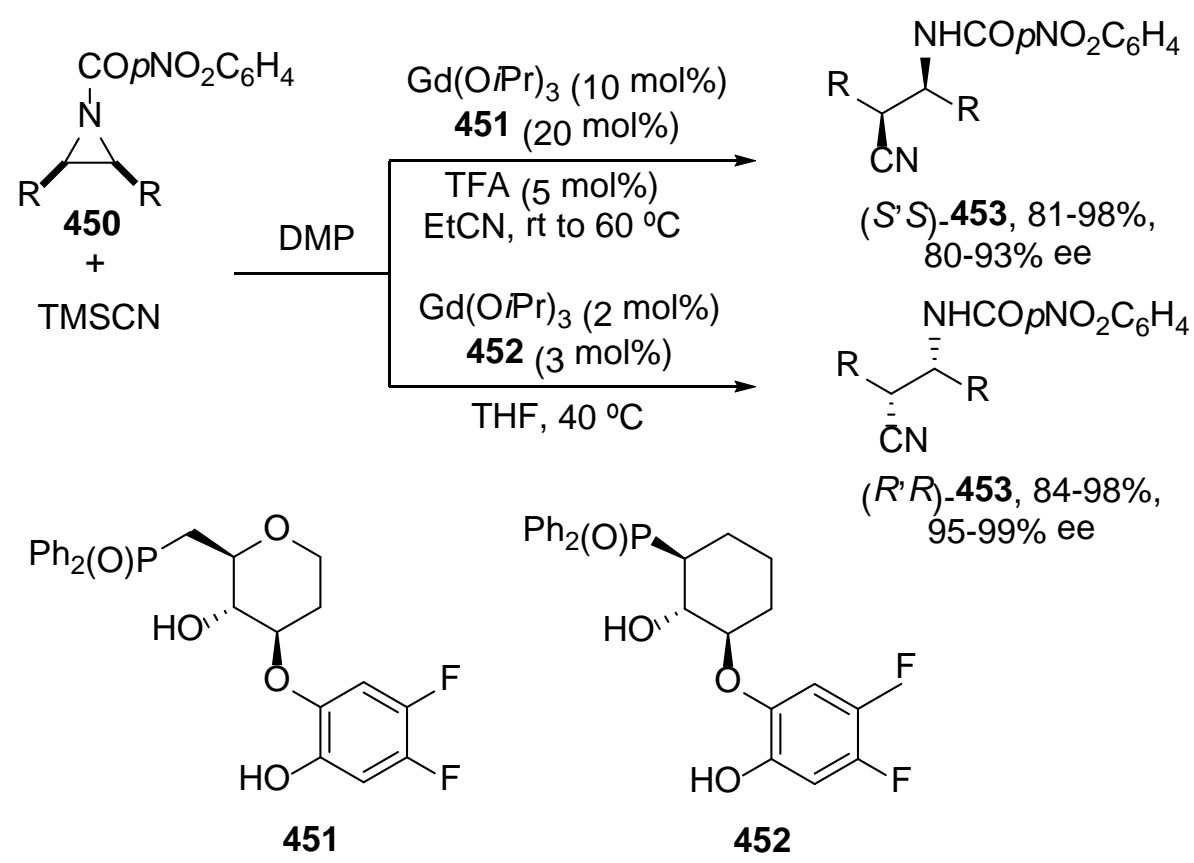

In conclusion, very few examples of enantiodivergent ring opening of oxiranes and aziridines have been reported. Different metal complexes either with different metals or different ligands control the enantiodivergence.

\subsection{Allylic Substitution Reactions}

Asymmetric allylic substitution has been performed mainly with $\mathrm{Pd}$, Ir, Mo and Ru complexes and different carbon, nitrogen and oxygen nucleophiles. ${ }^{392-396}$ Pd-allylic complexes are the most important catalysts and play a special role in the study and understanding of the Pd-catalysis. The nucleophilic attack on these $\pi$-allyl palladium intermediates takes place usually at the less hindered carbon atom giving linear products. Depending on the nucleophile the process can take place by inversion or retention of the configuration. Therefore, it is possible to expect examples of stereodivergence. In the case of Pd-catalyzed allylic substitutions with soft nucleophiles, through an outher-sphere attack to the allylpalladium intermediate, it occurred with an inversion of the configuration (global retention), which has been represented with a cyclic acetate (Scheme 167). On the other hand, hard nucleophiles attack the metal center and after reductive elimination provide a retention of the configuration (global inversion) (Scheme 167). According to Mayr nucleophilicity parameters, carbon nucleophiles are softer nucleophiles than amines. ${ }^{397}$ Allylic esters and carbonates are typical substrates but other types of allylic electrophilic reagents such as carbamates, phenyl ethers, vinyloxiranes, halides and alcohols can be used. Basic reaction conditions are only used for allylic acetates and neutral conditions for the other substrates.

Scheme 167. Inversion and Retention of the Configuration in Pd-Catalyzed Allylic Substitutions 


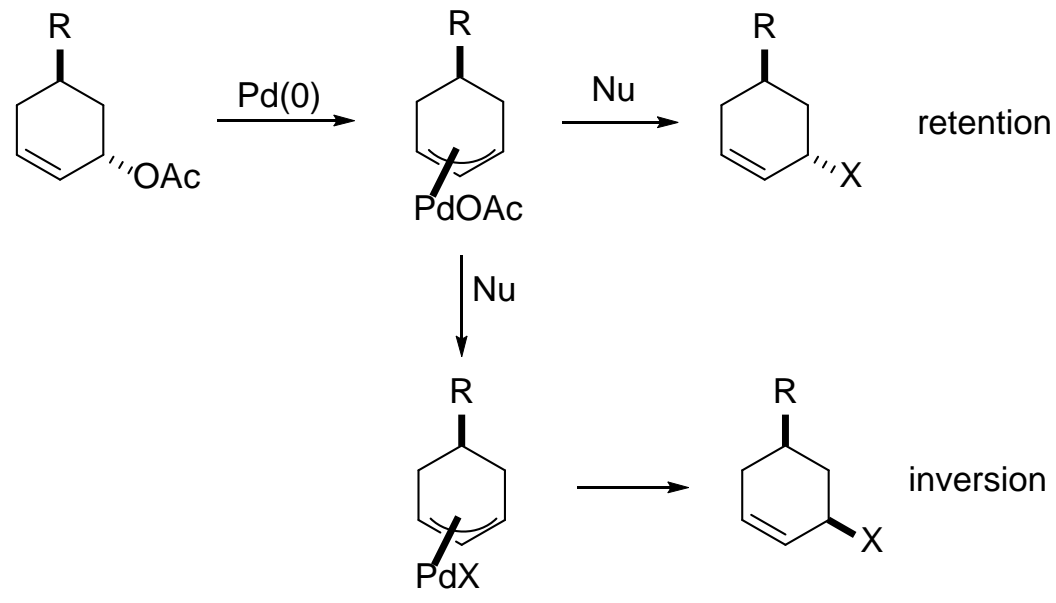

2.6.1. Carbon Nucleophiles. Carbanions, with pKa values for the conjugate acid in the 10-20 range especially malonates, have been extensively used in the asymmetric allylic alkylation (AAA), Tsuji-Trost reaction, mainly under palladium-catalysis and chiral ligands. The reaction of 1,3-diphenylallyl acetate with diethyl sodium malonate has been a benchmark reaction to test, and then design, chiral ligands like in the asymmetric hydrogenation of alkenes. Ligand substituent effects on the enantiodivergence using different bisphosphinites 454 and 455 have been studied. ${ }^{398,399}$ Using electron-deficient substituents such as in $\mathbf{4 5 4 a}$, compound (S)-456 is formed in 55\% ee, whereas steric bulky ones such as in $\mathbf{4 5 4 b}$ afforded the enantiomeric compound $(R)-\mathbf{4 5 6}$ in 59\% ee. Higher enantioselectivities for both enantiomers have been achieved using BINOL-derived ligands 455a and 455b, 87\% ee for $(R)-\mathbf{4 5 6}$ and $80 \%$ ee for $(S)-\mathbf{4 5 6}$ being obtained, respectively, by just changing the electronic properties of these ligands (Scheme 168).

Scheme 168. Enantiodivergent Allylic Substitution of 1,3-Diphenylallyl Acetate with Diethyl Sodium Malonate Catalyzed by Pd(II) and Differently Substituted Bisphosphonites 454 and 455

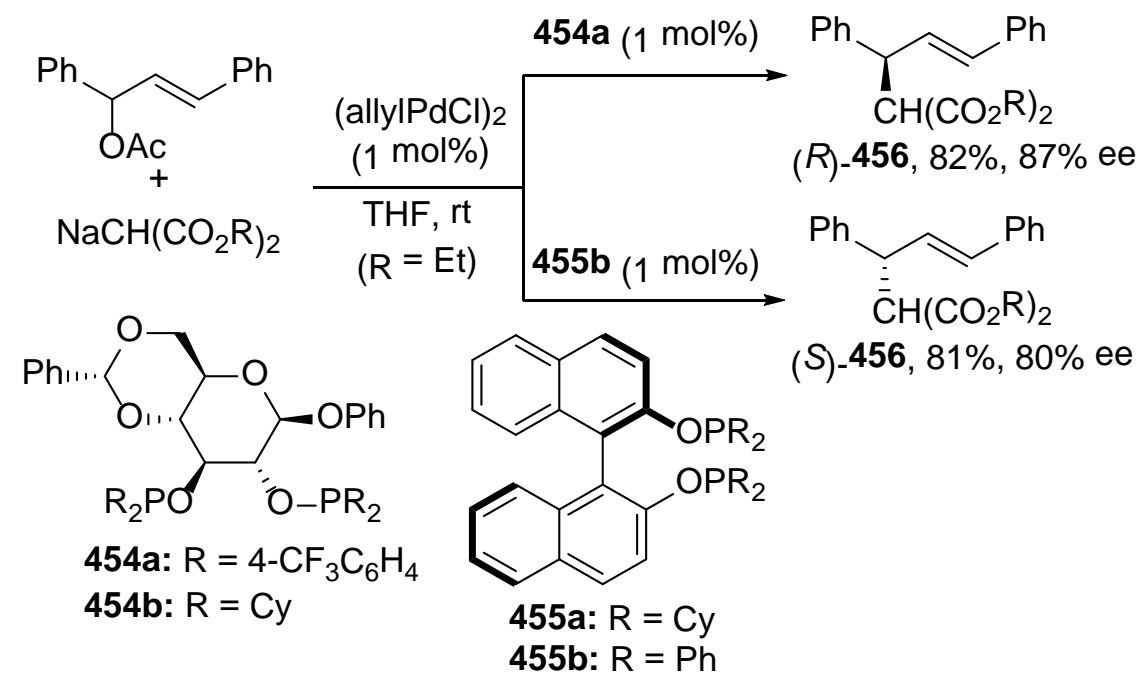


A strong switching of enantioselectivity was found by Balavoine and Aït-Haddou group $^{400,401}$ using $C_{2}$-symmetric bis(oxazoline) (BOX) ligands 457 with different substituents in the same Pd-catalyzed AAA (Figure 16$)$. Ligand 457 a gave $(R)-456(\mathrm{R}=$ $\mathrm{Me})$ in $90 \%$ ee through TS $\mathbf{4 5 7 A}$, whereas $\mathbf{4 5 7 b}$ provided $(S)-456(\mathrm{R}=\mathrm{Me})$ in $92 \%$ ee by means of TS 457B or 457'B. In the TS 457B a hydrogen-bond of the $\mathrm{OH}$ with the nucleophile directs the attack closer to the $\mathrm{C}-3$ of the allylic intermediate. Alternatively, in the TS 457' $\mathbf{B}$ the $\mathrm{OH}$ group blocks the C-1 position favoring the attack at C-3.

P,N-Ligands 458 (Figure 16) derived from L-Val can reverse the enantioselectivity by a simple change of the substituents at the nitrogen atom. ${ }^{402}$ For the ligand $458 \mathrm{a}$, a W-

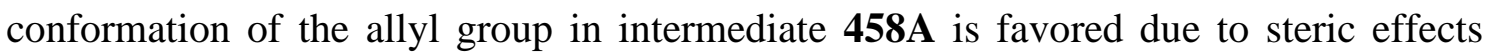
giving $(S)-456(\mathrm{R}=\mathrm{Me})$ in $\mathbf{8 3 \%}$ ee. In the case of ligand $\mathbf{4 5 8 b}$, the M-conformation 458B was preferred affording $(R)-456(\mathrm{R}=\mathrm{Me})$ in 92\% ee. Pyridylmethyloxazoline 459a and its benzocondensed quinolyl derivative 459b (Figure 16) gave $(R)-\mathbf{4 5 6}(\mathrm{R}=$ $\mathrm{Me})$ and $(\mathrm{S})-\mathbf{4 5 6}(\mathrm{R}=\mathrm{Me})$ in $16 \%$ and $78 \%$ ee, respectively. ${ }^{403,404}$

Hou and co-workers have applied $N, S$ - and $N, S e$-planar chiral [2,2]-paracyclophane ligands 460 to this allylic substitution with ee up to $93 \% .{ }^{405}$ Ligands 460 a and $460 \mathbf{b}$ with the same $S_{P}$ planar chirality afforded $(R)-\mathbf{4 5 6}(\mathrm{R}=\mathrm{Me})$ with $54 \%$ and $57 \%$ ee, respectively. However, 460c and 460d with $R_{P}$ configuration gave $(S)-456(\mathrm{R}=\mathrm{Me})$ in $63 \%$ and $73 \%$ ee, respectively.

In the case of a phosphine/oxazoline bidentate ligand 461, a switch of enantioselectivity has been observed by a simple change of the Pd:ligand ratio. ${ }^{406}$ With a 1:1 Pd:ligand ratio, $(S)-456(\mathrm{R}=\mathrm{Me})$ was obtained in $95 \%$ ee, whereas with a 1:2 ratio the corresponding enantiomer was formed in $88 \%$ ee. The $1: 1$ complex formed structure 461A, which afforded mainly (S)-456. However, in the 1:2 complex, the catalytic species provided structure $461 \mathrm{~B}$ to minimize the steric repulsion between the two ligands (Figure 16).

Modest results were obtained when the BOX was changed by an aziridine unit. Thus, $\eta^{3}$-allyl Pd complex (10 mol\%) derived from ligand 462a gave $(R)-456(\mathrm{R}=\mathrm{Me})$ in a miserable $<5 \%$ ee. On the other hand, $\mathbf{4 6 2 b}$ gave $(S)-456(\mathrm{R}=\mathrm{Me})$ in $23 \%$ ee. $^{407}$

Axially chiral P,S-ligands related to BINAP 463 (BINAPS) with different alkyl groups on the sulfur atom gave a reversal of enantioselectivity in this AAA. The alkylation using 463a gave $(S)-456(\mathrm{R}=\mathrm{Me})$ in 82\% ee, whereas $463 \mathrm{~b}$ afforded $(R)-456$ $(\mathrm{R}=\mathrm{Me}$ ) in $72 \%$ ee by models $463 \mathrm{~A}$ and $\mathbf{4 6 3 B}$, respectively, according to the X-ray and NMR data. ${ }^{408}$

Modest reversal of enantioselectivity has been achieved between 1,3-diphenylallyl and 1,3-dimethylallyl acetates using the same $\eta^{3}$-allyl palladium catalyst formed with a $\mathrm{P}, \mathrm{N}$ ligand $\mathbf{4 6 4} 4^{409}$ (Figure 16). In this case, the former acetate gave (S)-product 456 with ee up to $97 \%$, whereas the latter acetate yielded the $(R)$-product in $43 \%$ ee. According to the ONIOM calculations the AAA with 1,3-diphenylallyl acetate takes place through an exo-syn-syn intermediate 464A and the dimethylallyl substrate through an exo-syn-anti intermediate 464B.

Figure 16. Ligands for the Pd-Catalyzed Enantiodivergent Allylic Substitution of 1,3-Diphenylallyl Acetate with Dimethyl Sodium Malonate 


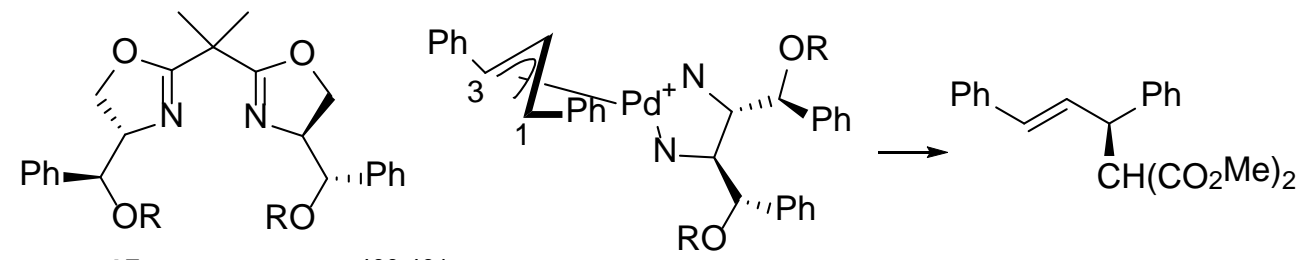

457a: $R=C O P h^{400,401}$

457b: $\mathrm{R}=\mathrm{H}^{400,401}$

457A

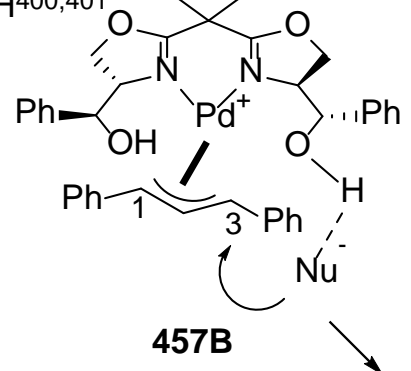

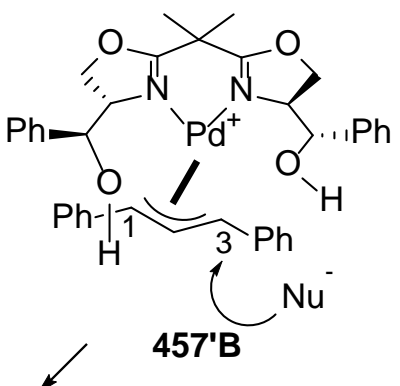

$\mathrm{CH}\left(\mathrm{CO}_{2} \mathrm{Me}\right)_{2}$

$\mathrm{Ph} \curvearrowright \overbrace{\mathrm{Ph}}$<smiles>CC(C)C(CPc1ccccc1)C(C)CP</smiles>

458a: $\mathrm{R}=\mathrm{Me}^{402}$

458b: $\mathrm{R}=\mathrm{Ph}^{402}$

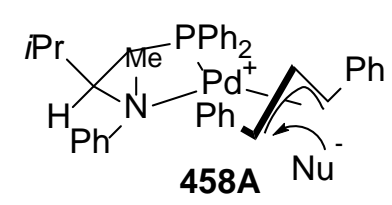

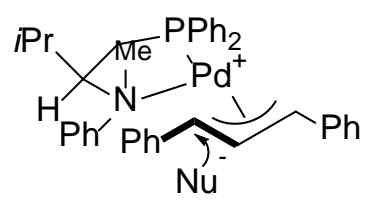

458B<smiles></smiles><smiles>CC(C)(C)c1ccccc1</smiles>

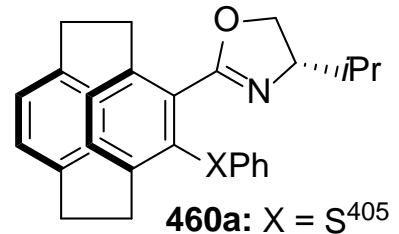

460b: $X=S e^{405}$

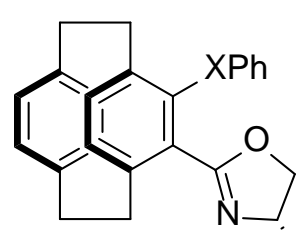

460c: $\mathrm{X}=\mathrm{S}^{405}$ "i $\mathrm{Pr}$ 460d: $X=S^{405}$<smiles>CC(C)(C)CC1=NC(C(C)(C)C)CO1</smiles><smiles>CC(C)C1CN1C(c1ccc2ccccc2n1)C(C)C</smiles>
$462 \mathbf{a}^{407}$<smiles>CN1[Pb]=C(CC(C)(C)C)C(C(C)(C)C)COP1(C)(C)C</smiles>

$461 \mathrm{~A}$<smiles>C1CCOC1</smiles><smiles>CCCCCCCCCC</smiles>
$-t \mathrm{Bu}$ tBu'<smiles>CC(C)C1CN1C(c1cccc2cccnc12)C1CC1</smiles>

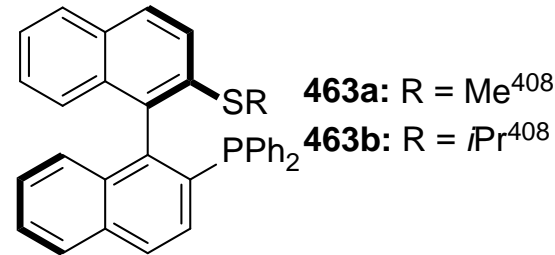

(S)-456

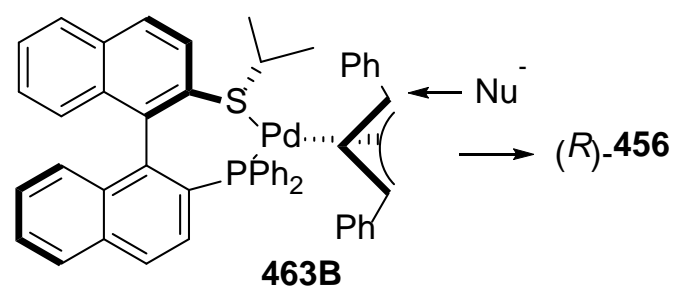


<smiles>COC[C@H]1OC(c2ccccc2P)=N[C@@H]1c1ccccc1</smiles><smiles></smiles>

$464 \mathrm{~A}$

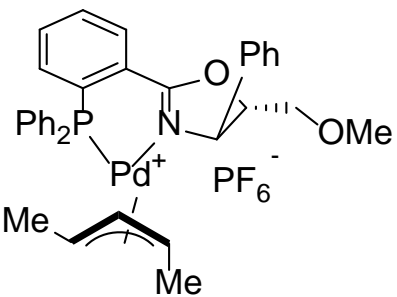

464B

Diastereodivergent Ir-catalyzed allylation of tert-butyl diphenylmethyleneglycinate has been performed with chiral phosphite $\mathbf{4 6 5}$ as ligand, derived from BINOL (Scheme 169). ${ }^{410}$ In contrast to Pd catalysis, the reaction with diethyl cinnamyl phosphate led to the formation of highly substituted compounds 466 instead of to the linear one. The diastereoselectivity of this reaction can be controlled by the base. Thus, with $50 \%$ aqueous $\mathrm{KOH}$ in toluene, (2S,3S)-466 was mainly formed in $64 \%$ de and $97 \%$ ee, whereas the formation of (2R,3S)-466 was directed using LiHMDS in THF, obtaining a $64 \%$ de and $92 \%$ ee. The observed diastereoselectivity was explained by the enolate geometry, $E$ for $\mathrm{KOH}$ and $Z$ for the lithium enolate. A plausible allyl $\operatorname{Ir}(\mathrm{III})$ complex 465A has been postulated. ${ }^{411}$

Scheme 169. Diastereodivergent Asymmetric Allylic Alkylation of tert-Butyl Diphenylmethyleneglycinate with Diethyl Cinnamyl Phosphate Catalyzed by Ir(III) and Chiral Phosphite 465 Using Different Bases

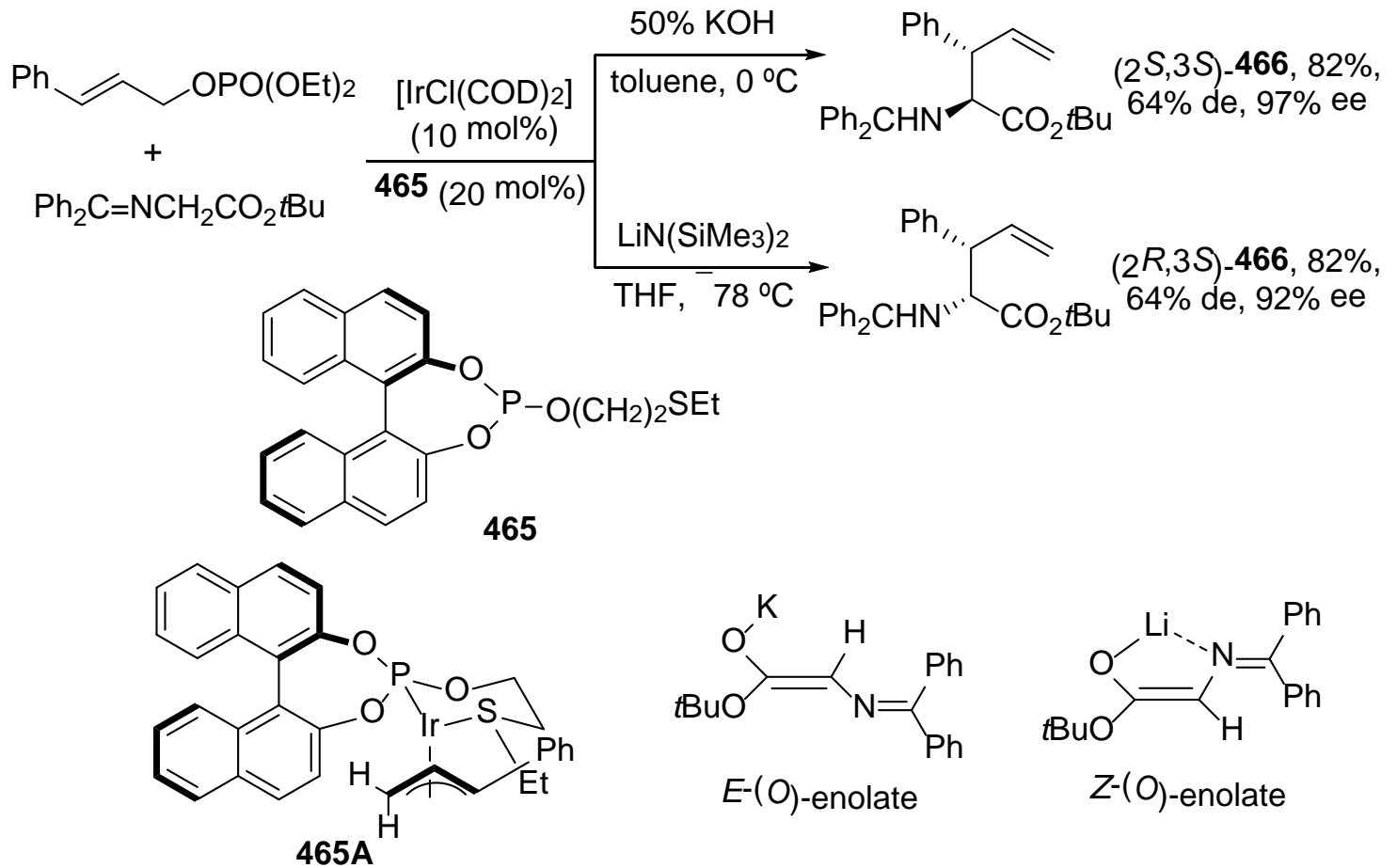

A similar base effect has been found by Trost and co-workers in the Pd-catalyzed decarboxylative asymmetric allylic alkylation (DAAA) of $\beta$-keto esters (Scheme 170). ${ }^{412}$ For instance, in the case of the reaction of tetralone 467 with allyl 
chloroformate, $(R)-469$ was formed in $88 \%$ ee using LiHMDS as base at $-78{ }^{\circ} \mathrm{C}$ and ligand 468. On the contrary, working at rt in THF and $\mathrm{Cs}_{2} \mathrm{CO}_{3}$ as base, $(S)-\mathbf{4 6 9}$ was obtained in $89 \%$ ee. Because 1.6 eq of LiHMDS must be used, it has been proposed the formation of an aggregate 469A, whereas for the other approach enolate 469B will afford (S)-469.

Scheme 170. Enantiodivergent Decarboxylative Asymmetric Allylic Alkylation of Ethoxycarbonyl Tetralone 467 with Allyl Chloroformate Catalyzed by $\operatorname{Pd(II)}$ and Ligand 468 Using Different Bases

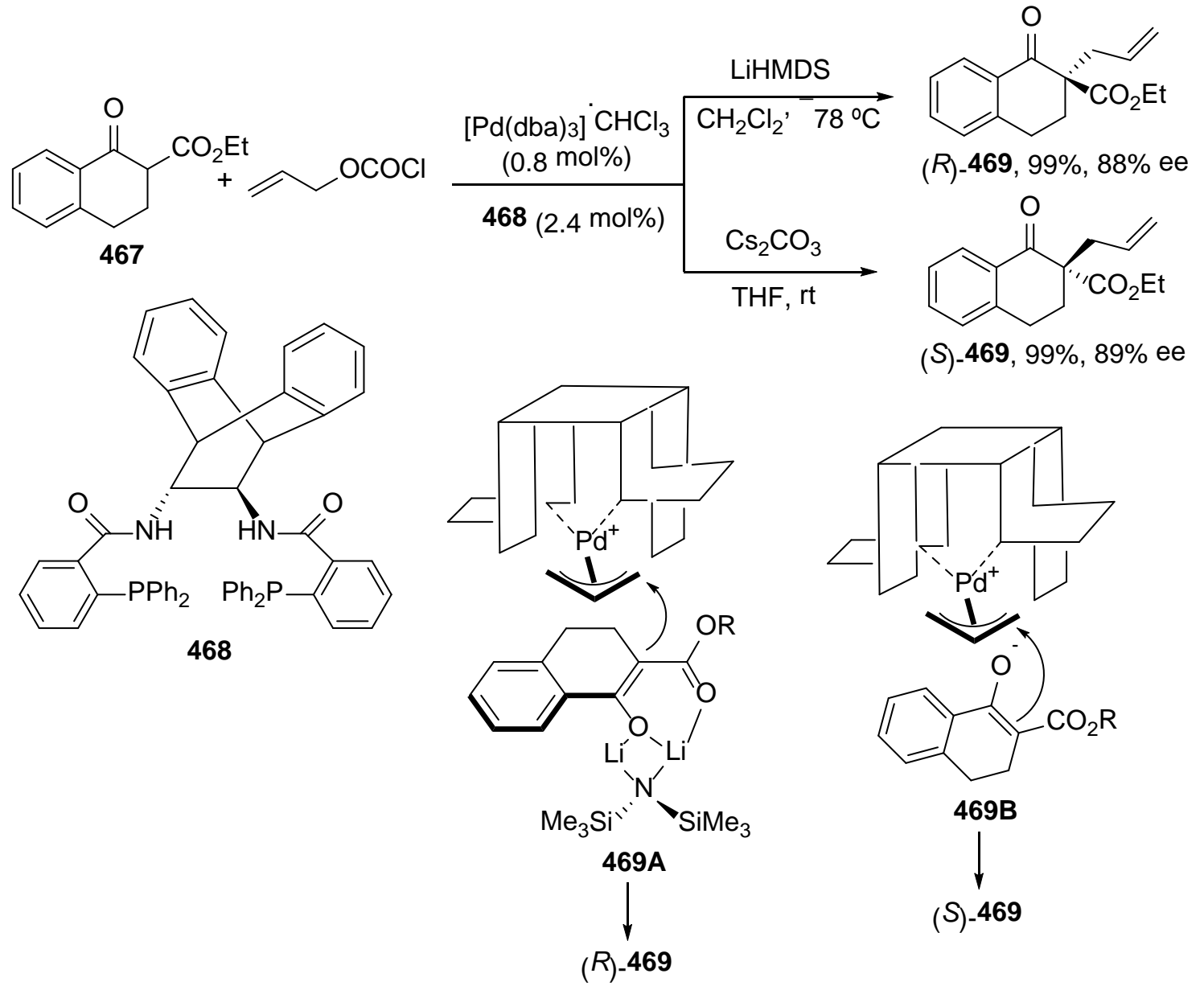

A dual catalysis or synergistic catalysis strategy has been used by Carreira and coworkers for the amine- and Ir-catalyzed $\alpha$-allylation of $\alpha$-branched aldehydes with allylic 1-aryl alcohols. ${ }^{413}$ For instance, hydratropaldehyde (470) reacted with 1-phenyl2-propen-1-ol to afford all the diastereomers 474 with high diasteroselectivity and total enantioselectivity depending on the catalysts combination (Scheme 171). Both enantiomers of phosphoramidite $\mathbf{4 7 1}$ were used as chiral ligands for the $\operatorname{Ir}(\mathrm{I})$ complex and pseudo enantiomeric amines $\mathbf{4 7 2}$ and $\mathbf{4 7 3}$ for the formation of the enamines. This methodology has been applied to different $\alpha, \alpha$-disubstituted aldehydes. 
Scheme 171. Diastereodivergent Asymmetric Allylic Alkylation of Branched Aldehydes with Allylic Alcohols Catalyzed by Ir-Phosphoramidite Complexes and Chiral Primary Amines

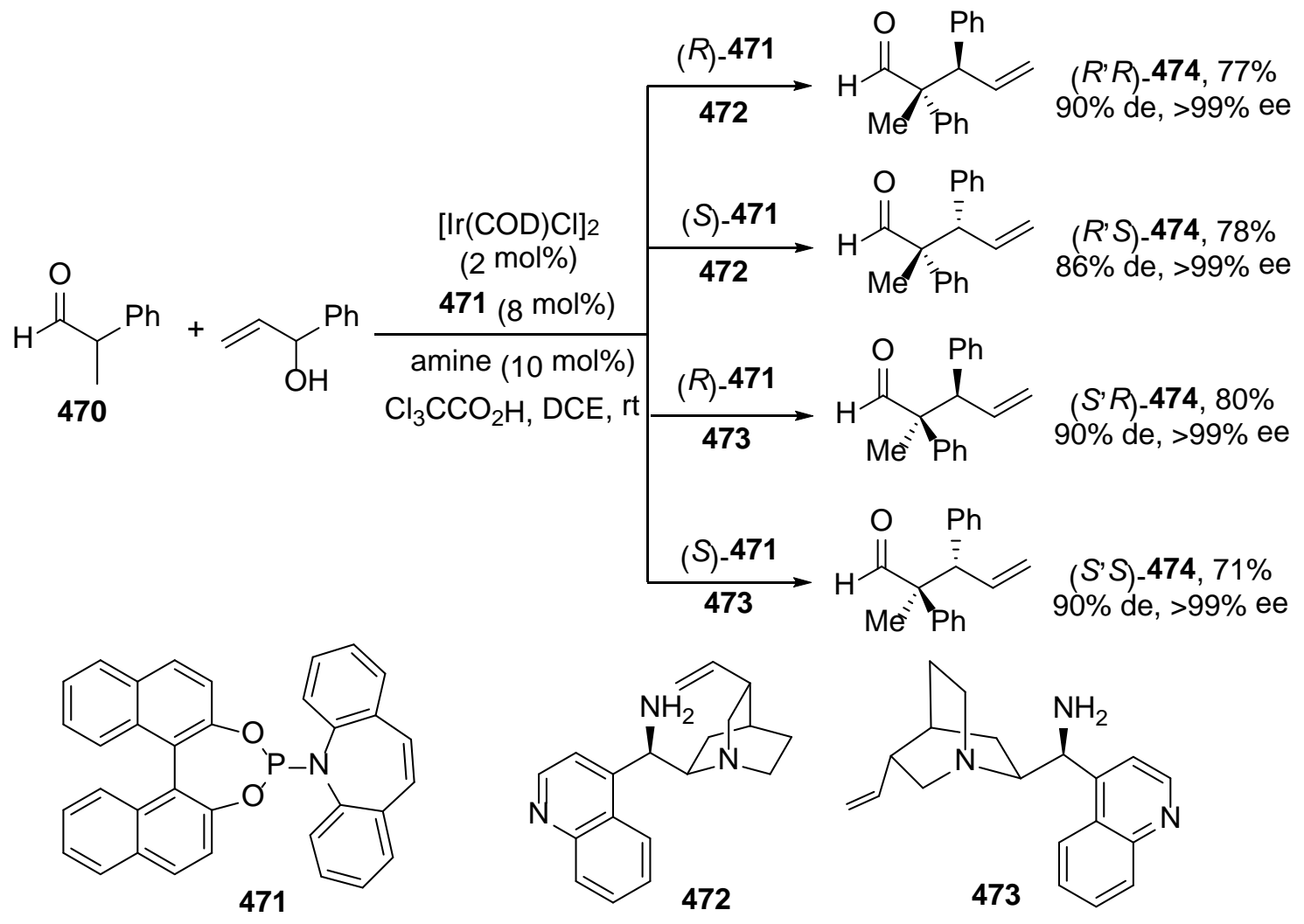

The DFT calculations have been performed to explain the origin of stereodivergence with involvement of the catalysts. ${ }^{414}$ All four stereoisomers of $\Delta^{9}$-tetrahydrocannabinol ( $\Delta^{9}$-THC) have been synthesized using the same dual catalysis in five steps. ${ }^{415}$ This diastereodivergent dual catalysis has been applied to $\alpha$-amino and $\alpha$ hydroxyacetaldehyde. ${ }^{416}$ For linear aldehydes a secondary amine diarylsilylprolinol 475 was used in the diastereodivergent $\alpha$-allylation of $n$-hexanal in the presence of dimethyl hydrogenephosphate giving the corresponding stereoisomers 476 in high ee (>99\%) and de $>90 \%$ (Scheme 172). ${ }^{417}$ This methodology has been applied to a concise synthesis of the selective serotonin reuptake inhibitor (-)-paroxetine, commonly used in the treatment of depression, obsessive compulsive disorders and panic disorders.

Scheme 172. Diastereodivergent Asymmetric Allylic Alkylation of Linear Aldehydes with Allylic Alcohols Catalyzed by Ir/Phosphoramidite 471 Complexes and Silylated Prolinol 475 


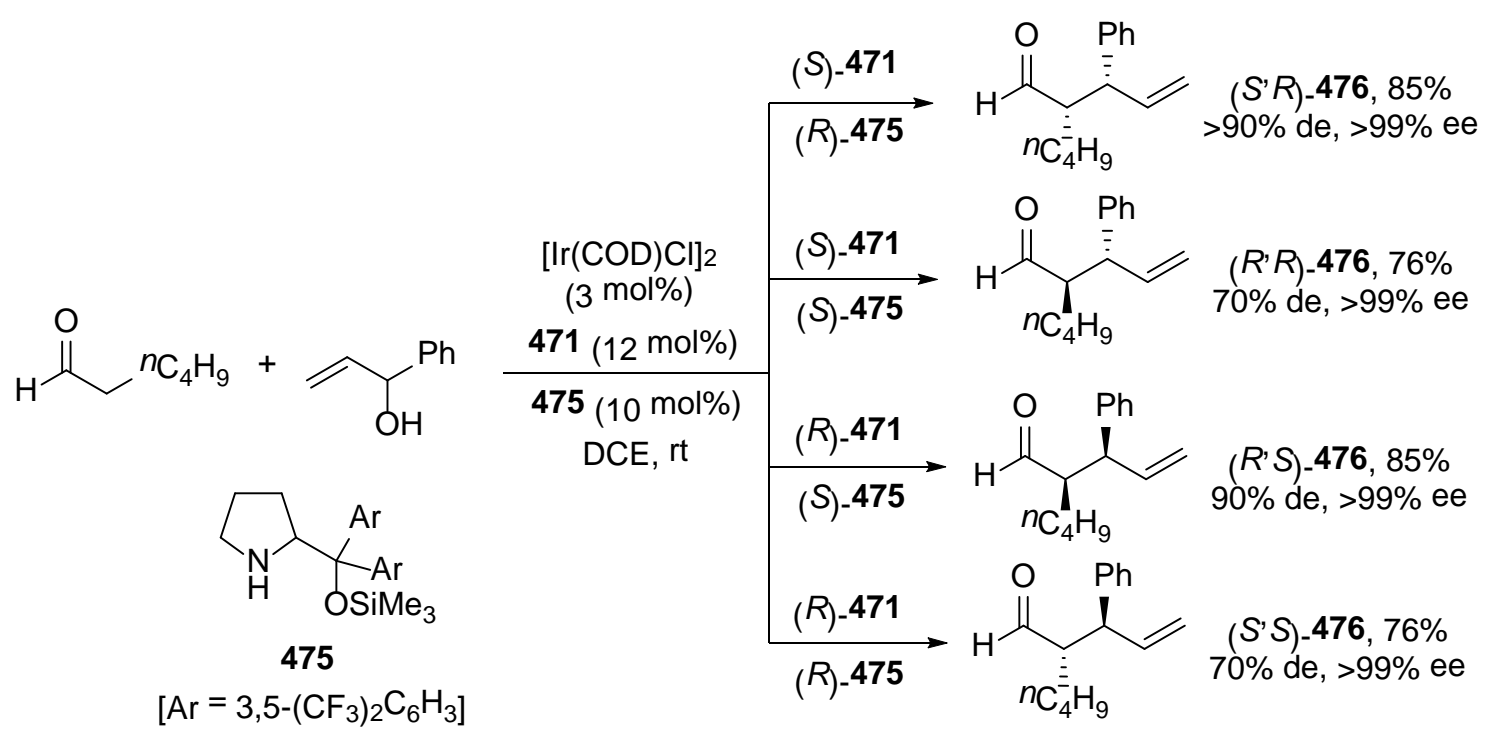

A formal $\alpha$-allylation of aldehydes with alkynes catalyzed by $\mathrm{Rh}$ has been recently reported by Cruz and Dong. ${ }^{418}$ The intermediate Rh- $\pi$-allyl species can be generated by the Rh-catalyzed isomerization of alkyne to an allene followed by the Rh-hydride insertion. The Rh- $\pi$-allyl intermediate reacted with the enamine of the aldehyde generated in situ by an amine organocatalyst. The diastereodivergent allylation has been performed using $(R)$-DTBM-BINAP 477 and Jacobsen's amines 482 (Scheme 173). In the case of amine $(S, S)-\mathbf{4 7 8}$, anti-products $(S, S)$-479 were obtained in high de and ee, whereas excellent results were achieved for the syn-products $(R, S)-\mathbf{4 7 9}$ when the amine $(R, R)-\mathbf{4 7 8}$ was used as organocatalyst.

Scheme 173. Diastereodivergent Asymmetric Allylic Alkylation of Aldehydes with Acetylenes Catalyzed by Rh/(R)-DTBM-BINAP 477 and Jacobsen's Amines 478 


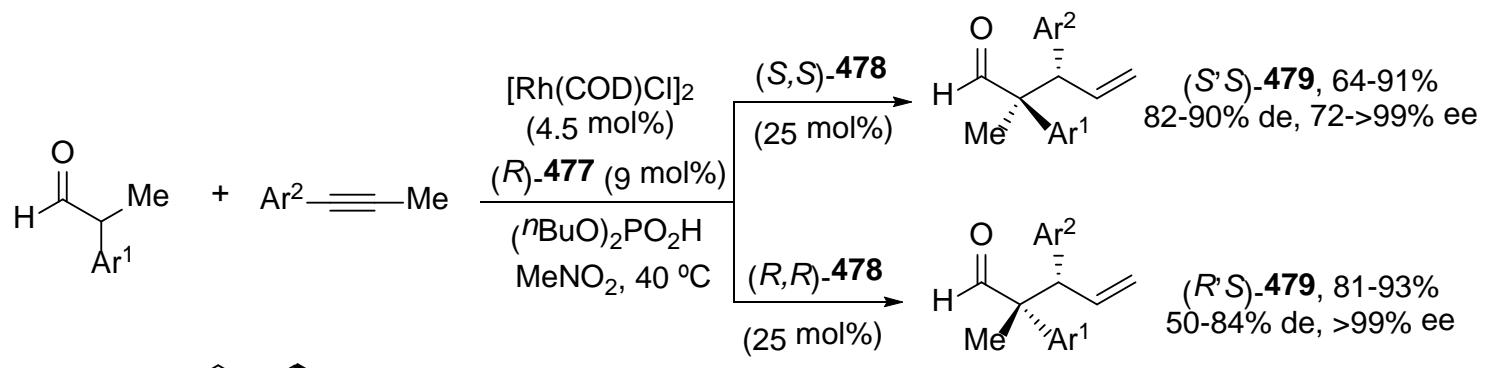<smiles>[R2]c1ccc2ccccc2c1-c1c([R2])ccc2ccccc12</smiles><smiles>N[C@@H]1CCCC[C@H]1NC(=O)c1c(-c2ccccc2)cccc1-c1ccccc1</smiles>

(S'S)-478<smiles>N[C@@H]1CCCC[C@H]1NC(=O)c1c(-c2ccccc2)cccc1-c1ccccc1</smiles>

$\left(R^{\prime} R\right)-478$

$(R)$-DTBM-BINAP (477)<smiles>[R][Y][14c]1cc([14CH3])c(OC)c(C(C)(C)C)c1</smiles>

In the case of $\alpha$-allylation of $\alpha$-hydroxy ketones, the Ir-phosphoramidite 187 was used in combination with a chiral Zn complex 221 (Scheme 79) derived from ligand 91 (Scheme 27), in this way all four stereoisomers have been prepared. ${ }^{419}$ For instance, in the $\alpha$-allylation of $\alpha$-hydroxyacetophenone with cinnamyl methyl carbonate the corresponding four diastereomers $\mathbf{4 8 0}$ were synthesized (Scheme 174).

Scheme 174. Diastereodivergent Asymmetric Allylic Alkylation of $\alpha$ Hydroxyacetophenone with Cinnamyl Methyl Carbonate Catalyzed by Ir/187 and Zn 221 Catalysts

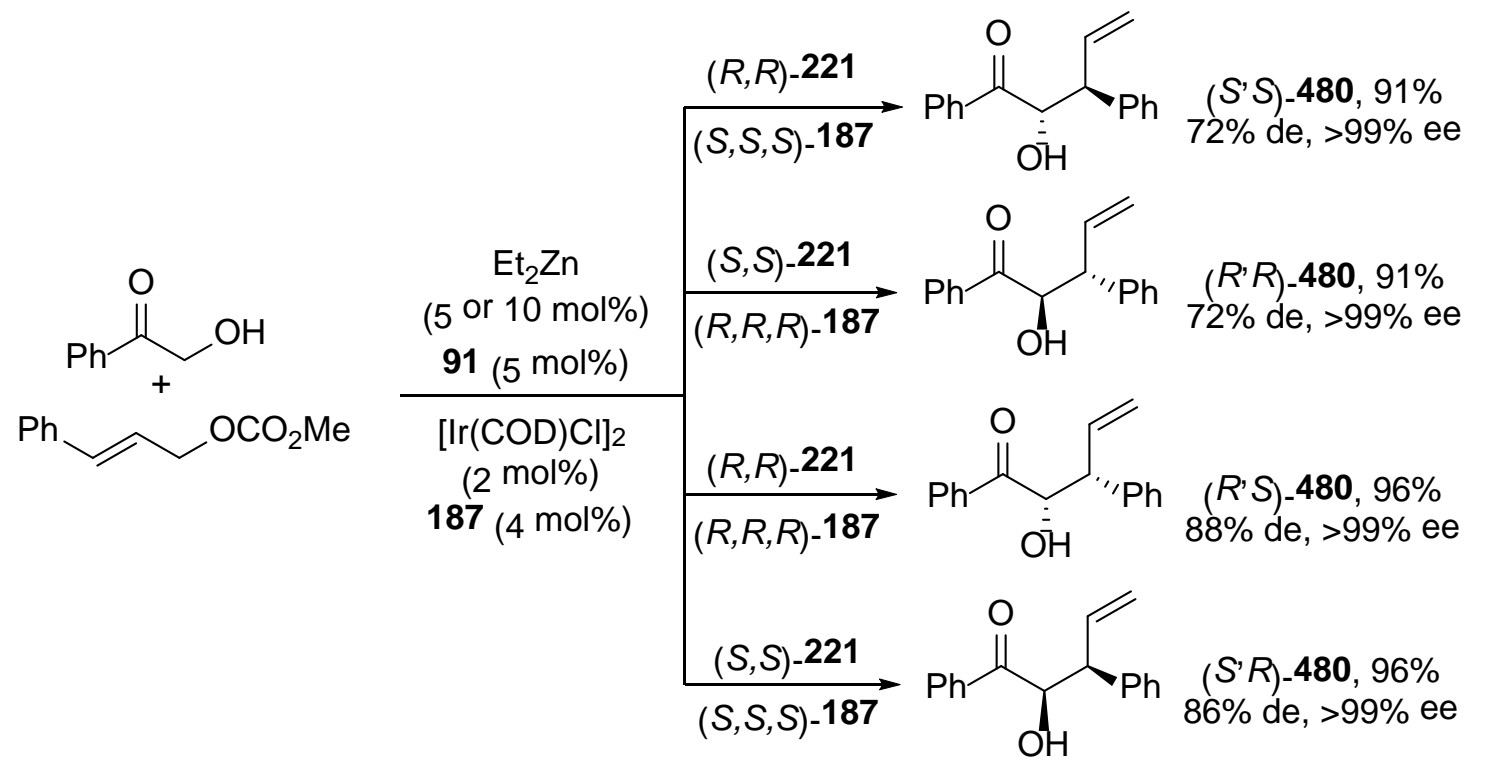

Formaldehyde $N, N$-dialkylhydrazone $\mathbf{4 8 1}$ derived from Enders' hydrazine (S)-RAMP has been widely used as a formyl anion equivalent in the diastereodivergent Ir-catalyzed allylic substitution. ${ }^{420}$ The reaction of $\mathbf{4 8 1}$ with Boc-protected 1-phenyl-2-propen-1-ol 
482 and $(R, R, R)-\mathbf{1 8 7}$ (Scheme 66) as chiral ligand provided $(S, R)-\mathbf{4 8 7}$ in 38\% yield, $90 \%$ de and $>99 \%$ ee, as well as enantiopure $(S)-\mathbf{4 8 2}$ in 50\% yield (Scheme 175). On the other hand, enantiopure $(S, S)-\mathbf{4 8 3}$ can be prepared diastereodivergently in 36\% yield and $90 \%$ de using ligand $(S, S, S)-\mathbf{1 8 7}$ together with enantiopure $(R)-\mathbf{4 8 2}$ in 50\% yield. Compounds $\mathbf{4 8 3}$ were further transformed into the corresponding diastereomeric amines 484.

Scheme 175. Diastereodivergent Asymmetric Allylic Alkylation of Formaldehyde (S)-RAMP Hydrazone 481 with Boc Allyl Carbonate 482 Catalyzed by Ir and Phosphoramidite 187 Complexes

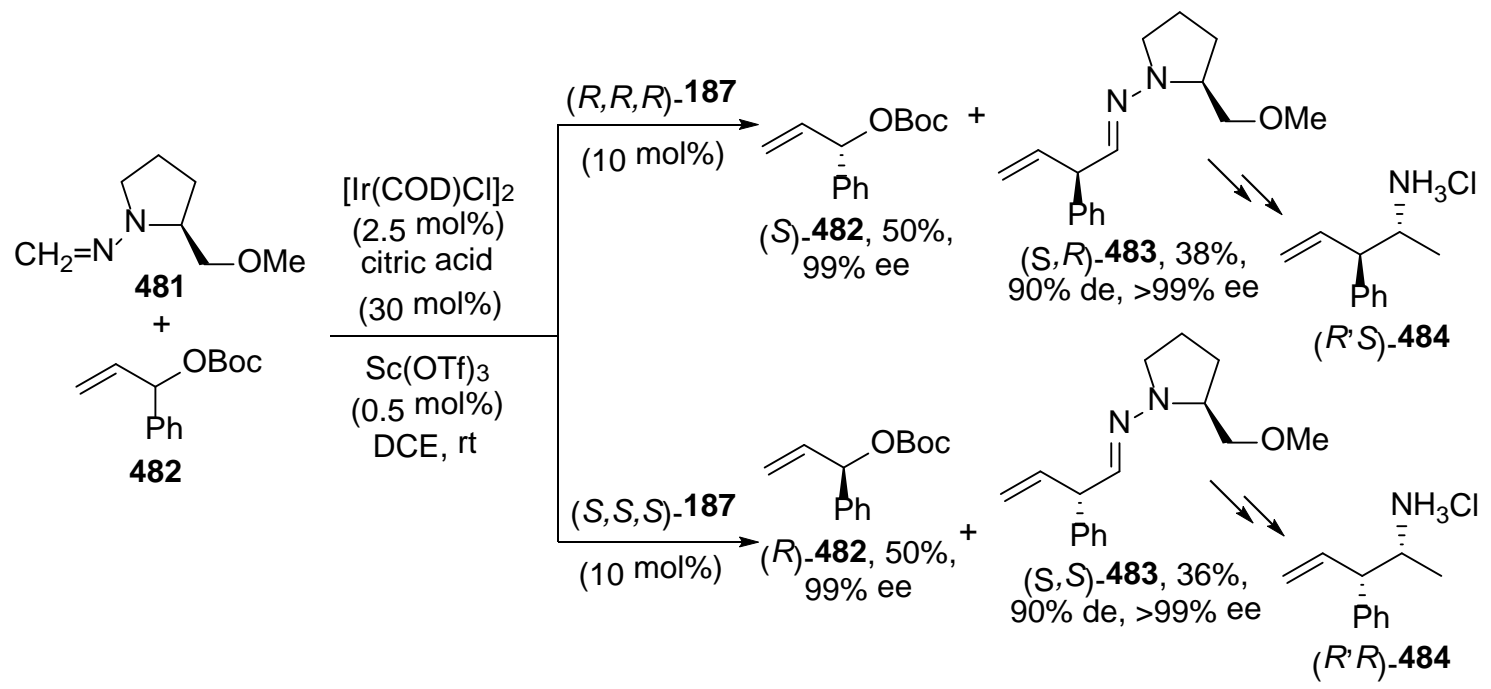

In the case of Pd-catalyzed AAA of indole in the presence of chiral $P$,S-ligands $\mathbf{4 6 3}$ (Sulfur-MOP) it was found that the substituents at the S-atom promoted a switch of enantioselectivity. ${ }^{421} 1,3$-Diphenylallyl acetate reacted with indol in the presence of $(R)$ 463c $(\mathrm{R}=2-i \mathrm{Pr})$ affording the corresponding product 486 in $92 \%$ ee. However, using $(R)-463 d(\mathrm{R}=\mathrm{Cy})$ the corresponding ent-486 was formed in lower $48 \%$ ee (Scheme 176). Inversion of the enantioselectivity was also observed with ligand $(R)$-MeOMOP $\mathbf{4 8 5}$ providing ent-486 in $45 \%$ yield and $\mathbf{8 5 \%}$ ee. The absolute configuration of products 486 was not assigned.

Scheme 176. Enantiodivergent Allylic Alkylation of Indole with 1,3-Diphenylallyl Acetate Catalyzed by Pd-(R)-Sulfur MOP 463 


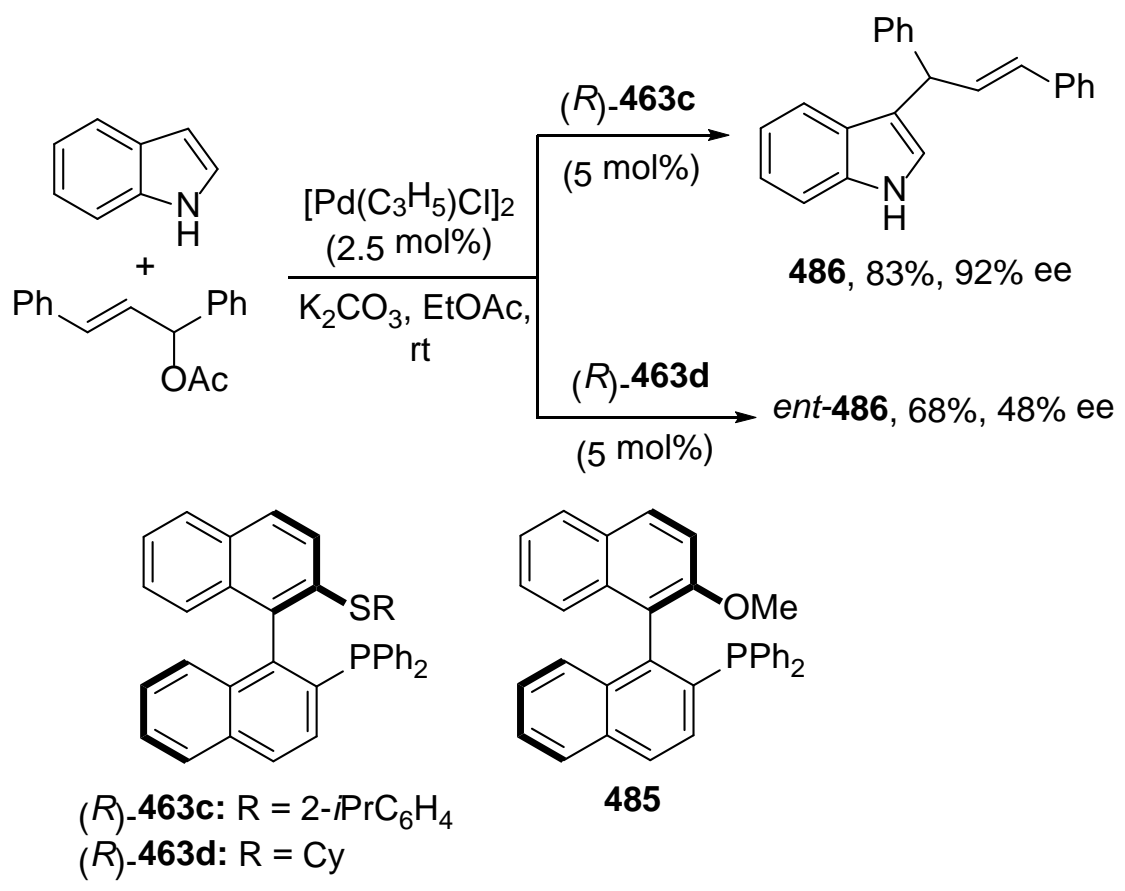

In conclusion, several examples of enantiodivergent Pd or Ir-catalyzed AAA can be controlled by changes in the structure of the chiral ligand and in some cases by the base. The diastereodivergent Ir-catalyzed AAA is carried out successfully changing the configuration of the ligand and the base.

2.6.2. Nitrogen Nucleophiles. Metal-catalyzed asymmetric allylic amination is the most direct way for the synthesis of allylic amines. ${ }^{396}$ This process is also used as benchmark reaction for the evaluation of new chiral ligands. Some stereodivergent processes follow.

Enantiodivergent Pd-catalyzed allylic amination of ethyl 1,3-diphenylallyl carbonate with benzylamine was firstly described by Togni and co-workers. ${ }^{422}$ Ferrocenylpyrazole ligands 487a-e gave an amine (R)-488 in high ee (94-99\%), whereas the more hindered ligand $\mathbf{4 8 7 f}$ provided inversion of the configuration (Scheme 177). From the X-ray and 2D NMR data of the Pd $\eta^{3}$-allylic intermediates it can be deduced that 487a,c complexes adopted an exo-syn-syn conformation 487A and in the case of the anthryl (Anth) substituted ligand 487f, the conformation was exo-syn-anti 487F. Therefore, due to the nucleophilic attack by benzylamine anti to the $\mathrm{P}$ atom of the ligand in intermediates $\mathbf{4 8 7} \mathrm{A}$ and $\mathbf{4 8 7 F}$, the enantiomeric amines 488 were formed enantiodivergently.

Scheme 177. Enantiodivergent Allylic Amination of Ethyl 1,3-Diphenylallyl Carbonate with Benzylamine Catalyzed by Pd and Ligands 487 
<smiles>CCOC(=O)C([PH2+])/C=C/c1ccccc1</smiles>
$\mathrm{PhCH}_{2} \mathrm{NH}_{2}$

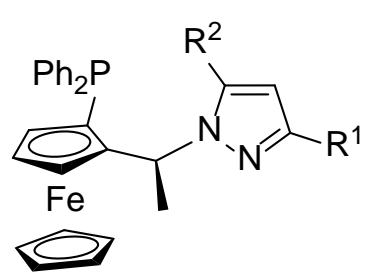

487a: $R^{1}=1-A d, R^{2}=H$

487b: $R^{1}=C y, R^{2}=M e$ 487c: $R^{1}=P h, R^{2}=M e$ 487d: $R^{1}=4-P y, R^{2}=M e$ 487e: $R^{1}=M e, R^{2}=M e$ 487f: $R^{1}=$ 9-Anth, $R^{2}=M e$

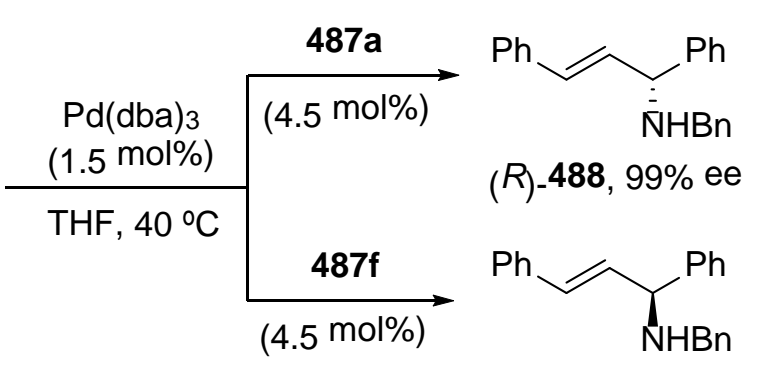

(S)-488, 40\% ee

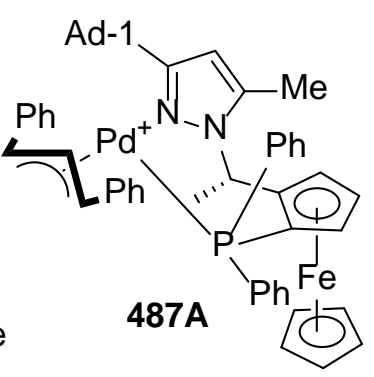

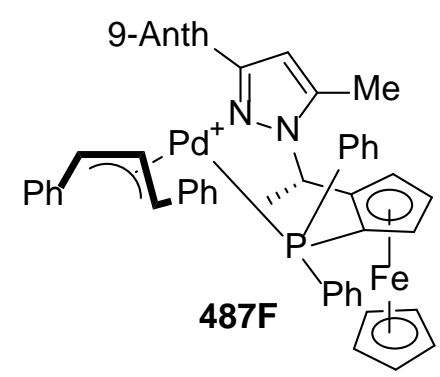

Due to the reversibility of Pd-catalyzed allylic amination of ethyl 1,3-diphenylallyl carbonate and benzylamine the group of Bunt analyzed 12 different chiral ligands observing an increase in the enantioselectivity in the presence of DBU or $\mathrm{Cs}_{2} \mathrm{CO}_{3}$ as bases. ${ }^{423}$ Working with Trost's ligands $\mathbf{4 8 9}$ and $\mathbf{4 9 0}$, compounds $(S)-\mathbf{4 8 8}$ and $(R)-\mathbf{4 8 8}$ were isolated in $30 \%$ and $78 \%$ ee, respectively, in the absence of base and in $68 \%$ and $28 \%$, respectively, in the presence of $\mathrm{Cs}_{2} \mathrm{CO}_{3}$ (Figure 17).

Zang and co-workers ${ }^{424}$ observed a reversal of enantioselectivity in the Pd-catalyzed allylic amination of 1,3-diphenylallyl acetate with benzylamine using chiral ferrocenyl phosphines with a different substituent such as 491 and 492 (Figure 17). In the case of ligand 491, (S)-488 was obtained in 99\% ee (yield no reported). However, the ferrocene with hydroxymethyl groups $\mathbf{4 9 2 a}(\mathrm{R}=\mathrm{H})$ gave $(R)-\mathbf{4 8 8}$ in $\mathbf{8 8 \%}$ ee, whereas the acetate 492b $(\mathrm{R}=\mathrm{Ac})$ and 496c $(\mathrm{R}=\mathrm{Me})$ provided $(R)-\mathbf{4 8 8}$ in lower $33 \%$ and $22 \%$ ee, respectively. These results showed that the hydroxyl group is not crucial for the observed switch of enantioselectivity.

Figure 17. Chiral Ligands used in the Pd-Catalyzed Enantiodivergent Allylic Amination of 1,3-Diphenylallyl Derivatives with Benzylamine 

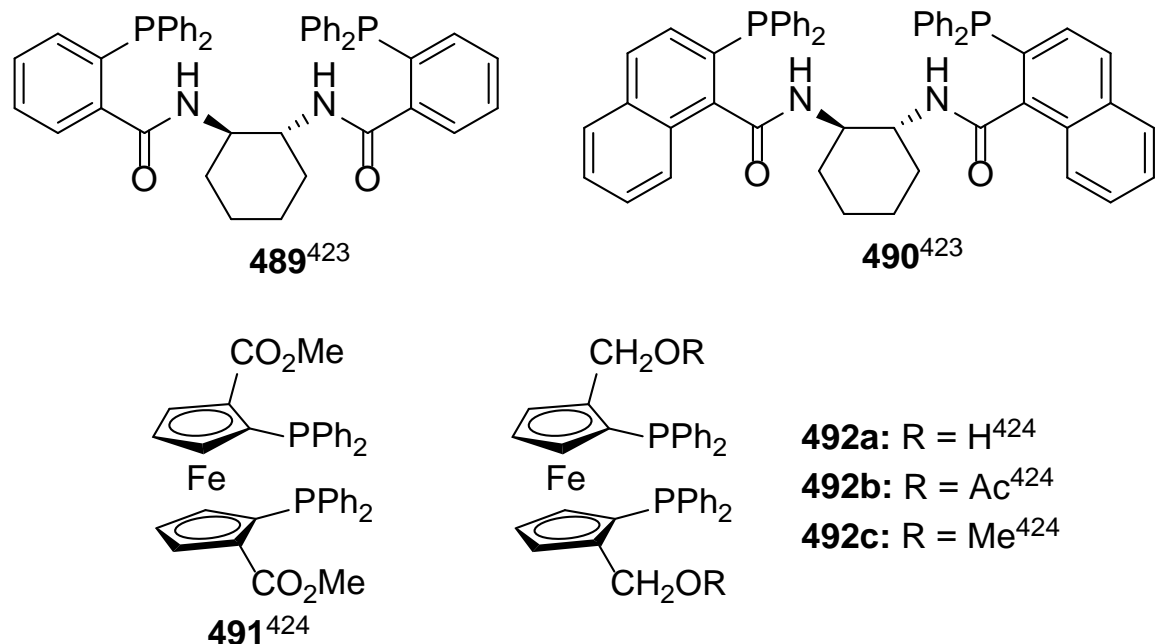

Asymmetric desymetrization of meso-diamide derivatives of cis-cyclohexane-1,2diamine 493 has been achieved through a Pd-catalyzed $N$-allylation with allyl acetate. Depending on the sulfonyl substituents and by means of Trost's ligand $(R, R)-\mathbf{4 9 0}$ it was possible to prepare compounds $\mathbf{4 9 4}$ from the corresponding sulfonamides. Product $(1 R, 2 S)-494$ a substituted by a 2,4,6-trimethylphenylsulfonyl (Trs) group was obtained from 493a and compound (1S,2R)-494b substituted by 4-methylphenylsulfonyl (Ts) was produced from $\mathbf{4 9 3 b}$ (Scheme 178). ${ }^{425}$

Scheme 178. Enantiodivergent Allylic Amination of Allyl Acetate with cisCyclohexane-1,2-diamine Sulfonamides Catalyzed by Pd and Ligands 490<smiles>[R]N[C@@H]1CCCC[C@H]1N[R]</smiles>

493a: $R=\operatorname{Trs}$ 493b: $R=T s$<smiles>C=CCOC(C)=O</smiles>

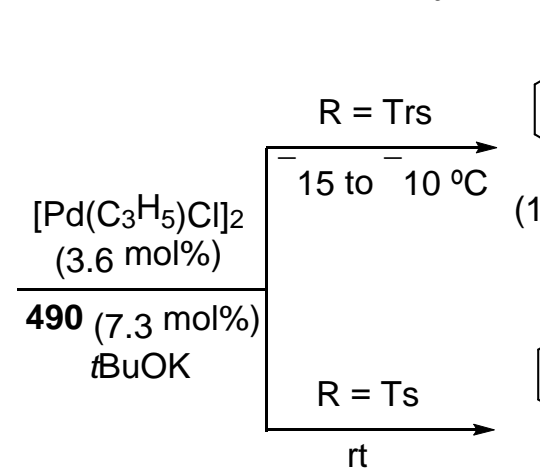

$$
(1 S, 2 R)
$$

The same reversal of enantioselectivity was observed also in the case of ciscyclopentane-1,2-diamine in $85 \%$ and $43 \%$ ee for the corresponding $(1 R, 2 S)$ - and $(1 S, 2 R)$-derivatives, respectively.

In conclusion, in general, different substitutions in the ligand of the Pd- complex determine the enantiodivergence of allylic aminations.

2.6.3. Oxygen Nucleophiles. Due to the lower nucleophilicity of alcohols, only recently one enantiodivergent allylic etherification has been described. Liao and coworkers found out that simple changes of the substituent positions in the aryl group of 
chiral sulfinylphosphines 495 promoted a moderate switching of enantioselectivity. ${ }^{426}$ 1,3-Diphenylallyl acetate reacted with benzyl alcohol under Pd catalysis giving (S)-496 when ligand 495c ( $\mathrm{Ar}=2-\mathrm{Tol}$ ) was used. On the other hand, with ligand 495d, bearing a methyl substituent at the para-position of the aryl groups of the phosphine unit, $(R)$ 496 was obtained. The scope of the reaction has been studied with different benzylic alcohols and ligands $\mathbf{4 9 5 c}$ and 495d providing moderate ee and good yields (Scheme 179). The steric bulkiness of the P-aryl groups controlled the orientation of the nucleophilic attack of benzyl alcohol.

Scheme 179. Enantiodivergent Allylic Etherification of 1,3-Diphenylallyl Acetate with Benzylic Alcohols Catalyzed by Pd and Ligands 495

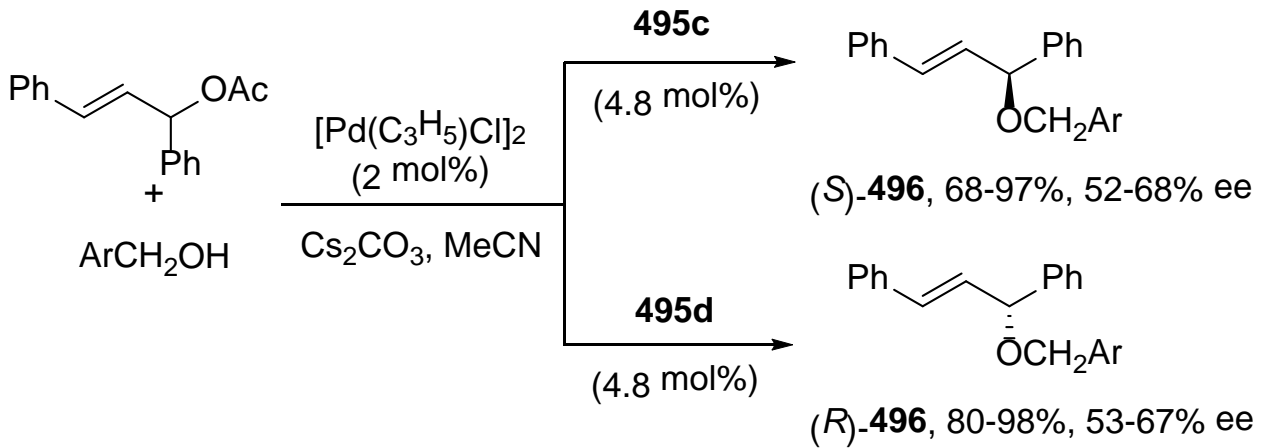<smiles>COc1cccc(S(=O)[18OH])c1P(=O)(Br)c1ccccc1</smiles>

$495 a$

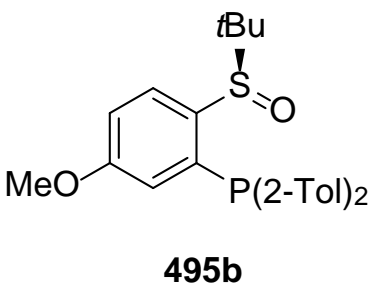

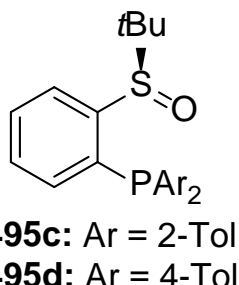

In conclusion, different substitution in the ligand of the Pd-complex determines the enantiodivergence of allylic etherification.

2.6.4. Other Nucleophiles. Miyaura borylation of allylic systems gave access to allylic boronates which are useful synthetic intermediates. Watson and co-workers recently reported the stereospecific enantiodivergent Ni-catalyzed borylation of allylic pivalates 497 (Scheme 180). ${ }^{427}$ During the optimization studies they found out a solvent dependent as well as ligand-dependent switch of the enantioselectivity from retention to inversion of the absolute configuration at the allylic stereocenter. When toluene was used as solvent and $t \mathrm{Bu}$-XantPHOS $\mathbf{4 9 8}$ as ligand, a retention of the configuration took place affording product 499. However, in acetonitrile and $\mathrm{BnPPh}_{2}$ as ligand, products ent-499 resulting from an inversion pathway were obtained. In both stereospecific reactions high $\alpha / \gamma$ diastereoselectivity was observed. Mechanistic studies revealed that the solvent effect was attributed to a competitive oxidative addition mechanism due to acetonitrile coordination to $\mathrm{Ni}$.

Scheme 180. Enantiodivergent Ni-Borylation of Allylic Pivalates 497 in Different Solvents and Ligands 


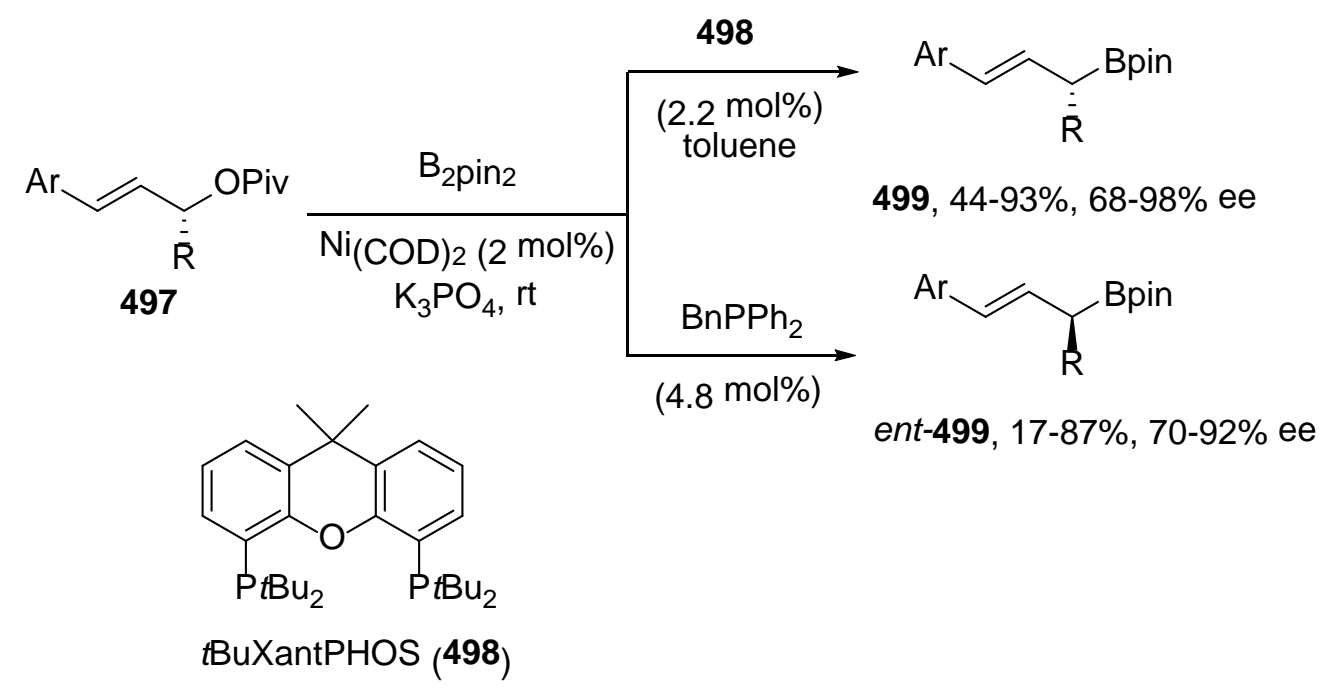

\subsection{Diastereodivergent Synthesis of Alkenes}

Transition-metal catalyzed addition to alkynes is a powerful strategy for accessing to diand trisubstituted unfunctionalized and functionalized alkenes in a steroselective manner. In this section, diastereodivergent reactions directed to the synthesis of diastereomeric alkenes will be considered. Hydrometallation of terminal and internal alkynes with boron, silicon, tin and germanium hydrides allows the regio- and stereoselective synthesis of vinylmetals, which can be further transformed stereodivergently into other functionalized alkenes. ${ }^{141,428}$ Semihydrogenation of internal alkynes can be stereocontrolled to give either (Z)- or (E)-alkenes by transfer hydrogenation. ${ }^{141}$ Hydroarylation and carbometalation of alkynes can also be stereocontrolled depending on the metal and/or the transition-metal catalyst. Other diastereodivergent reactions such as 1,3-rearrangements of propargylic derivatives and epoxides deoxygenation will be also considered in this section.

2.7.1. Hydrometallation of Alkynes. The addition of boranes, silanes, and stannanes to alkynes catalyzed by Lewis acids, generally transition metal complexes, can be diastereodivergently carried out allowing the synthesis of functionalized alkenes, which can be further submitted to cross-coupling reactions.

2.7.1.1. Hydroboration of Alkynes. Uncatalyzed hydroboration of terminal alkynes gave, by syn-addition of the borane reagent, $(E)$-alkenyl boranes, which are very useful synthetic intermediates in organic synthesis. ${ }^{429}$ However, noble transition-metal complexes derived from $\mathrm{Rh}$, Ir, and $\mathrm{Ru}$ catalyze the hydroboration ${ }^{430}$ of terminal alkynes giving (Z)-alkenyl boranes by a rearrangement of a metal vinylidene complex (Scheme 181). ${ }^{431-433}$

Scheme 181. Transition Metal-Catalyzed anti-Hydroboration of Terminal Alkynes 


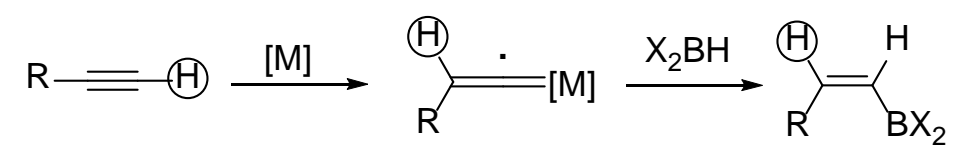

Using Wilkinson's catalyst, the addition of pinalcolborane (HBpin) to terminal alkynes gave (Z)- $\beta$-vinylboronates in high yields. ${ }^{434}$ Specially, $\mathrm{Rh}(\mathrm{CO})\left(\mathrm{PPh}_{3}\right)_{2} \mathrm{Cl}$ provided better regioselectivity than $\mathrm{Rh}\left(\mathrm{PPh}_{3}\right)_{3} \mathrm{Cl}$, and $\mathrm{CpNi}\left(\mathrm{PPh}_{3}\right) \mathrm{Cl}$ gave the same results. ${ }^{435}$ Miyaura and co-workers found out that $[\mathrm{Rh}(\mathrm{COD}) \mathrm{Cl}]_{2}$ and $[\operatorname{Ir}(\mathrm{COD}) \mathrm{Cl}]_{2}$ provided (Z)-vinyl boranes. ${ }^{431}$ Ruthenium hydride pincer complex [Ru(PNP)(H) $\left.{ }_{2} \mathrm{H}_{2}\right]$ [PNP = 1,3-bis(di-tert-butylphosphinomethyl)pyridine $]$ also afforded (Z)alkenylboronates. $^{432}$ Hydroboration of enynes with catecholborane (HBcat) led to the formation of (Z)-dienylboronates under Pd(0) catalysis with dppf as ligand. ${ }^{436}$ Swartz's reagent $\left(\mathrm{Cp}_{2} \mathrm{ZrHCl}\right), \quad \mathrm{Cp}_{2} \mathrm{Ti}(\mathrm{CO})_{2}$ and $\mathrm{Cp}_{2} \mathrm{ZnHCl}$ gave syn-addition by hydrometallation followed by transmetallation with HBpin. ${ }^{437}$

Recently, a Co-catalyzed hydroboration of terminal alkynes has been achieved by a syn-addition through a Co-acetylide hydroboration instead of via a vinylidene intermediate. ${ }^{438}$ In the initial experiments with 1-octyne and HBpin using 3 mol\% of complex 500a at rt for $6 \mathrm{~h}$, the corresponding (E)-alkenylborate 501 was obtained. However, when complex 500b, in which the 2,6-diisopropylphenyl substituent has been replaced by a cyclohexyl group, (Z)-501 was diastereodivergently formed (Scheme 182). The proposed mechanism for the catalysis with $\mathbf{5 0 0 b}$ postulates the formation of the Co acetylide $\mathbf{A}$ and then the oxidative addition of HBpin affording $\mathbf{B}$, which after reductive elimination will give the alkynylboronate cobalt hydride complex $\mathbf{C}$. Final syn-hydroboration provided the $(Z)$-alkenylboronate ester 501. In the case of catalyst 500a, the Co-hydride was kinetically preferred and $(E)-501$ was formed.

Scheme 182. Diastereodivergent Hydroboration of 1-Octyne with Pinacolborane Catalyzed by Different Co(II) Complexes 500 


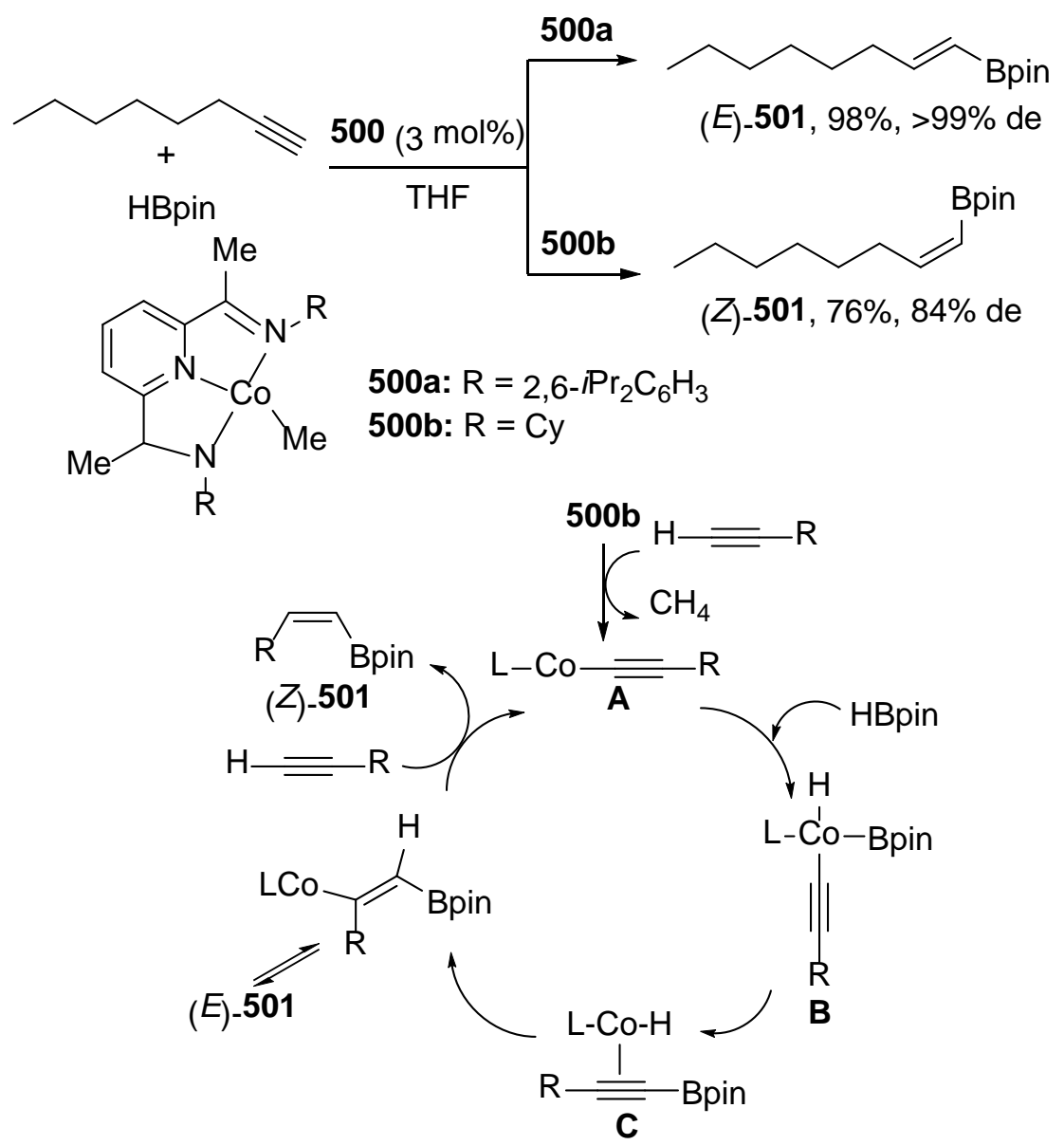

A diastereodivergent $\mathrm{Cu}$-catalyzed syn- and anti-hydroboration of terminal alkynes controlled by the ligand has been recently described. ${ }^{439}$ The addition of 1,8naphthalenediamine to borane gave HBdan, which added to arylacetylenes catalyzed by a copper NHC bulky ligand complex 502 giving (E)-alkenylboron derivatives 504 (Scheme 183). On the contrary, copper(I)-thiophene-2-carboxylate (CuTC) and the bidentate ligand DPEPHOX 503 afforded (Z)-alkenylboron products 504.

Scheme 183. Diastereodivergent Hydroboration of Arylacetylenes with HBdan Catalyzed by Different Cu(II) Complexes 


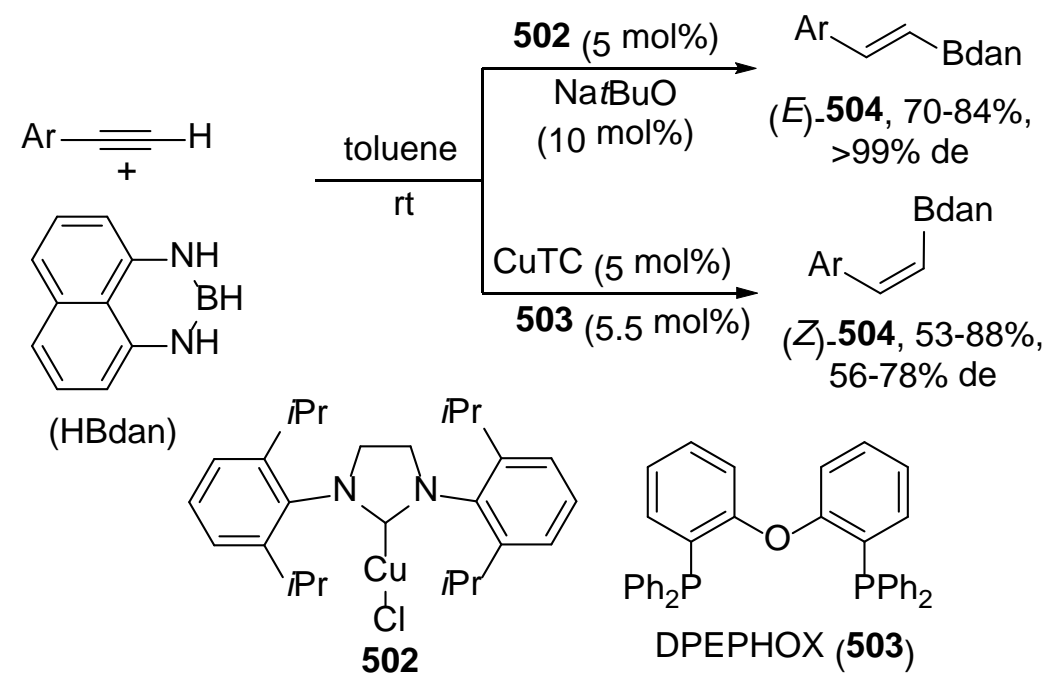

Internal alkynes gave syn-addition of boranes under uncatalyzed and metal-catalyzed reaction conditions. Regioselective reactions resulted in the hydroboration of alkylthio substituted acetylenes with HBcat catalyzed by $\mathrm{Pd}$, Rh, and $\mathrm{Ni}$ complexes. ${ }^{440}$ Zirconocene chloride hydride $\left(\mathrm{Cp}_{2} \mathrm{HZrCl}\right)(5 \mathrm{~mol} \%)$ has been also used in the synhydroboration of internal alkynes. ${ }^{441}$ Copper(I) chloride can generate $\mathrm{Cu}-\mathrm{H}$ or $\mathrm{Cu}-\mathrm{B}$ species in the syn-hydroboration using HBpin and $\mathrm{B}_{2}$ pin $_{2}$, respectively. ${ }^{442-444}$ Recently, the bis(iminopyridine)iron(II) complex and tolylmagnesium bromide provided synhydroboration of internal alkynes. ${ }^{445}$ The first anti-hydroboration with HBpin has been described using $\left[\mathrm{Cp} * \mathrm{Ru}\left(\mathrm{CH}_{3} \mathrm{CN}\right)_{3}\right] \mathrm{PF}_{6}$ as catalyst giving the corresponding (E)alkenylboronates with de up to $96 \%$ and in good yields for symmetrical alkynes. ${ }^{446}$

In conclusion, diastereodivergent hydroboration reactions for terminal alkynes can be controlled by using the metal complexes derived from $\mathrm{Co}(\mathrm{II})$ or $\mathrm{Cu}$ (I) with different ligands. However, for internal alkynes, $\mathrm{Pd}, \mathrm{Rh}, \mathrm{Ni}$, and $\mathrm{Fe}(\mathrm{II})$ complexes gave synaddition, whereas cationic Ru complexes afforded anti-addition.

2.7.1.2. Hydrosilylation of Alkynes. Hydrosilylation of alkynes can be catalyzed by transition metal complexes usually in a syn-manner giving the corresponding $(E)$ vinylsilanes. ${ }^{428,447}$ Vinylsilanes can be further transformed into other alkenes by crosscoupling reactions. They are easy to handle, non-toxic and shown an excellent functional group compatibility. Terminal alkynes can be regio- and diastereoselectively syn-hydrosilylated under $\mathrm{Pt}$ catalysis by $\mathrm{Et}_{3} \mathrm{SiH}, \mathrm{Me}_{2} \mathrm{ClSiH}$, and $\mathrm{Cl}_{3} \mathrm{SiH}$ using bulky trialkylphosphines giving (E)-vinylsilanes. ${ }^{448}$ Complexes such as $\mathrm{Pt}\left(\mathrm{Cy}_{3} \mathrm{P}\right)(\text { ethylene) })_{2}$ and $\left[\mathrm{Pt}\left(\mathrm{Cy}{ }_{3} \mathrm{P}\right)\left(\mathrm{R}_{3} \mathrm{Si}\right)(\mu-\mathrm{H})\right]_{2}$ can be used as catalysts with only $0.01 \mathrm{~mol} \%$ loading. The use of tri-tert-butylphosphine gave excellent syn-selectivity even with alkoxysilanes. ${ }^{449,450}$ This methodology has been applied to the synthesis of a HMG-CoA reductase inhibitor by hydrosilylation with $\mathrm{Me}_{2} \mathrm{ClSiH}$ followed by a Hiyama crosscoupling arylation. ${ }^{449}$ On the other hand, anti-hydrosilylation of terminal alkynes can be performed under cationic Rh complexes catalysis using trialkylsilanes, providing (Z)vinylsilanes. ${ }^{451-453}$ 
Diastereodivergent hydrosilylation of terminal alkynes was resulted under $\mathrm{Rh}$ catalysis using alkoxysilanes. ${ }^{454-456}$ Mori and co-workers performed the syn-addition under $\mathrm{RhCl}\left(\mathrm{PPh}_{3}\right)_{3} / \mathrm{NaI}$ catalysis premixed with (EtO) ${ }_{3} \mathrm{SiH}$ giving $(\mathrm{Z})-505$ with up to $>99: 1 \mathrm{Z} / \mathrm{E} \mathrm{dr}$ (Scheme 184). However, when the terminal alkynes and the alkoxysilane were added to $0.1 \mathrm{~mol} \%$ of $\mathrm{RhI}\left(\mathrm{PPh}_{3}\right)_{3}$ and the mixture was heated at $55^{\circ} \mathrm{C},(E)$-silanes 505 were obtained quantitatively.

Scheme 184. Diastereodivergent Hydrosilylation of Phenylacetylene Catalyzed by Cationic Rh(I) Complexes Under Different Reaction Conditions

$$
\begin{aligned}
& \mathrm{HSiX}_{3}+\mathrm{RhCl}\left(\mathrm{PPh}_{3}\right) 3(0.1 \mathrm{~mol} \%)+\mathrm{Nal}(5 \mathrm{~mol} \%) \\
& \mathrm{rt} \mid \mathrm{Ph}=\mathrm{H} \\
& \mathrm{Ph} \mathrm{Six}_{3} \\
& \text { (Z)-505, 20-95\%, 20-98\% de }
\end{aligned}
$$

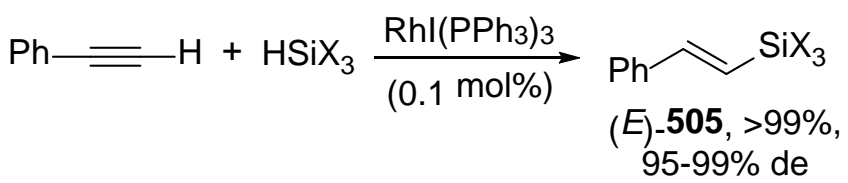

$$
\begin{aligned}
& {\left[\mathrm{SiX}_{3}=\mathrm{SiMe}_{2} \mathrm{OSiMe}_{3}, \mathrm{SiMe}(\mathrm{OEt})_{2}, \mathrm{Si}(\mathrm{OEt})_{3}\right. \text { ] }}
\end{aligned}
$$

Depending on the silane substituents diastereodivergent results have been observed under Rh catalysis. ${ }^{457,458}$ With trialkylsilanes such as $\mathrm{Et}_{3} \mathrm{SiH}$, anti-addition took place giving mainly (Z)-silanes $\mathbf{5 0 6}$ as it is shown for 1-hexyne in Scheme 185. After switching to electron-poor silanes such as $(\mathrm{MeO})_{3} \mathrm{SiH}$ or $\mathrm{ClMe}_{2} \mathrm{SiH}$, syn-addition occurred affording (E)-silanes 507.

Scheme 185. Diastereodivergent Hydrosilylation of 1-Hexyne Catalyzed by Rh Complexes Using Different Silanes

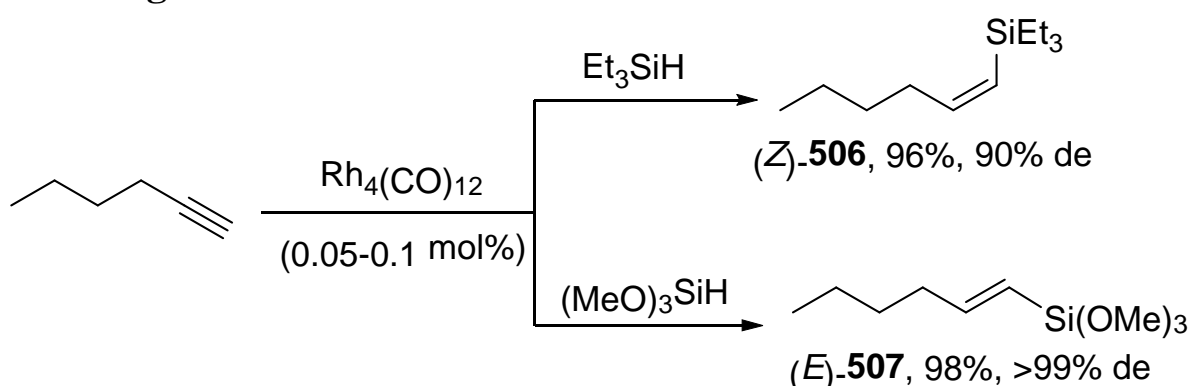

Faller and co-workers described reliable Rh catalysts for the diastereodivergent hydrosilylation either with $\mathrm{Et}_{3} \mathrm{SiH}$ or with $(\mathrm{EtO})_{3} \mathrm{SiH}^{459}$ In the case of $[\mathrm{Cp} * \mathrm{Ru}(\mathrm{BINAP})]\left(\mathrm{SbF}_{6}\right)_{2}(5 \mathrm{~mol} \%)$ syn-addition took place giving $(E)-505\left[\mathrm{SiX}_{3}=\right.$ $\left.\mathrm{SiEt}_{3}, \mathrm{Si}(\mathrm{OEt})_{3}\right]$ with total diastereoselectivity in $97 \%$ and $81 \%$ yield, respectively. Opposite anti-hydrosilylation took place with $\left(\mathrm{Cp} * \mathrm{RhCl}_{2}\right)_{2}$ giving mainly (Z)-505 
$\left[\mathrm{SiX}_{3}=\mathrm{SiEt}_{3}, \mathrm{Si}(\mathrm{OEt})_{3}\right]$ in $93 \%$ de. In the case of the cationic catalyst a Chalk-Harrod mechanism has been proposed, while for the neutral $\left(\mathrm{Cp} * \mathrm{RhCl}_{2}\right)_{2}$ a monohydride complex such as $\left[\mathrm{Cp}^{*} \mathrm{Rh}(\mathrm{SiH})\left(\mathrm{SiX}_{3}\right)_{2}\right]$ has been proposed as the active catalyst (Scheme 186).

Scheme 186. Proposed Mechanism for the anti-Hydrosilylation of Phenylacetylene with $\mathrm{X}_{3} \mathrm{SiH}$ Catalyzed by $\left(\mathrm{Cp}^{*} \mathrm{RhCl}_{2}\right)_{2}$

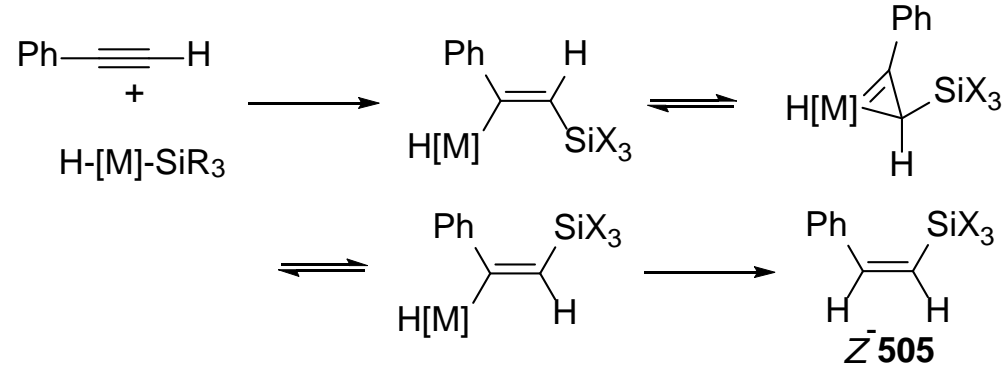

The ruthenium-catalyzed hydrosilylation of terminal alkynes can be diastereodivergently carried out according to the structure of the complex. Under $\mathrm{RuHCl}(\mathrm{CO})\left(\mathrm{PPh}_{3}\right)_{3}$ catalysis, hydrosilanes $\mathrm{ArMe}_{2} \mathrm{SiH}$ directed the syn-addition, whereas $\mathrm{Ru}\left(\mathrm{SiMe}_{2} \mathrm{Ph}\right) \mathrm{Cl}(\mathrm{CO})\left(\mathrm{PiPr}_{3}\right)_{2}$ provided anti-addition at rt in high yields (Scheme 187). ${ }^{460,461}$ Compounds (E)-508 were obtained in over 99\% selectivity and stereoisomers $(Z)-\mathbf{5 0 8}$ in $91-99 \%$ de. The proposed mechanism is based on the two catalytic cycles A and B. In the first cycle A, after alkyne insertion into a Ru hydride the intermediate $(E)$-alkenylsilane $\mathbf{5 0 8}$ was obtained. In the case of cycle B, a silyl ruthenium intermediate can be formed, which afforded $(Z)-\mathbf{5 0 8} .^{462,463}$

Scheme 187. Diastereodivergent Hydrosilylation of Terminal Alkynes with $\mathrm{HSiMe}_{2} \mathrm{Ar}$ Catalyzed by Different Ru Complexes 

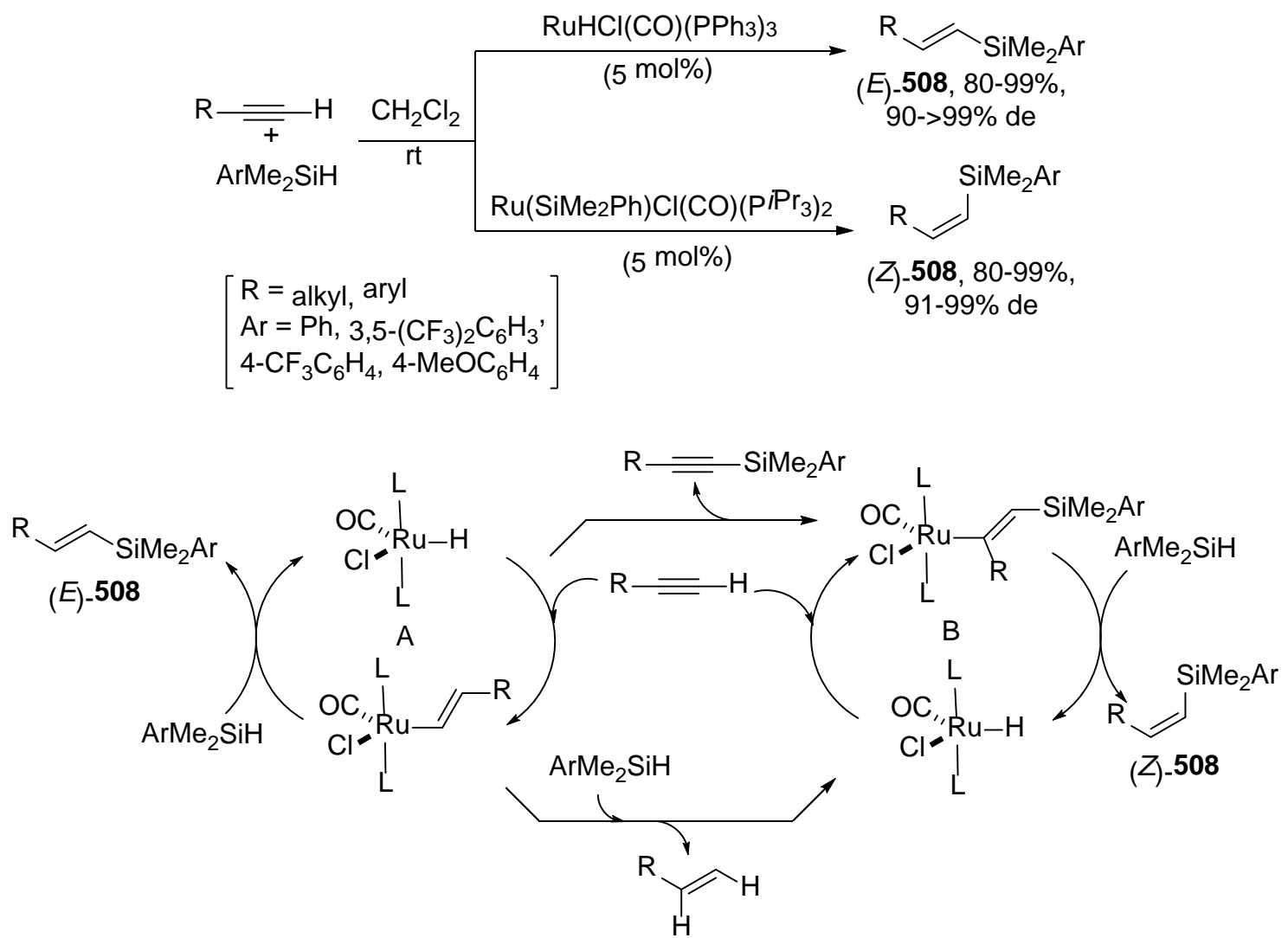

Metal(0) nanoparticles (NPs) derived from Pt, Ir, Rh, and Ru embedded in a solid matrix of ammonium salts have been used as catalysts in the stereodivergent hydrosilylation of phenylacetylene with trimethoxysilane. In the case of M@CTBA (cetyltrimethylammonium bromide, $0.1 \mathrm{~mol} \%$ ) of Pt gave mainly $(E)-505(X=\mathrm{OMe})$ in $>99 \%$ de, whereas Ru afforded mainly $(Z)-505(X=0 M e)$ in only $40 \%$ de. $^{464}$

For hydrosilylation of internal alkynes the control of the regioselectivity is a more challenging task than in the case of terminal alkynes. The best sterically discriminating catalyst $\mathrm{Cp}_{2}{ }_{2} \mathrm{Y}\left[\mathrm{CH}(\mathrm{TMS})_{2}\right]$ was able to react regioselectively with phenylsilane $\left(\mathrm{PhSiH}_{3}\right)$ giving exclusively the corresponding $(E)$-vinylsilane according to a synhydrosilylation process. ${ }^{465}$ On the other hand, the Ru complex $\left[\mathrm{Cp} * \mathrm{Ru}\left(\mathrm{CH}_{3} \mathrm{CN}\right)_{3}\right] \mathrm{PF}_{6}$ gave anti-addition forming (Z)-vinylsilanes with moderate regioselectivity. Only $\alpha, \beta$ unsaturated alkynyl esters and ketones reacted with trialkylsilanes giving anti-addition with $90 \%$ regioselectivity. ${ }^{466,467}$

Denmark and co-workers have described the diastereodivergent hydrosilylation of homopropargylic ${ }^{468,469}$ and propargylic ${ }^{470}$ alcohols for the preparation, after Hiyama reaction, of trisubstituted homoallylic and allylic alcohols, respectively. In situ silylation of homopropargylic alcohols to the corresponding silyl ethers, for instance 509, gave after intramolecular hydrosilylation either by syn-addition under Pt Speier catalysis ${ }^{468}$ or by anti-addition under $\mathrm{Ru}$ catalysis ${ }^{469}$ regio- and stereoselectively cyclic alkylidenesilacyclopentanes $\mathbf{5 1 0}$ (Scheme 188). These compounds were further transformed stereospecifically into trisubstituted $(E)$ - and (Z)-homoallylic alcohols by Pd catalyzed cross-coupling reactions. ${ }^{468,469}$ 
Scheme 188. Diastereodivergent Hydrosilylation of Homopropargylic Alcohols Catalyzed by Pt or Ru Complexes

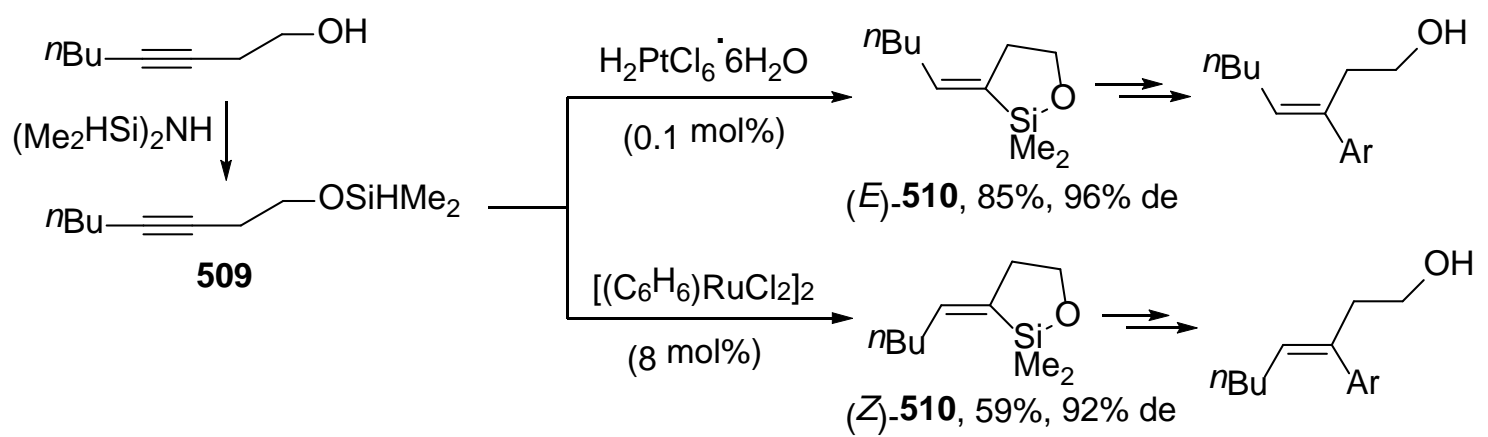

Similarly, through an appropriate choice of the hydrosilylation catalyst, propargylic alcohols were transformed diastereoselectively into (Z)- and (E)-allylic alcohols. ${ }^{470}$ In this case a disiloxane tether was used to perform the intramolecular syn and antihydrosilylation (Scheme 189). ${ }^{470}$ The previous silylation of 2-butyn-1-ol with tetraisopropyldisiloxane using Stryker's catalyst gave the silyl ether 511. Further hydrosilylation catalyzed by $\mathrm{Pt}(0)$-1,3-divinyl-1,1,3,3-tetramethyldisiloxane [Pt(DVDS)] led to the formation of (E)-6-ethylidenedioxadisilacyclohexane (512) in $60 \%$ yield. On the other hand, under Ru-catalysis (Z)-512 was diastereodivergently obtained in 82\% yield. Both compounds $\mathbf{5 1 2}$ were further transformed stereospecifically into $\beta$-arylated allylic alcohols by Hiyama reaction.

Scheme 189. Diastereodivergent Hydrosilylation of 2-Butyn-1-ol Disiloxane Under Pt or Ru Catalysis

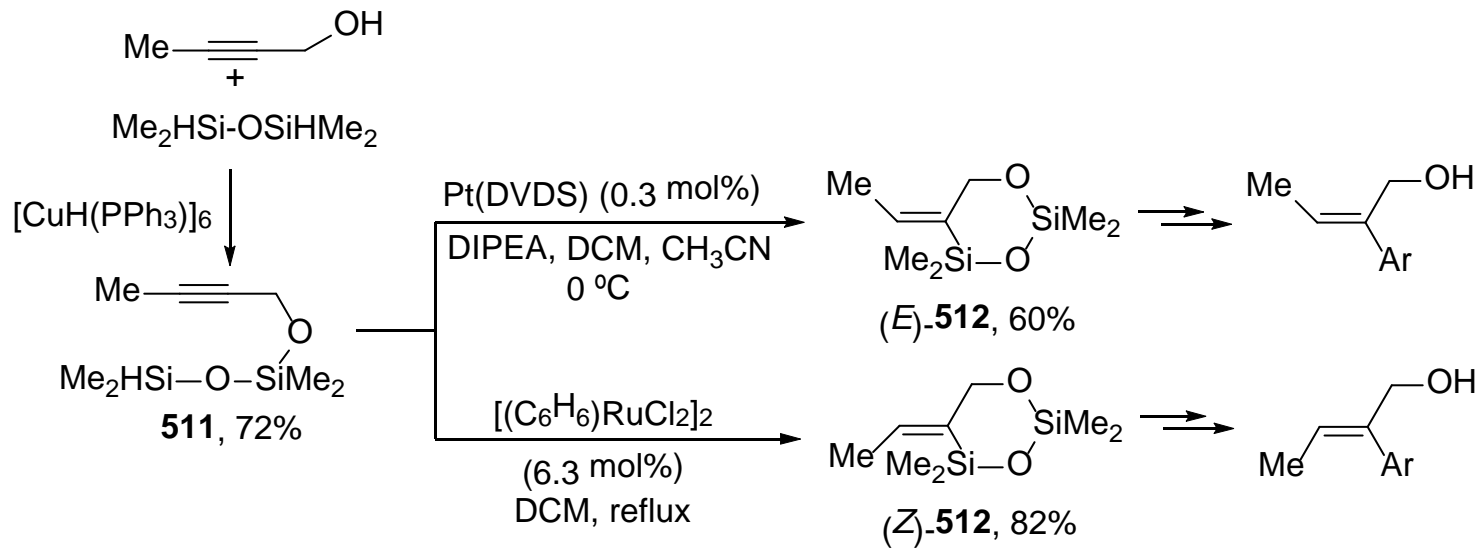

Only internal symmetrical alkynes have been hydrosilylated by means of Co(I) catalyst $\mathbf{5 1 3}$ in a diastereodivergent manner depending on the silane used. ${ }^{471}$ When triethylsilane was employed as a silylating agent syn-addition occured giving exclusively (E)-alkenylsilanes 514 (Scheme 190). On the other hand, anti-addition was achieved when trimethoxysilane was used as a silylating agent giving products (Z)-515 
in high de. In the latter case, a syn-hydrosilylation followed by isomerization has been proposed.

Scheme 190. Diastereodivergent Hydrosilylation of Symmetrical Internal Alkynes Catalyzed by Co(I) Complex 513 Using Different Silanes

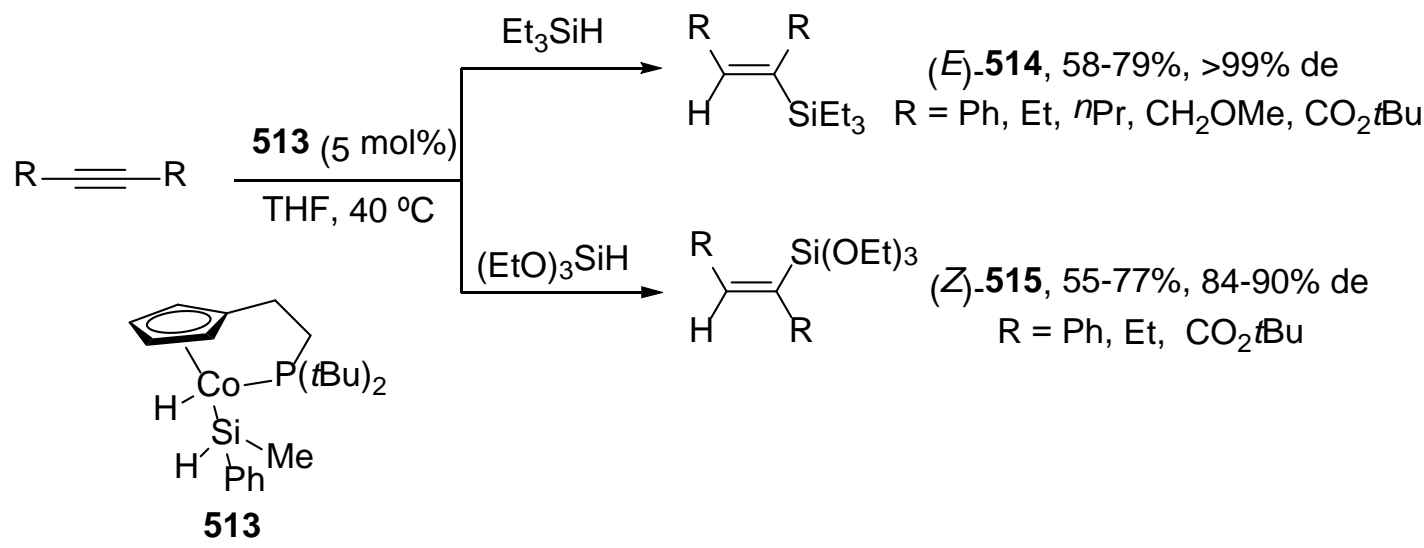

Silane-dependent diastereodivergent hydrosilylation of symmetrical internal alkynes has been also observed using the iron hydride complex $\mathrm{FeH}(\mathrm{CO})(\mathrm{NO})\left(\mathrm{PPh}_{3}\right)_{2}$ (516) as catalyst $(1 \mathrm{~mol} \%) .{ }^{472}$ Working in the presence of $\mathrm{Et}_{3} \mathrm{~N}(0.5 \mathrm{eq})$ in $\mathrm{THF}$ at $40-60{ }^{\circ} \mathrm{C}$, diphenylacetylene (tolane) reacted with phenylsilane through an anti-addition providing (Z)-phenylsilylstilbene 517 in 1:>20 E/Z ratio and 98\% yield (Scheme 191). In the case of dimethylphenylsilane or methylphenylvinylsilane syn-addition was mainly observed affording the corresponding $(E)$-isomer 518. This stereodivergent effect was attributed to steric reasons. This methodology has been applied to the synthesis of (E)- and (Z)combrestatin after further desilylation reactions in 50\% and $80 \%$ de, respectively.

\section{Scheme 191. Diastereodivergent Hydrosilylation of Tolane Catalyzed by Fe Complex 516 Using Different Silanes}

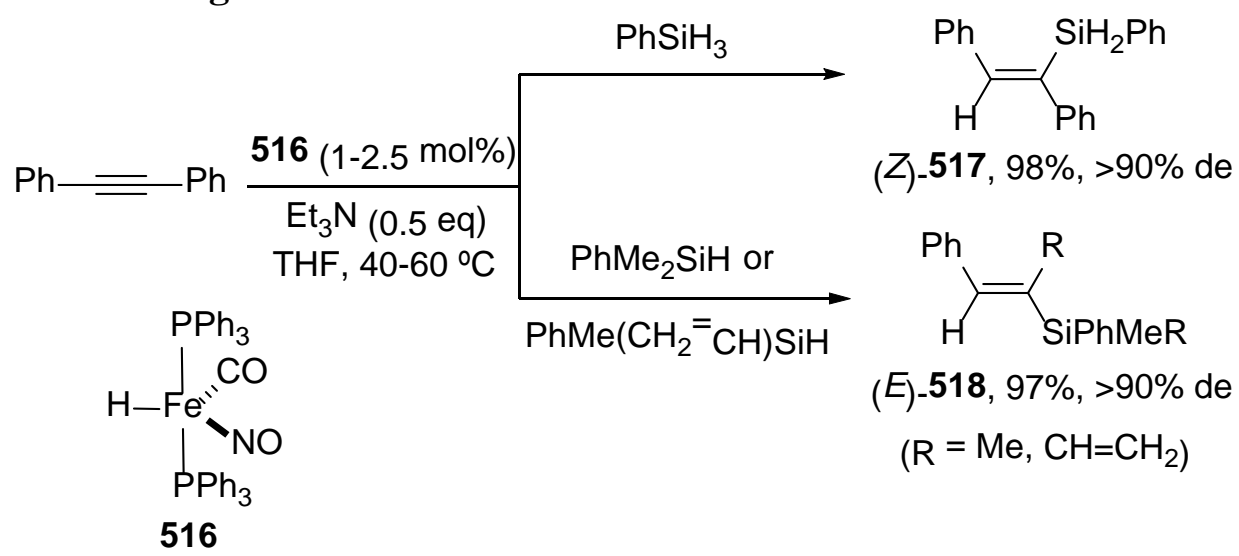

Regioselective and diastereodivergent hydrosilylation of methyl 2-hexynoate with dimethylphenylsilane has been studied using different transition-metal catalysts. Under $\mathrm{Pd}$ catalysis with $\left[\mathrm{Pd}(\mathrm{dba})_{2} \mathrm{PCy}_{3}\right]$, syn-addition was mainly observed, for the $(Z)$-isomer 
(>98\% de). ${ }^{473}$ However, anti-addition took place using $\mathrm{Rh}$ and $\mathrm{Ru}$ complexes giving methyl (E)-2-(dimethylphenylsilyl)-2-hexenoate.

In conclusion, several examples of diastereodivergent syn- or anti-hydrosilylation of alkynes either under Pt or Rh as well as Ru complexes have been shown, respectively. However, the most important role was played by the nature of the hydrosilylating agents.

2.7.1.3. Hydrostannation of Alkynes. Terminal alkynes, such as phenylacetylene, can be hydrostannylated under $\mathrm{Rh}, \mathrm{Ni}, \mathrm{Pd}, \mathrm{Pt}, \mathrm{Ru}, \mathrm{Co}$, and Mo catalysts giving mixtures of $\alpha$ - and (E)- $\beta$-alkenylstannanes. ${ }^{428,474}$ Only $\mathrm{Pd}$ catalysis gave exclusively (E)- $\beta$ alkenylstannane 519 and in the presence of $\mathrm{ZrCl}_{4}$ anti-addition took place giving the (Z)- $\beta$-alkenylstannane 519 (Scheme 192). ${ }^{475,476}$ However, simple aliphatic alkynes such as 1-octyne afforded low regioselectivity under the Pd catalysis. Only with bulky alkyl substituents at the propargylic position (E)-alkenylstannanes were exclusively formed. ${ }^{477}$ However, using $\mathrm{ZrCl}_{4}$ as Lewis acid, (Z)-alkenylstannanes were regio- and stereoselectively obtained. ${ }^{475,476}$

Scheme 192. Diastereodivergent Hydrostannylation of Phenylacetylene Catalyzed by $\mathrm{Pd}$ or $\mathrm{ZrCl}_{4}$

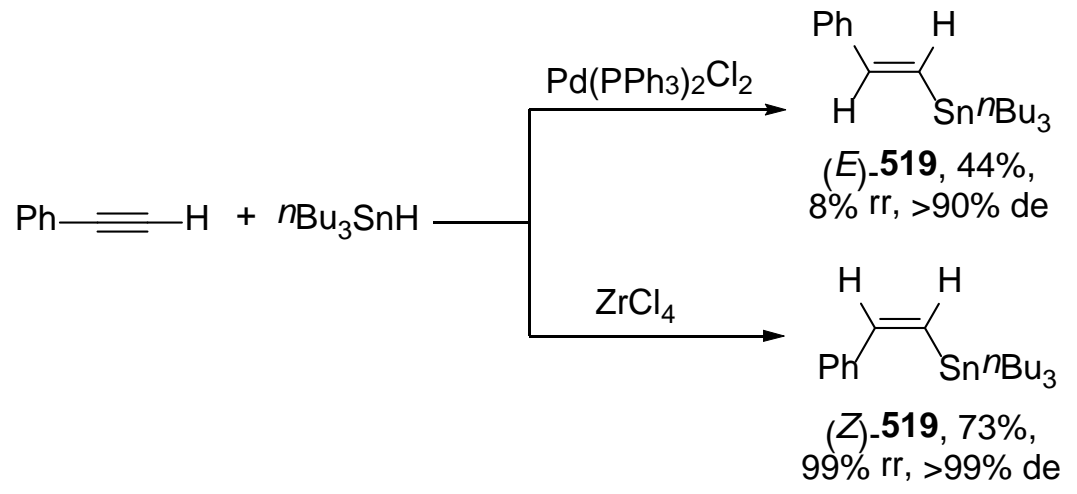

Due to the toxicity of stannanes, a one-pot tandem hydrostannylation and crosscoupling (Stille reaction) under Pd catalysis has been achieved with catalytic amounts of tin employing polymethylhydrosiloxane (PMHS) as reductant. ${ }^{478}$

In the case of internal aryl-alkyl alkynes, the hydrostannylation under Pd catalysis occurred in a syn-manner giving diastereoselectively (E)-alkenylstannanes, using hydrocarbons as solvents. ${ }^{479}$ Recently, anti-hydrostannylation of symmetrical and unsymmetrical internal alkynes with $\left[\mathrm{Cp} * \mathrm{Ru}\left(\mathrm{CH}_{3} \mathrm{CN}\right)_{3}\right] \mathrm{PF}_{6}$ has been reported. ${ }^{480}$ Both methods can be considered as diastereodivergent processes.

2.7.2. Reduction of Alkynes. Semihydrogenation of alkynes with molecular hydrogen has been usually performed with Lindlar's catalyst, $\mathrm{Pd}$ on $\mathrm{CaCO}_{3}$ doped with $\mathrm{Pb}$, allowing access to $(Z)$-alkenes. ${ }^{481,482}$ Some homogeneous catalysts also gave good (Z)-diastereoselectivity, namely $\mathrm{Pd}^{483,484} \mathrm{Rh}^{485}$ and $\mathrm{Ir}^{486}$ catalysts. Complementary trans-hydrogenation has been performed using homogeneous Rh catalysts $\mathbf{5 2 0}^{487}$ and $\mathbf{5 2 1}{ }^{488}$ (Figure 18). Ru catalyst 522 also reduced internal alkynes to (E)-alkenes. ${ }^{489}$ 
More recently, Fürstner and co-workers have described the Ru catalyst $\mathbf{5 2 3}$ for the trans-hydrogenation of different functionalized alkynes. ${ }^{490}$ The mechanistic studies revealed that both hydrogen atoms were delivered to the same sp carbon atom and the other alkyne carbon atom was converted into a metal carbene. ${ }^{491}$ Milstein and coworkers have described a highly selective trans-hydrogenation catalyzed by the iron pincer complex 524, ${ }^{492}$ due to the isomerization of the $(Z)$-alkene to the corresponding (E)-alkene. Heterobimetallic NHC complexes from Ag-Ru such as $\mathbf{5 2 5} 5^{493}$ also catalyzed the trans-hydrogenation of internal alkynes.

Figure 18. Metal Complexes for the trans-Hydrogenation of Alkynes

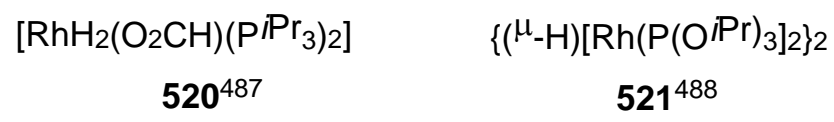

$\left[\mathrm{Cp} * \mathrm{Ru}\left(\eta^{4}-\mathrm{CH}_{3} \mathrm{CH}=\mathrm{CHCH}=\mathrm{CHCO}_{2} \mathrm{H}\right)\right] \mathrm{TfO}$

$\mathbf{5 2 2}^{489}$

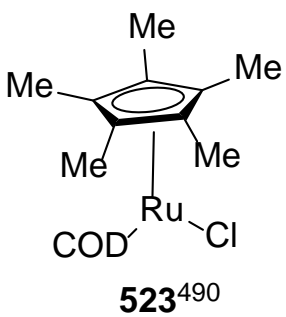

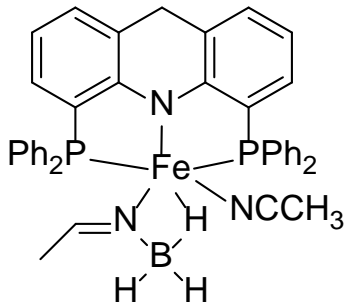

$524^{492}$<smiles>Cc1cc(C)c(C2C=CC(c3c(C)cc(C)cc3C)N2[Ge](C)(C)C(=O)C(C)C)c(C)c1</smiles>

$\mathbf{5 2 5} 493$

A metal-free diastereodivergent hydrogenation of alkynes promoted by pentafluorophenylethylene with $\left[\mathrm{HB}\left(\mathrm{C}_{6} \mathrm{~F}_{5}\right)_{2}\right]$ as catalyst has been recently described. ${ }^{494}$ In the presence of pentafluorophenylethylene $(20 \mathrm{~mol} \%)$ at $140{ }^{\circ} \mathrm{C}$ and $50 \mathrm{bar}$, the corresponding $(Z)$-alkenes were mainly obtained under strict control of the reaction time (Scheme 193). Under longer reaction times and a slight excess of [ $\left.\mathrm{HB}\left(\mathrm{C}_{6} \mathrm{~F}_{5}\right)_{2}\right]$, the $(E)$ isomers were mainly formed. Mechanistic studies proposed initial hydroboration of the alkyne giving borane $\mathbf{A}$, then a complex $\mathbf{B}$ with $\mathrm{C}_{6} \mathrm{~F}_{5} \mathrm{CH}=\mathrm{CH}_{2}$ through $\mathrm{H}-\mathrm{F}$ interactions is formed. Further hydrogenolysis via the TS $\mathbf{C}$ produced the $(Z)$-alkene. On the other hand, the $(E)$-alkene was formed by the $\mathrm{HB}\left(\mathrm{C}_{6} \mathrm{~F}_{5}\right)_{2}$ catalyzed isomerization of the $(Z)$ alkene.

Scheme 193. Diastereodivergent Hydrogenation of Alkynes Catalyzed by $\mathrm{HB}\left(\mathrm{C}_{6} \mathrm{~F}_{5}\right)_{2}$ and $\mathrm{C}_{6} \mathrm{~F}_{5} \mathrm{CH}=\mathrm{CH}_{2}$ 


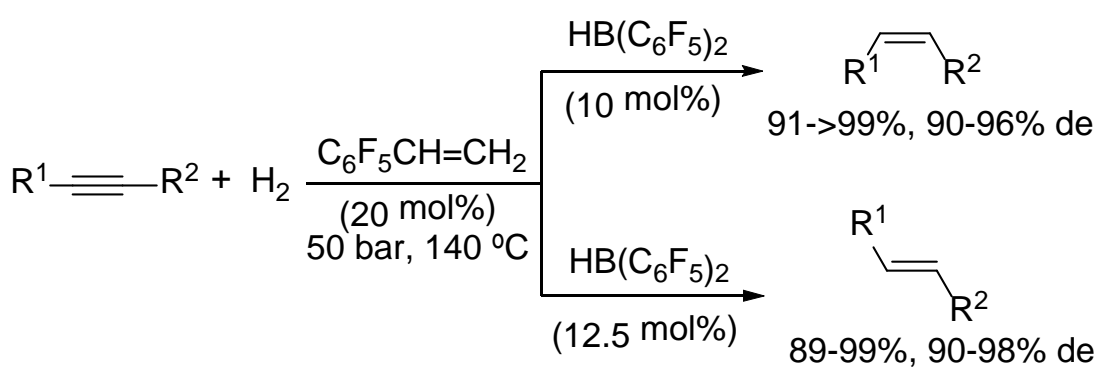

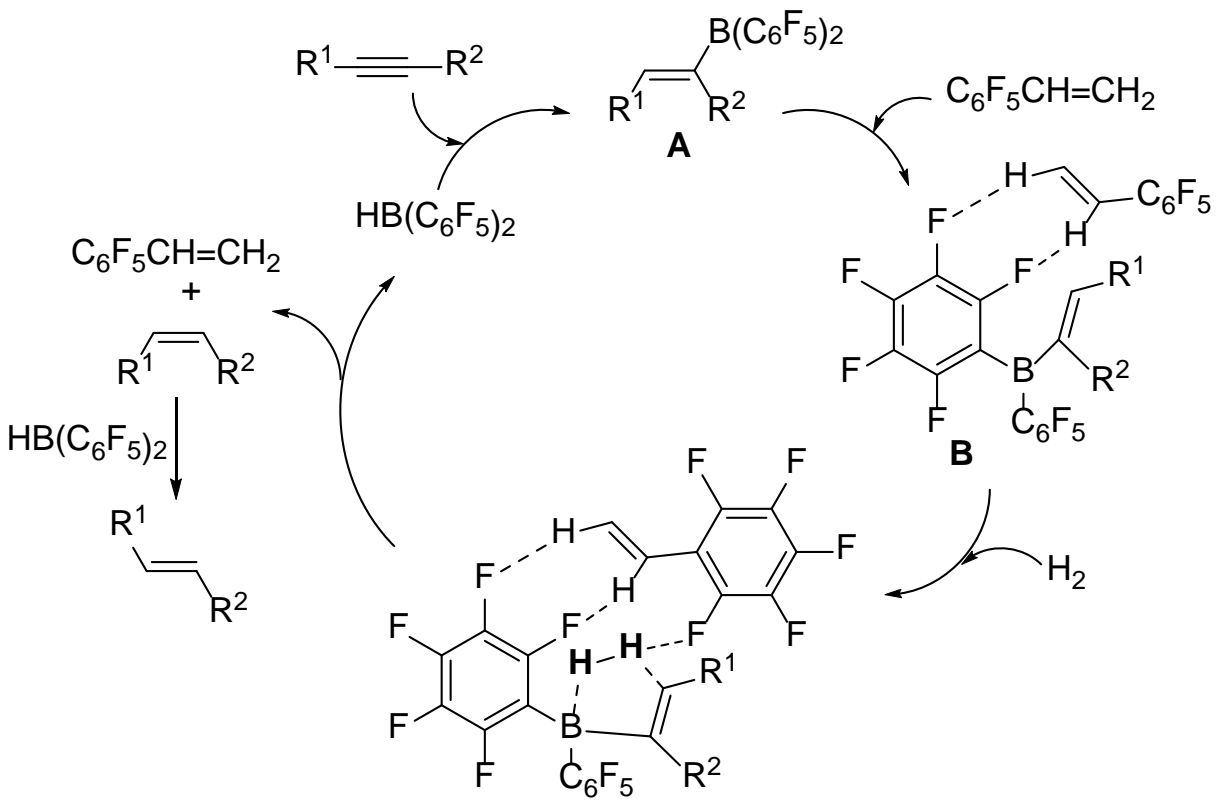

C

Diastereodivergent transfer hydrogenation of aromatic alkynes using the Ni complex 526 has been achieved using either triethylsilane $/ \mathrm{H}_{2} \mathrm{O}$ or $\mathrm{MeOH}$ as hydrogen sources. ${ }^{495}$ Under the first reaction conditions $(Z)$-alkenes were mainly obtained, whereas in $\mathrm{MeOH}(E)$-alkenes were the major diastereomers (Scheme 194).

Scheme 194. Diastereodivergent Transfer Hydrogenation of Aromatic Alkynes Catalyzed by Ni(II) Complex 526 Using $\mathrm{Et}_{3} \mathrm{SiH}$ and $\mathrm{H}_{2} \mathrm{O}$ or $\mathrm{MeOH}$

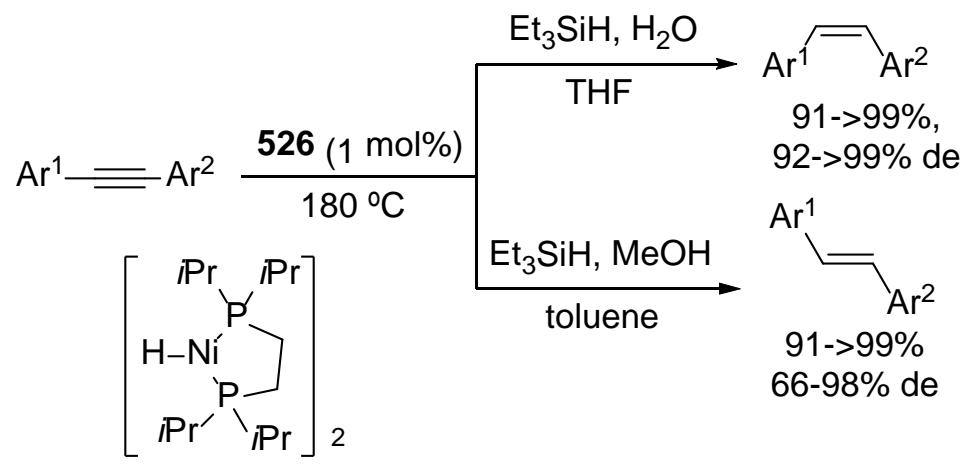


In the first case, catalytic amounts of $\mathrm{Et}_{3} \mathrm{SiH}$ and $\mathrm{H}_{2} \mathrm{O}$ were used and the diphosphine was oxidized in the process acting as a sacrificial reagent. In the presence of methanol, it was oxidized to formaldehyde during the process. The same group has described this reduction using ammonia-borane $\left(\mathrm{BH}_{3} \cdot \mathrm{NH}_{3}\right)$ or $\mathrm{NaBH}_{4}$ as hydrogen sources under the $\mathrm{Ni}(0)$ complex 526 catalysis. ${ }^{496}$ Diastereodivergent results were observed when $\mathrm{BH}_{3} \cdot \mathrm{NH}_{3}$ was used in $\mathrm{MeOH}$ or in THF, $(E)$-alkenes being formed in $\mathrm{MeOH}$ and (Z)-alkenes in THF. In the case of working with 1 eq of $\mathrm{NaBH}_{4}(Z)$-alkenes were mainly formed, whereas with 2 eq of $\mathrm{NaBH}_{4}(E)$-alkenes were obtained.

Palladium-catalyzed diastereodivergent reduction of alkynes has been performed using triethylsilane as reducing agent. ${ }^{497}$ In the absence of $\mathrm{CuSO}_{4}$, (Z)-alkenes were formed in aqueous $\mathrm{CH}_{2} \mathrm{Cl}_{2}$, whereas in the presence of $\mathrm{CuSO}_{4}(15 \mathrm{~mol} \%)$ and aqueous toluene the corresponding (E)-alkenes were obtained (Scheme 195).

Scheme 195. Diastereodivergent Reduction of Internal Alkynes with $\mathrm{Et}_{3} \mathrm{SiH}$ Catalyzed by Pd(II) in the Absence or Presence of $\mathrm{CuSO}_{4}$

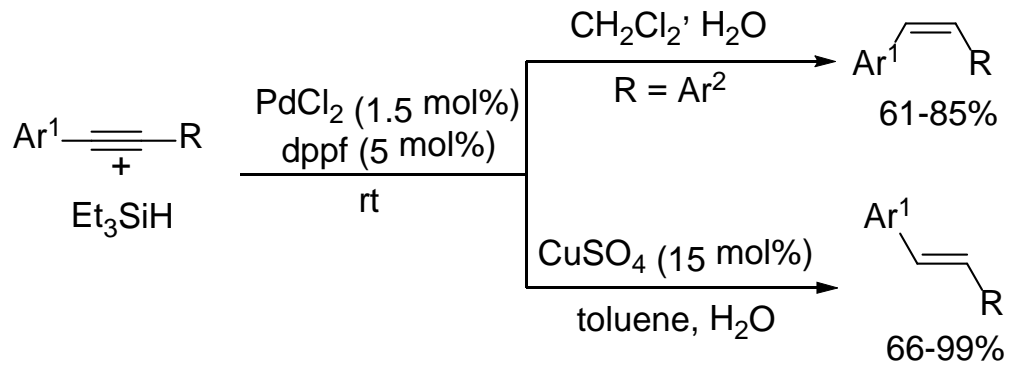

Transfer hydrogenation of diaryl alkynes has been performed using $\mathrm{Ru}_{3}(\mathrm{CO})_{13}$ as catalyst in DMF and $\mathrm{H}_{2} \mathrm{O}$ as hydrogen source. Diastereodivergent formation of $(Z)$ - or $(E)$-alkenes can be controlled by using $\mathrm{AcOH}$ or TFA as additives, respectively (Scheme 196). ${ }^{498}$ This methodology has been applied to the synthesis of $(Z)$ - and (E)combrestatin A-4 and (E)-resveratrol.

Scheme 196. Diastereodivergent Transfer Hydrogenation of Diaryl Alkynes Catalyzed by $\mathrm{Ru}_{3}(\mathrm{CO})_{13}$ in the Presence of AcOH or TFA

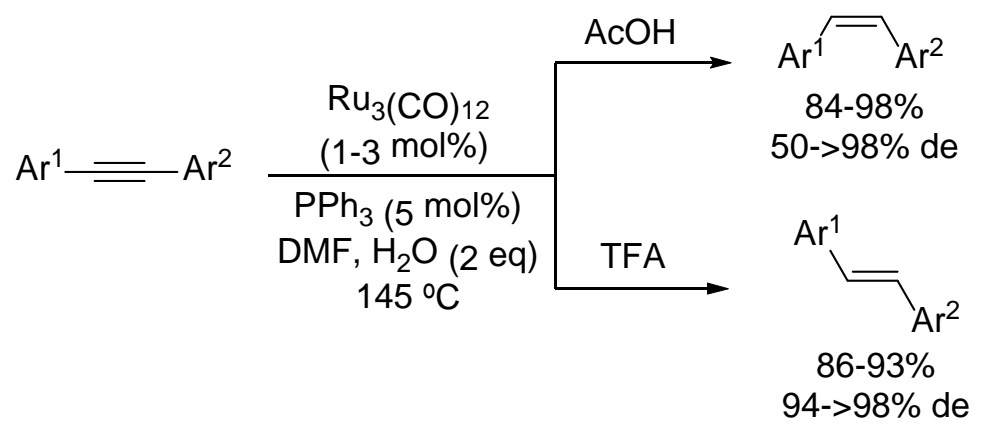

Moderate control of the $E / Z$ selectivity has been achieved using Ru complexes (2.5 mol\%), and $\mathrm{CuI}(10 \mathrm{~mol} \%)$ as catalysts and $\mathrm{Zn}(2 \mathrm{eq})$, in the presence of $\mathrm{H}_{2} \mathrm{O}$ (8 eq) at 
$100{ }^{\circ} \mathrm{C}$ in the transfer hydrogenation of different functionalized internal alkynes. ${ }^{499}$ Working in dioxane $\mathrm{RuCl}_{2}\left(\mathrm{PPh}_{3}\right)_{2}$ gave $(E)$-alkenes in $>90 \%$ de and using $\left(\mathrm{PPh}_{3}\right)_{3} \mathrm{Ru}(\mathrm{CO}) \mathrm{HCl}$, the corresponding $(Z)$-alkenes were obtained with de up to $80 \%$.

Ligand-controlled transfer hydrogenation of terminal and internal alkynes has been carried out under $\mathrm{Ni}(\mathrm{II})$ catalysis. $^{500}$ In the presence of formic acid and $\mathrm{Zn}$, internal alkynes were transformed into (E)-alkenes employing $\mathrm{NiCl}_{2} \cdot \mathrm{dme}$ as catalyst and bis(diphenylphosphinoethyl)phenylphosphine (TRIPHOS) as ligand. On the other hand, (Z)-alkenes were mainly formed under $\mathrm{NiBr}_{2}$ catalysis (Scheme 197). Mechanistic aspects have not been described.

\section{Scheme 197. Diastereodivergent Transfer Hydrogenation of Alkynes Under Ni(II)} Catalysis

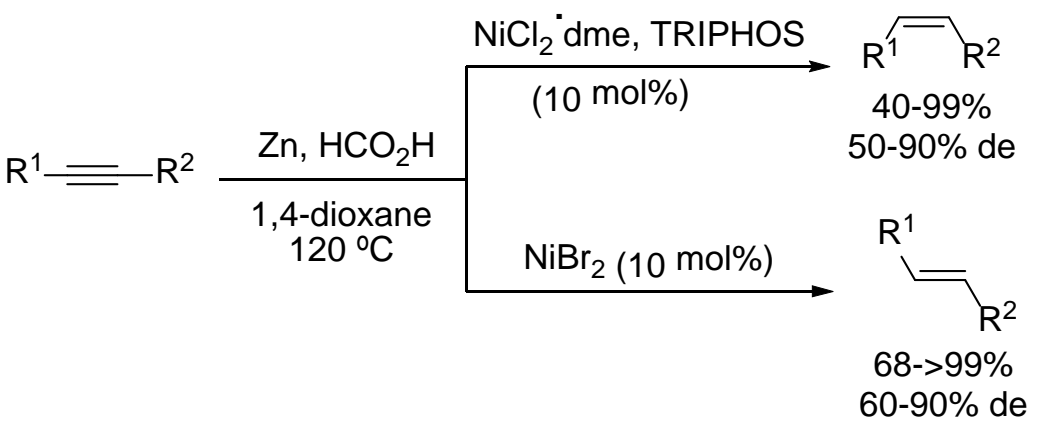

Another example of ligand-controlled diastereodivergent Co-catalyzed transfer hydrogenation of alkynes has been reported. ${ }^{501}$ Using $\mathrm{BH}_{3} \cdot \mathrm{NH}_{3}$ in $\mathrm{MeOH}$ at $50{ }^{\circ} \mathrm{C}$ and complex 527, (Z)-alkenes were mainly formed. However, in the presence of catalyst 528, (E)-alkenes were diastereoselectively obtained (Scheme 198). This methodology has been applied to different types of alkynes with good chemo- and diastereoselectivity. According to mechanistic studies an isomerization to the $(E)$-alkene took place after formation of $(Z)$-alkenes.

Scheme 198. Diastereodivergent Transfer Hydrogenation of Alkynes with Ammonia-Borane Catalyzed by Different Co Complexes 527 and 528

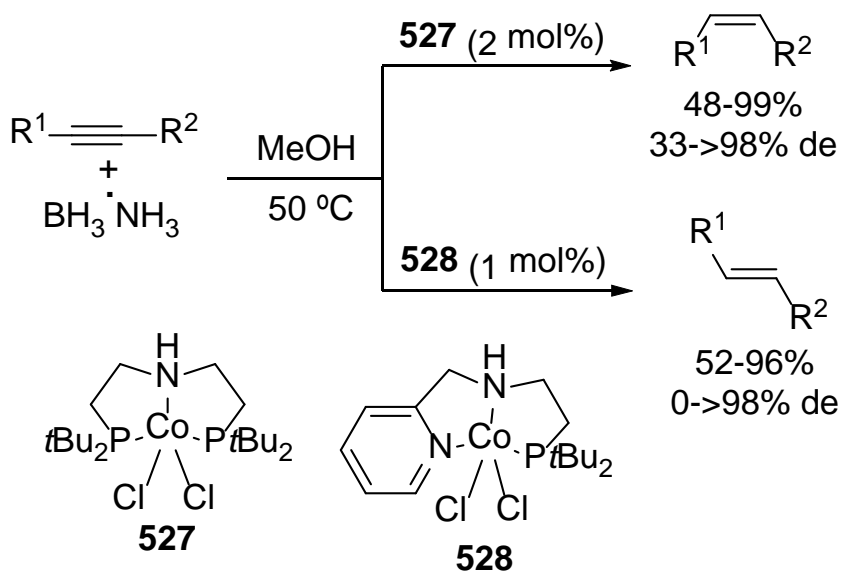


Diastereodivergent reduction of alkynes has been achieved by palladium-catalyzed transfer hydrogenation in the presence of formic acid by slightly tuning of the reaction conditions. ${ }^{502}$ In the presence of 1,4-bis(diphenylphosphino)butane (DPPB) (4 mol\%) as ligand and $\mathrm{Pd}\left(\mathrm{PPh}_{3}\right)_{4}(1 \mathrm{~mol} \%)$ as catalyst in dioxane at $80{ }^{\circ} \mathrm{C}(\mathrm{Z})$-alkenes were the main products (Scheme 199). However, using 25\% aqueous formic acid and $2 \mathrm{~mol} \%$ of DPPB, (E)-alkenes were obtained. A wide tolerance of the functional group in the alkyne has been found. The reduction directed to $(Z)$-alkenes has been rationalized on the basis of experimental studies by hydropalladation of the triple bond with formic acid forming the alkenylpalladium $\mathbf{A}$ followed by decarboxylation and final reductive elimination. The isomerization of intermediate $\mathbf{A}$ gives the corresponding $(E)$-alkenes in the presence of formic acid.

Scheme 199. Diastereodivergent Transfer Hydrogenation of Alkynes Catalyzed by $\operatorname{Pd}\left(\mathrm{PPh}_{3}\right)_{4}$ Using Formic Acid
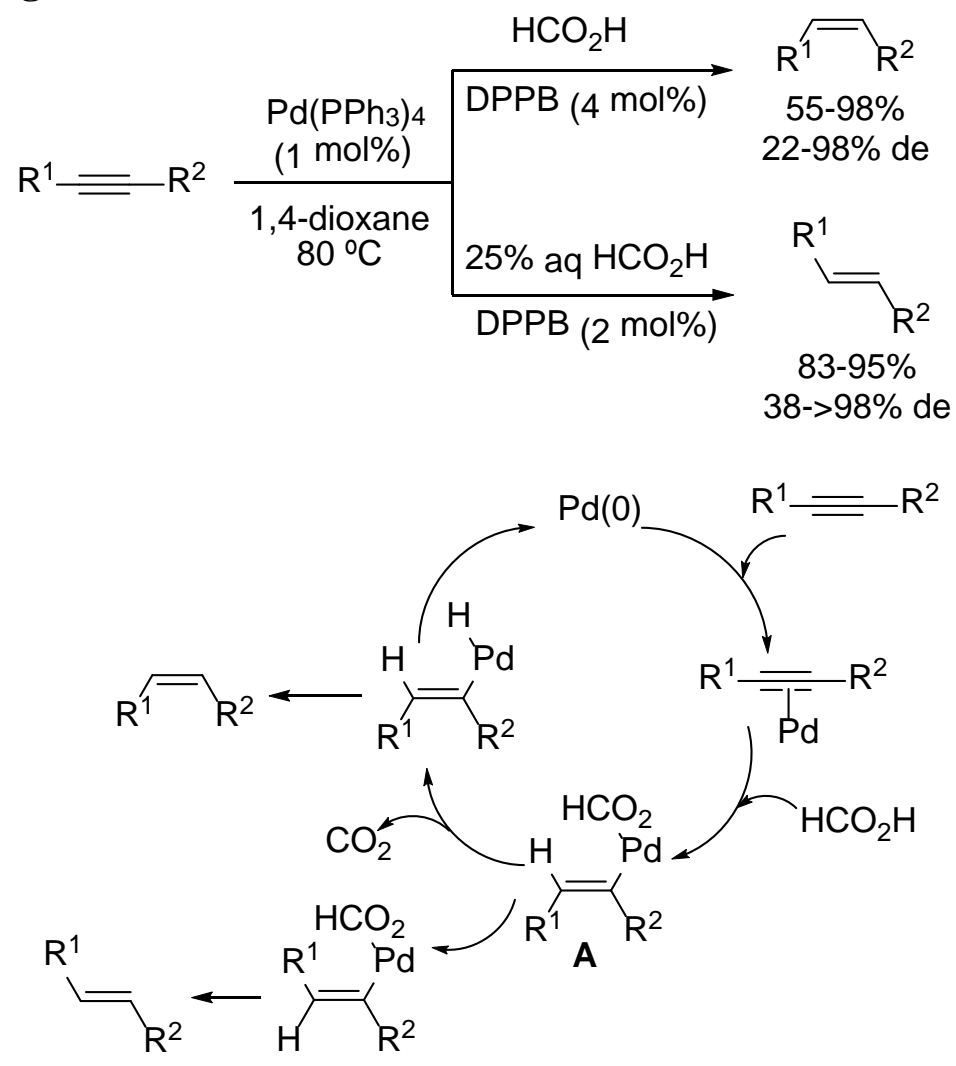

In conclusion, diastereodivergent transfer hydrogenations of alkynes catalyzed by Pd, $\mathrm{Ru}, \mathrm{Ni}$, and Co complexes are the most efficient methodologies for the synthesis of (Z)or $(E)$-alkenes in many cases just by adjusting the reaction conditions or the ligand. Under these reaction conditions, syn-hydrogenation gave (Z)-alkenes, which suffers from further isomerization to $(E)$-alkenes.

2.7.3. Other Reactions. Hydroarylation of alkynes catalyzed by transition metals enables the insertion of the $\mathrm{C}-\mathrm{C}$ triple bond into a $\mathrm{C}-\mathrm{H}$ bond of aromatic compounds allowing the synthesis of alkenyl arenes. ${ }^{503}$ Using $\mathrm{Ru}(\mathrm{II})$ complexes as catalysts 
trisubstituted $(E)$-alkenes were obtained by syn-addition. ${ }^{504}$ Recently, a switching in the E/Z stereoselectivity has been found by controlling the $\mathrm{AgSbF}_{6}$ loading. ${ }^{505} \mathrm{Ru}$-catalyzed hydroarylation of chromenes 529 in the presence of $\mathrm{AgSbF}_{6}(16 \mathrm{~mol} \%)$ and $\mathrm{Cu}(\mathrm{OAc})_{2}$ $(10 \mathrm{~mol} \%)$ and $\mathrm{AcOH}(2 \mathrm{eq})$ at $100{ }^{\circ} \mathrm{C}$ give $(E)$-isomers 530 (Scheme 200). When a 20 mol\% of $\mathrm{AgSbF}_{6}$ loading was used $(Z)-\mathbf{5 3 0}$ products were isolated. It has been demonstrated that $\mathrm{AgSbF}_{6}$ facilitated the isomerization of the $(E)$ - to the $(Z)$-alkene. In the proposed mechanism the cationic Ru complex formed the ruthenacycle intermediate A, which inserted the alkyne to form $\mathbf{B}$. Further protonation of $\mathbf{B}$ afforded $(E)-\mathbf{5 3 0}$, which in the presence of $\mathrm{AgSbF}_{6}$ suffered an isomerization at the allylic cation $\mathbf{C}$ to form the more stable $(Z)-530$ isomer.

Scheme 200. Diastereodivergent Hydroarylation of Alkynes with Chromenes 529 Catalyzed by a Ru(II) Complex and $\mathrm{Cu}(\mathrm{OAc})_{2}$ in the Presence of Different $\mathrm{AgSbF}_{6}$ Loadings

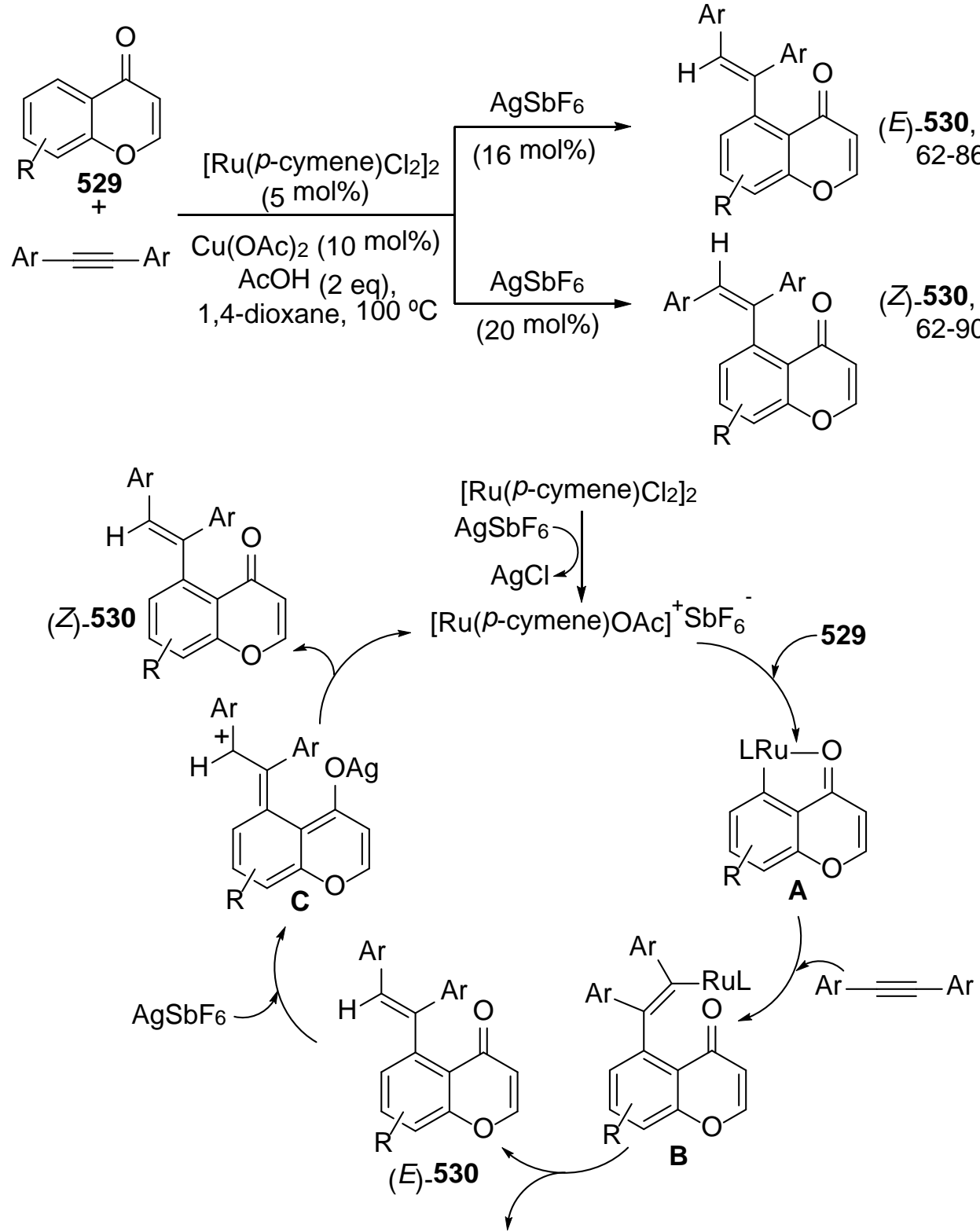

$[\mathrm{Ru}]$ 
The Pd-catalyzed hydroarylation of ynamides $\mathbf{5 3 1}$ with terminal alkynes took place diastereodivergently depending on the C-substituents in the ynamide. ${ }^{506}$ In the case of alkyl substituted 531, exclusively syn-addition occurred affording adducts ( $Z$ )-532 (Scheme 201). On the other hand, aryl ynamides gave only anti-addition of phenylacetylene providing ynenamides $(E)-532$.

\section{Scheme 201. Diastereodivergent Pd-Catalyzed Hydroalkynylation of Different Ynamides 531 with Phenylacetylene}

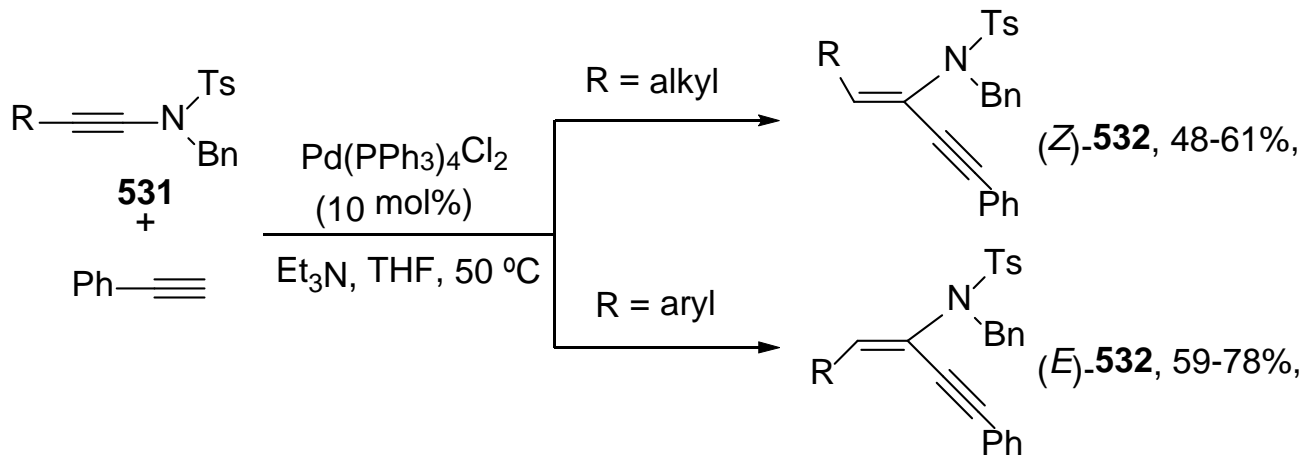

Solvent-dependent diastereodivergent anti/syn-addition of thiols to terminal alkynes catalyzed by CuI has been described by Trostyanskaya and Beletskaya. ${ }^{507}$ Steroselective trans-hydrothiolation of terminal alkynes with aromatic and aliphatic thiols gave regioselectively (Z)-alkenyl thioethers 533 in DMF or THF as solvents at 80 or $50{ }^{\circ} \mathrm{C}$, respectively, with excellent de (Scheme 202). The same reaction performed in a mixture of $i \mathrm{AmOH}$ and ethylene glycol (10:1) at $80^{\circ} \mathrm{C}$ provided products $(E)-533$ according to a $\mathrm{Cu}$-catalyzed isomerization of $Z$ - to $E$-isomers.

Scheme 202. Diastereodivergent Hydrothiolation of Terminal Alkynes Catalyzed by $\mathrm{CuI}$ in Different Solvents

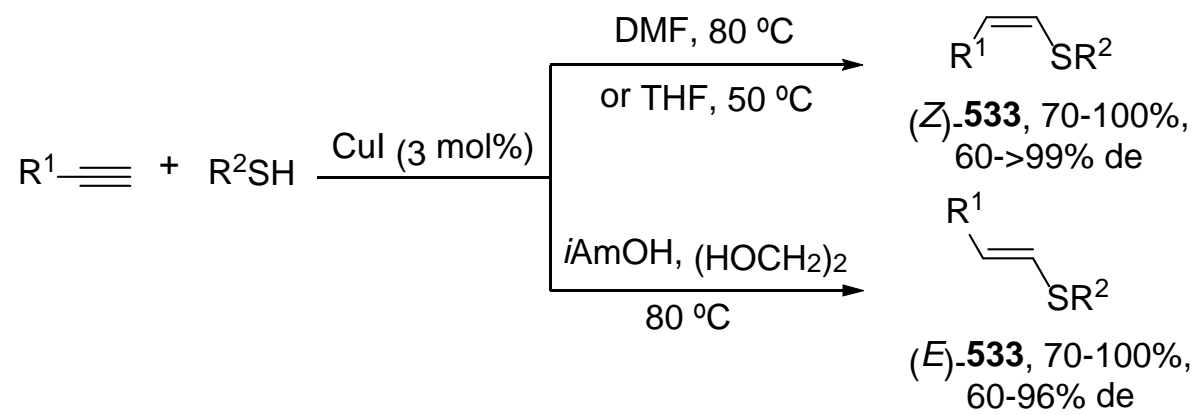

Rearrangement of propargyl esters 534 under Pt(II) or Cu(I)-catalyzed conditions allowed the diastereodivergent synthesis of $(Z)$ - and $(E)$-Knoevenagel derivatives 535, respectively (Scheme 203). ${ }^{508}$ Propargyl acetates, benzoates, acrylates, and carbonates were transformed efficiently into $\alpha$-benzylidene- $\beta$-keto esters in good yields and excellent stereoselectivities. The obtained results for the $\mathrm{Cu}(\mathrm{I})$-catalyzed rearrangement

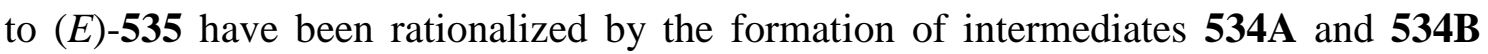
according to the Zhang's proposed mechanism for the Au-catalyzed process. ${ }^{509}$ In the 
case of $\mathrm{Pt}(\mathrm{II})$, intermediate 534C, where the Pt is coordinated to the oxygenatom acting as a Lewis acid, will form the species 534D, which suffers ring opening to provide the (Z)-isomer 535.

Scheme 203. Diastereodivergent Rearrangement of Propargyl Esters 534 to $\alpha$ Benzylidene- $\beta$-Keto Esters Catalyzed by $\mathrm{Cu}$ and $\mathrm{Pt}$

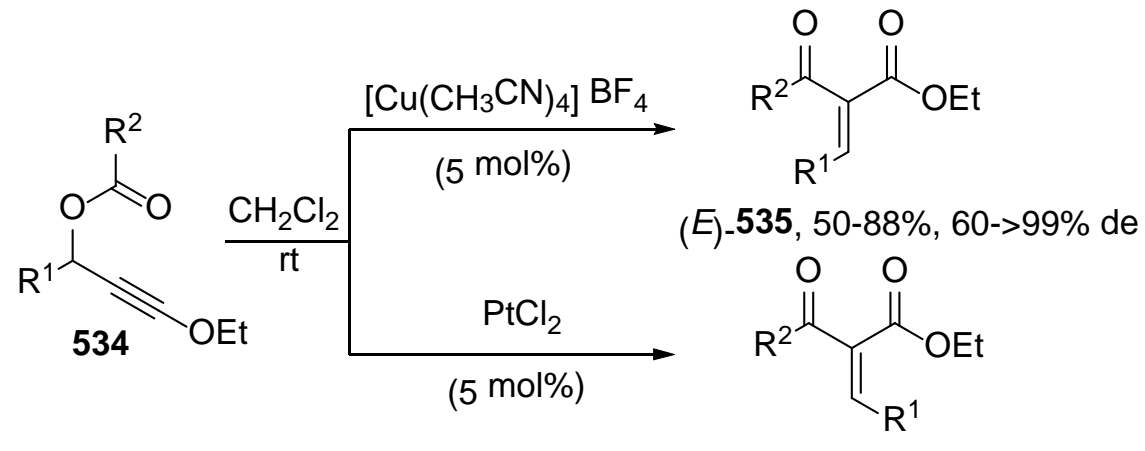

(Z)-535, 30-81\%, 40->99\% de

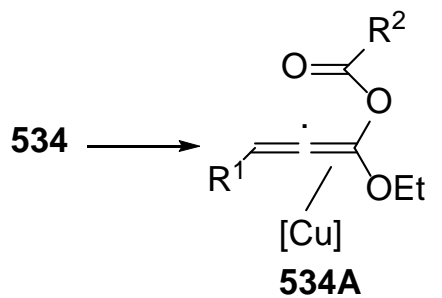<smiles>[R]C=CC(OC([R])=O)OC(C)(C)C</smiles>

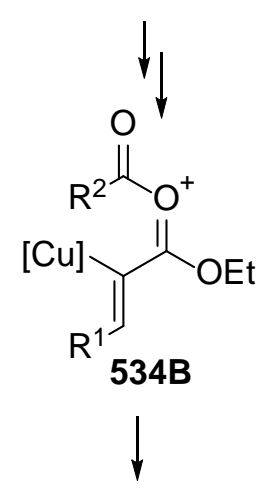

$(E)-535$

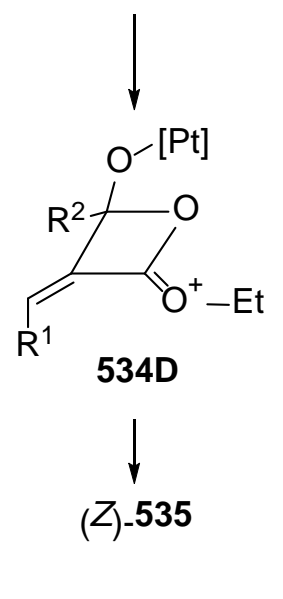

Stereocontrolled double 1,3-phosphatyloxy and 1,3-halogen migration on $\alpha$-halogen substituted propargyl phosphates 536 led to the formation of $(E)$ - or (Z)-1,3-dienes 537 (Scheme 204). ${ }^{510}$ Depending on the metal complexes (Z)-1,3-dienes 537 were obtained in the presence of $\mathrm{Cu}(\mathrm{OTf})_{2}$. However, in the presence of $\left(4-\mathrm{CF}_{3} \mathrm{C}_{6} \mathrm{H}_{4}\right)_{3} \mathrm{PAuCl}$ the corresponding $(E)$-537 were mainly formed. The proposed mechanism for the metalcatalyzed process started with the coordination to the alkyne, which promoted the 1,3migration of the phosphatyloxy group to give intermediate A. Further elimination of the metal gave the allenyl phosphate $\mathbf{B}$ (for $\mathrm{Cu}$ ). In the case of $\mathrm{Au}$ a $\pi$-allyl cation $\mathbf{E}$ was formed after halogen abstraction. In the case of $\mathrm{Cu}$, the metal was coordinated to the phosphate giving $\mathbf{B}$, which after halogen abstraction produced the $\pi$-allyl intermediate $\mathbf{C}$ and the halogen was transfered syn to the phosphate. The DFT calculations proposed the 
formation of the TS I by an associative mechanism and the complex TS II for the softer Au by a dissociative pathway. ${ }^{511}$

Scheme 204. Diastereodivergent Double Migration on $\alpha$-Halogen Propargyl Phosphates 537 Catalyzed by $\mathrm{Cu}$ and Au Complexes

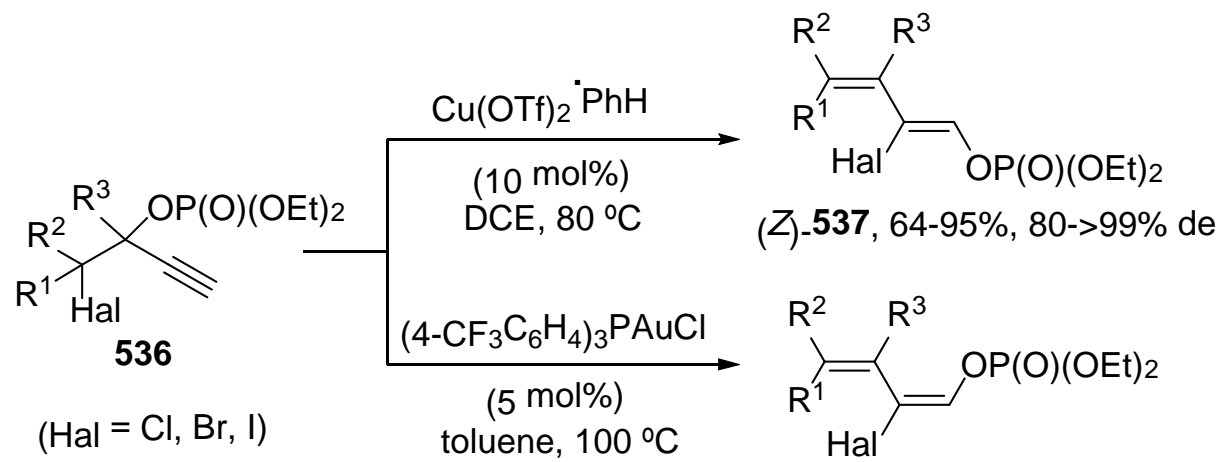

(E)-537, 89-98\%, 20-92\% de

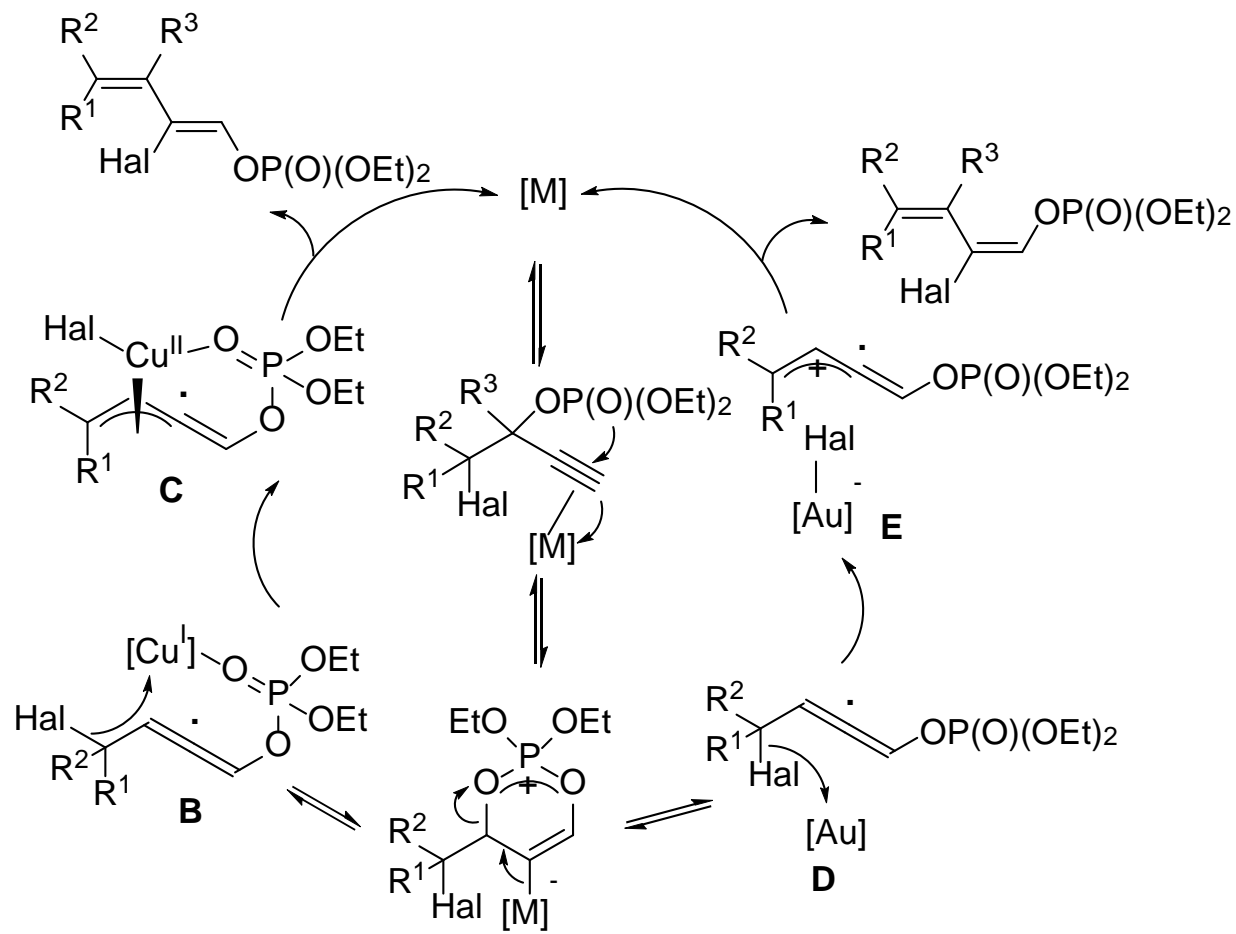

A

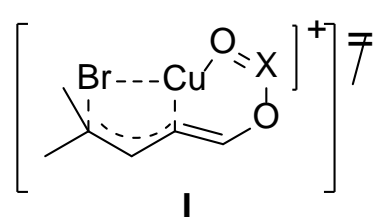

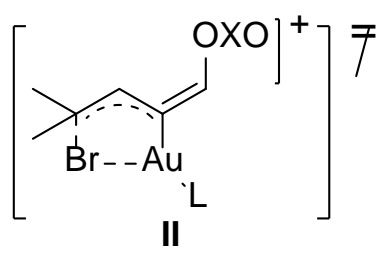

Recently, a diastereodivergent Mo-catalyzed deoxygenation of epoxides to alkenes has been reported. ${ }^{512}$ In the presence of different phosphines as reductants (Z)-alkenes were formed in the case of DPPE and (E)-alkenes with $\mathrm{PPh}_{3}$ (Scheme 205). Under the mild reaction conditions, several functional groups were tolerated. A plausible mechanism has been proposed based on the reduction of $\mathrm{Mo}^{\mathrm{VI}} \mathrm{O}_{2} \mathrm{Cl}_{2}$ by phosphines 
generating $\mathrm{Mo}^{\mathrm{IV}} \mathrm{OCl}_{2}$ as active species, which reacted with the epoxide forming a molybdena-2,5-dioxolane A. In the case of DPPE an extrusion of the (Z)-alkene formed a dioxomolybdenum(VI) complex $\mathbf{B}$, which after oxidation gave back the catalytic species. On the other hand, the anti-attack of $\mathrm{PPh}_{3}$ to dioxolane $\mathbf{A}$ gave an inversion of the stereochemistry forming the oxaphosphetane intermediate $\mathbf{C}$, which afforded the (E)-alkene and the catalytic species.

Scheme 205. Diastereodivergent Deoxygenation of Epoxides to Alkenes Catalyzed by $\mathrm{MoO}_{2} \mathrm{Cl}_{2}$ Using Different Phosphines
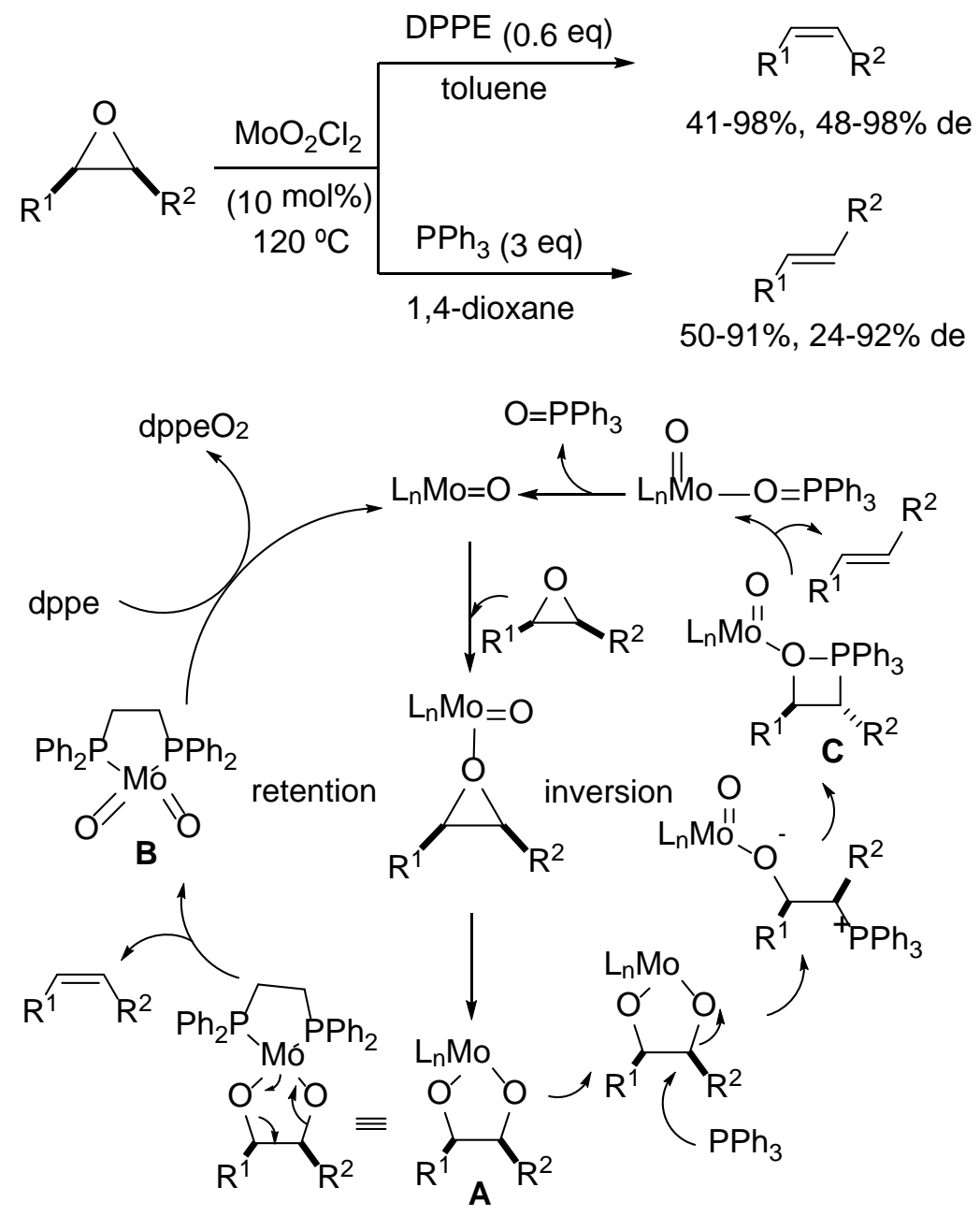

\section{STEREODIVERGENCE IN CYCLIC SYSTEMS}

In general, acyclic systems are more prone to stereodivergent catalytic processes than cyclic ones. In this Section additions to $\mathrm{C}-\mathrm{C}$ double bonds such as conjugate additions and hydrogenations are the most studied reactions. Other reactions already described in acyclic systems, such as hydroboration of cycloalkenes, $\alpha$-functionalization of 
cycloalkenones, ring opening of oxiranes, nucleophilic allylic substitutions and other reactions will be considered as well.

\subsection{Additions to $\mathrm{C}=\mathrm{C}$ Bonds}

Enantioselective stereodivergent metal-catalyzed addition of nucleophiles to cyclic alkenes, mainly cycloalkenones, is the most studied process of this section. In the case of cycloalkenes enantioselective hydrogenations have been used extensively as key step in the total synthesis of natural products and bioactive compounds.

3.1.1. Conjugate Additions. As it has been previously considered in Section 2.3.1 for acyclic systems, enantiocatalyzed conjugate additions (CA) of different carbon nucleophiles is the most widely used method for the construction of C-C bonds. ${ }^{280-288}$ In the case of cyclic compounds, cycloalkenones are usually employed as substrates. Stereodivergent asymmetric conjugate additions have been performed with cuprates, organomagnesium and organozinc compounds using copper-complexes as catalysts, and arylboronic acids as nucleophiles under Rh-catalyzed conditions. On the other hand, the stereodivergent asymmetric Michael reaction of 1,3-dicarbonyl compounds and enolates to cycloalkanones are also carried out using chiral metal complexes.

Enantiodivergent results were obtained by Tomioka and co-workers in the CA of cuprates, obtained from organomagnesium chlorides and copper iodide or from alkyllithiums and copper cyanide, ${ }^{282}$ to cyclohexenone using a stoichiometric amount of chiral amidophosphines 279 (Scheme 101). For instance, in the case of the addition of magnesium $n$-butylcyanocuprate in the presence of ligand 279e, (S)-538 was formed, whereas an opposite configuration was generated in the case of lithium $n$ butylcyanocuprate (Scheme 206). ${ }^{513,514}$ The conjugate addition of different alkyl Grignard reagents can be performed using only $8 \mathrm{~mol} \%$ of $\mathrm{CuI}$ and $32 \mathrm{~mol} \%$ of the chiral phosphine $\mathbf{2 7 9 b}$ in ether at $-78{ }^{\circ} \mathrm{C}$ giving $(S)-538$ with ee u to $91 \%$. ${ }^{514}$

\section{Scheme 206. Enantiodivergent Conjugate Addition of $n$-Butylcuprates to Cyclohexenone in the Presence of Phosphine 279e}

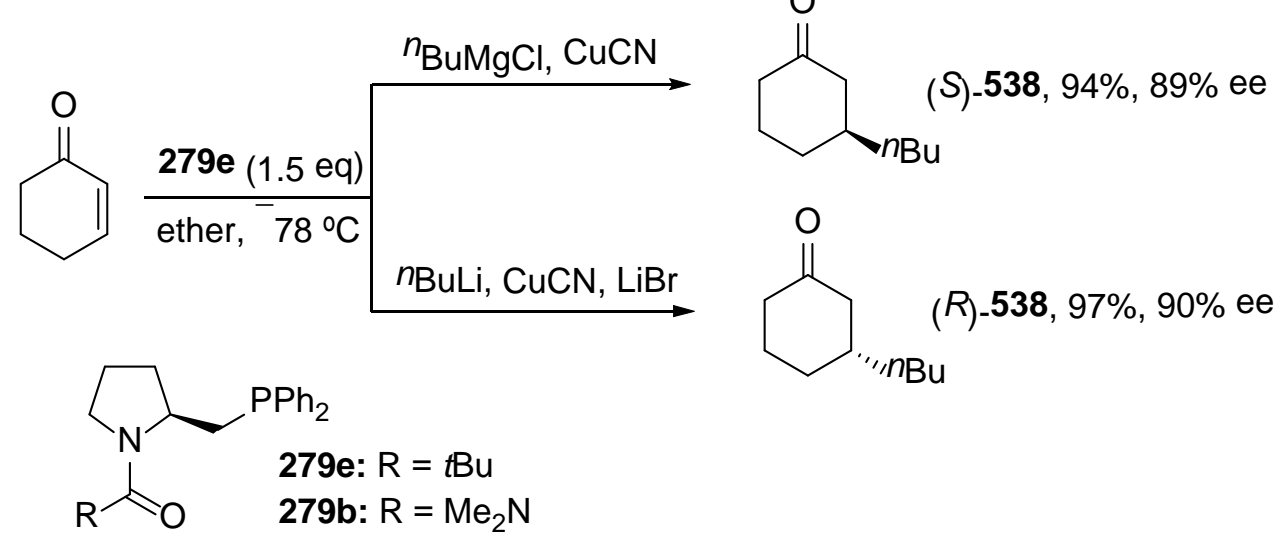


Grignard reagents gave enantiodivergent 1,4-addition to cycloalkenones when $\mathrm{Cu}(\mathrm{I}) /(R, R)$-TADDOL complexes $\mathbf{5 3 9}$ and $\mathbf{5 4 0}$ were used as catalysts (Scheme 207). ${ }^{515}$ Alkylmagnesium chlorides gave (S)-541 when complex 539 was used, whereas complex 540 gave enantiomeric products $(R)-\mathbf{5 4 1}$.

Scheme 207. Enantiodivergent Conjugate Addition of Alkylmagnesium Chlorides to Cycloalkenones Catalyzed by Cu Complexes 539 and 540

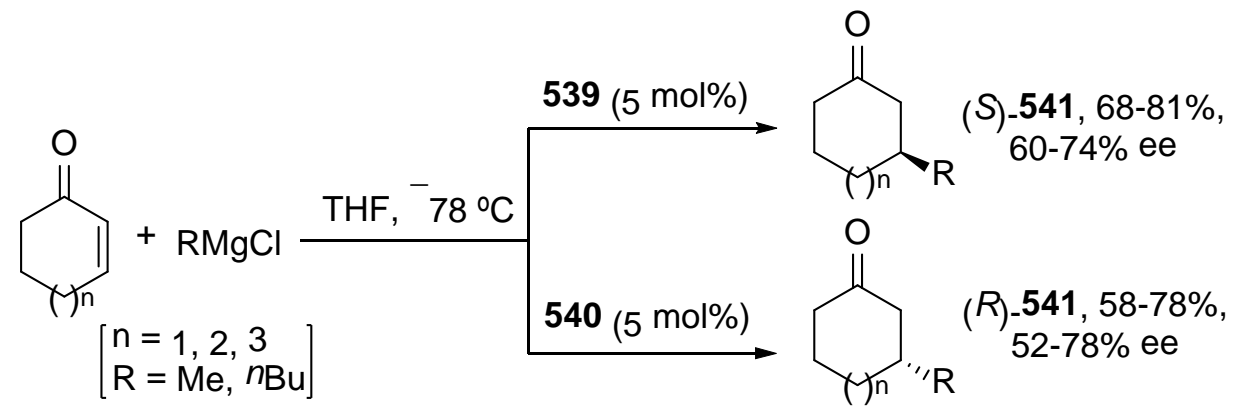<smiles>CC1(C)O[C@H]2[C@@H](O1)C(c1ccccc1)(c1ccccc1)O[Si](Cl)(c1ccccc1)C2(c1ccccc1)c1ccccc1</smiles>

539<smiles>CN1CC(Cl)(c2ccccc2)C(c2ccccc2)(c2ccccc2)OC2C(C)(C)OC2(c2ccccc2)C1c1ccccc1</smiles>

540

A reversal of enantioselectivity was observed by Sakaguchi and co-workers in the copper-catalyzed conjugate addition of alkylzincs to cycloalkenones using the same carbene derived from the chiral ligand 542. ${ }^{516-520}$ The addition of diethyl or di- $n$ butylzinc to cyclic enones catalyzed by copper complexes of the carbene derived from the azolium salt 542a gave adducts $(S)-543$ in the case $\mathrm{Cu}(\mathrm{OTf})_{2}$ with ee up to $84 \%$ (Scheme 208). However, changing the copper salt by $\mathrm{Cu}(\text { асас) })_{2}$ the corresponding enantiomers $(R)-543$ with ee up to $82 \%$ were found. ${ }^{516}$ In the case of the carbene derived from theazolium salt $\mathbf{5 4 2 b}$ products $(R)$-543 were obtained with ee up to $91 \% .^{517}$

Scheme 208. Enantiodivergent Conjugate Addition of Dialkylzinc to Cycloalkenones Catalyzed by Different Cu Complexes with Ligands 542 


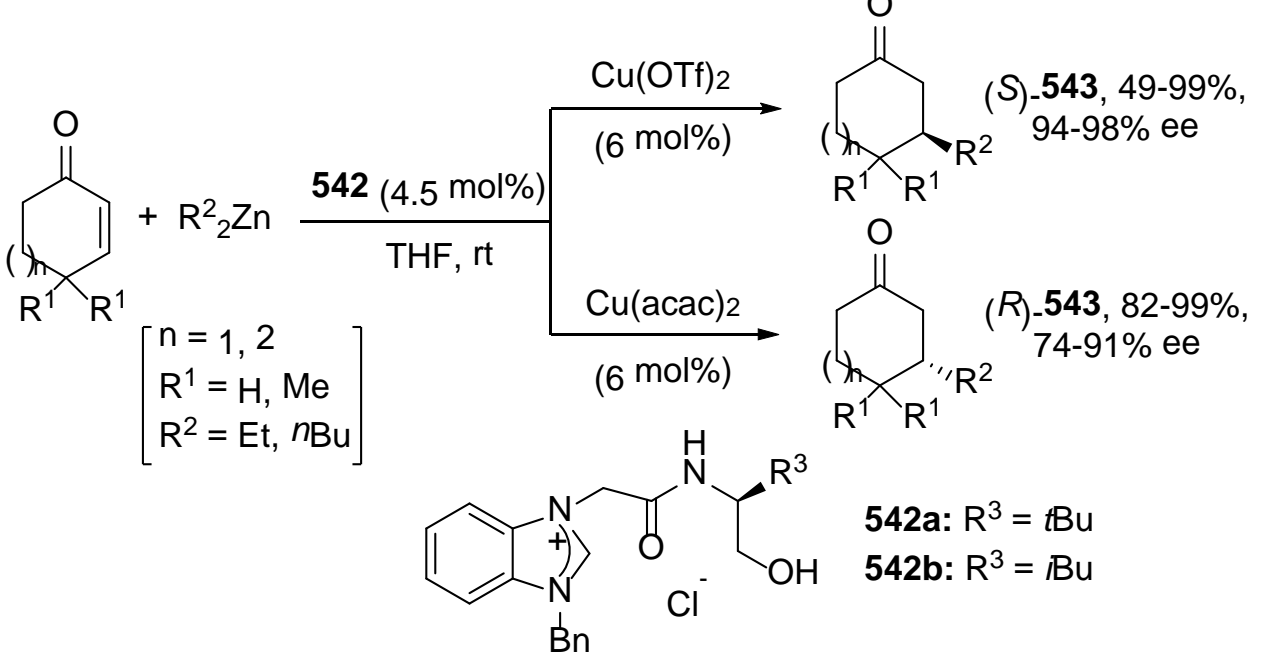

Sakaguchi and co-workers recently observed an unexpected reversal of enantioselectivity based on the addition order of the reagents in the reaction of diethylzinc with cyclohexenone employing $\mathrm{Cu}(\mathrm{OTf})_{2} \cdot \mathrm{PhH}(2-6 \mathrm{~mol} \%)$ and a $\mathrm{N}$ heterocyclic carbene (NHC)-Ag complex derived from the azolium salt 542a (4-10 mol\%) in THF at rt. Upon adding $\mathrm{Et}_{2} \mathrm{Zn}$ to a solution of the same catalyst and cyclohexenone, $(R)-538$ was obtained in $72 \%$ ee. However, when $\mathrm{Et}_{2} \mathrm{Zn}$ was added first and then the cyclohexenone (S)-538 was isolated in $88 \%$ ee. ${ }^{521}$

Rhodium-catalyzed asymmetric conjugate addition of arylboronic acids to electrondeficient alkenes was initially reported by Miyaura, Hayashi and coworkers. ${ }^{522}$ Recently, the use of chiral sulfoxide-olefin as ligands has shown interesting enantiodivergent effects in the CA of arylboronic acids to cycloalkenones. ${ }^{523}$ The position of the substituents on the alkene ligand $\mathbf{5 4 4}$ has a dramatic effect on the stereocontrol (Scheme 209). Compounds 541 were obtained with (R)-configuration using ligand 544a, whereas the (S)-enantiomers were formed with ligand 544c in high yield and ee. The facial coordination bias was proposed according to X-ray structural analysis of the Rh-complexes with ligands 544b and 544d. In the case of 544b, by means of the corresponding TS 544B the $\alpha$-Re face was preferred, while for ligand 544d TS 544D showed that the $\alpha$-Si face was the favored one. Similar results were observed by the same group with ligand $(E)-544 c$ and $(Z)-544 c$ providing $(S)$-541 and $(R)-541$, respectively with comparable yields and enantioselectivities. ${ }^{524}$

Scheme 209. Enantiodivergent Conjugate Addition of Arylboronic Acids to Cycloalkenones Catalyzed by Different Rh Complexes of Ligands 544 


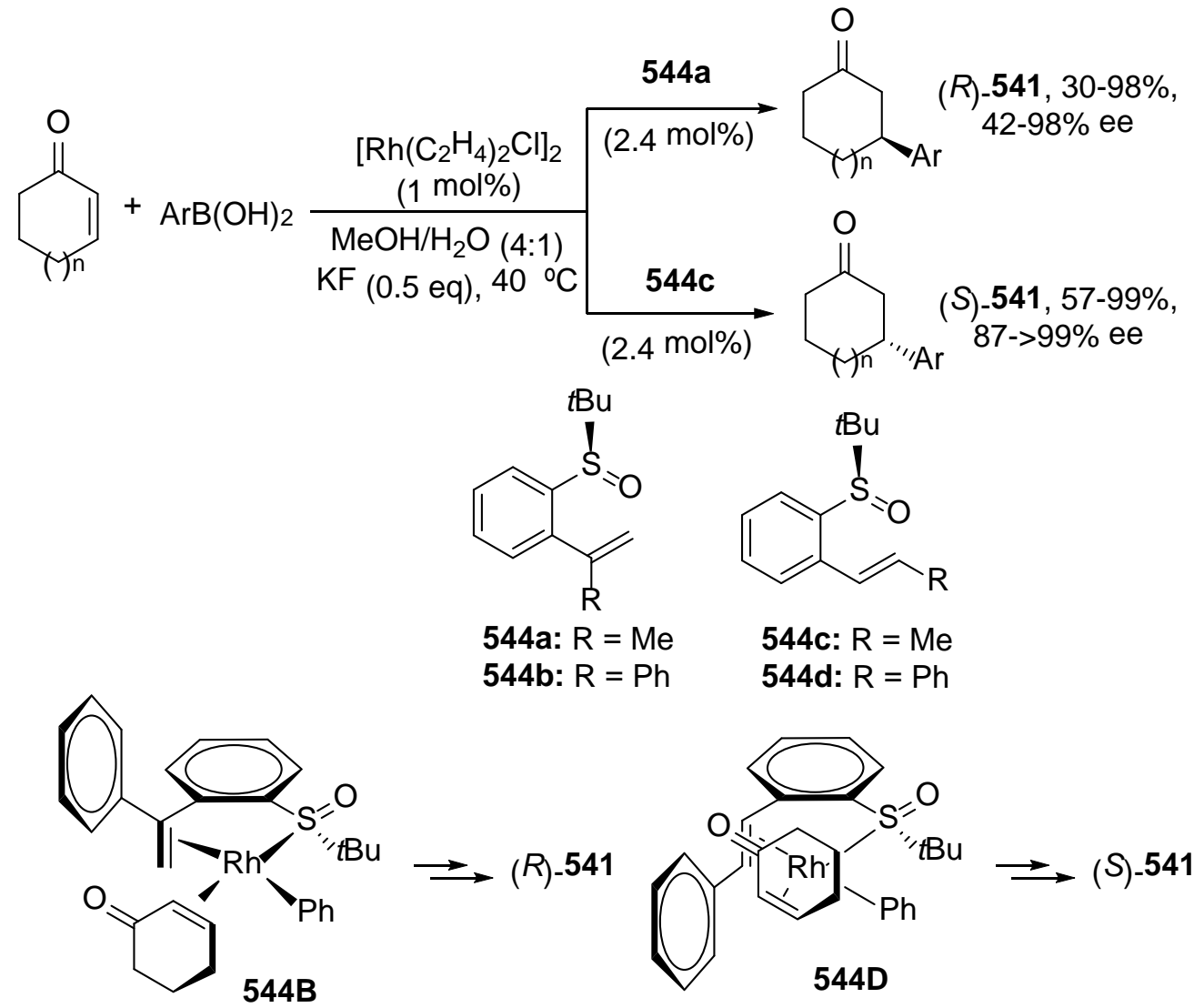

Diastereodivergent asymmetric CA of $\alpha$-cyanoacetates 545 to cycloalkenones catalyzed by chiral palladacycles has been described by Peters and co-workers. ${ }^{525}$ Bimetallic complex 546 gave in the presence of $\mathrm{AcOH}(0.2 \mathrm{eq})$ the corresponding adducts $(R, R)-548$ with de up to $84 \%$ and ee up to $99 \%$. This bimetallic catalyst could be recycled at least for 5 runs. On the other hand, monometallic palladacycle 547 (0.5-4 mol\%) and AcOH as co-catalyst provided products (S,R)-548 with de up to 82\% de and ee up to $99 \%$ (Scheme 210). The cooperativity of both metal centers explained the attack of the enolate to cyclohexanone in the TS 546A affording $(R, R)$-548. In the case of the monometallic catalyst the Pd coordinates the enolate and the pentaphenylcyclopentadienide ligand is shielding one face of the enolate in the TS 546A, which yielded intermediate 547B. Alternatively, the Pd can coordinate to the enone in 547'B and then the attack of the enolate will take place giving $(S, R)-548$.

Scheme 210. Diastereodivergent Asymmetric Conjugate Addition of $\alpha$ Cyanoacetates 545 to Cycloalkenones Catalyzed by Chiral Bis- and Monopalladacycles 546 and 547 


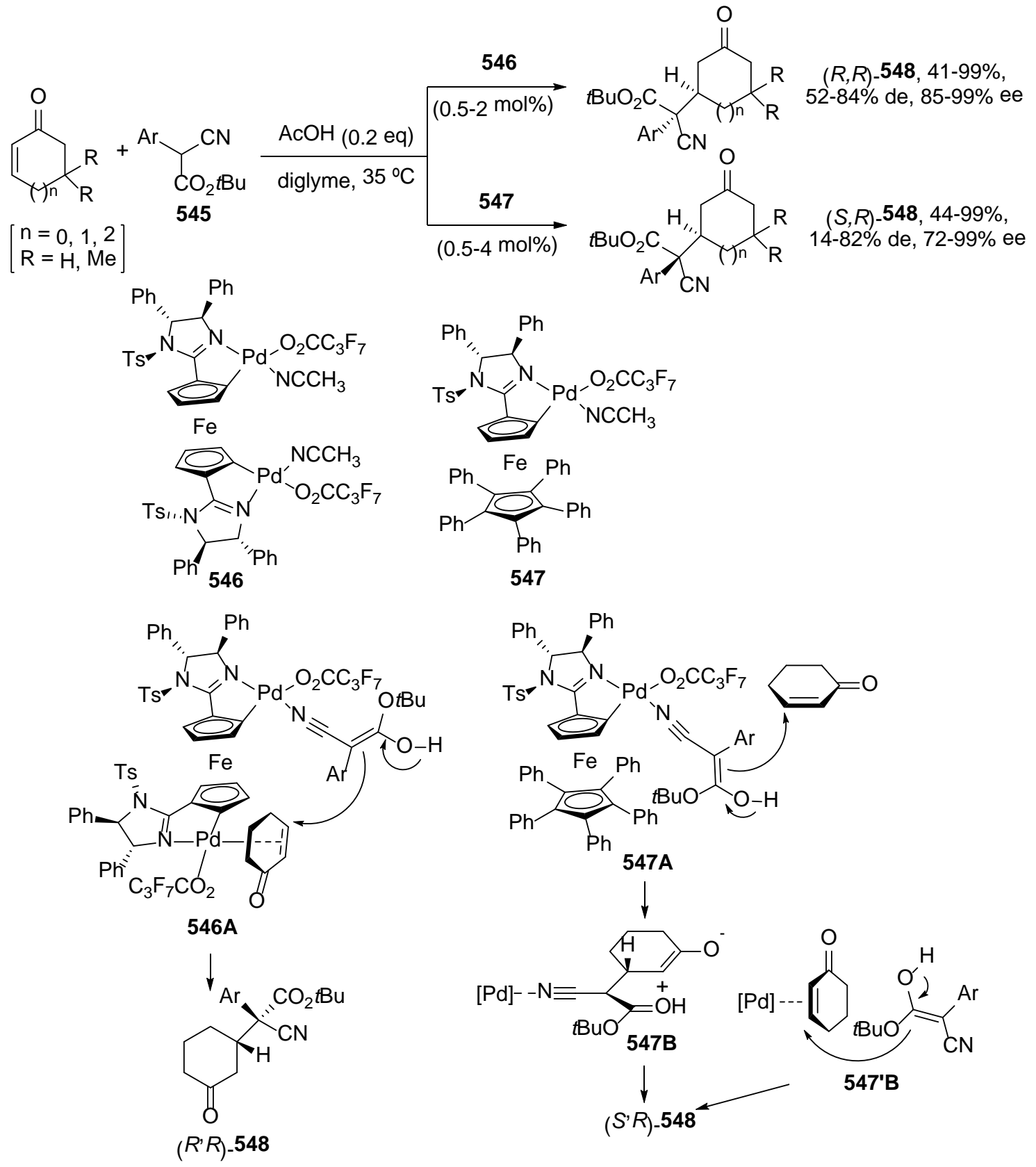

Modest level of enantioselectivity has been achieved in the Michael addition of 2methoxythiophenol to cyclohexenone using a light- and temperature-driven molecular motor, which by a $360^{\circ}$ unidirectional rotatory cycle it provoks the organocatalysts $\mathbf{5 4 9}$ to change its helicity. ${ }^{526}$ Wang and Feringa designed the compound 549 bearing a dimethylaminopyridine unit and a thiourea group acting as Brønsted base and hydrogen bonding donor, respectively. These units are connected by an alkene moiety able to rotate controlling the helical orientation. Organocatalyst $(M, M)$-cis-549 was formed after irradiation of the organocatalyst $(P, P)$-trans-549 at $312 \mathrm{~nm}$, giving adduct $(S)$-550 in $50 \%$ ee. After heating $(M, M)$-cis-549 at $70{ }^{\circ} \mathrm{C}$, the corresponding $(P, P)$-cis-549 was formed, which provided (R)-550 in 54\% ee (Scheme 211). The proposed ternary complex 549A explains the bifunctional behavior of these types of chiral organocatalysts. 
Scheme 211. Enantiodivergent Conjugate Addition of 2-Methoxythiophenol to Cyclohexenone Catalyzed by Molecular Motor Organocatalyst 549

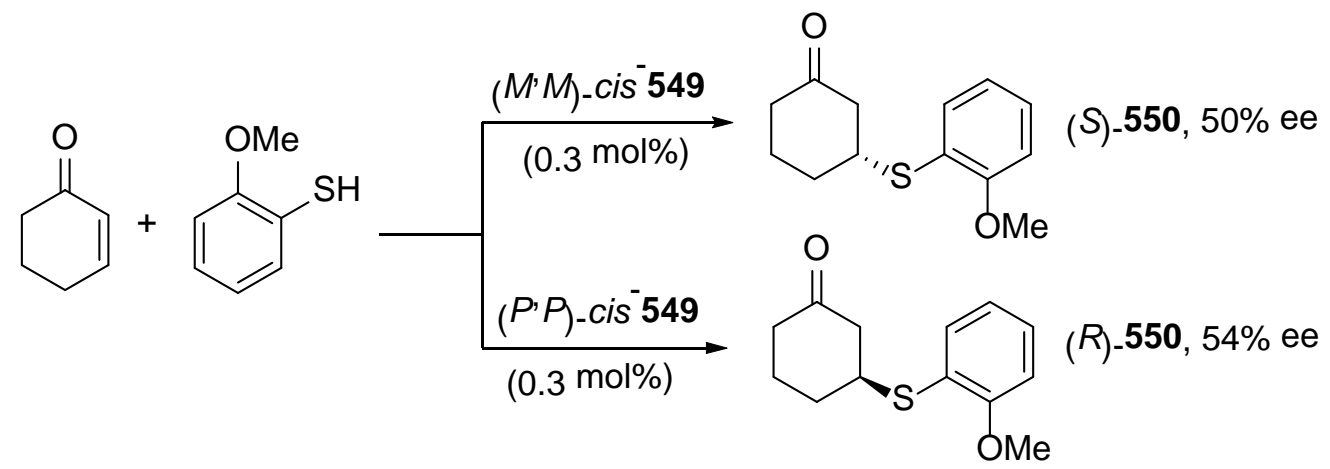<smiles>COc1ccnc(Nc2ccc(-c3cc(C)c4c(c3C)/C(=C3/c5c(C)c(-c6ccc(NC(=S)Nc7cc(C(F)(F)F)cc(C(F)(F)F)c7)cc6)cc(C)c5CC3C)C(C)C4)cc2)c1</smiles>
(P'P)-trans 549

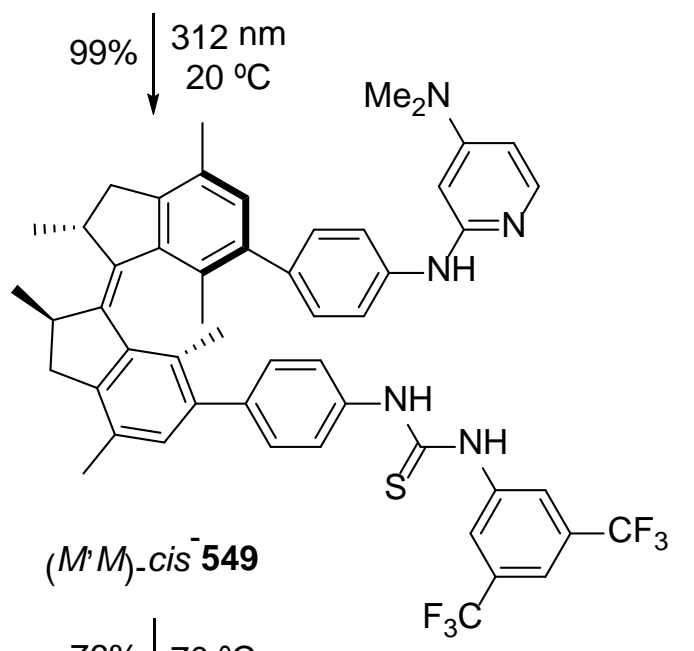<smiles>COc1ccccc1Sc1ncccc1NCCNC(=S)Nc1cc(N(C)C)cc[nH+]1</smiles>
$\left(M^{\prime} M\right)$-cis 549 $76 \% \sqrt{ } 70^{\circ} \mathrm{C}$<smiles>Cc1cc(-c2ccc(NC(=S)Nc3cc(C(F)(F)F)cc(C(F)(F)F)c3)cc2)cc(-c2cc(C)c3c4c2[C@@H](C)c2cc(C)c5c(c2-c2ccc(Nc6cc(N(C)C)ccn6)cc2)C(=C4[C@H](C)C3)[C@H](C)C5)c1</smiles> 
In conclusion, enantiodivergent conjugate additions of organocuprates or organozinc reagents to cycloalkenones are governed by the chiral ligand of the $\mathrm{Cu}(\mathrm{II})$ salt. In the case of the Rh-catalyzed conjugate addition of arylboronic acids to cycloalkenones, regio- or diastereomeric alkenes, acting as chiral ligands, controlled the reversal of enantioselectivity. Diastereodivergent conjugate additions of cyanoacetates to cycloalkenones were controlled by mono and dimetallic palladacycles as catalysts. A new concept in organocatalytic enantiodivergent conjugate addition of a thiophenol to cyclohexenone has been developed based on a helical bifunctional system covalently bonded to a molecular motor.

3.1.2. Hydrogenation. Several examples on stereodivergent hydrogenations of exoand endo-cyclic alkenes are related to the synthesis of natural products. A diastereodivergent deuteration of cyclic 2,3-dehydroamino acid derived from enantiopure diketopiperazines (DKP) 551 has been performed to provide threo or erythro isomers 552 depending on the protecting groups. ${ }^{527}$ For instance, starting from (Z)-phenylalanine dehydroamino acids the corresponding unprotected diketopiperazine 551a afforded threo-552a in $94 \%$ de and $91 \%$ ee by deuteration catalyzed by $10 \% \mathrm{Pd} / \mathrm{C}$ (Scheme 212). On the other hand, Boc-protected DKP 551b gave erythro-552b in 92\% de and $98 \%$ ee. These saturated DKPs were hydrolyzed to the corresponding dideuterated phenylalanines. This methodology has been applied to the synthesis of threo- and erythro-[2,3- $\left.{ }^{2} \mathrm{H}_{2}\right]$-tyrosine, DOPA and leucine.

\section{Scheme 212. Diastereodivergent Deuteration of Chiral Cyclic Diketopiperazines 551 Derived from Dehydroamino Acids}

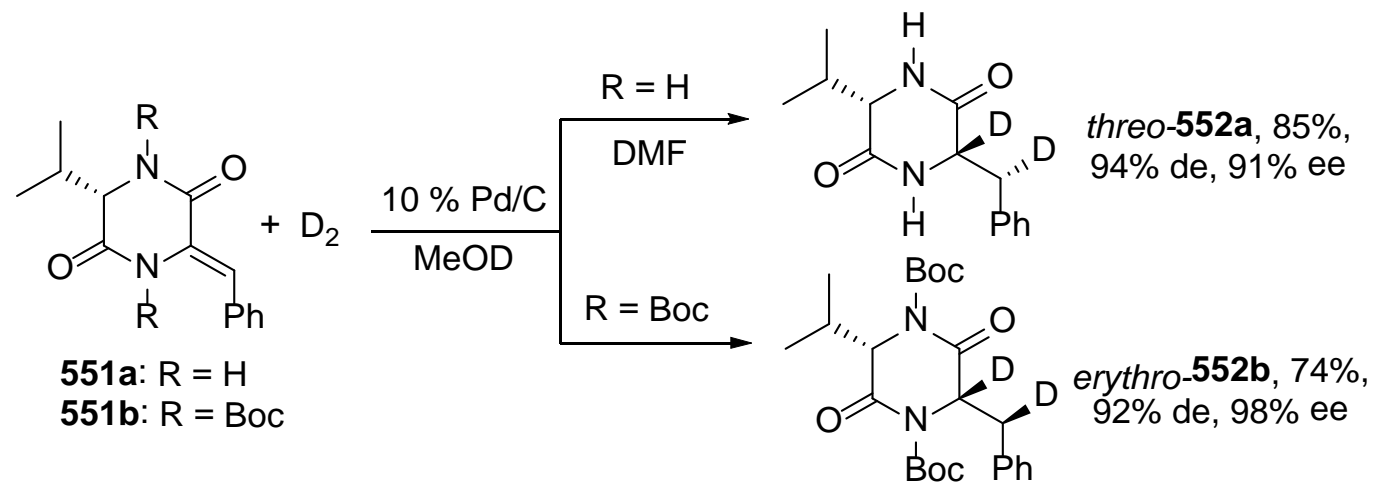

Another example of a diastereodivergent hydrogenation of an exocyclic alkene 553, which is an intermediate for the synthesis of 3,4- and 4-epi-eupomatilone 555, is shown in Scheme $213 .{ }^{528}$ Hall and co-workers prepared lactone 553 by a triflic acid-catalyzed allylboration of the corresponding aromatic aldehyde. Subsequent hydrogenation under heterogeneous conditions led to the formation of trans-554 in $71 \%$ de, whereas the homogeneous hydrogenation using Wilkinson's catalyst gave cis-554 in 67\% de. The C5 diastereomeric control took place in the case of using $\mathrm{Pd} / \mathrm{C}$ due to the approach to the catalyst surface. However, with Wilkinson's catalyst the facial selectivity was controlled by the methyl group in C4. Both products afforded after Suzuki-Miyaura 
reaction compounds 555. The synthesis of all four diastereomers of eupomatilone $\mathbf{5 5 5}$ allowed the stereochemical structural determination of all of them.

Scheme 213. Diastereodivergent Hydrogenation of the Methylene Lactone 553 Under Heterogeneous and Homogeneous Conditions

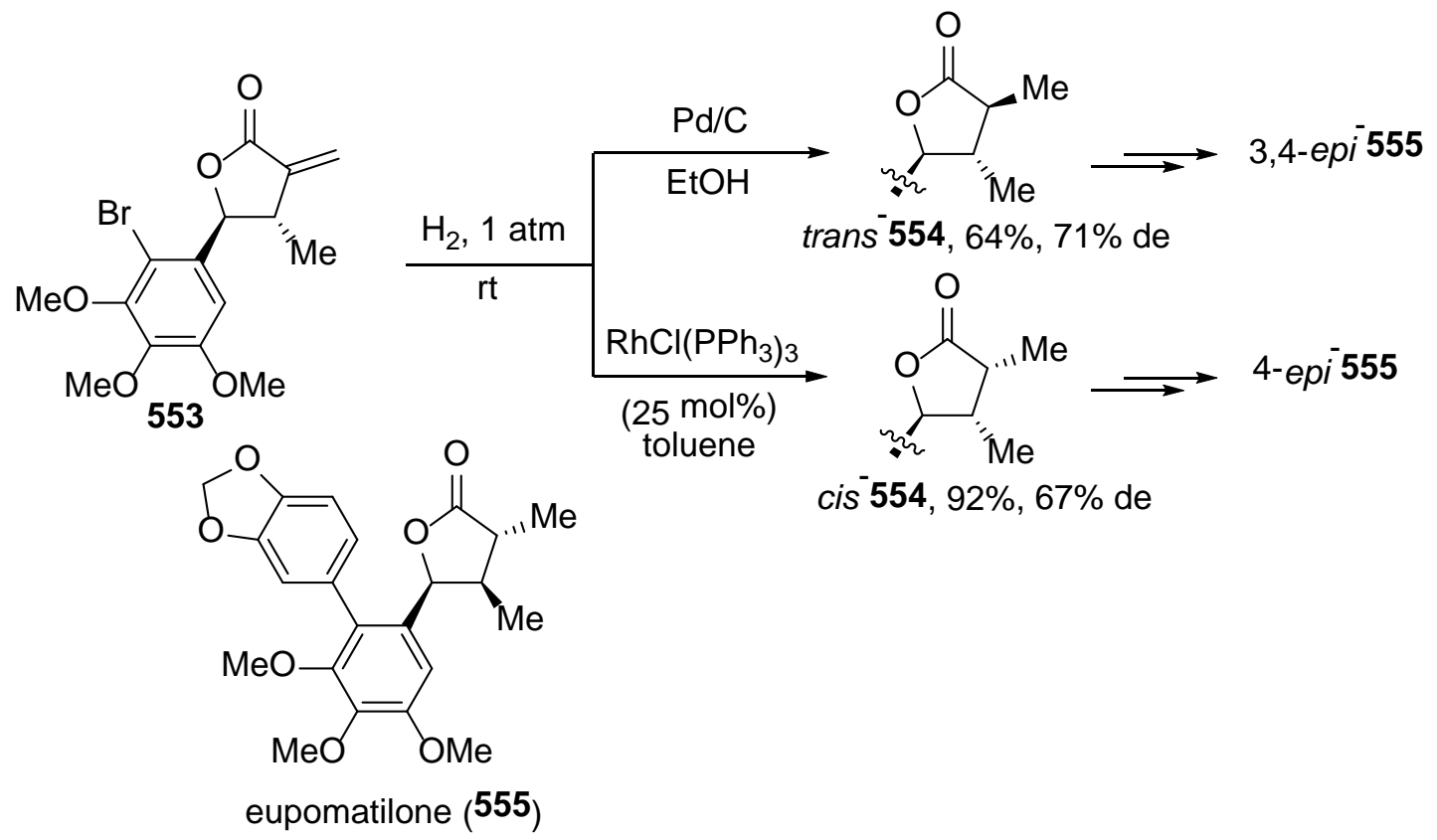

A diastereodivergent reduction step was crucial in the total synthesis of cis and trans Lycopodium alkaloids (+)-serratezomine E and huperzine $\mathrm{N}^{529}$ The selectivity of the hydrogenation of 556 using $\mathrm{Pd} / \mathrm{C}$ was directed by the methyl group located at the axial position giving quantitatively the kinetic product 557 in 94\% de (Scheme 214). Again, under the homogeneous hydrogenation conditions with Wilkinson's catalyst decahydroisoquinoline 558 was quantitatively obtained in $92 \%$ de. According to the DFT calculations a less energetic TS 558A has been postulated for the hydrogenation under homogeneous conditions, derived from the hydrorhodation step. In this TS the phenyl group of the $N$-tosyl moiety forms at least three strong $\pi$-stacking interactions with one of the phenyl groups of the triphenylphosphine.

Scheme 214. Diastereodivergent Hydrogenation of the Vinylpyridine 556 Under Heterogeneous and Homogeneous Conditions 


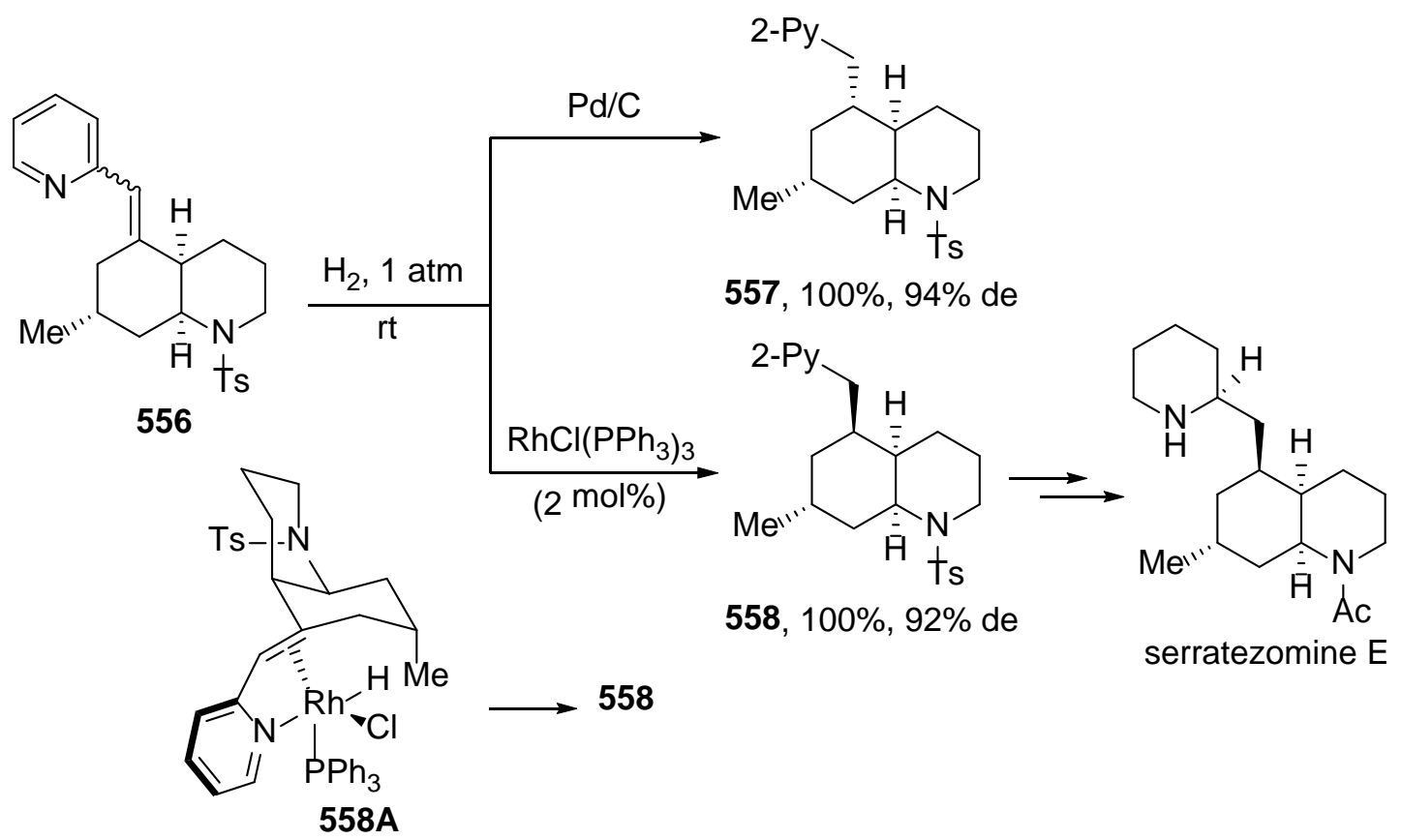

In the total synthesis of thailanstatin A, a component of Thailandensis burkholderia, with nano- to subnanomolar cytotoxicities against several human cancer cell lines, a diastereodivergent hydrogenation of intermediate $\mathbf{5 5 9}$ is one of the key step giving access to highly functionalized tetrahydropyrans $\mathbf{5 6 0}$ (Scheme 215). ${ }^{530}$ Under heterogeneous conditions the hydrogen delivery took place from the $\alpha$-face of 559, less hindered than the $\beta$-face, providing 560, after hydrolysis of the acetal unit, in $54 \%$ overall yield. In contrast, using a counteranion analogue of Crabtree's catalyst, compound epi-560 was formed in $85 \%$ yield. In this case, the hydrogen delivery occurred at the $\beta$-face facilitated by the oxygen atoms of the acetal unit.

Scheme 215. Diastereodivergent Hydrogenation of Dihydropyran 559 Under Heterogeneous and Homogeneous Conditions

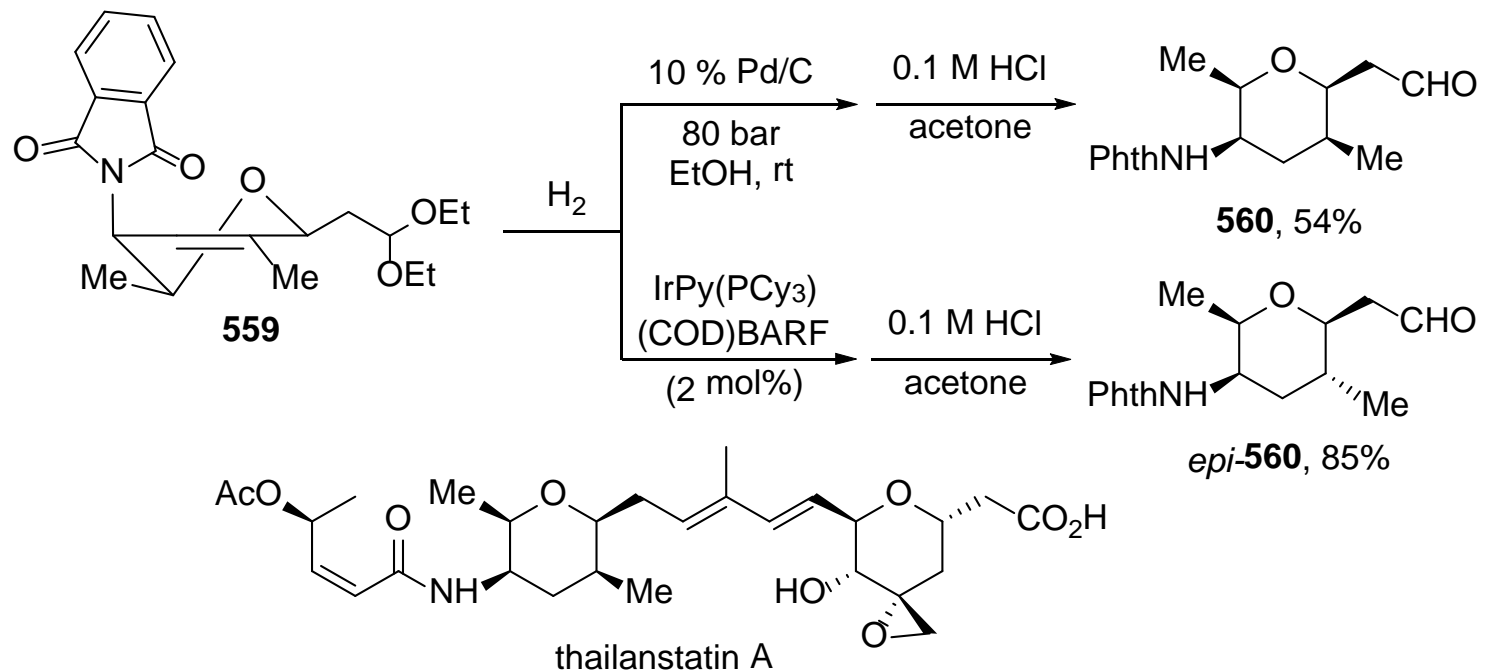


Pyrroloindolines have been obtained by an enantioselective formal Rh-catalyzed [3+2] cycloaddition of C(3)-substituted indoles with 4-vinyl-1-sulfonyl-1,2,3triazoles. $^{531}$ Diastereodivergent hydrogenation of the pyrroloindoline $\mathbf{5 6 1}$ was performed under different heterogeneous conditions. Hydrogenation with $\mathrm{Pd} / \mathrm{C}$ took place from the concave face affording 562 in 73\% de (Scheme 216). On the other hand, when $\mathrm{PtO}_{2}$ was used as catalyst the diastereomeric product 563 resulted in $87 \%$ de coming from the hydrogenation at the convex face of $\mathbf{5 6 1}$.

Scheme 216. Diastereodivergent Hydrogenation of Pyrroloindoline 561 Under $\mathrm{Pd} / \mathrm{C}$ or $\mathrm{PtO}_{2}$

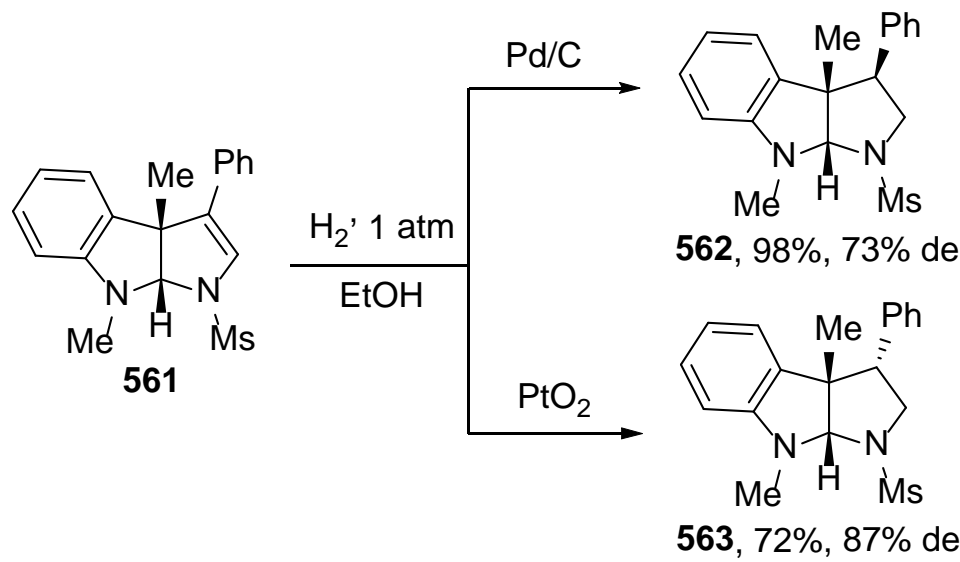

In conclusion, the diastereodivergent asymmetric hydrogenation of exocyclic and endocyclic alkenes can be controlled by using homogeneous or heterogeneous conditions.

3.1.3. Hydroboration. Metal-catalyzed hydroboration-oxidation of meso-substrates, such as the cycloadduct of cyclopentadiene $\mathbf{5 6 4}$ enantiodivergently provided alcohols 566 (Scheme 217). ${ }^{532}$ In the case of using the complex formed by $[\mathrm{Rh}(\mathrm{COD}) \mathrm{Cl}]_{2}$ and $(S, S)$-DIOP (565), compound 564 was hydroborated with catBH at $-50{ }^{\circ} \mathrm{C}$ affording, after oxidation, the corresponding $(1 R, 4 R, 5 R)$-alcohol 566 in modest $46 \%$ yield and $54 \%$ ee. When the metal complex used as catalyst was $[\operatorname{Ir}(\mathrm{COD}) \mathrm{Cl}]_{2}$, and the same chiral ligand, the corresponding enantiomer was isolated in $40 \%$ yield and $44 \%$ ee. These products $\mathbf{5 6 6}$ can be transformed into enantioenriched 2,4diaminocyclopentanols. Based on the theoretical studies on bond dissociation energies it has been found that in the Rh-catalyzed hydroboration, the $\mathrm{Rh}-\mathrm{H}$ migratory insertion step is favored, whereas in the case of Ir the Ir-Bcat insertion is the rate-determining step.

Scheme 217. Enantiodivergent Hydroboration-Oxidation of Bicyclic Alkene 564 with Different Rh and Ir Complexes and (S,S)-DIOP 565 as Ligand 


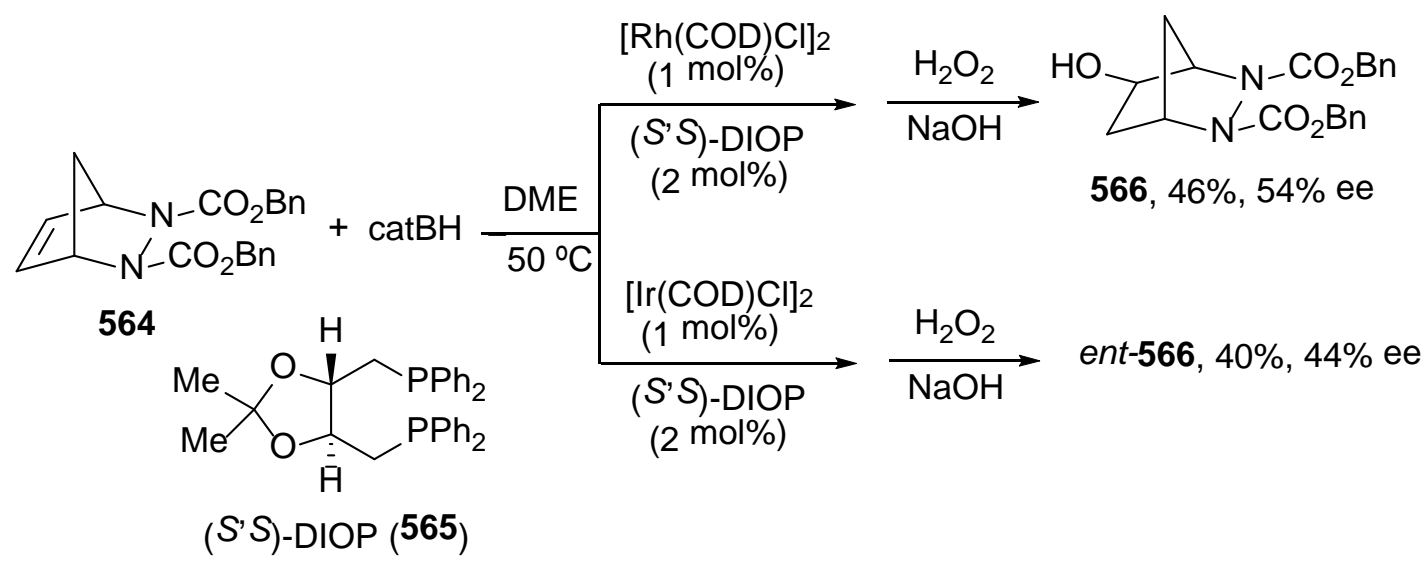

\section{2. $\alpha$-Functionalization of Carbonyl Compounds}

Enol derived from cyclic carbonyl compounds with a stereogenic center at the $\alpha$ position can undergo catalytic asymmetric protonation using chiral ligands or by metalbased decarboxylative protonation. In the case of the asymmetric protonation of achiral metal enolates the corresponding $\alpha$-substituted carbonyl compounds can be prepared starting from the racemic ones using a chiral ligand and an achiral proton source. ${ }^{53-535}$ Reversal of the enantioselectivity has been observed in the protonation of 2-methyl-1tetralone lithium enolate generated from the corresponding enol acetate 567 using a $C_{2}$ symmetric sulfonamide 568 as an internal proton source (Scheme 218). ${ }^{536}$ (R)-2Methyl-1-tetralone (570) was formed in 64\% ee using 568 in stoichiometric amounts. When dilithiated sulfonamide $\mathbf{5 6 9}$ was added and the reaction mixture was quenched with $\mathrm{AcOH}$ as an external proton source followed by addition of $\mathrm{Me}_{3} \mathrm{SiCl}$ the corresponding (S)-tetralone $\mathbf{5 7 0}$ was isolated in $45 \%$ ee.

\section{Scheme 218. Enantiodivergent Protonation of 2-Methyl-1-tetralone Lithium Enolate Using Chiral Sulfonamides 568 y 569}

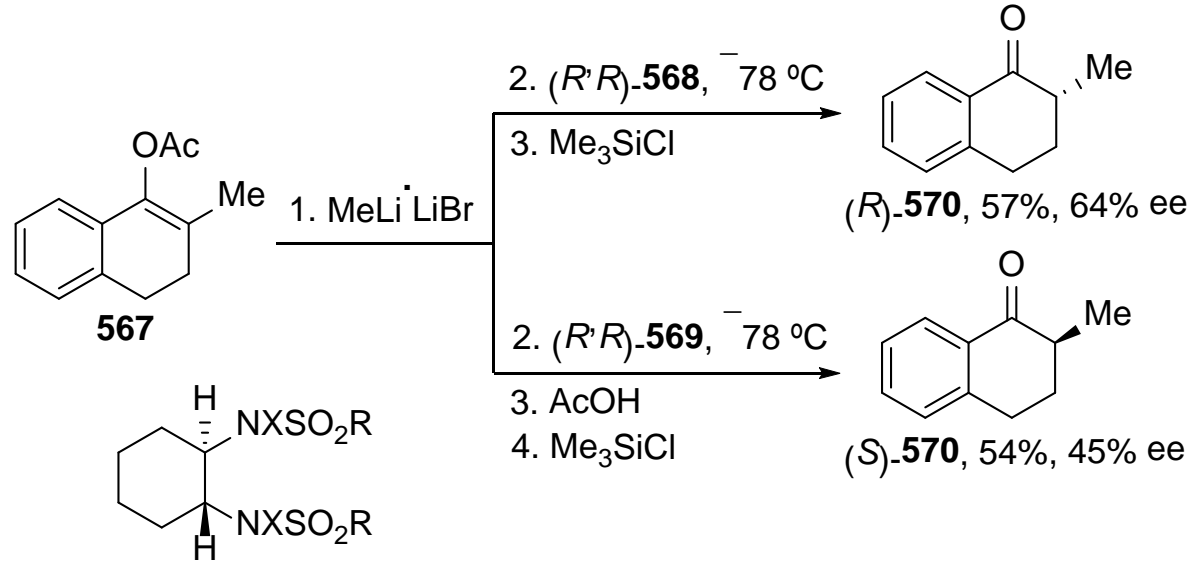

568: $\mathrm{R}=$ 2-naphthyl, $X=\mathrm{H}$

569: $R=2$-naphthyl, $X=L i$ 
Enantioselective Pd-catalyzed decarboxylative protonations have been carried out with cyclic $\alpha$-alkylated $\beta$-keto esters. ${ }^{537,538}$ This methodology generates in situ a chiral enolate using the complex $\mathrm{Pd}(\mathrm{OAc})_{2}(10 \mathrm{~mol} \%)$ and (S)-tBuPHOX 573 (12.5 mol\%) as chiral ligand in the presence of formic acid as proton source. Depending on the structure of the $\beta$-keto ester different absolute configurations were observed. For instance, cyclohexanone derivative 571 afforded (R)- $\alpha$-methylcyclohexanone (574), whereas tetralone 572 gave $(S)$ - $\alpha$-methyltetralone (570) (Scheme 219). This process is not a typical example of stereodivergent synthesis, but it has been included here because they are close related starting materials.

\section{Scheme 219. Enantiodivergent Decarboxylative $\alpha$-Protonation of Different $\beta$-Keto Esters Catalyzed by Pd and (S)-tBuPHOX 573}

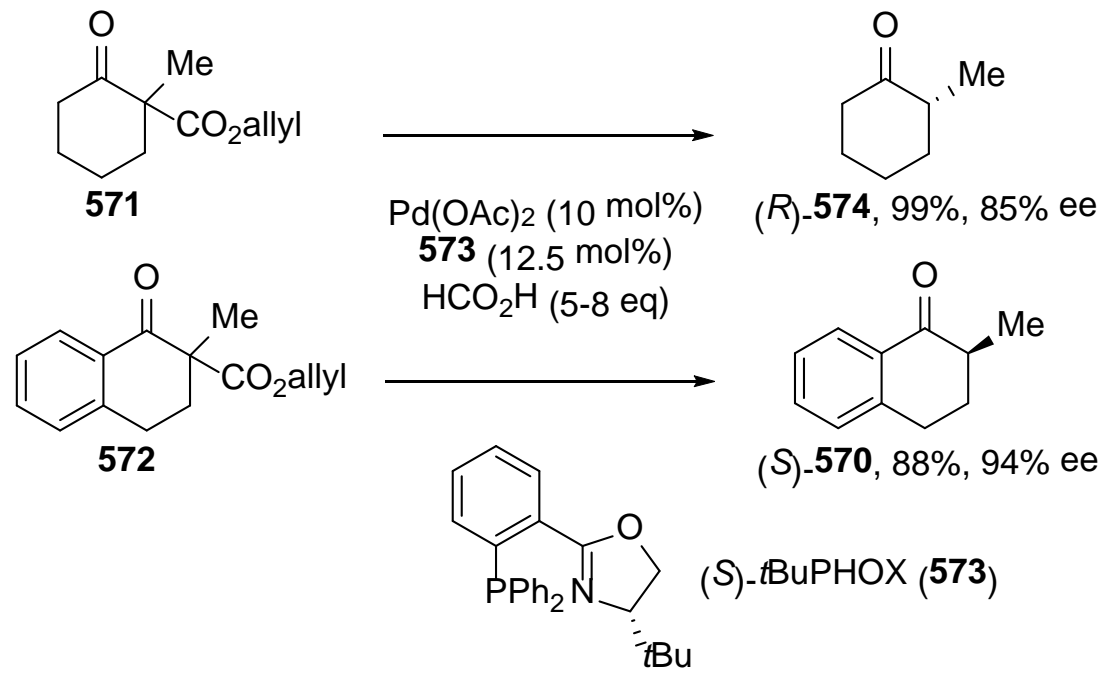

Guiry and co-workers discovered a similar enantioselective switch in the case of the isoflavone derivatives. For instance, compound $\mathbf{5 7 5}$ gave, after Pd-catalyzed decarboxylative protonation, (R)-isoflavone 577 using $(S)-\mathrm{CF}_{3}-\mathrm{tBuPHOX} 576$ as ligand and Meldrum's acid as an external proton source (Scheme 220). ${ }^{539}$ However, when formic acid was used as proton source the corresponding enantiomeric isoflavone $(S)$ 577 was prepared. Further studies about the enantiodivergent synthesis of tertiary $\alpha$-aryl 1 -indanones by a decarboxylative asymmetric protonation of the corresponding $\alpha$-aryl$\beta$-keto allyl esters under these reaction conditions have been performed by the same group. ${ }^{540}$

Scheme 220. Enantiodivergent Decarboxylative Protonation of Compounds 575 Catalyzed by Pd and (S)-CF $3-$-BuPHOX 576 


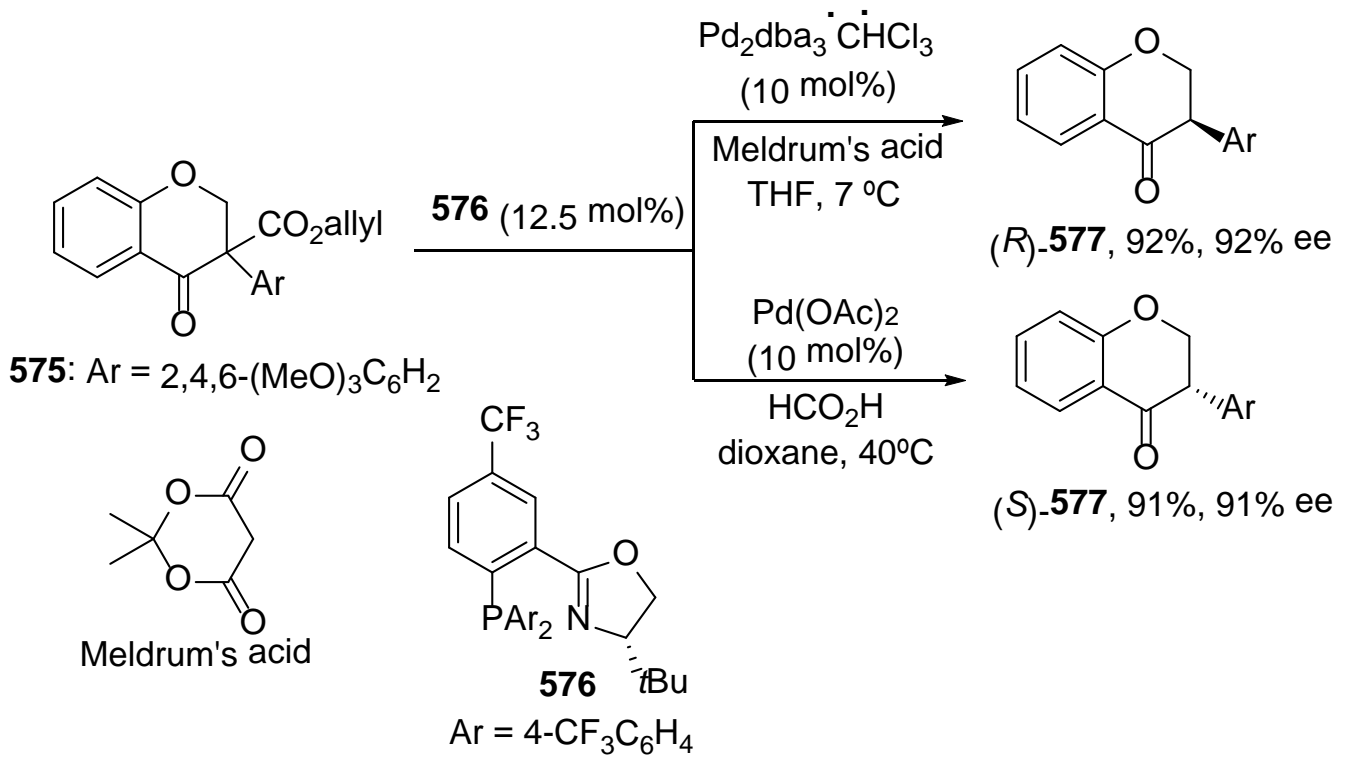

$\alpha$-Fluorination of cyclic $\beta$-keto esters has been performed by means of $N$ fluorobenzenesulfonimide (NFSI) catalyzed by the metal-box complex formed with $(S, S)-B O X-P h(433)$ as chiral ligand. Reversal of enantioselectivity has been observed depending on the metal salts used. Thus, in the case of $\mathrm{Cu}(\mathrm{OTf})_{2}$ fluorinated $\beta$-keto esters (S)-578 were obtained with ee up to $84 \%$ (Scheme 221). ${ }^{541}$ However, using $\mathrm{Ni}\left(\mathrm{ClO}_{4}\right)_{2} \cdot 6 \mathrm{H}_{2} \mathrm{O}$ and $4 \AA$ MS the enantiomers $(R)-578$ were formed with ee up to $93 \%$.

\section{Scheme 221. Enantiodivergent $\alpha$-Fluorination of $\beta$-Keto Esters Catalyzed by $\mathrm{Cu}(\mathrm{II})$} or $\mathrm{Ni}(\mathrm{II})$ and $(S, S)$-BOX-Ph 433 Complexes

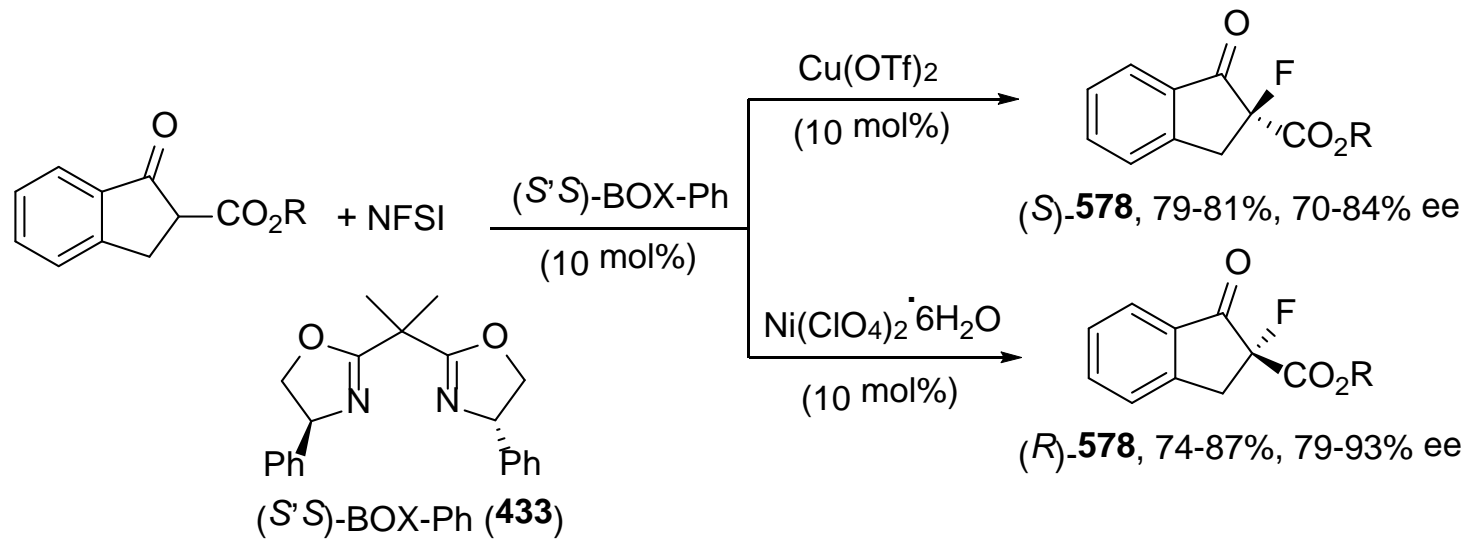

Enantiodivergent metal-catalyzed amination of 3-oxindoles 304 with tert-butyl azodicarboxylate using di- and monometallic Schiff base (related to 234) derived Ni complexes 579 and $\mathbf{5 8 0}$ has been reported by Shibasaki and co-workers. ${ }^{542}$ In the case of the homodinuclear complex 579, products $(R)$-581 were mainly formed in excellent yields and ee (Scheme 222). A reversal of the enantiofacial selectivity was obtained when monometallic complex $\mathbf{5 8 0}$ was used affording compounds (S)-581 also with comparable results. This methodology has been applied to the synthesis of a key intermediate for the synthesis of the therapeutic agent AG-041R (582), a gastrin/CCK-B receptor agonist and SSR-149415 for the treatment of anxiety and depression. 
Scheme 222. Enantiodivergent $\alpha$-Amination of 3-Oxindoles 304 by $\mathrm{Di}$ - and Monometallic Ni Complexes 579 and 580

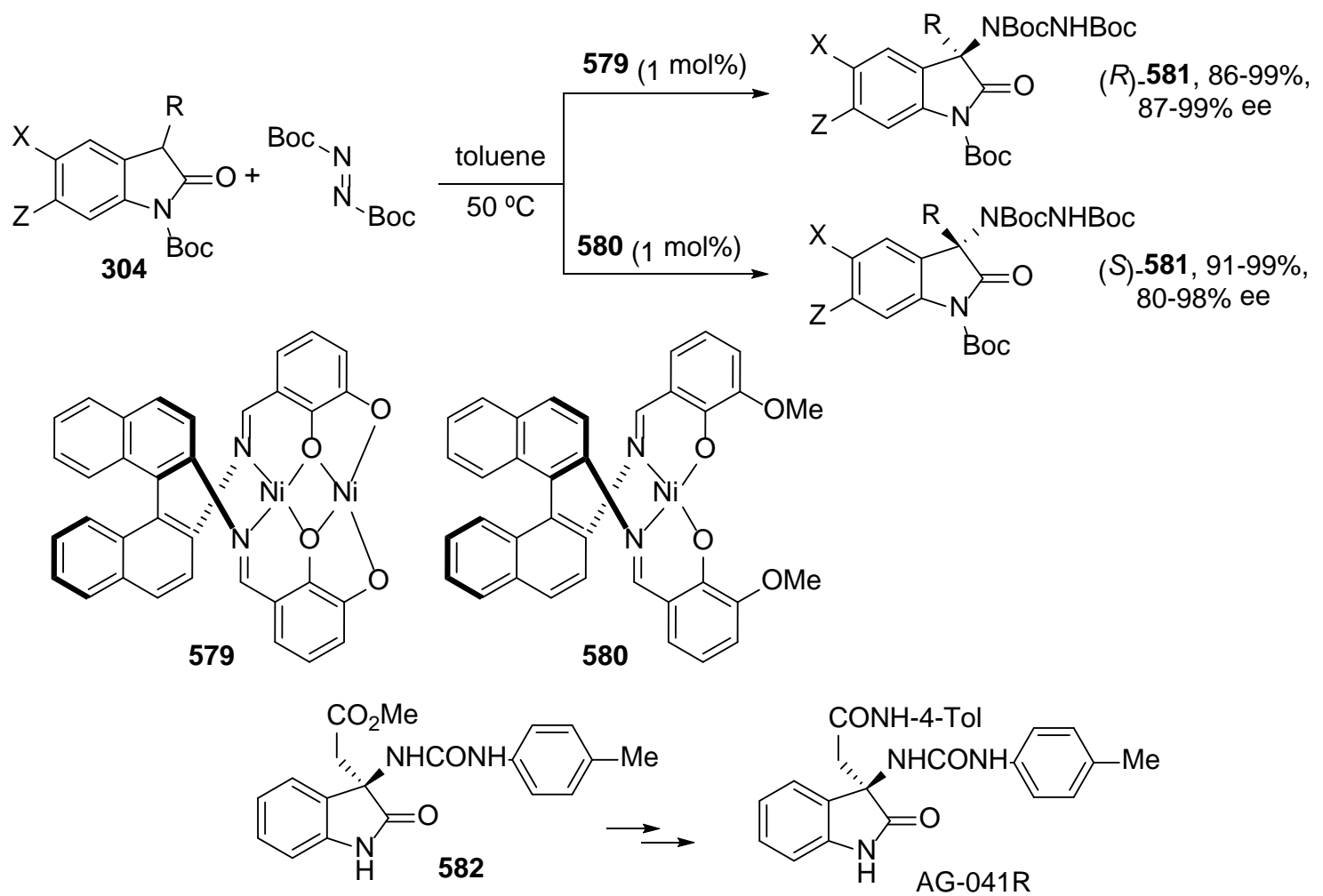

Organocatalyzed aminoxylation of cyclohexanone has been performed in an enantiodivergent manner, as in the case of acyclic aldehydes (see, Section 2.4), using the secondary binaphthylamines 435 and 436 (Scheme 159). ${ }^{376}$ When nitrosobenzene was used as electrophile and the organocatalysts 435 , the corresponding product $(R)$ $\mathbf{5 8 3}$ was obtained in $99 \%$ ee (Scheme 223). On the other hand, using the catalyst $\mathbf{4 3 6}$, (S)-583 was formed in $74 \%$ ee.

Scheme 223. Enantiodivergent Aminoxylation of Cyclohexanone Organocatalyzed by Amines 435 and 436

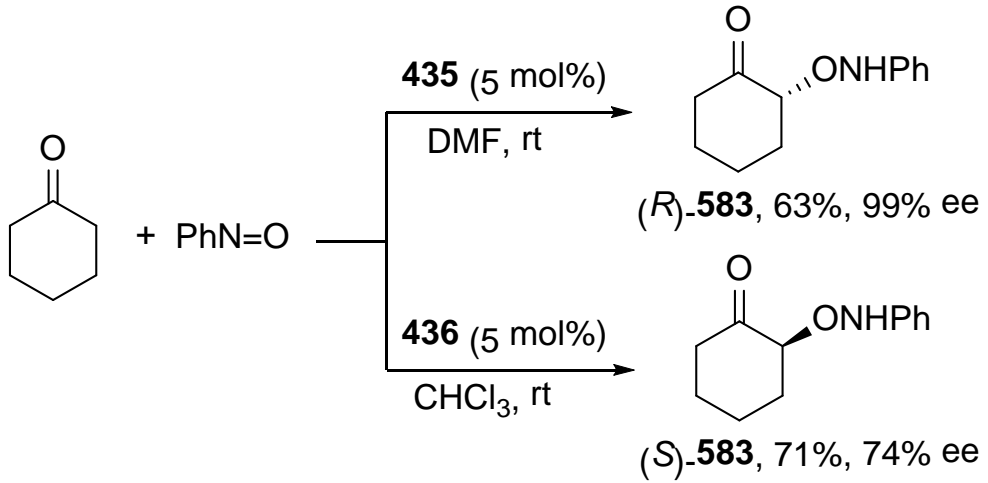

Iminium-enamine catalysis has been used for the diastereodivergent fluorination of pyrrolidinecarbaldehydes $\mathbf{5 8 4}$ with $N$-fluorobenzenesulfonimide (NFSI) using complementary organocatalysts. ${ }^{543}$ When the prolinol trimethylsilyl ether $\mathbf{4 7 5}$ (Scheme 
172) was employed as organocatalyst, trans-585 were prepared with de up to $99 \%$ (Scheme 224). On the contrary, using MacMillan's organocatalyst (R)-372 (Scheme 137) the corresponding fluorinated products cis-585 resulted with de up to 99\%. The observed stereochemical outcome was explained by the preference of the enamine double bond to adopt a (Z)-configuration.

Scheme 224. Diastereodivergent Fluorination of Pyrrolidinecarbaldehydes 584 with NFSI Organocatalyzed by Prolinol 475 and Imidazolidinone 372

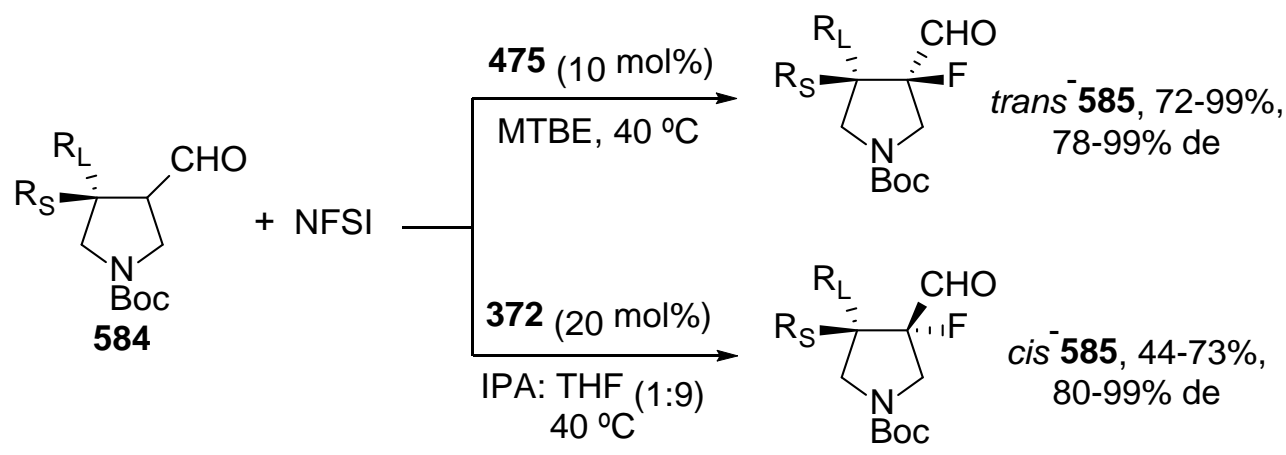

In conclusion, the enantiodivergent Pd-catalyzed decarboxylative protonation of cyclic $\beta$-keto esters can be performed by using different achiral proton sources. For the metal-catalyzed fluorination of $\beta$-keto esters the use of different metal salts with the same chiral ligand gave enantiodivergent results. For the amination of 2-oxindoles mono and bimetallic $\mathrm{Ni}(\mathrm{II})$ complexes control the enantiodivergence of the reaction. The use of different organocatalysts induces the reversal of enantio- or diastereoselectivity in the $\alpha$-functionalization of carbonyl compounds.

\subsection{Allylic Substitution Reactions}

Diastereodivergent processes in the Pd-catalyzed allylic alkylation of cyclic acetates were described by Farthing and Kočovský. ${ }^{544}$ Depending on the substituents on the amino group of racemic substrates cis-586, the reaction with diethyl sodium malonate took place with retention or inversion of the stereochemistry (Scheme 225). When the substituent is a benzhydryl group, cis-587 was formed, while in the case of a diphenylphosphino substituent trans-587 was obtained. The inversion of the configuration has been explained by the formation of the $s y n-\eta^{3}$-intermediate 586B through a coordination of the phosphino group to Pd.

Scheme 225. Diastereodivergent Pd-Catalyzed Allylic Alkylation of Cyclic Acetates 586 Bearing Different Substituted Amino Groups with Malonate 


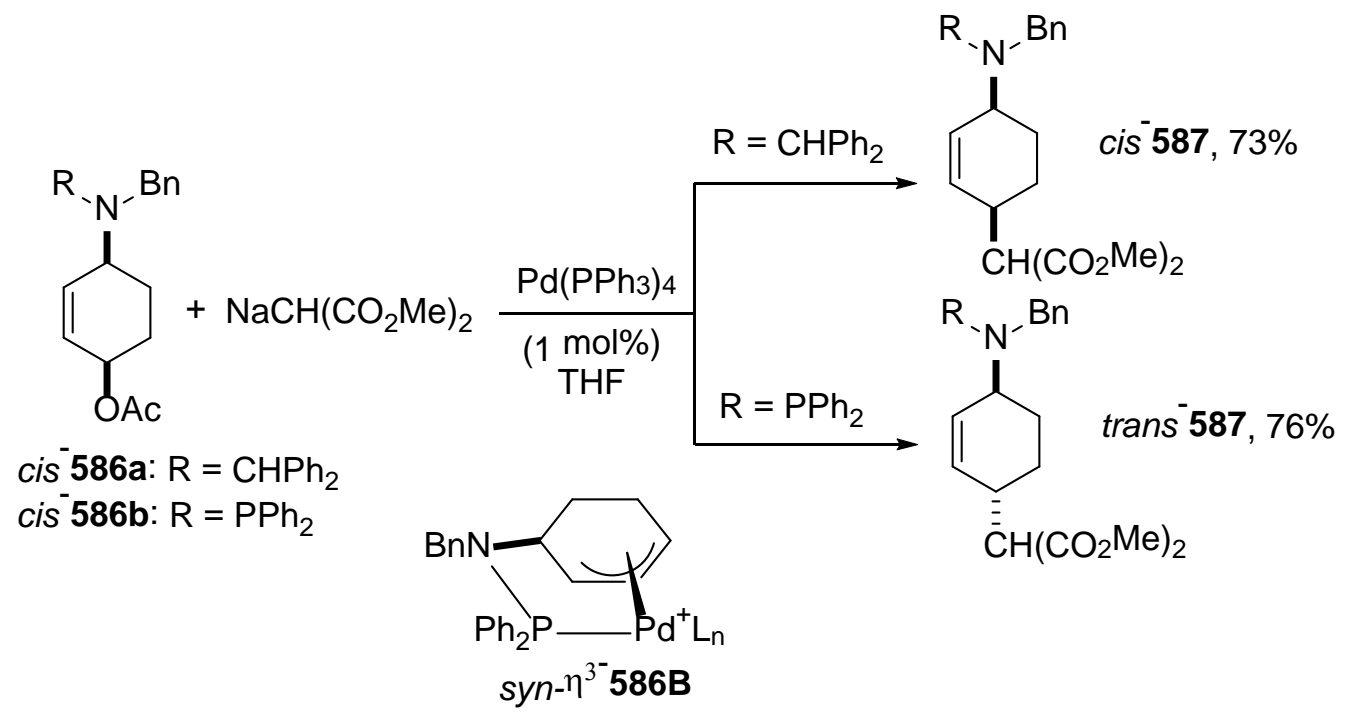

Solvent effects have been observed in the Pd-catalyzed cross-coupling of trans-588 with $\mathrm{PhSn}(n \mathrm{Bu})_{3}$ by Kurosawa and co-workers. ${ }^{545,546}$ syn-Oxidative addition was observed using maleic anhydride as ligand, instead of phosphines, in low polar solvents giving the product trans-589 (Scheme 226). However, the cis-product resulted in high polar solvents such as acetonitrile due to the prevention of the $\mathrm{Pd}-\mathrm{Cl}$ interaction.

Scheme 226. Diastereodivergent Pd-Catalyzed Cross-Coupling of Allyl Chloride trans-588 with $\mathrm{PhSn}(n \mathrm{Bu})_{3}$ in Different Solvents

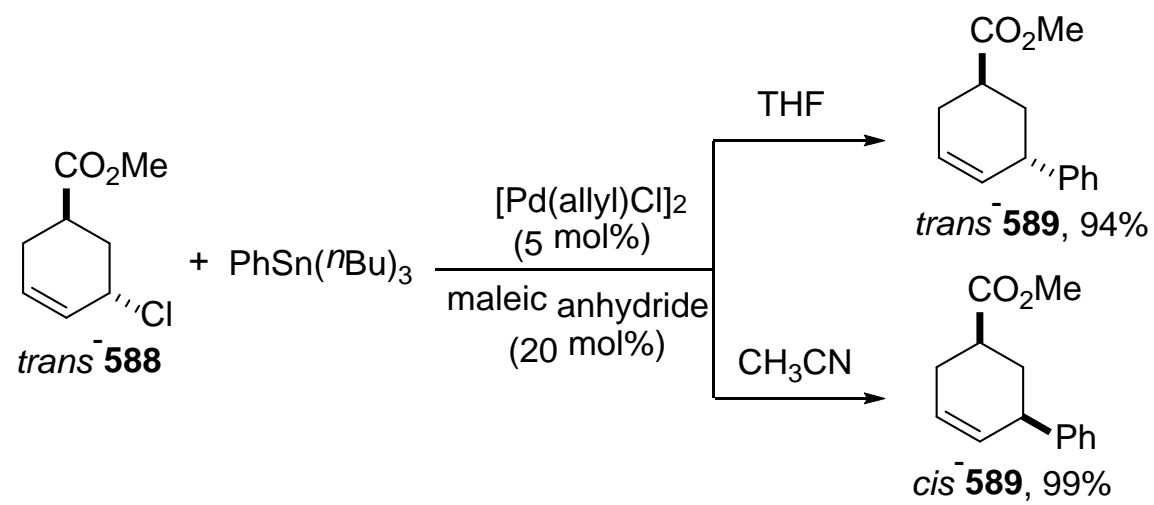

Ligand effects have been described in the diastereodivergent Pd-catalyzed deracemization of dienyl acetate $\mathbf{5 9 0}$ with malonates. For example, cis-592 was formed in the case of dimethyl methylmalonate sodium salt by using (-)-BINAP as ligand in $90 \%$ de but in $16 \%$ ee. On the other hand, with iPr-PHOX 591 as ligand the overall inversion was observed giving trans-592 in 10\% de and in 58\% ee (Scheme 227). ${ }^{547}$

Scheme 227. Diastereodivergent Pd-Catalyzed Allylic Alkylation of Dienyl Acetate 590 with Malonates Using Different Chiral Ligands 


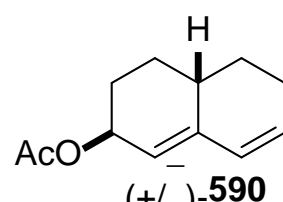

$(+/)-590$

$\mathrm{MeCH}(\mathrm{Na})\left(\mathrm{CO}_{2} \mathrm{Me}\right)_{2}$

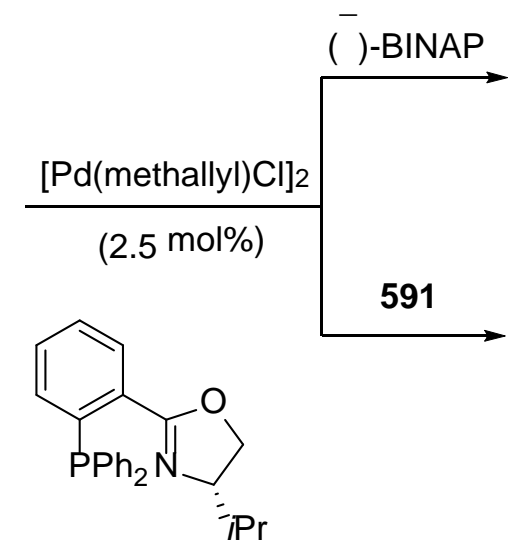

iPr-PHOX (591)

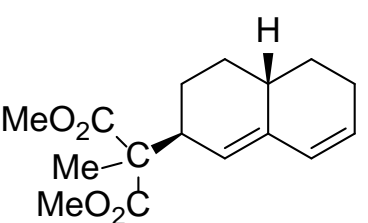

cis ${ }^{-592,}$, 95\% de, 16\% ee<smiles>COCC(C)(OC)[C@H]1C=C2C=CCC[C@H]2CC1</smiles>

trans $592,10 \%$ de, $58 \%$ ee

Diastereodivergent Pd-catalyzed deracemization of cis-593 has been performed also using different chiral ligands by Maulide and co-workers. ${ }^{548}$ In the presence of phosphoramidite $(R, R, R)-\mathbf{1 8 7}$ (Scheme 66), the reaction of cis-593 with malonates afforded the corresponding enantioenriched diastereomers cis-595 in moderate yields and good de and ee (Scheme 228). However, using ligand Ph-PHOX 594 the corresponding trans-595 derivatives were mainly formed with comparable results. Similar diastereodivergent results were obtained with trans-593: products trans-595 resulted in 59-76\% yield with $90 \%$ de and $88-94 \%$ ee when phosphoramidite 187 was employed as chiral ligand. However, in this case, the ligand Ph-PHOX 594 afforded very poor results.

Scheme 228. Diastereodivergent Pd-Catalyzed Allylic Alkylation of cis-4Chlorocyclobut-2-enecarboxylic Acid 593 with Malonates Using Different Chiral Ligands

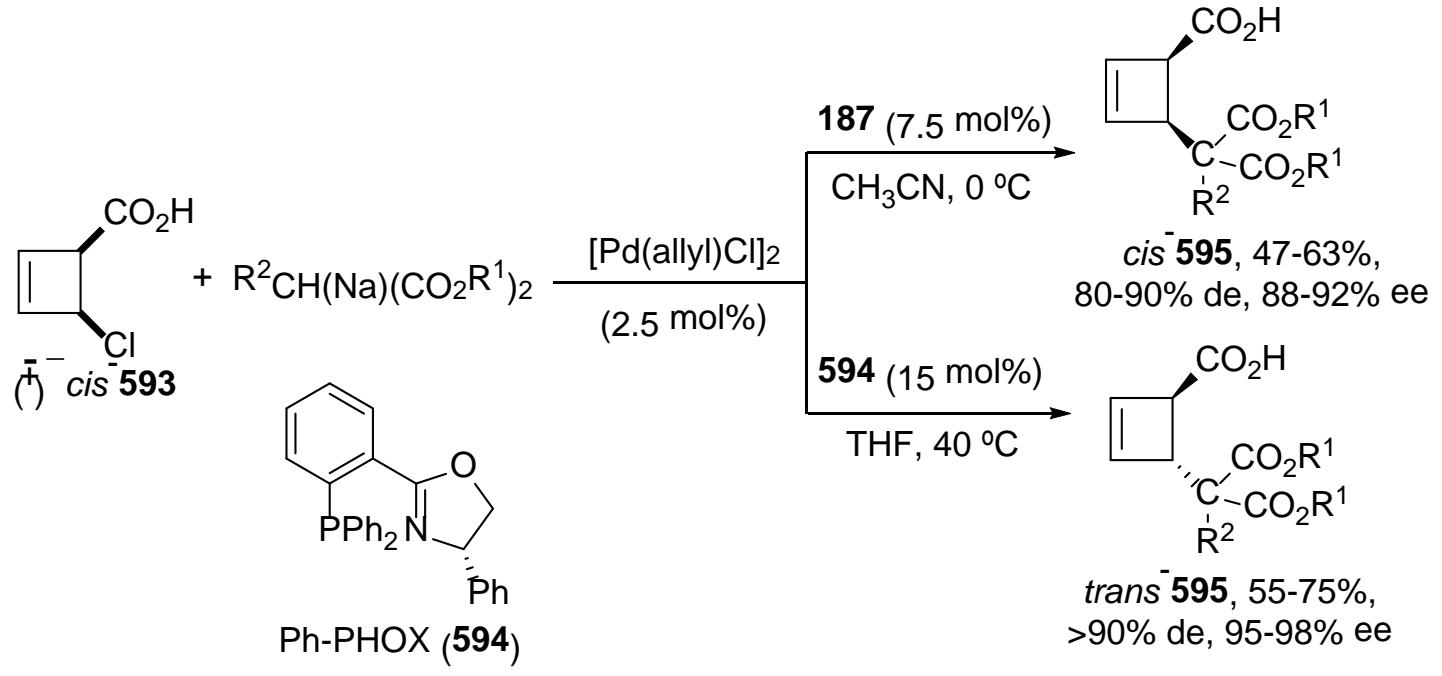

In conclusion, solvent effects played an important role in the Pd-catalyzed crosscoupling allylic arylation and mono and bidentante ligands gave diastereodivergent results in the Pd-catalyzed deracemization of allylic systems. 


\subsection{Other Reactions}

Asymmetric hydrogenation of the cyclic ketones 596 has been performed using different $\mathrm{RuCl}_{2}$ (diphosphine)(diamine) complexes as chiral catalysts. In the case of RuBIPOP (597a)/diamine complex, product (S)-598 was quantitatively formed using 2(aminomethyl)pyridine (AMPY) as base in $82 \%$ ee (Scheme 229). ${ }^{549}$ By changing the substitution in the ligand $\mathrm{RuCl}_{2}[\mathrm{MeO}-\mathrm{BIPOP}(\mathbf{5 9 7 b})]$ and using the same amine, the corresponding enantiomer $(R)$-598, a precursor for potential cholesteryl ester transfer protein inhibitors, was obtained in $>98 \%$ ee.

Scheme 229. Enantiodivergent Ru-Catalyzed Hydrogenation of Ketone 596 Using BIPOPs 597 Ligands

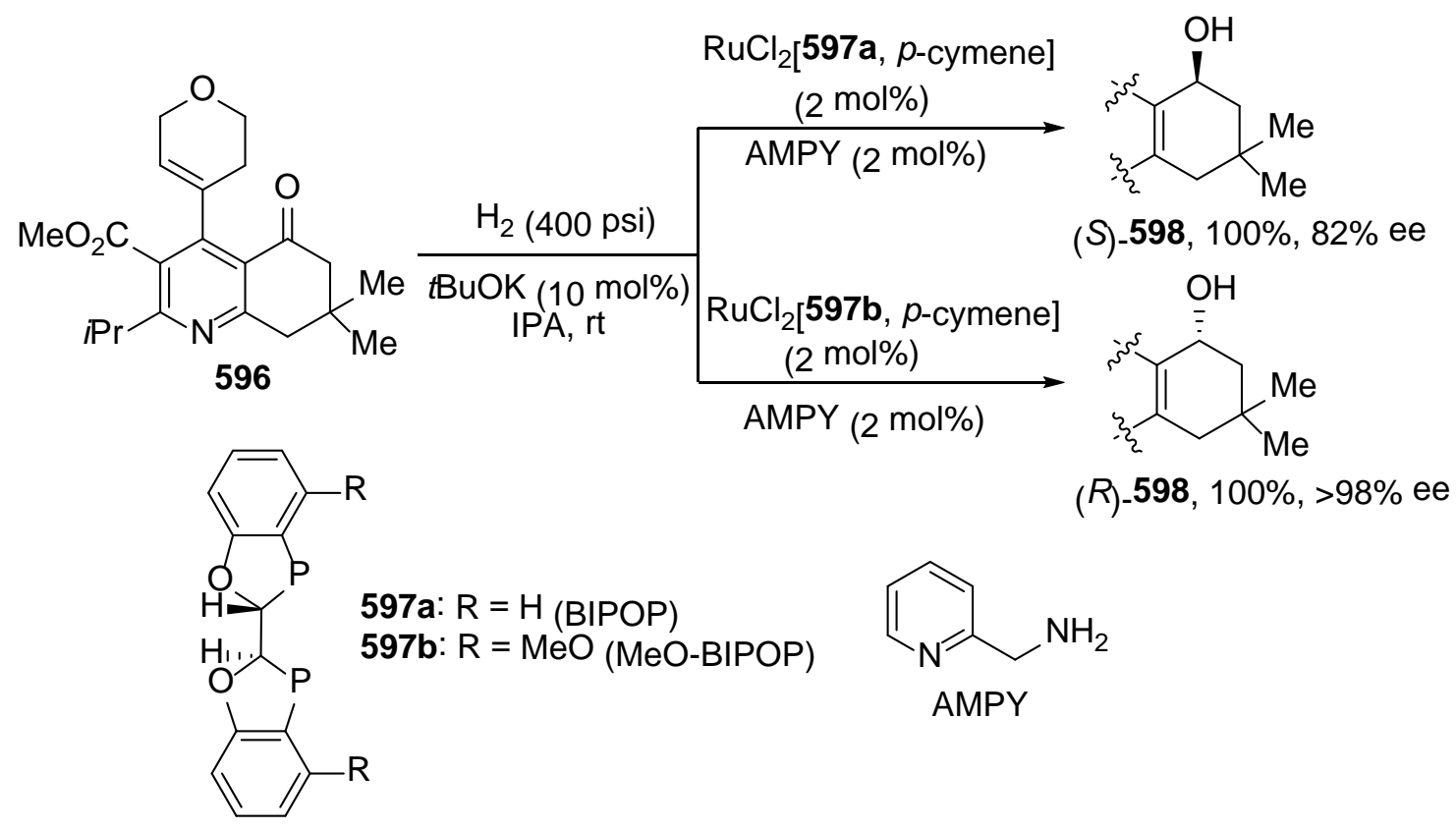

In the Ru-catalyzed hydrogenation of tetrahydro-5-quinolinone 599, the alcohol $(R)$ 600 was quantitatively prepared in 98\% ee when diphosphine MeO-BIPOP (597b) and $(R, R)$-1,2-diphenyl-1,2-ethanediamine (352) were employed as chiral ligands. However, by changing the amine to 8-aminoquinoline, the corresponding enantiomer ( $S$ )-600 was obtained in $80 \%$ ee (Scheme 230). ${ }^{549}$ These $\mathrm{Ru}$ catalysts have been used in the enantiodivergent reduction of several cyclic ketones, such as tetralone and pyridinethiophene-furan-pirazole-fused cyclohexanones. ${ }^{549}$

Scheme 230. Enantiodivergent Ru-Catalyzed Hydrogenation of Ketone 599 Using BIPOPs 597 Ligands 


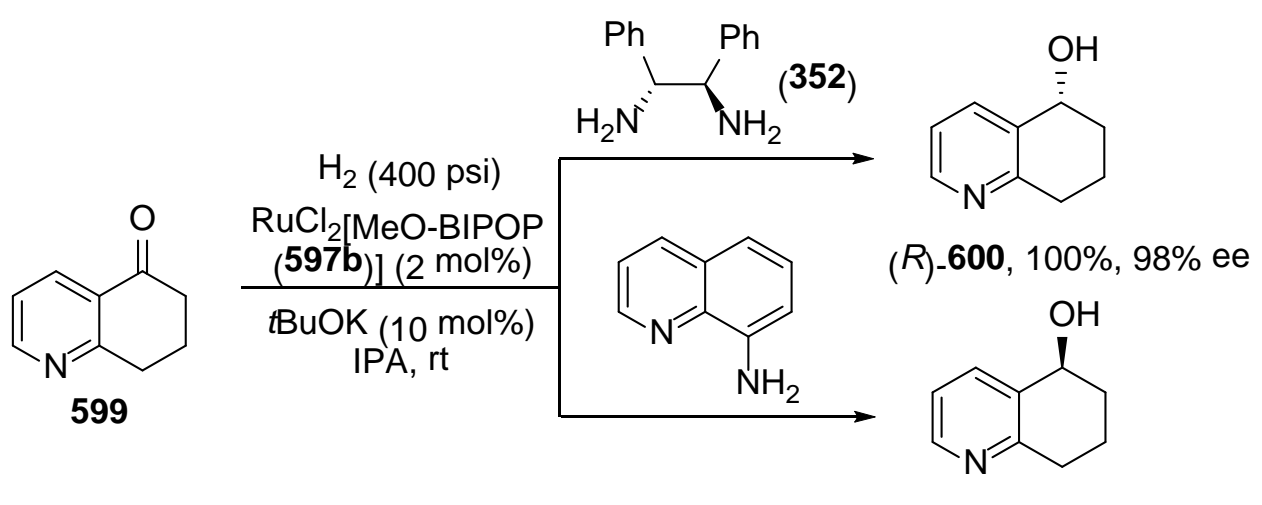

(S)-600, 100\%, 80\% ee

Zinc-dependent alcohol dehydrogenase (ADH) mutate enzymes 186A and W110T from Thermoanaerobacter brockii (TbSADH) were able to reduce enantiodivergently the ketone 601 to the corresponding alcohols $(S)-602$ and $(R)-602$, respectively (Scheme 231). ${ }^{550}$ Molecular dynamic simulations indicated that the introduced mutations induce dramatic changes in the shape of the active site and also in the substrate-enzyme interactions. ${ }^{551}$

Scheme 231. Enantiodivergent Biocatalytic Reduction of Ketone 601 Using Different Mutate Alcohol Dehydrogenase TbSADH

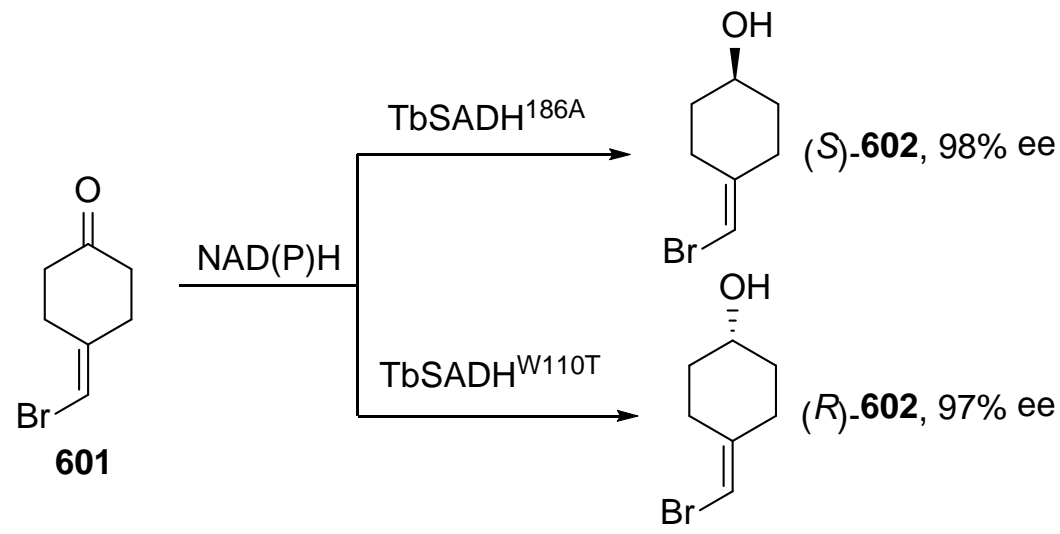

Moderate enantiodivergent solvent effects have been found in the hydrogenation of heterocyclic ketone-derived hydrazones under the Rh-catalyzed conditions using $(R, S)$ MandyPHOS 604 as chiral ligand. ${ }^{552}$ During the optimization experiments it was found out that compound $\mathbf{6 0 3}$ gave quantitatively $(R)-\mathbf{6 0 5}$ in $85 \%$ ee working in $\mathrm{MeOH}$ (Scheme 232). However, in 1,2-dichloroethane (S)-605 was obtained in 50\% yield but only in $27 \%$ ee. These results are probably due to the coordination of the solvent to the oxygen of the tetrahydropyran unit.

Scheme 232. Enantiodivergent Rh-Catalyzed Hydrogenation of Hydrazone 603 Using MandyPHOS 604 in Different Solvents 


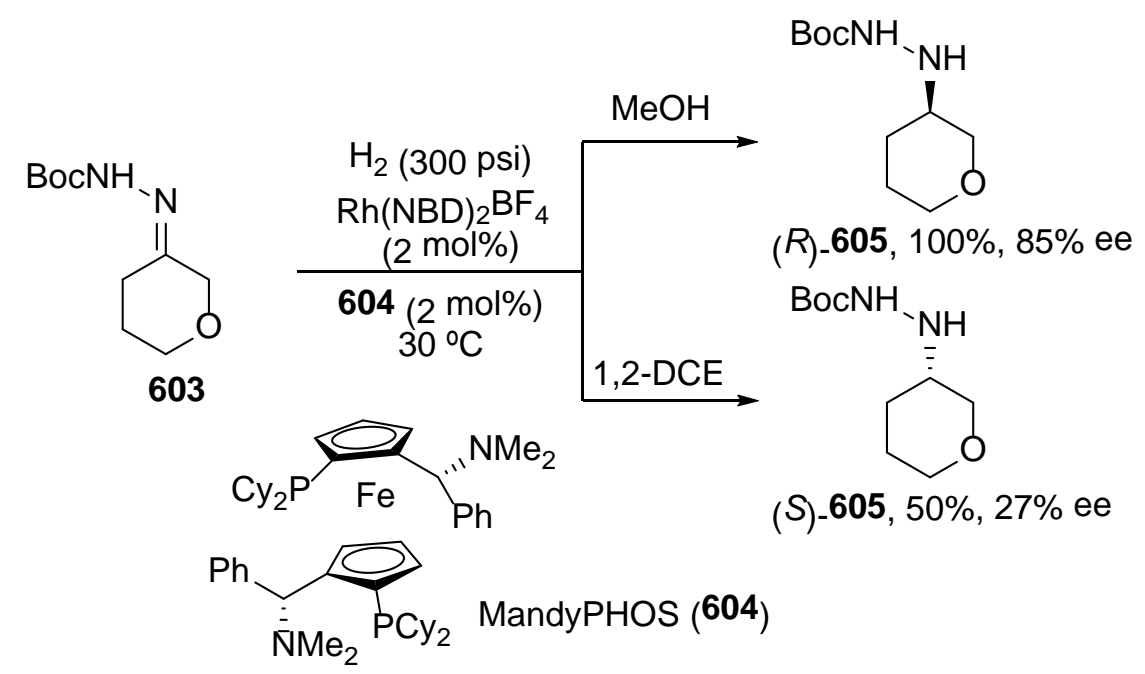

In the asymmetric hydrogenation of 2,4-disubstituted 1,5-benzodiazepines 606 using the $\mathrm{Ru}$ catalyst $(R, R)-\mathbf{6 0 7}$ an efficient reversal of enantioselectivity was resulted depending on the counteranion of the metal complex. ${ }^{553}$ Thus, working with tetrakis[3,5-(bistrifluoromethyl)phenyl]borate (BARF) a complete conversion to the corresponding products $(R, R)-\mathbf{6 0 8}$ was observed in high de and ee (Scheme 233). On the other hand, using diphenylphosphate the opposite enantiomer $(S, S)-608$ was formed. The authors proposed that in the last case a TS 608A, in which the phosphate is participating in the hydrogen bonding, is operating. On the other hand, with a weakly coordinating anion such as BARF, an electrostatic ion pair with the substrate can be formed, which is less effective than the previous one giving a lower efficiency.

Scheme 233. Enantiodivergent Hydrogenation of 1,5-Benzodiazepines 606 Catalyzed by Cationic Ru-Diamine Complex 607 with Different Counteranions

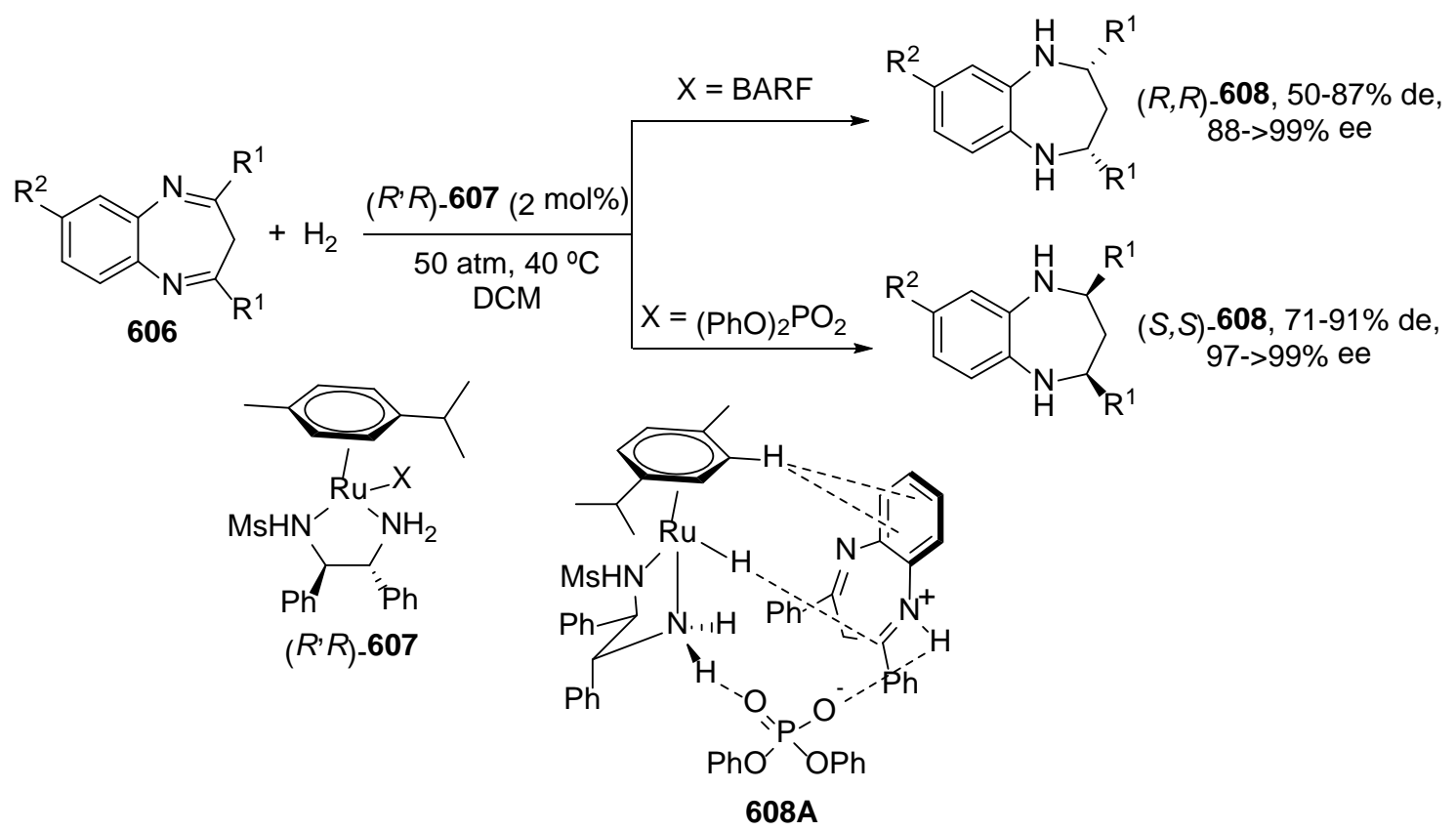


A Ru-catalyzed nucleophilic ring-opening of the hetero-Diels-Alder adduct 3-aza-2oxabicyclo[2.2.1]hept-5-ene $\mathbf{6 0 9}$ with alcohols can be regioselectively and diastereodivergently directed to the formation of cis and trans isomers $\mathbf{6 1 0}$ (Scheme 234). ${ }^{554}$ By using [Cp*Ru(COD)Cl] as catalyst, products trans-610 were obtained with de up to $100 \%$. On the other hand, by means of the cationic complex $\left[\mathrm{CpRu}\left(\mathrm{CH}_{3} \mathrm{CN}\right)\right] \mathrm{PF}_{6}$ the corresponding cis-610 was formed also with de up to $100 \%$. According to the tendency of the neutral complex to coordinate the oxygen atom of $\mathbf{6 0 9}$, an intermediate 609A is formed and the nucleophile will add to the less hindered exoface giving the trans product. When the cationic complex was used, the Ru coordinates the alkene, which evolves to the $\pi$-allyl complex 609B. This intermediate will be attacked by $\mathrm{MeOH}$ by the endo-face affording cis-610.

Scheme 234. Diastereodivergent Ru-Catalyzed Ring Opening of Bicycle 609 with Alcohols Using Different Ru Complexes

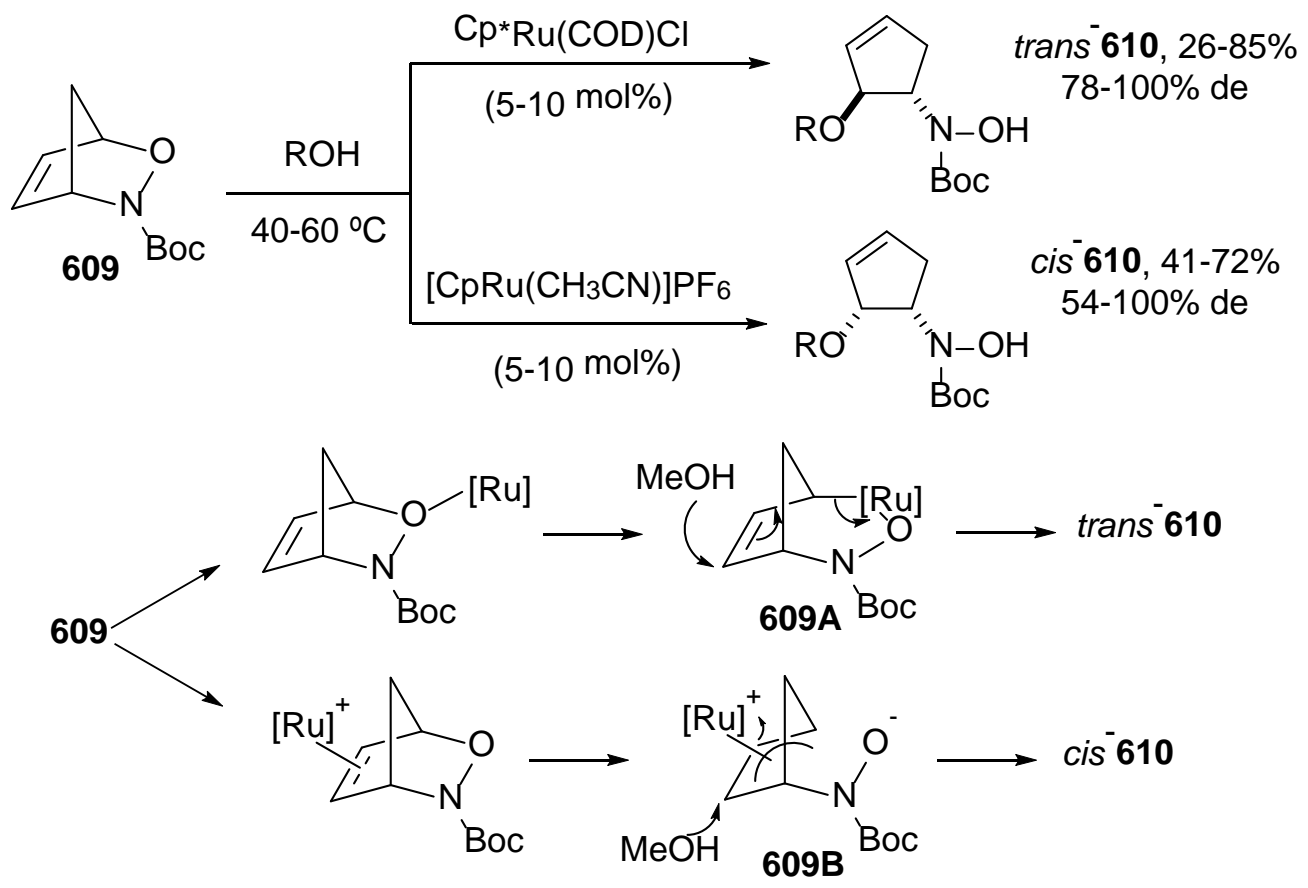

In the asymmetric Heck reaction of 2,3-dihydrofuran with aryl and cyclohexenyl triflates catalyzed by the Pd and PHOX ligands, a switch of enantioselectivity was observed depending on the substituents in the ligand. ${ }^{555}$ Ligands 611 a gave products (R)-612 with ee up to 95\%, whereas ligands with two methyl groups at the benzylic position 611b afforded products (S)-612 in lower ee (Scheme 235).

Scheme 235. Enantiodivergent Heck Reaction of 2,3-Dihydrofuran with Triflates Using PHOX Ligands 611 


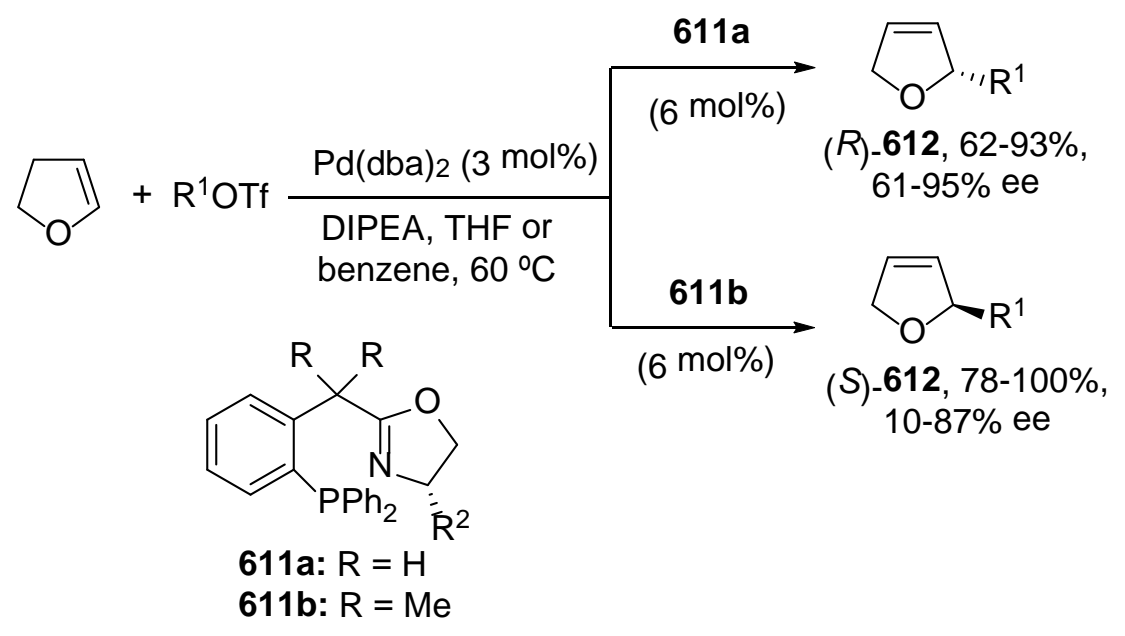

The synthesis of 2-arylcyclopropylamines (ACPAs), by Yamaguchi and coworkers, ${ }^{556}$ has been performed by a sequential Ir-catalyzed borylation of $N$-Boc protected cyclopropylamine $\mathbf{6 1 3}$ followed by a Suzuki-Miyaura cross-coupling reaction with retention of the configuration. However, the epimerization of the C-N stereocenter took place in the last step depending on the reaction conditions. Under oxygen atmosphere products cis-614 were formed with de up to 92\% (Scheme 236). On the contrary, under nitrogen atmosphere epimerization occurred and the diastereomeric compounds trans-614 were mainly obtained with de up to $76 \%$.

\section{Scheme 236. Diastereodivergent Suzuki-Miyaura Reaction of Compound 613 under Oxygen or Nitrogen Atmosphere}

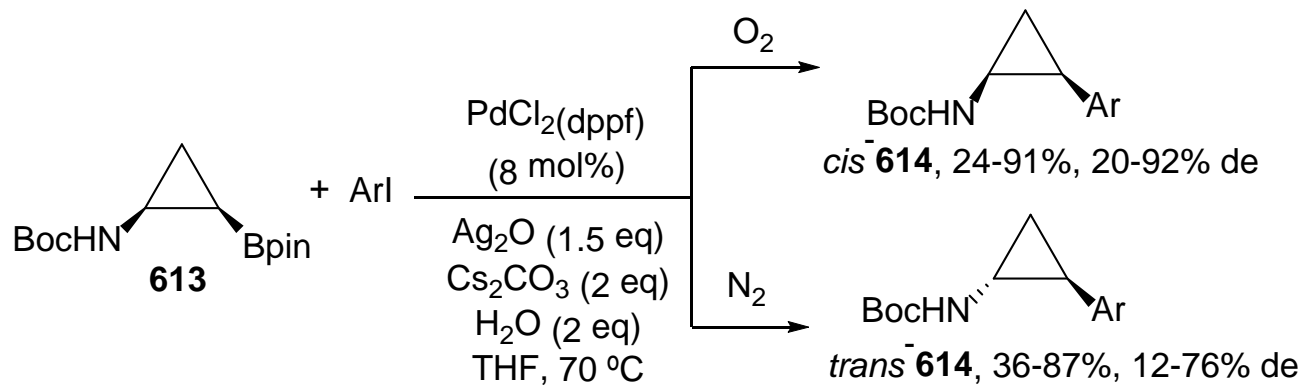

An enantiodivergent Steglich rearrangement of $O$-carboxylazlactones $\mathbf{6 1 5}$ has been recently described using optically switchable pseudoenantiomeric helicenes containing a 4-aminopyridine unit as catalysts (Scheme 237). ${ }^{557}$ These organocatalysts underwent a complementary photoswitching at 290 and $340 \mathrm{~nm}$ from $M$ to $P$. The corresponding $(R)-$ $C$-carboxylazlactones 617 were obtained by using $(P)$-616 and the $(S)$-enantiomers with (M)-616.

Scheme 237. Enantiodivergent Steglich Rearrangement of $\boldsymbol{O}$-Carboxylazlactones 615 Catalyzed by $(P)-616$ and $(M)-616$ 
<smiles>[R]C1N=C(c2ccc(OC)cc2)OC1OC(=O)Oc1ccccc1</smiles><smiles>COCC1c2ccc(Cl)cc2C(=C2CCN(C)c3ccncc32)c2cc(Cl)ccc21</smiles>

$(P)-616$<smiles>COC[C@H]1c2ccc(Cl)cc2C(=C2CCN(C)c3ccc(Cl)cc32)c2ccncc21</smiles>

$(M)-616$

An inversion of enantioselectivity depending on the catalyst loading has been observed in the quinine (QN)-mediated desymmetrization of glutaric meso-anhydrides 618 in the presence of an alcohol (Scheme 238). ${ }^{558}$ By using 0.1 eq of QN and xanthene-9-carboxylic acid (0.2 eq) at rt the corresponding $(R)$-glutaric mono esters 619 were mainly formed with ee up tp $72 \%$, whereas using 1 eq of QN at $-30{ }^{\circ} \mathrm{C}$, products (S)-619 were obtained with ee up to $69 \%$.

Scheme 238. Enantiodivergent Desymmetrization of Glutaric meso-Anhydrides with Different QN Loadings

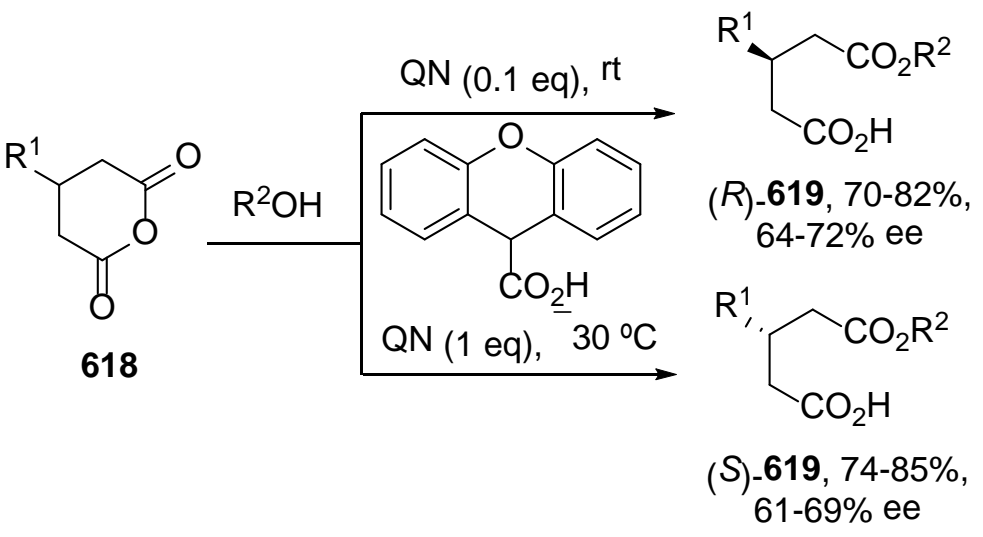

In conclusion, the enantio- and diastereodivergent metal-catalyzed processes, mainly hydrogenation and cross-coupling reactions, can be controlled by the structure of the metal complex or the organocatalysts, but also by the reaction conditions such as additives and solvents. 


\section{STEREODIVERGENCE IN INTRAMOLECULAR CYCLIZATIONS}

Intramolecular reactions allow the formation of cyclic systems with a high degree of stereocontrol, although the preparation of appropriate starting compounds can be rather troublesome. In this section, stereodivergent metal- or organocatalyzed processes such as intramolecular nucleophilic addition to carbonyl compounds and to $\mathrm{C}=\mathrm{N}$ bonds, conjugate additions to alkenes, allylic substitutions, hydroaminations, [3+2] annulations, ene cyclizations, ring-closing metathesis, enynes cyclizations, and other reactions will be considered.

\subsection{Intramolecular Nucleophilic Additions}

In this section, enantiodivergent organocatalyzed intramolecular aldol and Morita-Baylis-Hillman reactions are the only described procedures. Lewis or Brønsted acids have been used as promoters in the diastereodivergent intramolecular addition of vinyl silanes or alkenes to aldehydes. Diastereodivergent propargylation of aldehydes has been studied using different Lewis acids or under Pd-catalyzed conditions. Rhcatalyzed intramolecular hydroacylations allow the synthesis of cyclopentanones and $\gamma$ lactones in an enantio- and diastereodivergent manner. However, in the case of intramolecular diastereodivergent addition to $\mathrm{C}=\mathrm{N}$ bonds, only hydrazones have been used as substrates. In addition, the Pictet-Spengler reaction with in situ generated $\mathrm{N}$ acyl iminium ions has been described.

4.1.1. Addition to Carbonyl Compounds. Intramolecular aldol cyclization catalyzed by L-Pro and other organocatalysts has been used for the synthesis of carbobicyclic enediones, so-called Wieland-Miescher ketones, precursors for natural products such as steroids and other bioactive compounds. ${ }^{559,560}$ An enantiodivergent synthesis of a Wieland-Miescher ketone analog $\mathbf{6 2 2}$ bearing a seven-membered ring, has been described starting from the triketone $\mathbf{6 2 0}$ and the pyridinylmethylamine derivative 621 (1 eq) as chiral organocatalysts (Scheme 239). ${ }^{561}$ Although in moderate enantioselectivities, an inversion of configuration was observed based on the amount of trifluoroacetic acid (TFA) used as additive. Using 1-1.7 eq of TFA (S)-622 was formed in $43-48 \%$ ee, while in the presence of 2-3.5 eq of TFA product $(R)-\mathbf{6 6 2}$ was obtained in $30-54 \%$ ee. Among the transition states, the trans-anti-622B with the protonated DMAP moiety oriented away from the C-C bond forming site is the most favorable to form $(R)$ 622 in the presence of 1.8 eq of TFA. However, when less amount of TFA was used the trans-syn transition state 622A was the most favored one. This enantiodivergent Hajos-Parrish-Eder-Sauer-Wiechert reaction has been only observed with this bicyclic enedione 620.

Scheme 239. Enantiodivergent Intramolecular Aldol Reaction of Trione 620 Organocatalyzed by Diamine 621 Using Different Amounts of TFA as Additive 


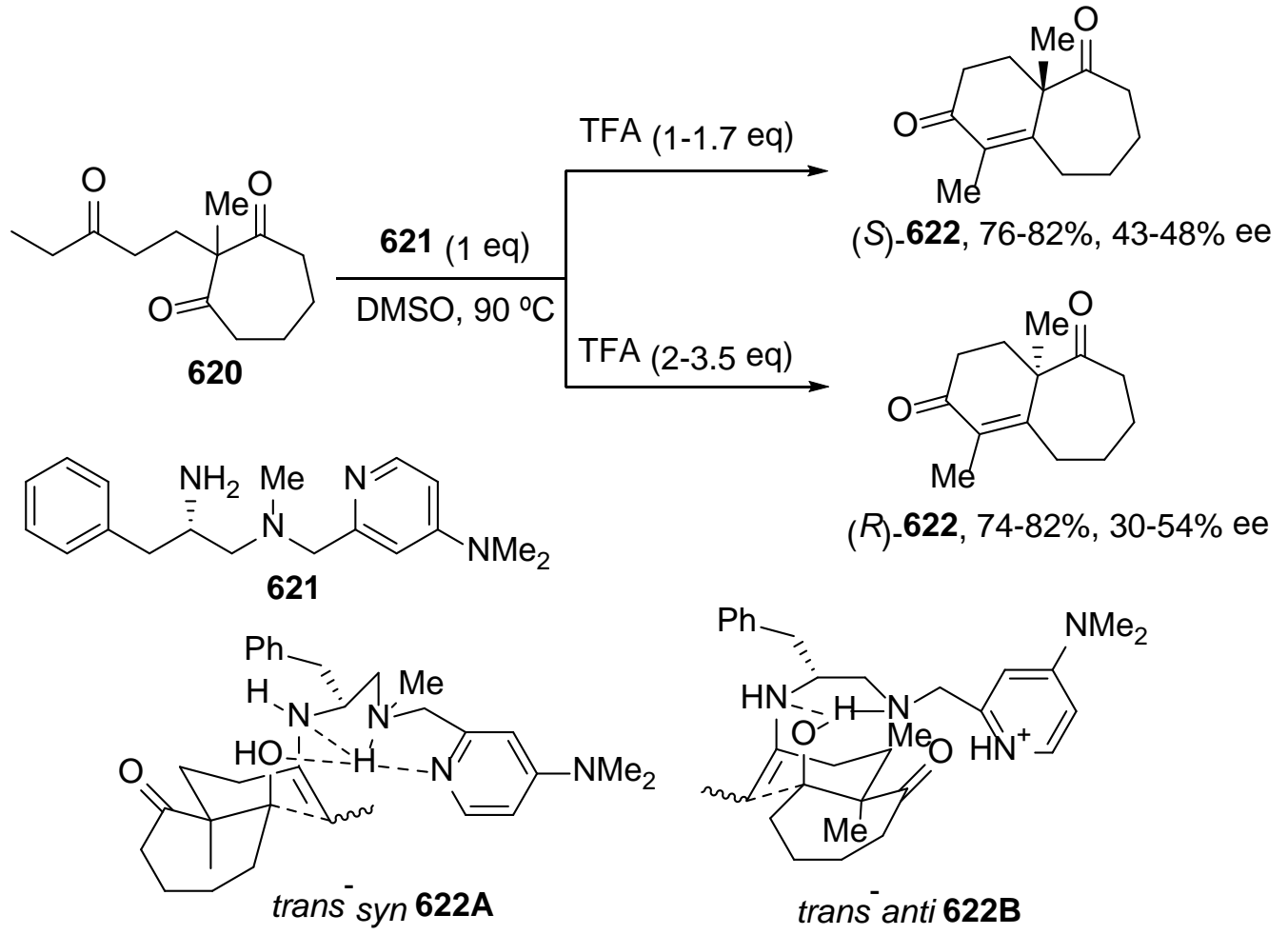

An intramolecular acid-catalyzed aldol cyclization of 2,3,7-triketoesters 623 gave highly functionalized cyclopentanones 624. This unit is present in several natural products such as prezawlskin B and picrotoxanes. In the presence of Lewis acids, such as $\mathrm{Yb}(\mathrm{OTf})_{3}$, the corresponding products 1,2-anti-624 were obtained in good diastereoselectivity. However, using $30 \mathrm{~mol} \%$ of mesitylenesulfonic acid ( $\mathrm{MesSO}_{3} \mathrm{H}$ ) cyclopentanones 1,2-syn-624 were formed diastereodivergently (Scheme 240). ${ }^{562}$ These stereodivergent results were explained by the different conformations of intermediates as in 623A and 623B using Brønsted and Lewis acids, respectively. In the case of the Brønsted acid the 1,2-diketo unit adopted an anti-orientation, whereas the Lewis acid coordinated both carbonyl groups in a syn-orientation via the intermediate 623B.

Scheme 240. Diastereodivergent Intramolecular Aldol Reaction of 2,3,7Triketoesters 623 Catalyzed by Brønsted and Lewis Acids 

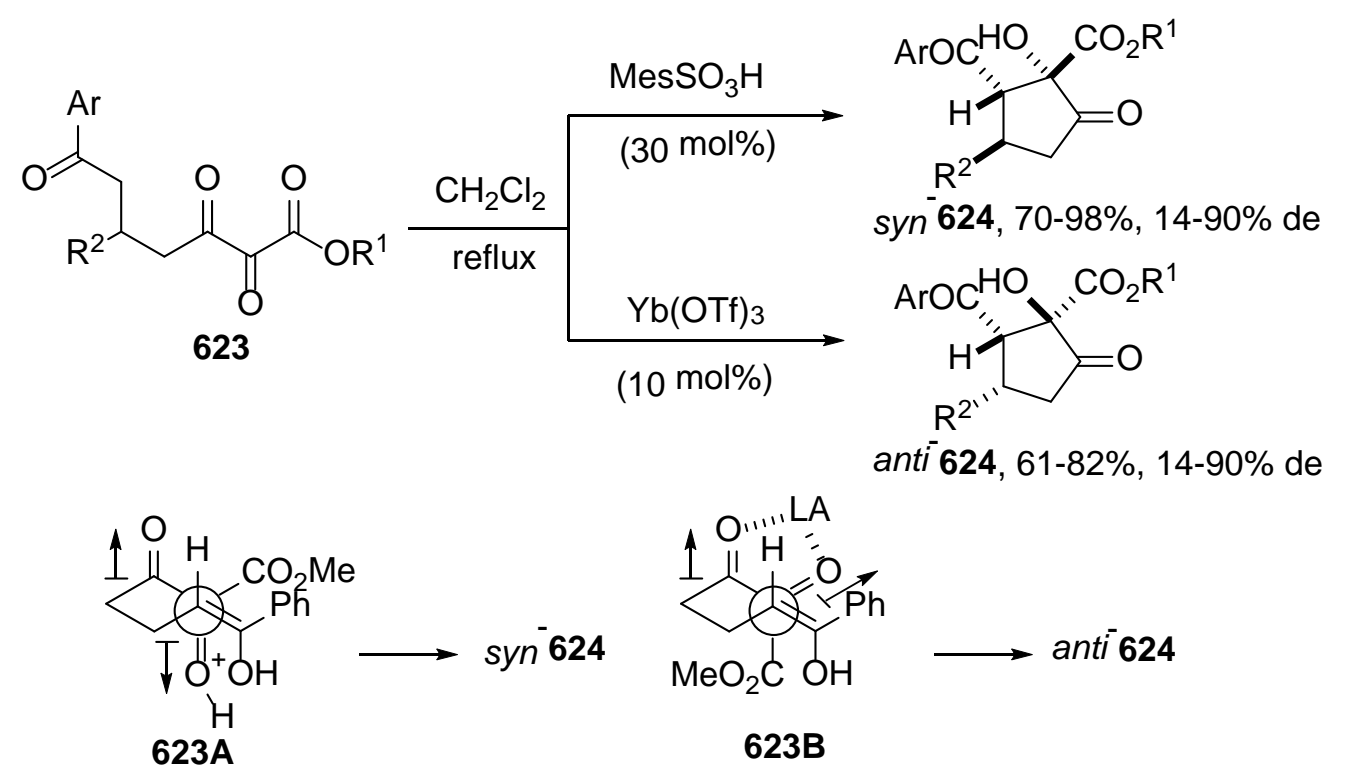

Intramolecular Morita-Baylis-Hillman reaction of hept-2-enedial (625) organocatalyzed by L-Pro in DMF at $70{ }^{\circ} \mathrm{C}$ gave the corresponding cyclohexanone $(S)$ 626 in $60 \%$ ee. On the contrary, by the addition of 1 eq of imidazole as additive at $0{ }^{\circ} \mathrm{C}$ in acetonitrile the enantiomer (R)-626 was formed in high 93\% ee (Scheme 241). ${ }^{563}$ In the case of using just L-Pro, a Zimmerman-Traxler TS 625A has been proposed, whereas in the presence of imidazole a Michael addition takes place giving the transition state 625B.

Scheme 241. Enantiodivergent Morita-Baylis-Hillman Reaction of Hept-2-enedial 625 Organocatyzed by L-Pro in the Absence and in the Presence of Imidazole

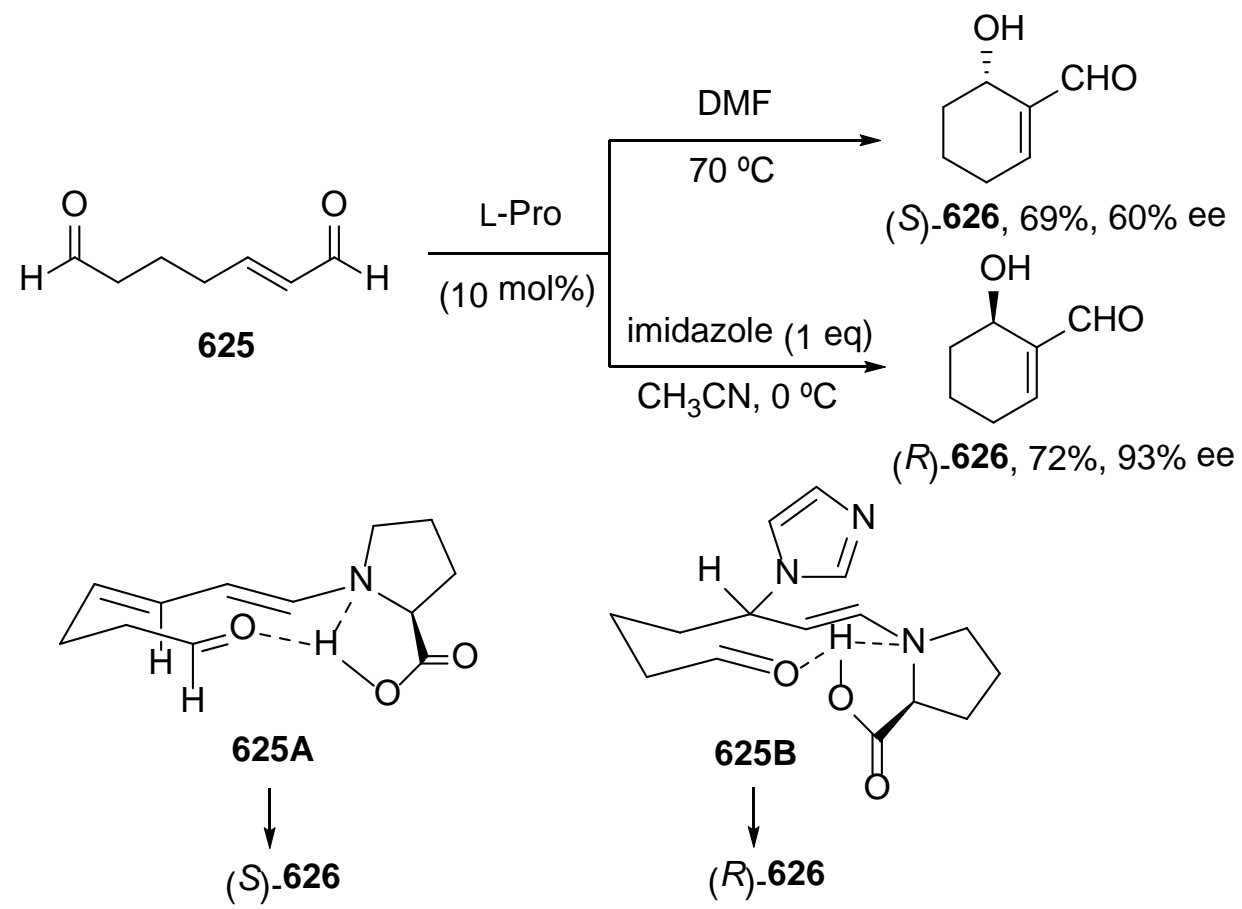


Lewis acid-promoted addition of vinylsilanes to aldehydes allows the synthesis of allylic alcohols. The intramolecular version of this reaction has been applied to the diastereodivergent synthesis of conduritols (1,2,3,4-cyclohexenetetraols), which are the aglycone unit of aminocyclitol antibiotics, as well as precursors for bioactive products. Compound $\mathbf{6 2 7}$ derived from L-arabinose led to the formation of 1,2-anti-cyclohexenol 628 in $94 \%$ de using $\mathrm{BF}_{3} \cdot \mathrm{OEt}_{2}$ at rt (Scheme 242). ${ }^{564}$ However, by treatment of $\mathbf{6 2 7}$ with $\mathrm{SnCl}_{4}$ at $-78{ }^{\circ} \mathrm{C}$ the corresponding 1,2-syn-cyclohexenol 629 was obtained in $68 \%$ yield and similar high diastereoselectivity. To explain the observed diastereodivergence in this cyclization promoted by $\mathrm{BF}_{3} \cdot \mathrm{OEt}_{2}$ and $\mathrm{SnCl}_{4}$, the participation of nonchelate intermediate 627A and chelate 627B, respectively, has been proposed.

Scheme 242. Diastereodivergent Intramolecular Vinylsilane-Aldehyde Reaction of 627 in the Presence of Different Lewis Acids

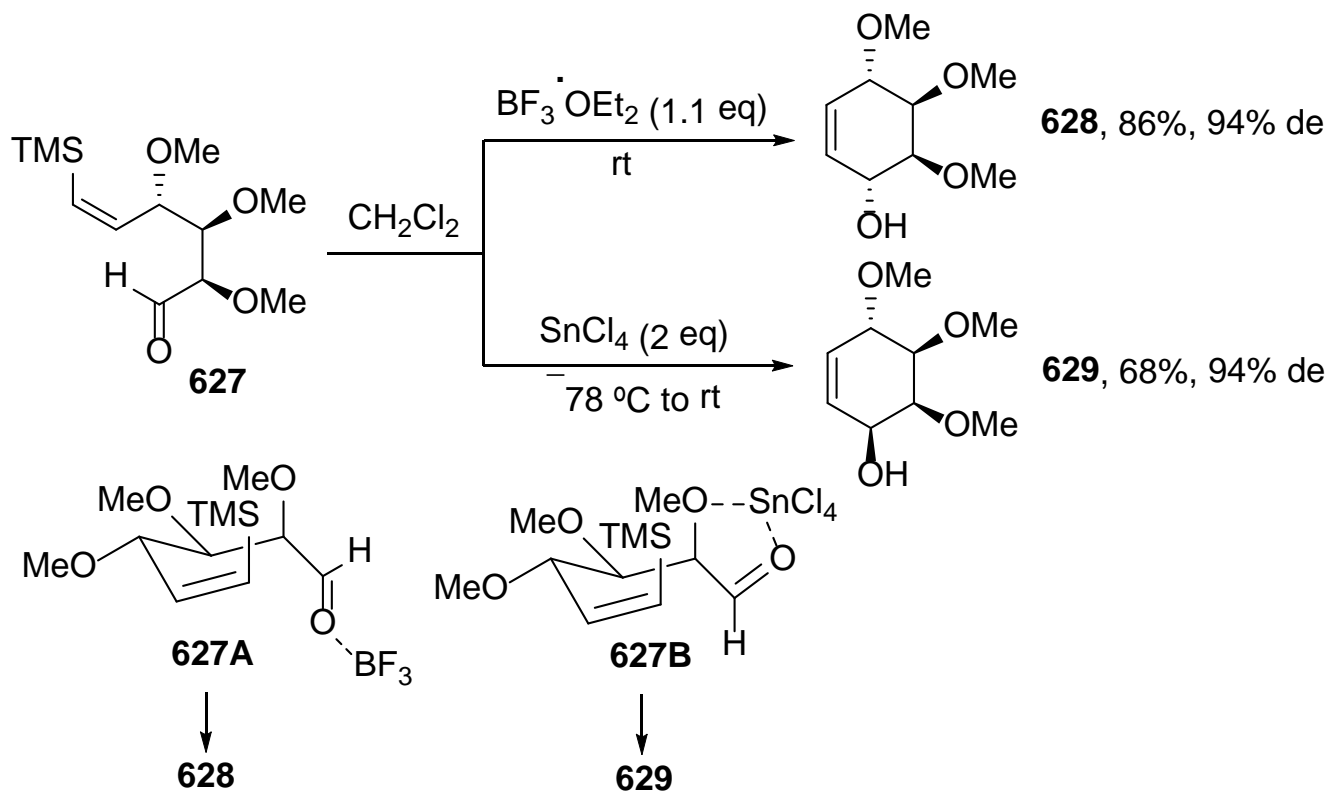

A unique example about the switch of the diastereoselectivity in the cyclization of aldehydes 630 promoted by Lewis or Brønsted acids has been described. Using $\mathrm{MeAlCl}_{2}$ an intramolecular carbonyl-ene reaction took place giving trans-3,4disubstituted piperidines 631 with de up to 86\% (Scheme 243). ${ }^{565}$ However, under acidic conditions a Prins cyclization occurred affording products cis-631 with de up to $96 \%$.

Scheme 243. Diastereodivergent Cyclization of Aldehydes 630 in the Presence of Lewis or Brønsted Acids 


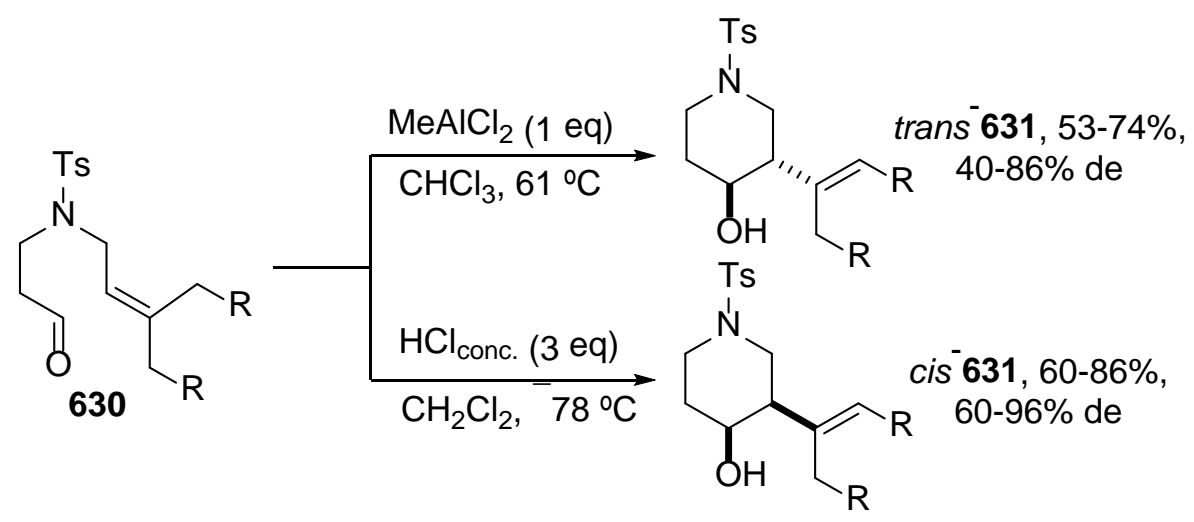

Intramolecular nucleophilic addition of propargylstannanes to the carbonyl group is promoted by Lewis acids. However, only in the case of compound $\mathbf{6 3 2}$ the diastereoselectivity could be modulated depending on the Lewis acidity. ${ }^{566}$ In the presence of $\mathrm{SnCl}_{4}$ the corresponding trans-tetrahydropyran 633 was obtained in $78 \%$ de (Scheme 244). On the other hand by using $\mathrm{ZnCl}_{2}$, cis-633 was formed in moderate $48 \%$ diastereoselectivity.

Scheme 244. Diastereodivergent Intramolecular Propargylation of Compound 632 in the Presence of Different Lewis Acids

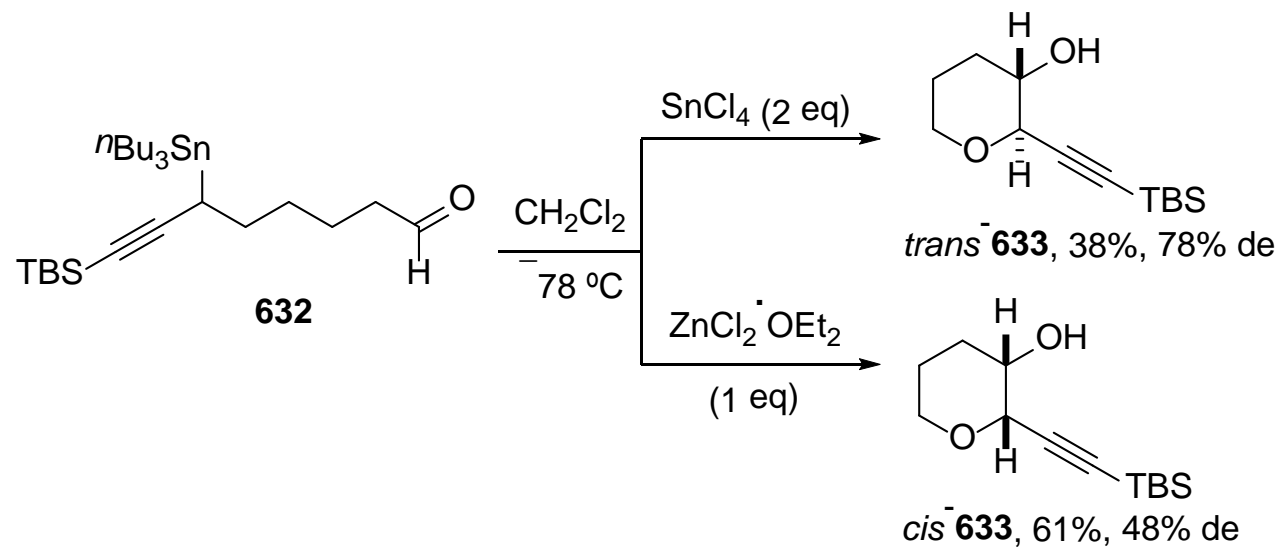

Diastereodivergent intramolecular Pd-catalyzed propargylation of aldehydes by the reaction of propargyl benzoates with diethylzinc has been modulated using different Pd complexes. In this process the corresponding allenylpalladium intermediates were formed. For instance, benzoate 634 gave cis-cyclopentanol 635 using $\mathrm{Pd}\left(\mathrm{PPh}_{3}\right)_{4}$, whereas $\mathrm{Pd}(\mathrm{OAc})_{2} / \mathrm{PnBu}_{3}$ afforded trans-635 (Scheme 245). ${ }^{567}$ Solvent and temperature effects have been also observed, so in a non-coordinating solvent such as benzene with electron-donating phosphines an increased tendency towards the trans-product was observed. On the other hand, the combination of a coordinating solvent such as THF and triphenylphosphine (an easily dissociated phosphine) afforded cis-products. According to the DFT calculations trans-cyclopentanols were formed through an open transition state 634A involving an allenylpalladium intermediate. However, in the case of cis-cyclopentanols a transmetallation Pd-Zn occurred and a chelated transition state 634B was proposed. 
Scheme 245. Diastereodivergent Intramolecular Pd-Catalyzed Propargylation of Compound 634 Using $\mathrm{Et}_{2} \mathrm{Zn}$

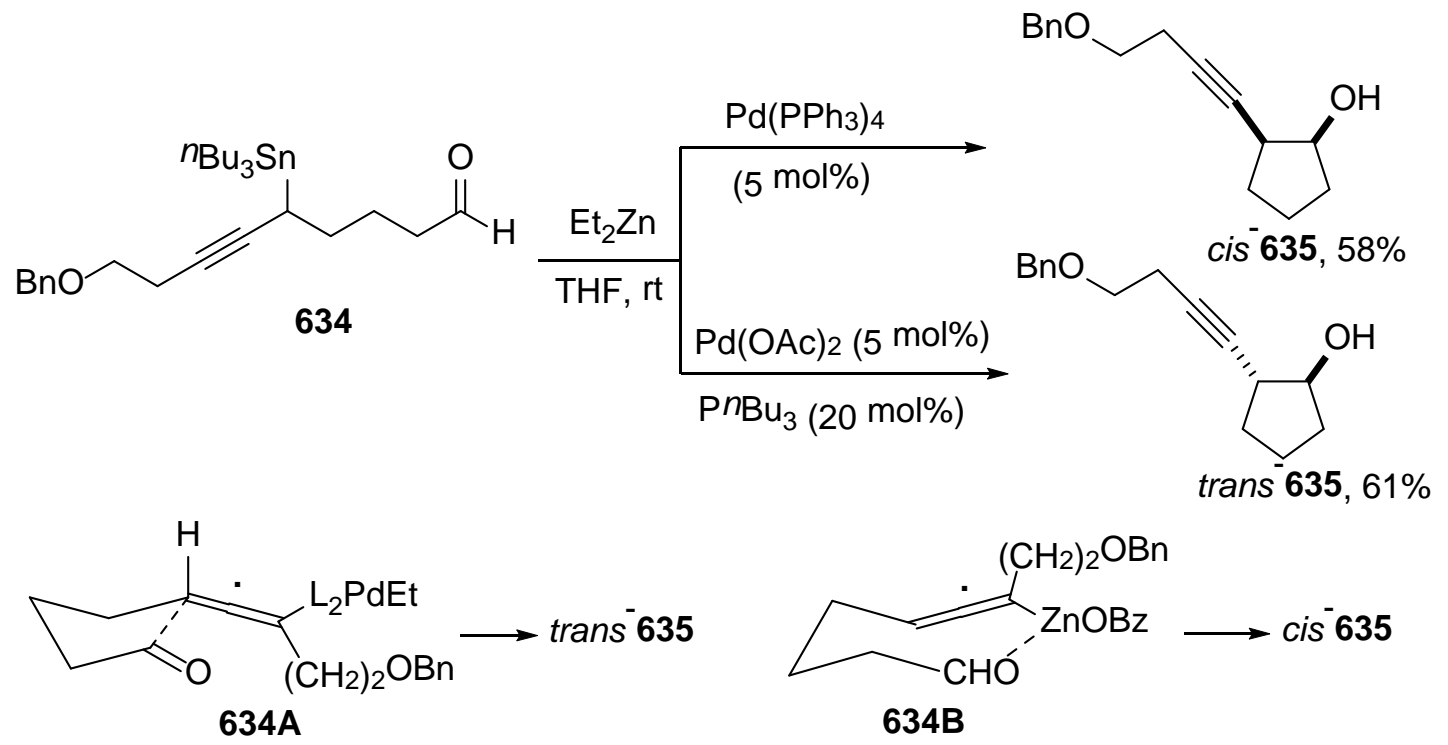

The first example of a Rh-mediated intramolecular hydroacylation was described by Sakai and co-workers en route to prostanoids, ${ }^{568}$ and the catalytic version was developed by Lochow and Miller using a Wilkinson's complex. ${ }^{569}$ A diastereodivergent asymmetric intramolecular hydroacylation was reported by Sakai ${ }^{570}$ and Suemune ${ }^{571}$ groups. When the cationic $\mathrm{RhClO}_{4} / \mathrm{BINAP}$ complex was used as catalysts, aldehydes 636 delivered trans-cyclopentanones 637 (Scheme 246). ${ }^{571}$ On the other hand, neutral $\mathrm{Rh}$ complexes gave the cis-product $637(\mathrm{R}=\mathrm{Me})$ in excellent diastereo- and enantioselectivity. An example of excellent diastereodivergent synthesis of spiro[4.4]nonanediones was described by Sakai and co-workers starting from the enal 638 (Scheme 246). ${ }^{572,573}$ Intramolecular cyclization using cationic $[\operatorname{Rh}((S)$ BINAP) $\mathrm{ClO}_{4}$ afforded dione 639, whereas Wilkinson's complex gave the diastereomeric product $\mathbf{6 4 0 .}$

Scheme 246. Diastereodivergent Intramolecular Hydroacylation of Aldehydes 636 and 638 Catalyzed by Chiral Cationic or Neutral Rh Complexes 

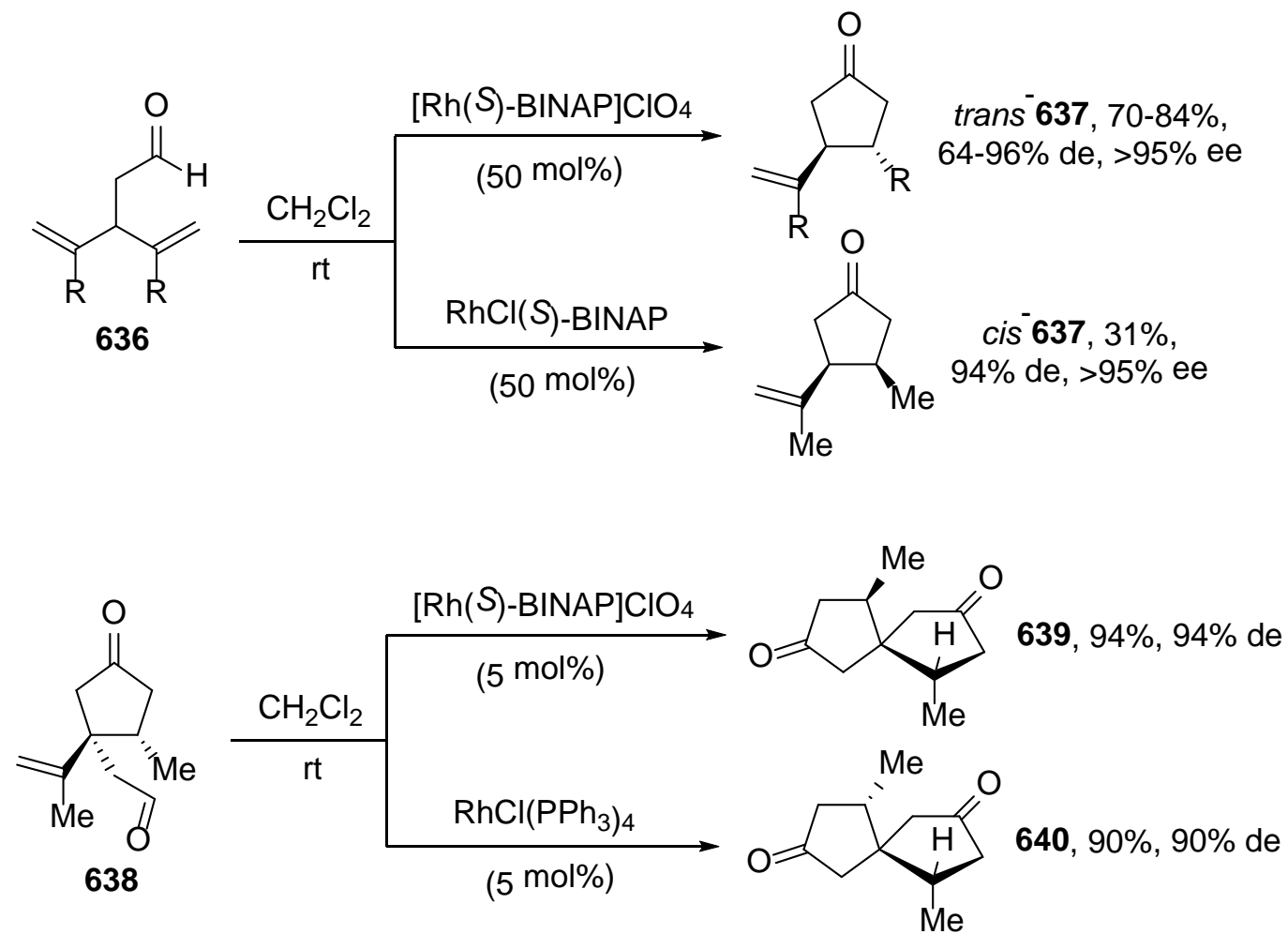

The enantioselective synthesis of bicyclic $\gamma$-lactones via intramolecular hydroacylation can be performed diastereodivergently according not only to the Rh catalyst but also to the reaction conditions. Starting from diketoaldehydes 641, neutral $[\mathrm{Rh}(\mathrm{NBD}) \mathrm{Cl}]_{2}$ and JoSPOphos 642 as chiral ligand in DME at $10{ }^{\circ} \mathrm{C}$ provided antilactones 643 in high enantioselectivity (Scheme 247). ${ }^{574}$ For the syn diastereoselective conditions, cationic $\left[\mathrm{Rh}(\mathrm{COD})_{2}\right] \mathrm{SbF}_{6}$ and the same ligand 642 in tert-amyl alcohol at $80{ }^{\circ} \mathrm{C}$ afforded lactones syn-644 in excellent results. According to the DFT calculations it was found out that the syn-bicyclic lactone $\mathbf{6 4 4}$ was thermodynamically more stable than the anti-643. This methodology has been applied to the formal synthesis of (-)mesembrine, a potent natural serotonine reuptake inhibitor isolated from Sceledium tortuosum.

Scheme 247. Diastereodivergent Intramolecular Hydroacylation of Diketoaldehydes 641 Catalyzed by Chiral Neutral or Cationic Rh Complexes and Chiral JoSPOphos 642 as Ligand 


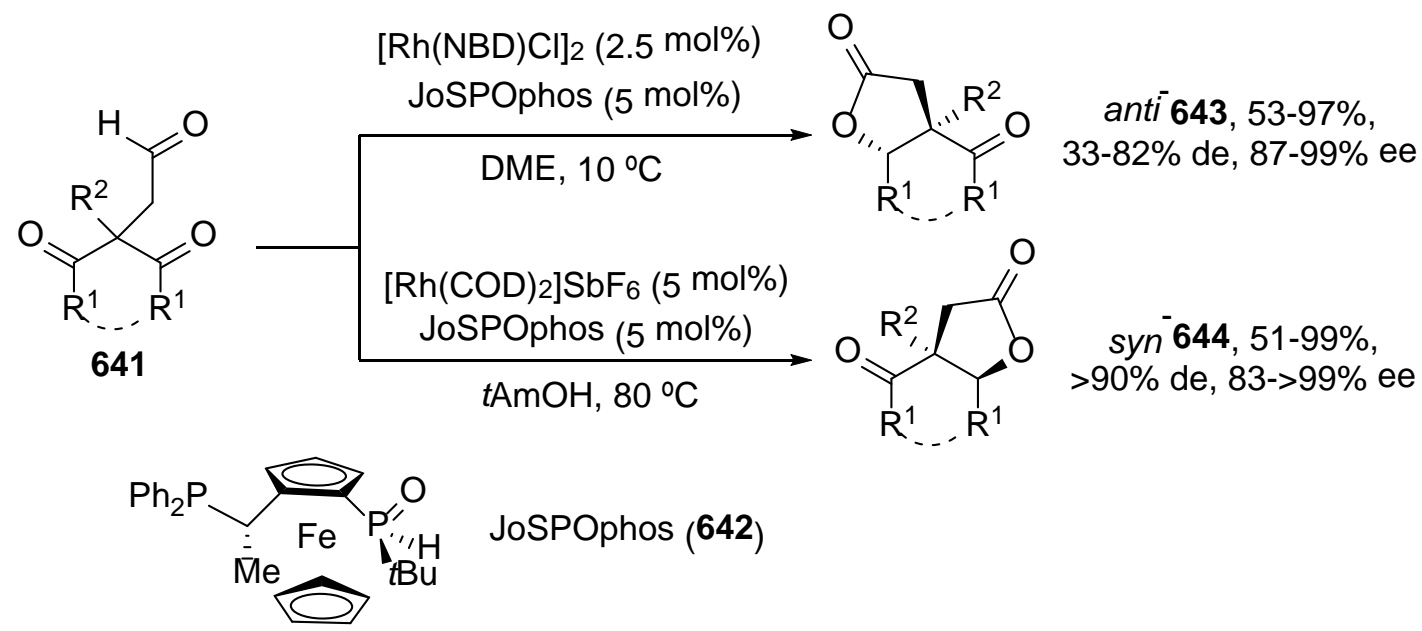

In conclusion, for intramolecular aldol reactions, the amount of Brønsted or Lewis acids as catalysts controls the stereodivergence of these processes as well as in the cyclization of unsaturated dialdehydes through a $\mathrm{MBH}$ reaction. The nature of the Lewis acid controls the intramolecular diastereodivergent vinylsilane-aldehyde reaction and the propargylation. In the case of asymmetric intramolecular hydroacylation, cationic and neutral Rh complexes cause diastereodivergent results.

4.1.2. Addition to $\mathbf{C}=\mathbf{N}$ Bonds. Intramolecular reactions of in situ generated hydrazones from enantiopure hydrazines 645 tethered to a 1,1-cyclopropanediester took place in the presence of catalytic amounts of $\mathrm{Yb}(\mathrm{OTf})_{3}$. Depending on the order of addition of the aldehyde and the Lewis acid the process can be diastereodivergently performed. ${ }^{575}$ When the reaction mixture was heated under $\mathrm{CH}_{2} \mathrm{Cl}_{2}$ reflux, intermediate hydrazones 646 cyclized providing 2,5-trans-pyrazolidines 647 (Scheme 248). This result has been explained by the formation of the iminium ion 646A through a ring opening of the cyclopropane ring which further cyclized to afford products 2,5-trans647. A sequential treatment of $\mathbf{6 4 5}$ with the Lewis acid first, and then with the aldehyde under refluxing $\mathrm{CH}_{2} \mathrm{Cl}_{2}$ provided the formation of products 2,5-cis-647 in a moderate de through an intermediate 648A.

Scheme 248. Diastereodivergent Intramolecular Cyclization of Hydrazine 645 with Aldehydes Catalyzed by $\mathrm{Yb}(\mathrm{OTf})_{3}$ Under Different Reaction Conditions 


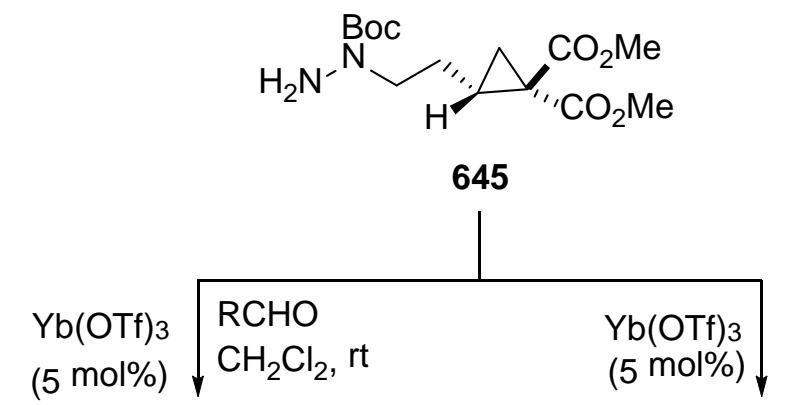

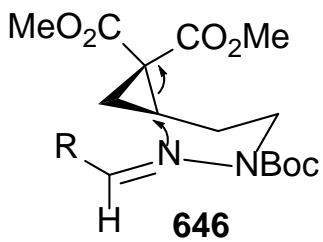

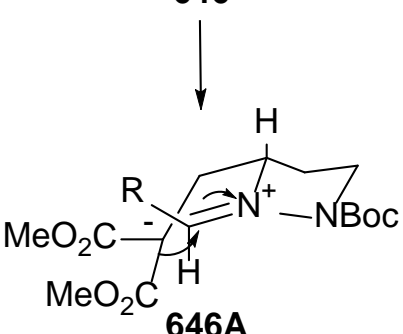

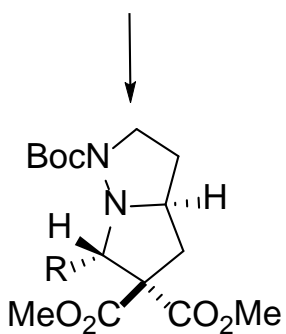

2,5-trans-647, 48-90\%, $66-99 \%$ de

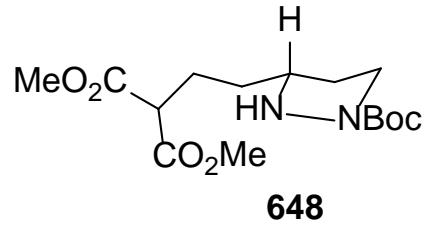

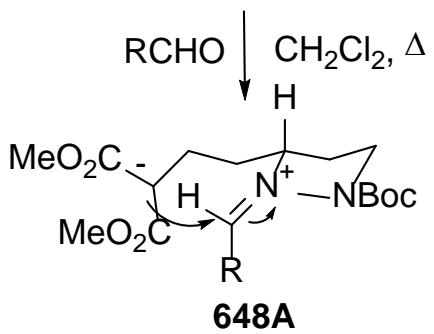

648A

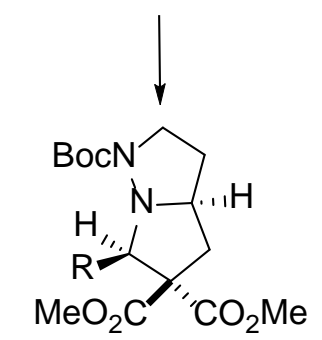

2,5-cis-647, 16-75\%, $24-61 \%$ de

Diastereodivergent Pictet-Spengler cyclization of bicyclic $N$-acyliminium ions has been performed by Cossy and co-workers using methanesulfonic acid ( $\mathrm{MsOH})$. As an example, a 4:1 mixture of $Z / E$ diastereomeric bicyclic enamides 649 gave under kinetic control compound $\mathbf{6 5 0}$ in $84 \%$ yield and $92 \%$ de (Scheme 249). ${ }^{576}$ On the other hand, under toluene reflux a reversal of diastereoselectivity was observed providing the corresponding epimer $\mathbf{6 5 1}$ with similar high de through an equilibration process leading to the thermodynamic product. Similar switch of diastereoselectivity was observed with other enamides bearing different aromatic substituents such as indol-3-yl, 1methylpyrrol-2-yl and 3,5-dimethoxyphenyl.

Scheme 249. Diastereodivergent Pictet-Spengler Cyclization of Bicyclic Enamides 649 Under Kinetic or Thermodynamic Control 
<smiles>O=C1[C@H]2C[C@H]2/C(=C/c2ccccc2)N1CCc1cc2ccccc2[nH]1</smiles>

649

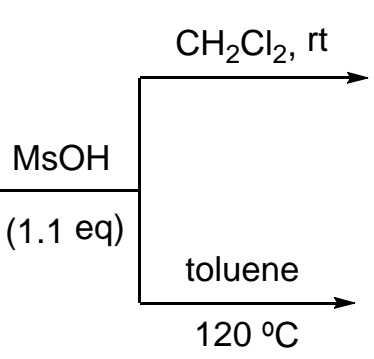

$120^{\circ} \mathrm{C}$

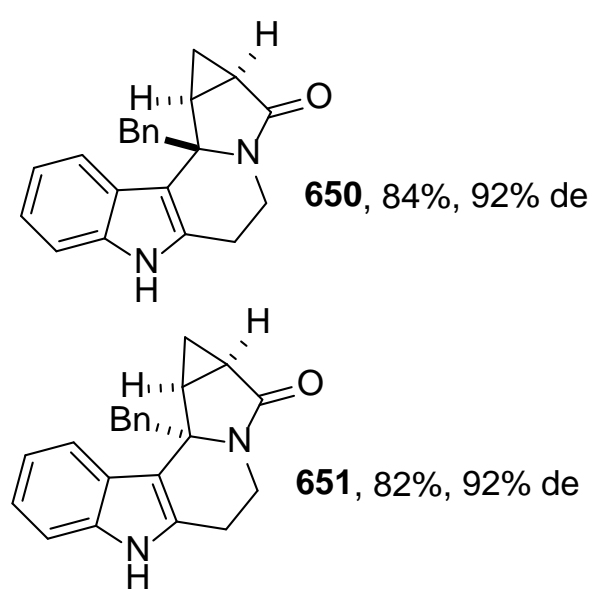

In conclusion, intramolecular nucleophilic additions to $\mathrm{C}=\mathrm{N}$ bond have received less attention than intermolecular reactions. Only a couple of diastereodivergent reactions have been described concerning the addition to hydrazones catalyzed by a Lewis acid with different orders of addition, as well as a temperature controlled Pictet-Spengler cyclization.

\subsection{Intramolecular Conjugate Additions}

Diastereodivergent asymmetric conjugate addition/lactonization of carboxylic acid enolates to enones followed by a nucleophilic ring opening allowed the synthesis of dihydrobenzofurans and tetrahydrofurans using different chiral bases as organocatalysts. Following the methodology previously described by Smith and co-workers, ${ }^{577}$ cis-2,3substituted dihydrobenzofurans $\mathbf{6 5 6}$ have been prepared by the same group, in excellent de and ee, starting from acids 652 and (S)-(-)-tetramisole hydrochloride (653) as organocatalyst (Scheme 250). ${ }^{578}$ On the other hand, using a Cinchona alkaloid-derived tertiary amine 654, the corresponding trans-diastereomers 656 could be obtained in moderate diastereoselectivity and high enantioselectivity. In the case of tetrahydrofurans similar stereodivergent effects were observed with excellent levels of stereocontrol.

Scheme 250. Enantio and Diastereodivergent Intramolecular Conjugate Addition/Lactonization of Acids 652 Organocatalyzed by 653 and 654 


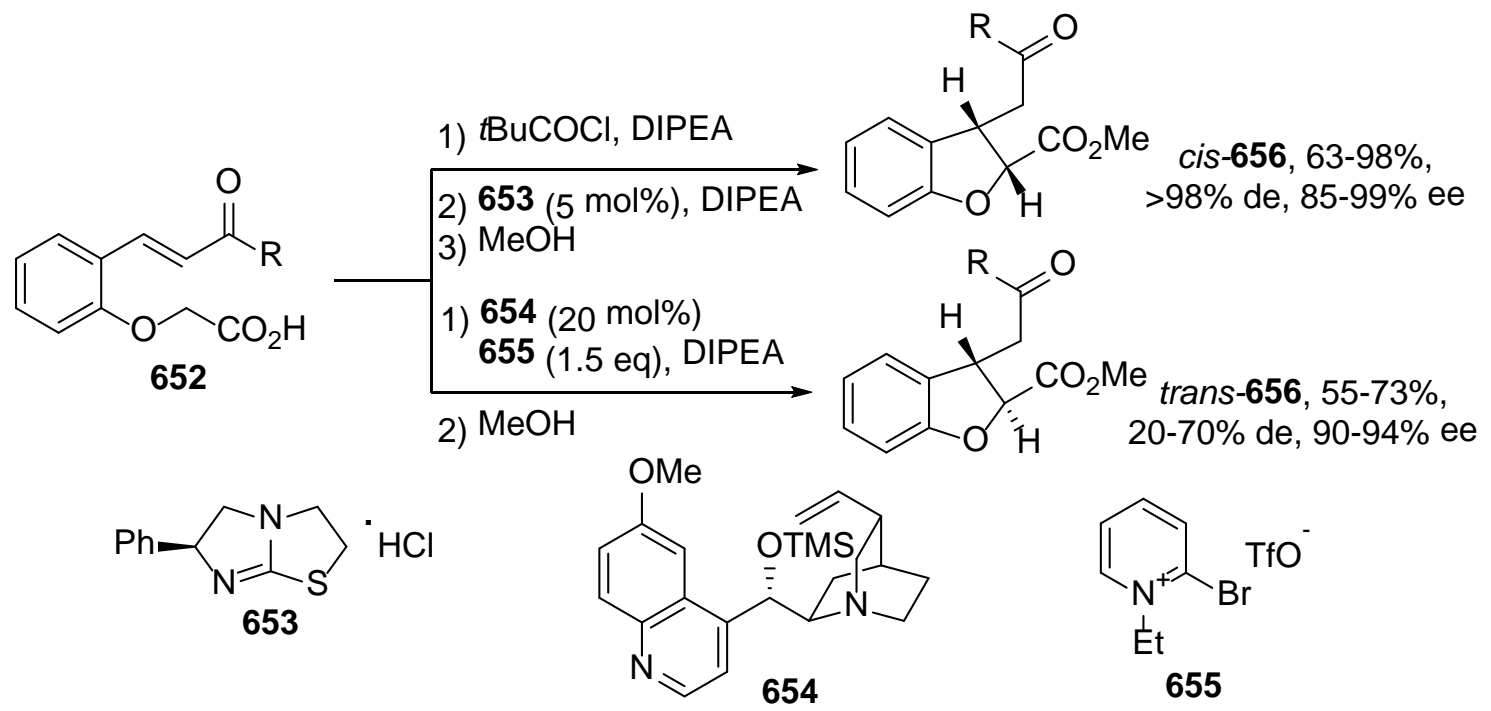

The solvent-dependent enantiodivergent lactonization initiated by an intermolecular sulfa-Michael addition has been described by Matsubara and co-workers ${ }^{579}$ using Takemoto's thiourea 658 as organocatalyst. $\gamma$-Hydroxy- $\alpha, \beta$-unsaturated thioesters reacted with thiophenols giving $\beta$-mercapto- $\gamma$-lactones by the conjugate addition of the thiol followed by lactonization. The influence of the solvent in the switch of enantioselectivity was studied where the formation of $(R)-\mathbf{6 5 9}$ has been observed in $78 \%$ ee in $\mathrm{CH}_{2} \mathrm{Cl}_{2}$ at rt, whereas in a mixture 2:1 of acetonitrile and water product $(S)$ 659 was formed in low ee (Scheme 251).

\section{Scheme 251. Enantiodivergent Intramolecular Conjugate Addition/Lactonization of $\gamma$-Hydroxy- $\alpha, \beta$-unsaturated Thioesters 657 Organocatalyzed by Thiourea 658}<smiles>Cc1cccc(C)c1SC(=O)/C=C/CO</smiles>

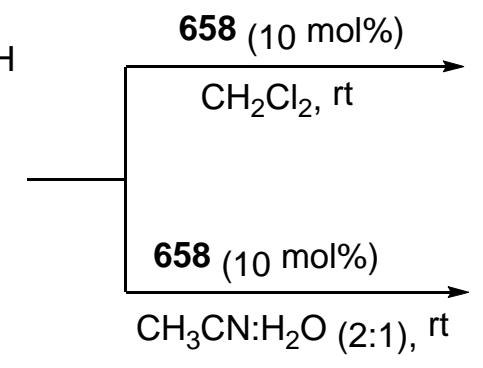<smiles>Cc1cccc(C)c1SC1COC(=O)C1</smiles>

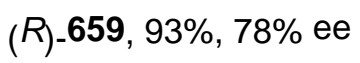<smiles>Cc1cc(NC(=S)N[C@H]2CCCC[C@H]2N(C)C)cc(C(F)(F)F)c1</smiles><smiles>Cc1cccc(C)c1SC1COC(=O)C1</smiles>

(S)-659, 95\%, 21\% ee

The tandem Michael-Henry reaction of 2-mercaptobenzaldehydes $\mathbf{6 6 0}$ with nitroolefins gave thiochromanes 663, using cupreine 661 as organocatalyst (Scheme 252). ${ }^{580}$ However, Arai and Yamamoto ${ }^{581}$ previously described that in the same reaction 
in the presence of an imidazoline-aminophenol (IAP) 662 nickel complex, the epimeric products 664 were obtained (Scheme 252). Recently, Xie and co-workers ${ }^{582}$ reported a diastereodivergent version of this process using 2-mercaptoquinoline-3-carbaldehydes 665 and different quinine derivatives as bifunctional organocatalysts. Using amine BnO-661 as catalyst, 3,4-dihydro-2H-thiopyrano[2,3-b]quinolines $\mathbf{6 6 6}$ were diastereoselectively formed, whereas with thiourea 347 (Scheme 126) the corresponding epimers 667 were obtained with excellent results (Scheme 252). To explain this diastereodivergent behavior model 666A, with the carbonyl group activated by the phenolic $\mathrm{OH}$ group, and 667A, with a $\mathrm{Re}$ face approach, have been postulated, respectively.

Scheme 252. Diastereodivergent Michael-Henry Reactions of 2-Mercaptobenzaldehydes with Nitroolefins Catalyzed by Different Organocatalysts 


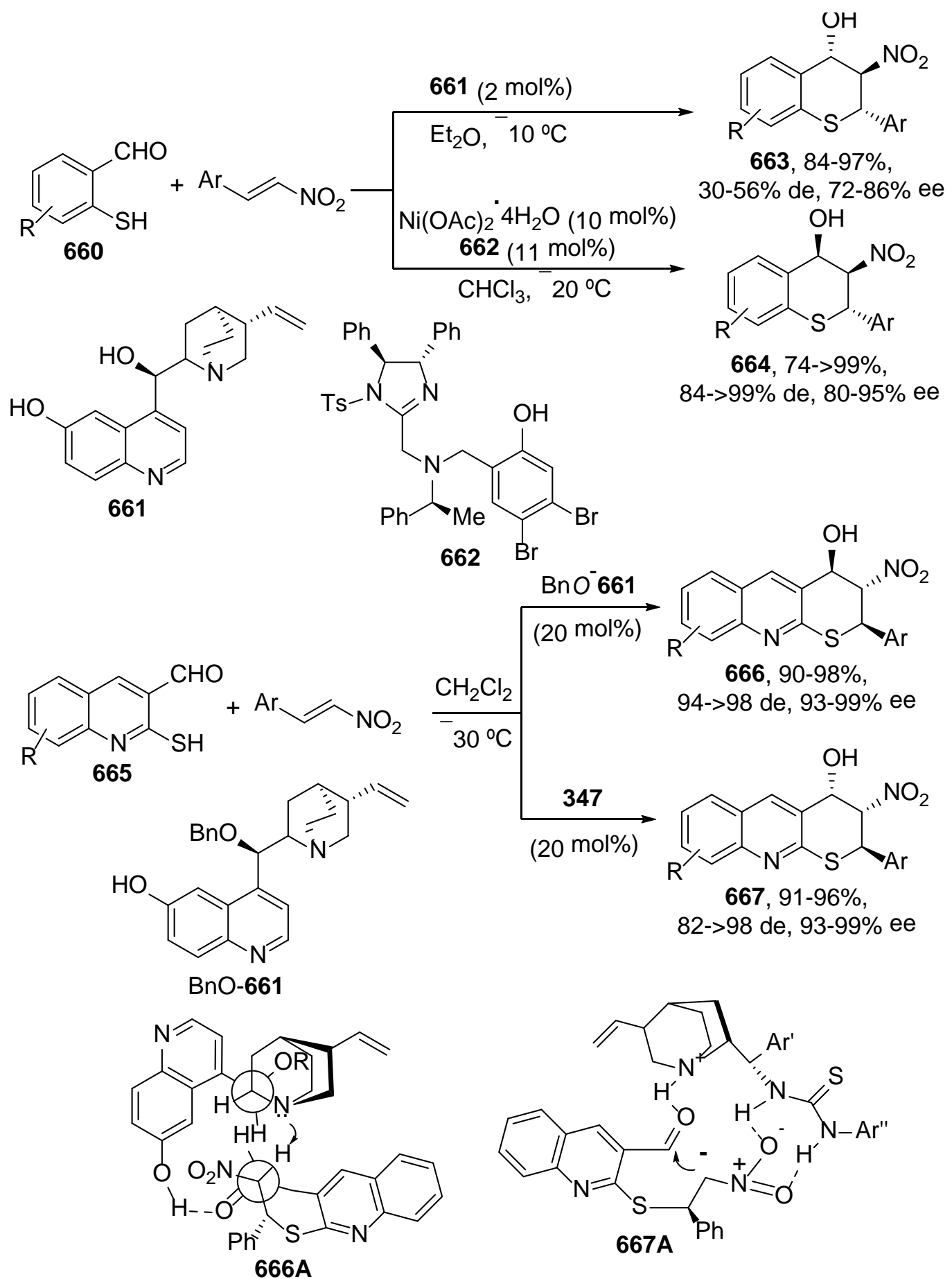

Starting from the syn-racemate 668 (90\% de), obtained from cyclohexanone and $\beta$ nitrostyrene, a Michael-intramolecular Henry tandem reaction with cinnamaldehyde afforded diastereodivergent results depending on the presence or absence of an achiral thiourea as co-catalyst. When prolinol $475(\mathrm{Ar}=\mathrm{Ph})$ (Scheme 172) was used as organocatalyst, product 669a was obtained in 49\% yield, $42 \%$ de and $90 \%$ ee. However, in the presence of the Schreiner's bis(3,5-trifluoromethyl) thiourea, the diastereomer 669b was isolated in $80 \%$ yield, $74 \%$ de and $90 \%$ ee (Scheme 253 ). ${ }^{583}$ This process has been studied with different nitroalkenes and $\alpha, \beta$-unsaturated aldehydes. In the proposed mechanism intermediates 669A and 669B have been postulated to explain the formation 
of products $669 \mathrm{a}$ and $669 \mathrm{~b}$, respectively. In the case of $668 \mathrm{~B}$, the thiourea is acting as hydrogen-bond-donating catalysts.

Scheme 253. Diastereodivergent Michael-Henry Reactions of 668 with Cinnamaldehyde Catalyzed by Prolinol 475 and with or without Schreiner's Thiourea
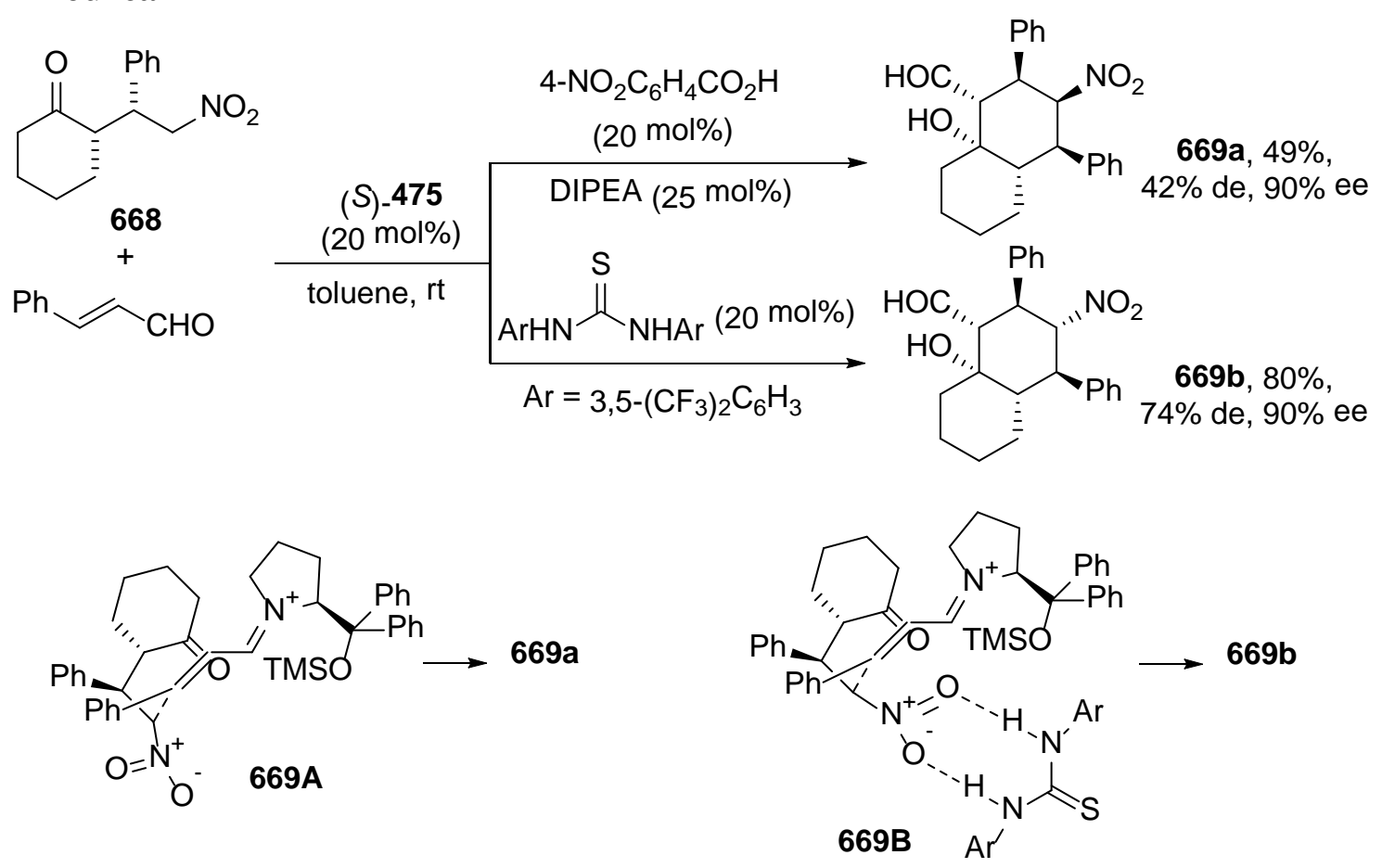

Brønsted acids with different strengths provided different diastereomers in the Pdcatalyzed intramolecular aza-Michael reactions of enones $670 .^{584}$ Trifluoroacetic acid controlled the formation of morpholines cis-671 with de up to 90\% (Scheme 254), while triflic acid catalyzed the formation of products trans-671 with de up to $86 \%$.

Scheme 254. Diastereodivergent Intramolecular aza-Michael Reaction of Amino Enones 670 Catalyzed by Pd and Different Brønsted Acids
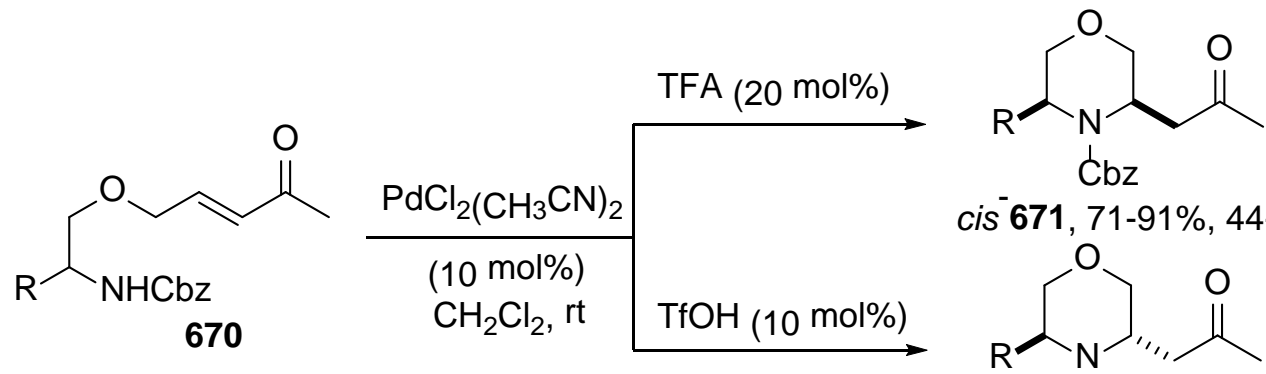

cis ${ }^{-671}, 71-91 \%, 44-90 \%$ de<smiles>[R]C1COC[C@@H](CC(C)=O)N1C(=O)O</smiles>

trans ${ }^{-} 671,64-95 \%, 44-86 \%$ de

Del Pozo, Fustero and co-workers have described a diastereodivergent intramolecular aza-Michael reaction of $\alpha, \beta$-unsaturated $\omega$-amino esters 672 promoted 
by TBAF or $\mathrm{BF}_{3} \cdot \mathrm{OEt}_{2} \cdot{ }^{585}$ Under basic conditions, fluorinated trans-homoprolines 673 were obtained, whereas using $\mathrm{BF}_{3} \cdot \mathrm{OEt}_{2}$ as Lewis acid products cis-673 were mainly formed (Scheme 255). The enantiopure sulfinyl group at the ortho-position controlled the formation of three stereocenters and can be finally removed by means of Raney Ni. The high selectivity with $\mathrm{BF}_{3} \cdot \mathrm{OEt}_{2}$ has been attributed by the authors to the formation of a chelate transition state 672A with the Lewis acid coordinating the carbonyl oxygen, the sulfoxide and the nitrogen atom. In the case of TBAF, the transition state 672B with a fluorinated group located in a pseudoequatorial arrangement controlled the nucleophilic addition.

Scheme 255. Diastereodivergent Intramolecular aza-Michael Reaction of $\omega$-Amino Esters 672 Promoted by TBAF or $\mathrm{BF}_{3} \cdot \mathrm{OEt}_{2}$

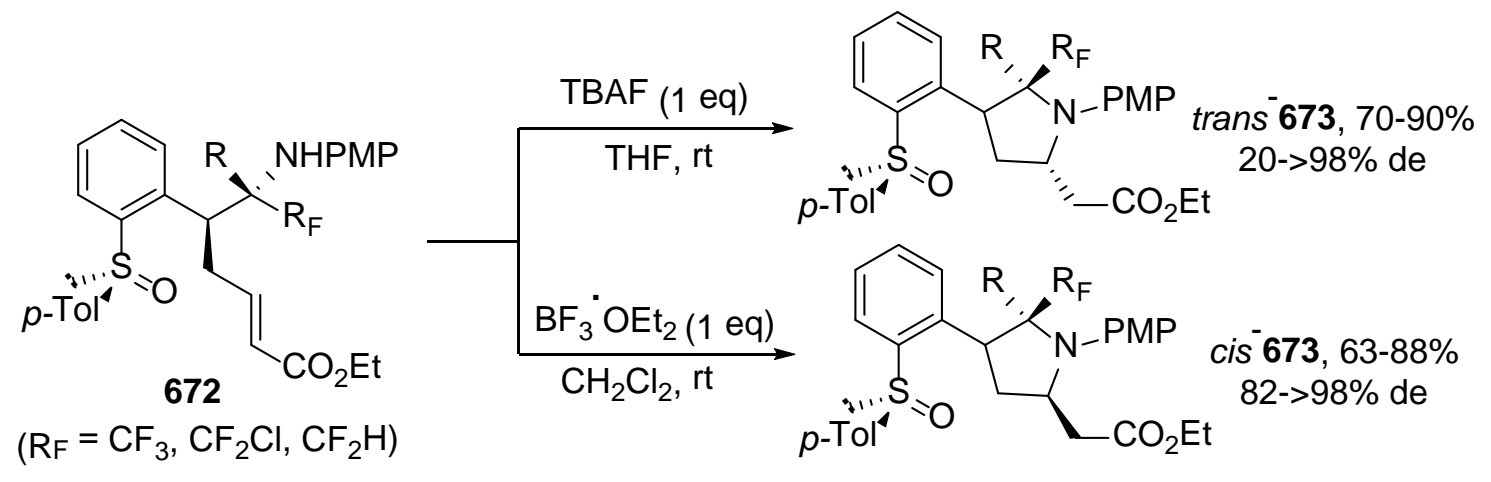

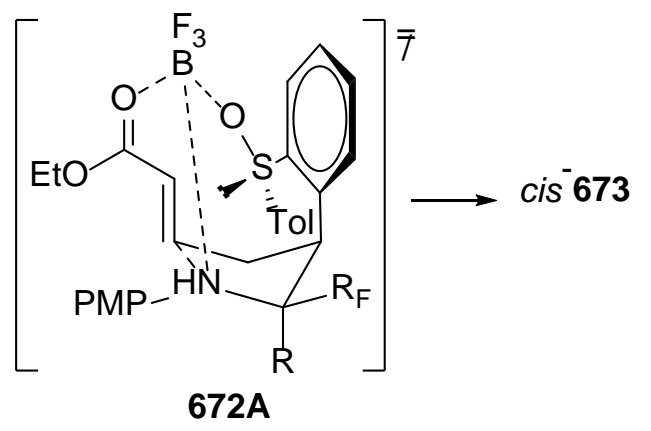

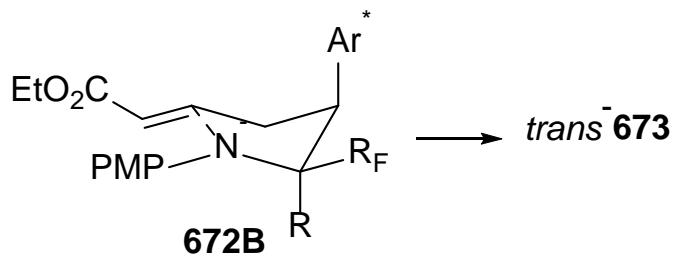

In conclusion, only few stereodivergent intramolecular conjugate additions have been described by different homochiral amines or by changing the solvent or the acidity of the media. The Pd-catalyzed diastereodivergent reactions were controlled by the strength of the Brønsted acid.

\subsection{Intramolecular Nucleophilic Substitution}

Stereodivergent Pd-catalyzed intramolecular allylation is the nucleophilic substitution mainly described by using amines as nucleophiles. A nucleophile-dependent stereodivergence has been observed by Llebaria and co-workers in the Pd-catalyzed cyclization of differently protected 4-acetoxy-5-(p-tolylsulfinyl)-5-hexenylamines $\mathbf{6 7 4}$ (Scheme 256). ${ }^{586}$ In the case of the sodium salt of $N$-Boc protected $674 a$, the corresponding pyrrolidine $\left(2 R, S_{\mathrm{S}}\right)$-675a was preferentially formed in $80 \%$ de, while the use of trifluoroacetamide $674 \mathbf{b}$ led to the formation of $\left(2 S, S_{S}\right)-675 \mathbf{b}$ with the same 
diastereoselectivity. However, in the case of piperidines the diastereoselectivity was very low: $50 \%$ and $10 \%$, respectively. These diastereodivergent results have not been rationalized.

Scheme 256. Diastereodivergent Pd-Catalyzed Intramolecular Allylic Amination of Different $N$-Protected Amines 674

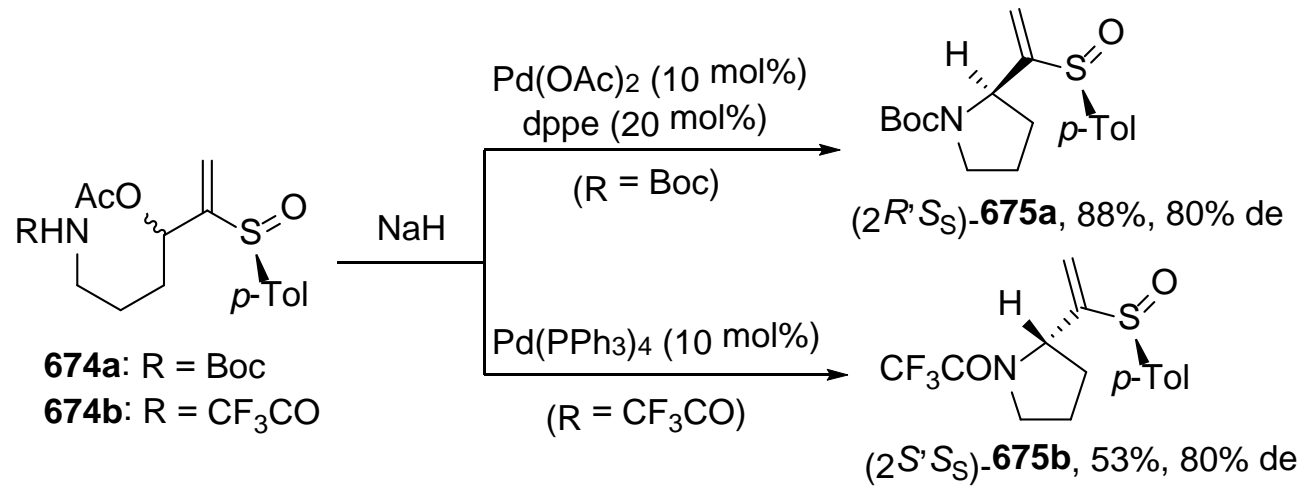

A leaving group-dependent diastereodivergent intramolecular allylic amination has been found by Tanaka and co-workers. ${ }^{587}$ Starting from allylic $N$-2,4,6trimethylphenylsulfonyl (Mts) protected $\delta$-amino alcohols 676a, the corresponding cisvinylaziridines 677 were formed under Pd-catalyzed conditions when methyl carbonates were used as substrates (Scheme 257). However, using the sodium salts of the corresponding mesylates the direct nucleophilic substitution exclusively provided the thermodynamically less stable trans-aziridines 677. The kinetically favored transselective cyclization was explained by the allylic 1,3-strain of aza-anionic intermediates 676B.

Scheme 257. Diastereodivergent Intramolecular Allylic Amination of Differently Protected $\delta$-Amino Allylic Alcohols 676

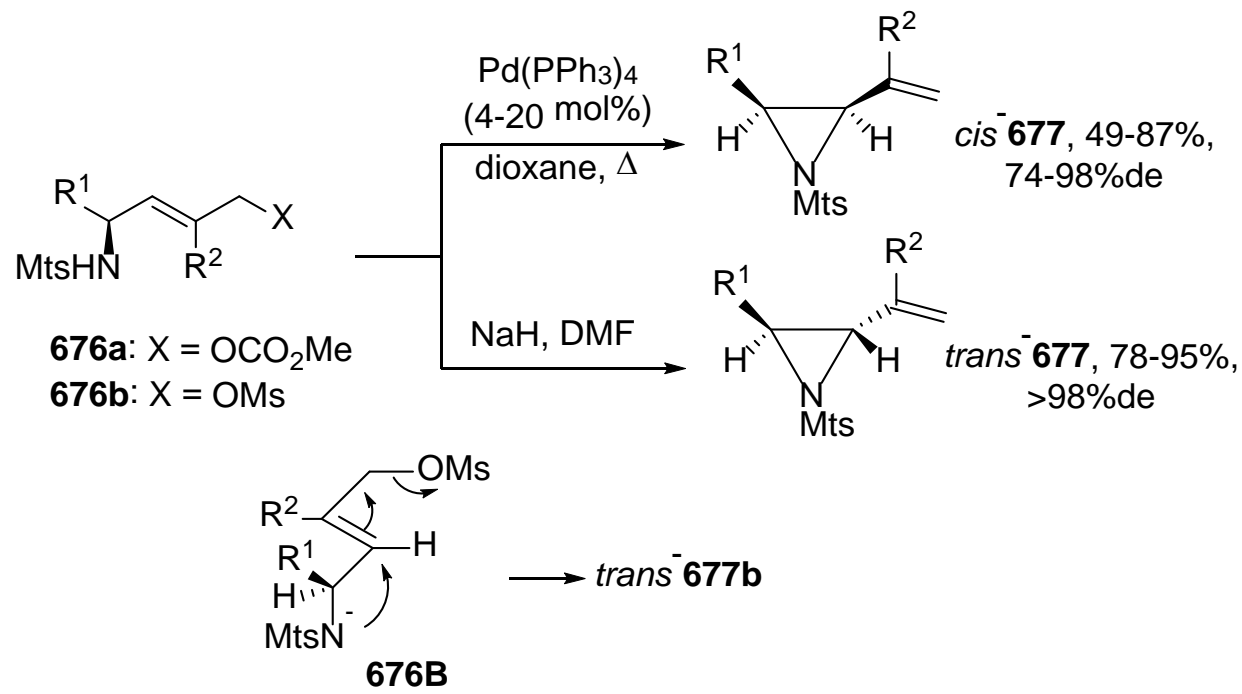

The diastereodivergent intramolecular Pd-catalyzed allylic amination of ureas 678 has been achieved under different reaction conditions. ${ }^{588}$ In THF, cis-1,3- 
tetrahydropyrimidones 679 were formed with de up to $90 \%$, whereas in $\mathrm{CH}_{2} \mathrm{Cl}_{2}$ 1,3trans-products 679 were obtained also with de up to 90\% (Scheme 258). These products were further transformed into syn- and anti-1,3-diamines.

Scheme 258. Diastereodivergent Pd-Catalyzed Intramolecular Allylic Amination of Ureas 678 in Different Solvents

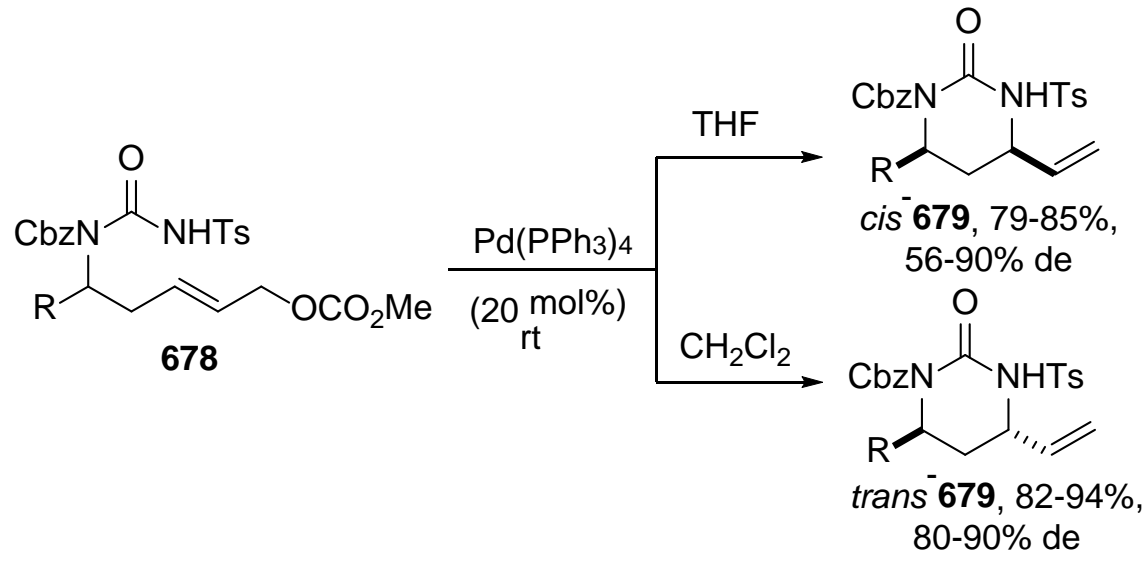

The iridium-catalyzed intramolecular asymmetric allylic amination of enantiopure amino carbonate $\mathbf{6 8 0}$ gave diastereodivergent results depending on the configuration of Feringa's phosphoramidite 187 (Scheme 66). ${ }^{589}$ When the $(S, S, S)$-ligand was used, the 2,5-trans-pyrrolidine $\mathbf{6 8 1}$ was mainly formed, while the enantiomeric $(R, R, R)$-ligand provided 2,5-cis-681 (Scheme 259). Pyrrolidine trans-681 was further transformed into (+)-bulgecinine, a nonproteinogenic amino acid which occurs in bulgecins A, B and C antibiotic glycopeptides, and cis-682 was converted into the antifugal agent (+)preussin.

Scheme 259. Diastereodivergent Asymmetric Ir-Catalyzed Intramolecular Allylic Amination of Compound 680 Using Enantiomeric Feringa's Phosporamidites 187

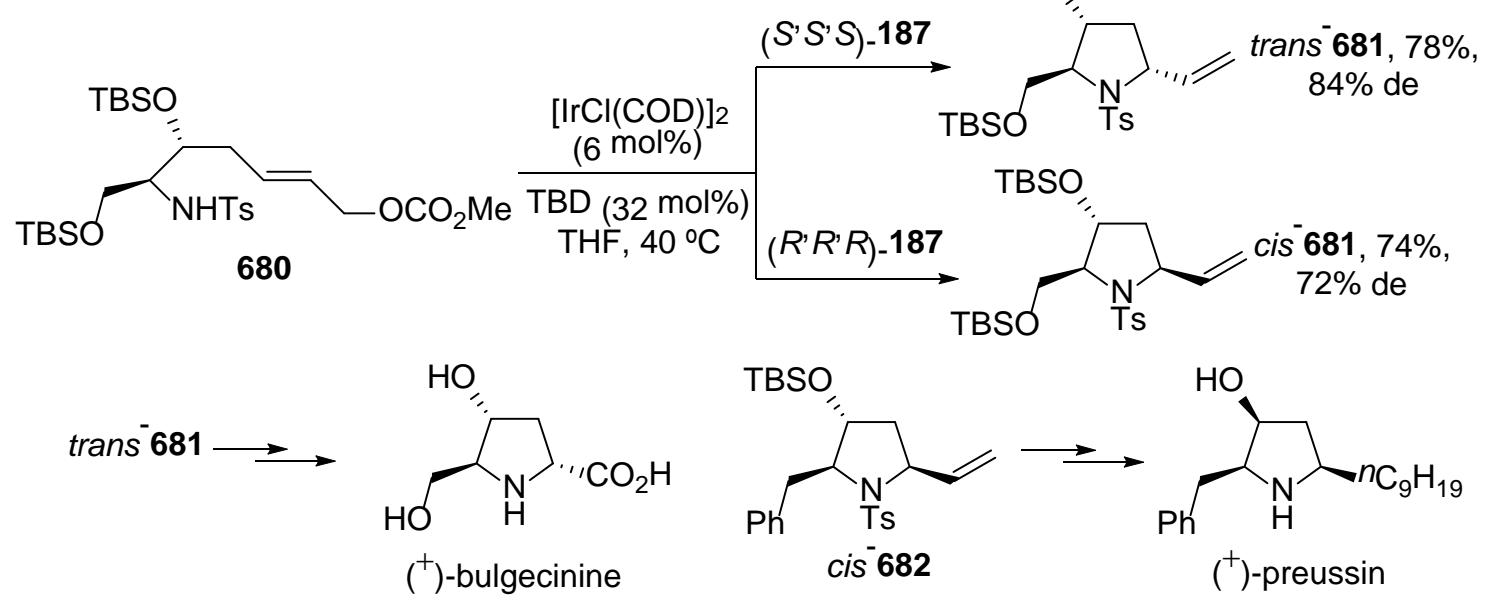

The Pd-catalyzed asymmetric allylic cyclization of allylic meso-diol diacetate $\mathbf{6 8 3}$ afforded diastereodivergent results depending on the structure of Trost's ligands ent-489 
and ent-490 (Figure 17). In the case of ligand ent-489, tetrahydrofuran cis-684 was obtained in moderate de (60\%) and excellent 96\% ee (Scheme 260). ${ }^{590}$ Changing the phenyl to a naphthyl group, the ligand ent-490 led to the formation of trans-684 in low $26 \%$ de and high $\mathbf{8 9 \%}$ ee. The tetrahydrofuran cis-684 is the key precursor for 7-epi-ST$\Delta^{8}$-10-neurofuran, a valuable neuronal oxidative stress biomarker.

Scheme 260. Diastereodivergent Asymmetric Pd-Catalyzed Intramolecular Allylic Etherification of Diol Acetate 683 with Different Chiral Trost's Ligands ent-489 and ent-490

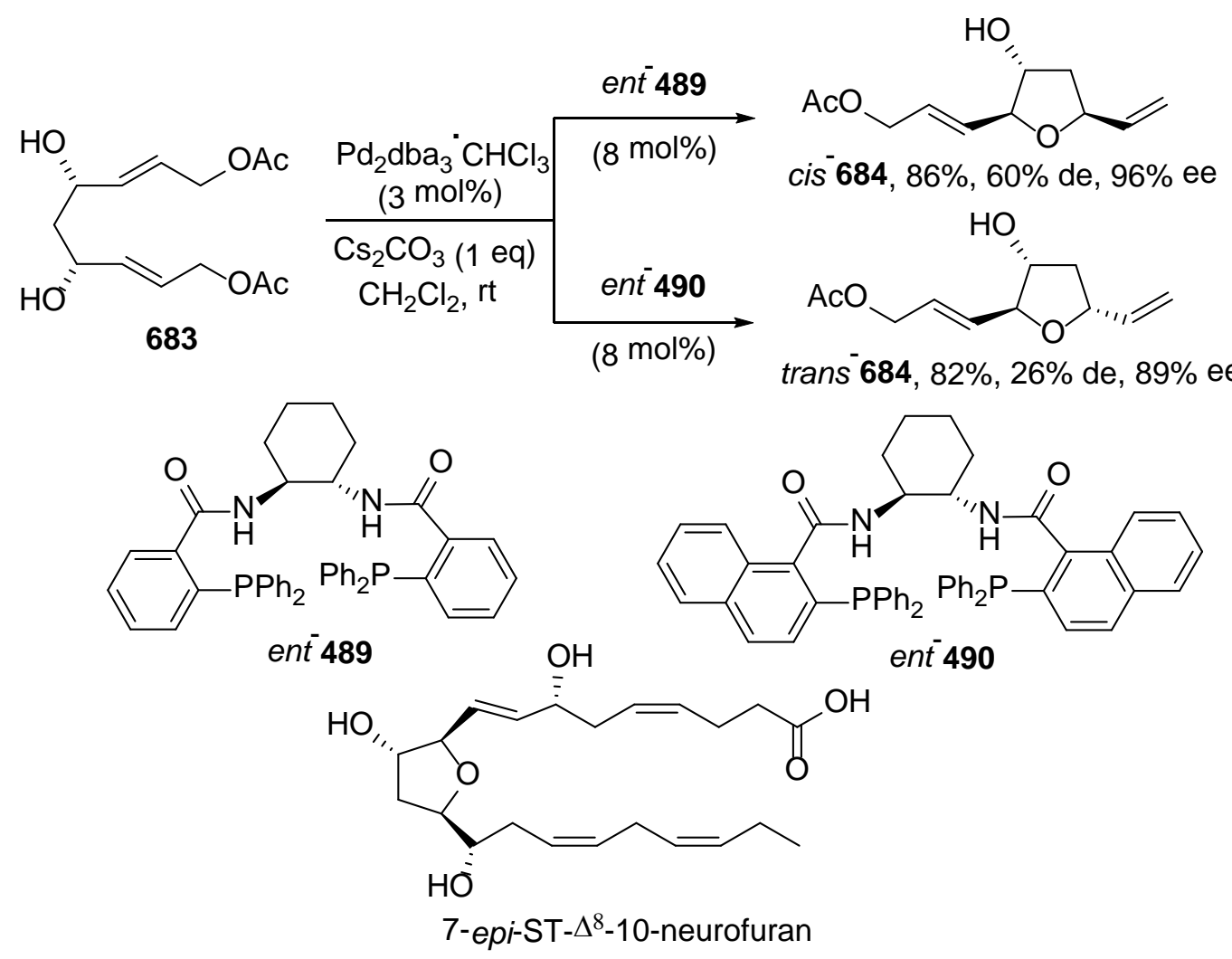

In conclusion, the intramolecular metal-catalyzed allylic nucleophilic substitutions can be diastereodivergently performed depending on the reaction conditions and the structure of the substrate. In the case of the asymmetric diastereodivergent processes the chiral ligand structure can provide means to control the diastereo- and enantioselectivity.

\subsection{Other Intramolecular Cyclizations}

Several stereodivergent metal-catalyzed intramolecular cyclizations will be considered in this Section, such as hydroaminations, [3+2] annulations, ene reactions, ring-closing metathesis, and other reactions.

Rare-earth metal complexes based on linked bis( $\beta$-diketiminato) ligands have been used as catalysts in the asymmetric intramolecular hydroamination of aminoalkenes 685. ${ }^{591}$ Pyrrolidines (S)-688 were obtained in moderate enantioselectivities with yttrium 
complex $(R, R)$-686, whereas lanthanum complex $(R, R)$-687 provided $(R)$-688 in very low ee (Scheme 261). This enantiodivergent hydroamination lacks of practical interest.

\section{Scheme 261. Enantiodivergent Intramolecular Hydroamination of Aminoalkenes} 685 Catalyzed by Y and La Complexes

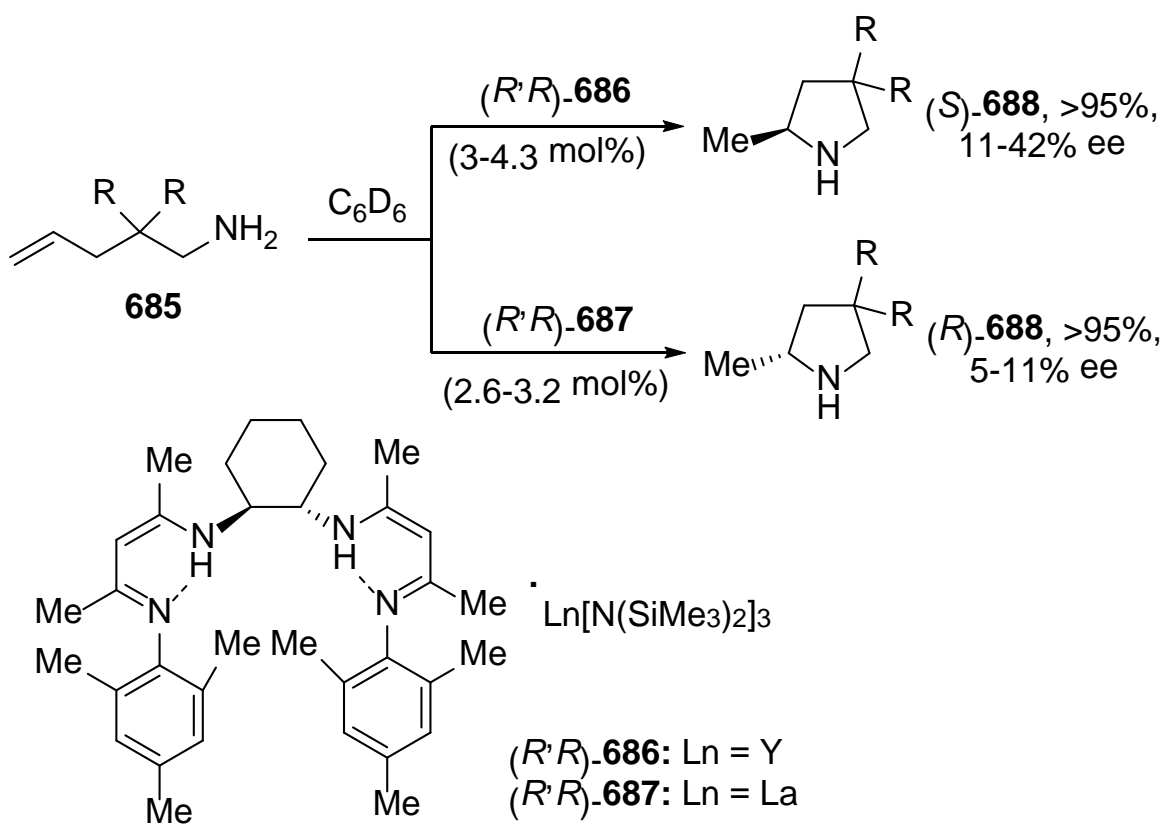

Recently, visible-light has been used as a promoter for the diastereodivergent intramolecular oxyamination of alkenes. ${ }^{592}$ Starting from unsaturated hydroxylamines 689 and the photocatalyst $\left[\operatorname{Ir}(\mathrm{ppy})_{2}\right.$ (dibbpy) $] \mathrm{PF}_{6} \quad\{[4,4$ '-bis(1,1-dimethylethyl)-2,2'bipyridine-N1,N1']bis[2-(2-pyridinyl-N)phenyl-C]iridium(III) hexafluorophosphate , controlling the basicity of the amine, it was possible to obtain anti-oxazolidinones 690 in the case of $\mathrm{Et}_{3} \mathrm{~N}$ and syn-690 with $\mathrm{Ph}_{3} \mathrm{~N}$ (Scheme 262). To explain this diastereodivergent effect it has been proposed that the benzoate anion attacks the aziridine intermediate using $\mathrm{Et}_{3} \mathrm{~N}$ to give the anti-690 products by a $\mathrm{S}_{\mathrm{N}} 2$ mechanism. Due to the weak basicity of $\mathrm{Ph}_{3} \mathrm{~N}$, it could not deprotonate benzoic acid and a $\mathrm{S}_{\mathrm{N}} 1$ reaction pathway might precede delivering syn-690.

Scheme 262. Diastereodivergent Visible-Light Promoted Intramolecular Oxyamination of Unsaturated Hydroxylamines 689 with Different Tertiary Amines 


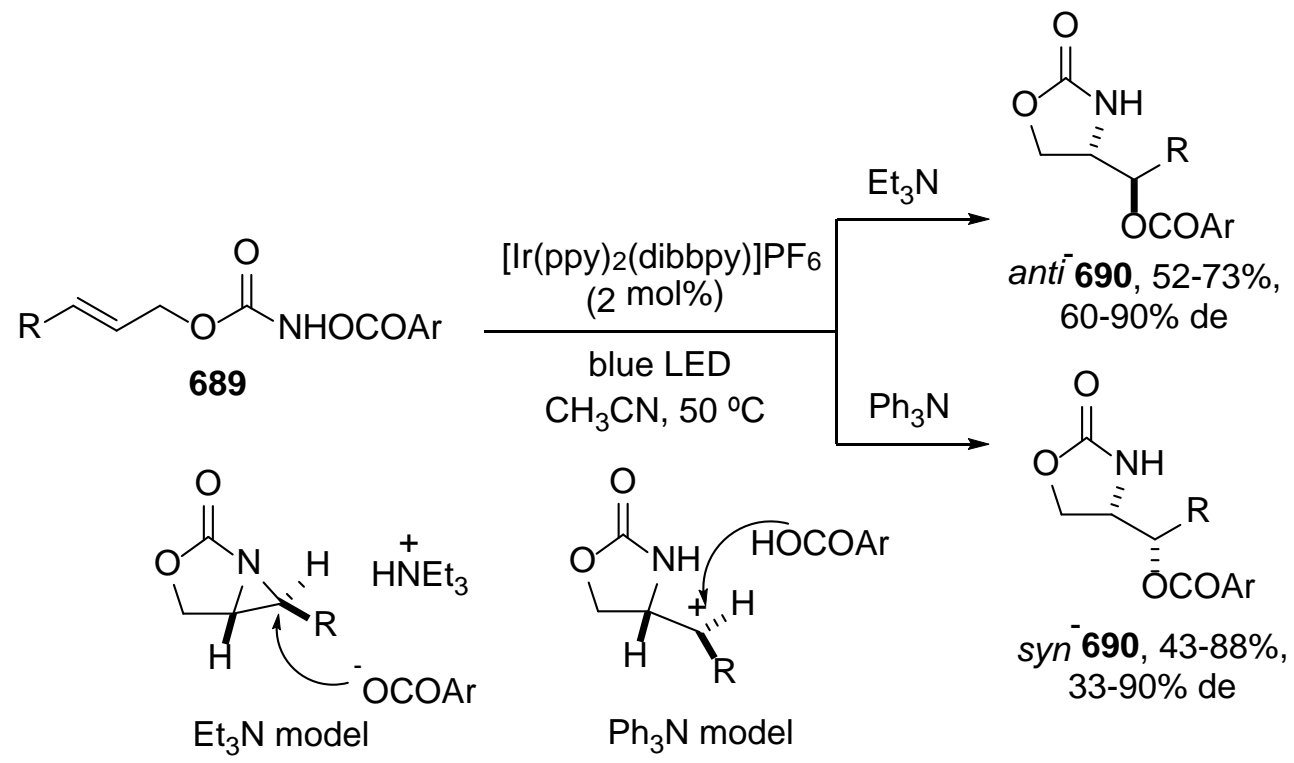

Diastereodivergent $\mathrm{Cu}(\mathrm{II})$-catalyzed intramolecular [3+2] annulations of cyclopropanes tethered to an indol unit can be carried out just by changing the ester group in the cyclopropane unit. ${ }^{593}$ With substrates 691a bearing isopropyl esters, tetracyclic spiroindolines trans-693a were obtained in the presence of ligand $\mathbf{6 9 2}$ and a $\mathrm{Cu}$ (II) salt with de up to $98 \%$ (Scheme 263). A switch of diastereoselectivity was achieved in the case of 2-adamantyl (Ad) esters 691b providing cis-693b with de up to $92 \%$. DFT calculations supported that the formation of the cis-isomers was preferred when steric repulsions become predominant. On the other hand, attractive interactions between the ester group and the arene of the indole favored the formation of the transisomers.

\section{Scheme 263. Diastereodivergent Intramolecular $\mathrm{Cu}(\mathrm{II})$-Catalyzed [3+2]} Annulations of Indolyl-Cyclopropanes 691 Bearing Different Ester Groups

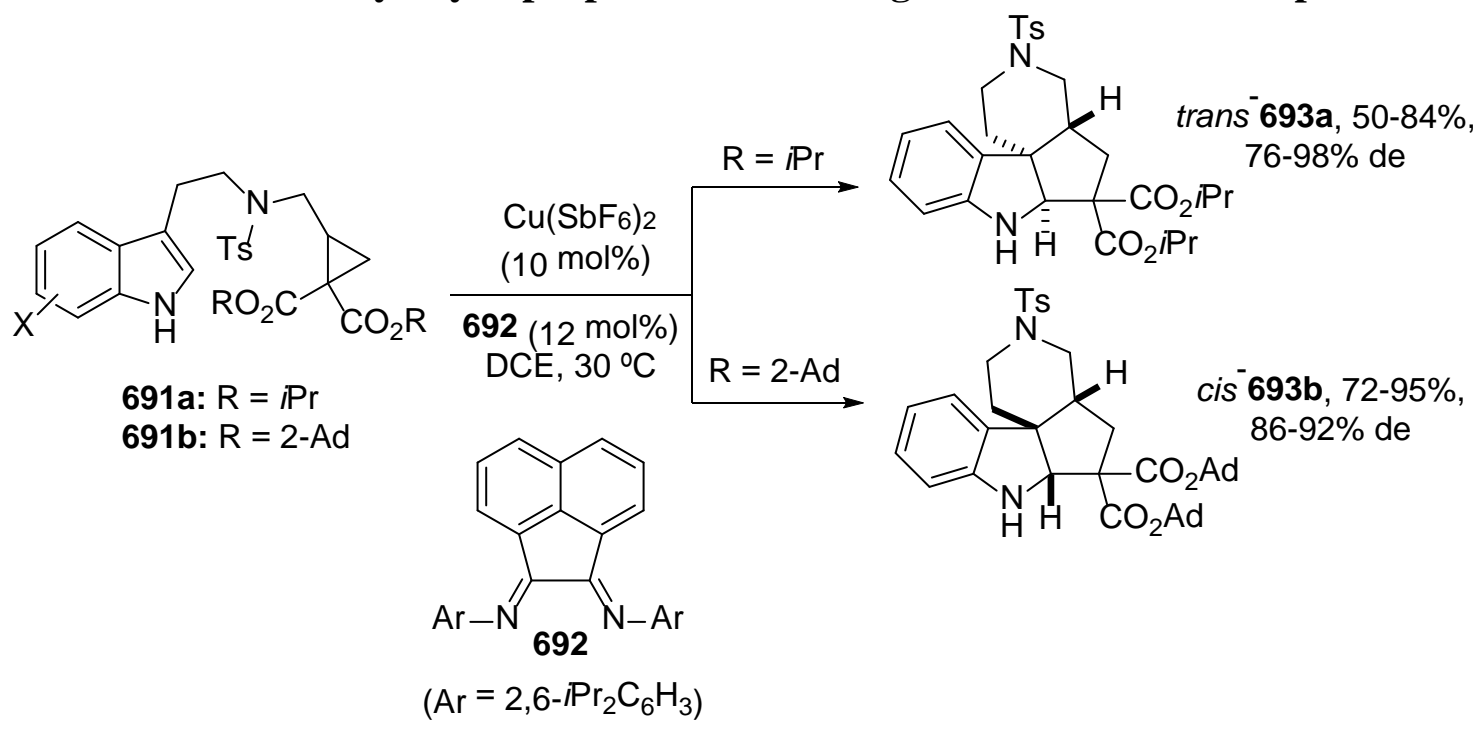


A metal catalyst-dependent [3+2] annulation of indolyl-allenes 694 has been recently described. ${ }^{594}$ Using $\mathrm{PtCl}_{2}$ as catalyst diazabenzo[a]cyclopenta[c,d]azulenes 696 were isolated (Scheme 264). However, when a cationic gold(I) complex [JohnPHOSAu]NTf 2 $\left(695 \cdot \mathrm{NTf}_{2}\right)$ was used as catalyst epimeric products 697 were formed. The proposed mechanism suggested the formation of heterocyclic intermediates 696A and 697A for the Pt- and Au-catalyzed processes, respectively. In the case of 696A a Pt-carbene intermediate 696B can participate in the formation of products $\mathbf{6 9 6}$, whereas in the case of 697A a Au-carbene 697B is the precursor for products 697.

Scheme 264. Diastereodivergent Intramolecular [3+2] Annulations of IndolylAllenes 694 Using $\mathrm{PtCl}_{2}$ or a Cationic Au Complex $695 \mathrm{NTf}_{2}$

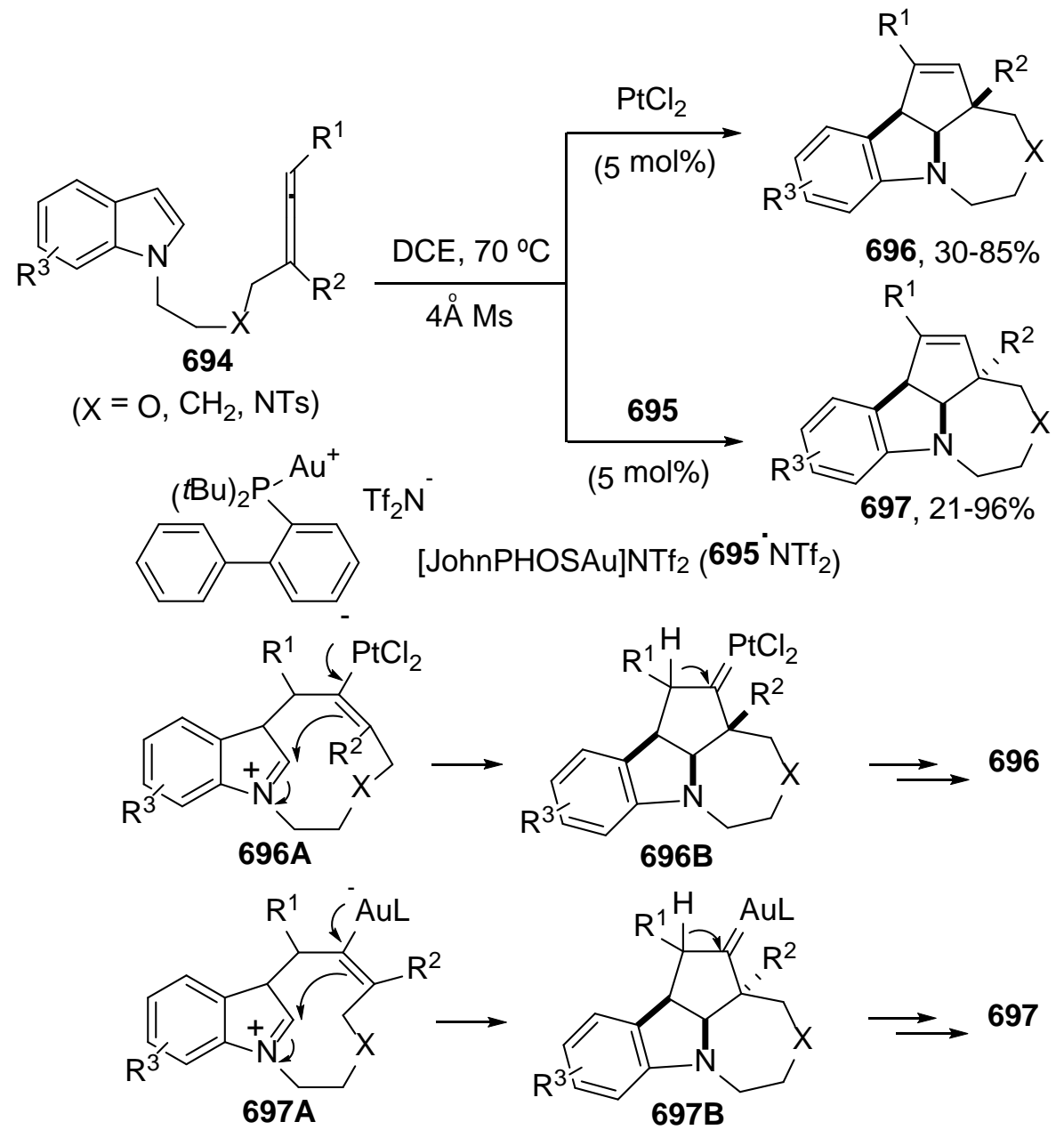

The metal-promoted ene cyclization of diene $\mathbf{6 9 8}$ has been used as key step in the total synthesis of kainic acid by Xia and Ganem. ${ }^{595}$ Modest diastereodivergent results have been observed during the cyclization of $\mathbf{6 9 8}$ in the absence or presence of $\mathrm{Mg}\left(\mathrm{ClO}_{4}\right)_{2}$ giving pyrrolidinone cis-699 or trans-699, respectively, in modest $33 \%$ de (Scheme 265).

Scheme 265. Diastereodivergent Ene Cyclization of Diene 698 Under Thermal Conditions or in the Presence of $\mathrm{Mg}\left(\mathrm{ClO}_{4}\right)_{2}$ 


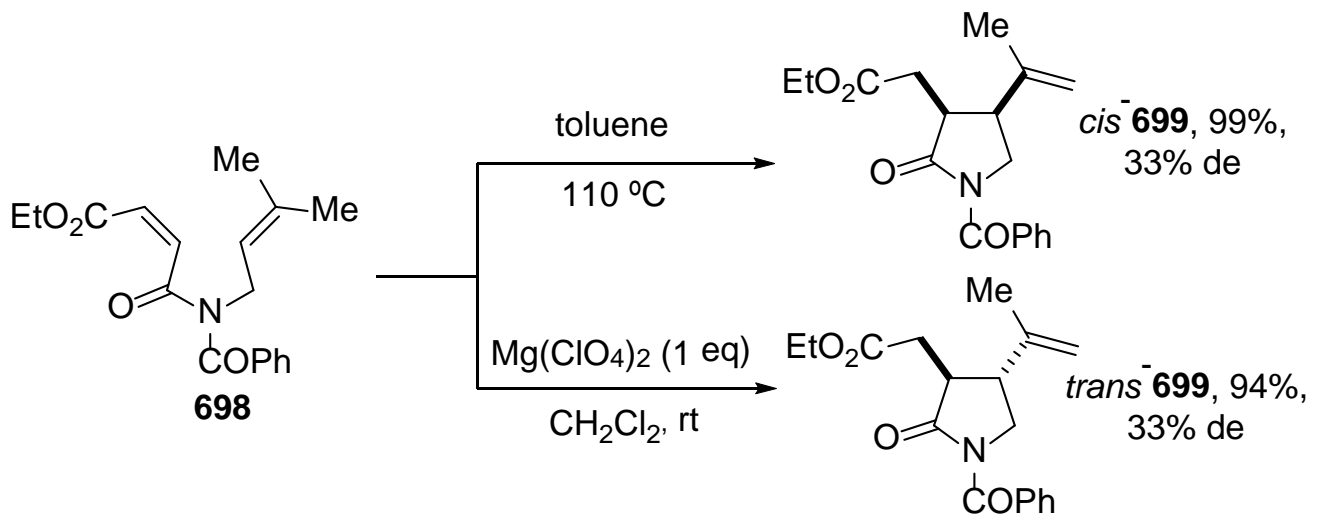

Only one example of the enantiodivergent ring-closing metathesis has been reported in the case of trienic phosphinates and phosphine oxides with chiral Mo catalysts. ${ }^{596}$ As a representative example, triene $\mathbf{7 0 0}$ provided the corresponding cyclic phosphine oxides (-)-702 and (+)-702 in 73\% and 96\% ee, respectively, using Mo catalysts 701a and 701b (Scheme 266). This reversal of enantioselectivity promoted by the structural modification of the achiral imido unit of the ligand, has been attributed to the different reactivity of both alkylidene isomers. In the case of catalyst 701a, the alkylidene intermediate might adopt a TS such as $\mathbf{7 0 2 A}$ and for catalyst 701b, with a tert-butyl group and the alkylidene in a trans position, the syn-intermediate 702B should have a more favorable TS.

Scheme 266. Enantiodivergent Ring-Closing Metathesis of Triene 700 Catalyzed by Different Mo-Catalysts 


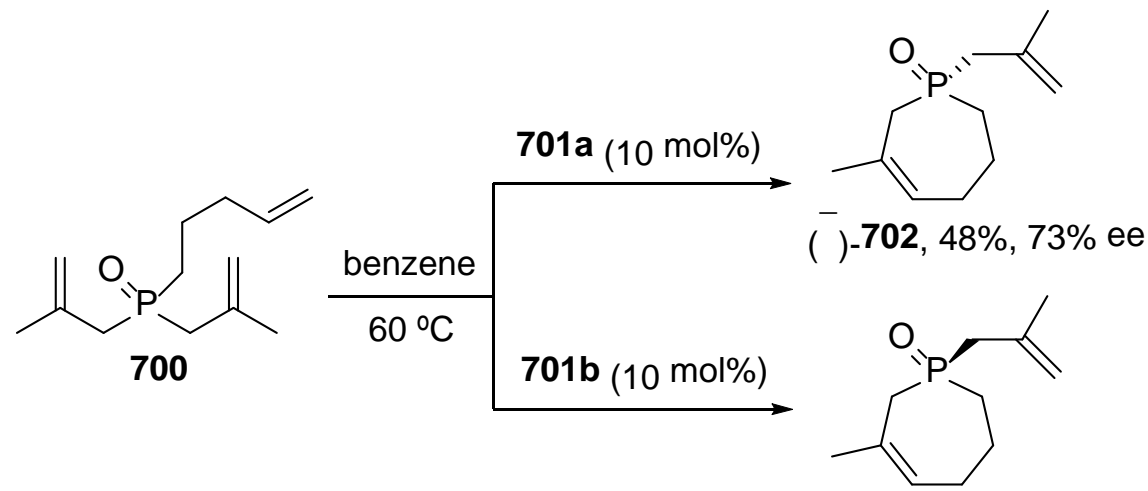

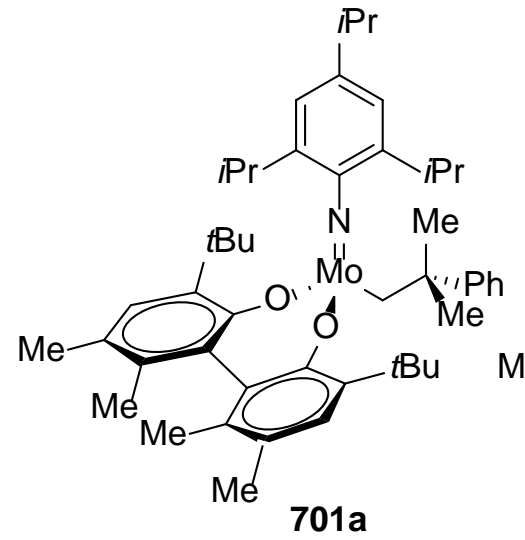

$\left(^{+}\right)-702,61 \%, 96 \%$ ee<smiles>C=C(C)CP(=O)(CCCC)C(=O)CCCCC</smiles>

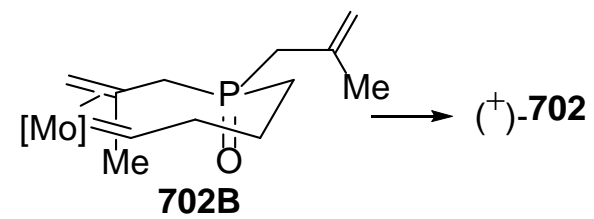

The intramolecular carbohalogenation of alkynes catalyzed by Pd and Q-PHOS (704) has shown diastereodivergent temperature-dependent effects. ${ }^{597}$ Aryl bromides bearing an alkyne unit $\mathbf{7 0 3}$ led to the formation of the corresponding Z-products $\mathbf{7 0 5}$ at $50{ }^{\circ} \mathrm{C}$ (Scheme 267). When the reaction was carried out at $100^{\circ} \mathrm{C}$ diastereomeric products $(E)$ 705 were obtained. According to the isomerization studies a plausible mechanism has been proposed. Under kinetic control (Z)-705 were preferentially formed and the $E$ products resulted under thermodynamic conditions. The isomerization processes took place under Pd-catalysis, and the oxidative addition of $\mathrm{C}\left(\mathrm{sp}^{2}\right)$-Hal and reductive elimination were reversible steps.

Scheme 267. Diastereodivergent Intramolecular Pd-Catalyzed Alkyne Carbohalogenation of 703 at Different Temperatures 


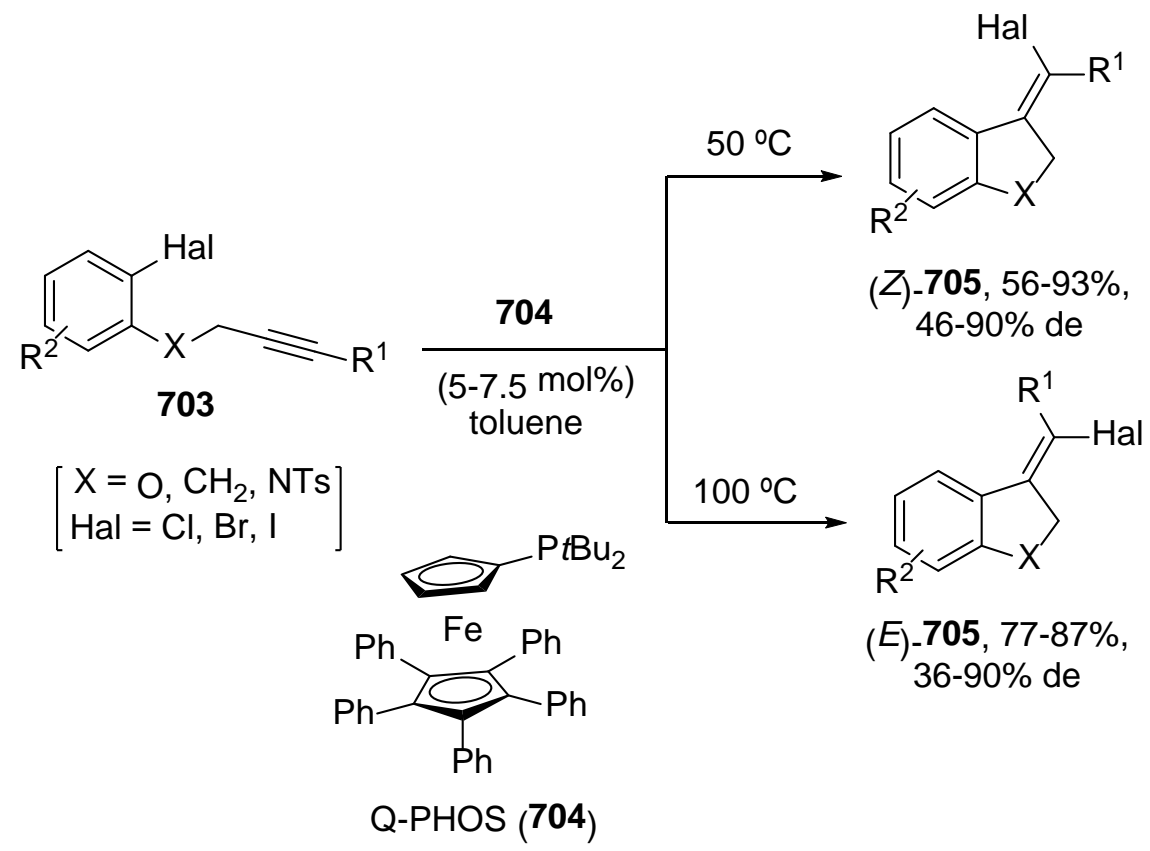

Only one example of the diastereodivergent gold(I)-catalyzed temperature-dependent cascade reaction of $(S, E)$-dienyne $\mathbf{7 0 6}$ with allyl alcohol has been described. In a reaction at rt with 10 eq of allyl alcohol the tricyclic ethers $\mathbf{7 0 7}$ were obtained in moderate de using a JohnPHOSAu (695) acetonitrile hexafluoroantimoniate complex (Scheme 268). However, at $-30{ }^{\circ} \mathrm{C}$ with 20 eq of allyl alcohol the formation of compound $\mathbf{7 0 8}$ with an opposite configuration at $\mathrm{C} 4$ was favored with similar modest de. The compound $\mathbf{7 0 7}$ has been employed by Echavarren and co-workers ${ }^{598}$ as a precursor in the total synthesis of the natural sesquiterpenoids (-)-epiglobulol and (-)$4 \beta, 7 \alpha$-aromadendranediol, and the compound $\mathbf{7 0 8}$ for (-)- $4 \alpha, 7 \alpha$-aromadendranediol.

Scheme 268. Diastereodivergent Intramolecular Au(I)-Catalyzed Cyclization of (S,E)-Dienyne 706 Under Different Reaction Conditions 


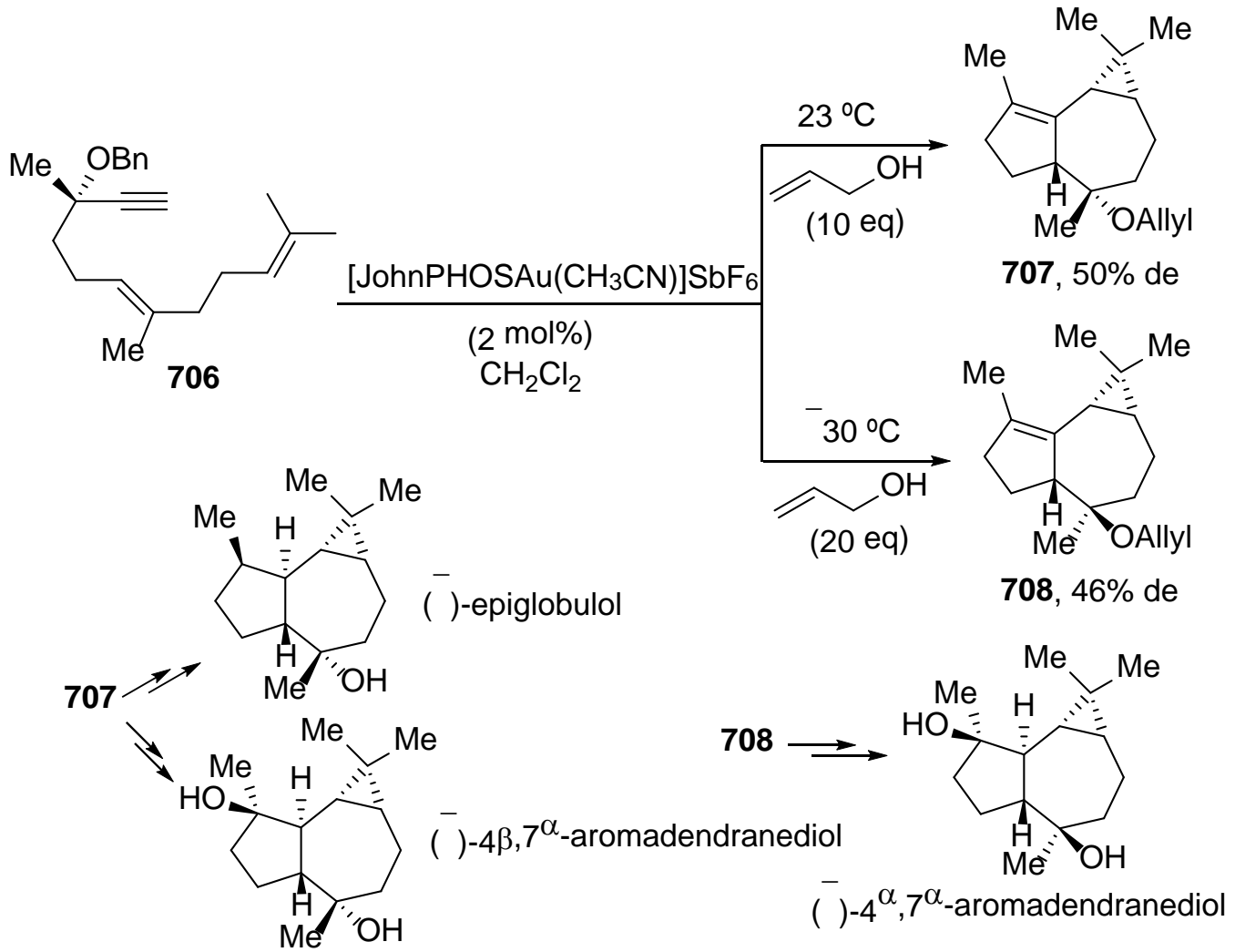

An example of the radical diastereodivergent cyclization-azidation cascade of 1,7enynes 709 using different metal complexes has been recently described. When $\mathrm{Cu}\left(\mathrm{ClO}_{4}\right)_{2}$ and bipyridine as ligand were used as catalyst and 1-[1'-(2-methylbenzyl)1,4'-bipiperidin-4-yl]-1H-benzo[d]imidazol-2(3H)-one (TBPB) as base, cis-fused pyrrolo[3,4-c]quinolines 710 were exclusively obtained (Scheme 269). ${ }^{599}$ On the other hand, the use of $\mathrm{Mn}(\mathrm{OAc})_{3}$ as catalyst afforded mainly products trans-710. To explain these results tentative models $\mathbf{7 1 0 A}$ and 710B have been proposed.

Scheme 269. Diastereodivergent Radical Cyclization-Azidation of 1,7-Enynes 709 Catalyzed by $\mathrm{Cu}\left(\mathrm{ClO}_{4}\right)_{2}$ or $\mathrm{Mn}(\mathrm{OAc})_{3}$ 


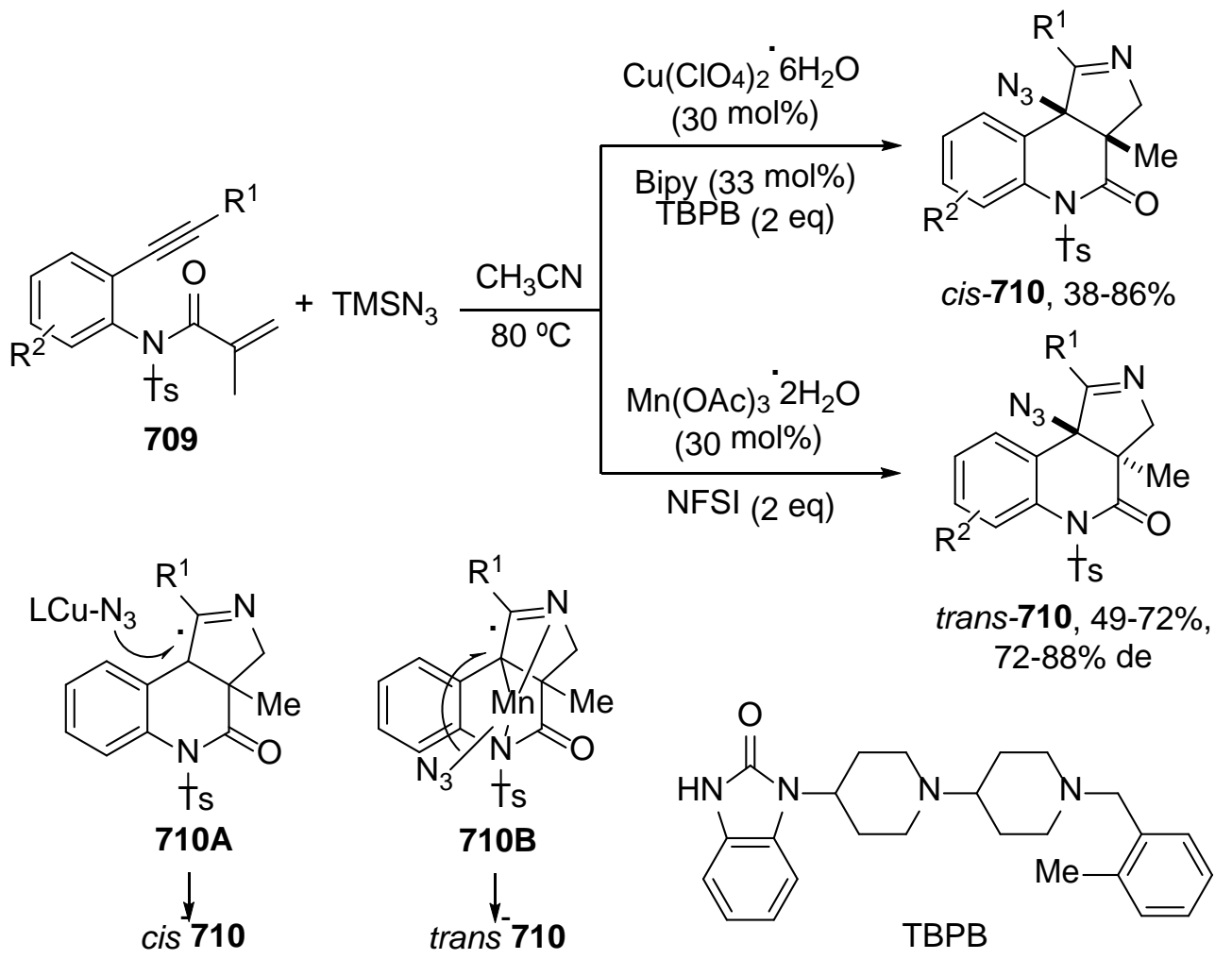

Sarpong and co-workers have recently described the intramolecular $\mathrm{C}\left(\mathrm{sp}^{3}\right)-\mathrm{H}$ functionalization of the Rh-azavinyl carbenes generated from the corresponding $\mathrm{N}$ sulfonyl triazoles, allowing the synthesis of saturated heterocycles (Scheme 270). ${ }^{600}$ The $\mathrm{Rh}$ (II) salts-dependent diastereodivergent processes can be performed affording cis- or trans-products 712. For instance, by treatment of triazole $\mathbf{7 1 1}$ with Rh(II) triphenylacetate (TPA) dimer in $\mathrm{CH}_{2} \mathrm{Cl}_{2}$ at $70{ }^{\circ} \mathrm{C}$ provided the tetrahydrofuran cis-712 after reduction with $\mathrm{LiAlH}_{4}$ of the intermediate imine unit. On the other hand, when $\mathrm{Rh}_{2}(\mathrm{OAc})_{4}$ was used as catalyst and after the same reduction, trans-712 was mainly obtained in 56\% de (Scheme 270). This effect can be explained by the different degree of freedom of the substituents around the new C-C bond. The smaller acetate anion allows the groups ( $\mathrm{Ph}$ and $\mathrm{C}=\mathrm{NTs}$ ) to be located in a more stable trans-configuration in the transition state.

Scheme 270. Diastereodivergent Intramolecular C(sp $\left.{ }^{3}\right)-\mathbf{H}$ Functionalization of RhAzavinyl Carbenes with Different Rh(II) Salts

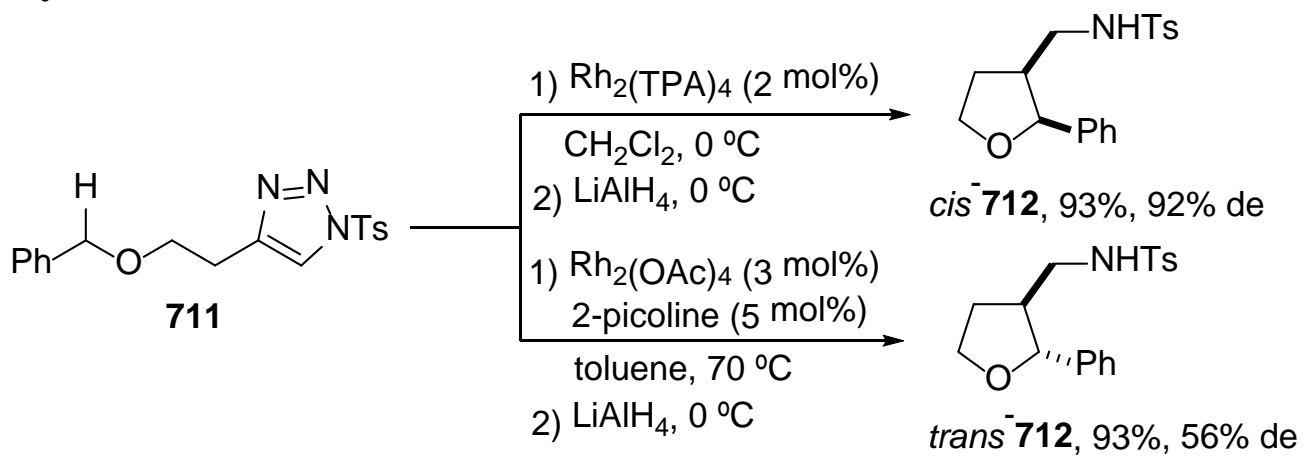


An unexpected inversion of enantioselectivity has been observed in the Cu-catalyzed intramolecular desymmetrization of $\alpha, \alpha$-bis(2-iodobenzyl)glycines $\mathbf{7 1 3}$ using an octahydro- $1 H$-indole-2-carboxylic acid 714 as chiral ligand (Scheme 271). ${ }^{601}$ In the presence of $\mathrm{K}_{3} \mathrm{PO}_{4}$ an aryl C-N coupling reaction took place affording indolines $(R)$ 715 with ee up to $84 \%$, whereas in the presence of 4-(dimetylamino)pyridine (DMAP) resulted enantiomers $(S)-715$ with ee up to $69 \%$.

\section{Scheme 271. Enantiodivergent Cu-Catalyzed Intramolecular Desymmetrization of Glycines 713 Using 714 as Chiral Ligand and Different Achiral Additives}

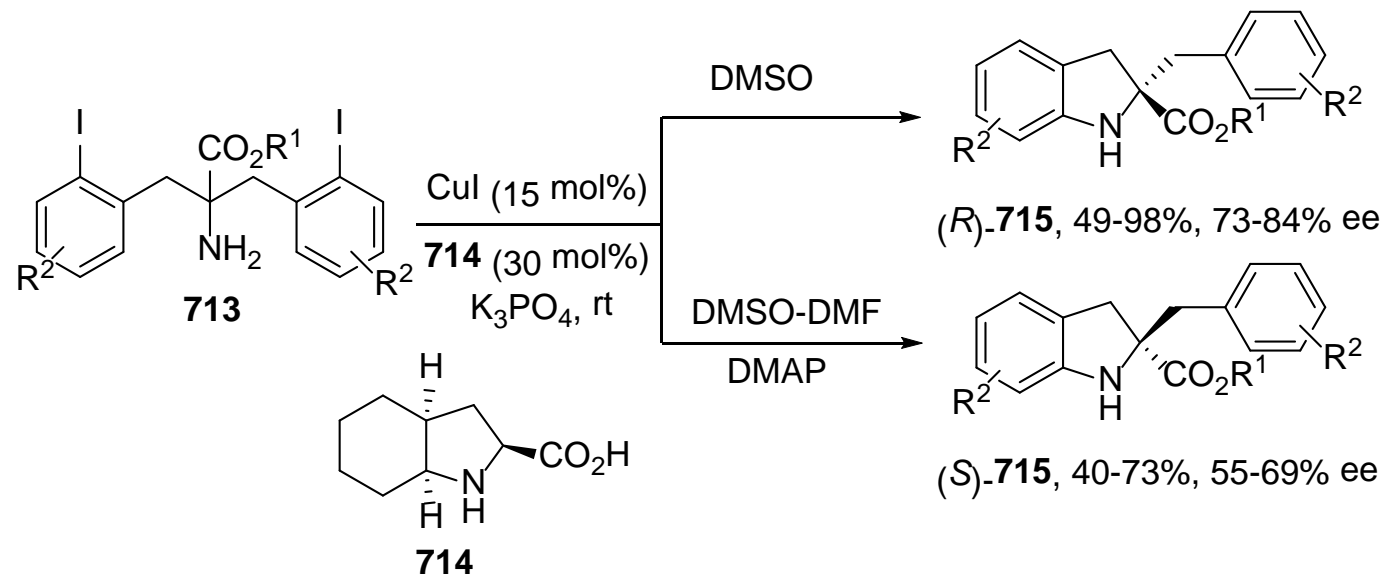

In conclusion for the above stereodivergent metal-catalyzed cyclization reactions, the metal salt or the complex played an important role in the diastereoselectivity of the different type of reactions, particularly with steric effects in the substrate or in the catalyst. In some cases, the reaction conditions, for example the reaction temperature, control the formation of either the kinetic or the thermodynamic diastereomer.

\section{STEREODIVERGENCE IN INTERMOLECULAR CYCLIZATIONS}

In this section, stereodivergent effects in the asymmetric intermolecular cyclizations such as [4+2], [3+2], and [2+2] cycloadditions under metal or organocatalyzed conditions will be considered. These reactions allow the formation of 6-, 5- and 4membered cyclic systems with a high degree of stereocontrol using simple starting compounds. The stereodivergent formation of five-membered rings by formal [3+2] annulations and six-membered rings by organocatalyzed cascade reactions, initiated by a Michael reaction, will be also included.

\section{1. [4+2] Cycloadditions}


Several types of stereodivergent [4+2] cycloadditons will be considered according to the nature of both components. Enantiodivergent normal electron-demand Diels-Alder (DA) reactions between dienes and $\alpha, \beta$-unsaturated carbonyl compounds are normally catalyzed by chiral metal complexes. In addition, diastereodivergent cycloadditions have been studied with chiral dienophiles. Some examples using chiral amines as organocatalysts have also been described. Enantiodivergent oxa-hetero DA reactions with normal and inverse electron-demand have been carried out generally with chiral metal complexes.

5.1.1. Diels-Alder Reactions. The first enantiodivergent Lewis acid-catalyzed [4+2] cycloadditions with normal electronic demand ${ }^{1 \mathrm{~g}}$ was described by Kobayashi and co-workers. ${ }^{602,603}$ In the DA reaction of cyclopentadiene with $\alpha, \beta$-unsaturated $N$-acyl 1,3-oxazolidin-2-ones using the complex 716, formed by $\mathrm{Yb}(\mathrm{OTf})_{3}$ and $(R)$-BINOL as chiral ligand, a reversal of enantioselectivity was observed depending on the achiral additive. For instance, $N$-crotonoyloxazolidinone 298 (Scheme 108) gave mainly the endo-adduct (2S,3R)-717 in the presence of $N$-acetyl-1,3-oxazolidinone (20 mol\%) in $77 \%$ yield and $93 \%$ ee (Scheme 272). However, in the presence of 3phenylacetylacetone the enantiomer (2R,3S)-717 was formed in $83 \%$ yield and $81 \%$ ee. This enantiodivergent effect was attributed to the different coordination number of the metal with the additives in complex $\mathbf{7 1 6 .}$

Scheme 272. Enantiodivergent Diels-Alder Reaction of Cyclopentadiene with $\mathrm{N}$ Crotonoyl Oxazolidinone 298 Catalyzed by $\mathrm{Yb}(\mathrm{OTf})_{3} /(\mathrm{R})-\mathrm{BINOL}$ in the Presence of Achiral Additives

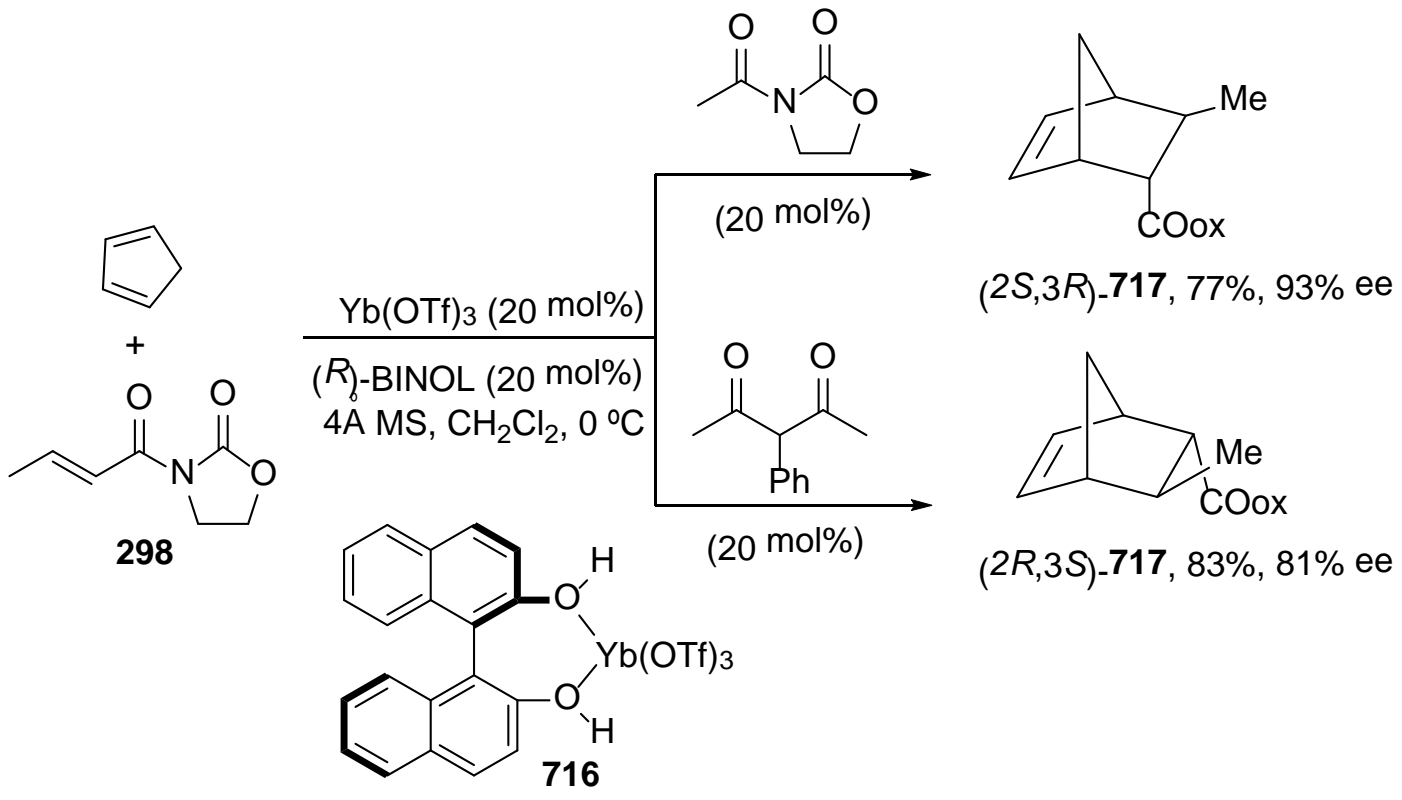

The influence of the lanthanide, in the above mentioned D-A reaction, using silylated PyBOX (299, Scheme 108) as chiral ligand has been studied by Desimoni and coworkers. $^{304}$ They observed a metal-dependent enantiodivergence in the Sc-based 
complex catalyzed DA with the formation of $(2 S, 3 R)-717$ in $99 \%$ ee, while the Y-based catalyst furnished $(2 R, 3 S)-717$ in $95 \%$ ee.

$C_{2}$-Symmetric chiral bis(oxazoline)-metal complexes have been successfully applied in the DA reaction. Ghosh and co-workers ${ }^{604}$ performed the [4+2] cycloaddition of cyclopentadiene with $\alpha$, $\beta$-unsaturated $N$-acyl 1,3-oxazolidin-2-ones using ligand 718 and $\mathrm{Cu}(\mathrm{OTf})_{2}$ obtaining in the case of the crotonoyl dienophile 298, adduct $(2 R, 3 S)-\mathbf{7 1 7}$ in $94 \%$ and $84 \%$ ee, and $84 \%$ and $80 \%$ de with 40 mol\% and 10 mol\% loading, respectively, through an intermediate with a square planar geometry. However, when the $\mathrm{Mg}$ (II) complex (10 mol\%) was used as catalyst the enantiomer (2S,3R)-717was obtained in 55\% ee through a tetrahedral geometry (Figure 19). Desimoni and coworkers observed a counteranion-dependent enantioselectivity effect when they used $\mathrm{Mg}\left(\mathrm{ClO}_{4}\right)_{2}$ or $\mathrm{Mg}(\mathrm{OTf})_{2}$ derived $\mathrm{BOX} \mathbf{7 1 9}$ complexes. ${ }^{605}$ In the first case the corresponding (2S,3R)-717 adduct was obtained in $72 \%$ ee and in the latter case the enantiomer $(2 R, 3 S)-717$ in $70 \%$ ee. This different behavior was attributed to the tetrahedral and octahedral geometry of the $\mathrm{Mg}\left(\mathrm{ClO}_{4}\right)_{2}$ and $\mathrm{Mg}(\mathrm{OTf})_{2}$ intermediates, respectively. Chiral bis(oxazoline) 433 derived complexes (Figure 19) from $\mathrm{Cu}(\mathrm{II})$, $\mathrm{Zn}(\mathrm{II})$, and $\mathrm{Mg}(\mathrm{II})$ triflates immobilized on a silica gel support via hydrogen-bonding interactions showed a moderate reversal of the enantioselectivity under homogeneous conditions. ${ }^{606}$ For instance, under homogeneous conditions $\mathrm{Mg}$ (II) complex gave $(2 R, 3 S)-717$ in $60 \%$ ee, whereas the supported catalyst provided $(2 S, 3 R)-717$ in only $30 \%$ ee.

Figure 19. Bis(oxazoline) Ligands Used in the Diels-Alder Reaction of Cyclopentadiene with $\mathrm{N}$-Crotonoyl Oxazolidinone 298<smiles>[H][Z10]#N</smiles><smiles>CC(C)(C1=NC(c2ccccc2)C(c2ccccc2)O1)C1=NC(c2ccccc2)C(c2ccccc2)O1</smiles><smiles>CC(C)(C1=NC(c2ccccc2)CO1)C1=NC(c2ccccc2)CO1</smiles>

Other achiral dienophiles such as 4-substituted 1,3-benzoxazol-2-(3H)-ones $\mathbf{7 2 0}$ have been used, by Renaud and co-workers, ${ }^{607}$ in the DA reaction of cyclopentadiene using the complex formed by $\mathrm{MgBr}_{2}$ and $\mathrm{BOX}-\mathrm{Ph}$ 433, providing compound (S)-721 (Scheme 273). However, only one example $(\mathrm{R}=\mathrm{H})$ showed a reversal of enantioselectivity when BOX 433/Mg( $\left(\mathrm{ClO}_{4}\right)_{2}$ was used as Lewis acid affording $(R)-721$ in $70 \%$ ee.

Scheme 273. Enantiodivergent Diels-Alder Reaction of Cyclopentadiene with $\mathrm{N}$ 1,3-Benzoxazol-2-(3H)-ones 720 Catalyzed by BOX 433 and $\mathrm{MgBr}_{2}$ or $\mathrm{Mg}\left(\mathrm{ClO}_{4}\right)_{2}$ 


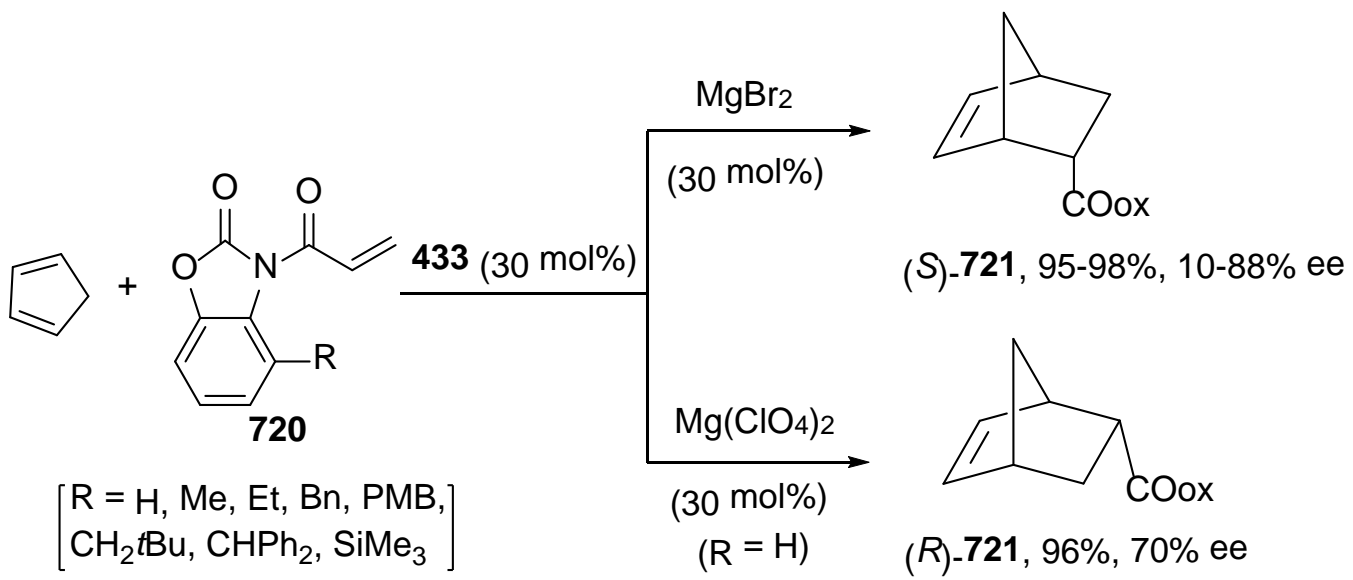

Diastereodivergent asymmetric DA reactions have been carried out using cyclopentadiene and chiral acrylamides as dienophiles depending on the stoichiometric amounts of Lewis acid. ${ }^{608}$ As an example, starting from (S)-722 the product endo-723 was exclusively formed when $\mathrm{TiCl}_{4}, \mathrm{Ti}(\mathrm{OiPr})_{4}, \mathrm{SnCl}_{4}$, or $\mathrm{ZrCl}_{4}$ were used as Lewis acids (Scheme 274). However, using $\mathrm{ZnCl}_{2}, \mathrm{Et}_{2} \mathrm{AlCl}$, or $\mathrm{BF}_{3} \cdot \mathrm{OEt}_{2}$ the corresponding endo-724 adduct was isolated. This excellent behavior can be attributed to the different coordination abilities of the two types of catalysts.

Scheme 274. Diastereodivergent Diels-Alder Reaction of Cyclopentadiene with Chiral Acrylamide 722 Catalyzed by Different Lewis Acids

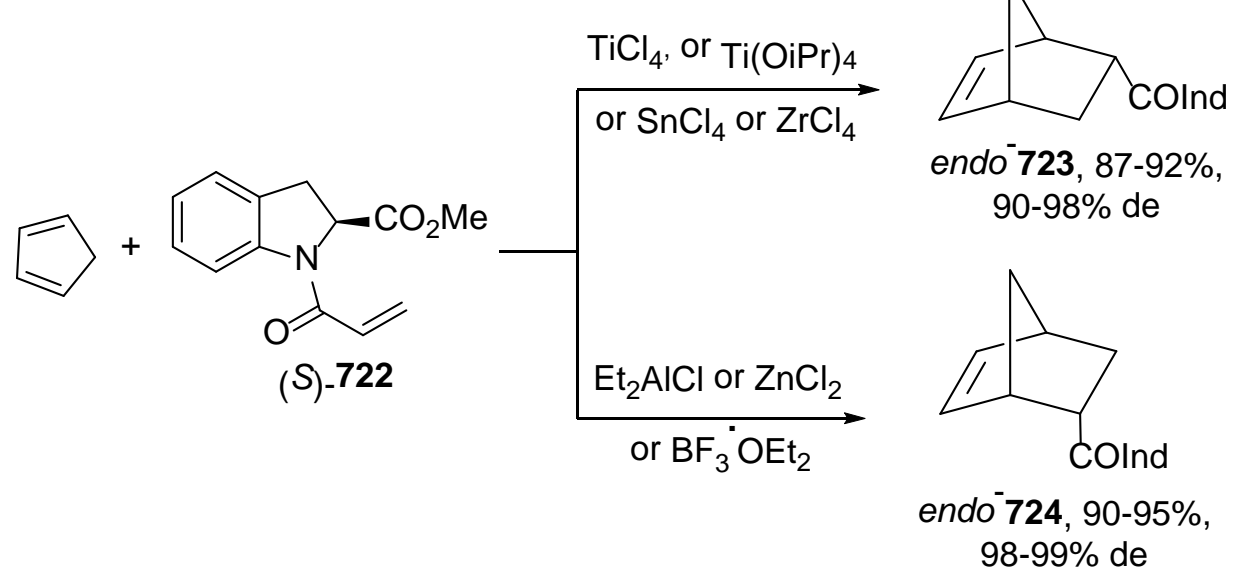

The complex $\mathrm{TiCl}_{2} / \mathrm{TADDOL}$ has been used as chiral catalyst in the enantiodivergent DA reaction between cyclopentadiene and (E)-crotonoyl-1,3oxazolidin-2-one (298). Seebach and co-workers studied the influence of substituents in the ligand. ${ }^{609}$ In the case of Ti(IV)-derived $(R, R)$-TADDOL 725a, the cycloadduct $(2 R, 3 S)-716$ was obtained in 72\% ee, whereas complex 726, bearing phenyl instead of naphthyl groups, provided the enantiomer (2S,3R)-716 in $88 \%$ ee (Figure 20). The influence of the substituents in the dioxolane ring exerted a decisive influence in the switching of enantioselectivity. For instance, Ti/TADDOL complex 725b gave mainly $(2 S, 3 R)-716$ in $82 \%$ ee, whereas complex 727 led to the formation of its enantiomer but 
only in 38\% ee. ${ }^{610}$ Modest enantiodivergent results have been observed with supported TADDOL- $\mathrm{TiCl}_{2}$ complexes. $^{611-613}$

Figure 20. Ti/TADDOLs Used in the Enantiodivergent Diels-Alder Reaction of Cyclopentadiene with $\mathrm{N}$-Crotonoyl Oxazolidinone 298<smiles>CC1(C)OC2C(O1)C([Al])([Te])O[Ge](Cl)(Cl)OC2([Al])[Al]</smiles>

725a: $\mathrm{Ar}=$ 1-naphthyl 725b: $\mathrm{Ar}=3,5-\mathrm{Me}_{2} \mathrm{C}_{6} \mathrm{H}_{3}$<smiles>CC1(c2ccccc2)OC2C(c3ccccc3)(O1)O[Ge](Cl)(Cl)OC2(c1ccccc1)c1ccccc1</smiles>

726

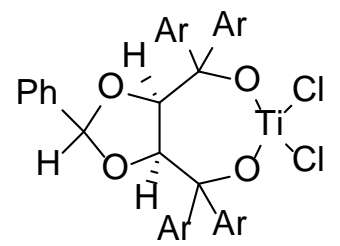

727: $\mathrm{Ar}=3,5-\mathrm{Me}_{2} \mathrm{C}_{6} \mathrm{H}_{3}$

Diastereodivergent results have been found in the DA reaction of cyclopentadiene with the acrylate $\mathbf{7 2 8}$ derived from levoglucosenone as chiral auxiliary. ${ }^{614}$ Under thermal conditions, endo-729 was obtained at rt in 56\% endo/exo de and 74\% ee for the endo-isomer (Scheme 275). However, in the presence of 2 eq of $\mathrm{Et}_{2} \mathrm{AlCl}$ at different temperatures, better results were obtained for the corresponding endo-730 adduct in 8492\% de and 84-94\% ee. According to the DFT calculations and NMR studies, one face of the acrylate is blocked due to $\pi$-stacking interactions, the endo approach being favored. The inversion of chirality in the presence of $\mathrm{Et}_{2} \mathrm{AlCl}$ is due to the chelation of the oxygen and of the 1,6-anhydro bridge, giving the kinetically favored cycloadduct endo-730.

\section{Scheme 275. Diastereodivergent Diels-Alder Reaction of Cyclopentadiene with} Chiral Acryloyl Ester 728 in the Absence or Presence of $\mathrm{Et}_{2} \mathrm{AlCl}$

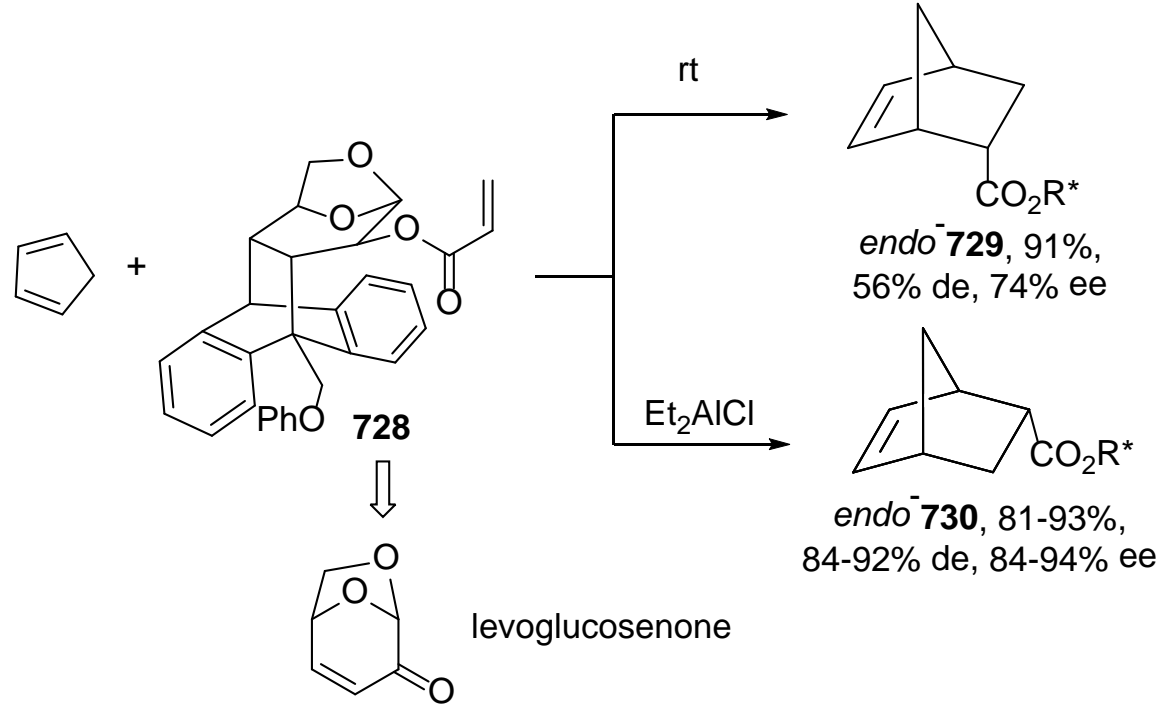

G-Quadruplex DNA (G4-DNA) requires monovalent ions for its structural formation. The use of $\mathrm{Na}^{+}$and $\mathrm{K}^{+}$ions tunes the configuration of human telomeric (HT)-G4-DNA metalloenzyme. A selected single-stranded G-rich DNA sequence from 
human telomere (ODN) can fold into various G-quadruplex motifs. Surprisingly, a G4DNA-based catalyst assembled by $\mathrm{Cu}^{2+}$ ions (ODN $\mathrm{Cu}^{2+}$ ) switched the enantioselectivity of the DA reaction of cyclopentadiene with cinnamoyl dienophiles 731 affording cycloadducts 732 in moderate ee (Scheme 276a). ${ }^{615,616}$ This effect was ascribed to the structural transformation of G-quadrupole motifs from an antiparallel to a hybrid type structure. Similar reversal of enantioselectivity was observed when $\alpha, \beta$ unsaturated ketones $\mathbf{7 3 3}$ were used as dienophiles and a higher order G-quadruplex DNA metalloenzyme as catalyst in the presence of $\mathrm{K}^{+}$or $\mathrm{NH}_{4}{ }^{+}$cations. The corresponding endo-products $\mathbf{7 3 4}$ were obtained in good de and ee (Scheme 276b)

\section{Scheme 276. Enantiodivergent Diels-Alder Reaction of Cyclopentadiene with Dienophiles 731 and 733 Catalyzed by G-Quadruplex DNA Metalloenzyme in the Presence of Different Cations}

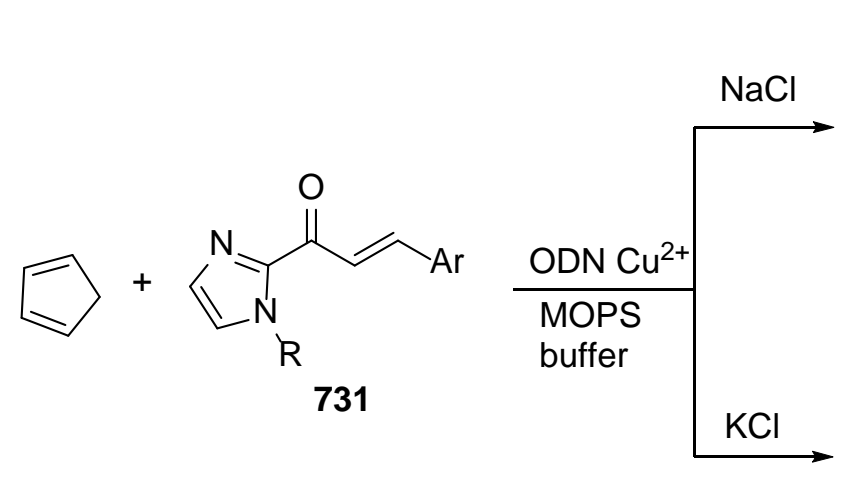

MOPS $=3-\left(N_{-}\right.$morpholino $)$propanesulfonic acid

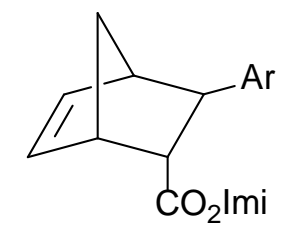

(2S,3S)-732, 17-80\%,

$82-92 \%$ de, $13-67 \%$ ee

(a)

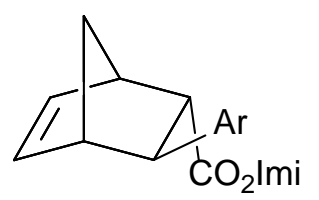

$(2 R, 3 R)-732,36-88 \%$, $72-90 \%$ de, $2-58 \%$ ee

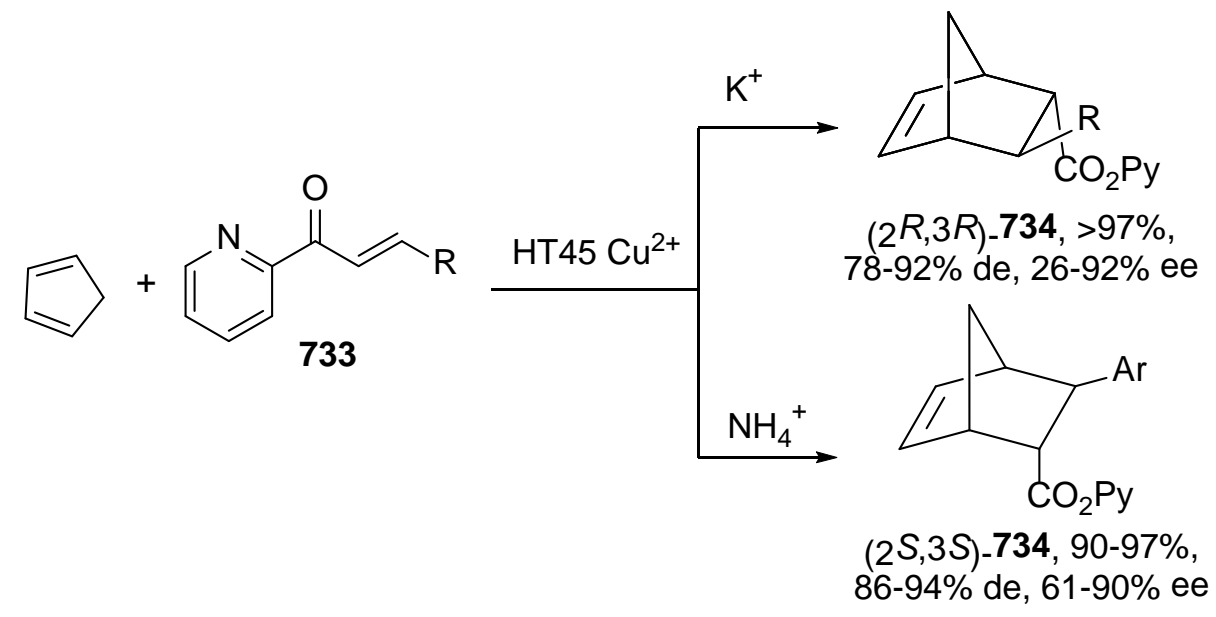

(b)

Alkaloid-catalyzed [4+2] annulations of polyconjugated alkylidene malononitriles 735 with $\beta$-substituted cyclohexenones 736 afforded highly substituted bicyclo[2.2.2] octanes 738 (Scheme 277). ${ }^{617}$ Cycloadducts endo-738 were produced using 9-amino-9-deoxyepiquinidine 737 and salicylic acid as catalysts in >19:1 dr and with ee up to 99\%. However, using the homochiral organocatalysts 365 and (S)- $N$-Bocphenylglycine, products exo-738 were obtained with de up to $90 \%$ and with ee up to 
85\%. These reactions took place via dienamine intermediates by a stepwise Michael-Michael cascade instead of a concerted DA cycloaddition. The observed diastereodivergence has been justified through the hydrogen-bonding interactions between the primary amine group and the oxygen atom of the ketone.

\section{Scheme 277. Diastereodivergent Formal [4+2] Cycloaddition of Alkylidene Malononitriles 735 with $\beta$-Substituted Cyclic Enones 736 Organocatalyzed by Chiral Cinchona Alkaloids}

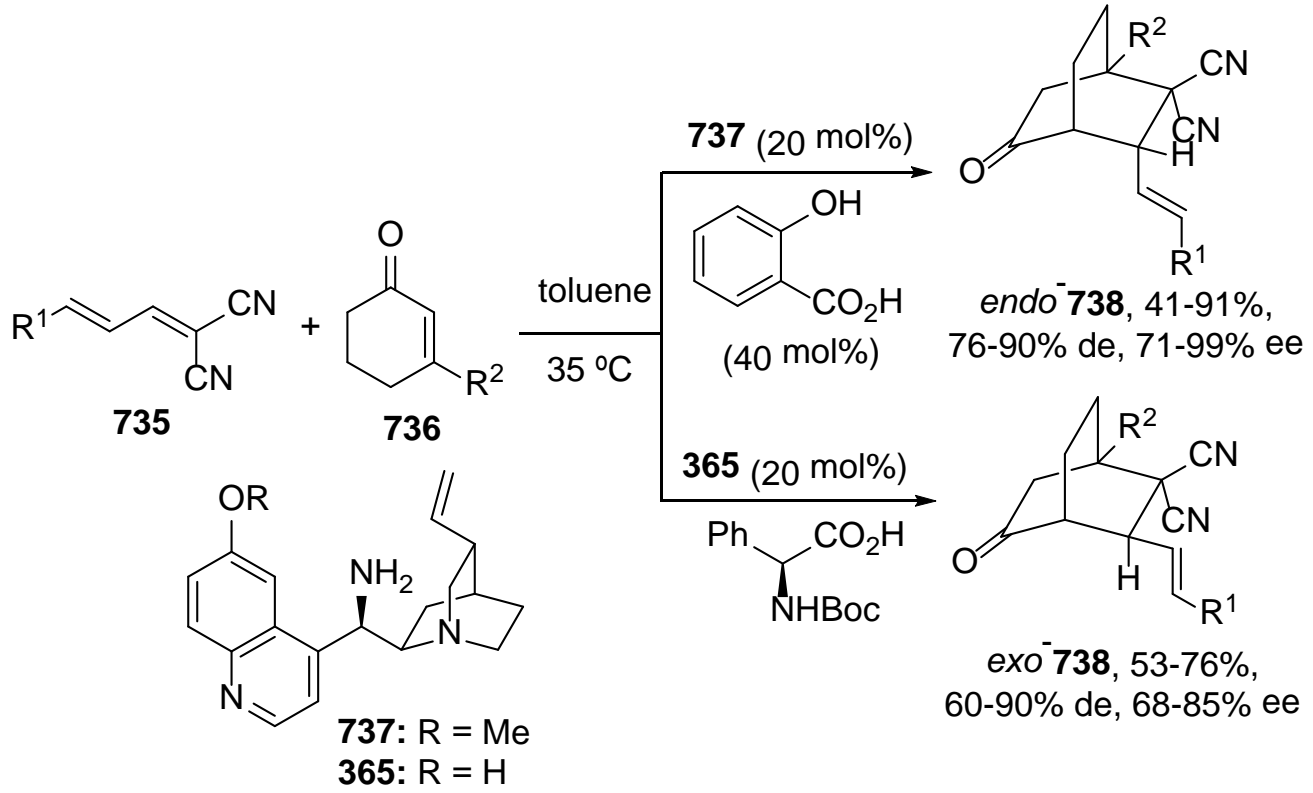

Searching for a more efficient organocatalyst in the DA reaction of anthrone enolate with maleimides, it was discovered that the substitution at the nitrogen atom of (S)-2-(1hydroxyethyl)benzimidazole $\mathbf{7 3 9}$ gave the corresponding adducts $\mathbf{7 4 0}$ with a reversal of enantioselectivity (Scheme 278). ${ }^{618}$ The pyridine derivative 739a afforded products $(S, S)-\mathbf{7 4 0}$ with ee up to $88 \%$, whereas the benzotriazole derivative organocatalyst $\mathbf{7 3 9 b}$ led to the formation of $(R, R)-\mathbf{7 4 0}$ with ee up to $73 \%$. The nitrogen atom of the heteroaryl ring attached to the nitrogen of the benzimidazole is protonated forming a hydrogen bond to the oxygen of the anthrone enolate and, on the other hand, the hydroxyl group of the benzimidazole ring will form a hydrogen bond with the maleimide giving transition states 739A and 739B, respectively. In the case of catalyst 739a the maleimide is approaching from the right hand side in TS 739A giving the $(S, S)-740$ stereoisomer. However, with catalyst 739b the benzotriazole ring, after protonation at $\mathrm{N}-3$, could not remain in the same place as the benzimidazole ring due to the repulsion between both heterocyclic units. Thus, the maleimide will approach by the left hand side affording the enantiomer $(R, R)-\mathbf{7 4 0}$.

Scheme 278. Enantiodivergent Diels-Alder Reaction of Anthrone Enolate with Maleimides Catalyzed by Different (S)-2-(1-Hydroxyethyl)benzimidazoles 739 


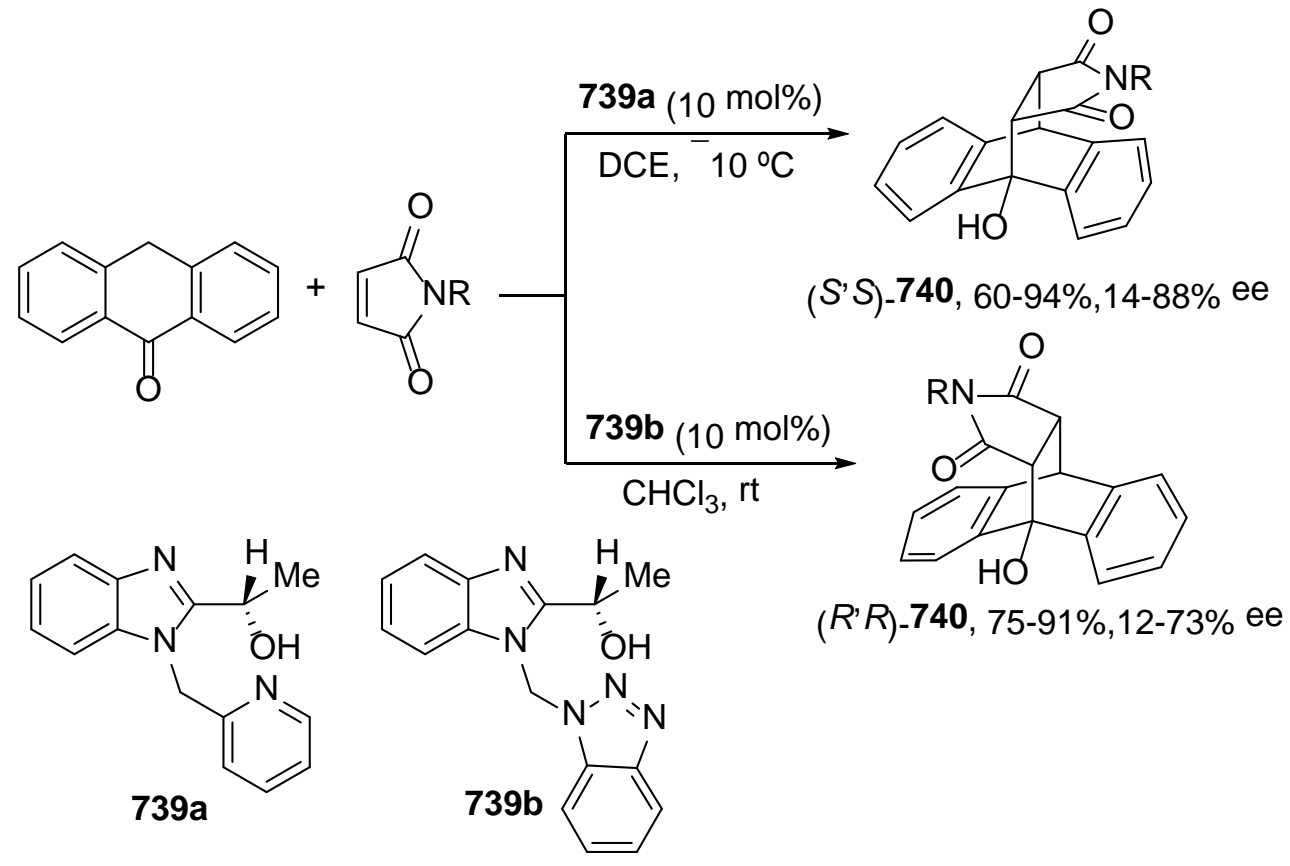

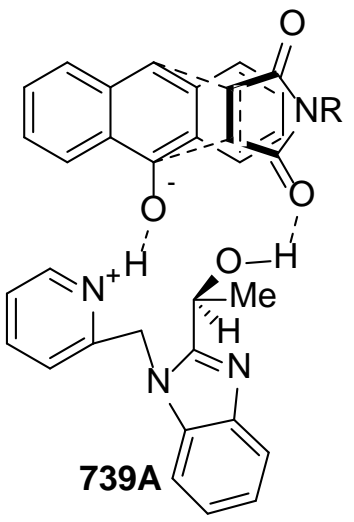

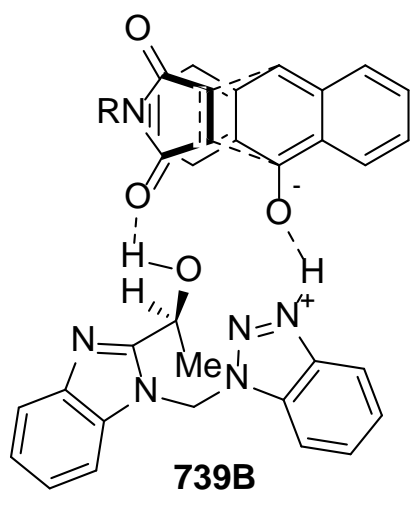

5.1.2. Hetero Diels-Alder Reactions. Asymmetric normal electron-demand oxa-Diels-Alder reactions were performed by Jørgensen and co-workers with $\alpha$-keto esters and 1,3-dienes catalyzed by $\mathrm{Cu}(\mathrm{II})$ /bis(oxazolines) complexes. ${ }^{619}$ Ghosh and coworkers studied the enantiodivergent hetero DA reactions of Danishefsky's diene and alkyl glyoxylates under the catalysis of $\mathrm{Cu} / \mathrm{BOX}$ complexes (Scheme 279). ${ }^{620}$ In the case of ligand $(S, S)-\mathrm{BOX}-\mathrm{Ph}$ (433, Scheme 158), product (2S)-741 was obtained in 40\% yield and $47 \%$ ee, whereas ligand $(S, S)$-BOX-tBu (80, Scheme 23) afforded (2R)-742 in $42 \%$ yield and $17 \%$ ee.

Scheme 279. Enantiodivergent Formal Hetero Diels-Alder Reaction of Danishefsky's Diene with Alkyl Glyoxylates Catalyzed by Different $\mathrm{Cu}(\mathrm{II}) /(\mathrm{S}, S)$ BOX Complexes 


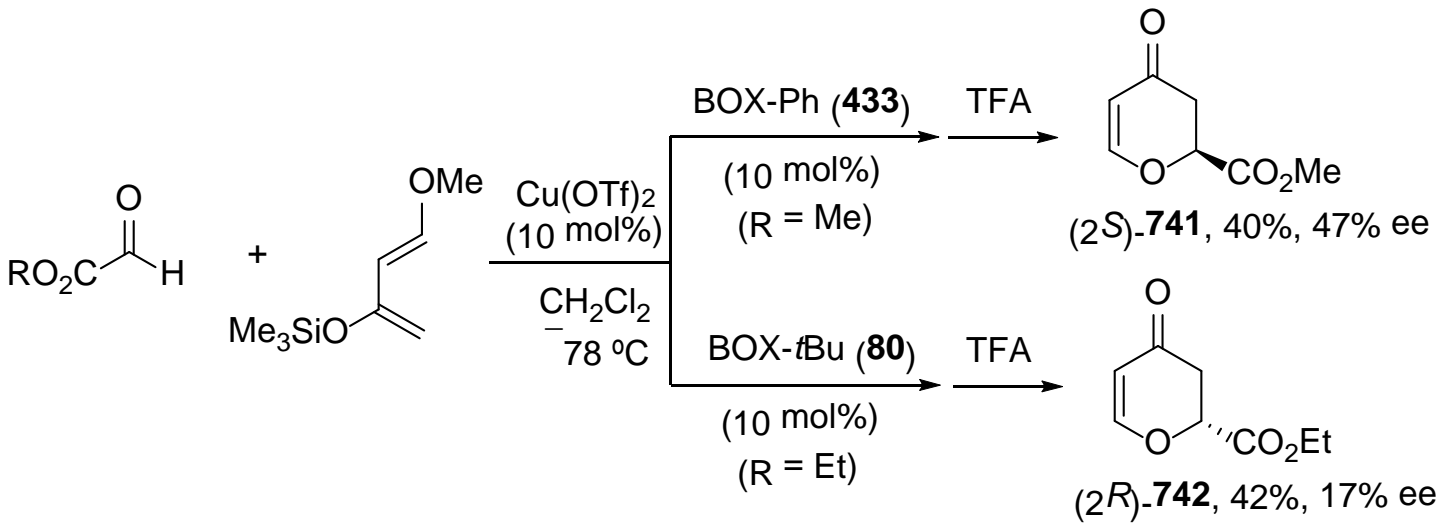

The attachment of a chiral ligand to a support such as chirasil-oxovanadium provided catalyst $\mathbf{7 4 3 b}$, which has shown a reversal effect with respect to monomeric 743a on the enantioselectivity in the hetero DA reaction between Danishefsky's diene and benzaldehyde giving (-)-744 and (+)-744, respectively (Scheme 280). ${ }^{621}$ This enantiodivergent effect was explained by the steric hindrance of the bulky polysiloxane (PS). The absolute configuration of cycloadducts $\mathbf{7 4 4}$ was not assigned.

\section{Scheme 280. Enantiodivergent Formal Hetero-Diels-Alder Reaction of Danishefsky's Diene with Benzaldehyde Catalyzed by Different Oxovanadium Complexes}

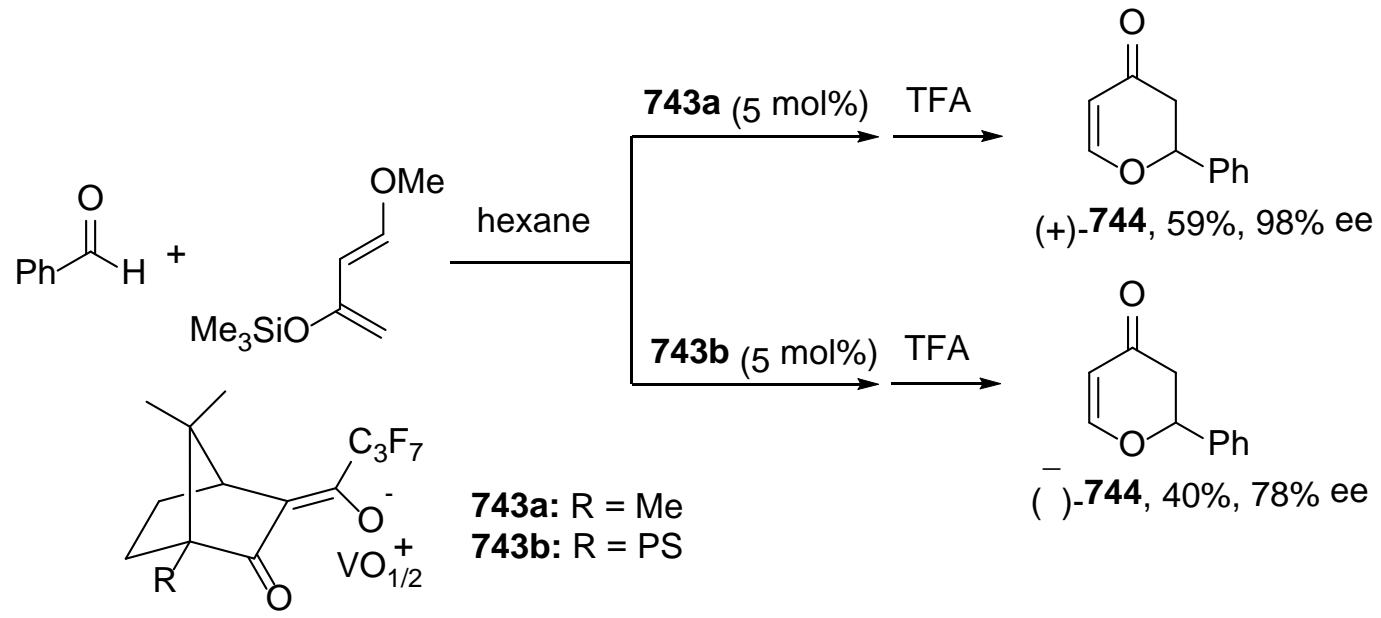

Kobayashi and co-workers observed a significant influence of the aggregation state of the chiral catalyst to the sense of enantioselectivity with the monomeric BINOL/Zr complex $\mathbf{7 4 6}$ and the dimer 218 (Scheme 78) (with $N$-methylimidazole as ligand) in the aza-Diels-Alder reaction of Danishefsky's dienes 745 with imines. ${ }^{62-624}$ The resulting piperidinones $(R)-\mathbf{7 4 7}$ were obtained with ee up to 91\%, whereas using complex 218 the products with opposite configuration were isolated with ee up to 93\% (Scheme 281). This switch of ee was explained by the formation of intermediate 746A in which one of the phenyl groups blocks one face of the imine, while in intermediate 218A, the diene will approach from the opposite side to the one with the naphthyl groups giving $(S)-747$. 
Scheme 281. Enantiodivergent Hetero Diels-Alder Reaction of Danishefsky's Dienes 745 with Imines Catalyzed by Zr/BINOLs Monomeric and Dimeric Complexes<smiles>[R]C=Nc1cc[cH+]ccc1O</smiles>

(S)-747, 47-98\%, 64-93\% ee<smiles>Oc1c(-c2ccccc2)cc(-c2ccccc2)c(O)c1C(c1ccc(Br)cc1)c1ccc(Br)cc1</smiles><smiles>[M]O[Ge](N)(Oc1ccc2cc(Br)ccc2c1-c1c(O)ccc2cc(Br)ccc12)Oc1ccc2cc(Br)ccc2c1-c1c(O[Al])ccc2cc(Br)ccc12</smiles>

218

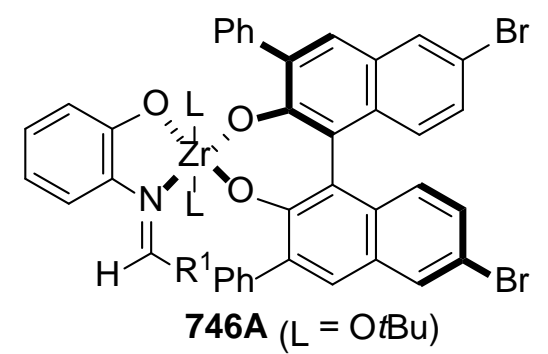

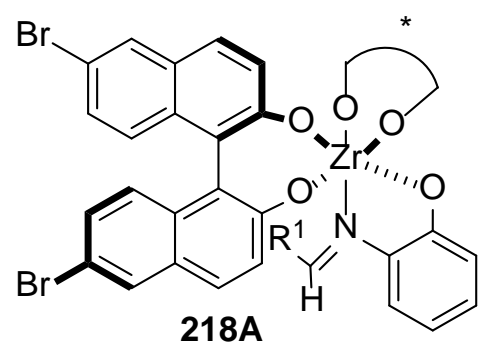

Inverse electron-demand hetero DA reaction of $\alpha, \beta$-unsaturated carbonyl compounds and electron-rich alkenes is a useful methodology for the synthesis of dihydropyrans. Evans and co-workers observed that the acyl phosphonate $\mathbf{7 4 8}$ reacted with ethyl vinyl ether under the catalysis of $\mathrm{Cu}(\mathrm{II}) / \mathrm{BOX}$ complexes providing the adducts $\mathbf{7 4 9}$ with opposite configuration (Scheme 282). ${ }^{625-627}$ For instance, acyl phosphonate 748 and ethyl vinyl ether, in the presence of $(S, S)$-BOX-Ph (433) and $(S, S)$-BOX-tBu (80) ligands, afforded enantiomeric products $(2 R, 4 R)-749$ and $(2 S, 4 S)-749$, respectively, both with the same $93 \%$ ee.

Scheme 282. Enantiodivergent Hetero Diels-Alder Reaction of Acyl Phosphonate 748 with Ethyl Vinyl Ether Catalyzed by $\mathrm{Cu} /(S, S)$-BOX Complexes 


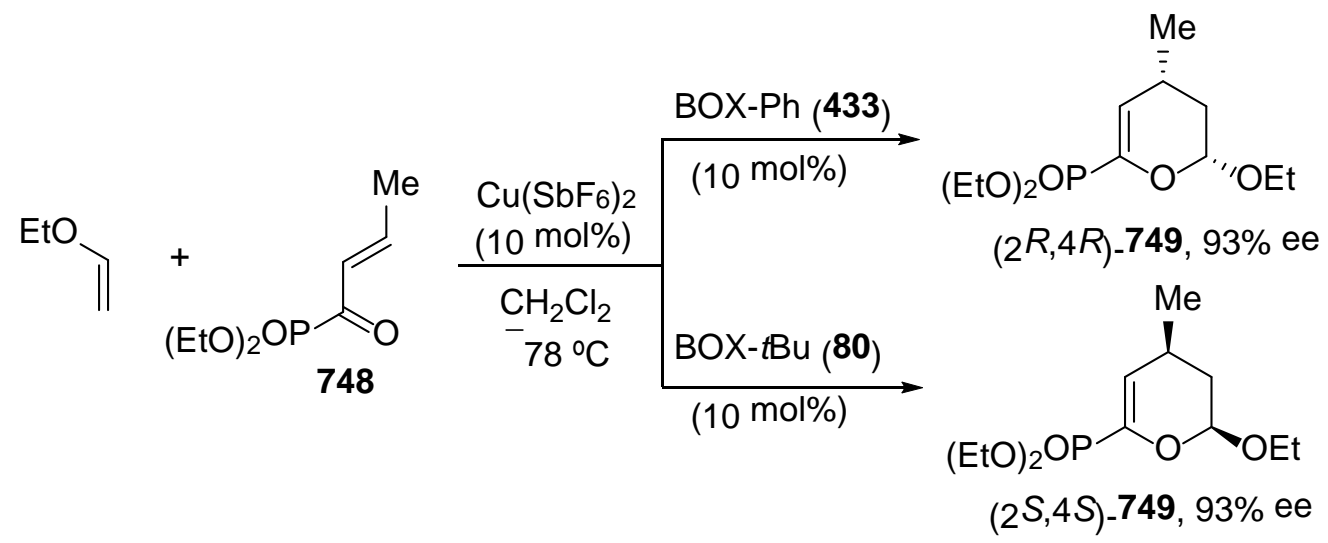

Similar reversal effect was observed by Jørgensen and co-workers using $\beta, \gamma$ unsaturated $\alpha$-keto esters and enol ethers. ${ }^{628}$ For instance, the reaction of the keto ester 335 with ethyl vinyl ether quantitatively gave the cycloadduct $(2 S, 4 S)-751$ in $64 \%$ ee under $\mathrm{Cu}(\mathrm{OTf})_{2} /(R, R)$-BOX-Ph (433) catalysis. However, the $\mathrm{Cu}(\mathrm{OTf})_{2} /(R, R)$-BOX$\mathrm{Ph}_{2}$ (750) complex gave the corresponding enantiomer $(2 R, 4 R)-751$ in $72 \%$ ee (Scheme 283).

Scheme 283. Enantiodivergent Hetero-Diels-Alder Reaction of $\beta, \gamma$-Unsaturated $\alpha$ Keto Ester 335 with Ethyl Vinyl Ether Catalyzed by Cu/BOX Complexes

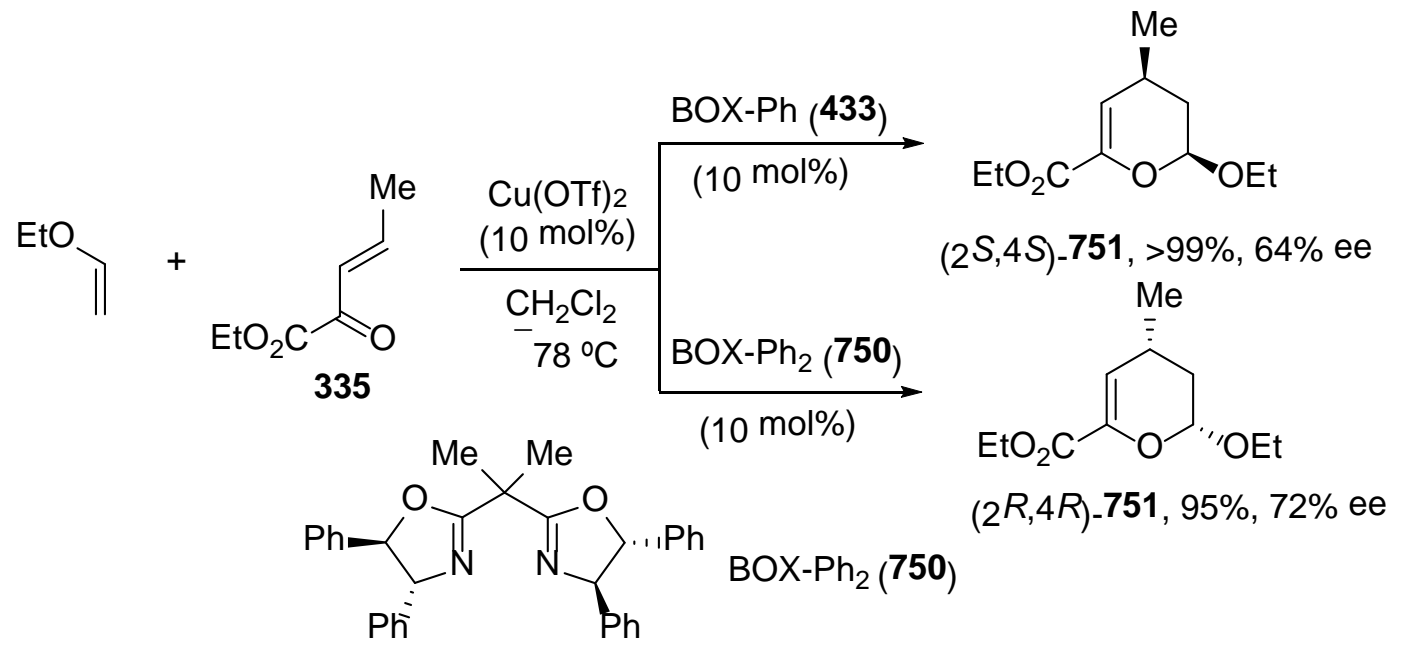

The diastereodivergent inverse electron-demand hetero DA reaction was described by Tietze and co-workers for the reaction of the enol ether $\mathbf{7 5 2}$ with the chiral oxabutadiene 753 promoted by different Lewis acids (Scheme 284). ${ }^{629}$ Using $\mathrm{Me}_{2} \mathrm{AlCl}$ the product endo-754 was formed in excellent 97\% de. In contrast, employing TMSOTf as Lewis acid the other endo-diastereomer 755 was obtained in lower $80 \%$ de. Mechanistic considerations proposed the formation of a chelated intermediate 754A with $\mathrm{Me}_{2} \mathrm{AlCl}$ favoring the approach of the dienophile by the front face. However, in the case of TMSOTf a nonchelated intermediate $\mathbf{7 5 5 A}$ with the two $\mathrm{C}=\mathrm{O}$ groups in an anti-arrangement will block the front face. 
Scheme 284. Diastereodivergent Hetero Diels-Alder Reaction of the Enol Ether 752 with the Oxabutadiene 753 Promoted by Different Lewis Acids

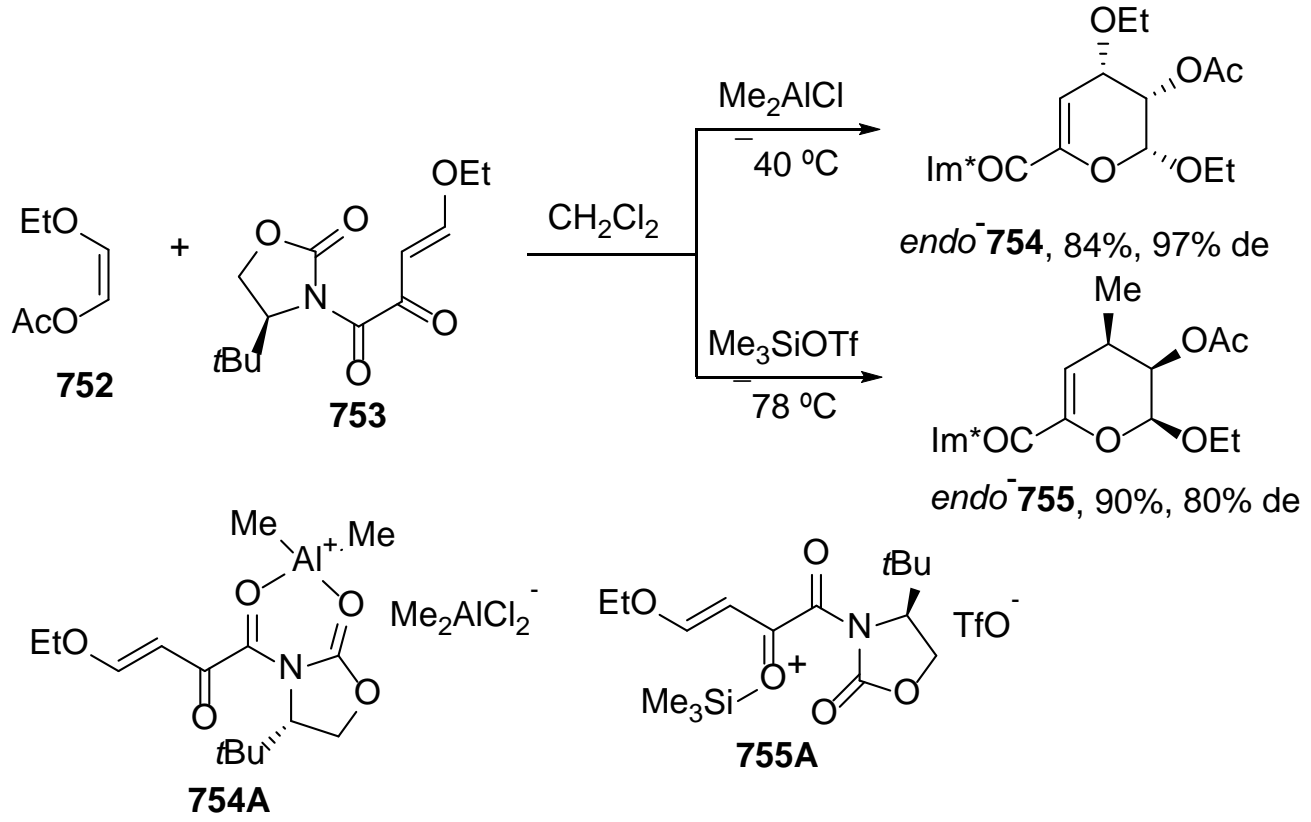

Similar diastereodivergent hetero DA reaction promoted by different Lewis acids has been performed with $\beta, \gamma$-unsaturated $\alpha$-keto esters 335 and $N$-vinyl-1,3-oxazolidin-2ones 756 (Scheme 285). ${ }^{630}$ In the presence of $\mathrm{SnCl}_{4}$, products endo-757 were mainly formed, whereas $\mathrm{Eu}(\mathrm{fod})_{3}$ gave endo-758 with very high diastereoselectivity. The reversed facial differentiation has been attributed to the different chelation modes of both Lewis acids. In the case of $\mathrm{SnCl}_{4}$, the two carbonyl groups of the pyruvic ester are chelated and the reaction took place through a stepwise mechanism, first by a Michael addition followed by cyclization. However, a concerted mechanism will take place with $\mathrm{Eu}(\mathrm{fod})_{3}$ forming an Eu(III) sandwiched chelate.

Scheme 285. Diastereodivergent Hetero Diels-Alder Reaction of $\beta, \gamma$-Unsaturated $\alpha$ Keto Esters 335 with Chiral $N$-Vinyl 1,3-Oxazolidin-2-ones 756 Promoted by Different Lewis Acids<smiles>[R]C=CC1[C@H](CC[R5])COC(=O)N1/C=C/[R]</smiles> 
Recently, a diastereodivergent asymmetric electron-demand oxa-hetero DA reaction has been developed by Zhao and co-workers. ${ }^{631}$ An excellent inversion of the diastereoselectivity was observed in the reaction of $\beta, \gamma$-unsaturated $\alpha$-keto esters 335 with $\alpha, \beta$-unsaturated 1,7-dicarbonyl compounds 759 using L-Pro and different Cinchona alkaloid-derived thioureas 760 and 761 (Scheme 286). In the case of thiourea $\mathbf{7 6 0}$ pyranopyrans cis-762 were predominantly formed and with the organocatalysts $\mathbf{7 6 1}$ diastereomers trans-763 were obtained.

Scheme 286. Diastereodivergent Oxa-Hetero Diels-Alder Reaction of $\beta, \gamma$ Unsaturated $\alpha$-Keto Ester 335 with Oxoaldehydes 759 Catalyzed by L-Pro and Different Thioureas

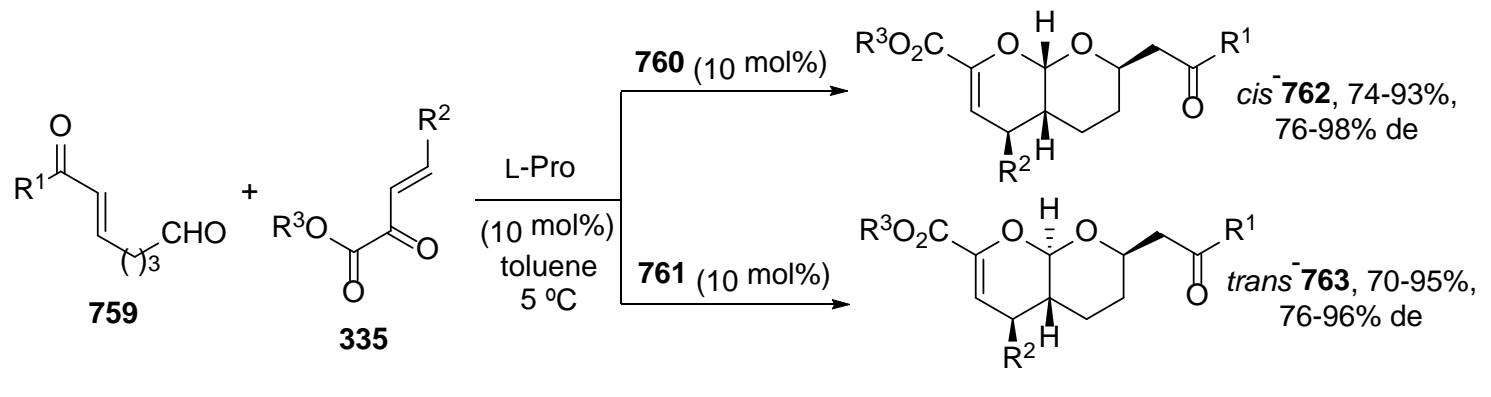<smiles>C=CC1C2CCN1C(C(NC(=S)Nc1cc(C(F)(F)F)cc(C(F)(F)F)c1)c1ccnc3ccc(OC)cc13)C2</smiles><smiles>C=CC1CC2CCC(C=C)(CN2)C1</smiles>

In conclusion, in the above described enantiodivergent [4+2] cycloadditons of cyclopentadiene and $\mathrm{N}$-alkenoyloxazolidinones catalyzed by chiral Lewis acids, the metal salt of the complex played an important role in the formation of endocycloadducts. Diastereodivergent examples also depend on the Lewis acid employed. Organocatalyzed diastereodivergent [4+2] cycloadditons are controlled by the additives and by the structure of the catalyst. Enantiodivergent hetero DA reactions with normal or inverse electron-demand are influenced by the structure of the metal complex, and the diastereodivergent examples by the ability of the Lewis acid to chelate the oxadiene.

\subsection{1,3-Dipolar Cycloadditions}

Catalytic 1,3-dipolar cycloadditions (1,3-DC) have been performed since 1990 in an asymmetric manner with different chiral metal complexes and more recently with chiral organocatalysts. ${ }^{632-641}$ Stereodivergent processes have been observed with different dipoles mainly nitrones, azomethine ylides, and diazo compounds. 
5.2.1. Nitrones. Nitrones are considered stable allyl type dipoles and can be prepared by the reaction of aldehydes with $N$-substitued hydroxylamines. Enantioselective reactions of nitrones take place with electron-poor and rich alkenes, and can be classified as normal and inverse electron demand 1,3-DC, respectively, affording isoxazolines, which are precursors for $\beta$-amino acids, $\beta$-lactams, and $\beta$-amino alcohols. The regiochemistry of this catalyzed $1,3-\mathrm{DC}$ is determined by the dipolarophile and by the Lewis acid employed. With respect to the endo/exodiastereoselectivity, depending on the dipolarophile different selectivity can be observed (Scheme 287). In the case of the 1,3-DC with normal electron-demand the 3,4- and 3,5-disubstituted isoxazolines and for inverse electron-demand the 1,3-DC 3,5-disubstituted isoxazolines are exclusively formed, in all cases with endo- or exo-diastereofacial selectivity.

\section{Scheme 287. Regio- and Diastereoselectivity Involved in 1,3-Dipolar Cycloadditions of Nitrones}

\section{1,3-DC: Normal Electron-Demand}

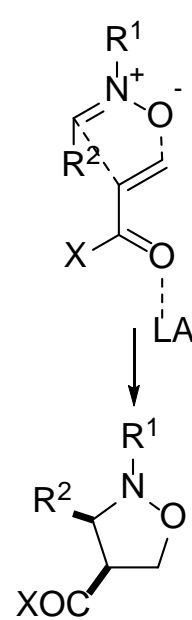

3,4-exo<smiles>[X]C(=O)c1c[R][n+]([R1])o1</smiles><smiles>[R2]C1C[C@H]([X])ON1[R]</smiles>

3,5-exo

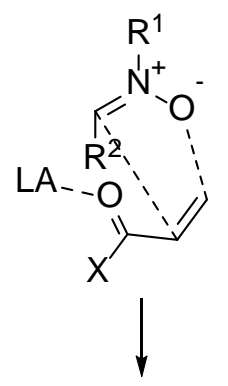

$\mathrm{R}^{1}$<smiles>[Y]C(=O)[C@@H]1CON([CH])C1[R]</smiles>

3,4-endo

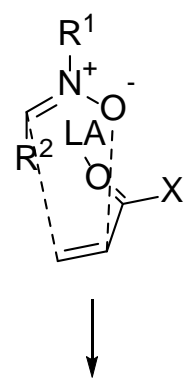<smiles>[R2]C1C[C@H]([X])ON1[R]</smiles>

3,5-endo

1,3-DC: Inverse Electron-Demand<smiles>[R7]Oc1c[R4]c[n+]([R1])c1[Ge]C</smiles>
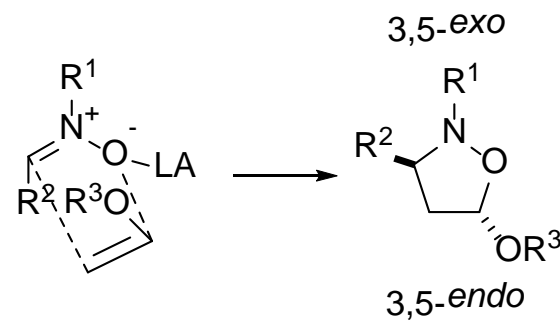

Gothelf and Jørgensen described for the first time that the 1,3-DC of nitrones with 3alkenoyl-2-oxazolidinones catalyzed by $\mathrm{Ti}(\mathrm{IV}) / \mathrm{TADDOL}$ complexes led to the formation of 3,4-exo-isoxazolidines with de up to $80 \%$ and ee up to $60 \%{ }^{642,643}$ They 
observed enantiodivergent effects when the $\mathrm{MgI}_{2} / \mathrm{BOX}-\mathrm{Ph}$ (433) complex was used as catalyst in the reaction of nitrones $764(\mathrm{R}=\mathrm{Ph})$ with $N$-acryloyl-1,3-oxazolidinone 298 (Scheme 288). ${ }^{644,645}$ In the presence of $4 \AA$ MS, 3,4-endo adduct (3S,4R)-765 was obtained in moderate de and good $82 \%$ ee, whereas in the absence of MS the corresponding enantiomer was formed in excellent 99\% de but moderate ee. It was postulated that octahedral $\mathrm{Mg}(\mathrm{II})$ is coordinated with two oxygen atoms from the MS giving intermediates $\mathbf{A}$ and $\mathbf{B}$ where the approach of the dipolarophile from its $R e$ face would give (3S,4R)-765. In the absence of MS intermediates $\mathbf{C}$ and $\mathbf{D}$ could be formed and the attack will occur from the Si face affording the enantiomer $(3 R, 4 S)-765$.

Scheme 288. Enantiodivergent 1,3-DC of Nitrone 764 with $N$-Acryloyl-1,3oxazolidinone 298 Catalyzed by $\mathrm{MgI}_{2} / \mathrm{BOX}-\mathrm{Ph} 433$ in the Presence or Absence of MS

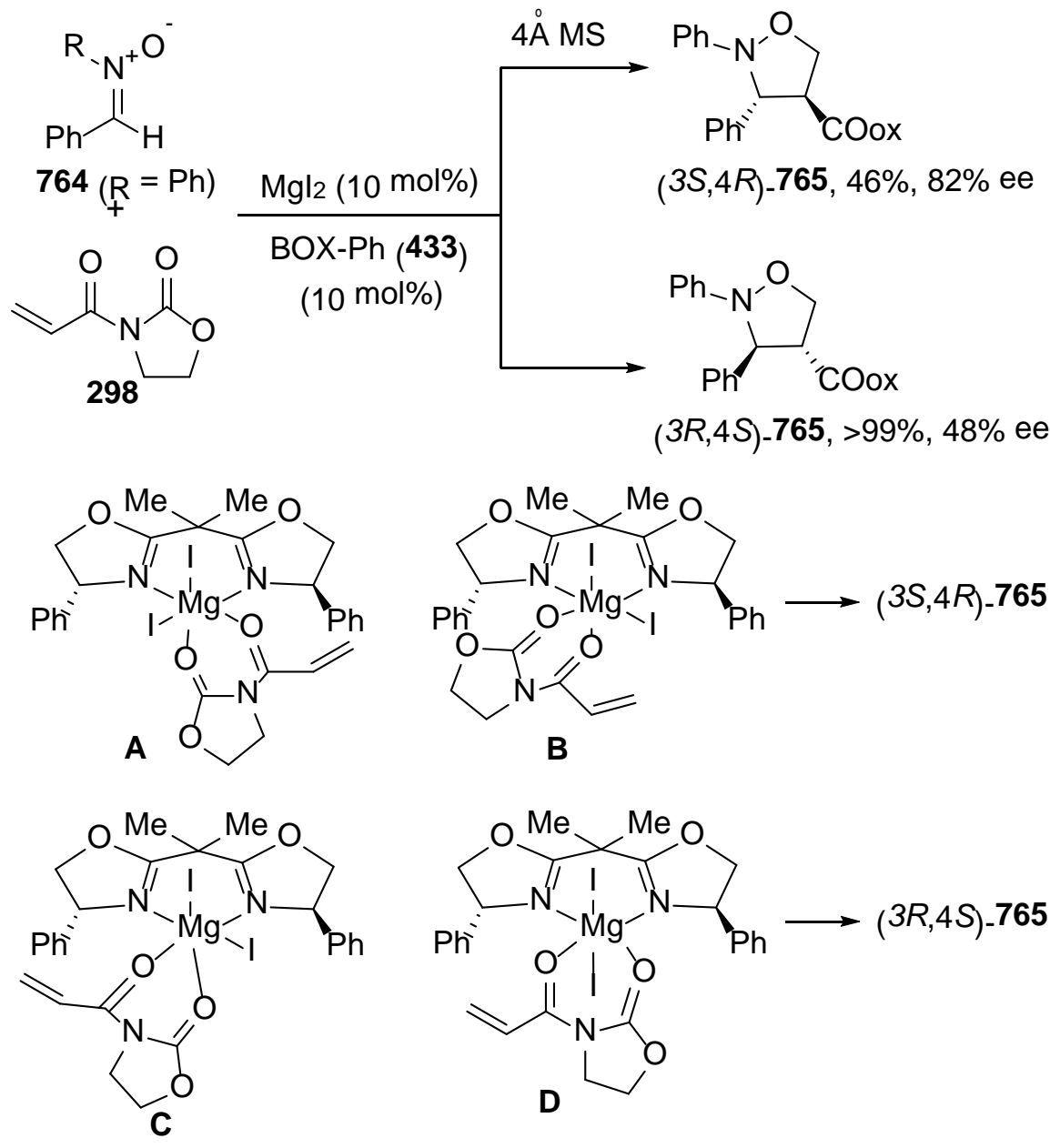

Desimoni and co-workers observed the influence of the $\mathrm{Mg}$ salt in the enantioselectivity using BOX-Ph (433) as chiral ligand. ${ }^{646,647}$ In the case of $\mathrm{Mg}\left(\mathrm{ClO}_{4}\right)_{2}$, 3,4-endo product $(3 R, 4 S)-765$ was formed in $72 \%$ ee, whereas $\mathrm{Mg}(\mathrm{OTf})_{2}$ gave the enantiomer in $86 \%$ ee. ${ }^{646}$ In addition, when $\mathrm{Zn}\left(\mathrm{ClO}_{4}\right)_{2}$ was used as metal salt-promoter an inversion of diastereoselectivity was observed and the corresponding 3,4-exodiastereomer $(3 R, 4 R)-765$ was obtained in $46 \%$ de and $84 \%$ ee. ${ }^{647}$ 
The influence of $4 \AA$ MS was also found by Kobayashi and Kawamura in the 1,3-DC of nitrone $764\left(\mathrm{R}^{1}=\mathrm{Bn}\right)$ with $N$-alkenoyl oxazolidinones 298 catalyzed by $\mathrm{Yb}(\mathrm{OTf})_{3} /(S)$-BINOL complex 716 and amine $(R)$-MNEA (767) (Scheme 289). ${ }^{648,649}$ Again enantiodivergent results were obtained, where 3,4-endo-compounds (3S,4R)-766 were isolated in the absence of $4 \AA$ MS with ee up to $88 \%$ and their enantiomers in the absence of MS with ee up to $96 \%$ ee, in both cases in excellent de.

Scheme 289. Enantiodivergent 1,3-DC of Nitrone 764 with $N$-Alkenoyl Oxazolidinones 298 Catalyzed by $\mathrm{Yb}(\mathrm{OTf})_{3} /(S)$-BINOL 716 in the Presence or Absence of MS

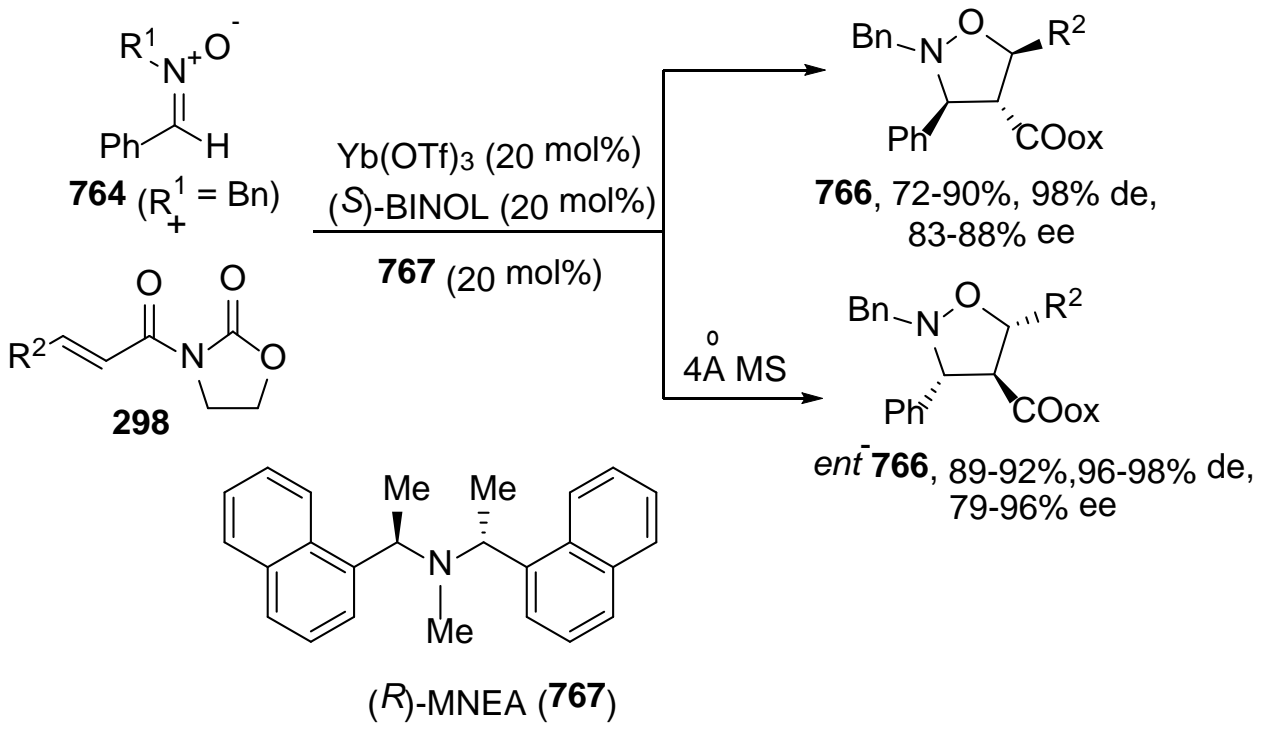

Other types of dipolarophiles such as alkylidene malonates reacted at $0{ }^{\circ} \mathrm{C}$ with nitrone $764\left(\mathrm{R}^{1}=\mathrm{Ph}\right)$ in the presence of trioxazolidine (TOX) 333 and $\mathrm{Co}\left(\mathrm{ClO}_{4}\right)_{2} \cdot 6 \mathrm{H}_{2} \mathrm{O}$ as chiral catalyst affording oxazolidines trans-768 with exoselectivity. ${ }^{650}$ However, at lower temperature endo-adducts cis-768 were formed in good diastereo- and enantioselectivity (Scheme 290). Mechanistic experiments revealed the reversibility of this 1,3-DC, the cis-diastereomer being obtained under kinetic control and the trans under thermodynamic conditions.

Scheme 290. Diastereodivergent 1,3-DC of Nitrone 764 with Alkylidene Malonates Catalyzed by Chiral $\mathrm{Co}\left(\mathrm{ClO}_{4}\right)_{2} / \mathrm{TOX} 333$ at Different Temperatures 


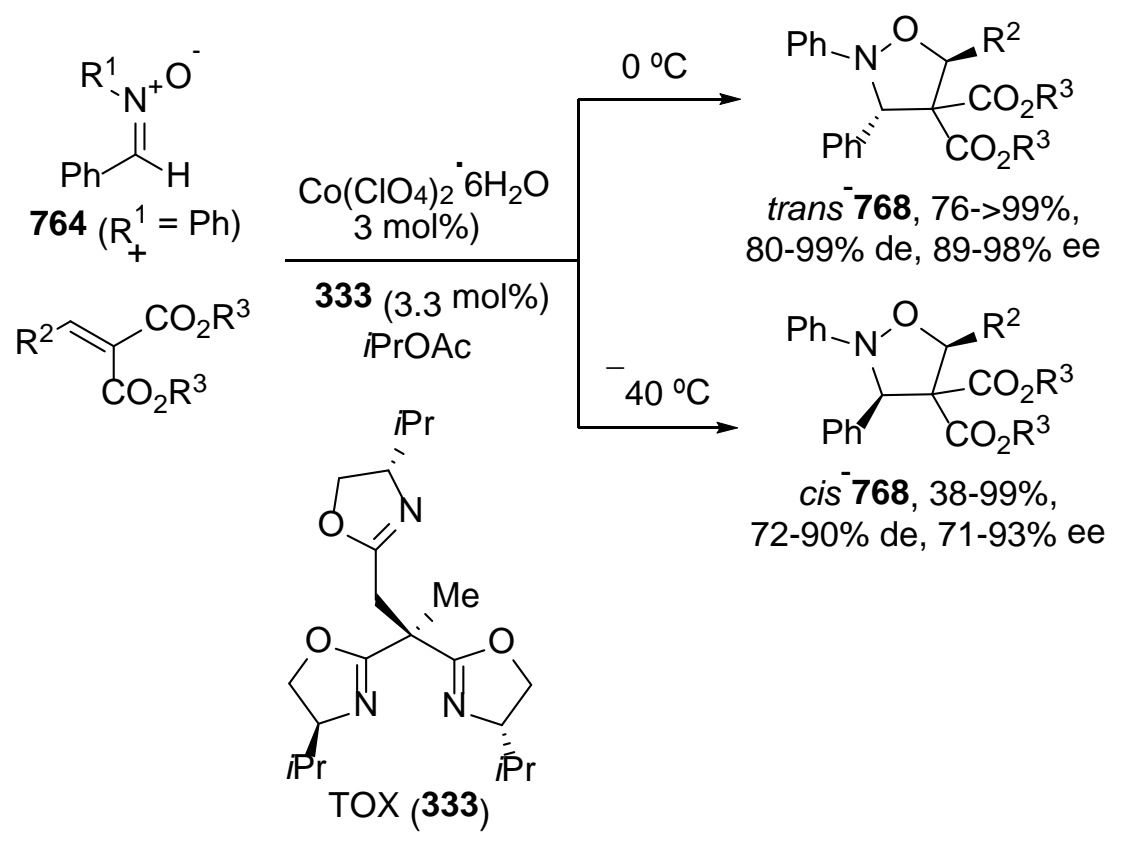

Kündig and co-workers disclosed in 2002 that Fe and Ru chiral complexes can be used as catalysts in the 3,5-endo-diastereo- and enantioselective 1,3-DC of diaryl nitrones with enals with moderate regioselectivity. ${ }^{651}$ Dujardin and co-workers described the application of Kündig's Ru catalyst $\mathbf{7 7 0}$ to the diastereodivergent 1,3-DC of nitrones 769 with methacrolein (Scheme 291). ${ }^{652}$ A significant solvent effect was observed in this 3,5-endo-or exo-diastereoselective 1,3-DC. Using MTBE endooxazolidines $\mathbf{7 7 1}$ were mainly isolated, whereas in toluene products exo-771 were formed in lower diastereo- and enantioselectivity. In addition, the substituents at the nitrogen and oxygen atoms on the nitrone controlled the observed diasteroselectivity. The corresponding DFT calculations revealed that the catalyst behaves as a frustrated Lewis ion pair. The resulting diastereodivergence was attributed to the competition between the counteranion and the solvent in the complexed methacrolein. The orientation of methacrolein and the solvent is shown in the intermediate 770 A based on the calculations. Product endo-771 $\left(\mathrm{R}^{2}=\mathrm{Me}\right)$ was transformed into the highly enantioenriched lactone $\mathbf{7 7 2}$.

Scheme 291. Diastereodivergent 1,3-DC of Differently Substituted Nitrones 769 with Methacrolein Catalyzed by Chiral Kündig’s Ru Complex 770 


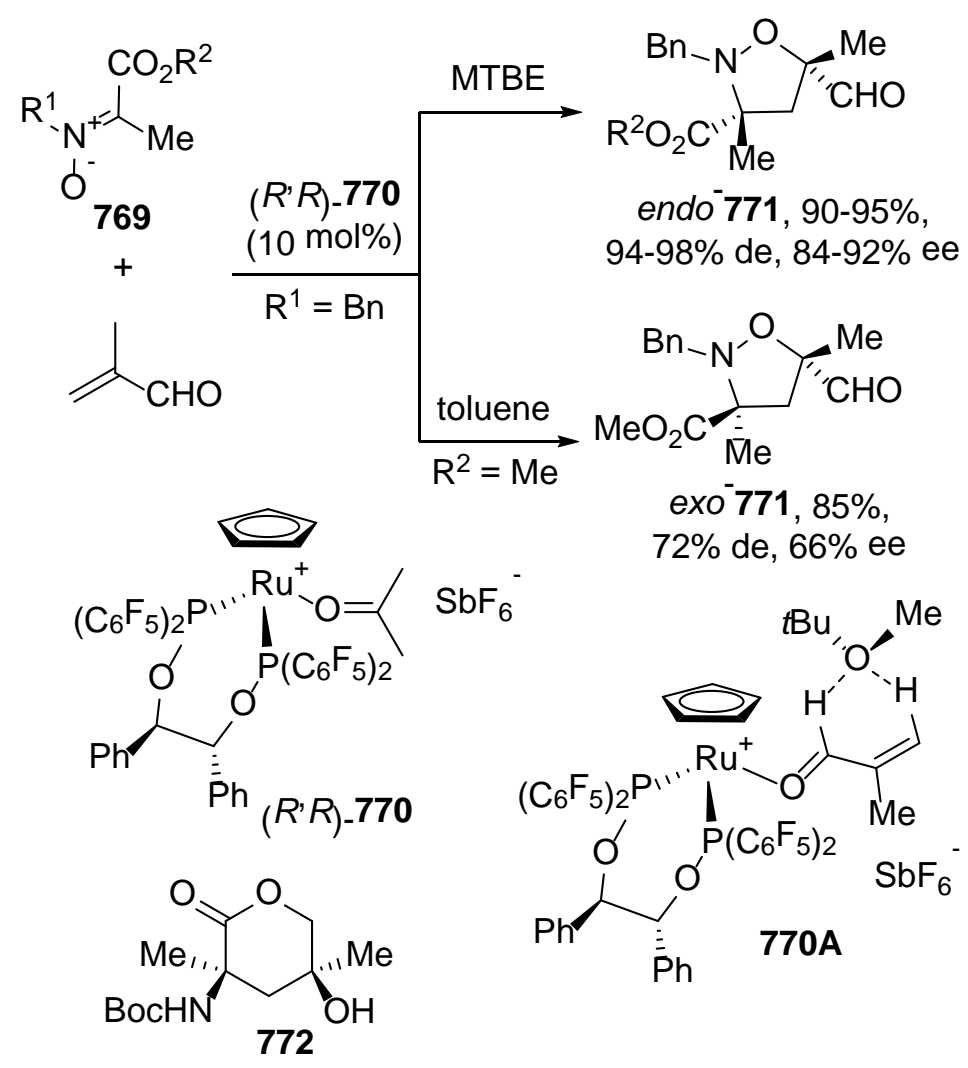

Complexation of the nitrone to a Lewis acid lowers its LUMO energy promoting an inverse electron-demand 1,3-DC with electron-rich alkenes. Scheeren and co-workers found out a solvent-dependent enantiodivergence in the reaction of diphenyl nitrone $\mathbf{7 6 4}$ with the ketene acetal 773 in $\mathrm{CH}_{2} \mathrm{Cl}_{2}$ at $-78{ }^{\circ} \mathrm{C} .{ }^{653,654}$ As achiral catalyst, oxazaborolidine $\mathbf{7 7 4}$, prepared from the corresponding amino alcohol and $\mathrm{BH}_{3} \cdot \mathrm{THF}$, was used giving 3,4-exo-oxazolidine 775 in diphenyl ether as co-solvent in 79\% ee. When the catalyst was prepared in THF ent-775 was obtained in moderate 62\% ee (Scheme 292). This reversed enantiofacial selectivity has been explained through conformers 774A and 774B in which the benzyl group of the catalyst shields one of the two faces of the nitrone.

Scheme 292. Enantiodivergent 1,3-DC of Nitrone 764 with Ketene Acetal 773 Catalyzed by Oxazaborolidine 774 in the Presence of Different Solvents 


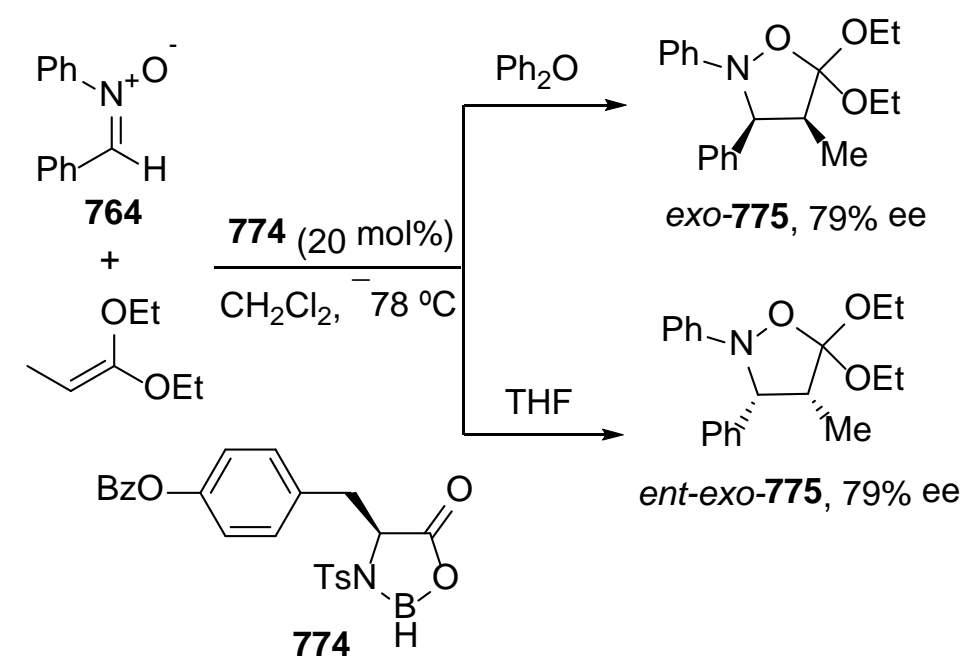

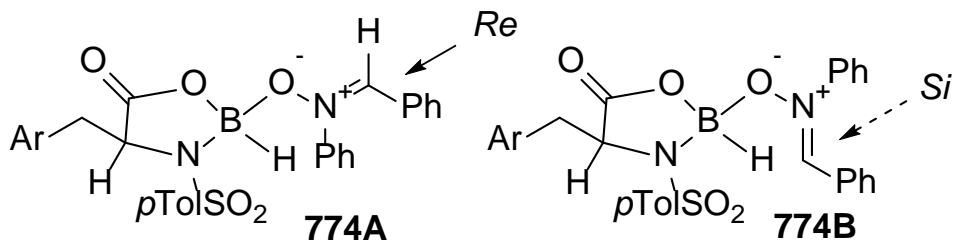

In conclusion, the presence or absence of $4 \AA$ MS has shown enantiodivergent effects in the 1,3-DC of alkenoyl oxazolidinones with nitrones catalyzed by chiral oxazoline metal complexes. In the case of using alkylidene malonates as dipolarophiles, the obtained diastereodivergent results depend on the reaction temperature. When acrolein was used as dipolarophile, solvent effects control the endo/exo diastereodivergence. Enantiodivergent solvent effects have been described in the reverse electron-demand 1,3-DC with a ketene acetal catalyzed by a chiral oxazaborolidine

5.2.2. Azomethine Ylides. Azomethine ylides are electron-rich allyl-type dipoles which react with electrophilic dipolarophiles giving, after a [3+2] cycloaddition. pyrrolidines. ${ }^{632-641}$ The most studied dipoles are the so-called stabilized azomethine ylides derived from $\alpha$-imino esters. These dipoles can be easily generated by metalcatalyzed reactions in the presence of a base. The 1,3-DC can take place through a concerted or stepwise mechanism depending mainly on the dipolarophile but also on the catalyst. In the case of metallo-dipoles the coordination of the metal with the nitrogen and oxygen atoms fixes the conformation of the dipole. There are three possible different dipole conformations, W-, U-, and S-shaped, which control the relative 2,5configuration in the pyrrolidine ring. In the case of $\mathrm{W}$ - and U-conformations 2,5-cis products are formed, and 2,5-trans substituted prolines in the case of the later. Usually, $\mathrm{Ag}$ and $\mathrm{Cu}$ complexes give endo- and exo-adducts, respectively, according to their sphere coordination and their ability to coordinate with the dipolarophile. Consequently, the endo- and exo-diastereoselectivity determines the 4,5-cis- and 4,5-trans-relative configuration, respectively (Scheme 293). Control of the enantioselectivity can be achieved by using chiral metal complexes or organocatalysts. 
Scheme 293. Regio- and Diastereoselectivity of 1,3-DC with Stabilized Azomethine Ylides
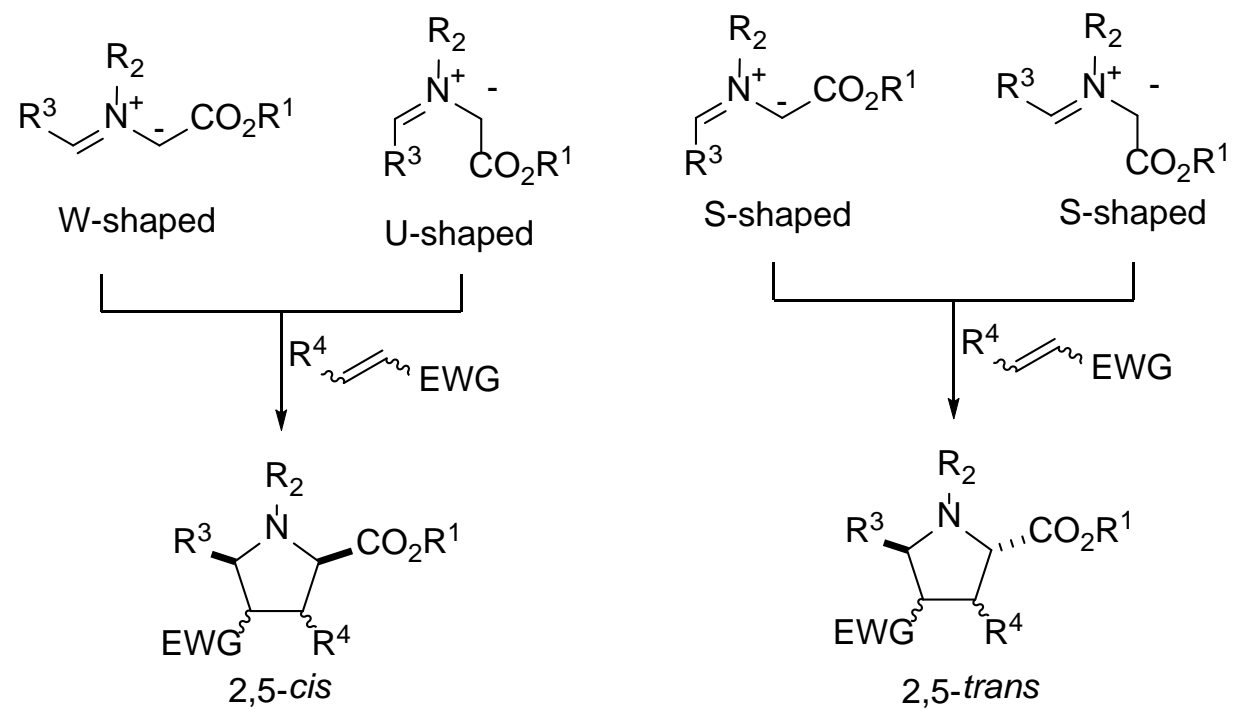

S-shaped S-shaped

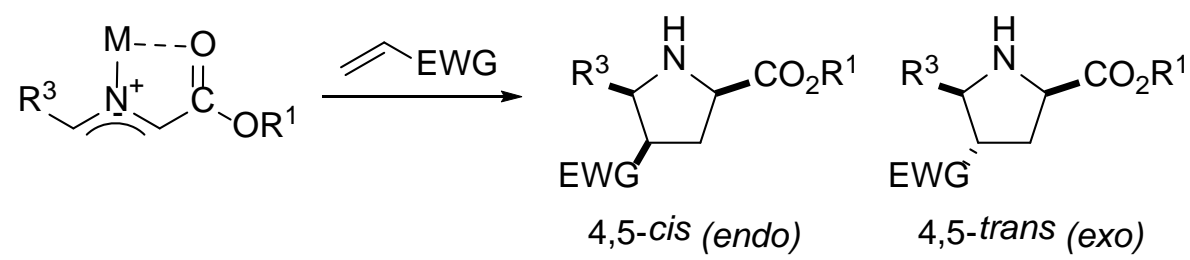

A hydrogen bonding-directed enantiodivergent Ag-catalyzed 1,3-DC of azomethine ylides was achieved using bidentate ferrocenyl ligands $776 .{ }^{655}$ The reaction of imino esters with dimethyl maleate using AgOAc and the ligand 776a, bearing a primary amine, afforded endo-prolinates all-cis-777 with total diastereoselectivity and in general high enantioselectivity (Scheme 294). In addition, homochiral ligand 776b with a tertiary amine gave the corresponding enantiomers ent-endo-777 with similar de and ee. According to the DFT calculations, in transition state 776A the two carbonyl groups of the maleate are coordinated to the Ag atom and with the two $\mathrm{NH}$ groups of the ligand 776a on the top face. However, ligand 776b will favor the approach of the maleate from the bottom face in transition state $\mathbf{7 7 6 B}$ giving adducts ent-endo-777.

Scheme 294. Enantiodivergent 1,3-DC of Imino Esters and Dimethyl Maleate Catalyzed by AgOAc and Ligands 776 


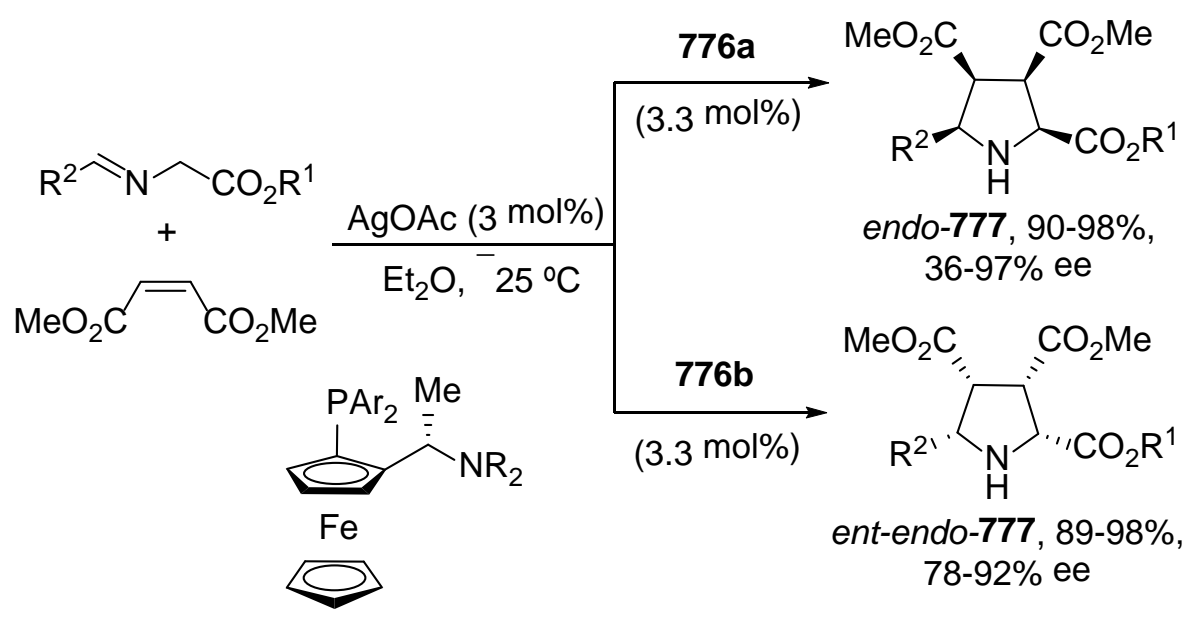

776a: $\mathrm{Ar}=3,5-\mathrm{Me}_{2} \mathrm{C}_{6} \mathrm{H}_{3}, \mathrm{R}=\mathrm{H}$

776b: $\mathrm{Ar}=3,5-\mathrm{Me}_{2} \mathrm{C}_{6} \mathrm{H}_{3}, \mathrm{R}=\mathrm{Me}$

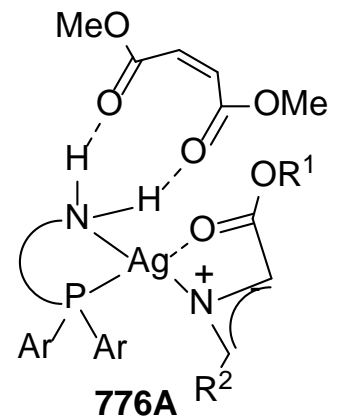

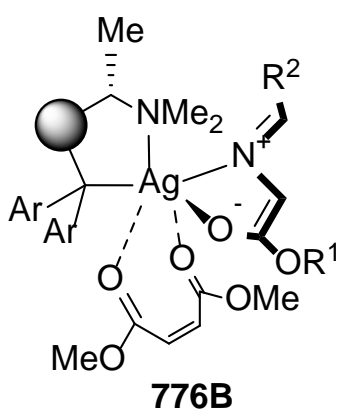

Diastereodivergent synthesis of nitroprolinates was achieved by 1,3-DC of glycine imino esters with $\beta$-nitroalkenes catalyzed by $\mathrm{Cu}$ complexes. Hou and co-workers described that ferrocenylphosphine-oxazoline 227c and $\mathrm{Cu}\left(\mathrm{ClO}_{4}\right)_{2}$ gave adducts exo778 in general with high de and ee (Scheme 295). ${ }^{656}$ Surprisingly, changing the diphenylphosphino group of ligand 227c by a 3,5-bis(trifluoro)phenylphosphino unit in ligand 227d, the corresponding endo-778 were obtained in good de and excellent ee. Generally, nitroalkenes react through a stepwise mechanism as it was previously proposed by Cossío and co-workers. ${ }^{657,658}$ From the computational studies a transition state $\mathbf{7 7 8 C}$ has been postulated for the formation of products exo-778. In this structure, the nitro group occupies far from the phenyl group of the phosphine unit. However, in the case of transition state $\mathbf{7 7 8 D}$ the nitro group, which carries a partial negative charge, is in between two 3,5-bis(trifluoromethyl)phenyl substituents and is stabilized by the electrostatic interactions. The TS models $\mathbf{7 7 8 C}$ and $\mathbf{7 7 8 D}$ are the most favorable ones for the exo- and endo-products, respectively.

Scheme 295. Diastereodivergent 1,3-DC of Imino Esters with $\beta$-Nitroalkenes Catalyzed by $\mathrm{Cu}\left(\mathrm{ClO}_{4}\right)_{2}$ and Differently Substituted Chiral Ligands 227 


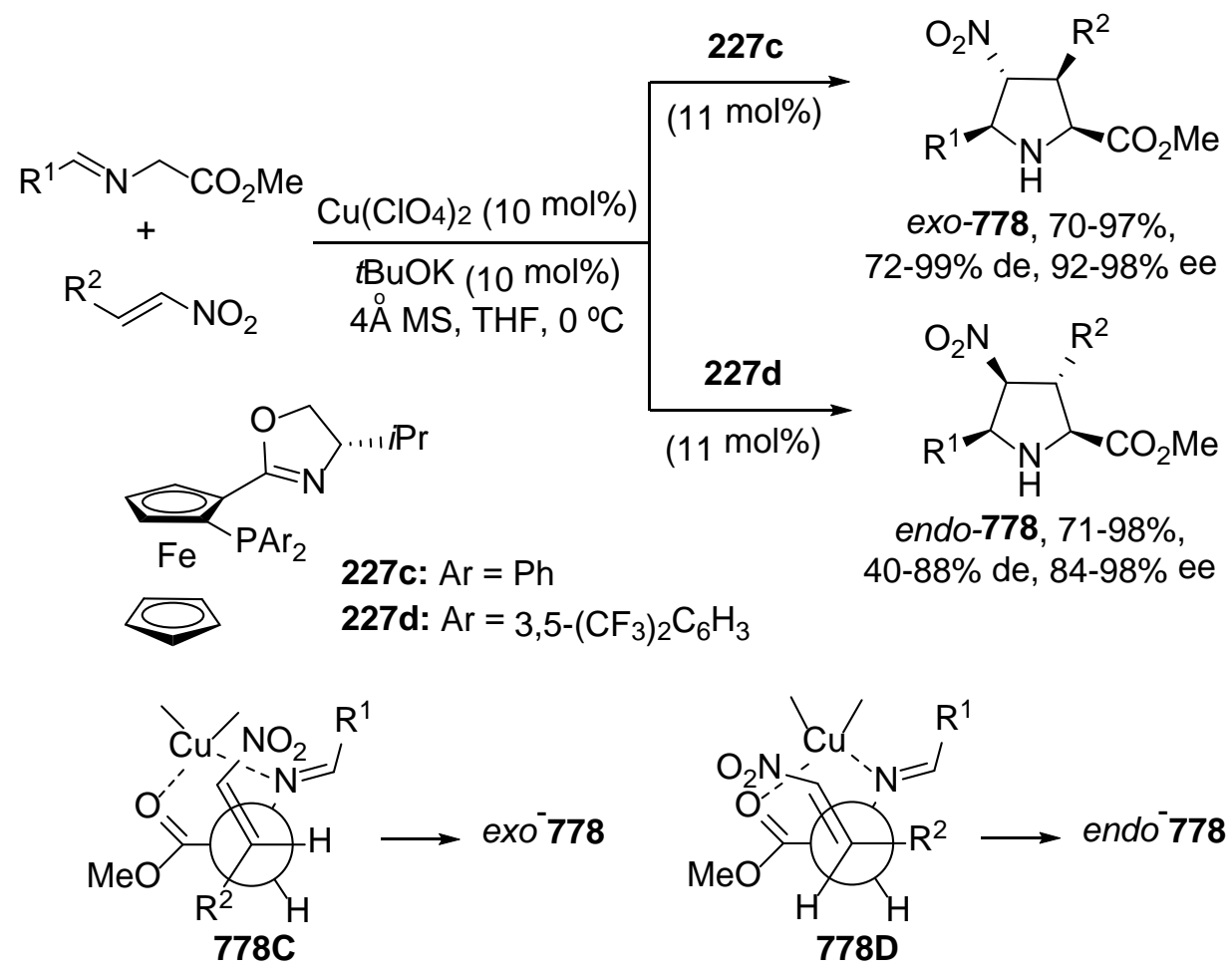

Diastereodivergent endo/exo results were observed by Cossío and co-workers when $\mathrm{Cu}(\mathrm{I})$ and ferrocenylphosphine ligands $\mathbf{7 7 9}$ were used as catalysts for the same cycloaddition (Scheme 296). ${ }^{659}$ When ligand 779a was used, endo-778 cycloadducts were obtained, whilst $\mathbf{7 7 9 b}$ gave exo-778 products, both in good de and excellent ee. The DFT calculations showed that a stepwise mechanism was operating, the first step being responsible for the observed stereocontrol. In addition, $\mathrm{Cu}(\mathrm{I})$ is coordinated to the phosphorous of the ligand and to the nitrogen and oxygen atoms of the azomethine ylide in the calculated endo-TS. Because there are no interactions between the $\mathrm{Cu}$ metal and the $\mathrm{N}$ atom of the pyrrolidine unit of the ligand, the $\mathrm{Cu}$ atom has a vacant coordination site available to interact with the nitro group. In the case of the exo-TS, the catalyst acts as a bidentate ligand and the $\mathrm{Cu}$ is bonded to the phosphine and to pyrrolidine units. Consequently, the fourth coordination position is not available for the nitro group favoring the formation of exo-778.

Scheme 296. Diastereodivergent 1,3-DC of Imino Esters with $\beta$-Nitroalkenes Catalyzed by $\mathrm{Cu}\left(\mathrm{ClO}_{4}\right)_{2}$ and Chiral Ligands 779 


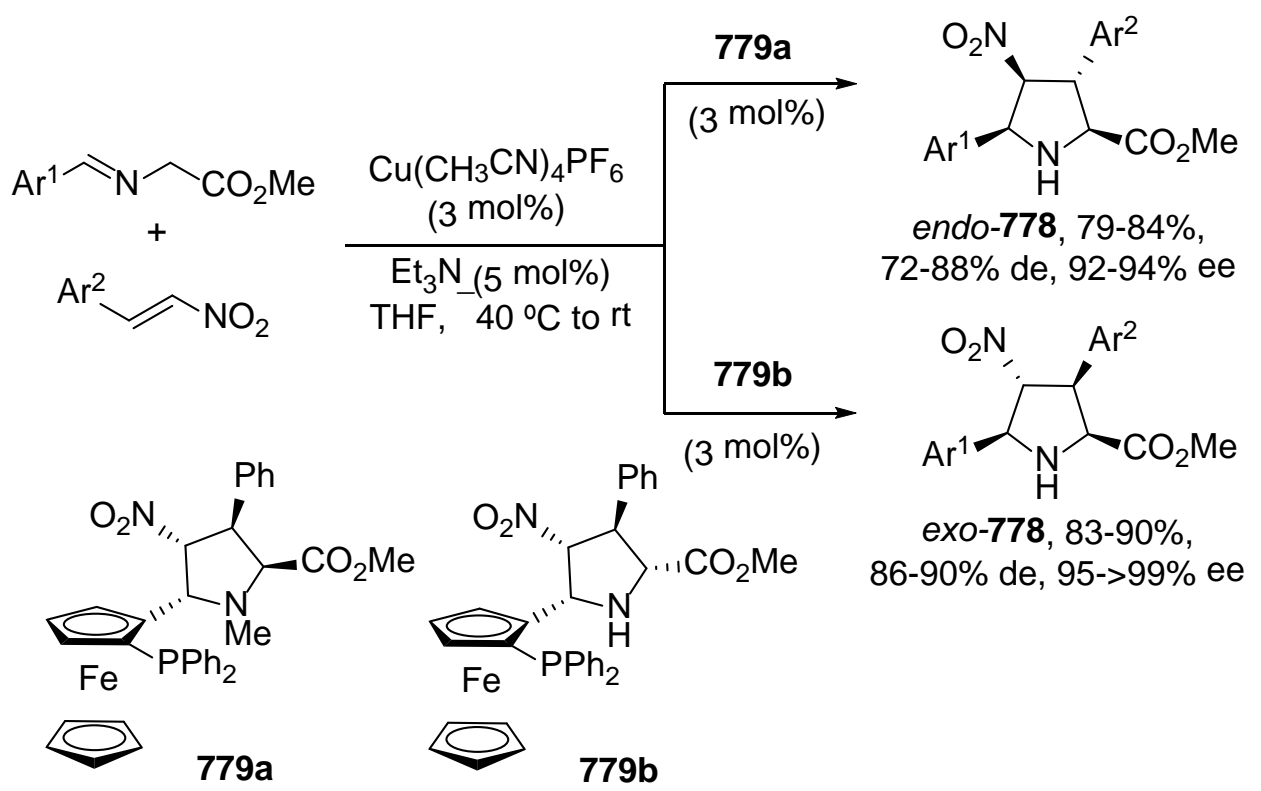

Martín and co-workers have described that the 1,3-DC of $\mathrm{C}_{60}$ with the 3benzaldehyde glycinimino methyl ester provided highly diastereodivergent 2,5-cis and trans-selectivity when $\mathrm{Ag}$ or $\mathrm{Cu}(\mathrm{II})$ complexes with chiral diphosphines were used as chiral catalysts. ${ }^{660}$ Thus, AgOAc and $(R, R)$-BPE $\mathbf{7 8 0}$ gave the 2,5-cis-782 adduct in $98 \%$ de and $86 \%$ ee (Scheme 297). However, the same reaction catalyzed by $\mathrm{Cu}(\mathrm{OTf})_{2}$ and (R)-DTBM-SEGPHOS $\mathbf{7 8 1}$ provided 2,5-trans-782 in 98\% de and 95\% ee.

Scheme 297. Diastereodivergent 1,3-DC of 3-Methoxybenzylidene Methyl Glycinate with $\mathrm{C}_{60}$ Catalyzed by Ag or Cu Complexes 

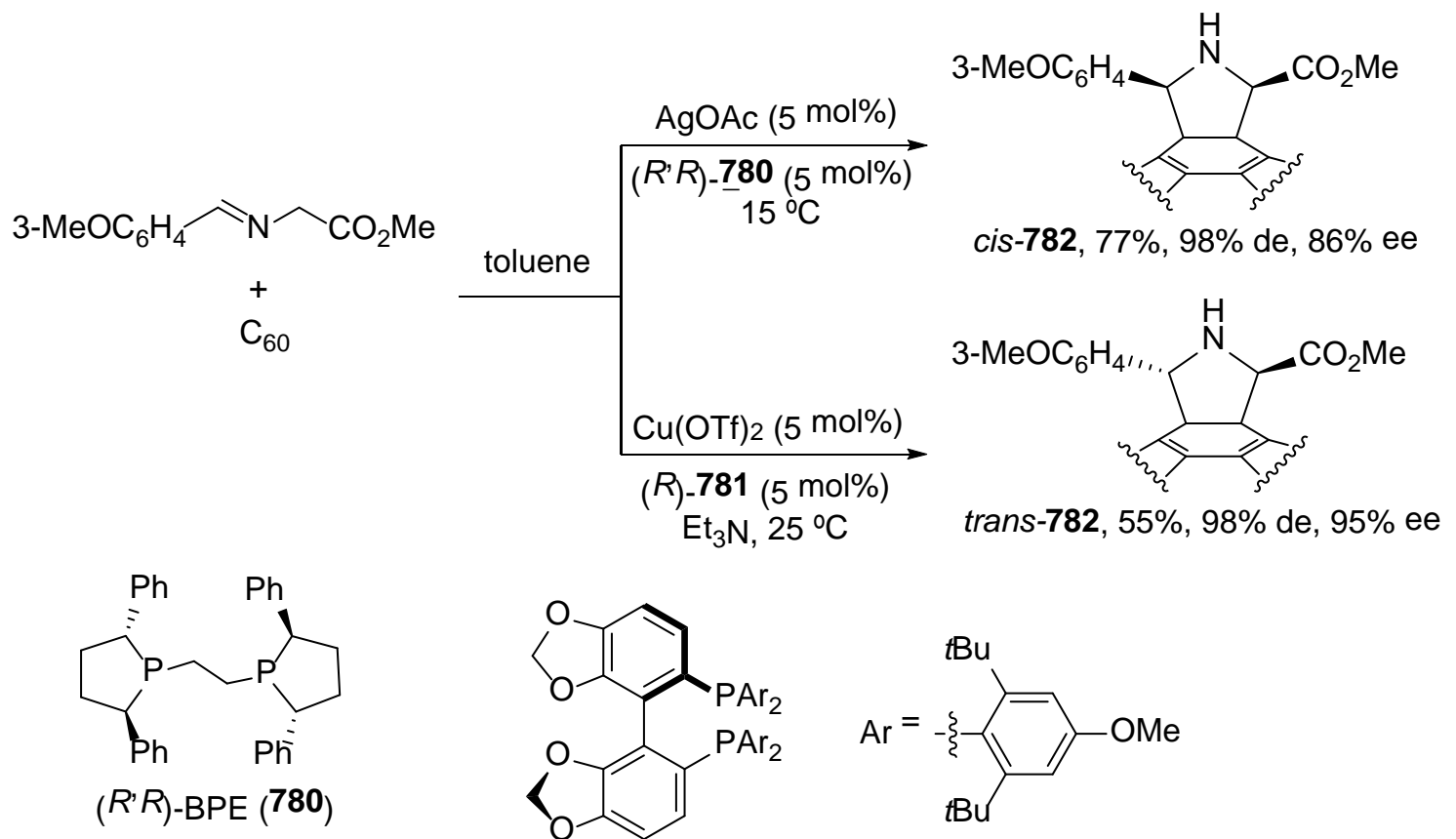<smiles>[R16]c1ccc2c(c1-c1c(P)ccc3c1OCO3)OCO2</smiles><smiles>COc1cc(C(C)(C)C)c(C(C)(C)C)c(C(C)(C)C)c1</smiles>

(R)-DTBM-SEGPHOS (781)

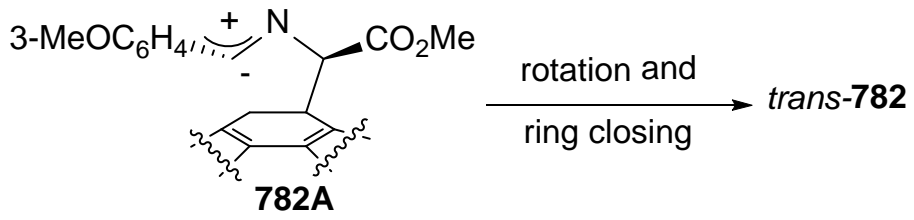

Fulleropyrrolidines have been prepared diastereodivergently by the same group using the same type of $\mathrm{Ag}^{661,662}$ and $\mathrm{Cu}^{663}$ chiral catalysts. In the case of $\mathrm{C}_{60}$, cis-782 ${ }^{661}$ was mainly formed and in the case of Cu trans-782 ${ }^{663}$ was isolated. The 1,3-DC of $\mathrm{C}_{70}$ as dipolarophile provided cis-adducts in 70-98\% de and $80-89 \%$ ee with moderate to high regiochemistry (60-94\%) in the presence of the Ag catalyst. ${ }^{662}$ Using the $\mathrm{Cu}$ complex trans-adducts were formed in $80-94 \%$ de, $90-95 \%$ ee and $64-94 \%$ regioselectivity. ${ }^{663}$ This methodology has been also applied to the 1,3-DC of endohedral fullerene $\mathrm{H}_{2} @ \mathrm{C}_{60}$ providing cis- and trans-adducts with ee up to $94 \% .{ }^{664}$

The diastereodivergent cis/trans selectivity with fullerenes has been rationalized by the competition of two reaction pathways depending on the ligand structure. The bulky $\mathrm{Cu}(\mathrm{II}) / \mathrm{SEGPHOS} \mathbf{7 8 1}$ complex allows the dipolarophile to attack by an exo-approach without the secondary interaction of the metal with the dipolarophile. Therefore, this process will occur through a stepwise mechanism giving an intermediate 782A with a negative charge in the fullerene and a positive charge in the benzylic substituent of the imino ester. This intermediate has already the $(R)$-configuration at C2 and can rotate though the $\mathrm{N}-\mathrm{C} 2$ bond to give the trans-isomer. ${ }^{663}$

The same Ag and $\mathrm{Cu}$ catalysts gave excellent endo/exo diastereodivergence in the 1,3-DC of methyl benzylideneglycinate with methyl acrylate and $N$-phenylmaleimide (Scheme 298). ${ }^{663}$ These results were explained by the bulkiness of $\mathrm{Cu}(\mathrm{OTf})_{2} / 781$ complex and the previously mentioned tetrahedral coordination of the $\mathrm{Cu}$ with the two 
phosphorous atoms and the nitrogen and oxygen atoms of the azomethine ylide affording exo-783 and 784. On the other hand, the square planar AgOAc/780 complex allows a secondary interaction of the cation with the dipolarophile giving the endoprolinates $\mathbf{7 8 3}$ and $\mathbf{7 8 4}$. The formation of trans-adducts was excluded because in this case the zwitterionic intermediate is not sufficiently stabilized to give free rotation as in the case of fullerenes.

\section{Scheme 298. Diastereodivergent 1,3-DC of Imino Esters with Dipolarophiles Catalyzed by Chiral Ag and Cu(II) Complexes}

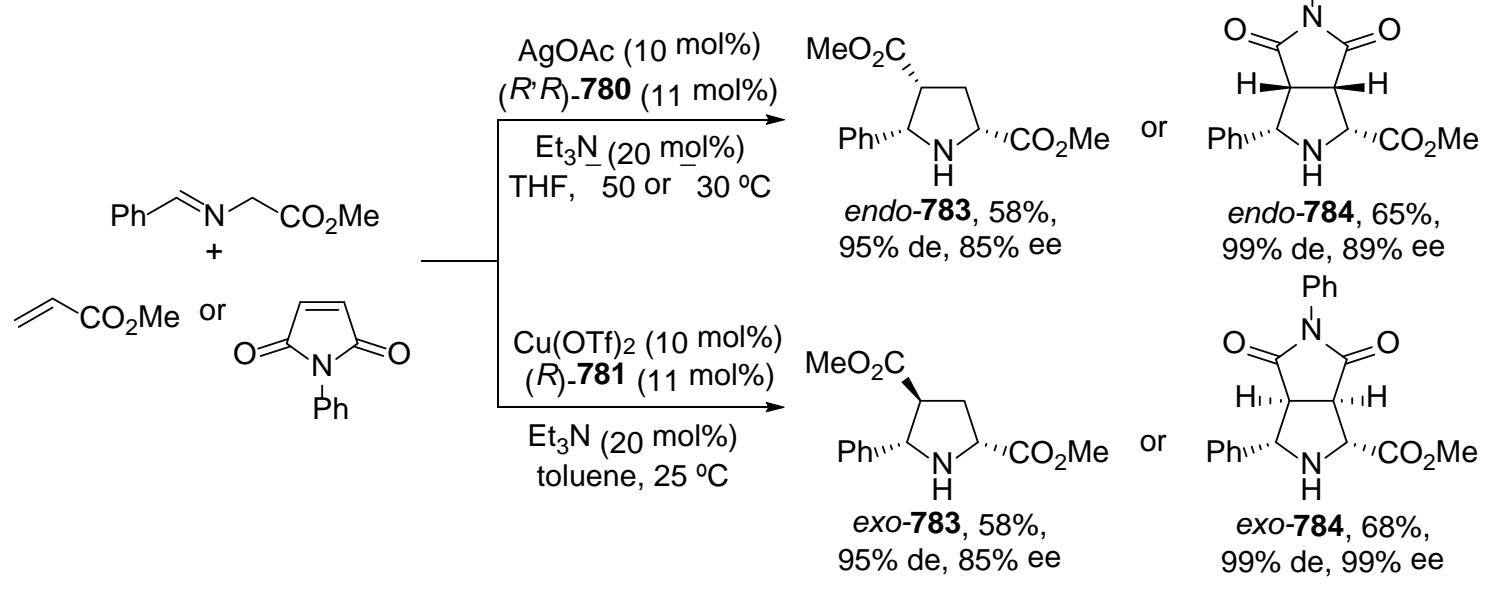

An example of the metal-controlled switching of enantioselectivity has been described by $\mathrm{Oh}$ and co-workers using brucine-derived amino diol 115 (Figure 7) as chiral ligand. ${ }^{665}$ This ligand presents different mode of coordination with Ag and $\mathrm{Cu}(\mathrm{I})$ giving in both cases products ent-endo-783 and endo-783, respectively (Scheme 299). In the case of the Ag-catalyzed 1,3-DC, the proposed model 115A, with a conformational change in the tert-butyl acrylate, will form a hydrogen bonding with the hydroxyl group at C21 in a 2:1 ligand:AgOAc complex. In the structure 115B a possible model to explain the endo-approach of the dipolarophile by a hydrogen bonding of the carbonyl group with the hydroxyl group at C22 of the ligand in the $\mathrm{Cu}$ complex is shown.

Scheme 299. Enantiodivergent 1,3-DC of Imino Esters with tert-Butyl Acrylate Catalyzed by Ag and Cu(I) with Chiral Ligand 115 Complexes 

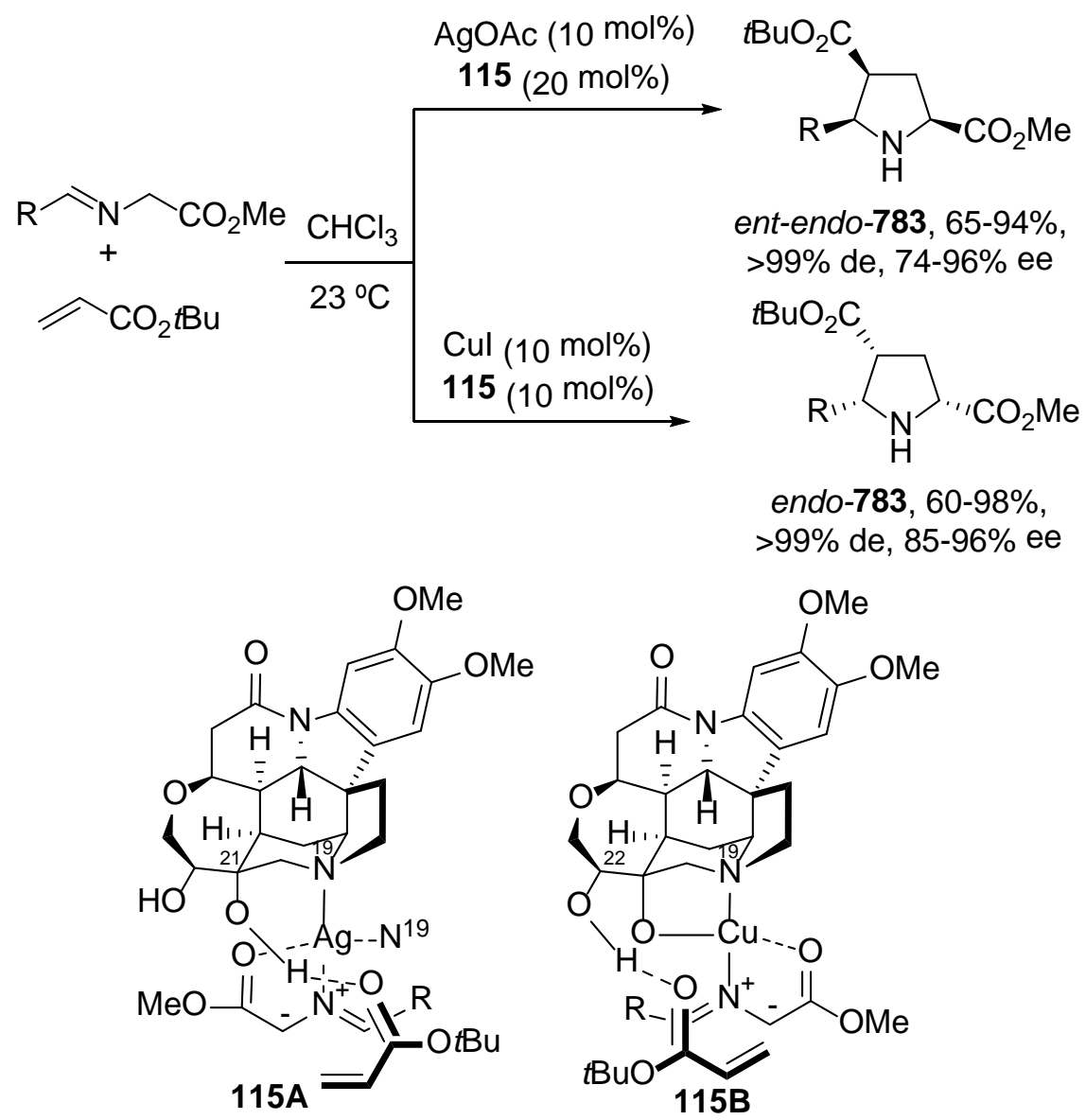

The same group has found that the reaction of aldimines from $\alpha$-amino esters with $\beta$ nitroalkenes catalyzed by CuOTf and brucine diol $\mathbf{1 1 5}$ led to the formation of ent-endo778 prolinates through a concerted mechanism (Scheme 300). ${ }^{666,667}$ However, the reaction with benzophenone imines took place stepwise giving endo-778 adducts.

Scheme 300. Enantiodivergent 1,3-DC of Imino Esters with $\beta$-Nitroalkenes Catalyzed by Chiral CuOTf and Ligand 115 Complex 


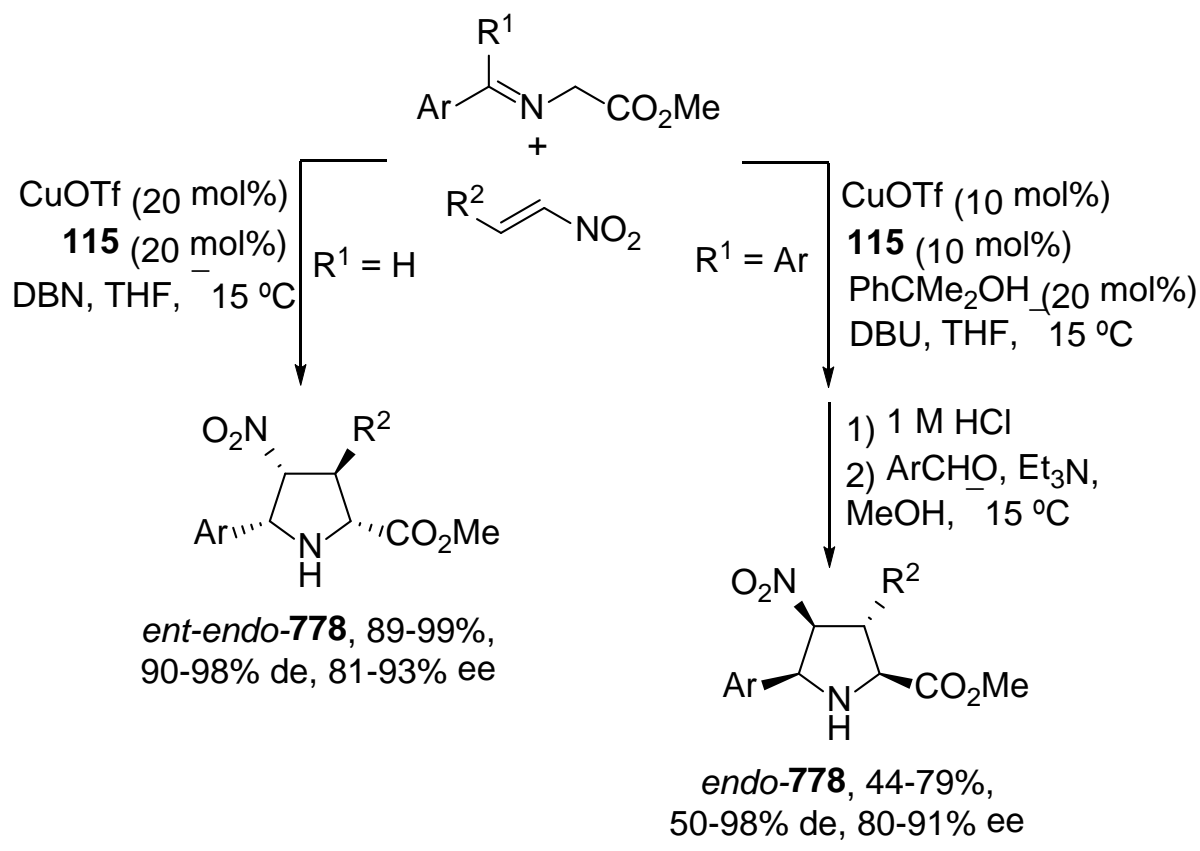

Another example of enantiodivergent 1,3-DC catalyzed by AgF/ and CuOTf/brucine diol 115 complexes has been recently described by the same group using chalcones as dipolarophiles (Scheme 301). ${ }^{668}$ Both catalysts gave enantiomeric endo-products 785 with ee up to $98 \%$ based on to the model described in Scheme 299 for the reaction with tert-butyl acrylate. This unique enantiodivergent behavior has been only described with the ligand 115.

\section{Scheme 301. Enantiodivergent 1,3-DC of Imino Esters with Chalcones Catalyzed by $\mathrm{Ag}$ and $\mathrm{Cu}(\mathrm{I})$ with Chiral Ligand 115 Complexes}

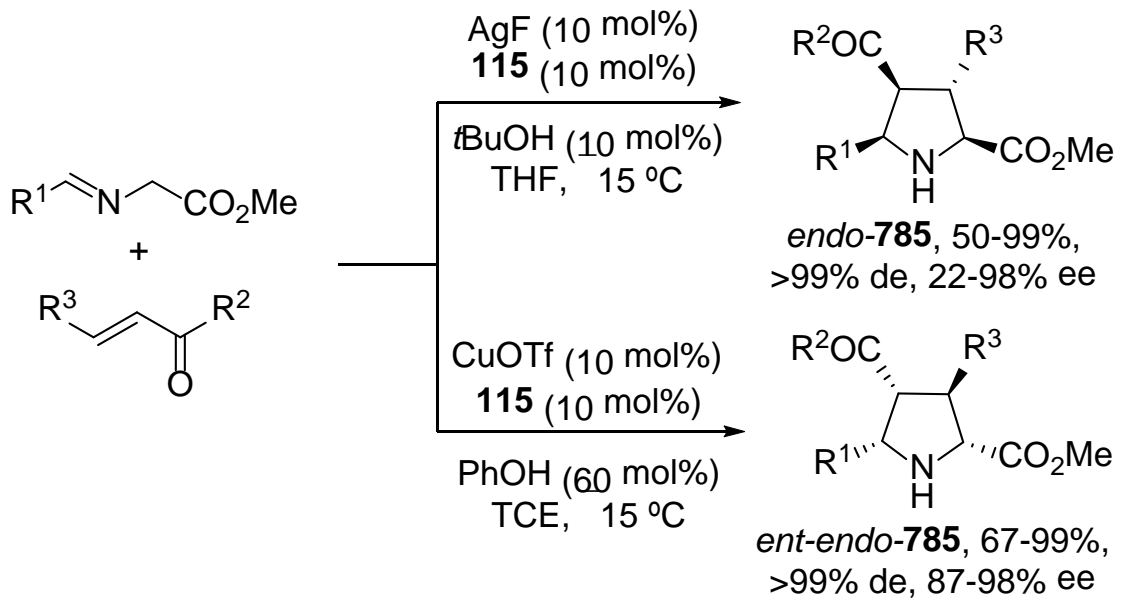

The chiral $\mathrm{Cu}(\mathrm{OTf})_{2}$ /bis(imidazoline)pyridine $\mathbf{7 8 6}$ complex has been used as catalyst in the unusual endo-selective 1,3-DC of imino esters with $\beta$-nitroalkenes providing nitroprolinates endo-778 with high de and ee (Scheme 302). ${ }^{669}$ However, the complex formed by imidazoline-aminophenol (IAP) ligand 662 and $\mathrm{Ni(OAc})_{2}$ catalyzes the formation of exo'-adducts 787, which posess a 2,5-trans configuration, in good de and 
excellent ee. ${ }^{670}$ These two chiral ligands are derived from (1S,2S)-1,2diphenylethanediamine as a chiral starting material. A stepwise mechanism has been proposed to explain this unusual diastereodivergence. Firstly, the Michael adduct is formed and then the $\mathrm{Ni}$ atom can coordinate with the nitro group in model 787A and the $\mathrm{C}-\mathrm{N}$ bond can rotate to give 787B before the intramolecular Mannich reaction takes place. The DFT calculations support the formation of this intermediate 787B which will afford the most stable exo'-787 diastereomer. Both $\mathrm{Cu}(\mathrm{II})$ and $\mathrm{Ni}(\mathrm{II})$ complexes have been used in the diastereodivergent 1,3-DC of imino esters with indolylnitroalkenes giving endo- and exo'-indolylpyrrolidines, respectively. ${ }^{671}$

Scheme 302. Diastereodivergent 1,3-DC of Imino Esters with $\beta$-Nitroalkenes Catalyzed by $\mathrm{Cu}(\mathrm{OTf})_{2} / 786$ and by $\mathrm{Ni}(\mathrm{OAc})_{2} / 662$ Chiral Complexes

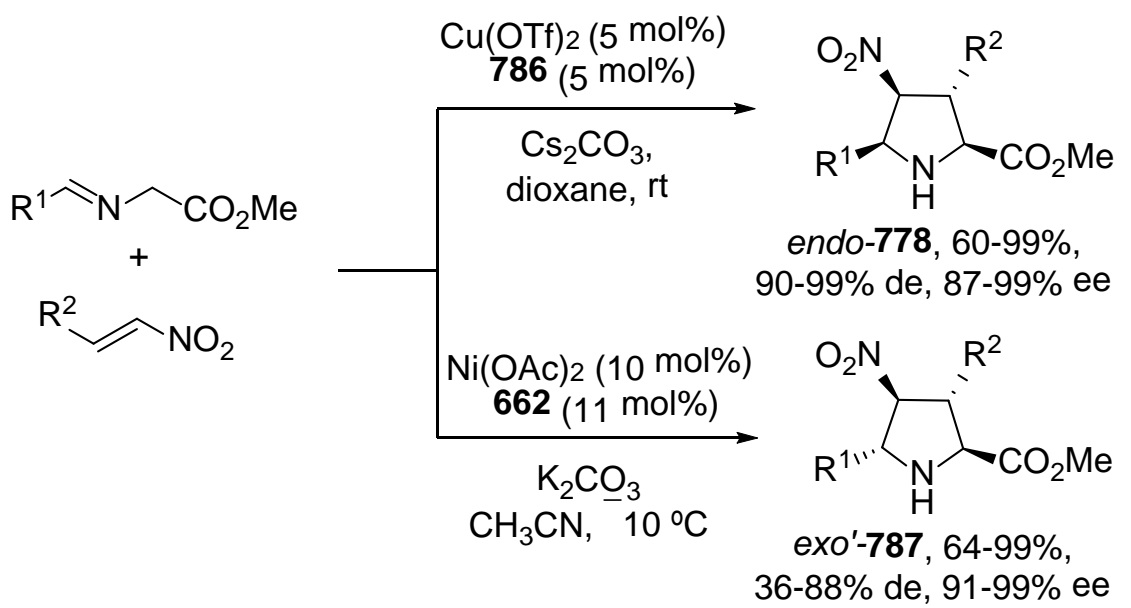<smiles>c1ccc(CC(c2ccccc2)N2C(c3ccccc3)NC(c3ccccc3)N2[C@@H]2NC(c3ccccc3)[C@H](c3ccccc3)N2Cc2ccccc2)cc1</smiles>

PyBidine (786)

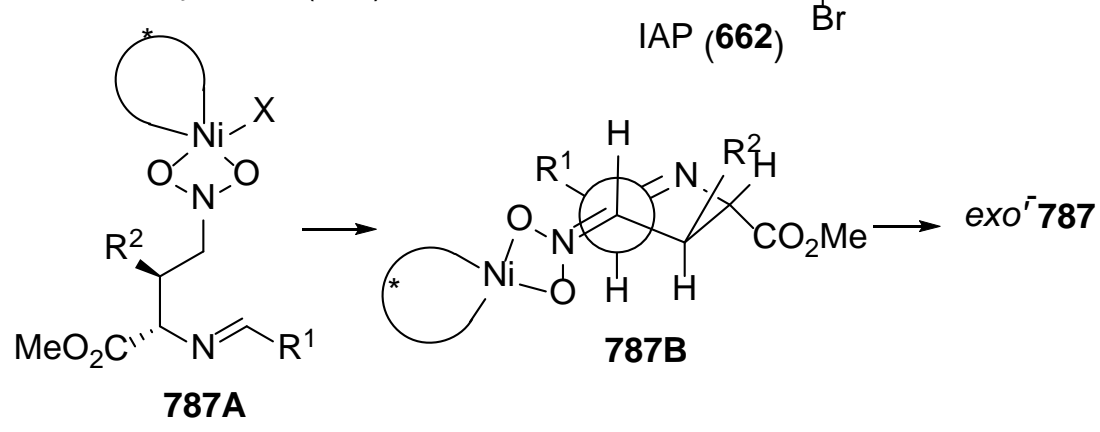

In the case of the $\mathrm{Cu}(\mathrm{I})$-catalyzed asymmetric $1,3-\mathrm{DC}$ of azomethine ylides with $\beta$ phenylsulfonyl enones $\mathbf{7 8 8}$ it was observed by Adrio, Carretero and coworkers a liganddependent reversal of the diastereoselectivity. ${ }^{672}$ Using $(R)$-SEGPHOS as chiral ligand endo-789 adducts were obtained in moderate yields, high de and excellent ee (Scheme 303). On the other hand, $\mathrm{Cu}(\mathrm{I}) /(R)$-DTBM-SEGPHOS 781 complex afforded exo-789 
products. In both cases the regioselectivity was controlled by the carbonyl group of the dipolarophile. The observed endo/exo diastereodivergence can be attributed to steric effects of the DTBM-SEGPHOS ligand.

Scheme 303. Diastereodivergent 1,3-DC of Imino Esters with $\beta$-Phenylsulfonyl Enones 788 Catalyzed by $\mathrm{Cu}\left(\mathrm{CH}_{3} \mathrm{CN}\right)_{4} \mathrm{PF}_{6}$ and by Chiral Ligands SEGPHOS and DTBM-SEGPHOS

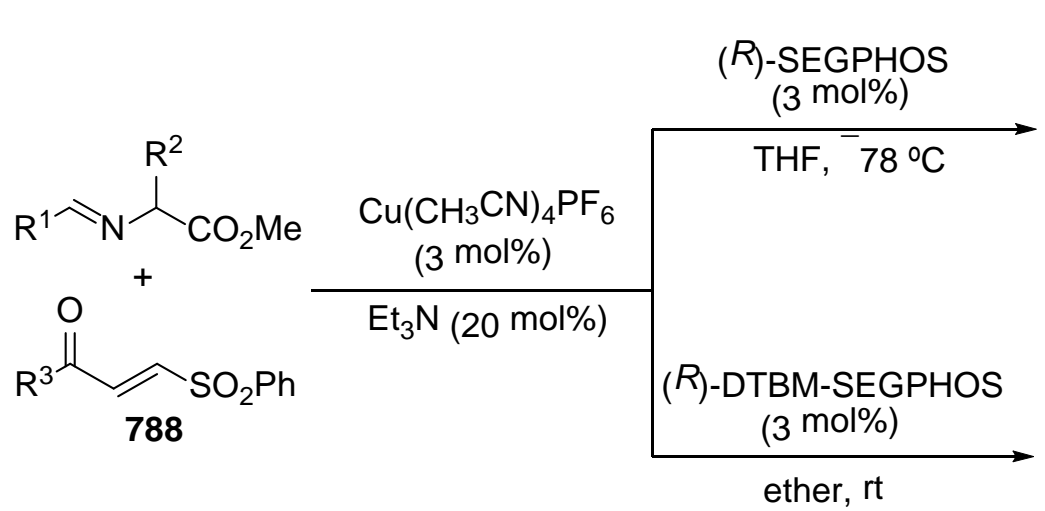

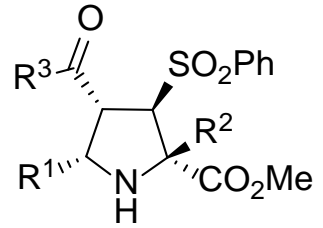

endo-789, 49-71\%, $84-98 \%$ de, $87->99 \%$ ee<smiles>[R]C(=O)C1C([R12])NC(C(C)=O)(C(=O)O)C1[R5]</smiles>

exo-789, 41-71\%, $72-98 \%$ de, $92->99 \%$ ee

Waldman and co-workers performed the 1,3-DC of imino esters with 3alkylideneoxindoles $\mathbf{7 9 0}$ promoted by a $\mathrm{Cu}(\mathrm{I})$ complex with ligand 776c (Scheme 304). ${ }^{673}$ The resulting spirooxindoles 791 were obtained in good ee with total diastereoselectivity. This methodology was applied to the enantioselective synthesis of spirotryprostatin $A^{674}$ by using the Carretero's FesulPHOS (793) ${ }^{675}$ as achiral ligand. Arai and co-workers carried out later the same 1,3-DC employing $\mathrm{Ni}(\mathrm{OAc})_{2} / \mathrm{IAP}$ (662) as catalyst, resulting diastereodivergent results. ${ }^{676}$ Again, the exo'-diastereomer 792 was formed in excellent de and ee (Scheme 303). A plausible mechanism, similar to the already described in Scheme 302, was proposed. Again, using $\mathrm{Cu}(\mathrm{I}) / \mathbf{7 8 6}$ the corresponding products ent-endo-789 were diastereodivergently obtained. ${ }^{677}$

Scheme 304. Diastereodivergent 1,3-DC of Imino Esters with 3-Alkylidenoxindoles 790 Catalyzed by $\mathrm{Cu}(\mathrm{I}) / 776 \mathrm{c}$ and by $\mathrm{Ni}(\mathrm{OAc})_{2} / 662$ Chiral Complexes 


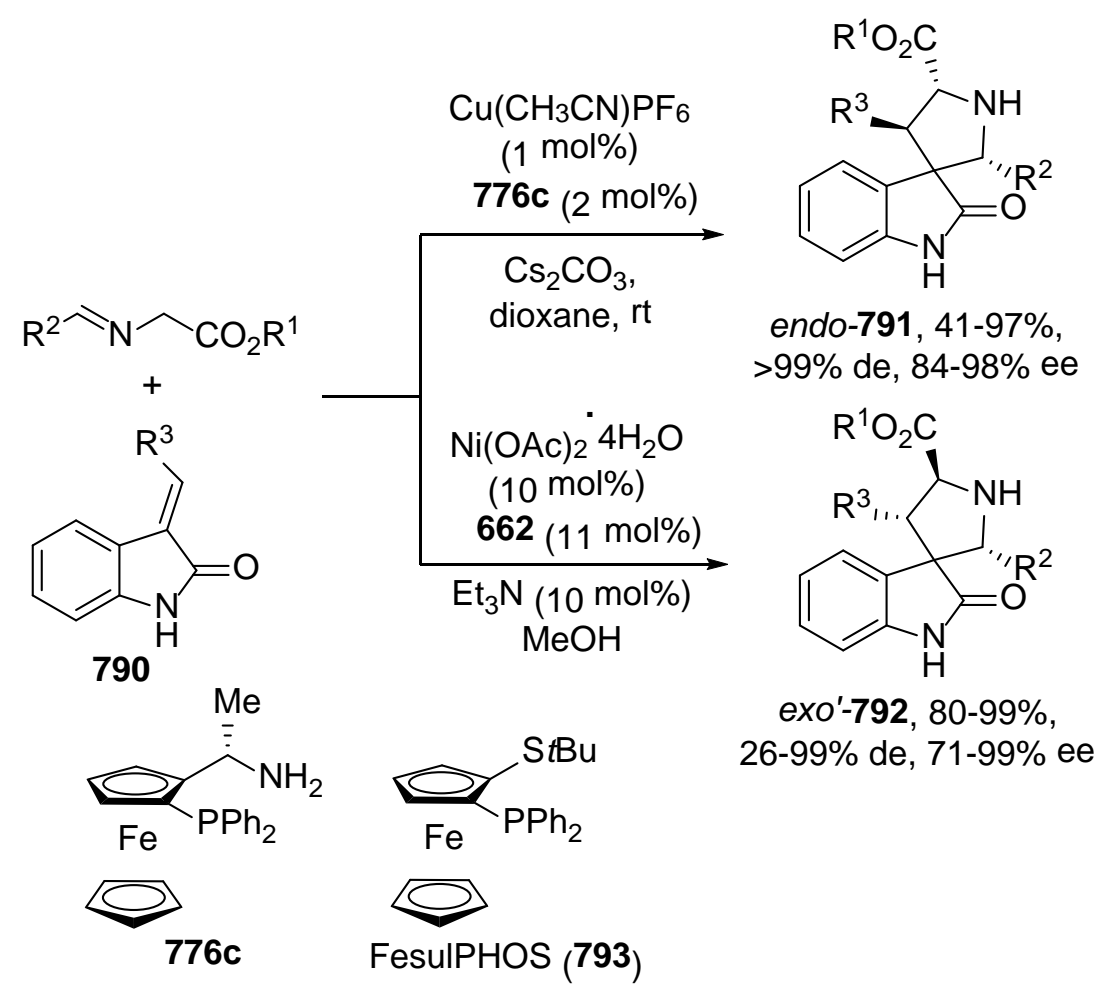

Antonchick, Waldmann and co-workers have studied the 1,3-DC of tropanes rac-794 with azomethine ylides catalyzed by $\left[\mathrm{Cu}\left(\mathrm{CH}_{3} \mathrm{CN}\right)_{4}\right] \mathrm{BF}_{4}$ and $\left(R_{P}\right)-3,5-t \mathrm{Bu}_{2}-$ MeOBIPHEP 795 as chiral ligand. ${ }^{678}$ Starting from rac-794, this process afforded not only the cycloadduct (-)-exo-796 in excellent ee but also a kinetic resolution took place affording tropanes (+)-794 in excellent ee (Scheme 305). These enantioenriched tropanes were allowed to react in situ with an appropriate glycine imino ester in the presence of rac-BINAP affording (+)-exo-797 with ee up to 97\%. Both (-)-796 and (+)exo-797 were prepared with different polarity to enable the chromatographic separation.

Scheme 305. Enantiodivergent Synthesis of Tropane 794 Cycloadducts by 1,3-DC with Imino Esters Catalyzed by $\mathrm{Cu}(\mathrm{I}) / 795$ 


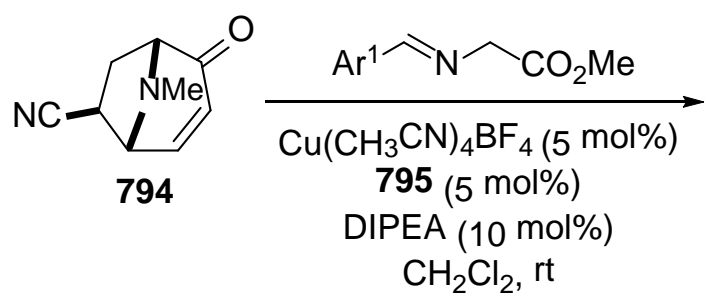

(PAr2

(R)-3,5-tBu 2 -MeOBIPHEP (795)

$\mathrm{Ar}=3,5-t \mathrm{Bu}_{2} \mathrm{C}_{6} \mathrm{H}_{3}$

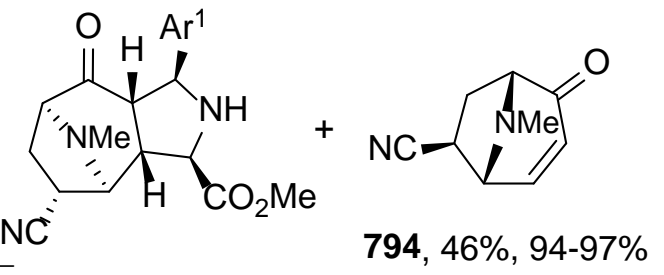

( ) - exo-796, 40-49\%, $95-99 \%$ ee

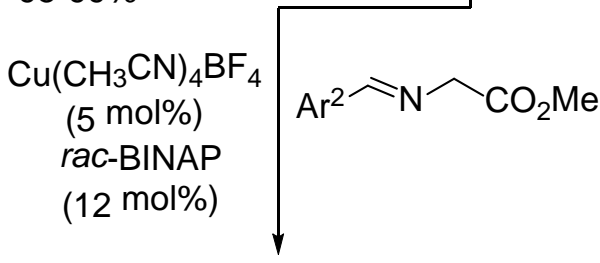

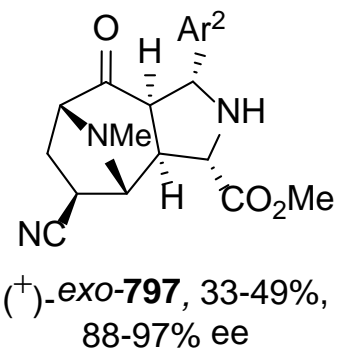

Organocatalyzed 1,3-DC of azomethine ylides provided endo-adducts regardless of the type of organocatalysts used. ${ }^{632-641}$

Antonchick, Waldman, and co-workers have described the first enantioselective [6+3] dipolar cycloaddition of azomethine ylides with fulvenes. ${ }^{679}$ Diastereodivergent results were found depending on the chiral metal complex used as catalyst. When $\mathrm{Cu}(\mathrm{I}) /\left(R_{P}\right)$-FesulPHOS 793 was employed, endo-selectivity was achieved giving products 799 in both variable de and ee (Scheme 306). ${ }^{680}$ However, exo-products 800 were formed with $\mathrm{Cu}(\mathrm{I}) /(R)$-DifluoroPHOS 798 in moderate de and good ee. Due to the lability of the cyclopentadiene unit, these cycloadducts were allowed to react with dienophiles affording the corresponding Diels-Alder adducts.

Scheme 306. Diastereodivergent [6+3] Cycloaddition of Imino Esters with Fulvenes Catalyzed by $\mathrm{Cu}(\mathrm{I})$ and Different Chiral Ligands 793 and 798 

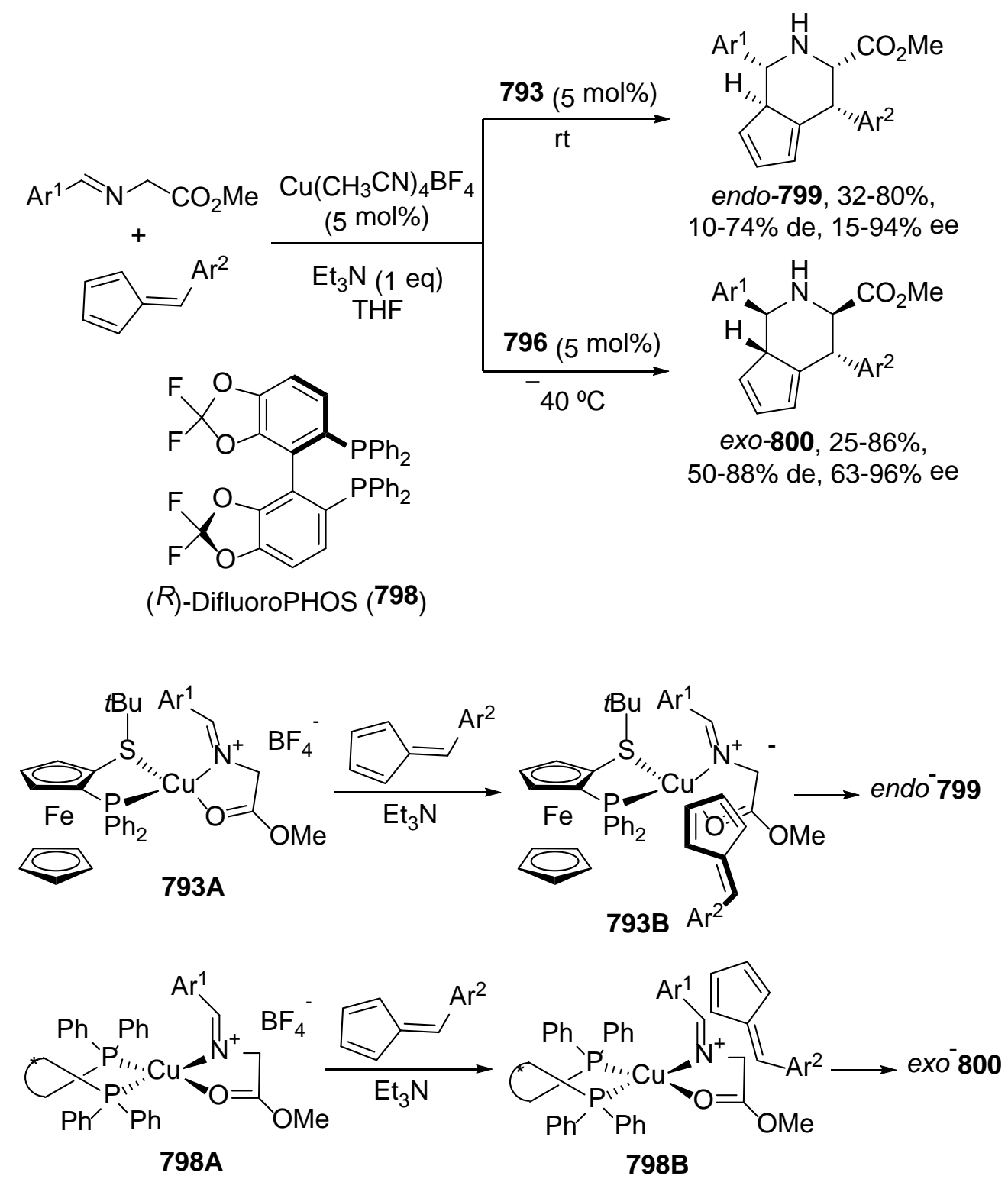

The steric course of the reaction under $\mathrm{Cu}(\mathrm{I}) / \mathrm{FesulPHOS}$ (793) catalysis was explained by the formation of 793A based on the previous NMR studies of Carretero. After the deprotonation of 793A with $\mathrm{Et}_{3} \mathrm{~N}$ the resulting metallodipole will react with a fulvene from the less hindered face in order to avoid steric interactions with the tertbutyl group in model 793B. ${ }^{680}$ In the diastereodivergent process with $(R)$-difluoroPHOS (798) as ligand complex, the intermediate $\mathbf{7 9 8 A}$ is formed and consequently the metallodipole is able to undergo the 1,3-DC with fulvene, which will approach from the back side to avoid steric interactions between the phenyl group of fulvene and the diphenylphosphino group as it is shown in model 798B.

In conclusion, azomethine ylides are able to give diastereodivergent endo/exo-[3+2] dipolar cycloadditions with electron-deficient alkenes modulating the substituents in the chiral ligand, but also changing metal sources. exo'-Cycloadditions are observed in the case of fullerenes with Cu/DTBM-SEGPHOS (781) complex and for $\mathrm{Ni}(\mathrm{OAc})_{2} / \mathrm{IAP}$ (662) complexes. In few cases enantiodivergent [3+2] cycloadditions have been controlled by the small modification of the ligand structure or by changing the metal 
salt from Ag to $\mathrm{Cu}$. Only one example about endo/exo-diastereodivergent [6+3] cycloaddition has been described by using $\mathrm{Cu}(\mathrm{I})$ and different ligands.

5.2.3. Diazo Compounds. In 2000 Kanemasa and co-workers described the first enantioselective 1,3-DC of trimethylsilyldiazomethane with $N$-crotonoyl-2oxazolidinones 298 catalyzed by $\mathrm{Zn}, \mathrm{Ni}$, and $\mathrm{Mg}$ perchlorates and $(R, R)$-DBFOX-Ph 316 (Scheme 113) as ligand. ${ }^{681}$ Enantiodivergent results were observed using $\mathrm{Mg}\left(\mathrm{ClO}_{4}\right)_{2}$ depending on the achiral template. Oxazolidinone 298 reacted with trimethylsilyldiazomethane affording trans-pyrazoline (4S,5R)-801, whereas in the case of oxazolidinone 320 the $(4 R, 5 S)-\mathbf{8 0 2}$ pyrazoline was obtained (Scheme 307). The formation of pyrazoline (4S,5R)-801 can be explained through an approach of trimethylsilyldiazomethane to the less hindered face of oxazolidinone $\mathbf{2 9 8}$, the top face in model 298A. On the other hand, the 4,4-dimethyloxazolidinone 320 adopts a different coordination in model 320A. Thus, the approach of trimethylsilyldiazomethane is through the opposite side giving product $(4 R, 5 S)-\mathbf{8 0 2}$.

Scheme 307. Enantiodivergent 1,3-DC of Trimethylsilyldiazomethane with $N$ Crotonoyl 2-oxazolidinones 298 Catalyzed by $\mathrm{Mg}\left(\mathrm{ClO}_{4}\right)_{2} /(R, R)-\mathrm{DBFOX}-\mathrm{Ph} 316$

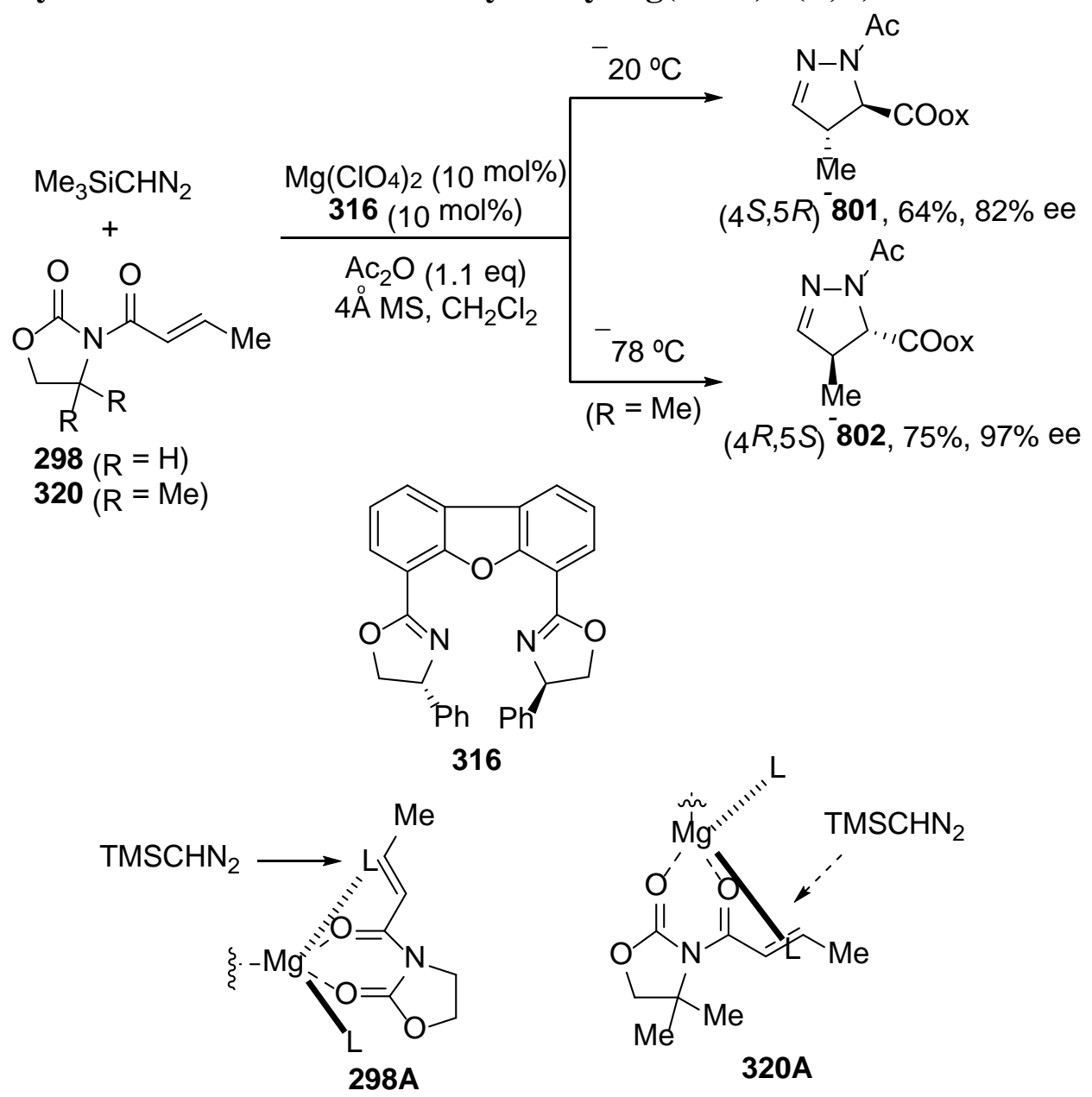

The diastereodivergent 1,3-DC of diazoalkanes with (S)-3-(4-tolylsulfinyl)furan2(5H)-one 803a and its 4-methyl derivative $\mathbf{8 0 3} \mathbf{b}$ was obtained in the absence or in the 
presence of Lewis acids. ${ }^{682,683}$ Reactions performed in $\mathrm{THF}$ at $-40{ }^{\circ} \mathrm{C}$ provided pyrazolines $\mathbf{8 0 4}$ in high de, whereas in the presence of 1 eq of $\mathrm{Yb}(\mathrm{OTf})_{3}$, pyrazolines 805 were formed (Scheme 308). The facial selectivity depends on the involved rotamers 803A or 803B in the absence or presence of the Lewis acid, respectively. The exoselectivity could be rationalized by the intermediacy of transition state exo-A, which is lower in energy than endo-A. Denitrogenation has been performed under $\mathrm{Yb}(\mathrm{OTf})_{3}$ catalysis yielding the corresponding cyclopropanes.

Scheme 308. Diastereodivergent 1,3-DC of Diazoalkanes with $\alpha$-Sulfinyl Butenolides 803 in the Absence or Presence of $\mathrm{Yb}(\mathrm{OTf})_{3}$

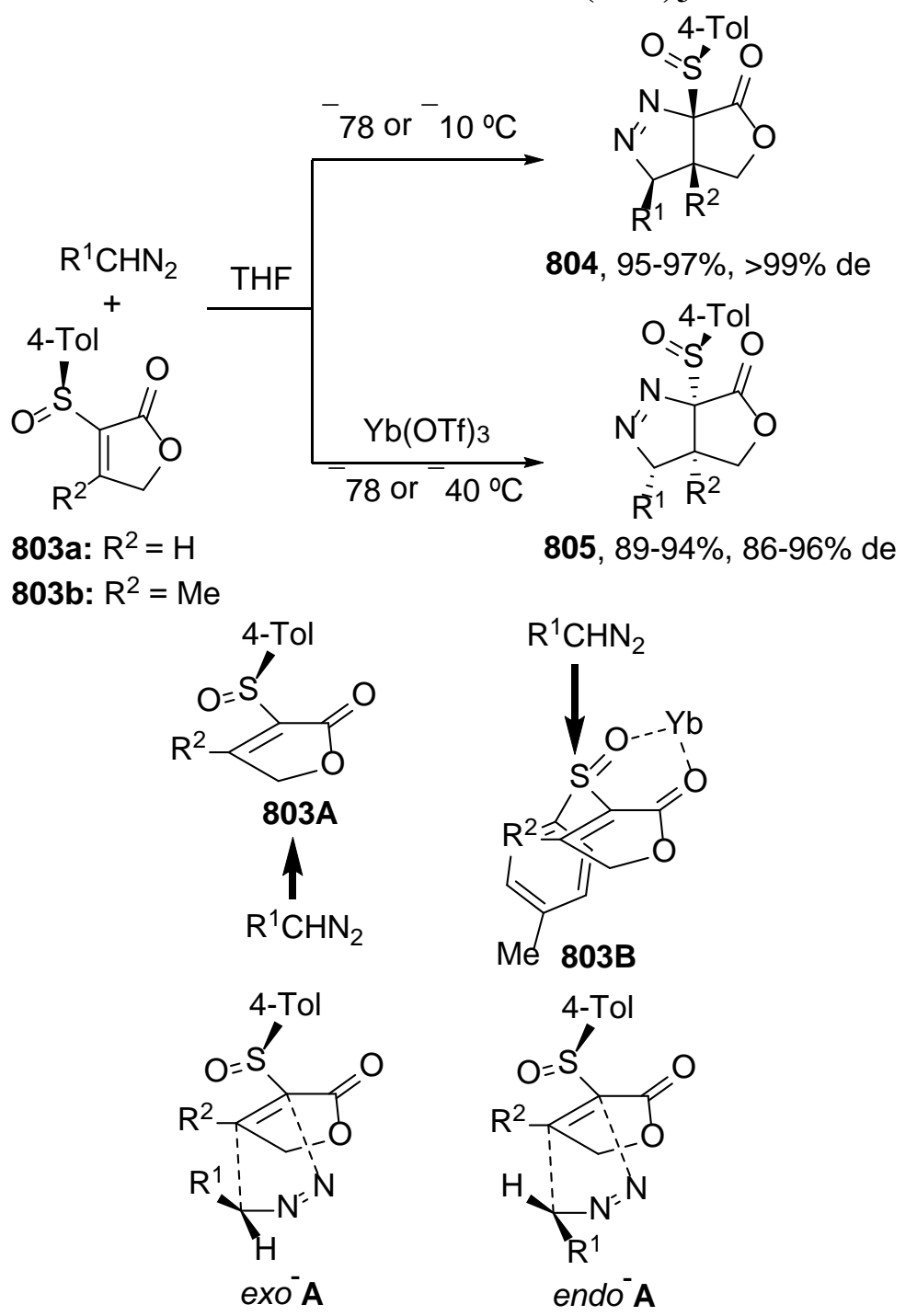

5.2.4. Other [3+2] Cycloadditions. Palladium-catalyzed [3+2] cycloaddition of trimethylenemethane (TMM) dipole equivalents allowed the synthesis of cyclopentanes. Complexes Pd-TMM, generated from 3-acetoxy-2-trimethylsilylmethyl-1-propene, reacted with electron-deficient alkenes giving exo-methylene cyclopentanes. ${ }^{684}$ The diastereodivergent [3+2] cycloaddition has been described by Trost and co-workers using the cyano-substituted TMM precursor 806 and 3-alkylidene-2-oxindoles $\mathbf{8 0 0}$ catalyzed by Pd and different phosphoramidites 807 (Scheme 309). ${ }^{685}$ In the case of 
ligand $\mathbf{8 0 7 a}$, products cis-808 were diastereo- and enantioselectivity obtained, while ligand $\mathbf{8 0 7 b}$ afforded compounds trans-809. This reversal of diastereoselectivity depends on the naphthyl substituents in the pyrrolidine unit. These diastereodivergent results have been explained by the bulky 1-naphthyl substituent of $\mathbf{8 0 7}$, which is preferentially oriented to the oxindole part close to the binaphthol unit. In the case of the 2-naphthyl substituent in ligand $\mathbf{8 0 7 a}$ the orientation of the oxindole should be with the oxindole far apart from the binaphthyl group.

Scheme 309. Diastereodivergent 1,3-DC of the Trimethylenemethane Precursor 806 with 3-Alkylidene-2-oxindoles 800 Catalyzed by Chiral Pd(0)/Phosphoramidites 807

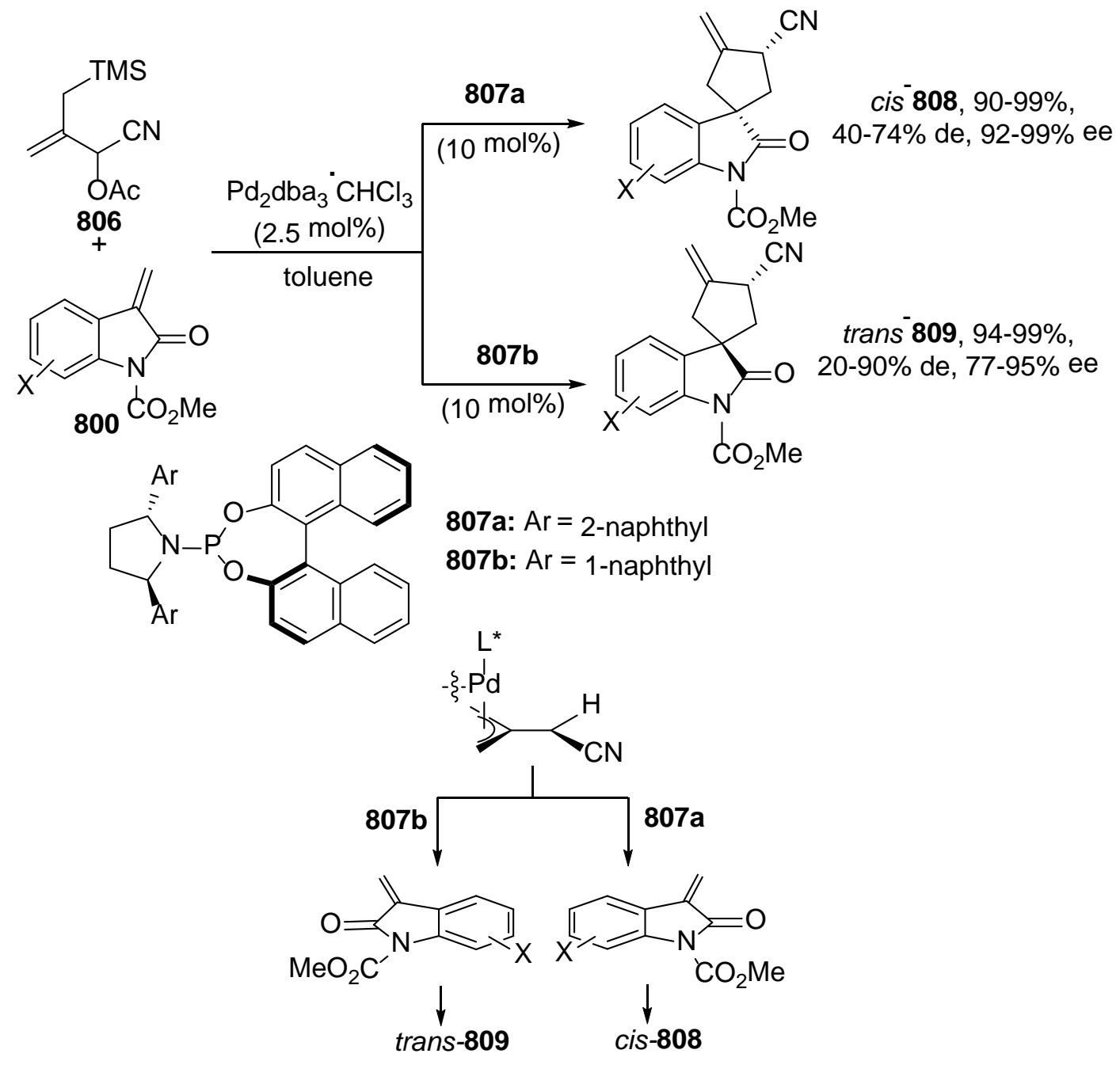

Recently, it has been described that the vinylaziridine-derived Pd-stabilized 1,3dipole reacted diastereodivergently with differently substituted 3-nitroindoles $\mathbf{8 1 1}{ }^{686}$ The reaction of $\mathrm{N}$-tosyl-2-vinylaziridine (810) in the presence of a catalytic amount of $\mathrm{Pd}_{2} \mathrm{dba}_{3} \cdot \mathrm{CHCl}_{3}$ /bathophenanthroline (BPHEN) afforded trans-adducts 812 (Scheme 310). Surprisingly, when the 3-nitroindole $\mathbf{8 1 1}$ has a substituent at the 4-position the corresponding pyrroloindolines cis-813 were formed. This reversal of 
diastereoselectivity was explained by the reversible nucleophilic attack of metallodipole $\mathbf{8 1 0}$ ' at C2 position of the nitroindole giving transition states $\mathbf{A}$ and $\mathbf{B}$ favored by the unsubstituted and 4-substituted indol unit, respectively.

Scheme 310. Diastereodivergent 1,3-DC of $N$-Tosyl-2-vinylaziridine 810 with Differently Substituted 3-Nitroindoles 811 Catalyzed by $\mathrm{Pd}_{2} \mathrm{dba}_{3} \cdot \mathrm{CHCl}_{3} / \mathrm{BPHEN}$

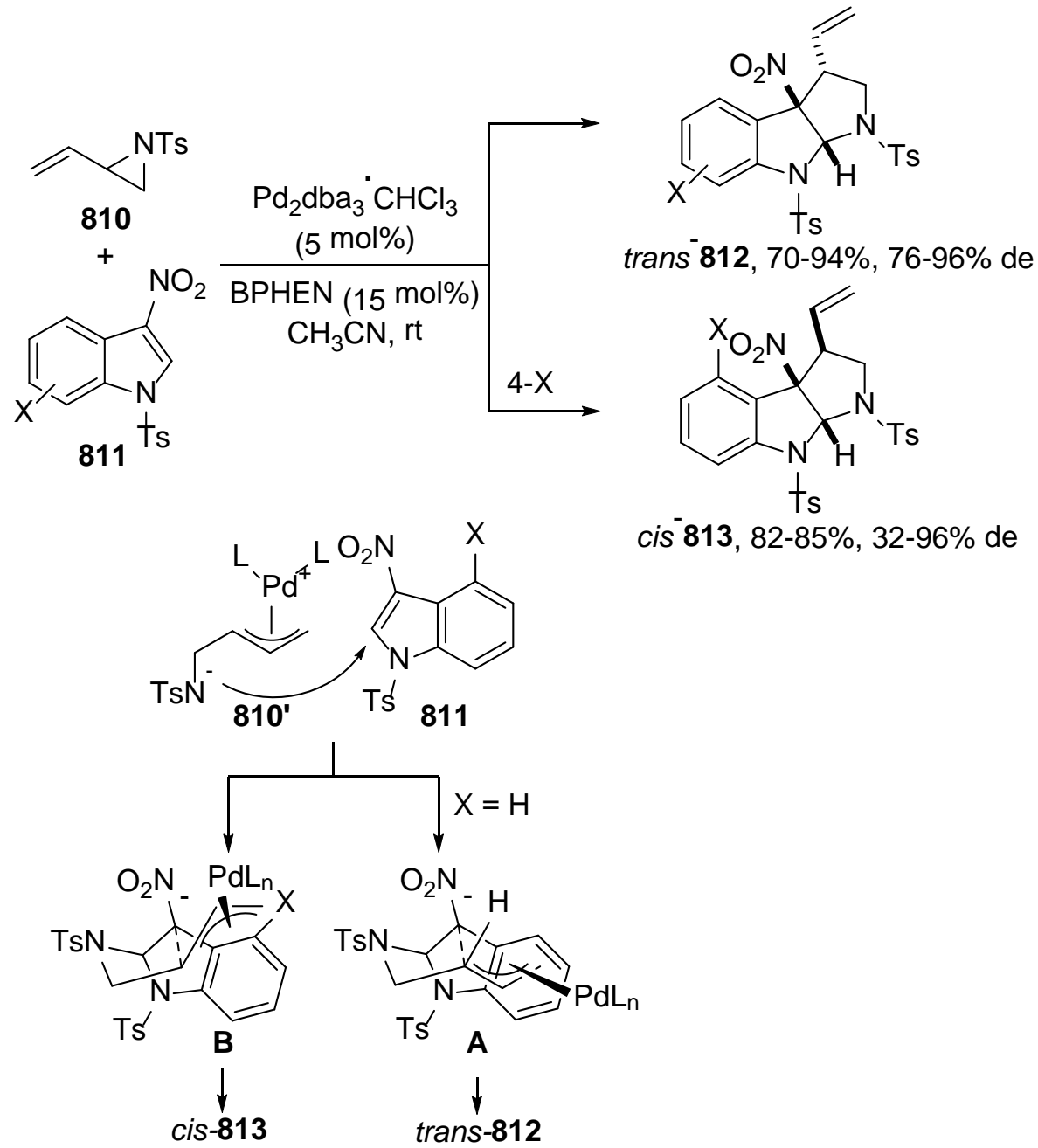

Recently, a diastereodivergent [3+2] cycloaddition between chiral $\mathrm{N}$-phosphonyl imines $\mathbf{8 1 4}$ and methyl isocyanoacetate in the absence and in the presence of AgF has been reported. ${ }^{687}$ When the reaction was carried out with $\mathrm{Cs}_{2} \mathrm{CO}_{3}$ as base, (4R,5S)imidazolines $\mathbf{8 1 5}$ were obtained, in general in excellent de (Scheme 311). On the other hand, in the presence of 5 mol\% of AgF, (4S,5R)-imidazolines 816 were mainly formed with de up to $92 \%$. The proposed mechanism postulates the participation of Newman projection transition state $\mathbf{8 1 5 A}$, which is attacked by the deprotonated isocyanoacetate from the $\mathrm{Si}$ face. In the case of the Ag-catalyzed cycloaddition a six-membered transition state 816A was proposed.

Scheme 311. Diastereodivergent [3+2] Cycloaddition of $N$-Phosphonoyl Imines 814 with Methyl Isocyanoacetate in the Absence and in the Presence of AgF 


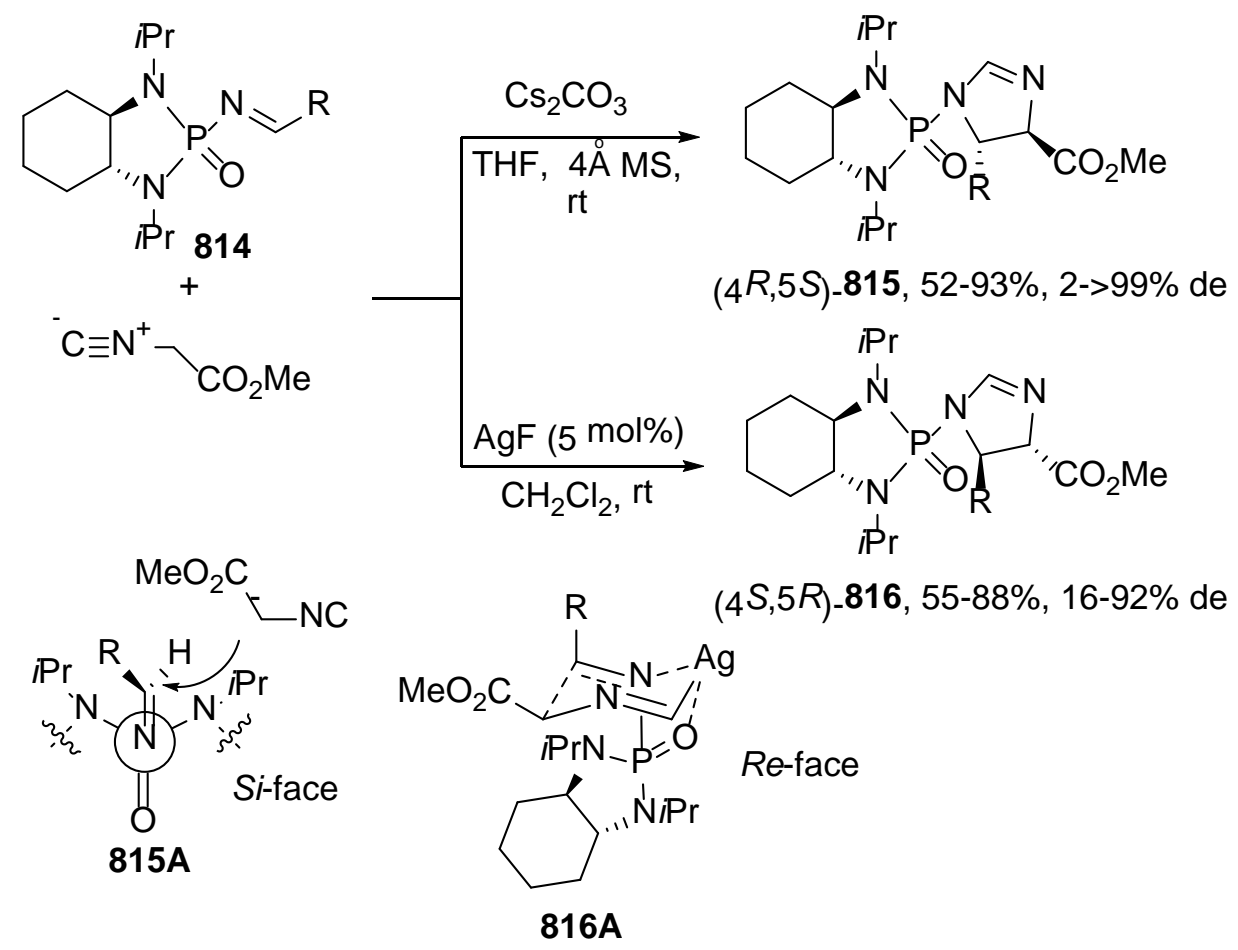

\section{3. [2+2] Cycloadditions}

The development of the Staudinger thermal [2+2] cycloadditon between ketenes and imines for the synthesis of $\beta$-lactams was based on their importance as biologically active compounds. ${ }^{688-690}$ From a mechanistic point of view this thermal cycloaddition takes place through a stepwise mechanism, which explains the diastereodivergent formation of cis- or trans- $\beta$-lactams depending on the substituents and reaction conditions. ${ }^{691-693}$ Organocatalyzed [2+2] cycloadditon between ketenes and imines was firstly described by Lectka and co-workers in 2000. ${ }^{694,695}$ Diastereodivergent results were found depending on the organocatalysts and the substrate structures. Enantioenriched cis- $\beta$-lactams $\mathbf{8 1 9}$ were produced when the in situ generated ketenes, from acyl chlorides, were allowed to react with $\mathrm{N}$-tosyl ethyl glyoxylate imine using $\mathrm{O}$ benzoylquinine (817) as catalyst. However, racemic trans- $\beta$-lactams were obtained using an anionic nucleophilic catalyst 818 (Scheme 312). ${ }^{696}$ The difference between the neutral and the charged catalyst is based on the presence of the anionic sulfonate unit. The diastereoselectivity was explained by the formation of the thermodynamically most stable $(\mathrm{Z})$-O-enolate under neutral conditions, whereas the $(E)$-O-enolate were the most favored under anionic catalysis due to the bulky ammonium cation closed to the negative charge of the oxygen atom affording the trans- $\beta$-lactam. The enantioselectivity of the first process has been explained by the participation of the most stable zwitterionic intermediate A. Thus, the imine approaches from the $R e$ face of the enolate.

Scheme 312. Diastereodivergent Formal [2+2] Cycloaddition of Ketenes with Imines Organocatalyzed by Chiral and Achiral Organocatalysts 


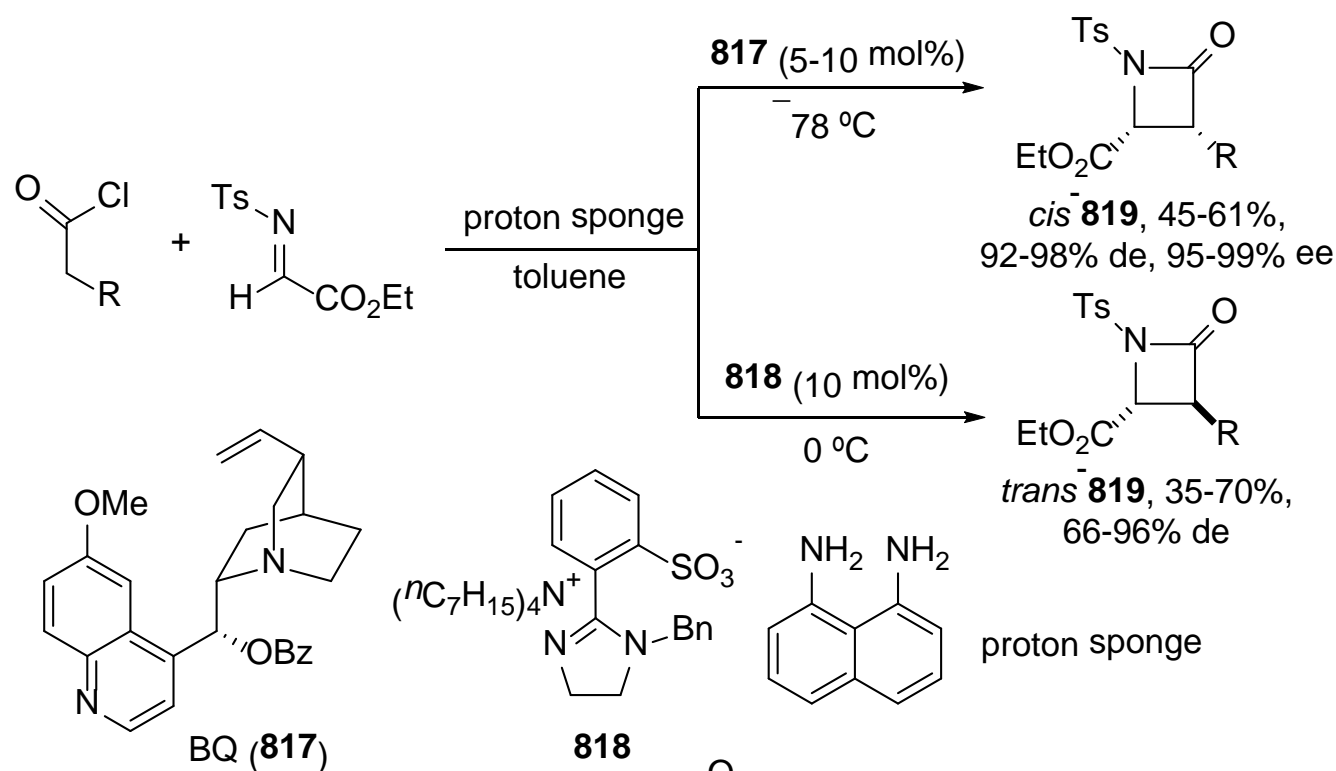

neutral $\mathrm{Nu}$<smiles>[R]/C=C/C=C/C=C/C=O</smiles>

(Z)-O-enolate favored

charged Nu
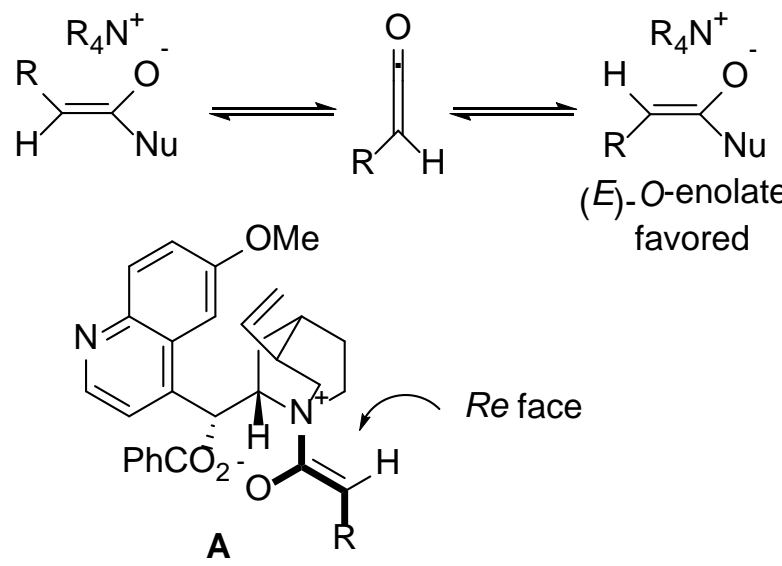

(E)-O-enolate favored

Fu and co-workers described the asymmetric synthesis of $\alpha, \alpha$-disubstituted cis- $\beta$ lactams 821 using 4-(pyrrolidino)pyridine $\mathbf{8 2 0}$ as chiral catalyst. ${ }^{697}$ During the optimization studies, they found out that changing the $N$-protecting group of the aldimines from tosyl to triflyl a diastereodivergent cyclization took place giving mainly trans- $\beta$-lactams 822 (Scheme 313). ${ }^{698}$ The authors proposed that the two classes of imines couple with ketenes by different mechanisms. Thus, $N$-tosyl imines reacted with the (Z)-O-enolate similar to the one described in Scheme 313 by Lectka. However, $N$ triflyl imines reacted with the chiral base $\mathbf{8 2 0}$ forming adduct $\mathbf{8 2 0 A}$, which is the intermediate that reacted with the ketene affording $\mathbf{8 2 0 B}$, providing lactams trans-822.

Scheme 313. Diastereodivergent Asymmetric Formal [2+2] Cycloaddition of Ketenes with Different $N$-Substituted Imines Organocatalyzed by Amine 820 


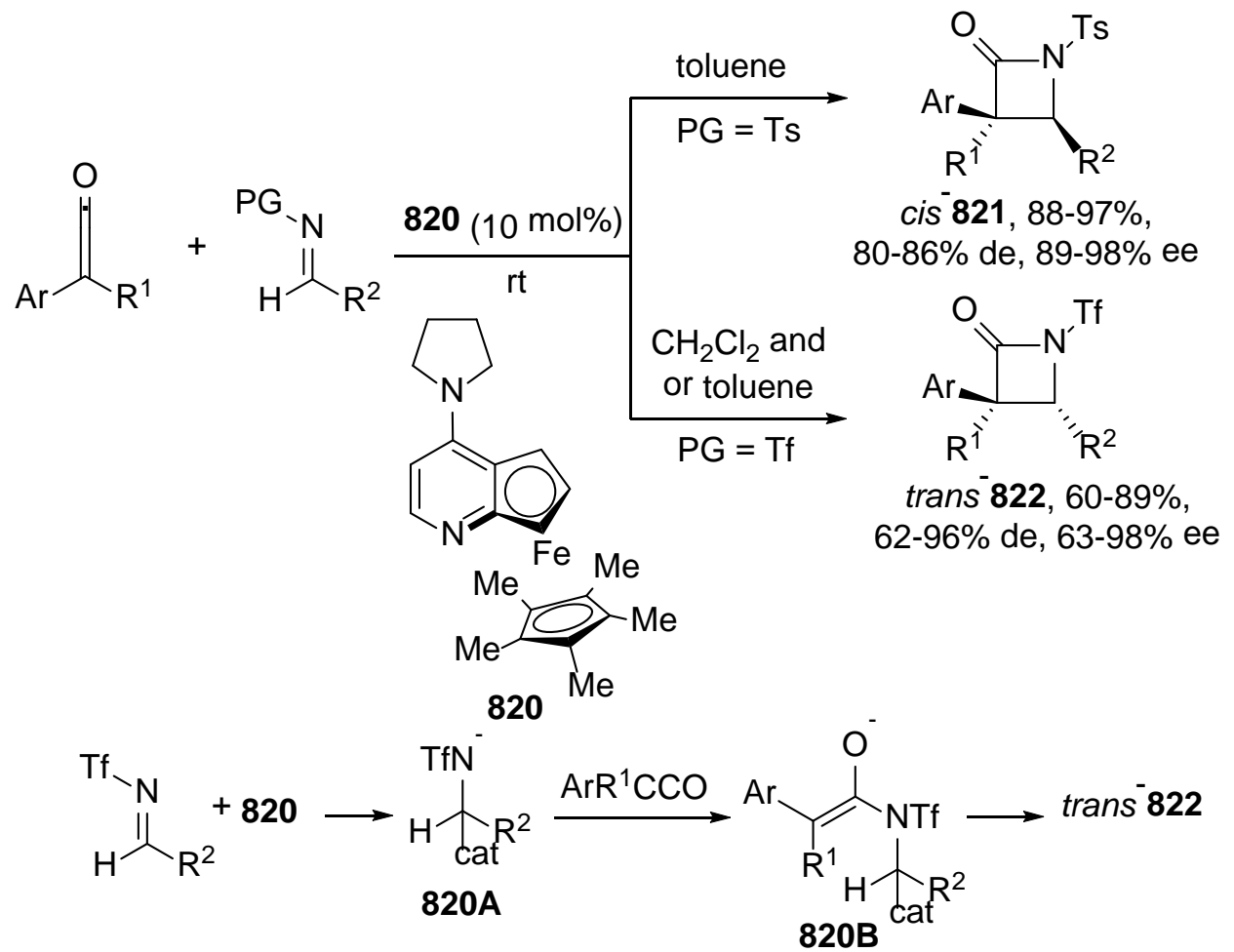

When acyl chlorides were allowed to react with a tertiary amine such as DIPEA, the corresponding ketene are generated in situ and using $O$-silylated quinidine derivative 654 as organocatalysts, the reaction with an aldehyde in the presence of a Lewis acid afforded $\beta$-lactones (Scheme 314). ${ }^{699}$ Diastereodivergent results were obtained depending on the substitution of the acyl chloride. $\alpha$-Alkoxyacetyl chlorides afforded cis-823, whereas aliphatic acid chlorides predominantly gave lactones trans-824, in good de and high ee. Because the intermediate ketenes are different starting materials, this methodology is not a proper diastereodivergent transformation, but it has been considered because it presents just a change in the structure of one of the substrates. Compounds cis-823or trans-824 were formed in high ee by means of a cooperative catalysis using $\operatorname{Er}(\mathrm{OTf})_{3}$ or $\mathrm{Sc}(\mathrm{OTf})_{3}$ as Lewis acids, respectively. The cisstereochemistry can be rationalized considering the formation of an acyl ammonium A, which through a closed TS 823A will form products cis-823. The formation of the acyl ammonium intermediate $\mathbf{B}$ will give an antiperiplanar open TS 824B, which minimizes non-bonded interactions, affording lactones trans-824.

Scheme 314. Diastereodivergent Asymmetric Formal [2+2] Cycloaddition of Different Ketenes with Aldehydes Catalyzed by Amine 654 and Lewis Acids 


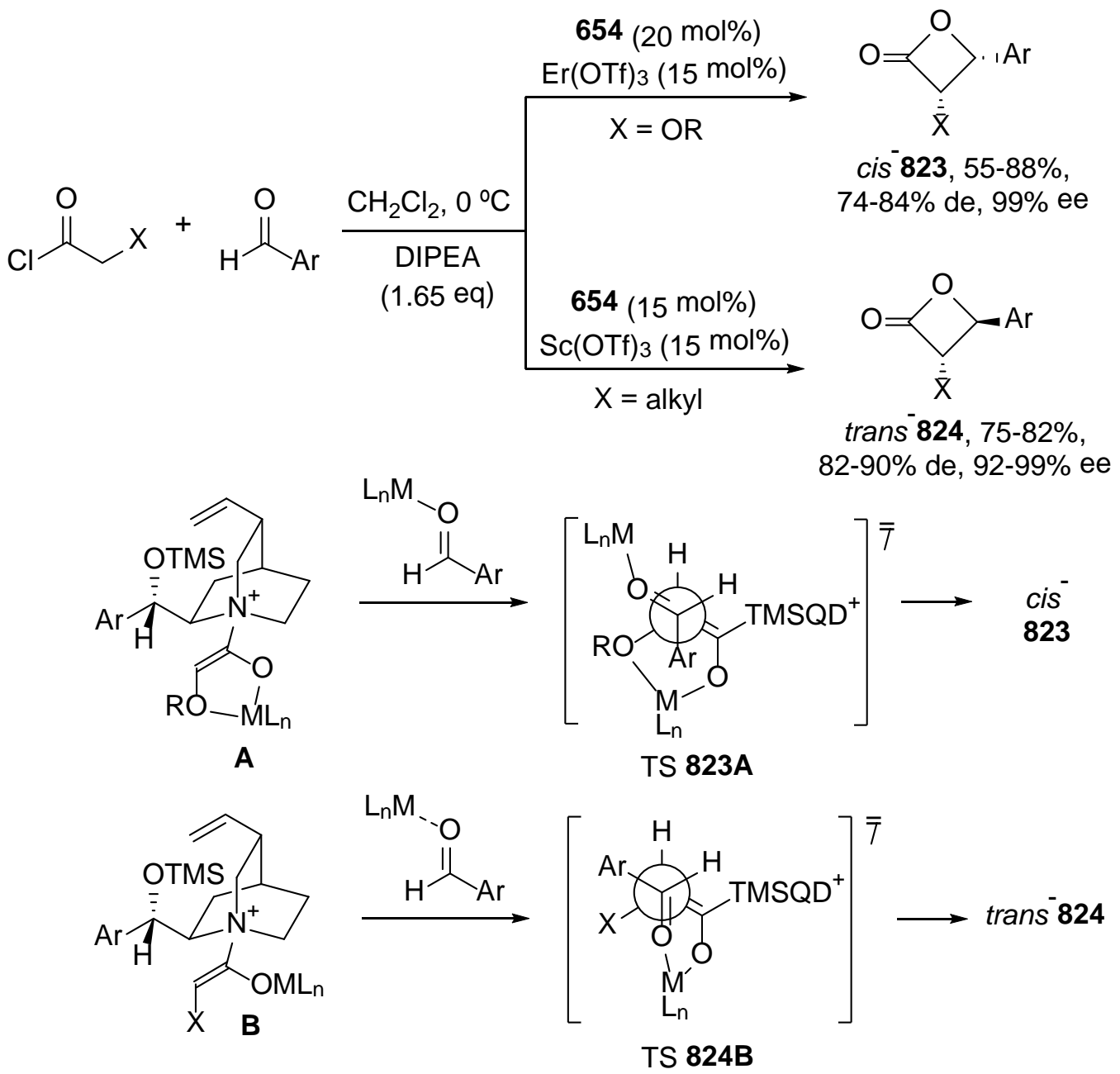

Lewis or Brønsted acids-catalyzed [2+2] cycloadditions of silyl enol ethers and $\alpha, \beta$ unsaturated esters lead to the formation of cyclobutanes. ${ }^{700-703}$ This cycloaddition takes place through a stepwise mechanism, first Michael addition followed by an intramolecular aldol reaction. Takasu and co-workers have found a temperaturedependent [2+2] cycloaddition of the estrone-derived silyl enol ether 825 with hexafluoroisopropyl (HFIP) acrylate catalyzed by $\mathrm{EtAlCl}_{2}{ }^{704}$ When the reaction was carried out at $-78{ }^{\circ} \mathrm{C}$ the trans-cyclobutane 826 was mainly resulted, while at $-40{ }^{\circ} \mathrm{C}$ or rt the product cis-827 was obtained (Scheme 315). From the crossover experiments the reversed diastereoselection has been explained by the kinetic or thermodynamic control. Thus, after Michael addition of $\mathbf{8 2 5}$ to the acrylate from the less hindered $\alpha$-face, the zwitterionic intermediate 825A gave at low temperature, through the corresponding TS 826A, the precursor of trans-826. However, at higher temperature TS 827A was formed giving the most stable product cis-827. This methodology allowed a diastereodivergent access to several stereoidal derivatives.

Scheme 315. Diastereodivergent Asymmetric Formal [2+2] Cycloaddition of Estrone-Derived Silyl Enol Ether 825 with Hexafluoroisopropyl Acrylate Catalyzed by EtAlCl 2 at Different Temperatures 


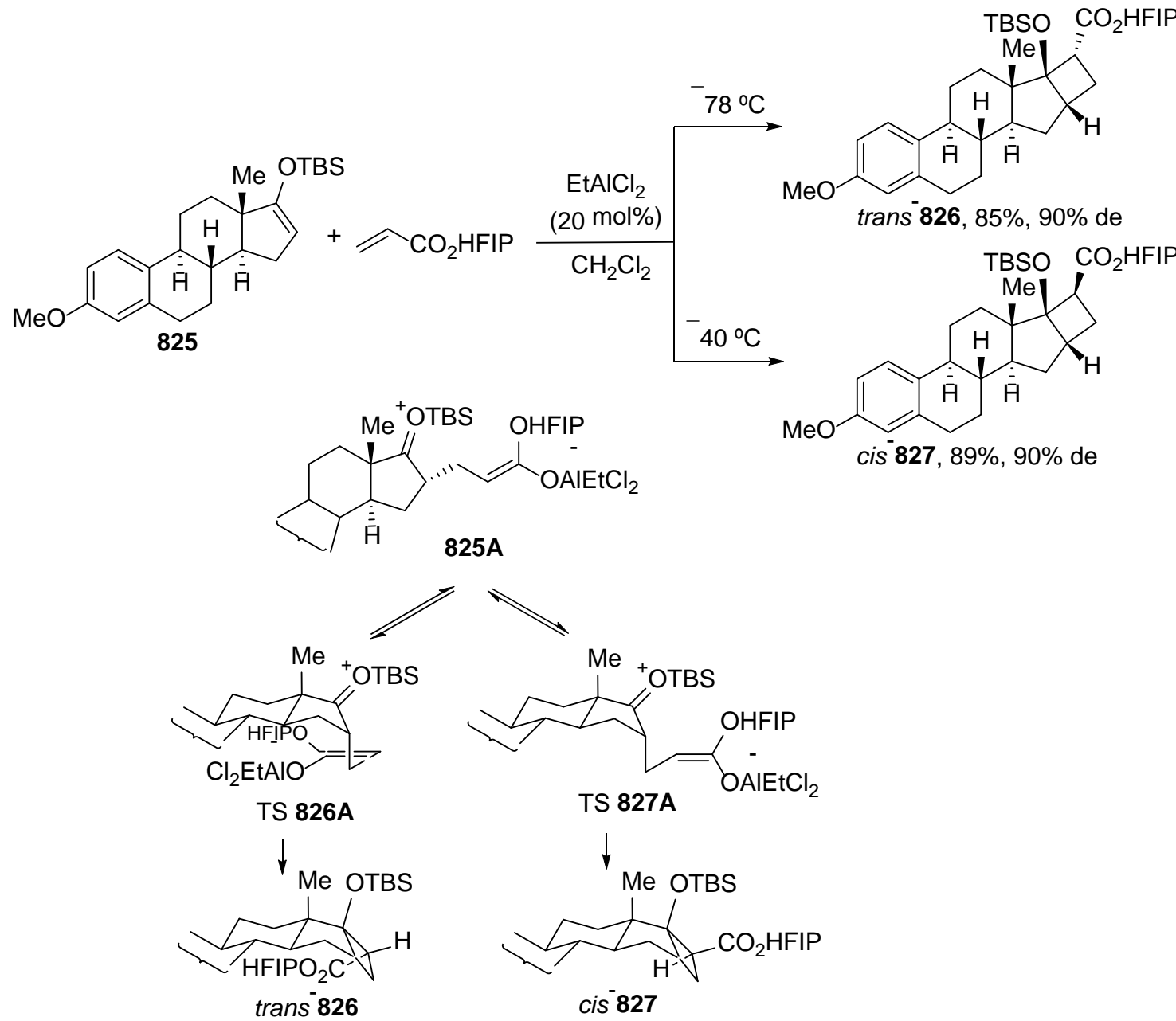

In conclusion, the diastereodivergent [2+2] cycloadditon of ketenes with imines to give the corresponding $\beta$-lactams (Staudinger reaction) can be controlled either by the organocatalyst or with the different protecting groups at the imine. In the case of the synthesis of $\beta$-lactones via the cycloaddition of ketenes with aldehydes, the Lewis acid controls the diastereodivergence. A temperature-dependent diastereodivergent [2+2] cycloaddition of a silyl enol ether and an acrylate has been found to give estronederived cyclobutanes.

\subsection{Other Intermolecular Cyclizations}

In this Section, the stereodivergent intermolecular cyclizations catalyzed by Lewis acids or bases to provide five-membered rings will be considered. Several organocatalyzed cascade reactions initiated by a Michael reaction have been found to promote the stereodivergent formation of six-membered rings.

5.4.1. Five-Membered Rings. Roush and co-workers have applied a [3+2] annulation of aldehydes with allylsilanes to the synthesis of bis-THF units present in annonaceous acetogenin natural products isolated from Annonaceae species. ${ }^{705-708}$ The diastereodivergent [3+2] annulation giving the tetrahydrofuran unit can be controlled 
either under $\mathrm{BF}_{3} \cdot \mathrm{OEt}_{2}$ or $\mathrm{SnCl}_{4}$ catalysis by nonchelate or chelate modes, respectively. For instance, using the allylsilane $\mathbf{8 2 8}$ and $\alpha$-benzyloxyacetaldehyde, the corresponding tetrahydrofurans $\mathbf{8 2 9}$ and $\mathbf{8 3 0}$ were prepared in two steps after further deprotection with TBAF (Scheme 316). ${ }^{708}$ The nonchelate and chelate models 829A and 830A have been proposed to rationalize the formation of $\mathbf{8 2 9}$ and $\mathbf{8 3 0}$, respectively. These compounds were further transformed into aldehydes and used again in a subsequent [3+2] annulation for the diastereodivergent synthesis of bis-THF fragments, and applied to the convergent synthesis of 10-hydroxytrilobacin and three more diastereomers.

Scheme 316. Diastereodivergent [3+2] Annulation of Allylsilane 828 with 2Benzyloxyacetaldehyde Catalyzed by Different Lewis Acids

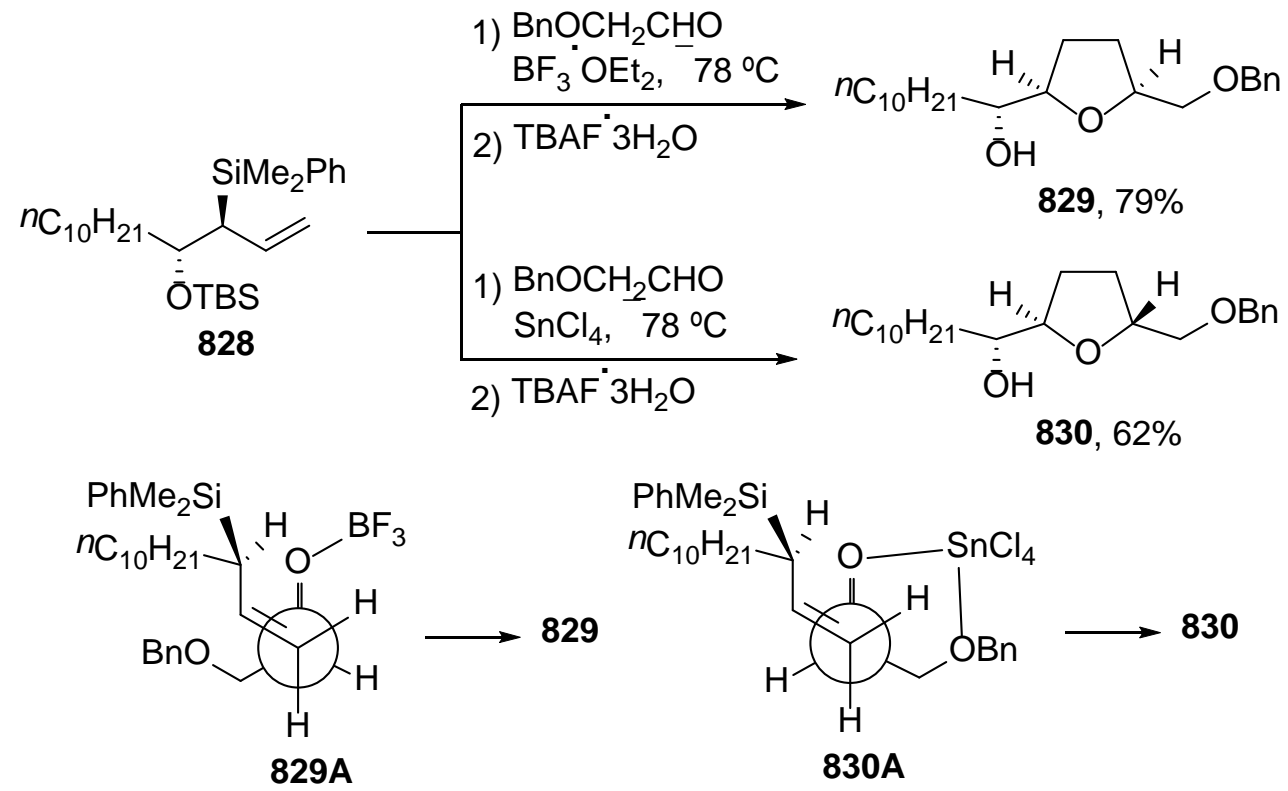

Diastereodivergent prenylations of indole and tryptophan derivatives have been achieved by Ir catalysis. ${ }^{709}$ The reaction of protected indoles 831 with Boc-protected 1,1-dimethylallyl alcohol by means of the Ir/phosphoramidite (R)-475 (Scheme 172) complex took place regioselectively giving the branched isomer 832 (Scheme 317). In this case, the stereoselectivity was controlled by means of different achiral borane additives. For instance, tryptophan derivative $\mathbf{8 3 1}$ gave endo-832 using the bulky 9$\mathrm{BBN}$-octyl, whereas in the presence of triphenylborane the exo-832 was formed preferentially after Fmoc-deprotection. The fused indole-prolinate exo-832 was employed in the total synthesis of amauromicine and its natural diastereomer epiamauromicine. On the other hand, endo-832 was transformed into novoamauromicine.

Scheme 317. Diastereodivergent Ir-Catalyzed Prenylation of Triptophan 831 Using Chiral Phosphoramidite $(R)-475$ as Ligand and Different Boranes as Additives 


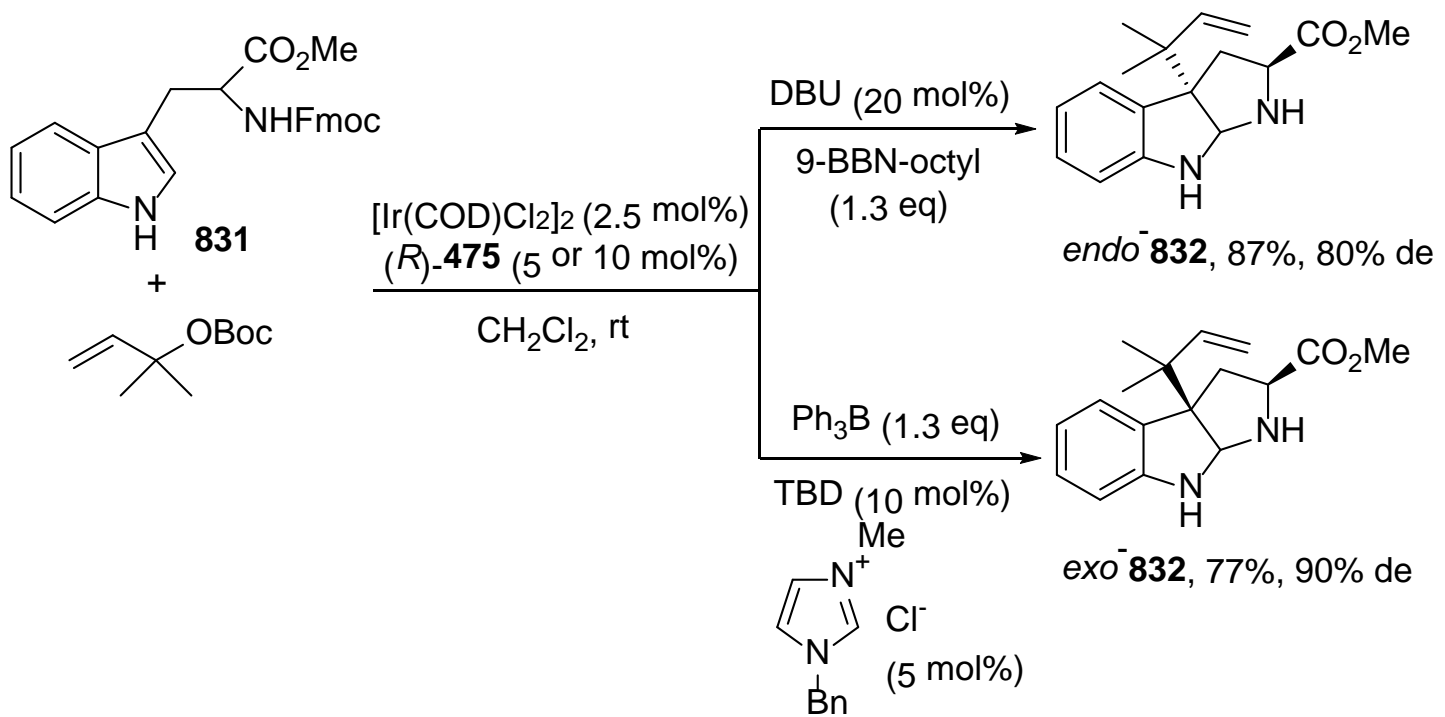

The enantiodivergent [3+2] annulation of ketimines with alkynes catalyzed by a cationic Ir/BINAP complex can be controlled by the absence or presence of catalytic amounts of benzoic acid. ${ }^{710}$ Starting from 3-hydroxy-3-arylisoindolin-1-ones (833), which generate in situ the $N$-acyl ketimine by dehydration, the corresponding (+)-834 spiroaminoindenes were formed (Scheme 318). However, in the presence of benzoic acid (10 mol\%) the enantiomers (-)-833 resulted with ee up to $87 \%$. This switch of enantioselectivity was attributed to a different reaction mechanism under both reaction conditions. The absolute configuration of these products has not been assigned.

Scheme 318. Enantiodivergent [3+2] Annulation of Ketimine Precursors 833 with Alkynes Catalyzed by Ir/BINAP in the Absence or Presence of Benzoic Acid

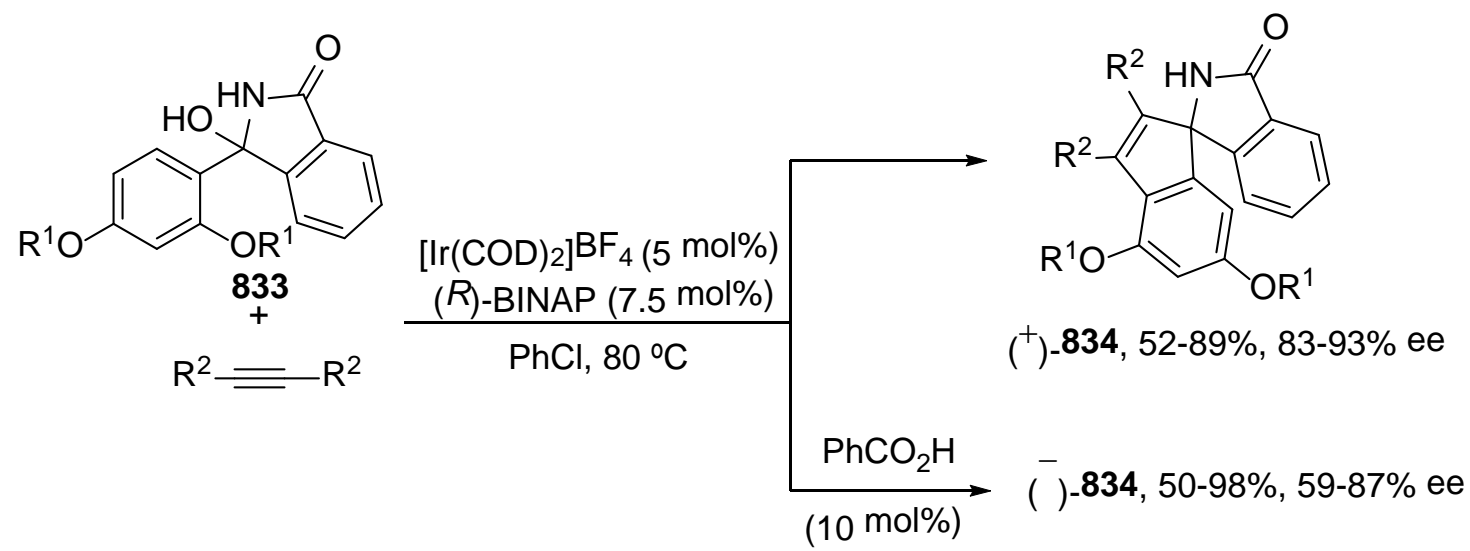

Asymmetric [4+1] annulation of vinylallenes 835 with carbon monoxide to provide 2-alkylidene-3-cyclopentenones $\mathbf{8 3 6}$ has been developed by Murakami, Itami, and Ito. ${ }^{710}$ Enantiodivergent metal-dependent results occurred with $\mathrm{Rh}$ and Pt catalysts using $(R, R)$-Me or Et-DUPHOS as chiral ligands. Thus, using cationic $\left[\mathrm{Rh}(\mathrm{COD})_{2}\right] \mathrm{PF}_{6}$ complex products $(S)$-836 were isolated in moderate ee, whereas $\operatorname{Pt}(\mathrm{COD})_{2}$ gave the enantiomeric products $(R)-\mathbf{8 3 6}$ with ee up to $79 \%$ (Scheme 319 ). For the Rh- and Pt- 
catalyzed cycloadditions the intermediates $\mathbf{A}$ and $\mathbf{B}$ were proposed, respectively, to explain the enantiofacial selectivity.

Scheme 319. Enantiodivergent [4+1] Annulation of Vinylallenes with CO Catalyzed by $\mathrm{Rh}(\mathrm{I}) /$ or Pt(0)/(R,R)-Me-DUPHOS Complexes

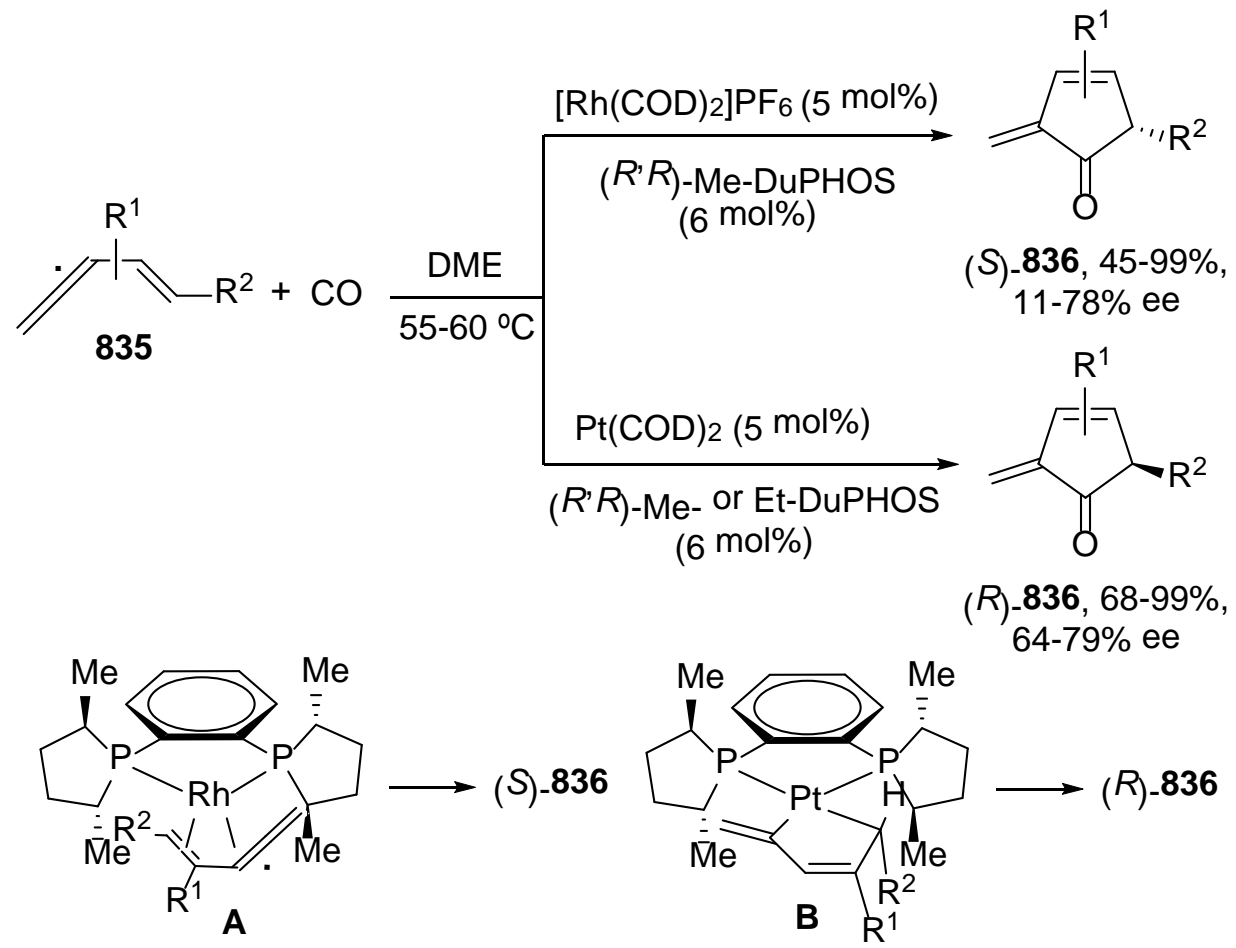

Morita-Baylis-Hillman (MBH) carbonates derived from isatins 837 reacted with 2alkylidene- $1 H$-indene-1,3(2H)-diones 838 affording products 841 or 842 depending on different chiral organocatalysts used (Scheme 320). ${ }^{712}$ When this asymmetric diastereodivergent [3+2] annulation was performed with the bifunctional phosphine 839, the corresponding dispirocyclopentenones $\mathbf{8 4 1}$ were formed in high ee. On the other hand, by using a chiral DMAP-type organocatalysts 840, diastereomers 842 were selectively obtained. The DFT calculations attributed the preferential formation of products $\mathbf{8 4 1}$ or $\mathbf{8 4 2}$ to the higher steric hindrance of the phosphine $\mathbf{8 3 9}$ compared to the amine 840.

Scheme 320. Diastereodivergent [3+2] Annulation of Isatin-Derived MBH-Adducts 837 with Alkylidenediones 838 Organocatalyzed by Phosphine 839 and Amine 840 


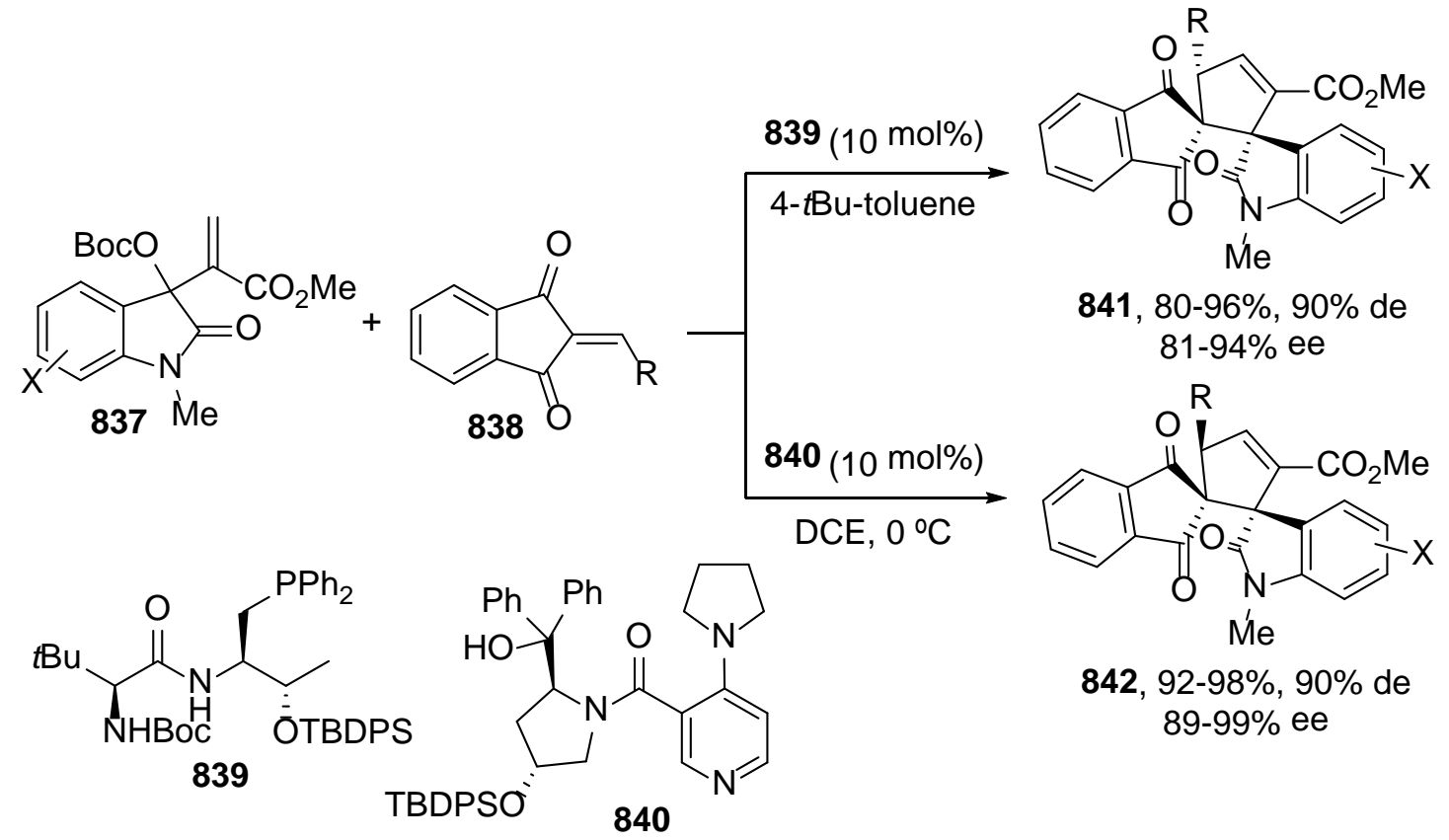

It can be concluded that the formation of 5-membered rings by the sterodivergent intermolecular cyclizations significantly depends on the catalyst structures and in some particular case, on the additives

5.4.2. Six-Membered Rings. Several tandem organocatalyzed asymmetric cyclizations starting by a Michael reaction will be firstly considered. One-pot diastereodivergent construction of the quinolizidine carbon skeleton has been achieved by reaction of $\alpha, \beta$-unsaturated aldehyde 843 with $\beta$-ketoamides 844 catalyzed by $O$ TMS-protected diphenyl prolinol $479(\mathrm{Ar}=\mathrm{Ph})$ (Scheme 321). ${ }^{713,714}$ Under thermodynamic conditions, compound $\alpha \mathbf{- 8 4 7}$ was formed in excellent de and ee, while under kinetic control diasteromer $\beta$-847 was isolated in moderate de and $95 \%$ ee. The proposed mechanisms for these transformations involved the conjugate addition of the iminium cation formed by the aldehyde 843 and the prolinol 479 to give the intermediate lactol $\mathbf{8 4 5}$ after intramolecular cyclization. After reaction of $\mathbf{8 4 5}$ with acetyl chloride at $-20^{\circ} \mathrm{C}$, the $N$-acyliminium 846 can be formed, which after cyclization with the indol unit gave product $\alpha$-847. Intermediate 845 can react with benzoyl chloride at $-78{ }^{\circ} \mathrm{C}$ giving $\beta$-847. The proposed TS $\mathbf{8 4 6 A}$ and $\mathbf{8 4 6 B}$ for the thermodynamically (Re-face addition) and kinetically (Si-face addition) favored last cyclizations will afford products $\alpha-\mathbf{8 4 7}$ and $\beta-847$, respectively.

Scheme 321. Diastereodivergent Asymmetric Michael Addition of Aldehyde 843 with $\beta$-Keto Amides 844 Organocatalyzed by $O$-Silylated Diphenylprolinol 479 Followed by Cyclization at Different Temperatures 


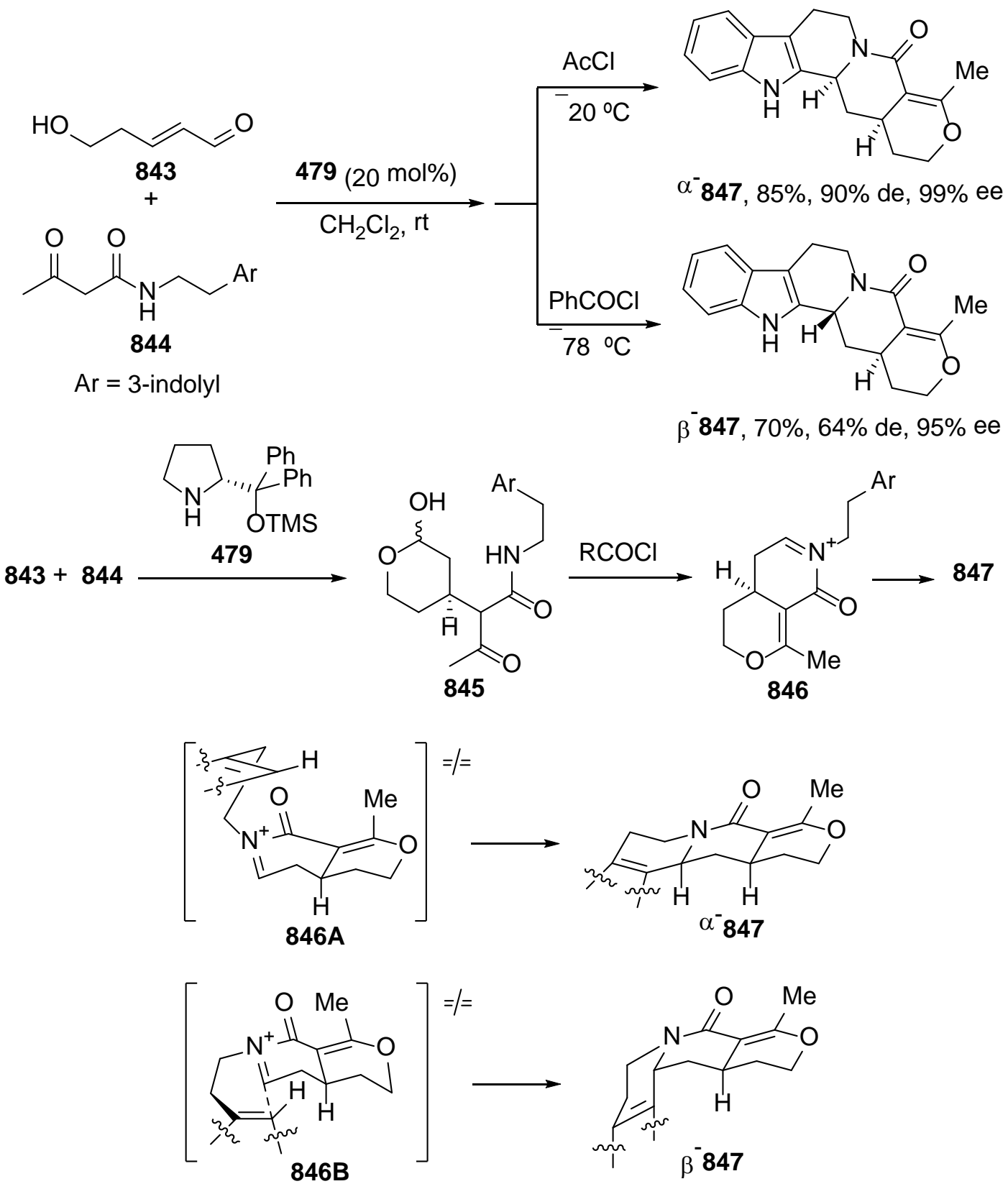

This methodology has been applied to the total synthesis of corynantheine and Ipecac alkaloids such as (-)-dihydrocorynantheol, (-)-hirsutinol, (-)-corynantheol, (-)protoemetinol, (-)-dihydrocorynantheal, (-)-corynantheal, (-)-protoemetine, (-)-(15S)hydroxydihydrocorynantheol, and several non-natural epimers.

A tandem diastereodivergent Michael-Michael asymmetric reaction of aldehydes 848 with $\beta$-nitroalkenes organocatalyzed by a quinidine thiourea 347 (Scheme 126) and L- or D-Pro gave cyclohexanes $\mathbf{8 4 9}$ or $\mathbf{8 5 0}$, respectively (Scheme 322). ${ }^{715}$ Similarly, the quinine thiourea $\mathbf{8 5 1}$ and D- or L-Pro afforded the corresponding enantiomers ent-849 and ent-850, respectively. This methodology has been used for the enantioselective synthesis of (-)- $\alpha$ - and $\beta$-lycoranes. 
Scheme 322. Diastereodivergent Asymmetric Michael-Michael Cyclization of Aldehyde 848 with $\beta$-Nitroalkenes Organocatalyzed by Quinidine Thiourea 347 and L- or D-Pro

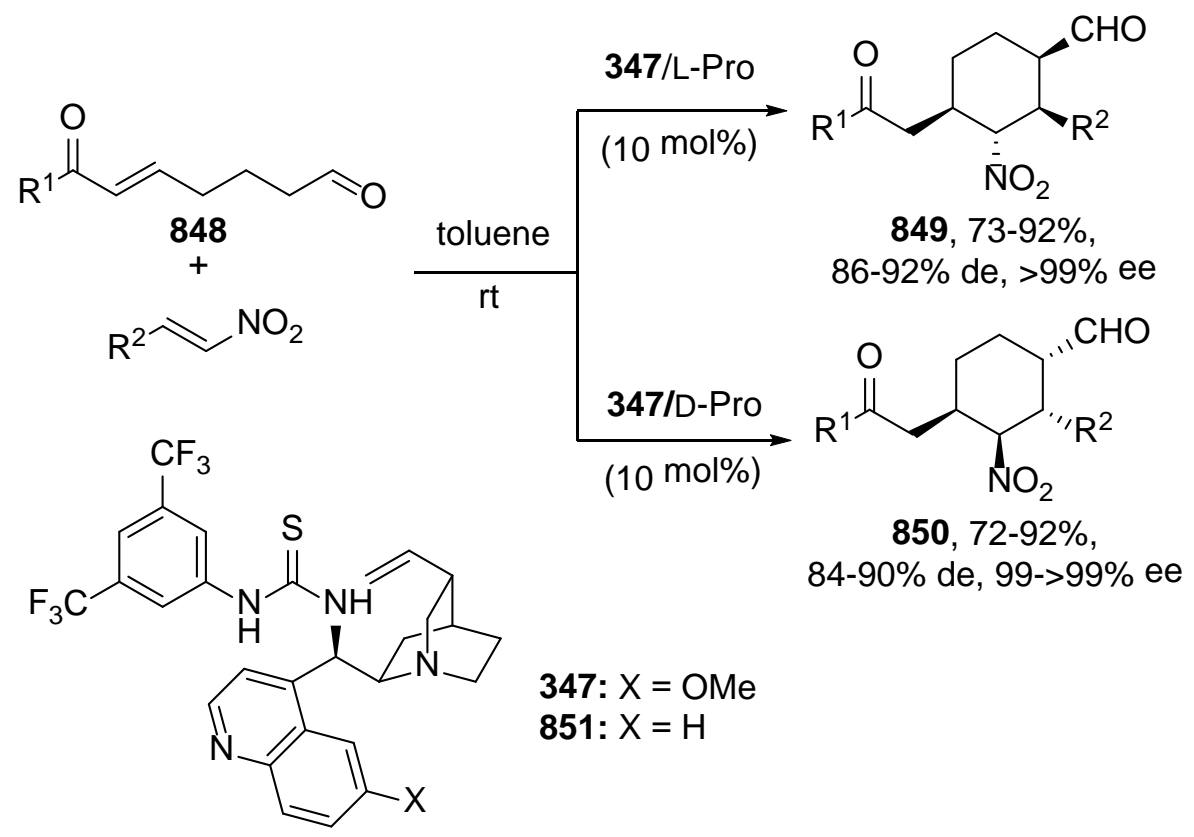

The asymmetric diastereodivergent Michael-Henry tandem reactions of $\alpha$-nitro- $\delta$ keto esters 852 with $\beta$-nitroalkenes, catalyzed either by cinchonidine-derived $\mathbf{8 5 3}$ or by trans-1,2-cyclohexanediamine-derived 367 (Scheme 136) squaramides, lead to the formation of functionalized cyclohexanes $\mathbf{8 5 4}$ and $\mathbf{8 5 5}$, respectively (Scheme 323). ${ }^{716}$ These enantioselective reactions organocatalyzed by the bifunctional cinchonidinederived $\mathbf{8 5 3}$ or by $\mathbf{3 6 7}$ took place with moderate to high de and ee. In order to explain the diastereoselectivity in the Michael addition, an example using methyl $\alpha$ nitropropionate as nucleophile was chosen. In this case, the diastereoselectivity of this first step has been attributed to the two different intermediates 853A and 367A of the organocatalysts with methyl $\alpha$-nitropropionate through hydrogen bonding based on the DFT calculations, giving products 856 and 857, respectively.

Scheme 323. DiastereodivergentAsymmetric Michael-Henry Cyclization of $\alpha$ Nitro- $\delta$-keto Esters 852 with $\beta$-Nitroalkenes Organocatalyzed by Squaramides 853 and 367 


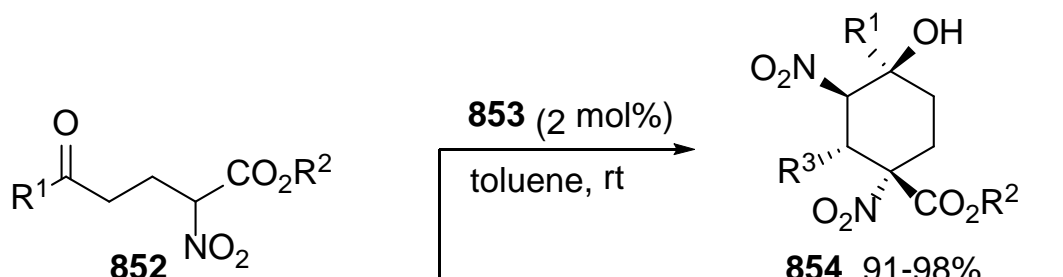

854, 91-98\%,

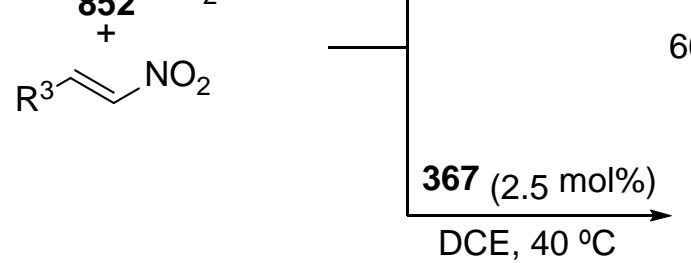

$66-90 \%$ de, $96->99 \%$ ee<smiles>C=CC1CC2CCN1C2[C@H](Nc1c(NCc2cc(C(F)(F)F)cc(C(F)(F)F)c2)c(=O)c1=O)c1ccnc2ccccc12</smiles><smiles>[R]C1[C@H]([N+](=O)[O-])[C@@]([R])(O)CC[C@]1([R])[N+](=O)[O-]</smiles>

855, 77-94\%,

$38-90 \%$ de, $75-86 \%$ ee

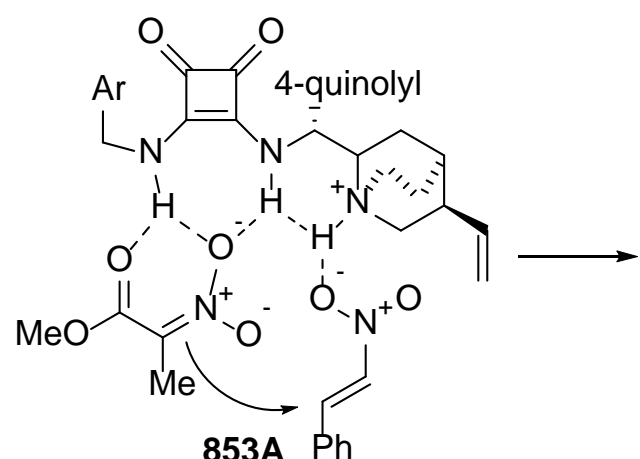

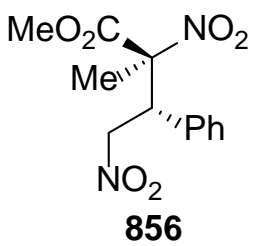

853A Ph

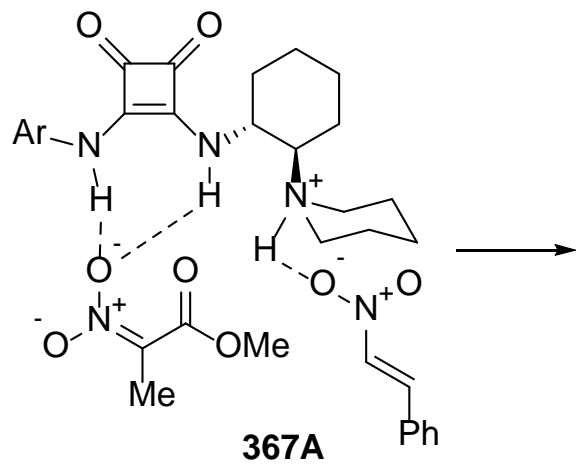<smiles>COC(=O)[C@H]([C@H](CNC(=O)O)c1ccccc1)[N+](=O)[O-]</smiles>

Another example of diastereodivergent cascade reactions, by changing the order of addition of the reagents and catalysts, allowed the synthesis of fully substituted tetrahydropyrans. Hayashi and co-workers performed a tandem reaction in which a Michael addition of aldehydes to nitroalkenes catalyzed by $O$-silylated diphenylprolinol 479 followed by intramolecular Henry reaction with ethyl glyoxylate and further acetalization provided products 858 (Scheme 324). ${ }^{717}$ However, Peng and co-workers carried out firstly a MBH reaction, between the $\beta$-nitroalkene and ethyl glyoxylate 
catalyzed by an achiral tertiary amine, followed by a Michael addition of the aldehyde catalyzed by $O$-silylated diphenylprolinol 479 and the final intramolecular acetalization providing diastereomeric products 859. ${ }^{718}$ In addition, the MBH alcohols $(S)$-860 were kinetically resolved and isolated in high ee.

Scheme 324. Diastereodivergent Asymmetric Cascade Reactions of $\boldsymbol{\beta}$-Nitroalkenes, Aldehydes, and Ethyl Glyoxylate Organocatalyzed by Tertiary Amines and $O$ Silylated Diphenylprolinol 479

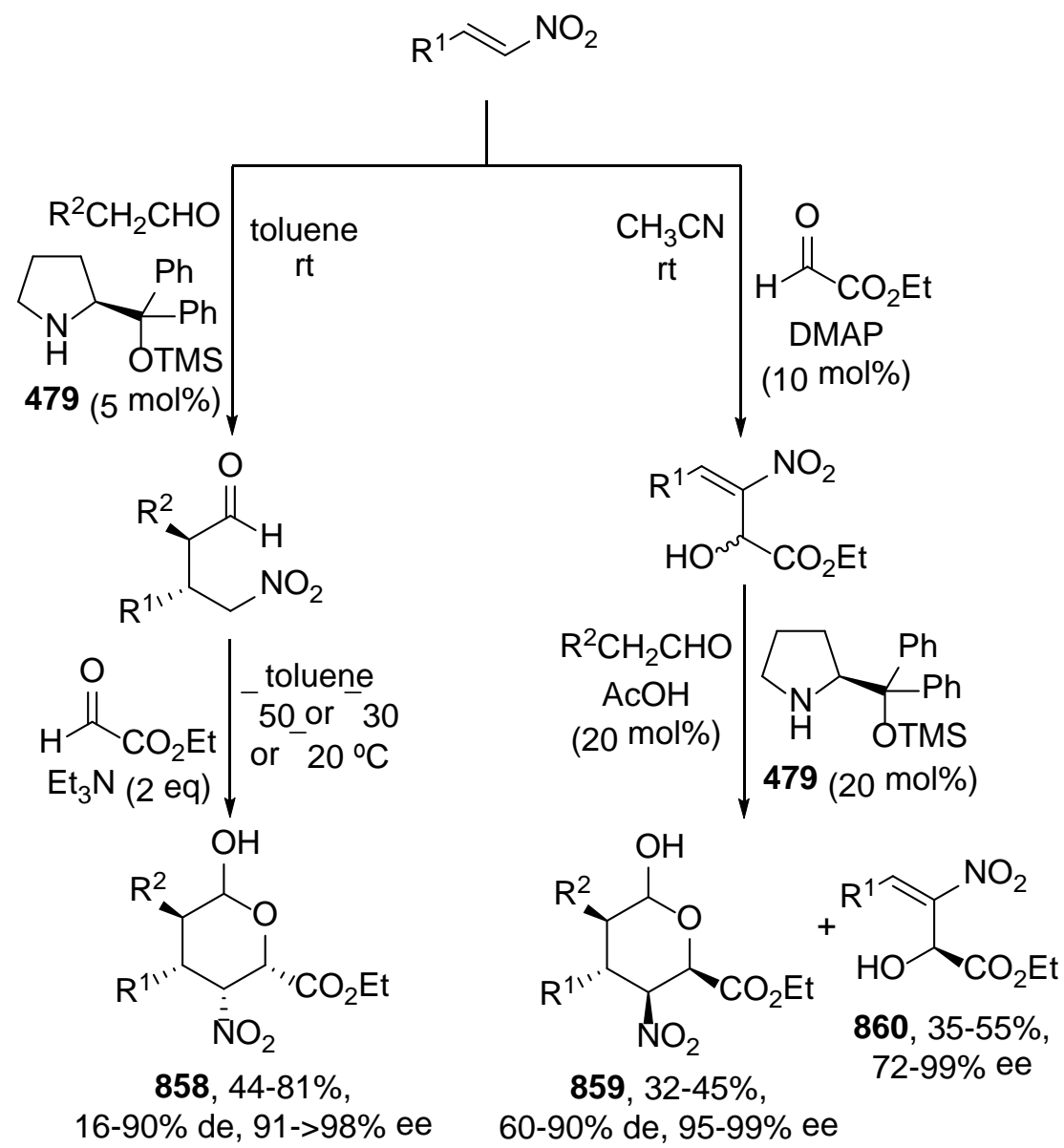

Enantiodivergent Biginelli reaction of aldehydes, thiourea, and $\beta$-keto esters gave the corresponding dihydropyrimidinethiones 861 depending on the chiral phosphoric acid used as organocatalyst. ${ }^{719}$ For example, 4-nitrobenzaldehyde, thiourea, and ethyl 3oxobutanoate gave $(R)$-861 when 3,3'-diphenyl-H8-binaphthol-derived phosphoric acid 64 (Scheme 18) was used. However, the 3,3'-bis(triphenylsilyl) derivative 276 (Scheme 100 ) afforded the enantiomer (S)-861 (Scheme 325). The proposed models 861A and 861B are supported by the DFT calculations. This methodology has been applied to the synthesis of chiral thioureas, dihydropyimidines, guanidines, and the precursor of (S)-L771688, a selective $\alpha_{1 \mathrm{a}}$ receptor antagonist.

Scheme 325. Enantiodivergent Biginelli Reaction of 4-Nitrobenzaldehyde, Thiourea, and Ethyl 3-Oxobutanoate Organocatalyzed by Different Phosphoric Acids 


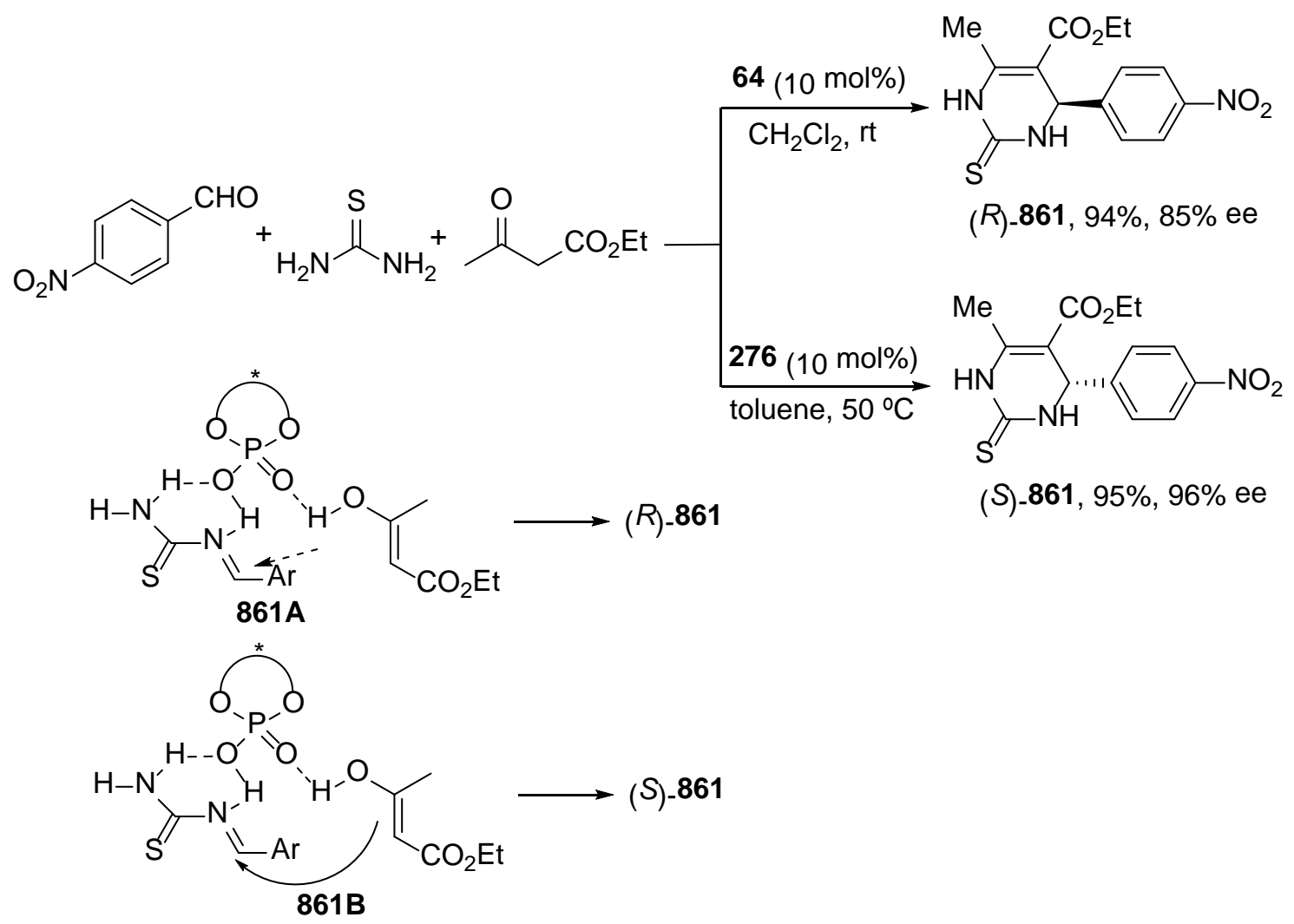

A temperature-dependent enantiodivergent [3+3] annulation of 4-hydroxycoumarins 862 with $\mathrm{MBH}$ acetates 863 using $\mathrm{Pd}(\mathrm{OAc})_{2} /(S)$-tol-BINAP as catalyst has been recently discovered (Scheme 326). ${ }^{720}$ When the reaction was carried out at $10{ }^{\circ} \mathrm{C}$, products (-)-864 were obtained in variable yields with ee up to $99 \%$, whereas at $60{ }^{\circ} \mathrm{C}$ the corresponding enantiomers (+)-864 were obtained in ee up to $99 \%$. The absolute configuration of these products was not assigned.

Scheme 326. Enantiodivergent [3+3] Annulation of 4-Hydroxycoumarins 862 with MBH Adducts 863 Catalyzed by $\operatorname{Pd}(\mathrm{OAc})_{2} /(S)$-tol-BINAP at Different Temperatures<smiles>[X]c1ccc2oc(=O)cc(O)c2c1</smiles>

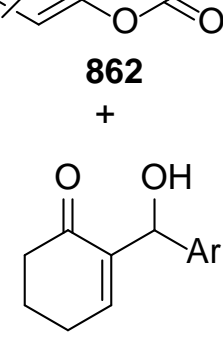

$\mathrm{Pd}(\mathrm{OAc}) 2(5 \mathrm{~mol} \%)$ (S)-tol-BINAP (15 mol\%) $\mathrm{K}_{2} \mathrm{CO}_{3}(2 \mathrm{eq})$, toluene<smiles>[Te]</smiles><smiles>[X]c1ccc2oc(=O)c3c(c2c1)OC1CCCC(=O)C1C3[Al]</smiles>
( )-864, 23-94\%, 51-99\% ee

$60^{\circ} \mathrm{C}$ 
In conclusion, in the different intermolecular diastereodivergent cyclizations giving 5-membered rings catalyzed by Lewis acids or bases, the catalysts and the additives play an important role in the control of diastereoselectivity and enantioselectivity. In the case of the diastereodivergent formation of six-membered rings several organocatalyzed cascade reactions, based mainly on Michael additions are controlled by the catalyst structure, as well as by the temperature and by the order of the addition of the components.

\section{CONCLUSIONS}

Although along this review particular conclusions have been added in every section, some general conclusions can be summarized. Catalytic stereodivergent processes have been described mainly in the last 30 years parallel to the development of enantio- and diastereoselective catalytic reactions. Unexpected inversion of the enantio- and/or diastereoselectivity can be now rationalized due to the deeper understanding of the mechanism of metal- and organocatalyzed transformations. Theoretical calculations have contributed to explain these amazing phenomena, but some rationales should be very important in futur studies in order to overcome serendipity. We can deduce that the synthesis of enantiomeric pairs from the same chiral staring material is not anymore a dream. Small changes in the structure of the organocatalysts and in the ligand of the metal complex can be crucial to achieve an efficient reversal of the stereoselectivity. The use of additives and the modification of the reaction conditions can produce dramatic effects in the switch of the enantio- and diastereoselectivity. Stereodivergent effects, when using metal complexes in homogeneous catalysis, can be modulated not only by the ligand but also by the metal and the counteranion. Cooperative catalysis by means of two organocatalysts or by a metal complex and an organocatalysis is designed as a very efficient strategy for stereodivergent processes. In addition, small changes in the substrates such as different protecting groups also play an important role. Stereodivergent results are very important in acyclic systems and in cyclization reactions as well. The development of designable processes will be crucial for controlling the stereodivergence and we hope that this review will contribute in the future to focuse efforts in this direction. However, many sterodivergent transformations need to be rationalized in order to understand these processes. Several fields such as enantiodivergent biocatalysis using engineered enzymes, photocatalysis and electrocatalysis will contribute to the development of new sterodivergent reactions. During the preparation of our manuscript new examples of stereo- and enantiodivergence appeared, and certainly this will further continue. Many researchers now are still far away from the possibility to predict and control these results. Let we have another dream!

\section{AUTHOR INFORMATION}

\section{Corresponding Author}

*E-mail: cnajera@ua.es 


\section{Biographies}

Irina Beletskaya was born in Leningrad (St-Petersburg). She was graduated from the Department of Chemistry at Lomonosov Moscow State University (MSU), where she started her career. In 1963 she reached the D.Sci. degree and soon after became a Professor. In 1958 she obtained first the Candidate of Chemistry (analogous to Ph.D.) degree (1958), and then the Doctor of Chemistry degree (1963). Since 1971 she served as a Professor of Chemistry in the same the Department. In 1974 Beletskaya was elected a corresponding member of the Academy of Science of USSR, and in 1992 she became a full member (an academician) of the Russian Academy of Science. Since 1989 Beletskaya has been head of the Organoelement Chemistry laboratory at the Department of Chemistry at MSU. Professor Beletskaya is an emeritus academician of the Bashkir National Academy of Science, an emeritus professor of StPetersburg State University, and Cordoba University (Argentina). Among others, she has won the Mendeleev, Lomonosov, Nesmeyanov and Demidov Prizes for creativity and achievements in chemistry. Abroad, she also won the Kapitza Award Fellowship (UK), and Women in the Engineering Science award (Sweden). She was named Doctor Honoris Causa by the Universities of Córdoba (Argentina), Technical of St. Petersburg (Russia), Alicante (Spain) and the Royal Institute of Technology of Stockholm (Sweden). Irina Beletskaya served for many years as an editor-in-chief of the Russian Journal of Organic Chemistry. She has been a member of the editorial boards of a number of leading journals including The Bulletin of Russian Academy of Science (Izvestiya RAN), Mendellev Communications, The Proceedings of Russian Academy of Science (Vestnik RAN), Organometallics, Chemistry-A European Journal, and the Journal of Organometallic Chemistry. For many years, Beletskaya also participated in the activities of the International Union of Pure and Applied Chemistry (IUPAC) serving for 8 years first as a secretary, then a vice-president, and in 1991-1993 as a president of the Division of Organic Chemistry. Until 2001 she was working on the IUPAC Committee on chemical weapons destruction technologies (CWDT). Her publications list contains more than 1000 papers, cited more than 18.000 times and her h-index is 51. She has supervised more than $100 \mathrm{Ph} . \mathrm{D}$. students and has delivered more than 100 lectures abroad. She has interest in general organic chemistry but especially concerning organometallic intermediates and transition metal catalysis, as well as mechanistic aspects of these processes. More recently she has been interested in new areas as the development of new methodologies based on reactions in aqueous media (green chemistry), transition metal nanoparticles, and dendrimers (supramolecular chemistry).

Carmen Nájera was born in Nájera (La Rioja) in 1951 and was graduated from the University of Zaragoza in 1973, obtaining her doctorate in chemistry from the University of Oviedo in 1979. She spent postdoctoral stays at the ETH (Zurich), the Dyson Perrins Laboratory (Oxford), Harvard University, and Uppsala University. She became Associate Professor in 1985 at the University of Oviedo and Full Professor in 1993 at the University of Alicante. She is coauthor of more than 400 papers (h 64), 6 patents and 30 book chapters and has supervised more than $45 \mathrm{PhD}$ students. She has been awarded with the 2006 Organic Chemistry Prize from the Spanish Royal Chemical Society of Chemistry, the 2006 Rosalind Franklin International Lectureship from the English Royal Society, the SCF 2010 French-Spanish Prize from the Société Chimique de France, the IUPAC 2015 Distinguished Women in Chemistry or Chemical Engineering Award and the 2018 Serratosa lectureship. In 2012 she was named full Member of the Royal Spanish Academy of Sciences and was appointed as Active Member of the European Academy of Sciences and Arts. Professor Najera has been in the Advisory Board of several international journals, among others, Tetrahedron, Tetrahedron Letters, Tetrahedron Asymmetry, Synthesis, European Journal of Organic Chemistry, Chemistry Letters and ChemCatChem. Professor Nájera is Manager Director of the chemical company MEDALCHEMY S.L. for the development of APIs.

Miguel Yus was born in Zaragoza (Spain) in 1947, and received his BSc (1969), MSc (1971) and PhD (1973) degrees from the University of Zaragoza. After spending two years as a postdoctoral fellow at the Max Planck Institut für Kohlenforschung in Mülheim a.d. Ruhr he returned to Spain to the University of Oviedo where he became associate professor in 1977, being promoted to full professor in 1987 at the same university. In 1988 he moved to a chair in Organic Chemistry at the University of Alicante. 
Professor Yus has been visiting professor at different institutions and universities among them ETHZentrum, Oxford, Harvard, Uppsala, Marseille, Tucson, Okayama, Paris, Strasbourg, Bolonia, Sassari, Tokyo and Kyoto. He is co-author of more than 600 papers (h 68) and five patents, and has supervised more than 60 Doctoral Theses (already presented), and delivered more than 200 lectures, most of them abroad. Among others, he has received the Spanish-French Prize (1999), twice the Japan Society for the Promotion of Science Prize (Okayama 2000, Kyoto 2007), the Stiefvater Memorial Lectureship Award (Lincoln 2001), the Nagase Science and Technology Foundation fellowship (Kyoto 2003), the Cellchem Lectureship (Sheffield 2005), the Singenta Lectureship (Basel 2007), the Fundeun-Iberdrola Prize (Alicante 2007), the Serratosa Lectureship (Barcelona 2010), the Conferencia Lourenço-Madinaveitia (Lisboa 2012), the Medalla Felix Serratosa from the RSEQ (Madrid 2012), being also named recently Active Academician from the European Academy of Sciences and Arts (Salzburg 2012) and Academic member of the Athens Institute for Education \& Research. Professor Yus has been in the Advisory Board of 30 international journals, among others, Tetrahedron, Tetrahedron Letters, European Journal of Organic Chemistry, Chemistry Letters, The Chemical Record, Current Organic Chemistry, Current Chemical Biology, Jordan Journal of Chemistry, Applied Sciences, and Trends in Organic Chemistry, being also Editor-in-Chief of Letters in Organic Chemistry and Open Chemistry, as well as Regional Editor of The World Journal of Chemistry. Professor Yus founded the new chemical company MEDALCHEMY S.L. for the development of APIs.

\section{ACKNOWLEDGMENTS}

We thank to the Spanish Ministerio de Economia y Competitividad (MINECO) (projects CTQ201343446-P, CTQ2014-53695P and CTQ2014-51912-REDC), the Spanish Ministerio de Economía, Industria y Competitividad, Agencia Estatal de Investigación (AEI) and Fondo Europeo de Desarrollo Regional (FEDER, EU) (projects CTQ2016-76782-P and CTQ2016-81797-REDC), the Generalitat Valenciana (PROMETEO2009/039 and PROMETEOII/ 2014/017) and the University of Alicante for financial support. Dedicated to Professor E. J. Corey on occasion of his $90^{\text {th }}$ birthday

\section{ABBREVIATIONS}

Ad

$\mathrm{ADH}$

ALB

AMPY

Ant

BARF

bipy

BINAP

BINOL

Boc

BOX

BPHEN

BIPOP

BozPHOS adamantly

alcohol dehydrogenase

AlLibis(binaphtoxide)

2-aminomethylpyridine

anthryl

tetrakis[3,5-bis(trifluoromethyl)phenyl]borate

2,2'-bipyridine

1,1'-Binaphthalene-2,2'-diyl)bis(diphenylphosphine

1,1'-bi-2-naphthol

tert-butoxycarbonyl

bis(oxazoline)

bathophenanthroline

6-bis [4- (2-pyridyl)methyl) -1, 3-oxazolin-2-yl]pyridine

1,2-bis[(2S,5S)-2,5-dimethylphospholano]benzene monoxide 


\begin{tabular}{|c|c|}
\hline CD & cinchonidine \\
\hline $\mathrm{CN}$ & cinchonine \\
\hline DA & Diels-Alder \\
\hline DABCO & 1,4-diazabicyclo[2.2.2]octane \\
\hline DBFOX & 4,4'-disubstituted (dibenzofuran-4,6-diyl)-2,2'-bioxazolines \\
\hline $\mathrm{DC}$ & dipolar cycloaddition \\
\hline DCE & 1,2-dichloroethane \\
\hline de & diastereomeric excess \\
\hline DEAD & diethyl diazodicarboxylate \\
\hline DFT & density functional theory \\
\hline DIOP & $\begin{array}{l}\text { 2,3-O-isopropylidene-2,3-dihydroxy-1,4- } \\
\text { bis(diphenylphosphino)butane }\end{array}$ \\
\hline DIPEA & $N, N$-diisopropylethylamine \\
\hline DKP & diketopiperazine \\
\hline DME & 1,2-dimethoxyethane \\
\hline DMSO & dimethyl sulfoxide \\
\hline DPPB & 1,4-bis(diphenylphosphino)butane \\
\hline DPPE & 1,2-bis(diphenylphosphino)ethane \\
\hline DPPF & 1,1'-ferrocenediyl-bis(diphenylphosphine \\
\hline DPP & diphenylphosphinoyl \\
\hline DVDS & 1,3-divinyl-1,1,3,3-tetramethyldisiloxane \\
\hline ee & enantiomeric excess \\
\hline FesulPHOS & 2-(tert-butylthio)-1-(diphenylphosphino)ferrocene \\
\hline HFIP & hexafluoroisopropanol \\
\hline ICN & isocinchonine \\
\hline ICP & isocupreidine \\
\hline IPA & isopropanol \\
\hline JohnPHOS & 2-biphenyl)di-tert-butylphosphine \\
\hline LA & Lewis acid \\
\hline $\mathrm{MBH}$ & Morita-Baylis-Hillman \\
\hline Mes & mesityl \\
\hline MNEA & methyldi(1-naphthylethyl)amine \\
\hline MOF & metal-organic frameworks \\
\hline MOPS & 3-( $N$-morpholino)propanesulfonic acid \\
\hline MTBE & methyl tert-butyl ether \\
\hline MS & molecular sieves \\
\hline Mts & (2,4,6-trimethylphenyl)sulfonyl \\
\hline NFSI & $N$-fluorobenzenesulfonimide \\
\hline NHC & N-heterocyclic carbene \\
\hline NME & $N$-methylephedrine \\
\hline NPs & nanoparticles \\
\hline pin & pinacolate \\
\hline PHMS & polymethylhydrosiloxane \\
\hline PHOX & phosphinooxazolines \\
\hline
\end{tabular}




$\begin{array}{ll}\text { PMP } & \text { p-methoxyphenyl } \\ \text { PS } & \text { polysiloxane } \\ \text { PTC } & \text { phase-transfer catalyst } \\ \text { QD } & \text { quinidine } \\ \text { QN } & \text { quinine } \\ \text { Q-PHOS } & \text { 1,2,3,4,5-pentaphenyl-1'-(di-tert-butylphosphino)ferrocene } \\ \text { RCM } & \text { ring-closing metathesis } \\ \text { Rt } & \text { room temperature } \\ \text { SEGPHOS } & \text { 5,5'-bis(diphenylphosphino)-4,4'-bi-1,3-benzodioxole } \\ \text { TADDOL } & \text { 2,2-dimethyl- } \alpha, \alpha, \alpha \text { ', } \alpha^{\prime} \text {-tetraaryl-1,3-dioxolane-4,5-dimethanol } \\ \text { TBAF } & \text { tetra- } n \text {-butylammonium fluoride } \\ \text { TBD } & \text { 1,5,7-triazabicyclo[4.4.0]dec-5-ene } \\ \text { TCE } & \text { tetrachloroethane } \\ \text { TESA } & \text { triethylmethylsulfinamide } \\ \text { TFE } & \text { trifluoroethanol } \\ \text { THF } & \text { tetrahydrofuran } \\ \text { TMS } & \text { trimetylsilyl } \\ \text { Tol } & p \text {-metylphenyl } \\ \text { TPA } & \text { triphenylacetate } \\ \text { TRIPHOS } & \text { phenylbis(diphenylphosphinoethyl)phosphine } \\ \text { Trs } & \text { 2,4,6-trimethylphenylsulfonyl } \\ \text { Ts } & p \text {-toluenesulfonyl } \\ \text { TS } & \text { transition state }\end{array}$

\section{REFERENCES}

(1) Lin, L.; Feng, X. Catalytic Strategies for Diastereodivergent Synthesis. Chem.-Eur. J. 2017, 23, 6464-6482.

(2) Kim, H. Y.; Oh, K. Stereodivergent Asymmetric Reactions Catalyzed by Brucine Diol. Synlett 2015, 26, 2067-2087.

(3) Sohtome, Y.; Nagasawa, K. Sequential Stereodivergent Organocatalysis and Programmed Organocascades. Org. Biomol. Chem. 2014, 12, 1681-1685.

(4) Escorihuela, J.; Burguete, M. I.; Luis, S.V. New Advances in Dual Stereocontrol for Asymmetric Reactions. Chem. Soc. Rev. 2013, 42, 5595-5617.

(5) Miller, L. C.; Sarpong, R. Divergent Reactions on Racemic Mixtures. Chem. Soc. Rev. 2011, 40, 4550-4562.

(6) Pineschi, M.; Di Bussolo, V.; Crotti, P. Copper-Catalyzed Divergent Kinetic Resolution of Racemic Allylic Substrates. Chirality 2011, 23, 703-710.

(7) Bartók, M. Unexpected Inversions in Asymmetric Reactions: Reactions with Chiral Metal Complexes, Chiral Organocatalysts, and Heterogeneous Chiral Catalysts. Chem. Rev. 2010, 110, 1663-1705.

(8) Flanagan, S. P.; Guiry, P. J. Substituent Electronic Effects in Chiral Ligands for Asymmetric Catalysis. J. Organomet. Chem. 2006, 691, 2125-2154.

(9) Kim, Y. H. Dual Enantioselective Control in Asymmetric Synthesis. Acc. Chem. Res. 2001, 34, 955-962. 
(10) Kagan, H. B. Various Aspects of the Reaction of a Chiral Catalyst or Reagent with a Racemic or Enantiopure Substrate. Tetrahedron 2001, 57, 2449-2468.

(11) Sibi, M. P.; Liu, M. Reversal of Stereochemistry in Enantioselective Transformations. Can they be Planned or are they Just Accidental? Curr. Org. Chem. 2001, 5, 719-755.

(12) Yamada, J.; Abe, H.; Yamamoto, Y. Stereodivergent Synthesis of 1,2-Diol Derivatives via $\alpha$ Alkoxy Organolead Compounds: $\mathrm{S}_{\mathrm{E}}$ 2-Retention Pathway. J. Am. Chem. Soc. 1990, 112, 6118-6120.

(13) Luchetti, G.; Ding, K.; d'Alarcao, M.; Kornienko, A. Enantio- and Diastereodivergent Synthetic Route to Multifarious Cyclitols from D-Xylose via Ring-Closing Metathesis. Synthesis 2008, 3142-3147.

(14) Yamamoto, Y.; Asao, N. Selective Reactions Using Allylic Metals. Chem. Rev. 1993, 93, 2207-2293.

(15) Denmark, S. E.; Fu, J. Catalytic Enantioselective Addition of Allylic Organometallic Reagents to Aldehydes and Ketones. Chem.Rev. 2003, 103, 2763-2793.

(16) Charette, A. B.; Mellon, C.; Rouillard, L.; Malenfant, E. Design, Synthesis, and Applications of New Oxygenated Chiral Auxiliaries. Pure Appl. Chem. 1992, 64, 1925-1931.

(17) Charette, A. B.; Mellon, C.; Motamedi, M. The 2-Benzyloxytetrahydropyranyl Group as Chiral Auxiliary for the Nucleophilic Addition to $\alpha$-Alkoxy Aldehydes. Tetrahedron Lett. 1995, 37, 8561-8564.

(18) Charette, A. B.; Mellon, C.; Rouillard, L.; Malenfant, E. Design of New Chiral Auxiliaries for the Stereoselective Nucleophilic Addition to $\alpha$-Alkoxy Aldehydes. A Remarkable Remote Protecting Group Effect. Synlett 1993, 81-82.

(19) Canielli, G.; Giacomini, D.; Galletti, P.; Orioli, P. Dynamic Solvation Effects in Ethylmagnesium Bromide Addition to (S)-O-(tert-Butyldimethylsilyl) Lactal. Eur. J. Org. Chem. 2001, 4509-4515.

(20) Canielli, G.; Giacomini, D.; Galletti, P.; Quintavalla, A. Solvent and Temperature Effects on Diastereofacial Selectivity: Amines as Co-Solvents in $n$-Butyllithium Addition to $\alpha$-Chiral Aldehydes. Eur. J. Org. Chem. 2003, 1993-2000.

(21) Coleman, R. S.; Carpenter, A. J. Diastereoselective Addition of Vinyl Organometallic Reagents to L-Serinal. Tetrahedron Lett. 1992, 33, 1697-1700.

(22) Murakami, T.; Furusawa, K. Efficient Stereodivergent Synthesis of erythro- and threoSphingoshines: Unprecedent Reversal of the Stereochemistry in the Addition. Tetrahedron 2002, 58, 9257-9263.

(23) Ginesta, X.; Pastó, M.; Pericás, M. A.; Riera, A. New Stereodivergent Approach to 3-Amino2,3,6-trideoxysugars. Enantioselective Synthesis of Daunosamine, Ristosamine, Acosamine and Epidaunosamine. Org. Lett. 2003, 5, 3001-3004.

(24) Tanaka, T.; Hayashi, M. Approach for Complete Reversal of Enantioselectivity Using a Single Chiral Source. Synthesis 2008, 3361-3376.

(25) Soai, K.; Niwa, S. Enantioselective Addition of Organozinc Reagents to Aldehydes. Chem. Rev. 1992, 92, 833-856.

(26) $\mathrm{Pu}, \mathrm{L} . ; \mathrm{Yu}, \mathrm{H} .-\mathrm{B}$. Catalytic Asymmetric Organozinc Additions to Carbonyl Compounds. Chem. Rev. 2001, 101, 757-824.

(27) Pu, L. Asymmetric Alkynylzinc Additions to Aldehydes and Ketones. Tetrahedron 2003, 59, 9873-9886.

(28) Walsh, P. J. Titanium Catalyzed Enantioselective Additions of Alkyl Groups to Aldehydes. Mechanistic Studies and New Concepts in Asymmetric Catalysis. Acc. Chem. Res. 2003, 36, 739-749.

(29) Kimura, K.; Sugiyama, E.; Ishizuka, T.; Kunieda, T. Dramatic Reversal of Enantioselectivity in $\beta$ Aminoalcohol-Catalyzed Addition of Diethylzinc to Aldehydes. Tetrahedron Lett. 1992, 33, 3147-3148.

(30) Sibi, M. P.; Chen, J.; Cook, G. R. Reversal of the Stereochemistry in Diethylzinc Addition to Aldehydes by a Simple Change of the Backbone Substituent in L-Serine Derived Ligands. Tetrahedron Lett. 1999, 40, 3301-3304.

(31) Goldfuss, B.; Steigelmann, M.; Rominger, F. Increasing Selectivities and Reactivities by Stereochemical Tuning: Fenchone-Based Catalysts in Dialkylzinc Additions to Benzaldehyde. Eur. J. Org. Chem. 2000, 1785-1792. 
(32) Cobb, A. J. A.; Marson, C. M. Reversal of Enantioselectivity Using CatalystsContaining Multiple Stereogenic Centers.Tetrahedron: Asymmetry 2001, 12, 1547-1550.

(33) Cobb, A. J. A.; Marson, C. M. Asymmetric Synthesis Using Catalysts Containing Multiple Stereogenic Centers and a trans-1,2-Diaminocyclohexane Core; Reversal of Predominant Enantioselectivity Upon $N$-Alkylation. Tetrahedron 2005, 61, 1269-1279.

(34) Huang, H.; Chen, H.; Hu, X.; Bai, C.; Zheng, Z. Structural Probing of D-Fructose Derived Ligands for Asymmetric Addition of Diethylzinc to Aldehydes. Tetrahedron: Asymmetry 2003, 14, 297-304.

(35) Shi, M.; Satoh, Y.; Makihara, T.; Masaki, Y. Chiral $\mathrm{C}_{2}$-Symmetric 2,5-Disubstituted Pyrrolidine Derivatives as Chiral Catalyst Ligands in the Reaction of Diethylzinc with Arylaldehydes. Tetrahedron: Asymmetry 1995, 6, 2109-2112.

(36) Shi, M.; Satoh, Y.; Masaki, Y. Chiral $\mathrm{C}_{2}$-Symmetric 2,5-Disubstituted Pyrrolidine Derivatives as Catalytic Chiral Ligands in the Reactions of Diethylzinc with Arylaldehydes. J. Chem. Soc., Perkin Trans. 1 1998, 2547-2552.

(37) Alvarez-Ibarra, C.; Collados Lujan, J. F.; Quiroga-Feijóo, M. L. Straightforward Synthesis of [(2S,4R)-1-Cyclohexyl-4-methylpiperidin-2-yl]methanol and [(2S,4R)-1-Cyclohexyl-4-methylpiperidin-2yl](diphenyl)methanol: Novel Chiral Ligands for the Catalytic Addition of Diethylzinc to Benzaldehyde to Give Rise to an Extensive Turn in the Sense of Asymmetric Induction. Tetrahedron: Asymmetry 2010, 21, 2334-2345.

(38) Wang, X.-B.; Kodama, K.; Hirose, T.; Yang, X.-F.; Zhang, G.-Y. Chirality Control in the Enantioselective Arylation of Aromatic Aldehydes Catalyzed by cis-(1R,2S)-2Benzamidocyclohexanecarboxylic Acid Derived 1,3-Aminoalcohols. Tetrahedron: Asymmetry 2010, 21, 75-80.

(39) Hirose, T.; Sugawara, K.; Kodama, K. Switching of Enantioselectivity in the Catalytic Addition of Diethylzinc to Aldehydes by Regioisomeric Chiral 1,3-Amino Sulfonamide Ligands. J. Org. Chem. 2011, 76, 5413-5428.

(40) Yamakawa, M.; Noyori, R. Asymmetric Addition of Dimethylzinc to Benzaldehyde Catalyzed by (2S)-3-exo-(Dimethylamino)isoborneol.A Theoretical Study on the Origin of Enantioselection. Organometallics 1999, 18, 128-133.

(41) Yamakawa, M.; Noyori, R. An Ab Initio Molecular Orbital Study on the Amino Alcohol-Promoted Reaction of Dialkylzincs and Aldehydes. J. Am. Chem. Soc. 1995, 117, 6327-6335.

(42) Kang, S. Y.; Park, Y. S. Discovery of Dipeptide-Derived Catalysts for the Enantioselective Addition of Dimethylzinc to Aldehydes. Eur. J. Org.Chem. 2012, 1703-1706.

(43) Márquez-Sánchez-Carnereo, E. M.; De las Casas Engel, T.; Lora Maroto, B.; Dela Moya Cerero, S. Dual Stereoselection in the Addition of Diethylzinc to Benzaldehyde by Using Highly Structurally Close Ligands. Chirality 2012, 24, 255-261.

(44) Celik, S.; Cakici, M.; Kilic, H.; Sahin, E. Synthesis of Quinazolinone-Based Aziridine Diols as Chiral Ligands: Dual Stereoselectivity in the Asymmetric Ethylation of Aryl Aldehydes. Tetrahedron: Asymmetry 2015, 26, 152-157.

(45) Sasaki, S.; Yamauchi, T.; Kanai, M.; Ishii, A.; Higashiyama, K. Bisoxazoline-Catalyzed Asymmetric Nucleophilic Addition of Diethyl Zinc to Fluorinated Ketones: Enantiofacial Control by Changing the Bisoxazoline Substitutent. Bull. Chem. Soc. Jpn. 2015, 88, 200-208.

(46) Lake, F.; Moberg, C. Ti-Mediated Addition of Diethylzinc with Benzaldehyde. The Effect of Chiral Additives. Tetrahedron: Asymmetry 2001, 12, 755-760.

(47) Mao, J.; Wan, B.; Zhang, Z.; Wang, R.; Wu, F.; Lu, S. Reversal of the Enantioselectivity by Adding $\mathrm{Ti}(\mathrm{OiPr})_{4}$ : Novel Sulfamide-Amine Alcohol Ligands for the Catalytic Asymmetric Addition of Diethylzinc to Aldehydes. J. Mol. Catal. A: Chem. 2005, 225, 33-37.

(48) Dean, M. A.; Hitchcock, S. R. Divergent Enantioselective Pathwaysin the Catalytic Asymmetric Addition of Diethylzinc to Aldehydes in the Presence and Absence of Titanium Tetraisopropoxide. Tetrahedron: Asymmetry 2008, 19, 2563-2567. 
(49) Lutz, F.; Igarashi, T.; Kawasaki, T.; Soai, K. Small Amounts of Achiral $\beta$-Amino Alcohols Reverse the Enantioselectivity of Chiral Catalysts in Cooperative Asymmetric Autocatalysis. J. Am. Chem. Soc. 2005, 127, 12206-12207.

(50) Lutz, F.; Igarashi, T.; Kinoshita, T.; Asahina, M.; Tsukiyama, K.; Kawaski, T.; Soai, K. Insights in the Reversal of Enantioselectivity of Chiral Catalysts by Achiral Catalysts in Asymmetric Autocatalysis. J. Am. Chem. Soc. 2008, 130, 2956-2958.

(51) Shibata, T.; Tarumi, H.; Kawaski, T.; Soai, K. Reversal in Enantioselectivity Induced by Achiral Alcohols in Asymmetric Autocatalysis in the Presence of a Chiral Diol as a Chiral Initiator. Tetrahedron: Asymmetry 2012, 23, 1023-1027.

(52) Matusmoto, A.; Fujiwara S.; Hiyoshi, Y.; Zawatzky, K.; Makarov, A. A.; Welch, C. J.; Soai, K. Unusual Reversal of Enantioselectivity in the Asymmetric Autocatalysis of Pyrimidyl Alkanol Triggered by Chiral Aromatic Alkanols and Amines. Org. Biomol. Chem. 2015, 13, 555-558.

(53) Matsumoto, A.; Yonemitsu, K.; Ozaki, H.; Mišek, J.; Stary, I.; Stará, I. G.; Soai, K. Reversal of the Sense of Enantioselectivity between 1- and 2-Aza[6]helicenes Used as Chiral Inducers of Asymmetric Autocatalysis. Org. Biomol. Chem. 2017, 15, 1321-1324.

(54) Burguete, M. I.; Collado, M.; Escorihuela, J.; Luis, S. V. Efficient Chirality Switching in the Addition of Diethylzinc to Aldehydes in the Presence of Simple Chiral $\alpha$-Amino Amides. Angew. Chem. Int. Ed. 2007, 46, 9002-9005.

(55) Escorihuela, J.; Altava, B.; Burguete, M. I.; Luis, S. V. $C_{2}$ Symmetrical Nickel Complexes Derived from $\alpha$-Amino Amides as Efficient Catalysts for the Enantioselective Addition of Dialkylzinc Reagents to Aldehydes. Tetrahedron 2013, 69, 551-558.

(56) Morimoto, H.; Harada, T. Catalytic Enantioselective Synthesis of Trisubstituted Secondary Allylic Alcohols Starting from Terminal Alkynes and Aldehydes. Eur. J. Org. Chem. 2015, 7378-7383.

(57) Adate, P. A.; Matsunaga, T.; Shin, H.; Harada, T. Chiral Aluminum Catalyst System for the Enantioselective Addition of Vinylaluminum Reagents to Aldehydes: Metal Controlled Reversal of Enantioselectivity. Adv. Synth. Catal. 2016, 358, 3688-3693.

(58) Herold, P. Synthesis of D-erythro- and D-threo-Sphingoshine Derivatives from L-Serine. Helv. Chim. Acta 1988, 71, 354-362.

(59) Fujisawa, T.; Nagai, M.; Koike, Y.; Shimizu, M. Diastereoface Discrimination in the Addition of Acetylide to a Chiral Aldehyde, Leading to a Synthesis of (+)-Deoxybiotin in Enantiomerically Pure Form Starting from L-Cysteine. J. Org. Chem. 1994, 59, 5865-5867.

(60) Utimoto, K.; Nakamura, A.; Matsubara, S. Highly Diastereoselective Reactions of YtterbiumMediated Alkynyllithium Reagents with Chiral 2-Acyl-1,3-Oxathianes: Reversal of Diastereoselectivity.

J. Am. Chem. Soc. 1990, 112, 8189-8190.

(61) Eliel, E. L.; Morris-Natschke, S. Asymmetric Synthesis Based on 1,3-Oxathianes. 1. Scope of the Reaction. J. Am. Chem. Soc. 1984, 106, 2937-2942.

(62) Lynch, J. E.; Eliel, E. L. Asymmetric Synthesis Based on 1,3-Oxathianes. 2. Synthesis of Chiral Tertiary $\alpha$-Hydroxy Aldehydes, $\alpha$-Hydroxy Acids, Glycols (RR' $\mathrm{C}(\mathrm{OH}) \mathrm{CH}_{2} \mathrm{OH}$ ), and Carbinols (RR' $\left.(\mathrm{OH}) \mathrm{CH}_{3}\right)$ in High Enantiomeric Purity. J. Am. Chem. Soc. 1984, 106, 2943-2948.

(63) Maezaki, N.; Kojima, N.; Asai, M.; Tominaga, H.; Tanaka, T. Highly Steroselective and Stereodivergent Synthesis of Four Typesof THF Cores in Acetogenins Using a $\mathrm{C}_{4}$-Chiral Building Block. Org. Lett. 2002, 4, 2977-2980.

(64) Maezaki, N.; Kojima, N.; Tominaga, H.; Yanai, M.; Tanaka, T. Systematic Synthesis of Bis-THF Ring Cores in Annonaceous Acetogenins. Org. Lett. 2003, 5, 1411-1414.

(65) Kojima, N.; Maezaki, N.; Tominaga, H.; Asai, M.; Yanai, M.; Tanaka, T. Systematic Construction of a Monotetrahydrofuran-Ring Library in Annonaceous Acetogenins by Asymmetric Alkynylation and Stereodivergent Tetrahydrofuran-Ring Formation. Chem. - Eur. J. 2003, 9, 4980-4990.

(66) Kojima, N.; Maezaki, N.; Tominaga, H.; Yanai, M.; Urabe, D.; Tanaka, T. Stereodivergent and Reiterative Synthesis of Bistetrahydrofuran Ring Cores of Annonaceous Acetogenins. Chem.-Eur. J. 2004, 10, 672-680. 
(67) Frantz, D. E.; Fässler, R.; Tomooka, C. S.; Carreira, E. M. The Discovery of Novel Reactivity in the Development of C-C Bond-Forming Reactions: In Situ Generation of Zinc Acetylides with $\mathrm{Zn}^{\mathrm{II}} / \mathrm{R}_{3} \mathrm{~N}$. Acc. Chem. Res. 2000, 33, 373-381.

(68) Mao, J.; Wan, B.; Wu, F.; Wang, R.; Lu, S. Reversal of the Stereochemistry by Adding Ti(OiPr) 4 in the Enantioselective Phenylacetylene Addition to Aldehydes Using L-Prolinol-Backbone Ligand. $J$. Mol. Cat. A: Chem. 2005, 232, 9-12.

(69) Mao, J.; Zhang, Z.; Wan, B.; Wu, F.; Lu, S. Reversal of Predominant Enantioselectivity in the Asymmetric Alkynylation Reaction Using Sulfamide-Amine Alcohols. Cat. Commun. 2006, 7, 550-553.

(70) Song, T.; Zheng, L.-S.; Ye, F.; Deng, W.-H.; Wei, Y.-L.; Jiang, K.-Z.; Xu, L.-W. Modular Synthesis of Ar-BINMOL-Phos for Catalytic Asymmetric Alkynylation of Aromatic Aldehydes with Unexpected Reversal of Enantioselectivity. Adv. Synth. Catal. 2014, 356, 1708-1718.

(71) Hameury, T.; Guillemont, J.; Van Hijfte, L.; Bellosta, V.; Cossy, J. Diastereodivergent Addition of Allenylzincs to Aryl Glyoxylates. Org. Lett. 2009, 11, 2397-2400.

(72) Rauniyar, V.; Hall, D. G. Catalytic Enantioselective and Catalyst Controlled DiastereofacialSelective Additions of Allyl- and Crotylboronates to Aldehydes Using Chiral Brønsted Acids. Angew. Chem. Int. Ed. 2006, 45, 2426-2428.

(73) Marek, I.; Sklute, G. Creation of Quaternary Stereocenters in Carbonyl Allylation Reactions. Chem. Commun. 2007, 1683-1691.

(74) Yus, M.; González-Gómez, J. C.; Foubelo, F. Catalytic Enantioselective Allylation of Carbonyl Compounds and Imines. Chem. Rev. 2011, 111, 7774-7854.

(75) Yus, M.; González-Gómez, J. C.; Foubelo, F. DiastereoselectiveAllylation of Carbonyl Compounds and Imines. Application to the Synthesis of Natural Products. Chem. Rev. 2013, 113, 5595-5698.

(76) Bandini, M.; Cozzi, P. G.; Umani-Ronchi, A. Salen as a Chiral Activator: anti Versus syn Switchable Diastereoselection in the Enantioselective Addition of Crotyl Bromide to Aromatic Aldehydes. Angew. Chem. Int. Ed. 2000, 39, 2327-2330.

(77) Zbieg, J. R.; Yamaguchi, E.; McInturff, E. L.; Krische, M. J. Enantioselective C-H Crotylation of Primary Alcohols via Hydrohydroxyalkylation of Butadiene. Science 2012, 336, 324-327.

(78) McInturff, E. L.; Yamaguchi, E.; Krische, M. J. Chiral-Anion-Dependent Inversion of Diastereoand Enantioselectivity in Carbonyl Crotylation via Ruthenium-Catalyzed Butadiene Hydrohydroxyalkylation. J. Am. Chem. Soc. 2012, 134, 20628-20631.

(79) Grayson, M. N.; Krische, M. J.; Houk, K. N. Ruthenium-Catalyzed Asymmetric Hydrohydroxyalkylation of Butadiene: The Role of the Formyl Hydrogen Bond in Stereochemical Control. J. Am. Chem. Soc. 2015, 137, 8838-8850.

(80) Modern Aldol Reactions, Mahrwald, R., Ed.; Wiley-VCH: Weinheim, 2004, Vol. 1 and 2.

(81) Modern Methods in Stereoselective Aldol Reactions, Mahrwald, R., Ed.; Wiley-VCH: Weinheim, 2013.

(82) Mukaiyama, T.; Narasaka, K.; Banno, K. New Aldol Type Reaction. Chem. Lett. 1973, 1011-1014.

(83) Mukaiyama, T.; Banno, K.; Narasaka, K. New Cross-Aldol Reactions. Reactions of Silyl Enol Ethers with Carbonyl Compounds Activated by Titanium Tetrachloride. J. Am. Chem. Soc. 1974, 96, 7503-7509.

(84) Evans, D. A.; Dart, M. J.; Duffy, J. L.; Yang, M. G. A Stereochemical Model for Merged 1,2- and 1,3-Asymmetric Induction in Diastereoselective Mukaiyama Aldol Addition Reactions and Related Processes. J. Am. Chem. Soc. 1996, 118, 4322-4343.

(85) Evans, D. A.; Allison, B. D.; Yang, M. G.; Masse, C. E. The Exceptional Chelating Ability of Dimethylaluminum Chloride and Methylaluminum Dichloride. The Merged Stereochemical Impact of $\alpha$ and $\beta$-Stereocenters in Chelate-Controlled Carbonyl Addition Reactions with Enolsilanes and Hydride Nucleophiles. J. Am. Chem. Soc. 2001, 123, 10840-10852. 
(86) Brazeau, J.-F.; Mochirian, P.; Prévost, M.; Guindon, Y. Stereopentads Derived from a Sequence of Mukaiyama Aldolization and Free Radical Reduction on $\alpha$-Methyl- $\beta$-alkoxy Aldehydes: A General Strategy for Efficient Polypropionate Synthesis. J. Org. Chem. 2009, 74, 64-74.

(87) Kobayashi, S.; Horibe, M. Highly Enantioselective Synthesis of Enantiomeric Proline. J. Am. Chem. Soc. 1994, 116, 9805-9806.

(88) Kobayashi, S.; Horibe, M. Highly Enantioselective Synthesis of Both Enantiomers of 2-Methyl-3hydroxythioesters by Asymmetric Aldol Reactions Using Similar Types of Chiral Sources Derived from L-Proline. Chem. Lett. 1995, 1029-1030.

(89) Kobayashi, S.; Horibe, M. Chiral Tin(II) Lewis Acid-Mediated Enantioselective Aldol Reactions: Synthesis of Both Enantiomers Using Similar Types of Chiral Sources. Tetrahedron: Asymmetry 1995, 6, 2565-2569.

(90) Kobayashi, S.; Horibe, M. Preparation of Both Enantiomers of 2-Methyl-3-hydroxythioesters Based on Chiral Lewis Acid-Controlled Synthesis. Tetrahedron 1996, 52, 7277-7286.

(91) Kobayashi, S.; Horibe, M. Chiral Lewis Acids Controlled Synthesis (CLAC Synthesis): Chiral Lewis Acids Influence the Reaction Course in Asymmetric Aldol Reactions for the Synthesis of Enantiomeric Dihydroxythioester Derivatives in the Presence of Chiral Diamines Derived from L-Proline. Chem. - Eur. J. 1997, 3, 1472-1481.

(92) Evans, D. A.; Kozlowski, M. C.; Murry, J A.; Burgey, C. S.; Campos, K. R.; Connell, B. T.; Staples, R. J. $\mathrm{C}_{2}$-Symmetric Copper (II) Complexes as Chiral Lewis Acids. Scope and Mechanism of Catalytic Enantioselective Aldol Additions of Enolsilanes to (Benzyloxy)acetaldehyde. J. Am. Chem. Soc. 1999, 121, 669-685.

(93) Evans, D. A.; MacMillan, D. W. C.; Campos, K. R. C $_{2}$-Symmetric Copper (II) Complexes as Chiral Lewis Acids. Catalytic Enantioselective anti Aldol Additions of Enolsilanes to Glyoxylate and Pyruvate Esters. J. Am. Chem. Soc. 1997, 119, 10859-10860.

(94) Evans, D. A.; Burgey, C. S.; Kozlowski, M. C.; Tregay, S. W. C 2 -Symmetric Copper (II) Complexes as Chiral Lewis Acids. Scope and Mechanism of the Catalytic Enantioselective Aldol Additions of Enolsilanes to Pyruvate Esters. J. Am. Chem. Soc. 1999, 121, 686-699.

(95) Jefford, C. W.; Jaggi, D.; Boukouvalas, J. Condensation of 2-Trimethylsilyloxyfuran with Aldehydes. A Stereodivergent Route to threo and erythro $\delta$-Hydroxy- $\gamma$-lactones. Tetrahedron Lett. 1987, 28, 4037-4040.

(96) Landsberg, D.; Hartmann, O.; Eggert, U.; Kalesse, M. Diastereodivergent Vinylogous Mukaiyama Aldol Reaction. Synlett 2013, 24, 1105-1108.

(97) Trost, B. M.; Fettes, A.; Shireman, B. T. Direct Catalytic Asymmetric Aldol Additions of Methyl Ynones. Spontaneous Reversal in the Sense of Enantioinduction. J. Am. Chem. Soc. 2004, 126, 2660-2661.

(98) Li, X.-J.; Zhang, G.-W.; Wang, L.; Hua, M.-Q.; Ma, J.-A. Molecular-Sieves Controlled Diastereoand Enantioselectivity: Unexpected Effect in the Organocatalyzed Direct Aldol Reaction. Synlett 2008, 1255-1259.

(99) Kutzscher, C.; Nickerl, G.; Senkovska, I.; Bon, V.; Kaskel, S. Proline Functionalized UiO-67 and UiO-68 Type Metal-Organic Frameworks Showing Reversal Diastereoselectivity in Aldol Addition Reactions. Chem. Mater. 2016, 28, 2573-2580.

(100) Zhong, L. Xiao, J.; Li, C. An Unexpected Inversion of Enantioselectivity in Direct Asymmetric Aldol Reactions on a Unique L-Proline $\gamma-\mathrm{Al}_{2} \mathrm{O}_{3}$ Catalyst. J. Catal. 2006, 243, 442-445.

(101) Szőllősi, G.; Fekete, M.; Gurka, A. A.; Bartók, M. Reversal of Enantioselectivityin Aldol Reaction: New Data on Proline/ $\gamma$-Alumina Organic-Inorganic Hybrid Catalysts. Cat. Lett. 2014, 144, 478-486.

(102) Gurka, A. A.; Szőri, K.; Szőllősi, G.; Bartók, M.; London, G. Tuning the Sense of Product Stereochemistry in Aldol Reactions of Acetone and Aromatic Aldehydes in the Presence of Water with Single Chiral Catalyst. Tetrahedron Lett. 2015, 56, 7201-7205. 
(103) Gurka, A. A.; Szőri, K.; Bartók, M.; London, G. Dual Stereocontrol in Aldol Reactions Catalysed by Hydroxyproline Derivatives in the Presence of a Large Amount of Water. Tetrahedron: Asymmetry 2016, 27, 936-942.

(104) Luppi, G.; Monari, M.; Corrêa, R. J.; Violante, F. de A.; Pinto, A. C.; Kaptein, B.; Broxterman, Q. B.; Garden, S. J.; Tomasini, C. The First Total Synthesis of $(R)$-Convolutamydine A. Tetrahedron 2006, 62, 12017-12024.

(105) Luppi, G.; Cozzi, P. G.; Monari, M.; Kaptein, B.; Broxterman, Q. B.; Tomasini, C. DipeptideCatalyzed Asymmetric Aldol Condensation of Acetone with ( $N$-Alkylated) Isatins. J. Org. Chem. 2005, 70, 7418-7421.

(106) Corrêa, R. J.; Garden, S. J.; Angelici, G.; Tomasini, C. A DFT and AIM Study of the ProlineCatalyzed Asymmetric Cross-Aldol Addition of Acetone to Isatins: A Rationalization for the Reversal of Chirality. Eur. J. Org. Chem. 2008, 736-744.

(107) Gao, J.; Bai, S.; Gao, Q.; Liu, Y.; Yang, Q. Acid Controlled Diasteroselectivity in Asymmetric Aldol Reactionof Cycloketones with Aldehydes using Enamine-Based Organocatalysis. Chem. Commun. 2011, 47, 6716-6718.

(108) Moteki, S. A.; Han, J.; Arimitsu, S.; Akakura, M.; Nakayama, K.; Maruoka, K. An Achiral-AcidInduced Switch in the Enantioselectivity of a Chiral cis-Diamine-Based Organocatalyst for Asymmetric Aldol and Mannich Reactions. Angew. Chem. Int. Ed. 2012, 51, 1187-1190.

(109) Wu, F.-C.; Da, C.-S.; Du, Z.-X.; Guo, Q.-P.; Li, W.-P.; Yi, L.; Jia, Y.-N.; Ma, X. N-PrimaryAmine-Terminal $\beta$-Turn Tetrapeptides as Organocatalysts for Highly Enantioselective Aldol reaction. $J$. Org. Chem. 2009, 74, 4812-4818.

(110) Nießing, S.; Czekelius, C.; Janiak, C. Immobilization of Catalytically Active Proline on $\mathrm{H}_{2} \mathrm{~N}-$ MIL-101 (Al) Accompanied with Reversal in Enantioselectivity. Cat. Commun. 2017, 95, 12-15.

(111) Messerer, M.; Wennemers, H. Reversing the Enantioselectivity of a Peptide Catalyst by Changing the Solvent. Synlett 2011, 499-502.

(112) Martínez-Castañeda, A.; Poladura, B.; Rodríguez-Solla, H.; Concellón, C.; del Amo, V. Direct Aldol Reactions Catalyzed by a Heterogeneous Guanidinium Salt/Proline System Under Solvent-Free Conditions. Org. Lett. 2011, 13, 3032-3035.

(113) Martínez-Castañeda, A.; Rodríguez-Solla, H.; Concellón, C.; del Amo, V. Switching Diastereoselectivity in Proline-Catalyzed Aldol Reaction. J. Org. Chem. 2012, 77, 10375-10381.

(114) Szőllősi, G.; Csámpai, A.; Somlai, C.; Fekete, M.; Bartók, M. Unusual Enantioselectivities in Heterogeneous Organocatalyzed Reaction: Reversal of Direction Using Proline Di-versus Tri-peptides in the Aldol Addition. J. Mol. Catal. A.: Chem. 2014, 382, 86-92.

(115) Gurka, A.; Bucsi, I. Kovácks, L.; Szőllősi, G.; Bartók, M. Reversal of the Enantioselectivity in Aldol Addition over Immobilized Di- and Tripeptides: Studies Under Continuous Flow Conditions. RSC Adv. 2014, 4, 61611-61618.

(116) Denmark, S. E.; Wong, K. T.; Stavenger, R. A. The Chemistry of Trichlorosilyl Enolates. 2. Highly-Selective Asymmetric Aldol Additions of Ketone Enolates. J. Am. Chem. Soc. 1997, 119, 2333-2334.

(117) Denmark, S. E.; Su, X.; Nishigaichi, Y. The Chemistry of Trichlorosilyl Enolates. 6. Mechanistic Duality in the Lewis Base-Catalyzed Aldol Addition Reaction. J. Am. Chem. Soc. 1998, 120, 12990-12991.

(118) Denmark, S. E.; Stavenger, R. A. The Chemistry of Trichlorosilyl Enolates. Aldol Addition Reactions of Methyl Ketones. J. Am. Chem. Soc. 2000, 122, 8837-8847.

(119) Palomo, C.; Oiarbide, M.; Laso, A. Recent Advances in the Catalytic Asymmetric Nitroaldol (Henry) Reaction. Eur. J. Org. Chem. 2007, 2561-2574.

(120) Boruwa, J.; Gogoi, N.; Saikia, P.; Barua, N. C. Catalytic Asymmetric Henry Reaction. Tetrahedron: Asymmetry 2006, 17, 3315-3226.

(121) Ono, N. The Nitro Group in Organic Synthesis; Wiley-VCH: New York, 2001. 
(122) Lu, S. F.; Du, D. M.; Zhang, S. W.; Xu, J. X. Facile Synthesis of $\mathrm{C}_{2}$-Symmetric Tridentate Bis(thiazoline) and Bis(oxazoline) Ligands and their Application in the Enantioselective Henry Reaction. Tetrahedron: Asymmetry 2004, 15, 3433-3441.

(123) Du, D. M.; Lu, S. F.; Fang, T.; Xu, J. X. Asymmetric Henry Reaction Catalyzed by $\mathrm{C}_{2}$-Symmetric Tridentate Bis(oxazoline) and Bis(thiazoline) Complexes: Metal-Controlled Reversal of Enantioselectivity. J. Org. Chem. 2005, 70, 3712-3715.

(124) Spangler, K. Y.; Wolf, C. Asymmetric Copper(I)-Catalyzed Henry Reaction with an Aminoindanol-Derived Bisoxazolidine Ligands. Org. Lett. 2009, 11, 4724-4727.

(125) Kim, H. Y.; Oh, K. Brucine-Derived Amino Alcohol Catalyzed Asymmetric Henry Reaction: An Orthogonal Enantioselectivity Approach. Org. Lett. 2009, 11, 5682-5685.

(126) Wu, S.; Tang, J.; Han, J.; Mao, D.; Liu, X.; Gao, X.; Yu, J.; Wang, L. Design of $\mathrm{C}_{2}$-Symmetric Salen Ligands and their $\mathrm{Co}(\mathrm{II})$ or $\mathrm{Yb}(\mathrm{III})-$ Complexes, and their Role in the Reversal of Enantioselectivity in the Asymmetric Henry Reaction. Tetrahedron 2014, 70, 5986-5992.

(127) Dimroth, J.; Weck, M. Co-Salen Complexes as Catalysts for the Asymmetric Henry Reaction - Reversed Enantioselectivity through Simple Ligand Modification. RSC Adv. 2015, 5, 29108-29113.

(128) Sohtome, Y.; Sato, Y.; Handa, S.; Aoyama, N.; Nagawa, K.; Masunaga, S.; Shibasaki, M. Stereodivergent Catalytic Doubly Diastereoselective Nitroaldol Reactions Using Heterobimetallic Complexes. Org. Lett. 2008, 10, 2231-2234.

(129) Hayashi, M.; Miyamoto, Y.; Inoue, T.; Oguni, N. Enantioselective Trimethylsilylcyanation of Some Aldehydes by Chiral Titanium Schiff's Base Complexes. J. Chem. Soc., Chem. Commun. 1991, 1752-1753.

(130) Hayashi, M.; Miyamoto, Y.; Inoue, T.; Oguni, N. Enantioselective Trimethylsilylcyanation of Some Aldehydes by Chiral Titanium Schiff's Base-Titanium Alkoxide Complexes. J. Org. Chem. 1993, 58, 1515-1522.

(131) Hayashi, M.; Inoue, T.; Miyamoto, Y.; Oguni, N. Asymmetric Carbon-Carbon Bond Forming Reactions Catalyzed by Chiral Schiff Based-Titanium Alkoxide Complexes. Tetrahedron 1994, 50, 4385-4398.

(132) Yabu, K.; Masumoto, S.; Yamasaki, S.; Hamashima, Y.; Kanai, M.; Du, W.; Curran, D. P.; Shibasaki, M. Switching Enantiofacial Selectivities Using One Chiral Source: Catalytic Enantioselective Synthesis of the Key Intermediate for (20S)-Camptothecin Family by (S)-Selective Cyanosilylation of Ketones. J. Am. Chem. Soc. 2001, 123, 9908-9909.

(133) Hamashima, Y.; Kanai, M.; Shibasaki, M. Catalytic Enantioselective Cyanosilylation of Ketones. J. Am. Chem. Soc. 2000, 122, 7412-7413.

(134) Hamashima, Y.; Kanai, M.; Shibasaki, M. Catalytic Enantioselective Cyanosilylation of Ketones: Improvements of Enantioselectivity and Catalyst Turn-over by Ligand Tuning. Tetrahedron Lett. 2001, 42, 691-694.

(135) Yokomatsu, T.; Yamagishi, T.; Shibuya, S. Sterodivergent Synthesis of $\beta$-Amino- $\alpha$ hydroxyphosphonic Acid Derivatives by Lewis Acid Mediated Stereoselective Hydrophosphonylation of $\alpha$-Amino Aldehydes. Tetrahedron: Asymmetry 1993, 4, 1401-1404.

(136) Yamagishi, T.; Suemune, K.; Yokomatsu, T.; Shibuya, S. Diastereoselective Synthesis of $\beta$ Amino- $\alpha$-hydroxy- $H$-phosphinates Through Hydrophosphonylation of $\alpha$-Amino Aldehydes. Tetrahedron Lett. 2001, 42, 5033-5036.

(137) Xhou, X.; Liu, X.; Yang, X.; Shang, D.; Xin, J.; Feng, X. Highly Enantioselective Hydrophosphonylation of Aldehydes Catalyzed by Tridentate Schiff Base Aluminum (III) Complexes. Angew. Chem. Int. Ed. 2008, 47, 392-394.

(138) Yang, K.-S.; Chen, K. Diastereoselective Baylis-Hillman Reactions: The Design and Synthesis of a Novel Camphor-Based Chiral Auxiliary. Org. Lett. 2000, 2, 729-731.

(139) Haghshenas, P.; Gravel, M. Chemo- and Diastereoselective N-Heterocyclic Carbene-Catalyzed Cross-Benzoin Reactions Using N-Boc- $\alpha$-Amino Aldehydes. Org. Lett. 2016, 18, 4518-4521. 
(140) Haghshenas, P.; Quail, J. W.; Gravel, M. Substrate-Controlled Diastereoselectivity Reversal in NHC-Catalyzed Cross-Benzoin Reactions Using $N$-Boc- $N$-Bn-Protected $\alpha$-Amino Aldehydes. J. Org. Chem. 2016, 81, 12075-12083.

(141) Modern Reduction Methods. Andersson, P. G.; Munslow, I. J., Eds.; Wiley-VCH: Weinheim, 2008.

(142) Kim, Y. H.; Park, D. H.; Byun, I. S. Stereocontrolled Catalytic Asymmetric Reduction of Ketones with Oxazaborolidines Derived from New Chiral Amino Alcohols. J. Org. Chem. 1993, 58, 4511-4512.

(143) Riant, O.; Mostefaï, N.; Courmarcel, J. Recent Advances in the Asymmetric Hydrosilylation of Ketones, Imines and Electrophilic Double Bonds. Synthesis 2004, 2943-2958.

(144) Rendler, S.; Oestreich, M. in Modern Reduction Methods. Andersson, P. G.; Munslow, I. J., Eds.; Wiley-VCH: Weinheim, 2008, pp 109-134.

(145) Malacea, R.; Poli, R.; Manoury; E. Asymmetric Hydrosilylation, Transfer Hydrogenation and Hydrogenation of Ketones Catalyzed by Iridium Complexes. Coord. Chem. Rev. 2010, 254, 729-752.

(146) Kinting, A.; Kreuzfeld, H.-J.; Abicht, H.-P. Metal Controlled Enantioselectivity in the Catalytic Asymmetric Hydrosilylation. J. Organomet. Chem. 1989, 370, 343-349.

(147) Faller, J. W.; Chase, K. J. Enantioselective Hydrosilylation of Ketones with $\mathrm{L}^{*} /[\mathrm{Rh}(\mathrm{COD}) \mathrm{Cl}]_{2}$ and $\mathrm{L}^{*} /[\mathrm{Ir}(\mathrm{COD}) \mathrm{Cl}]_{2}$ Catalysts $\left(\mathrm{L}^{*}=\mathrm{Ph}_{2} \mathrm{P}(\mathrm{O})_{\mathrm{n}} \mathrm{CH}_{2} \mathrm{CH}\left(\mathrm{NMe}_{2}\right)\left(\mathrm{CH}_{2}\right)_{\mathrm{m}} \mathrm{SMe}(\mathrm{n}=0,1 ; \mathrm{m}=1,2)\right)$. Organometallics 1994, 13, 989-992.

(148) Nishibayashi, Y.; Segawa, K.; Ohe, K.; Uemura, S. Chiral Oxazolinylferrocene-Phosphine Hybrid Ligand for the Asymmetric Hydrosilylation of Ketones. Organometallics 1995, 14, 5486-5487.

(149) Frölander, A.; Moberg, C. Ag ${ }^{+}$-Assisted Hydrosilylation: Complementary Behavior or Rh and Ir Catalysts (Reversal of Enantioselectivity). Org. Lett. 2007, 9, 1371-1374.

(150) Inagaki, T.; Ito, A.; Ito, J.; Nishiyama, H. Asymmetric Iron-Catalyzed Hydrosilane Reduction of Ketones: Effect of Zinc Metal upon the Absolute Configuration. Angew. Chem. Int. Ed. 2010, 49, 9384-9387.

(151) Kawabata, S.; Tokura, H.; Chiyojima, H.; Okamoto, M.; Sakaguchi, S. Asymmetric Hydrosilane Reduction of Ketones Catalyzed by an Iridium Complex Bearing a Hydroxyamide-Functionalized NHC Ligand. Adv. Synth. Catal. 2012, 354, 807-812.

(152) Chiyojima, H.; Sakaguchi, S. Iridium Complex Bearing a Chiral Hydroxy-Amide Functionalized N-Heterocyclic Carbene: a Catalyst Precursor for Asymmetric Transfer Hydrogenation. Tetrahedron Lett. 2011, 52, 6788-6791.

(153) Mallat, T.; Orglmeister, E.; Baiker, A. Asymmetric Catalysis at Chiral Metal Surfaces. Chem. Rev. 2007, 107, 4863-4890.

(154) Blaser, H.-U.; Studer, M. Cinchona-Modified Platinum Catalysts: From Ligand Acceleration to Technical Processes. Acc. Chem. Res. 2007, 40, 1348-1356.

(155) Collier, P. J.; Hall, T. J.; Iggo, J. A.; Johnston, P.; Slipszenko, J. A.; Wells, P. B.; Whyman, R. Solvent and Substituent Effects on the Sense of the Enantioselective Hydrogenation of Pyruvate Esters Catalysed by Pd and Pt in Colloidal and Supported Forms. Chem. Commun. 1998, 1451-1452.

(156) von Arx, M.; Mallat, T.; Baiker, A. Inversion of Enantioselectivity During the Platinum-Catalyzed Hydrogenation of an Activated Ketone. Angew. Chem. Int. Ed. 2001, 40, 2302-2305.

(157) Bartók; M.; Sutyinski, M.; Felföldi, K.; Szőllősi, G. Unexpected Change of the Sense of the Enantioselective Hydrogenation of Ethyl Pyruvate Catalyzed by a Pt-Alumina-Cinchona Alkaloid System. Chem. Commun. 2002, 1130-1131.

(158) Felföldi, K.; Varga, T.; Forgó, P. Bartók, M. Enantioselective Hydrogenation of Trifluoromethylcyclohexyl Ketone on Cinchona Alkaloid Modified Pt-Alumina Catalyst. Cat. Lett. 2004, 97, 65-70.

(159) Bartók, M.; Sutyinszki, M.; Bucsi, I.; Felföldi, K.; Szőllősi, G.; Bartha, F.; Bartók, T. Enantioselective Hydrogenation of Ethyl Pyruvate Catalyzed by $\alpha$ - and $\beta$-Isocinchonine-Modified $\mathrm{Pt} / \mathrm{Al}_{2} \mathrm{O}_{3}$ in Toluene. J. Catal. 2005, 231, 33-40. 
(160) Szőri, K.; Balázsik, K.; Cserényi, S.; Szöllősi, G. Bartók, M. Inversion of Enantioselectivity in the 2,2,2-Trifluoroacetophenone Hydrogenation over Pt-Alumina Catalyst Modified by Cinchona Alkaloids. Appl. Cat. A: Gen. 2009, 362, 178-184.

(161) Hess, R.; Vargas, A.; Mallat, T.; Bürgi, T.; Baiker, A. Inversion of Enantioselectivity in the Platinum-Catalyzed Hydrogenation of Substituted Acetophenones. J. Catal. 2004, 222, 117-128.

(162) Hess, R.; Diezi, S.; Mallat, T.; Baiker, A. Chemo-and Enantioselective Hydrogenation of the Activated Keto Group of Fluorinated $\beta$-Diketones. Tetrahedron: Asymmetry 2004, 15, 251-257.

(163) Diezi, S.; Szabo, A.; Mallat, T.; Baiker, A. Inversion of Enantioselectivity in the Hydrogenation of Ketopantolactone on Platinum Modified by Ether Derivatives of Cinchonidine. Tetrahedron: Asymmetry 2003, 14, 2573-2577.

(164) Bartók, M.; Balázsik, K.; Bucsi, I.; Szöllősi, G. Inversion of Enantioselectivity in the Hydrogenation of Ketopantolactone on a Pt- $\beta$-ICN Chiral Catalyst. J. Catal. 2006, 239, 74-82.

(165) Diezi, S.; Mallat, T.; Szabo, A.; Baiker, A. Fine Tuning the "Chiral Sites" on Solid Enantioselective Catalysts. J. Catal. 2004, 228, 162-173.

(166) Bonalumi; N.; Vargas, A.; Ferri, D.; Bürgi, T.; Mallat, T.; Baiker, A. Competition of Chiral Metal Surfaces: Fundamental Aspects of the Inversion of Enantioselectivity in Hydrogenations on Platinum. $J$. Am. Chem. Soc. 2005, 127, 8467-8477.

(167) Hess, R.; Krumeich, F.; Mallat, T.; Baiker, A. Rhodium-Catalyzed Heterogeneous Enantioselective Hydrogenation of 3,5-Di(trifluoromethyl)-Acetophenone. J. Mol. Cat. A: Chem. 2004, 212, 205-209.

(168) Toukoniitty, E.; Busygin, I.; Leino, R.; Murzin, D. Y. Asymmetric Hydrogenation of 1Phenylpropane-1,2-dione over Cinchona-Modified Pt: Role of the C-9 OH Group of Cinchonidine. $J$. Catal. 2004, 227, 210-216.

(169) Busygin, I.; Wärnå, J.; Toukoniitty, E.; Murzin, D. Y.; Leino, R. Hydrogenation of 1-Phenyl-1,2propanedione over Pt Catalysts Modified by Cinchona Alkaloid O-Ester and the Kinetic Resolution of the 1-Hydroxyketones Generated. J. Catal. 2008, 254, 339-348.

(170) Jenkins, R. L.; Dummer, N. F.; Li, X.; Bawaked, S. M.; McMorn, P.; Wells, R. P. K.; Burrows, A.; Kiely, C. J.; Hutchings, G. J. Unexpected Inversion of Enantioselectivity During the Hydrogenation of Ethyl Pyruvate using Hydroquinine and Hydroquinidine Modified $\mathrm{Pt} / \mathrm{Al}_{2} \mathrm{O}_{3}$. Cat. Lett. 2006, 110, 135-138.

(171) Dummer, N. F.; Jenkins, R. L.; Li, X.; Bawaked, S. M.; McMorn, P.; Burrows, A.; Kiely, C. J.; Wells, R. P. K.; Willock, D. J.; Hutchings, G. J. Inversion of Enantioselectivity for the Hydrogenation of Ethyl Pyruvate in the Gas-Phase over $\mathrm{Pt}-\mathrm{SiO}_{2}$ Modified with Derivatives of Hydroquinidine. J. Catal. 2006, 243, 165-170.

(172) Gladiali, S.; Taras, R. in Modern Reduction Methods. Andersson, P. G.; Munslow, I. J. Eds.; Wiley-VCH: Weinheim, 2008, pp 135-157.

(173) Ikariya, T.; Blacker, A. J. Asymmetric Transfer Hydrogenation of Ketones with Bifunctional Transition Metal-Based Molecular Catalysts. Acc. Chem. Res. 2007, 40, 1300-1308.

(174) Samec, J.-E.; Bäckvall, J.-E.; Andersson, P. G. Mechanistic Aspects of Transition Metal-Catalyzed Hydrogen Transfer Reactions. Chem. Soc. Rev. 2006, 35, 237-248.

(175) Noyori, R.; Hashiguchi, S. Asymmetric Transfer Hydrogenation Catalyzed by Chiral Ruthenium Complexes. Acc. Chem. Rev. 1997, 30, 97-102.

(176) Furegati, M.; Rippert, A. J. Unusual Base Dependent Enantioselectivity in a Transfer Hydrogenation Using Axially Chiral Ir(III)-Catalysts. Tetrahedron: Asymmetry 2005, 16, 3947-3950.

(177) Zaitsev, A. B.; Adolfsson, H. Enantioswitchable Catalysts for the Asymmetric Transfer Hydrogenation of Aryl Alkyl Ketones. Org. Lett. 2006, 8, 5129-5132.

(178) Yu, W.; Zhao, M.; Du, W.; Jiang, Q.; Wu, K.; Wu, P.; Yu, Z. Highly Active Ruthenium(II) Complex Catalysts Bearing an Unsymmetrical NNN Ligand in the (Asymmetric) Transfer Hydrogenation of Ketones. Chem. - Eur. J. 2011, 17, 4737-4741.

(179) Endo, T.; Hayashi, Y.; Okawara, M. Syntheses of 1,4-Dihydropyridines Containing Amino Acids and Reduction of Ethyl Benzoylformate. Chem. Lett. 1997, 391-394. 
(180) Binay, P.; Dupas, G.; Bourguignon, J.; Quequiner, G. Inversion in the Enantioselectivity of Reductions Performed with NADH Models Bearing the Same Chiral Auxiliary. Tetrahedron Lett. 1998, 29, 931-932.

(181) Vasse, J.-L.; Levacher, V.; Bourguignon, J.; Dupas, G. Atropoisomeric Quinolinium Salt Promoting the Access to Both Enantiomers Forms of Methyl Mandelate: a Versatile NADH Mimic. Chem. Commun. 2002, 2256-2257.

(182) Saito, R.; Naruse, S.; Takano, K.; Fukuda, K.; Katoh, A.; Inoue, Y. Unusual Temperature Dependence of Enantioselectivity in Asymmetric Reduction by Chiral NADH Models. Org. Lett. 2006, 8, 2067-2070.

(183) Nakamura, K.; Matsuda, T. in Modern Reduction Methods. Andersson, P. G.; Munslow, I. J., Eds.; Wiley-VCH: Weinheim, 2008, pp 209-234.

(184) Feske, B. D.; Kaluzna, I. A.; Stewart, J. D. Enantiodivergent Biocatalytic Routes to Both Taxol Side Chain Antipodes. J. Org. Chem. 2005, 70, 9654-9657.

(185) Agudo, R.; Roiban, G.-D.; Reetz, M. T. Induced Axial Chirality in Biocatalytic Asymmetric Ketone Reduction. J. Am. Chem. Soc. 2013, 135, 1665-1668.

(186) Díaz-Rodríguez, A.; Borzecka, W.; Lavandera, I.; Gotor, V. Stereodivergent Preparation of Valuable $\gamma$ - or $\delta$-Hydroxy Esters and Lactones through One-Pot Cascade or Tandem Chemoenzymatic Protocols. ACS Catal. 2014, 4, 386-393.

(187) Zhang, D.; Chen, X.; Chi, J.; Feng, J.; Wu, Q.; Zhu, D. Semi-Rational Engineering of a Carbonyl Reductase for the Enantioselective Reduction of $\beta$-Amino Ketones. ACS Catal. 2015, 5, 2452-2457.

(188) Enders, D.; Reinhold, U. Asymmetric Synthesis of Amines by Nucleophilic 1,2-Addition of Organometallic Reagents to the CN-double Bond. Tetrahedron: Asymmetry 1997, 8, 1895-1946.

(189) Bloch, R. Addition of Organometallic Reagents to C=N Bonds: Reactivity and Selectivity. Chem. Rev. 1998, 98, 1407-1438.

(190) Adams, J. P. Imines, Enamines and Oximes. J. Chem. Soc., Perkin Trans.1 2000, 125-139.

(191) Kobayashi, S.; Ishitani, H. Catalytic Enantioselective Addition to Imines. Chem. Rev. 1999, 99, 1069-1094.

(192) Alvaro, G.; Savoia, D. Addition of Organometallic Reagents to Imines Bearing Stereogenic NSubstituents. Stereochemical Models Explaining the 1,3-Asymmetric Induction. Synlett 2002, 651-673.

(193) Ellman, J. A.; Owens, T. D.; Tang, T. P. N-tert-Butanesulfinyl Imines: Versatile Intermediates for the Asymmetric Synthesis of Amines. Acc. Chem. Res. 2002, 35, 984-995.

(194) Morton, D.; Stockman, R. A. Chiral Non-racemic Sulfinylimines: Versatile Reagents for Asymmetric Synthesis. Tetrahedron 2006, 62, 8869-8905.

(195) Ferreira, F.; Botoha, C.; Chemla, F.; Pérez-Luna, A. tert-Butanesulfinimines: Structure, Synthesis and Synthetic Applications. Chem. Soc. Rev. 2009, 38, 1162-1186.

(196) Robak, M. T.; Herbage, M. A.; Ellman, J. A. Synthesis and Applications of tert-Butane Sulfinamide. Chem. Rev. 2010, 110, 3600-3740.

(197) Foubelo, F.; Yus, M. Diastereoselective Indium-Promoted Allylation of Chiral N-Sulfinyl Imines. Eur. J. Org. Chem. 2014, 485-491.

(198) Cogan, D. A.; Liu, G.; Ellman, J. AsymmetricSynthesis of Chiral Amines by Highly Diastereoselective 1,2-Additions of Organometallic Reagents to Chiral $\mathrm{N}$-tert-Butanesulfinyl Imines. Tetrahedron 1999, 55, 8883-8904.

(199) Plobeck, N.; Powell, D. Asymmetric Synthesis of Diarylmethylamines by Diastereoselective Addition of Organometallic Reagents to Chiral N-tert-Butanesulfinylimines: Switchover of Diastereofacial Selectivity Tetrahedron: Asymmetry 2002, 13, 303-310.

(200) Han, Z.; Krishnamurthy, D.; Pflum, D.; Grover, P.; Wald, S. A.; Senanayake, C. H. First Application of Tunable Alkyl and Aryl Sulfinamides to the Stereoselective Synthesis of a Chiral Amine: Asymmetric Synthesis of $(R)$-Didesmethylsibutramine ((R)-DDMS) Using $(R)$-Triethylmethylsulfinamide ((R)-TESA). Org. Lett. 2002, 4, 4025-4028.

(201) Lu, B. Z.; Senanayake, C.; Li, N.; Han, Z.; Bakale, R. P.; Wald, S. A. Control of Diastereoselectivity by Solvent Effects in the Addition of Grignard Reagents to Enantiopure $t$ - 
Butylsulfinimine: Synthesis of the Stereoisomers of the Hydroxyl Derivatives of Sibutramine. Org. Lett. 2005, 7, 2599-2602.

(202) Jones, C. A.; Jones, I. G.; North, M.; Pool, C. R. Asymmetric Addition of Organolithium Reagents to Imines Favouring (S)-Amines. Tetrahedron Lett. 1995, 36, 7885-7888.

(203) Cabello, N.; Kizitian, J.-C.; Alexakis, A. Enantioselective Addition of Aryllithium Regents to Aromatic Imines Mediated by 1,2-Diamine Ligands. Tetrahedron Lett. 2004, 45, 4639-4642.

(204) Shao, Z.; Wang, J.; Ding, K.; Chan, A. S. C. Unprecedent Effects of Additives and Ligand-toMetal Ratio on the Enantiofacial Selection of Copper-Catalyzed Alkynylation of $\alpha$-Imino Ester and Arylacetylenes. Adv. Synth. Catal. 2007, 349, 2375-2379.

(205) Piazzuti, M. G.; Minnaard, A. J.; Feringa, B. L. Catalytic Enantioselective Addition of Organometallic Reagents to $\mathrm{N}$-Formylimines Using Monodentate Phosphoramidite Ligands. J. Org. Chem. 2008, 73, 940-947.

(206) Dondoni, A.; Franco, S.; Merchán, F. L.; Merino, P.; Tejero, T. Stereocontrolled Addition of 2Lithiothiazole to the Nitrone Derived from D-Glyceraldehyde Acetonide. A Revision and Extension. Tetrahedron Lett. 1993, 34, 5475-5478.

(207) Paddon-Row, M. N.; Rondan, N. G.; Houk, K. N. Staggered Models for Asymmetric Induction: Attack Trajectories and Conformations of Allylic Bonds from Ab Initio Transition Structures on Addition Reactions. J. Am. Chem. Soc. 1982, 104, 7162-7166.

(208) Dondoni, A.; Franco, S.; Merchán, F. L.; Merino, P.; Tejero, T. Stereocontrol by Diethylaluminum Chloride in the Addition of 2-Lithiofuran and $N$-Methyl-2-lithioimidazole to $\alpha$-Alkoxy Nitrones. Total Synthesis of 5-O-Carbamoylpolyoxamic Acid. Tetrahedron Lett. 1993, 34, 5479-5482.

(209) Dondoni, A.; Junquera, F.; Merchán, F. L.; Merino, P.; Tejero, T. Addition of 2-Lithiofuran to Chiral $\alpha$-Alkoxy Nitrones; a Stereoselective Approach to $\alpha$-Epimeric $\beta$-Alkoxy- $\alpha$-amino Acids. Synthesis 1994, 1450-1456.

(210) Dondoni, A.; Franco, S.; Junquera, F.; Merchán, F. L.; Merino, P.; Tejero, T.; Bertolasi, V. Stereoselective Homologation-Amination of Aldehydes by Addition of Their Nitrones to C-2 Metalated Thiazoles - A General Entry to $\alpha$-Amino Aldehydes and Amino Sugars. Chem.-Eur. J. 1995, 1, 505-520.

(211) Dondoni, A.; Franco, S.; Merchán, F. L.; Merino, P.; Tejero, T. Stereocontrolled Addition of 2Thiazolyl Organometallic Reagents to $C$-Galactopyranosylnitrone. A Formal Synthesis of Destomic Acid and Lincosamine. Synlett 1993, 78-80.

(212) Merino, P.; Anoro, S.; Castillo, E.; Merchán, F. L.; Tejero, T. Direct Vinylation and Ethynylation of Nitrones. Stereodivergent Synthesis of Alkyl and Propargyl Amines. Tetrahedron: Asymmetry 1996, 7, 1887-1890.

(213) Merino, P.; Franco, S.; Merchán, F. L.; Tejero, T. Diasteroselective Nucleophilic Addition of Acetylide to $N$-Benzyl-2,3-O-isopropyliden-D-glyceraldehyde Nitrone (BIGN). Stereodivergent Synthesis of $\beta$-Hydroxy- $\alpha$-(hydroxyamino)- and $\beta$-Hydroxy- $\alpha$-amino Acids. Tetrahedron: Asymmetry 1997, 8, 3489-3496.

(214) Merino, P.; Castillo, E.; Franco, S.; Merchán, F. L.; Tejero, T. Enantiodivergent Approach to Dand L-Secondary $N$-Hydroxy- $\alpha$-amino Acids by Using $N$-Benzyl-2,3- $O$-isopropyliden-D-glyceraldehyde Nitrone as an Effective $N$-Hydroxyglycine Cation Equivalent. J. Org. Chem. 1998, 63, 2371-2374.

(215) Merino, P.; Castillo, E.; Merchán, F. L.; Tejero, T. Stereocontrolled Addition of Grignard Reagents to $\alpha$-Alkoxy Nitrones. Synthesis of syn and anti 3-Amino-1,2-diols. Tetrahedron: Asymmetry 1997, 8, 1725-1729.

(216) Merino, P.; Mannucci, V.; Tejero, T. Exploring Nitrone Chemistry: Towards the Enantiodivergent Synthesis of 6-Substituted 4-Hydroxypipecolic Acid Derivatives. Eur. J. Org. Chem. 2008, 3943-3959.

(217) Merino, P.; Lanaspa, A.; Merchan, F. L.; Tejero, T. Stereoselective Grignard Reactions to $\alpha$ Amino Nitrones. Synthesis of Optically Active $\alpha$-Aminohydroxylamines and 1,2-Diamines. Tetrahedron: Asymmetry 1997, 8, 2381-2401.

(218) Merino, P.; Lanaspa, A.; Merchán, F. L.; Tejero, T. Stereocontrolled Synthesis of 2,3Diaminobutanoic Acids. Tetrahedron Lett. 1997, 38, 1813-1816. 
(219) Merino, P.; Franco, S.; Merchán, F. L.; Revuelta, J.; Tejero, T. Efficient Synthesis of (2R,3S)- and (2S,3S)-2-Amino-1,3,4-butanetriols Through Stereodivergent Hydroxymethylation of D-Glyceraldehyde Nitrones. Tetrahedron Lett. 2002, 43, 459-462.

(220) Dondoni, A.; Perrone, A. Stereocontrolled Synthesis of Pseudo $\mathrm{C}_{2}$-Symmetric 1,3-Diamino-2propanol Core Units of HIV Protease Inhibitors. Tetrahedron Lett. 1997, 38, 499-502.

(221) Schade, W.; Reissig, H.-U. A New Diastereoselective Synthesis of Enatiomerically Pure 1,2Oxazine Derivatives by Addition of Lithiated Methoxyallene to Chiral Nitrones. Synlett 1999, 632-634.

(222) Helms, M.; Shade, W.; Pulz, R.; Watanabe, T.; Al-Harrasi, A.; Fisera, L.; Hlobilová, I.; Zhan, G.; Reißig, H.-U. Stereodivergent Synthesis of Highly SubstitutedEnantiopure 4-Alkoxy-3,6-dihydro-2H-1,2oxazines by Addition of Lithiated Alkoxyallenes to Carbohydrate-Derived Aldonitrones. Eur. J. Org. Chem. 2005, 1003-1019.

(223) Pfrengle, F.; Reissig, H.-U. Internally Protected Amino Sugar Equivalents from Enantiopure 1,2Oxazines: Synthesis of Variably Configured Carbohydrates with C-Branched Amino Sugar Units. Chem.- Eur. J. 2010, 16, 11915-11925.

(224) Pfrengle, F.; Lentz, D.; Reissig, H.-U. A New Ring Closure Approach to Enantiopure 3,6Dihydro-2H-pyrans: Stereodivergent Access to Carbohydrate Mimetics. Org. Lett. 2009, 11, 5534-5537.

(225) Hart, D. J.; Ha, D.-C. The Ester Enolate-Imine Condensation Route to $\beta$-Lactams. Chem. Rev. 1989, 89, 1447-1465.

(226) Arend, H.; Westermann, B.; Risch, N. Modern Variants of the Mannich Reaction. Angew. Chem., Int. Ed. Engl. 1998, 37, 1044-1070.

(227) Córdova, A. The Direct Catalytic Asymmetric Mannich Reaction. Acc. Chem. Res. 2004, 37, 102-112.

(228) Verkade, J. M. M.; van Hermert, L. J. C.; Quaedflieg, P. J. L. M.; Rutjes, F. P. J. T. Organocatalyzed Asymmetric Mannich Reaction. Chem. Soc. Rev. 2008, 37, $29-41$.

(229) Gómez Arrayás, R.; Carretero, J. C. Catalytic Asymmetric Direct Mannich Reaction: A Powerful Tool for the Synthesis of $\alpha, \beta$-Diamino Acids. Chem. Soc. Rev. 2009, 38, 1940-1998.

(230) Kobayashi, S.; Mori, Y.; Fossey, J. S.; Salter, M. M. Catalytic Enantioselective Formation of C-C Bonds by Addition to Imines and Hydrazones. A Ten Years Update. Chem. Rev. 2011, 111, 2626-2704.

(231) Benohoud, M.; Hayashi, Y. in Asymmetric Organocatalysis1. Lewis Base and Acid Catalysis, List, B. Ed.; Thieme: Stuttgart 2012, pp 73-134.

(232) Shibata, N.; Nishima, T.; Shibata, N.; Tokunaga, E.; Kawada, K.; Kagawa, T.; Sorochinsky, A. E.; Soloshonok, V. A. Organic Base-Catalyzed Stereodivergent Synthesis of $(R)$ - and (S)-3-Amino-4,4,4trifluorobutanoic Acids. Chem. Commun. 2012, 48, 4124-4126.

(233) Shibata, N.; Nishima, T.; Shibata, N.; Tokunaga, E.; Kawada, K.; Kagawa, T.; Aceña, J. L.; Sorochinsky, A. E.; Soloshonok, V. A. Asymmetric Mannich Reaction Between (S)- $N$-(tertButanesulfinyl)-3,3.3-trifluoroacetaldimine and Malonic Acid Derivatives. Stereodivergent Synthesis of (R)- and (S)-3-Amino-4,4,4-trifluorobutanoic Acids. Org. Biomol. Chem. 2014, 12, 1454-1462.

(234) Gu, C.-L.; Liu, L.; Wang, D.; Chen, Y.-J. Tunable and Highly Regio- and Diastereoselective Vinylogous Mannich-Type Reaction of Dioxinone-Derived Silyl Dienolate. J. Org. Chem. 2009, 74, 5754-5757.

(235) Rondot, C.; Zhu, J. Synthesis of Chiral Vicinal Diamines by Highly Diastereoselective ThreeComponent Phenolic Mannich Reaction: Temperature-Dependent Stereodivergency. Org. Lett. 2005, 7, 1641-1644.

(236) Merino, P.; Jimenez, P.; Tejero, T. Enantiodivergent Synthesis of D- and L-erythro-Sphingosines Through Mannich-Type Reactions of $\mathrm{N}$-Benzyl-2,3-O-isopropylidene D-Glyceraldehyde Nitrone. J. Org. Chem. 2006, 71, 4685-4688.

(237) Yamasaki, S.; Iida, T.; Shibasaki, M. Direct Catalytic Asymmetric Mannich Reaction of Unmodified Ketones: Cooperative Catalysis of an AlLibis(binaphthoxide) Complex and $\mathrm{La}(\mathrm{OTf})_{3} \cdot \mathrm{nH}_{2} \mathrm{O}$. Tetrahedron 1999, 55, 8857-8867. 
(238) Matsunaga, S.; Kumagai, N.; Harada, S.; Shibasaki, M. anti-Selective Direct Catalytic Asymmetric Mannich-TypeReaction of Hydroxyketone Providing $\beta$-Amino Alcohols. J. Am. Chem. Soc. 2003, 125, 4712-4713.

(239) Matsunaga, S.; Yoshida, T.; Marimoto, H.; Kumagai, N.; Shibasaki, M. Direct Catalytic Asymmetric Mannich-Type Reaction of Hydroxyketone Using a $\mathrm{Et}_{2} \mathrm{Zn} /$ Linked-BINOL Complex: Synthesis of either anti- or syn- $\beta$-Amino Alcohols. J. Am. Chem. Soc. 2004, 126, 8777-8785.

(240) Kobayashi, S.; Ishitami, H.; Ueno, M. Catalytic Asymmetric Synthesis of Both syn- and anti- $\beta$ Amino Alcohols. J. Am. Chem. Soc. 1998, 120, 431-432.

(241) Trost, B. M.; Jaratjaroonphong, J.; Reutrakul, V. A Direct Catalytic Asymmetric Mannich-Type Reaction via a Dinuclear Zinc Catalyst: Synthesis of Either anti- or syn- $\alpha$-Hydroxy- $\beta$-Amino Ketones. $J$. Am. Chem. Soc. 2006, 128, 2778-2279.

(242) Harada, S.; Handa, S.; Matsunaga, S.; Shibasaki, M. Direct Catalytic Asymmetric Mannich-Type Reactions of $\mathrm{N}$-(2-Hydroxyacetyl)pyrrole as an Ester-Equivalent Donor. Angew. Chem. Int. Ed. 2005, 44, 4365-4368.

(243) Yan, X.-X.; Peng, Q.; Li, Q.; Zhang, K.; Yao, J.; Hou, X.-L.; Wu, Y.-D. Highly Diastereoselective Switchable Enantioselective Mannich Reaction of Glycine Derivatives with Imines. J. Am. Chem. Soc. 2008, 130, 14362-14363.

(244) Nojiri, A.; Kumagai, N.; Shibasaki, M. Asymmetric Catalysis via Dynamic Substrate/Ligand/Rare Earth Metal Conglomerate. J. Am. Chem. Soc. 2008, 130, 5630-5631.

(245) Nojiri, A.; Kumagai, N.; Shibasaki, M. Linking Structural Dynamics and Functional Diversity in Asymmetric Catalysis. J. Am. Chem. Soc. 2009, 131, 3779-3784.

(246) Sohtome, Y.; Nagasawa, K. Sequential Stereodivergent Organic Catalysis and Programmed Organocascades. Org. Biomol. Chem. 2014, 12, 1681-1685.

(247) Lu, G.; Yoshino, T.; Marimoto, H.; Matsunaga, S.; Shibasaki, M. Stereodivergent Direct Catalytic Asymmetric Mannich-Type Reactions of $\alpha$-Isothiocyanato Ester with Ketimines. Angew. Chem. Int. Ed. 2011, 50, 4382-4385.

(248) Matsunaga, S.; Yoshino, T. Construction of Contiguous Tetrasubstituted Chiral Carbon Stereocenters via Direct Catalytic Asymmetric and Mannich Reactions. Chem. Rec. 2011, 11, 260-268.

(249) Kumagai, N.; Shibasaki, M. Asymmetric Catalysis with Bis(hydroxyphenyl)diamides/Rare Earth Metal Complexes. Angew. Chem. Int. Ed. 2013, 52, 223-224.

(250) Liu, Z.; Shi, M. Efficient Chirality Switching in the Asymmetric Addition of Indole to $\mathrm{N}$ Tosylarylimines in the Presence of Axially Chiral Cyclometallated Bidentate N-Heterocyclic Carbene Palladium(II) Complexes. Tetrahedron: Asymmetry 2009, 20, 119-123.

(251) Matsubara, R.; Berthiol, F.; Nguyen, H. V.; Kobayashi, S. Catalytic Mannich-Type Reactions of Sulfonylimidates. Bull. Chem. Soc. Jpn. 2009, 9, 1083-1102.

(252) List, B. The Direct Catalytic Asymmetric Three-Component Mannich Reaction. J. Am. Chem. Soc. 2000, 122, 9336-9337.

(253) Yang, J. W.; Chandler, C.; Stadler, M.; Kampen, D.; List, B. Proline-Catalysed Mannich Reactions of Acetaldehyde. Nature 2008, 452, 453-456.

(254) Poulsen, T. B.; Alemparte, C.; Saaby, S.; Bella, M.; Jørgensen, K. A. Direct Organocatalytic and Highly Enantio- and Diastereoselctive Mannich Reactions of $\alpha$-Substituted $\alpha$-Cyanoacetates. Angew. Chem. Int. Ed. 2005, 44, 2896-2899.

(255) Hatano, M.; Moriyama, K.; Maki, T.; Ishihara, K. Which is the Actual Catalyst: Chiral Phosphoric Acid or Chiral Calcium Phosphate. Angew. Chem. Int. Ed. 2010, 49, 3823-3826.

(256) Rueping, M.; Koenigs, R. M.; Atodiresei, I. Unifying Metal and Brønsted Acid Catalysis - Concepts, Mechanisms, and Classifications. Chem.- Eur. J. 2010, 16, 9350-9365.

(257) Hahn, B. T.; Frölich, R.; Harms, K.; Glorius, F. Proline-Catalyzed Highly Enantioselective and anti-Selective Mannich Reaction of Unactivated Ketones: Synthesis of Chiral $\alpha$-Amino Acids. Angew. Chem. Int. Ed. 2008, 47, 9985-9988. 
(258) Sohtome, Y.; Tanaka, S.; Takada, K.; Yamaguchi, T.; Nagasawa, K. Solvent-Dependent Enantiodivergent Mannich-Type Reaction: Utilizing a Conformationally Flexible Guanidine/Bisthiourea Organocatalyst. Angew. Chem. Int. Ed. 2010, 49, 9254-9257.

(259) Sohtome, Y.; Nagasawa, K. Dynamic Asymmetric Organocatalysis: Cooperative Effects of Weak Interactions and Conformational Flexibility in Asymmetric Organocatalysts. Chem. Commun. 2012, 48, 7777-7789.

(260) Sohtome, Y.; Yamaguchi, T.; Tanaka, S.; Nagasawa, K. Sequential Enantiodivergent Organocatalysis: Reversibility in Enantioswitching Controlled by a Conformationally Flexible Guanidine/Bisthiourea Organocatalyst. Org. Biomol. Chem. 2013, 11, 2780-2786.

(261) Zheng, M.; Liu, Y.; Wang, C.; Liu, S.; Lin, W. Cavity-Induced Enantioselectivity Reversal in a Chiral Metal-Organic Framework Brønsted Acid Catalyst. Chem. Sci. 2012, 3, 2623-2627.

(262) Singh, A.; Yodar, R. A.; Shen, B.; Johnston, J. N. Chiral Proton Catalysis: Enantioselective Brønsted Acid Catalyzed Additions of Nitroacetic Acid Derivatives as Glycine Equivalents. J. Am. Chem. Soc. 2007, 129, 3466-3467.

(263) Singh, A.; Johnston, J. N. A Diastereo-and Enantioselective Synthesis of $\alpha$-Substituted syn- $\alpha, \beta$ Diamino Acids. J. Am. Chem. Soc. 2008, 130, 5866-5867.

(264) Lovick, H. M.; Michael, F. E. Reversal of Enantioselectivity Using Tethered Bisguanidine Catalysts in the aza-Henry Reaction. Tetrahedron Lett. 2009, 50, 1016-1019.

(265) Declerck, V.; Martínez, J.; Lamaty, F. Aza-Baylis-Hillman Reaction. Chem. Rev. 2009, 109, $1-48$.

(266) Shi, M.; Xu, Y.-M.; Shi, Y.-L. Catalytic Asymmetric Aza-Baylis-Hillman Reaction of $N$ Sulfonated Imines with Activated Olefins by Quinidine-Derived Chiral Amines. Chem.- Eur. J. 2005, 11, 1794-1802.

(267) Shi, M.; Qi, M.-J.; Liu, X.-G. Asymmetric Catalytic aza-Morita-Baylis-Hillman Reaction (azaMBH): An Interesting Functional Group-Caused Reversal of Asymmetric Induction. Chem. Commun. 2008, 6025-6027.

(268) Shi, M.; Xu, Y.-M. Catalytic Asymmetric Baylis-Hillman Reaction of Imines with Methyl Vinyl Ketone and Methyl Acrylate. Angew. Chem. Int. Ed. 2002, 41, 4507-4510.

(269) Abermil, N.; Masson, G.; Zhu, J. Invertible Enantioselectivity in 6'-Deoxy-6'-acylamino- $\beta$ isocupreidine-Catalyzed Asymmetric Aza-Morita-Baylis-Hillman Reaction: Key Role of Achiral Additive. Org. Lett. 2009, 11, 4648-4651.

(270) Gröger, H. Catalytic Enantioselective Strecker Reactions and Analogous Syntheses. Chem. Rev. 2003, 103, 2795-2828.

(271) Berkessel, A.; Mukherjee, S.; Lex, J. Reversal of Enantioselectivity by Catalyst Protonation: Asymmetric Hydrocyanation of Imines with Oxazaborolidines. Synlett 2006, 41-44.

(272) Wang, H.; Zhao, X.; Li, Y.; Lu, L. Solvent-Controlled Asymmetric Strecker Reaction: Steroselective Synthesis of $\alpha$-Trifluoromethyated $\alpha$-Amino Acids. Org. Lett. 2006, 8, 1379-1381.

(273) Masumoto, S.; Usuda, H.; Suzuki, M.; Kato, N.; Kanai, M.; Shibasaki, M. Catalytic Enantioselective Strecker Reaction of Ketoimines. J. Am. Chem. Soc. 2003, 125, 5634-5635.

(274) Fukuda, N.; Sasaki, K.; Sastry; T. V. R. S.; Kanai, M.; Shibasaki, M. Catalytic Asymmetric Total Synthesis of (+)-Lactacystin. J. Org. Chem. 2006, 71, 1220-1225.

(275) Kato, N.; Mita, T.; Kanai, M.; Therrien, B. Kawano, M.; Yamaguchi, K.; Danjo, H.; Sei, Y.; Sato, A.; Furusho, S.; Shibasaki, M. Assembly State of Catalytic Molecules as Chiral Switches in Asymmetric Strecker Amino Acid Synthesis. J. Am. Chem. Soc. 2006, 128, 6768-6769.

(276) Foubelo, F.; Yus, M. Catalytic Asymmetric Transfer Hydrogenation of Imines: Recent Advances. Chem. Rec. 2015, 15, 907-924.

(277) Shimizu, M.; Tsukamoto, K.; Fujisawa, T. Stereodivergent Approach to syn and anti-2-Amino1,2-diarylethanols Using Oxazaborolidine-Mediated Asymmetric Reduction. Tetrahedron Lett. 1997, 38, 5193-5196. 
(278) Buriak, J. M.; Osborn, J. A. Studies on Catalytic Asymmetric Imine Hydrogenation in the Presence of Reverse Micelles: Enhanced Enantioselectivity due to Surfactant Head Group Coordination. Organometallics 1996, 15, 3161-3169.

(279) Mori, T.; Itakura, T.; Akiyama, T. Enantiodivergent Atroposelective Synthesis of Chiral Biaryls by Asymmetric Transfer Hydrogenation: Chiral Phosphoric Acid Catalyzed Dynamic Kinetic Resolution. Angew. Chem. Int. Ed. 2016, 55, 11642-11646.

(280) Sibi, M. P.; Maryem, S. Enantioselective Conjugate Additions, Tetrahedron 2000, 56, 8033-8061.

(281) Krause, N.; Hoffmann-Röder, A. Recent Advances in Catalytic Enantioselective Michael Additions. Synthesis 2001, 171-196.

(282) Almaşi, D.; Alonso, D. A.; Nájera, C. Organocatalytic Asymmetric Conjugate Additions. Tetrahedron: Asymmetry 2007, 18, 299-365.

(283) Tsogoeva, S. B. Recent Advances in Asymmetric Organocatalytic 1,4-Conjugate Additions. Eur. J. Org. Chem. 2007, 1701-1716.

(284) Mukherjee, S.; Yang, J. W.; Hoffmann, S.; List, B. Asymmetric Enamine Catalysis. Chem. Rev. 2007, 107, 5471-5568.

(285) Alexakis, A.; Bäckvall, J. E.; Krause, N.; Pamiés, O.; Diéguez, M. Enantioselective CopperCatalyzed Conjugate Addition and Allylic Substitution Reactions. Chem. Rev. 2008, 108, 2796-2823.

(286) Vicario, J. L.; Badía, D.; Carrillo, L.; Reyes, E. Organocatalytic Enantioselective Conjugate Addition Reactions. A Powerful Tool for the Stereocontrolled Synthesis of Complex Molecules, RSC Publishing: Cambridge, 2010.

(287) Nas, N. Enamine Catalysis of Michael Reactions. In Science of Synthesis, Asymmetric Organocatalysis 1. Lewis Base and Acid Catalysis, List, B., Ed.; Thieme: Stuttgart 2012, pp 135-216.

(288) MacMillan, D. W. C.; Watson, A. J. B. Iminium Catalysis. In Science of Synthesis, Asymmetric Organocatalysis 1. Lewis Base and Acid Catalysis, List, B., Ed.; Thieme: Stuttgart 2012, pp 551-599.

(289) Kanai, M.; Koga, K.; Tomioka, K. Solvent Effect and NMR Behavior in a Chiral Amidophosphine Mediated Reaction of Organocuprate with Chalcone. J. Chem. Soc., Chem. Commun. 1993, 1248-1249.

(290) Nakagawa, Y.; Kanai, M.; Nagaoka, Y.; Tomioka, K. Structural Requirements for an External, Chiral Amidophosphine Ligand for Asymmetric Reaction of and Organocopper Reagent. Tetrahedron Lett. 1996, 37, 7805-7808.

(291) Nakagawa, Y.; Kanai, M.; Nagaoka, Y.; Tomioka, K. An External Chiral Amidophosphine Ligand for Asymmetric Conjugate Addition of Organocopper. Tetrahedron 1998, 54, 10295-10307.

(292) Kanai, M.; Nagaoka, Y.; Tomioka, K. An Asymmetric Conjugate Addition Reaction of Lithium Organocopper Reagent Controlled by a Chiral Amidophosphine. Tetrahedron 1999, 55, 3831-3842.

(293) Lum, T.-K.; Wang, S.-Y.; Loh, T.-P. A Highly Catalytic Asymmetric Conjugate Addition: Synthesis of C14-C20 Fragment of Antibiotic TMC-151A, Siphonarienal and Siphonarienone. Org. Lett. 2008, 10, 761-764.

(294) Lagoutte, R.; Šebesta, P.; Jiroš, P.; Kalinová, B.; Jirošova, A.; Straka, J.; Černá, K.; Šobotnik, J.; Cračka, J.; Jahn, U. Total Synthesis, Proof of the Absolute Configuration, and Biosynthetic Origin of Stylopsal, the First Isolated Sex Pheromone of Strepsiptera. Chem.- Eur. J. 2013, 19, 8515-8524.

(295) Schuppan, J.; Minnard, A. J.; Feringa, B. L. A Catalytic and Iterative Route to $\beta$-Unsubstituted Esters via Highly Enantioselective Conjugate addition of Dimethylzinc to Unsaturated Malonates. Chem. Commun. 2004, 792-793.

(296) Yu, H.; Xie, F.; Ma, Z.; Liu, Y.; Zhang, W. The Effects of Solvent on Switchable Stereoselectivity: Copper-Catalyzed Asymmetric Conjugate Additions Using $D_{2}$-Symmetric Biphenyl Phosphoramidite Ligands. Org. Biomol. Chem. 2012, 10, 5137-5142.

(297) Anderson, J. C.; Stepney, G. J.; Mills, M. R.; Horsfall, L. R.; Blake, A. J.; Lewis, W. Enantioselective Conjugate Addition Nitro-Mannich Reactions: Solvent Controlled Synthesis of Acyclic anti- and syn- $\beta$-Nitroamines with Three Contiguous Stereocenters. J. Org. Chem. 2011, 76, 1961-1971.

(298) Côté, A.; Lindlay, V. N. G.; Charette, A. B. Application of the Chiral Bis(phosphine) Monoxide Ligand to Catalytic Enantioselective Addition of Dialkylzinc Reagents to $\beta$-Nitroalkenes. Org. Lett. 2007, 9, 85-87. 
(299) Mampreian, D. M.; Hoveyda, A. H. Efficient Cu-Catalyzed Asymmetric Conjugate Addition of Alkylzinc Reagents to Aromatic and Aliphatic Acyclic Nitroalkenes. Org. Lett. 2004, 6, 2829-2832.

(300) Yu, H.; Xie, F.; Ma, Z.; Liu, Y.; Zhang, W. Switchable Stereoselectivity: The Effects of Substituents on the $D_{2}$-Symmetric Biphenyl Backbone of Phosphoramidites in Copper-Catalyzed Asymmetric Conjugate Addition Reactions with Triethylaluminium. Adv. Synth. Catal. 2012, 354, 1941-1947.

(301) Evans, D. A.; Rovis, T.; Kozlowski, M. C.; Tedrow, J. S. $C_{2}$-SymmetricCu(II) Complexes as Chiral Lewis Acids. Catalytic Enantioselective Michael Addition of Silylketene Acetals to Alkylidene Malonates. J. Am. Chem. Soc. 1999, 121, 1994-1995.

(302) Evans, D. A.; Rovis, T.; Kozlowski, M. C.; Downey, C. W.; Tedrow, J. S. Enantioselective Lewis Acid Catalyzed Michael Reactions of Alkylidene Malonates. Catalysis by $C_{2}$-Symmetric Bis(oxazoline)Copper(II) Complexes in the Synthesis of Chiral, Differentiated Glutarate Esters. J. Am. Chem. Soc. 2000, 122, 9134-9142.

(303) Johnson, J. S.; Evans, D. A. Chiral Bis(oxazoline) Copper(II) Complexes: Versatile Catalysts for Enantioselective Cycloaddition, Aldol, Michael, and Carbonyl Ene Reactions. Acc. Chem. Res. 2000, 33, 325-335.

(304) Desimoni, G.; Faita, G.; Guala, M.; Laurenti, A.; Mella, M. A New Pyridine-2,6-bis(oxazoline) for Efficient and Flexible Lanthanide-Based Catalysis of Enantioselective Reactions with 3-Alkenoyl-2oxazolidinones. Chem.- Eur. J. 2005, 11, 3816-3824.

(305) Duursma, A.; Peña, D.; Minnard, A. J.; Feringa, B. L. Improved Catalytic Asymmetric CarbonCarbon Bond Formation Using Combinations of Chiral and Achiral Monodentate Ligands. Tetrahedron: Asymmetry 2005, 16, 1901-1904.

(306) Mechler, M.; Peters, R. Diastereodivergent Asymmetric 1,4-Addition of Oxindoles to Nitroolefins by Using Polyfunctional Nickel-Hydrogen-Bond-Azolium Catalysts. Angew. Chem. Int. Ed. 2015, 54, 10303-10307.

(307) Koizumi, A.; Kimura, M.; Arai, Y.; Tokoro, Y.; Fukuzawa, S. Copper- and Silver-Catalyzed Diastereo- and Enantioselective Conjugate Addition Reaction of 1-Pyrroline Esters to Nitroalkenes: Diastereoselectivity Switch by Chiral Metal Complexes. J. Org. Chem. 2012, 80, 10883-10891.

(308) Mortezaei, S.; Catarineu, N. R.; Canary, J. W. A Redox-Reconfigurable, Ambidextrous Asymmetric Catalysis. J. Am. Chem. Soc. 2012, 134, 8054-8057.

(309) Wang, Z.; Yang, Z.; Chen, D.; Liu, X.; Lin, L.; Feng, X. Highly Enantioselective Michael Addition of Pyrazolin-5-ones Catalyzed by Chiral Metal/ $N, N$ '-Dioxide Complexes: Metal-Directed Switch in Enantioselectivity. Angew. Chem. Int. Ed. 2011, 50, 4928-4932.

(310) Kawato, Y.; Takahashi, N.; Kumagai, N.; Shibasaki, M. Catalytic Asymmetric Conjugate Addition of $\alpha$-Cyanoketones for the Construction of a Quaternary Stereogenic Center. Org. Lett. 2010, 12, 1484-1487.

(311) Kanemasa, S.; Oderaottoshi, Y.; Wada, E. Asymmetric Conjugate Addition of Thiols to a 3-(2Alkenoyl)-2-oxazolidinone Catalyzed by the DBFOX/Ph Aqua Complex of Nickel(II) Perchlorate. J. Am. Chem. Soc. 1999, 121, 8675-8676.

(312) Sibi, M. P.; Shay, J. J.; Liu, M.; Jasperse, C. P. Chiral Lewis Acid Catalysis in Conjugate Addition of $O$-Benzylhydroxylamine to Unsaturated Amides. Enantioselective Synthesis of $\beta$-Amino Acid Precursors. J. Am. Chem. Soc. 1998, 120, 6615-6616.

(313) Sibi, M. P.; Gorikunti, U.; Liu, M. Temperature-Dependent Reversal of Sterochemistry in Enantioselective Conjugate Amine Additions. Tetrahedron 2002, 58, 8357-8363.

(314) Chew, R. J.; Li, X.-R.; Li, Y. Pullakart, S. A.; Leung, P.-H. Pd-Catalyzed Enantiodivergent and Regiospecific Phospha-Michael Addition of Diphenylphosphine to 4-Oxoenamides: Efficient Access to Chiral Phosphinocarboxamides and Their Analogues. Chem.- Eur. J. 2015, 21, 4800-4804.

(315) Sibi, M. P.; Shay, J. J.; Ji, J. Enantioselective Intermolecular Free Radical Conjugate Additions. Application of a Pyrazole Template. Tetrahedron Lett. 1997, 38, 5955-5958. 
(316) Murakata, M.; Tsutsui, H.; Hoshima, O. Unprecedent Effects of Achiral Oxazolidinones on Enantioselective Radical-Mediated Conjugate Additions Using a Chiral Zinc Triflate. Org. Lett. 2001, 3, 299-302.

(317) Sibi, M. P.; Cgen, J. Enantioselective Tandem Radical Reactions: Vicinal Difunctionalization in Acyclic Systems with Control over Relative and Absolute Stereochemistry. J. Am. Chem. Soc. 2001, 123, 9472-9473.

(318) Zhou, J.; Ye, M.-C.; Huang, Z.-Z.; Tang, Y. Controllable Enantioselective Friedel-Crafts Reaction Between Indoles and Alkylidene Malonates Catalyzed by Pseudo- $C_{3}$-SymmetricTrisoxazoline Copper(II) Complexes. J. Org. Chem. 2004, 69, 1309-1320.

(319) Liu, Y. L.; Shang, D.; Zhou, X.; Zhu, Y.; Lin, L.; Liu, X. H.; Feng, X. AgAsF $F_{6} / \operatorname{Sm}(\mathrm{OTf})_{3}$ Promoted Reversal of Enantioselectivity for the Asymmetric Friedel-Crafts Alkylations of Indoles with $\beta, \gamma$-Unsaturated $\alpha$-Ketoesters. Org. Lett. 2010, 12, 180-183.

(320) Liu, Y. L.; Shang, D.; Zhou, X.; Liu, X. H.; Feng, X. Enantioselective Friedel-Crafts Alkylations of Indoles with Alkylidene Malonates Catalyzed by $N, N$ '-Dioxide-Scandium(III) Complexes: Asymmetric Synthesis of $\beta$-Carbolines. Chem.- Eur. J. 2009, 15, 2055-2058.

(321) Kim, H. Y.; Kim, S.; Oh, K. Orthogonal Enantioselectivity Approaches Using Homogeneous and Heterogeneous Catalyst Systems: Friedel-Crafts Alkylation of Indole. Angew. Chem. Int. Ed. 2010, 49, 4476-4478.

(322) For a review, see: Bandini, M. Eichholzer, A. Catalytic Functionalization of Indoles in a New Dimension. Angew. Chem. Int. Ed. 2009, 48, 9608-9644.

(323) Zhang, Y.; Yang, N.; Liu, X.; Guo, J.; Zhang, X.; Lin, L.; Hu, C.; Feng, X. Reversal of Enantioselectivity Friedel-Crafts C3-Alkylation of Pyrrole by Slightly Tuning the Amide Units of $N, N^{\prime}$ Dioxide Ligands. Chem. Commun. 2015, 51, 8432-8435.

(324) Dere, R. T.; Pal, R. R., Patil, P. S.; Salunkhe, M. M. Influence of Ionic Liquids on the Phase Transfer-Catalysed Enantioselective Michael Reaction. Tetrahedron 2003, 44, 5351-5353.

(325) Vijaya, P. K.; Murugesan, S.; Siva, A. Unexpected Solvent/Substitution-Dependent Inversion of the Enantioselectivity in Michael Addition Reaction Using Chiral Phase Transfer Catalysts. Tetrahedron 2015, 56, 5209-5212.

(326) Wang, B.; Wu, F.; Wang, Y.; Liu, X.; Deng, L. Control of the Diastereoselectivity in Tandem Asymmetric Reactions Generating Nonadjacent Stereocenters with Bifunctional Catalysis by Cinchona Alkaloids. J. Am. Chem. Soc. 2007, 129, 768-769.

(327) Hua, M.-Q.; Cui, H.-F.; Wang, L.; Nie, J.; Ma, J.-A. Reversal of Enantioselectivity by Tuning the Conformational Flexibility of Phase-Transfer Catalysts. Angew. Chem. Int. Ed. 2010, 49, 2772-2776.

(328) Shirakawa, S.; Maruoka, K. Phase Transfer Catalysis: Non-Natural-Product-Derived PTC. In Science of Synthesis Asymmetric Organocatalysis 2. Brønsted Base and Acid Catalysts, and Additional Topics. Maruoka, K., Ed.; Thieme: Stuttgart, 2012, pp 551-599.

(329) Rai, V.; Namboothiri, I. N. N. Effect of Achiral and Mixed Chiral Ligands on the Asymmetric Synthesis of $\gamma$-Nitrophosphonates via Michael Addition. Tetrahedron: Asymmetry 2008, 19, 767-772.

(330) Matsunaga, H.; Tajima, D.; Kawauchi, T.; Yasuyama, T.; Ando, S.; Ishizuka, T. A. A Dramatic Synergistic Effect of a Flexible Achiral Linker on a Rigid Chiral cis-1,2-Diamine Bifunctional Organocatalyst. Org. Biomol. Chem. 2017, 15, 2892-2896.

(331) Wei, Y.; Wen, S.; Liu, Z.; Wu, Z.; Wu, X.; Zeng, B.; Ye, J. Diastereodivergent Catalytic Asymmetric Michael Addition of 2-Oxindoles to $\alpha, \beta$-Unsaturated Ketones by Chiral Diamine Catalysts. Org. Lett. 2015, 17, 2732-2735.

(332) Qian, Y.; Xiao, S.; Liu, L.; Wang, Y. A Mild and Efficient Procedure for Asymmetric Michael Additions of Cyclohexanone to Chalcones Catalyzed by an Amino Acid Ionic Liquid. Tetrahedron: Asymmetry 2008, 19, 1515-1518.

(333) Demir, A. S.; Basceken, S. Self-Assembly of an Organocatalyst for the Enantioselective Synthesis of Michael Adducts and $\alpha$-Aminoxy Alcohols in a Nonpolar Medium. Tetrahedron: Asymmetry 2013, 24, $1218-1224$. 
(334) Flores-Ferrándiz, J.; Fiser, B.; Gómez-Bengoa, E.; Chinchilla, R. Solvent-Induced Reversal of Enantioselectivity in the Synthesis of Succinimides by the Addition of Aldehydes to Maleimides Catalyzed by Carbamate-Monoprotected 1,2-Diamines. Eur. J. Org. Chem. 2015, 1218-1225.

(335) Vizcaíno-Milla, P.; Sansano, J. M.; Nájera, C.; Fiser, B.; Gómez-Bengoa, E. Primary Amine-2Aminopyrimidine Chiral Organocatalysts for the Enantioselective Conjugate Addition of Branched Aldehydes to Maleimides. Synthesis 2015, 47, 2199-2206.

(336) Tian, X.; Cassani, C.; Liu, Y.; Morán, A.; Urakawa, A.; Galzerano, P.; Arceo, E.; Melchiorre, P. Diastereodivergent Asymmetric Sulfa-Michael Additions of $\alpha$-Branched Enones Using a Single Organic Catalyst. J. Am. Chem. Soc. 2011, 133, 17934-17941.

(337) Kuchurov, I. V.; Nigmatov, A. G.; Kryuchova, E. V.; Kostenko, A. A.; Kucherenko, A. S.; Zlotin, S. G. Stereodivergent Michael Addition of Diphenyl Phosphite to $\alpha$-Nitroalkenes in the Presence of Squaramide-Derived Tertiary Amines: An Enantioselective Organocatalytic Reaction in Supercritical Carbon Dioxide. Green Chem. 2014, 16, 1521-1526.

(338) Huang, Y.; Walji, A. M.; Larsen, C. H., MacMillan, D.W. C. Enantioselective Organo-Cascade Catalysis. J. Am. Chem. Soc. 2005, 127, 15051-15053.

(339) Simmons, B.; Walji, A. M.; MacMillan, D. W. C. Cycle-Specific Organocascade Catalysis: Application to Olefin Hydroamination, Hydro-oxidation, and Amino-oxidation, to Natural Product Synthesis. Angew. Chem. Int. Ed. 2009, 48, 4349-4353.

(340) Genet, J.-P.; Reduction of Functionalized Alkenes. In Modern Reduction Methods. Andersson, P. G.; Munslow, I. J., Eds.; Wiley-VCH: Weinheim 2008, pp 1-38.

(341) Diesen, J. S.; Hydrogenation of Unfunctionalized Alkenes. In Modern Reduction Methods. Andersson, P. G.; Munslow, I. J., Eds.; Wiley-VCH: Weinheim 2008, pp 39-64.

(342) Kuwano, R.; Sawamura, M.; Ito, Y. Catalytic Asymmetric Hydrogenation of $\alpha$ (Acetamido)acrylates Using TRAP trans-Chelating Chiral Bisphosphine Ligands: Remarkable Effects of Ligand P-Substituents and Hydrogen Pressure on Enantioselectivity. Bull. Chem. Soc. Jpn. 2000, 73, 2571-2578.

(343) Reetz, M. T.; Mehler, G. Mixtures of Chiral and Achiral Monodentate Ligands in Asymmetric RhCatalyzed Olefin Hydrogenation: Reversal of Enantioselectivity. Tetrahedron Lett. 2003, 44, 4593-4596.

(344) Arribas, I.; Vargas, S.; Rubio, M.; Suárez, A.; Domene, C.; Alvarez, E.; Pizzano, A. Chiral Phosphine-Phosphite Ligands with a Substituted Ethane Backbone. Influence of Conformational Effects in Rhodium-Catalyzed Asymmetric Olefin Hydrogenation and Hydroformylation Reactions. Organometallics 2010, 29, 5791-5804.

(345) Colston, N. J.; Wells, R. P. K.; Wells, P. B.; Hutchings, G. J. Unexpected Inversion in the Enantioselectivity in the Hydrogenation of $N$-Acetyl Dehydrophenylalanine Methyl Ester Using Cinchona-Modified $\mathrm{Pd} / \mathrm{Al}_{2} \mathrm{O}_{3}$ Catalyst. Cat. Lett. 2005, 103, 117-120.

(346) Nita, Y.; Shibata, A. Enantioselective Hydrogenation of (E)- $\alpha$-Phenylcinnamic Acid on $\mathrm{Pd} / \mathrm{TiO}_{2}$ Catalyst Modified by Cinchona Alkaloids: Effects of Modifier Structure. Chem. Lett. 1998, 161-162.

(347) Szöllősi, G.; Busygin, I.; Hermán, B.; Leino, I.; Murzin, D. Y.; Fülop, F.; Bartók, M. Inversion of the Enantioselectivity in the Hydrogenation of (E)-2,3-Diphenylpropenoic Acids over Pd Modified by Cinchonidine Silyl Ethers. ACS Catal. 2011, 1, 1316-1326.

(348) Sun, Y.; Leblond, C.; Wang, J.; Blackmond, D. G.; Laquidara, J.; Sowa, J. R., Jr. Observation of a $\left[\mathrm{RuCl}_{2}((\mathrm{~S})-(-) \text {-tol-BINAP) }]_{2} \cdot \mathrm{N}\left(\mathrm{C}_{2} \mathrm{H}_{5}\right)_{3}\right.$-Catalyzed Isomerization-Hydrogenation Network. J. Am. Chem. Soc. 1995, 117, 12647-12648.

(349) Zhu, Y.; Burgess, K. Asymmetric Hydrogenation Approaches to Valuable Acyclic 1,3Hydroxymethyl Chirons. J. Am. Chem. Soc. 2008, 130, 8894-8895.

(350) Pischl, M. C.; Weise, C. F.; Haseloff, S.; Müller, M.-A.; Pfaltz, A.; Schneider, C. A. Highly Steroselective and Flexible Strategy for the Convergent Synthesis of Long-Chain Polydeoxypropionates: Application Towards the Synthesis of Glycolipid Membrane Components Hydroxypthioceranic and Phthioceranic Acid. Chem.- Eur. J. 2014, 20, 17360-17374.

(351) Helmchen, G.; Pfaltz, A. Phosphinooxazolines-A New Class of Versatile, Modular, P,N-Ligands for Asymmetric Catalysis. Acc. Chem. Res. 2000, 33, 336-345. 
(352) Mengues, F.; Pfaltz, A. Threonine-Derived Phosphinite-Oxazoline Ligands for the Ir-Catalyzed Enantioselective Hydrogenation. Adv. Synth. Catal. 2002, 344, 40-44.

(353) Blankenstein, J.; Pfaltz, A. A New Class of Modular Phosphinite-Oxazoline Ligands: Ir-Catalyzed Enantioselective Hydrogenation of Alkenes. Angew. Chem. Int. Ed. 2001, 40, 4445-4447.

(354) Powell, M. T.; Hou, D.-R.; Perry, M. C.; Cui, X.; Burgess, K. Chiral Imidazolidine Ligands for Asymmetric Hydrogenation of Aryl Alkenes. J. Am. Chem. Soc. 2001, 123, 8878-8879.

(355) Perry, M. C.; Cui, M.; Powell, M. T.; Hou, D.-R.; Reibenspies, J. H.; Burgess, K. Optically Active Iridium Imidazol-2-ylidene-oxazoline Complexes: Preparation and Use in Asymmetric Hydrogenation of Arylalkenes. J. Am. Chem. Soc. 2003, 125, 113-123.

(356) Franke, R.; Selant, D.; Börner, A. Applied Hydroformylation. Chem. Rev. 2012, 112, 5675-5732.

(357) Kollár, L.; Bakos, J.; Tóth, I.; Heil, B. Temperature Dependence of the Asymmetric Induction in the $\quad \mathrm{PtCl}\left(\mathrm{SnCl}_{3}\right)[(-)-(2 S, 4 S)-2,4-\mathrm{Bis}($ diphenylphosphino)pentane]-Catalyzed Enantioselective Hydroformylation. J. Organomet. Chem. 1988, 350, 277-284.

(358) Casey, C. P.; Martins, S. C.; Fagan, M. A. Reversal of Enantioselectivity in the Hydroformylation of Styrene with [2S,4S-BDPP]Pt $\left(\mathrm{SnCl}_{3}\right) \mathrm{Cl}$ at High Temperature Arises from a Change in the Enantioselectivity-Determining Step. J. Am. Chem. Soc. 2004, 126, 5585-5592.

(359) Pongrácz, P.; Papp, T.; Kollár, L.; Kégl; T. Influence of the Substituents on the Reversal of Enantioselectivity in the Asymmetric Hydroformylation of 4-Substituted Styrenes with $\mathrm{PtCl}\left(\mathrm{SnCl}_{3}\right)[(2 S, 4 S)-\mathrm{BDPP}]$. Organometallics 2014, 33, 1389-1396.

(360) Wasernaar, J.; de Bruin, B.; Reek, J. N. H. Rhodium-Catalyzed Asymmetric Hydroformylation with Taddol-Based IndolPhos Ligands. Organometallics 2010, 29, 2767-2776.

(361) Rubio, M.; Suarez, A.; Alvarez, E.; Bianchini, C.; Oberhauser, W.; Peruzzini, M.; Pizzano, A. Asymmetric Hydroformylation of Olefins with Rh Catalysts Modified with Chiral Phosphine-Phosphite Ligands. Organometallics 2007, 26, 6428-6436.

(362) Hayashi, T.; Matsumoto, Y.; Ito, Y. Catalytic Asymmetric Hydroboration of Styrenes. J. Am. Chem. Soc. 1989, 111, 3426-3428.

(363) Smith; S. M.; Tackas, J. M. Remarkable Levels of Enantioswitching in Catalytic Asymmetric Hydroboration. Org. Lett.2010, 12, 4612-4615.

(364) Guo, H.; Shi, X.; Qiao, Z.; Hon, S.; Wang, M. Efficient Soluble Polymer-Supported Sharpless Alkene Epoxidation Catalysts. Chem. Commun. 2002, 118-119.

(365) Guo, H.; Shi, X.; Wang, X.; Liu, S.; Wang, M. Liquid-Phase Synthesis of Chiral Tartrate Ligand Library for Enantioselective Sharpless Epoxidation of Allylic Alcohols. J. Org. Chem. 2004, 69, 2042-2047.

(366) Reed, N. N.; Dickerson, T. J.; Boldt, G. E.; Janda, K. D. Enantioreversal in the Sharpless Asymmetric Epoxidation Reaction Controlled by the Molecular Weight of a Covalently Appended Achiral Polymer. J. Org. Chem. 2005, 70, 1728-1731.

(367) García, P. A.; Van Grieken, R.; Iglesias, J.; Sherrington, D. C.; Gibson, C. L. Modification of Chiral Dimethyl Tartrate Through Transesterification: Immobilization on POSS and Enantioselectivity Reversal in Sharpless Asymmetric Epoxidation. Chirality 2010, 22, 675-683.

(368) Fernández, A. I.; Fraile, J. M.; García, J. I.; Herrerías, C. I.; Mayoral, J. A.; Salvatella, L. Reversal of Enantioselectivity by Change of Solvent with Clay-Immobilized Bis(oxazoline) Copper Catalysts. Cat. Commun. 2001, 2, 165-170.

(369) García, J. L.; López-Sánchez, B.; Mayoral, J. A.; Pires, E.; Villalba, I. Surface Confinement Effects in Enantioselective Catalysis: Design of New Heterogeneous Chiral Catalysts: Design of New Heterogeneous Chiral Catalysts Based on $\mathrm{C}_{1}$-Symmetric Bisoxazolines and their Application in Cyclopropanation Reactions. J. Catal. 2008, 258, 378-385.

(370) Castillo, M. R.; Fousse, L.; Fraile, J. M.; García, J. I.; Mayoral, J. A. Supported Ionic-Liquid Films (SILF) as Two-Dimensional Nanoreactors for Enantioselective Reactions: Surface-Mediated Selectivity Modulation. Chem.- Eur. J. 2007, 13, 287-291. 
(371) Cornejo, A.; Fraile, J. M.; García, J. I.; Gil, M. J.; Herrerías, C. I.; Legarreta, G.; Martínez-Merino, V.; Mayoral, J. A. Surface-Mediated Improvement of Enantioselectivity with Clay-Immobilized Copper Catalysts. J. Mol. Catal. A: Chem. 2003, 196, 101-108.

(372) Nájera, C.; Sansano, J. M. Catalytic Asymmetric Synthesis of $\alpha$-Amino Acids. Chem. Rev. 2007, 107, 4584-4671.

(373) Park, H.-G. Phase-Transfer Catalysis: Natural Product-Derived PTC. In Science of Synthesis, Asymmetric Organocatalysis2. Brønsted Base and Acid Catalysts, and Additional Topics.Maruoka, K., Ed.; Thieme: Stuttgart, 2012, pp 499-549.

(374) Mazón, P.; Chinchilla, R.; Nájera, C.; Guillena, G.; Kreiter, R.; Gebbink, R. J. M. K.; van Koten, G. Unexpected Metal Base-Dependent Inversion of the Enantioselectivity in the Asymmetric Synthesis of $\alpha$-Amino Acids using Phase-Transfer Catalysts Derived from Cinchonidine. Tetrahedron: Asymmetry 2002, 13, 2181-2185.

(375) Porter, N. A.; Hongliu, J.; Zhang, G.; Reed, A. D. Enantioselective Free Radical Allyl Transfers from Allylsilanes Promoted by Chiral Lewis Acids. J. Org. Chem. 1997, 62, 6702-6703.

(376) Kano, T.; Yamamoto, A.; Shirozu, F.; Maruoka, K. Enantioselectivity Switch in Direct Aminoxylation Catalyzed by Binaphthyl-Based Chiral Secondary Amines. Synthesis 2009, 1557-1563.

(377) Blackmond, D. G.; Moran, A.; Hughes, M.; Armstrong, A. Unusual Reversal of Enantioselectivity in the Proline-Mediated $\alpha$-Amination of Aldehydes Induced by Tertiary Amine Additives. J. Am. Chem. Soc. 2010, 132, 7598-7599.

(378) Hein, J. E.; Armstrong, A.; Blackmond, D. G. Kinetic Profiling of Prolinate-Catalyzed $\alpha$ Amination of Aldehydes Org. Lett. 2011, 13, 4300-4303.

(379) Hein, J. E.; Burés. J.; Lam, Y.-h.; Hughes, M.; Houk, K. N.; Armstrong, A.; Blackmond, D. G. Enamine Carboxylates as Stereodetermining Intermediates in Prolinate Catalysis. Org. Lett. 2011, 13, 5644-5647.

(380) Sharma, A. K.; Sunoj, R. B. Stereocontrol in Proline-Catalyzed Asymmetric Amination: a Comparative Assessment of the Role of Enamine Carboxylic Acid and Enamine Carboxylate. Chem. Commun. 2011, 47, 5759-5761.

(381) Fu, A.; Tian, C.; Li, H.; Chu T.; Wang, Z.; Liu, J. A Revisit to Proline-Catalyzed Amination under Basic Conditions: Insight into the Key Intermediates and Stereocontrolling Transition State Models for the Reversal of Enantioselectivity. Chem. Phys. 2015, 455, 65-72.

(382) Macharia, J.; Wambua, V.; Hong, Y.; Harris, L.; Hirschi, J. S.; Evans, G. B.; Vetticatt, M. J. A Designed Approach to Enantiodivergent Enamine Catalysis. Angew. Chem. Int. Ed. 2017, 56, 8756-8760. (383) Wang, S.-G.; yin, Q.; Zhuo, C.-X.; You, S.-L. Asymmetric Dearomatization of $\beta$-Naphthols through an Amination Reaction Catalyzed by a Chiral Phosphoric Acid. Angew. Chem. Int. Ed. 2015, 54, 647-650.

(384) Changotra, A.; Das, S.; Sunoj, R. B. Reversing Enantioselectivity Using Noncovalent Interactions in Asymmetric Dearomatization of $\beta$-Naphthols: The Power of 3,3' Substituents in Chiral Phosphoric Acid Catalysts. Org. Lett. 2017, 19, 2354-2357.

(385) You, Y.; Zhang, L.; Luo, S. Reagent-Controlled Enantioselectivity Switch for the Asymmetric Fluorination of $\beta$-Ketocarbonyls by Chiral Primary Amine Catalysis. Chem. Sci. 2017, 8, 621-626.

(386) Boudou, M.; Ogawa, C.; Kobayashi, S. Chiral Scandium-Catalyzed Enantioselective RingOpening of meso-Epoxides with N-Heterocycles. Adv. Synth. Catal. 2006, 348, 2585-2589.

(387) Kobuko, M.; Naito, T.; Kobayashi, S. Metal-Controlled Reversal of Enantioselectivity in Catalyzed Asymmetric Ring Opening Reactions of meso-Epoxides in Water. Chem. Lett. 2009, 38, 904-905.

(388) Kobuko, M.; Naito, T.; Kobayashi, S. Chiral Zinc(II) and Copper(II)-Catalyzed Asymmetric Ring Opening Reactions of meso-Epoxides with Aniline and Indole Derivatives. Tetrahedron 2010, 66, 1111-1118.

(389) Karadeniz, L.; Koz, G.; Aydin, K.; Astley, S. T. Co(III) Catalyzed Asymmetric Ring-Opening of Epichlorohydrin by Salicylaldehyde Derivatives: Reversal of Enantioselectivity and Rate Acceleration on Addition of $\mathrm{AlCl}_{3}$. Turk. J. Chem. 2010, 34, 711-718. 
(390) Mita, T.; Fujimori, I.; Wada, R.; Wen, J.; Kanai, M.; Shibasaki, M. Catalytic Enantioselective Desymmetrization of meso-N-Acylaziridines with TMSCN. J. Am. Chem. Soc. 2005, 127, 11252-11253.

(391) Fujimori, I.; Mita, T.; Maki, K.; Shiro, M.; Sato, A.; Furusho, S.; Kanai, M.; Shibasaki, M. Key Role of the Lewis Base Position in Asymmetric Bifunctional Catalysis: Design and Evaluation of a New Ligand for Chiral Polymetallic Catalysts. J. Am. Chem. Soc. 2006, 128, 16438-16439.

(392) Trost, B. M.; Crawley, M. L. Asymmetric Transition-Metal Catalyzed Allylic Alkylations: Applications in Total Synthesis. Chem. Rev. 2003, 103, 2921-2943.

(393) Lu, Z.; Ma, S. M. Metal-Catalyzed Enantioselective Allylation in Asymmetric Synthesis. Angew. Chem. Int. Ed. 2008, 47, 258-297.

(394) Norsikan, S.; Chang, C.-W. Control of the Regioselectivity in Palladium(0)-Catalyzed Allylic Alkylation. Curr. Org. Synth. 2009, 6, 264-289.

(395) Helmchen, G.; Kazmaier, U.; Förster; S. In Catalytic Asymmetric Synthesis, $3^{\text {rd }}$ Ed.; Ojima, I., Ed.; John Wiley \& Sons, Inc.: Hoboken, NJ, 2010, pp 497-641.

(396) Grange, R. L.; Clizbe, E. A.; Evans, P. A. Recent Developments in Asymmetric Allylic Amination Reactions. Synthesis 2016, 48, 2911-2968.

(397) Lucius, R.; Loos, R.; Mayr, H. Kinetic Studies of Carbocation-Carbanion Combinations: Key to a General Concept of Polar Organic Reactivity. Angew. Chem. Int. Ed. 2002, 41, 91-95.

(398) Nomura, N.; Mermet-Bouvier, Y. C.; RajanBabu, T. V. Unprecedent Electronic and Steric Effects in Palladium-Catalyzed Asymmetric Allylation: Switching of Enantioselectivity with a Single Chiral Backbone. Synlett 1996, 745-746.

(399) Clyne, D. S.; Mermet-Bouvier, Y. C.; Nomura, N.; RajanBabu, T. V. Substituent Effects of Ligands on Asymmetric Induction in a Prototypical Palladium-Catalyzed Allylation Reaction: Making Both Enantiomers of a Product in High Optical Purity Using the Same Source of Chirality. J. Org. Chem. 1999, 64, 7601-7611.

(400) Hoarau, O.; Aït-Haddou, H.; Daran, J.-C.; Cramallère, D.; Balavoine, G. G. A. Asymmetric Palladium-Catalyzed Allylic Alkylation Using Bis(oxazoline) Ligands: Phenomenal Reversal of Enantioselectivity with a Single Chiral Backbone. Organometallics 1999, 18, 4718-4723.

(401) Aït-Haddou, H.; Hoarau, O.; Cramallère, D.; Pezet, F.; Daran, J.-C.; Balavoine, G. G. A. New Dihydroxy Bis(oxazoline) Ligands for the Palladium-Catalyzed Asymmetric Allylic Alkylation: Experimental Investigations of the Origin of the Reversal of the Enantioselectivity. Chem.- Eur. J. 2004, 10, 699-707.

(402) Anderson, J. C.; Cubbon, R. J.; Harling, J. D. Dramatic Reversal of Enantioselection in a Palladium-Catalyzed Allylic Substitution by Choice of Nitrogen Substituents in a $N-P$ Chiral Ligand. Tetrahedron: Asymmetry 1999, 10, 2829-2832.

(403) Chelucci, G.; Gladialy, S.; Saba, A. Chiral Pyridiylmethyl- and Quinolinyl-Oxazolines as Ligands for Enantioselective Palladium-Catalyzed Allylic Alkylation. Tetrahedron: Asymmetry 1999, 10, 1393-1400.

(404) Chelucci, G.; Pinna, G. A.; Saba, A.; Valenti, R. Unexpected Reversal of the Enantioselectivity Using Chiral Quinolylmethyl- and Acridinyl-Oxazolines as Ligands for Asymmetric Palladium-Catalyzed Allylic Alkylation. Tetrahedron: Asymmetry 2000, 11, 4027-4036.

(405) Hou, X.-L.; Wu, X.-W.; Dai, L.-X.; Cao, B.-X.; Sun, J. Novel N,S and N,Se-Planar Chiral [2,2]Paracyclophane Ligands: Synthesis and Application in Pd-Catalyzed Allylic Alkylation. Chem. Commun. 2000, 1195-1196.

(406) Danjo, H.; Higuchi, M.; Yada, M.; Imamoto, T. P-Stereogenic P/N Hybrid Ligands: a Remarkable Switch in Enantioselectivity in Palladium-Catalyzed Asymmetric Allylation. Tetrahedron Lett. 2004, 45, 603-606.

(407) Ferioli, F.; Florelli, C.; Martelli, G.; Monari, M.; Savoia, D.; Tobaldin, P. Steric Effects in Enantioselective Allylic Alkylation Catalysed by Cationic ( $\eta^{3}$-Allyl)palladium Complexes Bearing Chiral Pyridine-Aziridine Ligands. Eur. J. Org. Chem. 2005, 1416-1426.

(408) Zhang, W.; Shi, M. Axially Chiral P,S-Heterodonor Ligands with a Binaphthalene Framework for Palladium-Catalyzed Asymmetric Allylic Substitutions: Experimental Investigation on the Reversal of 
Enantioselectivity Between Different Alkyl Groups on Sulfur Atom. Tetrahedron: Asymmetry 2004, 15, 3467-3476.

(409) Popa, D.; Puigjaner, C.; Gómez, M.; Benet-Buchholz, J.; Vidal-Ferran, A.; Pericàs, M. A. Phosphinooxazolines Derived from 3-Amino-1,2-diols: Highly Efficient Modular $P-N$ Ligands. Adv. Synth. Catal. 2007, 349, 2265-2278.

(410) Kanayama, T.; Yoshida, K.; Miyabe, H.; Takemoto, Y. Enantio- and Diastereoselective IrCatalyzed Allylic Substitution for Asymmetric Synthesis of Amino Acid Derivatives. Angew. Chem. Int. Ed. 2003, 42, 2054-2056.

(411) Lipkowitz, K. B.; Cavanaugh, M. W.; Baker, B.; O’Donnell, M. J. Theoretical Studies in Molecular Recognition: Asymmetric Induction of Benzophenone Imine Ester Enolates by the Benzylcinchoninium Ion. J. Org. Chem. 1991, 56, 5181-5192.

(412) Trost, B. M.; Schäffner, B.; Osipov, M.; Wilton, D. A. A. Palladium-Catalyzed Decarboxylative Asymmetric Allylic Alkylation of $\beta$-Ketoesters: An Unusual Counterion Effect. Angew. Chem. Int. Ed. 2011, 50, 3548-3551.

(413) Krautwald, S.; Sarlah, D.; Schafroth, M. A.; Carreira, E. M. Enantio- and Diastereodivergent Dual Catalysis: $\alpha$-Allylation of Branched Aldehydes. Science 2013, 340, 1065-1068.

(414) Bhaskararao, B.; Sunoj, R. B. Origin of Stereodivergence in Cooperative Asymmetric Catalysis with Simultaneous Involvement of Two Chiral Catalysts. J. Am. Chem. Soc. 2015, 137, 15712-15722.

(415) Schafroth, M. A.; Zuccarello, G.; Krautwald, S.; Sarlah, D.; Carreira, E. M. Stereodivergent Total Synthesis of $\Delta^{9}$-Tetrahydrocannabinols. Angew. Chem. Int. Ed. 2014, 53, 13988-13901.

(416) Sandmeier, T.; Krautwald, S.; Zipfel, H. F.; Carreira, E. M. Stereodivergent Dual Catalytic $\alpha-$ Allylation of Protected $\alpha$-Amino- and $\alpha$-Hydroxyacetaldehydes. Angew. Chem. Int. Ed. 2015, 54, 14363-14367.

(417) Krautwald, S.; Schafroth, M. A.; Sarlah, D.; Carreira, E. M. Stereodivergent $\alpha$-Allylation of Linear Aldehydes with Dual Iridium and Amine Catalysis. J. Am. Chem. Soc. 2014, 136, 3020-3025.

(418) Cruz, F. A.; Dong, V. M. Stereodivergent Coupling of Aldehydes and Alkynes via Synergistic Catalysis Using Rh and Jacobsen's Amine. J. Am. Chem. Soc. 2017, 139, 1029-1032.

(419) Huo, X.; He, R.; Zhang, X.; Zhang, W. An Ir/Zn Dual Catalysis for Enantio- and Diastereodivergent $\alpha$-Allylation of $\alpha$-Hydroxyketones. J. Am. Chem. Soc. 2016, 138, 11093-11096.

(420) Breitler, S.; Carreira, E. M. Formaldehyde $N, N$-Dialkylhydrazones as Neutral Formyl Anion Equivalents in Iridium-Catalyzed Asymmetric Allylic Substitution. J. Am. Chem. Soc. 2015, 137, 5296-5299.

(421) Hoshi, T.; Sasaki, K.; Sato, S.; Ishii, Y.; Suzuki, T.; Hagiwara, H. Highly Enantioselective PdCatalyzed Allylic Alkylation of Indoles Using Sulfur-MOP Ligand. Org. Lett. 2011, 13, 932-935.

(422) Togni, A.; Burckhardt, U.; Gramlich, V.; Pregosin, P. S.; Salzmann, R. Palladium-Catalyzed Asymmetric Allylic Amination Using Ferrocenyl Pyrazole Ligands: Steric Control of $\eta^{3}$-Allyl Configuration and Site-Selective Nucleophilic Attack. J. Am. Chem. Soc. 1996, 118, 1031-1037.

(423) Caminiti, N. S.; Goodstein, M. B.; Leobler, I. N.-M.; Holtzman, B. S.; Jia, Z. B.; Martini, M. L.; Nelson, N. C., Bunt, R. C. Reversible Nucleophilic Addition Can Lower the Observed Enantioselectivity in Palladium-Catalyzed Allylic Amination Reactions with a Variety of Chiral Ligands. Tetrahedron Lett. 2015, 56, 5445-5448.

(424) Xie, F.; Liu, D.; Zhang, W. Reversal in Enantioselectivity for the Palladium-Catalyzed Asymmetric Allylic Substitution with Novel Metallocene-Based Planar Chiral Diphosphine Ligands. Tetrahedron Lett. 2008, 49, 1012-1015.

(425) Kitagawa, O.; Matsuo, S.; Yotsumoto, K.; Taguchi, T. Catalytic Asymmetric Desymmetrization of meso-Diamide Derivatives through Enantioselective $N$-Allylation with a Chiral $\pi$-Allyl Pd Catalyst: Improvement and Reversal of Enantioselectivity. J. Org. Chem. 2006, 71, 2524-2527.

(426) Xing, J.; Cao, P.; Liao, J. Chiral SO/P Hybrid Ligands: an Enantioselective Switch in PalladiumCatalyzed Asymmetric Allylic Etherifications. Tetrahedron: Asymmetry 2012, 23, 527-535. 
(427) Zhou, Q.; Srinivas, H. D.; Zhang, S.; Watson, M. P. Accessing Both Retention and Inversion Pathways in Stereospecific Nickel-Catalyzed Miyaura Borylations of Allylic Pivalates. J. Am. Chem. Soc. 2016, 138, 11989-11995.

(428) Trost, B. M.; Ball, Z. Addition of Metalloid Hydrides to Alkynes: Hydrometallation with Boron, Silicon, and Tin. Synthesis 2005, 853-887.

(429) Pelter, A.; Smith, K.; Brown, H. C. Borane Reagents. Academic Press: New York 1988.

(430) Beletskaya, I. P.; Pelter, A. Hydroborations Catalyzed by Transition Metal Complexes. Tetrahedron 1997, 53, 4957-5026.

(431) Ohmura, T.; Yamamoto, Y.; Miyaura, N. Rhodium- or Iridium-Catalyzed trans-Hydroboration of Terminal Alkynes Giving (Z)-1-Alkenylboron Compounds. J. Am. Chem. Soc. 2000, 122, 4990-4991.

(432) Gunanathan, C.; Holscher, M.; Pan, F.; Leitner, W. Ruthenium Catalyzed Hydroboration of Terminal Alkynes to (Z)-Vinylboronates. J. Am. Chem. Soc. 2012, 134, 14349-14352.

(433) Cid, J.; Carbó, J. J.; Fernández, E. Catalytic Non-Conventional trans-Hydroboration: A Terminal and Experimental Perspective. Chem.- Eur. J. 2012, 18, 1512-1521.

(434) Burgess, K.; Van der Donk, W.; Westcott, S. A.; Marder, T. B.; Baker, R. T.; Calabrese, J. C. Reactions of Catecholborane with Wilkinson's Catalyst: Implications for Transition Metal-Catalyzed Hydroboration of Alkenes. J. Am. Chem. Soc. 1992, 114, 9350-9359.

(435) Pereira, S.; Srebnik, M. A. Study of Hydroboration of Alkenes and Alkynes with Pinacolborane Catalyzed by Transition Metals. Tetrahedron Lett. 1996, 37, 3283-3286.

(436) Matsumoto, Y.; Naito, M.; Hayashi, T. Palladium(0)-Catalyzed Hydroboration of 1-Buten-3-ynes: Preparation of Alkenylboranes. Organometallics 1992, 11, 2732-2734.

(437) Cole, T. E.; Quintanilla, R.; Rodewald, S. Migration of 1-Alkenyl Groups from Zirconium to Boron Compounds. Organometallics 1991, 10, 3777-3781.

(438) Obligacion, J. V.; Neely, J. M.; Yazdani, A. N.; Pappas; I.; Chirik, P. J. Cobalt Catalyzed ZSelective Hydroboration of Terminal Alkynes and Elucidation of the Origin of Selectivity. J. Am. Chem. Soc. 2015, 137, 5855-5858.

(439) Jang, W. J.; Lee, W. L.; Moon, J. H.; Lee, J. Y.; Yun, J. Copper-Catalyzed trans-Hydroboration of Terminal Aryl Alkynes: Stereodivergent Synthesis of Alkenylboron Compounds. Org. Lett. 2016, 18, 1390-1393.

(440) Gridnev, I. D.; Miyaura, N.; Suzuki, A. Regio- and Stereospecific Preparation of $\beta$ (Alkylthio)alkenyl-1,3,2-benzodioxaboroles by Nickel-Catalyzed Hydroboration of Thioacetylenes with Catecholborane. Organometallics 1993, 12, 589-592.

(441) Pereira, S.; Srebnik, M. Hydroboration of Alkynes with Pinacolborane Catalyzed by $\mathrm{HZrCp}_{2} \mathrm{Cl}$. Organometallics 1995, 14, 3127-3128.

(442) Lipshutz, B. H.; Bošković, Z. V.; Aue, D. H. Synthesis of Activated Alkenylboronates from Acetylenic Esters by CuH-Catalyzed 1,2-Addition/Transmetallation. Angew. Chem. Int. Ed. 2008, 47, 10183-10186.

(443) Kim, H. R.; Yun, J. Highly Regio- and Steroselective Synthesis of Alkenylboronic Esters by Copper-Catalyzed Boron Addition to Disubstituted Alkynes. Chem. Commun. 2011, 47, 2943-2945.

(444) Semba, K.; Fujihara, T.; Tercio, J.; Tsuji, Y. Copper-Catalyzed Direct Hydroboration of Unsymmetrical Internal Alkynes: Controlling Regioselectivity by Choice of Catalytic Species. Chem.- Eur. J. 2012, 18, 4179-4184.

(445) Greenhalgh, M. D.; Thomas, S. P. Chemo-, Regio-, and Stereoselective Iron-Catalyzed Hydroboration of Alkenes and Alkynes. Chem. Commun. 2013, 49, 11230-11231.

(446) Sundararaju, B.; Fürstner, A. A trans-Selective Hydroboration of Internal Alkynes. Angew. Chem. Int. Ed. 2013, 52, 14050-14054.

(447) Lim, D. S. W.; Anderson, E. A. Synthesis of Vinylsilanes. Synthesis 2012, 44, 983-1010.

(448) Tsipis, C. A. Investigation of the Factors Controlling the Regioselectivity of the Hydrosilylation of Alkynes Catalyzed by trans-di- $\mu$-Hydridobis-(tricyclohexylphosphine)bis(silyl)diplatinum Complexes. $J$. Organomet. Chem. 1988, 187, 427-446. 
(449) Takahashi, K.; Minami, T.; Ohara, Y.; Hiyama, T. A New Synthesis of HMG-CoA Reductase Inhibitor NK-104 through Hydrosilylation-Cross Coupling Reaction. Tetrahedron Lett. 1993, 34, 8263-8266.

(450) Denmark, S. E.; Wang, Z. G. Highly Stereoselective Hydrocarbation of Terminal Alkynes via PtCatalyzed Hydrosilylation/Pd-Catalyzed Cross-Coupling Reactions. Org. Lett. 2001, 3, 1073-1076.

(451) Takeuchi, R.; Nitta, S.; Watanabe, D. A Selective Synthesis of (E)-Vinylsilanes by Cationic Rhodium Complex-Catalysed Hydrosilylation of 1-Alkynes and Tandem Hydrosilylation/Isomerization Reaction of Propargylic Alcohols to $\beta$-Silyl Ketones. J. Org. Chem. 1995, 60, 3045-3051.

(452) Robichaud, J.; Tremblay, F. Formal Enantioselective Synthesis of (+)-Compactin. Org. Lett. 2006, 8, 597-600.

(453) Hayashida, J.; Rawal, V. H. Total Synthesis of ( \pm )-Platencin. Angew. Chem. Int. Ed. 2008, 47, 4373-4376.

(454) Mori, A.; Takahisa, E.; Kajiro, H.; Hirabayashi, K.; Nishihara, Y.; Hiyama, T. RhCl( $\left(\mathrm{PPh}_{3}\right)_{3} / \mathrm{NaI}$ Catalyst System for Hydrosilylation of 1-Alkynes: Stereodivergent Synthesis of E- and Z-Alkenylsilanes with Heteroatom Substituents on Silicon. Chem. Lett. 1998, 443-444.

(455) Mori, A.; Takahisa, E.; Kajiro, H.; Nishihara, Y.; Hiyama, T. Stereodivergent Hydrosilylation of 1-Alkynes Catalyzed by $\mathrm{RhI}\left(\mathrm{PPh}_{3}\right)_{3}$ Leading to (E)- and (Z)-Alkenylsilanes and the Application to Polymer Synthesis. Polyhedron 2000, 19, 567-568.

(456) Mori, A.; Takahisa, E.; Yamamura, Y.; Kato, T.; Mudalige, A. P.; Kajiro, H.; Hirabayashi, K.; Nishihara, Y.; Hiyama, T. Stereodivergent Syntheses of (Z)- and (E)-Alkenylsilanes via Hydrosilylation of Terminal Alkynes Catalyzed by Rhodium(I) Iodide Complexes and Application to Silicon-Containing Polymer Syntheses. Organometallics 2004, 23, 1755-1765.

(457) Ojima, I.; Kumagai, M.; Nagai, Y. The Stereochemistry of the Addition of Hydrosilanes to Alkyl Acetylenes Catalyzed by Tris(triphenylphosphine)-chlororhodium. J. Organomet. Chem. 1974, 66, C14-C16.

(458) Ojima, I.; Clos, N.; Donovan, R. J.; Ingallina, P. Hydrosilylation of 1-Hexyne Catalyzed by Rhodium and Cobalt-Rhodium Mixed Metal Complexes. Mechanism of Apparent trans-Addition. Organometallics 1990, 9, 3127-3133.

(459) Faller, J. W.; D’Alliessi, D. G. Tunable Stereoselective Hydrosilylation of PhCCH Catalyzed by Cp*Rh Complexes. Organometallics 2002, 21, 1743-1746.

(460) Maruyama, Y.; Yamamura, K.; Nakayama, I.; Yoshiuchi, K.; Ozawa, F. Mechanistic Study of Ruthenium-Catalyzed Hydrosilylation of 1-(Trimethylsilyl)-1-buten-3-yne. J. Am. Chem. Soc. 1998, 120, 1421-1429.

(461) Katayama, H.; Taniguchi, K.; Kobayashi, M.; Sagawa, T.; Minami, T.; Ozawa, F. RutheniumCatalyzed Hydrosilylation of Terminal Alkynes: Stereodivergent Synthesis of (E)- and (Z)Alkenylsilanes. J. Organomet. Chem. 2002, 645, 192-200.

(462) Esteruelas, M. A.; Herrero, J.; Oro, L. A. Exclusive Formation of cis-PhCH:CH( $\left(\mathrm{SiEt}_{3}\right)$ by Addition of Trietylsilane to Phenylacetylene Catalyzed by Ruthenium Complex $\left[\left(\mathrm{Me}_{2} \mathrm{CH}\right)_{3} \mathrm{P}\right]_{2} \mathrm{RuHCl}(\mathrm{CO})$. Organometallics 1993, 12, 2377-2379.

(463) Maddock, S. M.; Richard, C. E. F. Roper, W. R.; Wright, L. J. Insertion of Ethyne into the Ru-Si Bonds of Coordinatively Unsaturated Ruthenium Silyl Complexes. X-ray Crystal Structures of $\mathrm{Ru}\left(\mathrm{CHCHSiMe}{ }_{2} \mathrm{OEt}\right) \mathrm{Cl}(\mathrm{CO})_{2}\left(\mathrm{PPh}_{3}\right)_{2}$ and $\quad\left[\mathrm{Ru}\left(\mathrm{CHCHSiMe}{ }_{2} \mathrm{OH}\right)(\mathrm{CN}-p\right.$-Tolyl $\left.)(\mathrm{CO})\left(\mathrm{PPh}_{3}\right)_{2}\right] \mathrm{ClO}_{4}$. Organometallics 1996, 15, 1793-1803.

(464) Pasau, O.; Liautard, V.; Vaultier, M.; Pucheault, M.; Aymonier, C. Catalyzed Stereodivergent Hydrosilylation with Onium Salts Stabilized $\mathrm{M}(0)$ Nanocatalysts Prepared in $\mathrm{scCO}_{2}$. RSC Adv. 2014, 4, 59953-59960.

(465) Molander, G. A.; Retsch, W. H. Selective Hydrosilylation of Alkynes Catalyzed by an Organoyttrium Complex. Organometallics 1995, 14, 4570-4575.

(466) Trost, B. M.; Ball, Z. T. Markovnikov Alkyne Hydrosilylation Catalyzed by Ruthenium Complexes. J. Am. Chem. Soc. 2001, 123, 12726-12727. 
(467) Trost, B. M.; Ball, Z. T.; Jöge, T. Regioselective Hydrosilylation of Propargylic Alcohols: An Aldol Surrogate. Angew. Chem. Int. Ed. 2003, 42, 3415-3418.

(468) Denmark, S. E.; Pan, W. Intramolecular Hydrosilylation and Silicon-Assisted Cross-Coupling: An Efficient Route to Trisubstituted Homoallylic Alcohols. Org. Lett. 2001, 3, 61-64.

(469) Denmark, S. E.; Pan, W. Intramolecular Anti-Hydrosilylation and Silicon-Assisted CrossCoupling: Highly Regio- and Stereoselective Synthesis of Trisubstituted Homoallylic Alcohols. Org. Lett. 2002, 4, 4163-4166.

(470) Denmark, S. E.; Pan, W. Intramolecular syn and anti Hydrosilylation and Silicon-Assisted CrossCoupling: Highly Regio- and Steroselective Synthesis of Trisubstituted Allylic Alcohols. Org. Lett. 2003, 5, 1119-1122.

(471) Yong, L.; Kirleis, K.; Butenschön, H. Stereodivergent Formation of Alkenylsilanes: syn or antiHydrosilylation of Alkynes Catalyzed by a Cyclopentadienylcobalt(I) Chelate Bearing a Pendant Phosphane Ether. Adv. Synth. Catal. 2006, 348, 833-836.

(472) Belger, C.; Plietker, B. Aryl-Aryl Interactions as Directing Motifs in the Stereodivergent IronCatalyzed Hydrosilylation of Internal Alkynes. Chem. Commun. 2012, 48, 5419-5421.

(473) Sumida, Y.; Kato, T.; Yoshida, S.; Hosoya, T. Palladium-Catalyzed Regio- and Steroselective Hydrosilylation of Electron-Deficient Alkynes. Org. Lett. 2012, 14, 1552-1555.

(474) Smith, N. D.; Mancuso, J.; Lautens, M. Metal-Catalyzed Hydrostannations. Chem. Rev. 2000, 100, 3257-3282.

(475) Asao, N.; Liu, J. X.; Sudoh, T.; Yamamoto, Y. Lewis Acid Catalysed trans-Hydrostannylation of Acetylenes. J. Chem. Soc., Chem. Commun. 1995, 2405-2406.

(476) Asao, N.; Liu, J. X.; Sudoh, T.; Yamamoto, Y. Lewis Acid-Catalyzed Hydrostannation of Acetylenes. Regio- and Steroselective trans-Addition of Tributyltin Hydride and Dibutyltin Dihydride. $J$. Org. Chem. 1996, 61, 4568-4571.

(477) Zhang, H. X.; Guibe, F.; Balavoine, G. Palladium- and Molybdenum-Catalyzed Hydrostannation of Alkynes. A Novel Access to Regio- and Stereodefined Vinylstannanes. J. Org. Chem. 1990, 55, 1857-1867.

(478) Meleczka, R. E.; Gallagher, W. P.; Terstiege, I. Stille Couplings Catalytic in Tin: Beyond Proofof-Principle. J. Am. Chem. Soc. 2000, 122, 384-385.

(479) Semmelhack, M. F.; Hooley, R. J. Palladium-Catalyzed Hydrostannylations of Highly Hindered Acetylenes in Hexane. Tetrahedron Lett. 2003, 44, 5737-5739.

(480) Rummelt, S. M.; Fürstner, A. Ruthenium-Catalyzed trans-Selective Hydrostannation of Alkynes. Angew. Chem. Int. Ed. 2014, 53, 3626-3630.

(481) Lindlar, H. Ein Neuer Katalysator für Selektive Hydrierungen. Helv. Chim. Acta 1952, 35, $446-450$.

(482) Oger, C.; Balas, L.; Durand, T.; Galano, J.-M. Are Alkyne Reductions Chemo-, Regio-, and Steroselective Enough to Provide Pure (Z)-Olefins in Polyfunctionalized Bioactive Molecules? Chem. Rev. 2013, 113, 1313-1350.

(483) Pelagatti, P.; Venturini, A.; Leporati, A.; Carcelli, M.; Costa, M.; Bacchi, A.; Pelizzi, G.; Pelizzi, C. Chemoselective Homogeneous Hydrogenation of Phenylacetylene Using Thiosemicarbazone and Thiobenzoylhydrazone Palladium(II) Complexes as Catalysts. J. Chem. Soc., Dalton Trans. 1998, 16, 2715-2722.

(484) Van Laren, M. W.; Elsevier, C. J. Selective Homogeneous Palladium(0)-Catalyzed Hydrogenation of Alkynes to (Z)-Alkenes. Angew. Chem. Int. Ed. 1999, 38, 3715-3717.

(485) Schrock, R. R.; Osborn, J. A. Catalytic Hydrogenation Using Cationic Rhodium Complexes II. The Selective Hydrogenation of Alkynes to cis Olefins. J. Am. Chem. Soc. 1976, 98, 2143-2147.

(486) Tani, K.; Iseki, A.; Yamagata, T. Efficent Transfer Hydrogenation of Alkynes and Alkenes with Methanol by Hydrido(methoxo)iridium(III) Complexes. Chem. Commun. 1999, 1821-1822.

(487) Yoshida, T.; Youngs, W. J.; Sakaeda, T.; Ueda, T.; Otsuka, S.; Ibers, J. A. Reactions of Dihydro(bicarbonato)bis(triisopropylphosphine)(diphenylacetylene)-carbonatodihydrodirhodium 
$\left[\mathrm{Rh}_{2} \mathrm{H}_{2}\left(\mathrm{OCO}_{2}\right)(\mathrm{PhCCPh})\left[\mathrm{P}\left(\mathrm{CHMe}_{2}\right)_{3}\right]_{3}\right.$ and the Stereoselective Hydrogenation of Alkynes to trans Olefins. J. Am. Chem. Soc. 1983, 105, 6273-6278.

(488) Burch, R. R.; Shusterman, A. J.; Muetterties, E. L.; Teller, R. G.; Williams, J. M. Coordinately Unsaturated Clusters. A Novel Catalytic Reaction. J. Am. Chem. Soc. 1983, 105, 3546-3556.

(489) Schleyer, D.; Niessen, H. G.; Bargon, J. In situ ${ }^{1}$ H-PHIP-NMR Studies of the Stereoselective Hydrogenation of Alkynes to (E)-Alkenes Catalyzed by a Homogeneous [Cp*Ru] ${ }^{+}$Catalyst. New. $J$. Chem. 2001, 25, 423-426.

(490) Radkowski, K.; Sundararaju, B.; Fürstner, A. A Functional Group-Tolerant Catalytic transHydrogenation of Alkynes. Angew. Chem. Int. Ed. 2013, 52, 355-360.

(491) Leutzsch, M.; Wolf, L. M.; Gupta, P.; Fuchs, M.; Thiel, M.; Farès, C.; Fürstner, A. Formation of Ruthenium Carbenes by gem-Hydrogen Transfer to Internal Alkynes: Implications for Alkyne transHydrogenation. Angew. Chem. Int. Ed. 2015, 54, 12431-12436.

(492) Srimani, D.; Diskin-Posner, Y.; Ben-David, Y.; Milstein, D. Iron Pincer Complex Catalyzed, Environmentally Benign, E-Selective Semi-Hydrogenation of Alkynes. Angew. Chem. Int. Ed. 2013, 52, 14131-14134.

(493) Karunamanda, M. K.; Mankad, N. P. E-Selective Semi-Hydrogenation of Alkynes by Heterobimetallic Catalysis. J. Am. Chem. Soc. 2015, 137, 14598-14601.

(494) Liu, Y.; Hu, L.; Chen, H.; Du, H. An Alkene-Promoted Borane-Catalyzed Highly Steroselective Hydrogenation of Alkynes to Give Z- and E-Alkenes. Chem.- Eur. J. 2015, 21, 3495-3501.

(495) Barrios-Francisco, R.; García, J. J. Stereoselective Hydrogenation of Aromatic Alkynes Using Water, Triethylsilane, or Methanol, Mediated and Catalyzed by $\mathrm{Ni}(0)$ Complexes. Inorg. Chem. 2009, 478, 386-393.

(496) Barrios-Francisco, R.; García, J. J. Semihydrogenation of Alkynes in the Presence of Ni(0) Catalyst Using Ammonia-Borane and Sodium Borohydride as Hydrogen Sources. Appl. Cat. A: Gen. 2010, 385, 108-113.

(497) Luo, F.; Pan, C.; Wang, W.; Ye, Z.; Cheng, J. Palladium-Catalyzed Reduction of Alkynes Employing $\mathrm{HSiEt}_{3}$ : Stereoselective Synthesis of trans- and cis-Alkenes. Tetrahedron 2010, 66, 1399-1403.

(498) Li, J.; Hua, R. Stereodivergent Ruthenium-Catalyzed Transfer Semihydrogenation of Diaryl Alkynes. Chem.- Eur. J. 2011, 17, 8462-8465.

(499) Schabel, T.; Belger, C.; Plietker, B. A Mild Chemoselective Ru-Catalyzed Reduction of Alkynes, Ketones, and Nitro Compounds. Org. Lett. 2013, 15, 2858-2861.

(500) Richmond, E.; Moran, J. Ligand Control of E/Z Selectivity in Nickel-Catalyzed Transfer Hydrogenation Alkyne Semireduction. J. Org. Chem. 2015, 80, 6922-6929.

(501) Fu; S.; Chen, N.-Y.; Liu, X.; Shao, Z.; Luo, S.-P.; Liu, Q. Ligand-Controlled Cobalt-Catalyzed Transfer Hydrogenation of Alkynes: Stereodivergent Synthesis of Z- and E-Alkenes. J. Am. Chem. Soc. 2016, 138, 8588-8594.

(502) Shen, R.; Chen, T.; Zhao, Y.; Qiu, R.; Zhou, Y.; Yin, S.; Wang, X.; Goto, M.; Han, L.-B. Facile Regio- and Stereoselective Hydrometalation of Alkynes with a Combination of Carboxylic Acids and Group 10 Transition Metal Complexes: Selective Hydrogenation of Alkynes with Formic Acid. J. Am. Chem. Soc. 2011, 133, 17037-17044.

(503) Yamamoto, Y. Synthesis of Heterocycles via Transition-Metal Catalyzed Hydroarylation of Alkynes. Chem. Soc. Rev. 2014, 43, 1575-1600.

(504) Arockiam, P.B.; Bruneau, C.; Dixneuf, P. H. Ruthenium(II)-Catalyzed C-H Bond Activation and Functionalization. Chem. Rev. 2012, 112, 5879-5918.

(505) Min, M.; Kim, D.; Hong, S. AgSbF ${ }_{6}$-Controlled Diastereodivergence in Alkyne Hydroarylation: Facile Access to Z- and E-Alkenyl Arenes. Chem. Commun. 2014, 50, 8028-8031.

(506) Dwivedi, V.; Babu, M. H.; Kant, R.; Reddy, M. S. N-Substitution Dependent Steroselectivity Switch in Palladium Catalyzed Hydroalkynylation of Inamides: a Regio and Stereoselective Synthesis of Ynenamides. Chem. Commun. 2015, 51, 14996-14999. 
(507) Trostyanskaya, I. G.; Beletskaya, I. P. Regio- and Steroselective Copper-Catalyzed Addition of Aromatic and Aliphatic Thiols to Terminal and Internal Alkynes. Synlett 2012, 23, 535-540.

(508) Barluenga, J.; Riesgo, L.; Vicente, R.; López, L. A.; Tomás, M. Rearrangement of Propargyl Esters: Metal-Based Stereospecific Synthesis of (E)- and (Z)-Knoevenagel Derivatives. J. Am. Chem. Soc. 2007, 129, 7772-7773.

(509) Wang, S.; Zhang, L. A Highly Efficient Preparative Method of $\alpha$-Ylidene- $\beta$-Diketones via Au ${ }^{\text {III }}$ Catalyzed Acyl Migration of Propargyl Esters. J. Am. Chem. Soc. 2006, 128, 8414-8415.

(510) Shiroodi, R. K.; Dudnik, A. S.; Gevorgyan, V. Stereocontrolled 1,3-Phosphatyloxy and 1,3Halogen Migration Relay Toward Highly Functionalized 1,3-Dienes. J. Am. Chem. Soc. 2012, 134, 6928-6931.

(511) Jiang, J.; Hou, C.; Zhang, S.; Luan, Z.; Zhao, C.; Ke, Z. The Effect of HSAB on Stereoselectivity: Copper- and Gold-Catalyzed 1,3-Phosphatyloxy and 1,3-Halogen Migration Relay to 1,3-Dienes. J. Org. Chem. 2015, 80, 1661-1671.

(512) Asako, S.; Sakae, T.; Murai, M.; Takai, K. Molybdenum-Catalyzed Stereospecific Deoxygenation of Epoxides to Alkenes. Adv. Synth. Catal. 2016, 358, 3966-3970.

(513) Kanai, M.; Tomioka, K. Asymmetric Conjugate Addition of Organomagnesium Cuprates Controlled by a Stoichiometric Amount of Chiral Phosphine. Tetrahedron Lett. 1995, 36, 4273-4274.

(514) Kanai, M.; Nakagawa, Y.; Tomioka, K. Catalytic Enantioselective Conjugate Addition of Grignard Reagents to Cyclic $\alpha, \beta$-Unsaturated Carbonyl Compounds. Tetrahedron 1999, 55, 3843-3854.

(515) Seebach, D.; Jaeschke, G.; Pichots, A.; Audergon, L. Enantioselective 1,4-Addition of Aliphatic Grignard Reagents to Enones Catalyzed by Readily Available Copper(I) Thiolates Derived from TADDOL. Helv. Chim. Acta 1997, 80, 2515-2519.

(516) Okamoto, M.; Yamamoto, Y.; Sakaguchi, S. A New Approach to Switching of Enantioselectivity in NHC-Cu-Catalyzed Conjugate Addition of Alkylzincs to Cyclic Enones. Chem. Commun. 2009, 7363-7365.

(517) Shibata, N.; Okamoto, M.; Yamamoto, Y. Sakaguchi, S. Reversal of Stereoselectivity in the CuCatalyzed Conjugate Addition Reaction of Dialkylzinc to Cyclic Enone in the Presence of a Chiral Azolium Compound. J. Org. Chem. 2010, 75, 5707-5715.

(518) Harano, A.; Sakaguchi, S. A New $C_{2}$-Symmetric Azolium Compound for Cu-Catalyzed Asymmetric Conjugate Addition of $\mathrm{R}_{2} \mathrm{Zn}$ to Cyclic Enone. J. Organomet. Chem. 2011, 696, 61-67.

(519) Yoshimura, M.; Shibata, N.; Kawakami, M.; Sakaguchi, S. Ligand Design for Dual Enantioselective Control in Cu-Catalyzed Asymmetric Conjugate Addition of $\mathrm{R}_{2} \mathrm{Zn}$ to Cyclic Enone. Tetrahedron 2012, 68, 3512-3518.

(520) Shibata, N.; Yoshimura, M.; Yamada, H.; Arakawa, R.; Sakaguchi, S. Hydroxy-amide Functionalized Azolium Salts for Cu-Catalyzed Asymmetric Conjugate Addition: Stereocontrol Based on Ligand Structure and Copper Precatalyst. J. Org. Chem. 2012, 77, 4079-4086.

(521) Matsumoto, K.; Nakano, Y.; Shibata, N.; Sakaguchi, S. Enantioselectivity Switch in CopperCatalyzed Conjugate Addition Reactions Under the Influence of a Chiral N-Heterocyclic Carbene-Silver Complex. RSC Adv. 2016, 6, 7755-7759.

(522) Takaya, Y.; Ogasawara, M.; Hayashi, T.; Sakai, M.; Miyaura, N. Rhodium-Catalyzed Asymmetric 1,4-Addition of Aryl- and Alkenylboronic Acids to Enones. J. Am. Chem. Soc. 1998, 120, 5579-5580.

(523) Chen, G.; Gui, J.; Li, L.; Liao, J. Chiral Sulfoxide-Olefin Ligands: Completely Switchable Stereoselectivity in Rhodium-Catalyzed Asymmetric Conjugate Additions. Angew. Chem. Int. Ed. 2011, 50, 7681-7685.

(524) Chen, G.; Gui, J.; Cao, P.; Liao, J. Chiral Sulfoxide-Olefin Ligands: Tunable Stereoselectivity in Rh-Catalyzed Asymmetric 1,4-Addition. Tetrahedron 2012, 68, 3220-3224.

(525) Eitel, S. H.; Jautze, S.; Frey, W.; Peters, R. Asymmetric Michael Additions of $\alpha$-Cyanoacetates by Soft Lewis Acid/Hard Brønsted Acid Catalysis: Stereodivergency with Bi-vs. Monometallic Catalyst. Chem. Sci. 2013, 4, 2218-2233.

(526) Wang, J.; Feringa, B. L. Dynamic Control of a Chiral Space in a Catalytic Asymmetric Reaction Using a Molecular Motor. Science 2011, 331, 1429-1432. 
(527) Oba, M.; Terauchi, T.; Owari, Y.; Imai, Y.; Motoyama, I.; Nishiyama, K. Stereodivergent Synthesis of L-threo and L-erythro-[2,3- $\left.{ }^{2} \mathrm{H}_{2}\right]$ Amino Acids Using Optically Active Dioxopiperazine as Chiral Template. J. Chem. Soc., Perkin Trans. 1 1998, 1275-1281.

(528) Yu, S. H.; Ferguson. M. J.; McDonald, R.; Hall, D. G. Brønsted Acid-Catalyzed Allylboration: Short and Stereodivergent Synthesis of All Four Eupomatilone Diastereomers with Crystallographic Assignments. J. Am. Chem. Soc. 2005, 127, 12808-12809.

(529) Bosch, C.; Fiser, B.; Gómez-Bengoa, E.; Bradshaw, B.; Bonjoch, J. Approach to cis-Phlegmarine Alkaloid via Stereodivergent Reduction: Total Synthesis of (+)-Serratezomine E and Putative Structure of (-)-Huperzine N. Org. Lett. 2015, 17, 5084-5087.

(530) Nicolaou, K. C.; Rhoades, D.; Lamani, M.; Pattanayak, M. R.; Kumar, S. M. Total Synthesis of Thailanstatin A. J. Am. Chem. Soc. 2016, 138, 7532-7535.

(531) Spangler, J. E.; Davies, H. M. L. Catalytic Asymmetric Synthesis of Pyrroloindolines via a Rhodium(II)-Catalyzed Annulation of Indoles. J. Am. Chem. Soc. 2013, 135, 6802-6805.

(532) Pérez-Luna, A.; Bonin, M.; Micouin, L.; Husson, H.-P. Reversal of Enantioselectivity in the Asymmetric Rhodium- versus Iridium-Catalyzed Hydroboration of Meso Substrates. J. Am. Chem. Soc. 2002, 124, 12098-12099 (Corrigendum: J. Am. Chem. Soc. 2002, 124, 14503).

(533) Fehr, C. Enantioselective Protonation of Enolates and Enols. Angew. Chem. Int. Ed. 1996, 35, 2566-2587.

(534) Eames, J.; Weerasooriya, N. Recent Advances into the Enantioselective Protonation of Prostereogenic Enol Derivatives. Tetrahedron: Asymmetry 2001, 12, 1-24.

(535) Duhamel, P.; Plaquevent, J.-C. Enantioselective Protonations: Fundamental Insights and New Concepts. Tetrahedron: Asymmetry 2004, 15, 3653-3691.

(536) Boy, E.; Coumbarides, G. S.; Eames, J.; Hay, A.; Jones, R. V. H.; Stenson, R. A.; Suggate; M. J. Reversal of Enantioselectivity on Protonation of Enol(ate)s Derived from 2-Methyl-1-tetralone Using $C_{2}$ Symmetric Sulfonamides. Tetrahedron Lett. 2004, 45, 9465-9468.

(537) Mohr, J. T.; Nishimata, T.; Behenna, D. C.; Stoltz, B. M. Catalytic Enantioselective Decarboxylative Protonation. J. Am. Chem. Soc. 2006, 128, 11348-11349.

(538) Marinescu, S. C.; Nishimata, T.; Mohr, J. T.; Stoltz, B. M. Homogeneous Pd-Catalyzed Enantioselective Decarboxylative Protonation. Org. Lett. 2008, 10, 1039-1042.

(539) Dorau, R.; Carroll, M. P.; Akula, R.; Hogan, B. F.; Martins, M.; Fanning, S.; Guiry, P. J. A Stereoselective Switch: Enantiodivergent Approach to the Synthesis of Isoflavones. Chem.-Eur. J. 2014, 20, 15354-15359.

(540) Kingston, C.; Guiry, P.J. Enantiodivergent Synthesis of Tertiary $\alpha$-Aryl 1-Indanones: Evidence toward Disparate Mechanisms in the Palladium-Catalyzed Decarboxylative Asymmetric Protonation. $J$. Org. Chem. 2017, 82, 3806-3819.

(541) Shibata, N.; Ishimaru, T.; Nagai, T.; Kohna, J.; Toru, T. First Enantio-Flexible Fluorination Reaction Using Metal-Bis(oxazoline) Complexes. Synlett 2004, 1703-1706.

(542) Mouri, S.; Chen, Z.; Mitsunuma, H.; Furutachi, M.; Matsunaga, S.; Shibasaki, M. Catalytic Asymmetric Synthesis of 3-Aminoxindoles: Enantiofacial Selectivity Switch in Bimetallic vs Monometallic Schiff Base Catalysis. J. Am. Chem. Soc. 2010, 132, 1255-1257.

(543) Fjelbye, K.; Marigo, M.; Clausen, R. P.; Juhl, K. Diastereodivergent Access to syn and anti 3,4Substituted $\beta$-Fluoropyrrolidines: Enhancing or Reversing Substrate Preference. Org. Lett. 2016, 18, 1170-1173.

(544) Farthing, C. N.; Kočovsky, P. The Stereochemical Dichotomy in Palladium(0)- and Nickel(0)Catalyzed Allylic Substitution. J. Am. Chem. Soc.1998, 120, 6661-6672.

(545) Kurosawa, H.; Ogoshi, S.; Kawaski, Y.; Murai, S.; Miyashi, M.; Ikeda, I. Novel Dependency of Stereochemistry upon Metal, Ligand and, Solvent in Oxidative Addition of Allylic Chloride to $\operatorname{Pd}(0)$ and Pt(0) Complexes. J. Am. Chem. Soc. 1990, 112, 2813-2814.

(546) Kurosawa, H.; Kajimaru, H.; Ogoshi, S.; Yoneda, H.; Miki, K.; Kasai, N.; Murai, S.; Ikeda, I. Novel syn Oxidative Addition of Allylic Halides to Olefin Complexes of Palladium(0) and Platinum(0). $J$. Am. Chem. Soc.1992, 114, 8417-8424. 
(547) Shimizu, I.; Matsumoto, Y.; Nishikawa, M.; Kawahara, T.; Satake, A.; Yamamoto, A. Enantioselective Nucleophilic Substitution of a 6,6-Bicyclic Dienyl Acetate by Pd-Chiral Phosphine Catalyst. Chem. Lett. 1998, 983-984.

(548) Audisio, D.; Luparia, M.; Oliveira, M. T.; Klütt, D.; Maulide, N. Diastereodivergent Deepimerization in Catalytic Asymmetric Allylic Alkylation. Angew. Chem. Int. Ed. 2012, 51, 7314-7317.

(549) Rodríguez, S.; Qu, B.; Fandrick, K. R.; Buono, F.; Haddad, N.; Xu, Y.; Herbage, M. A.; Zeng, X.; Ma, S.; Grinberg, N.; Lee, H.; Han, Z. S.; Yee, N. K.; Senanayake, C. H. Amine-Tunable Ruthenium Catalysts for Asymmetric Reduction of Ketones. Adv. Synth. Catal. 2014, 356, 301-307.

(550) Agudo, R.; Roiban, G.-D.; Reetz, M. T. Induced Axial Chirality in Biocatalytic Asymmetric Ketone Reduction. J. Am. Chem. Soc. 2013, 135, 1665-1668.

(551) Maria-Solano, M. A.; Romero-Rivera, A.; Osuna, S. Exploring the Reversal of Enantioselectivity on a Zinc-Dependent Alcohol Dehydrogenase. Org. Biomol. Chem. 2017, 15, 4122-4129.

(552) Haddad, N.; Qu, B.; Rodríguez, S.; Van der Veen, L.; Reeves, D. C.; Gonnella, N. C.; Lee, H.; Grinberg, N.; Ma, S.; Krishnamurthy, D.; Wunberg, T.; Senanayake, C. H. Catalytic Asymmetric Hydrogenation of Heterocyclic Ketone-Derived Hydrazones, Pronunced Solvent Effect on the Inversion of Configuration. Tetrahedron Lett. 2011, 52, 3718-3722.

(553) Ding, Z.-Y.; Chen, F.; Qin, J.; He, Y.-M.; Fan, Q.-H. Asymmetric Hydrogenation of 2,4Disubstituted 1,5-Benzodiazepines Using Cationic Ruthenium Diamine Catalysts: An Unusual Achiral Counteranion Induced Reversal of Enantioselectivity. Angew. Chem. Int. Ed. 2012, 51, 5706-5710.

(554) Machin, B. P.; Howell, J.; Mandel, J.; Blanchard, N.; Tam, W. Ruthenium-Catalyzed Nucleophilic Ring-Opening Reactions of a 3-Aza-2-oxabicyclo[2.2.1]hept-5-ene with Alcohols. Org. Lett. 2009, 11, 2077-2080.

(555) Wu, W.-Q.; Peng, Q.; Dong, D.-X.; Hou, X.-L.; Wu, Y.-D. A Dramatic Switch of Enantioselectivity in Asymmetric Heck Reaction by Benzylic Substituents of Ligands. J. Am. Chem. Soc. 2008, 130, 9717-9725.

(556) Miyamura, S.; Araki, M.; Suzuki, T.; Yamaguchi, J.; Itami, K. Stereodivergent Synthesis of Arylcyclopropylamines by Sequential C-H Borylation and Suzuki-Miyaura Coupling. Angew. Chem. Int. Ed. 2015, 54, 846-851.

(557) Chen, C.-T.; Tsai, C.-C.; Tsou, P.-K.; Huang, G.-T.; Yu, C.-H. Enantiodivergent Steglich Rearrangement of $O$-Carboxylazlactones Catalyzed by a Chirality Switchable Helicene Containing a 4Aminopyridine Unit. Chem. Sci. 2017, 8, 524-529.

(558) Ivšić, T.; Novak, J.; Došlić, N.; Hameršak, Z. One Catalyst for Both Enantiomers: Uncovering the Inversion of Enantioselectivity in Cinchona-Mediated Desymmetrization of Glutaric meso-Anhydrides. Tetrahedron 2012, 68, 8311-8317.

(559) Wang, X.-W.; Wang, Y.; Jia, J. Enamine Catalysis of Intramolecular Aldol Reactions. In Science of Synthesis, Asymmetric Organocatalysis 1. LewisBase and Acid Catalysis, List, B., Ed.; Thieme: Stuttgart 2012, pp 1-33.

(560) Bradshaw, B.; Bonjoch, J. The Wieland-Miescher Ketone: A Journey from Organocatalysis to Natural Product Synthesis. Synlett 2012, 23, 337-356.

(561) Honda, S.; Inomata, K.; Endo, Y. Enantiodivergent Synthesis of Wieland-Miescher Ketone Analog Mediated by a Chiral Pyridinmethylamine Derivative. Heterocycles 2015, 90, 950-966.

(562) Truong, P.; Shanahan, C. S.; Doyle, M. P. Divergent Stereocontrol of Acid Catalyzed Intramolecular Aldol Reactions of 2,3,7-Triketoesters: Synthesis of Highly Functionalized Cyclopentanones. Org. Lett. 2012, 14, 3608-3611.

(563) Chen, S.-H.; Hong, B.-C.; Su, C.-F.; Sarshar, S. An Unexpected Inversion of Enantioselectivity in the Proline Catalyzed Intramolecular Baylis-Hillman Reaction. Tetrahedron Lett. 2005, 46, 8899-8903.

(564) McIntosh, M.; Weinreb, S. M. A Strategy for Synthesis of Conduritols and Related Cyclitols via Stereodivergent Vinylsilane-Aldehyde Cyclizations. J. Org. Chem. 1991, 56, 5010-5012.

(565) Williams, J. T.; Bahia, P. S.; Snaith, J. S. Synthesis of 3,4-Disubstituted Piperidines by Carbonyl Ene and Prins Cyclizations: A Switch in Diastereoselectivity Between Lewis and Brønsted Acid Catalysts. Org. Lett. 2002, 4, 3727-3730. 
(566) Kadota, I.; Hatakeyama, D.; Seki, K.; Yamamoto, Y. IntramolecularReaction of Oxo-Substituted Allenyl- and Propargylstannane with Aldehyde. Tetrahedron Lett. 1996, 37, 3059-3062.

(567) Arrate, M.; Durana, A.; Lorenzo, P.; Rodríguez de Lera, A.; Alvarez, R.; Aurrecoechea, J. M. Catalyst- and Solvent-Dependent Stereodivergence in the Intramolecular $\mathrm{Et}_{2} \mathrm{Zn} / \mathrm{Pd}^{0}$-Promoted Carbonyl Propargylation: Mechanistic Implications. Chem. - Eur. J. 2013, 19, 13893-13900.

(568) Sakai, K.; Ide, J.; Oda, O.; Nakamura, N. SyntheticStudies on Prostanoids 1. Synthesis of Methyl 9-Oxoprostanoate. Tetrahedron Lett. 1972, 1287-1290.

(569) Lochow, C. F.; Miller, R. G. Transition-Metal-Promoted Aldehyde-Alkene Addition Reactions. J. Am. Chem. Soc. 1976, 98, 1281-1283.

(570) Wu, X.-M.; Funakoshi, K.; Sakai, K. Highly Diastereoselective Enantioselective Cyclization of Symmetrical 3,4-Disubstituted 4-Pentenal Using Chiral Rhodium(I)-Complex. Tetrahedron Lett. 1993, 34, 5927-5930.

(571) Tanaka,M.; Imai, M.; Fujio, M.; Sakamoto, E.; Takahashi,M.; Eto-Kato,Y.; Wu, X. M.; Funakoshi, K.; Sakai, K.; Suemune, H. Concurrent Induction of Two Chiral Centers from Symmetrical 3,4-Disubstituted and 3,3,4-Trisubstituted 4-Pentenals Using Rh-Catalyzed Asymmetric Cyclizations. J. Org. Chem. 2000, 65, 5806-5816.

(572) Takahashi, M.; Tanaka, M.; Sakamoto, E.; Imai, M.; Matsui, A.; Funakoshi, K.; Sakai, K.; Suemune, H. Application of Rh-Catalyzed Cyclization for the Construction of Three Consecutive Chiral Carbon in 4,9-Dimethylspiro[4,4]nonane-2,7-dione. Tetrahedron Lett. 2000, 41, 7879-7883.

(573) Tanaka, M.; Takahashi, M.; Sakamoto, E.; Imai, M.; Matsui, A.; Fujito, M.; Funakoshi, K.; Sakai, K.; Suemune, H. Enantio-and Diastereoselective Construction of 4,9-Dimethylspiro[4,4]nonane-2,7-dione Using Rh-Catalyzed Asymmetric Cyclization. Tetrahedron 2001, 57, 1197-1204.

(574) Wu, X.; Chen, Z.; Bai, Y.-B.; Dong, V. M. Diastereodivergent Construction of Bicyclic $\gamma$ Lactones via Enantioselective Ketone Hydroacylation. J. Am. Chem. Soc. 2016, 138, 12013-12016.

(575) Lebold, T. P.; Kerr, M. A. Stereodivergent Synthesis of Fused Bicyclopyrazolidines: Access to Pyrazolines and Pyrrolidines. Org. Lett. 2009, 11, 4354-4357.

(576) De Carré-Carnavaled, B.; Krieger, J.-P.; Folléas, B.; Brayer, J.-L.; Demonte, J.-P.; Meyer, C.; Cossy, J. Diastereodivergent Pictet-Spengler Cyclization of Bicyclic $N$-Acyliminium Ions: Controlling a Quaternary Stereocenter. Eur. J. Org. Chem. 2015, 1273-1282.

(577) Belmessieri, D.; Morrill, L. C.; Simal, C.; Slawin, A. M. Z.; Smith, A. D. Organocatalytic Functionalization of Carboxylic Acids: Isothiourea-Catalyzed Asymmetric Intra- and Intermolecular Michael-Addition-Lactonizations. J. Am. Chem. Soc. 2011, 133, 2714-2720.

(578) Belmessieri, D.; de la Houpliere, A.; Calder, E. D. D.; Taylor, J. E.; Smith, A. D. Stereodivergent Organocatalytic Intramolecular Michael Addition/Lactonization for the Asymmetric Synthesis of Substituted Dihydrobenzofurans and Tetrahydrofurans. Chem. - Eur. J. 2014, 20, 9762-9769.

(579) Fukata, Y.; Okamura, T.; Asano, K.; Matsubara, S. Asymmetric Isomerization of $\omega$-Hydroxy- $\alpha, \beta-$ unsaturated Thioesters into $\beta$-Mercaptolactones by a Bifunctional Aminothiourea Catalyst. Org. Lett. 2014, 16, 2184-2187.

(580) Dodda, R.; Goldman, J. J.; Mandal, T.; Zhao, C.-G.; Broker, G. A.; Tiekink, E. R. T. Synthesis of 2,3,4-Trisubstituted Thiochromanes Using an Organocatalytic Enantioselective Tandem Michael-Henry Reaction. Adv. Synth. Catal. 2008, 350, 537-541.

(581) Arai, T.; Yamamoto, Y. Diversity-Oriented Asymmetric Catalysis (DOAC): Stereochemically Divergent Synthesis of Thiochromanes Using an Imidazoline-Aminophenol-Nickel-Catalyzed Michael/Henry Reaction. Org. Lett. 2014, 16, 1700-1703.

(582) Ping, X.-N.; Wei, P.-S.; Zhu, X.-Q.; Xie, J.-W. Catalyst-Controlled Switch in Diastereoselectivities: Enantioselective Construction of Functionalized 3,4-Dihydro-2H-thiopyrano[2,3b]quinolines with Three Contiguous Stereocenters. J. Org. Chem. 2017, 82, 2205-2210.

(583) Ma, G.; Lin, S.; Ibrahem, I.; Kubik, G.; Liu, L.; Sun, J.; Córdova, A. Achiral Co-Catalyst Induced Switches in Catalytic Asymmetric Reactions on Racemic Mixtures (RRM): From Stereodivergent RRM to Stereoconvergent Deracemization by Combination of Hydrogen Bond Donating and Chiral Amine Catalysts. Adv. Synth. Catal. 2012, 354, 2865-2872. 
(584) Zhong, C.; Wang, Y.; O’Herin, C.; Young, D. W. Synthesis of Substituted Morpholines Using Stereodivergent Aza-Michael Reactions Catalyzed by Brønsted Acids. ACS Catal. 2013, 3, 643-646.

(585) Aparici, I.; Guerola, M.; Dialer, C.; Simón-Fuentes, A.; Sánchez-Roselló, M.; del Pozo, C.; Fustero, S. Diastereodivergent Synthesis of Fluorinated Cyclic $\beta^{3}$-Amino Acid Derivatives. Org. Lett. 2015, 17, 5412-5415.

(586) Henrich, M.; Delgado, A.; Molins, E.; Llebaria, A. Nucleophile-Dependent Stereodivergence in the Pd-Catalyzed Intramolecular Cyclization of 2-(p-Tolylsufinyl)allylacetates. Tetrahedron Lett. 1999, 40, 4259-4262.

(587) Ohno, H.; Takemoto, Y.; Fujii, N.; Tanaka, T.; Ibuka, T. Stereodivergent Synthesis of Chiral 2Alkenylaziridines: Palladium(0)-Catalyzed 2,3-cis-Selective Aziridination and Base-Mediated 2,3-transSelective Aziridination. Chem. Pharm. Bull. 2004, 52, 111-119.

(588) Morgen, M.; Bretzke, S.; Li, P.; Menche, D. Stereodivergent Synthesis of 1,3-syn and antiTetrahydropyrimidones. Org. Lett. 2010, 12, 4494-4497.

(589) Natori, Y.; Kikuchi, S.; Kondo, T.; Saito, Y.; Yoshimura, Y.; Takahata, H. Asymmetric Synthesis of 2,5-Disubstituted 2-Hydroxypyrrolidines Based on Stereodivergent Intramolecular Iridium-Catalyzed Allylic Aminations. Org. Biomol. Chem. 2014, 12, 1983-1994.

(590) Valli, M.; Bruno, P.; Sbarbada, D.; Porta, A.; Vidari, G.; Zanoni, G. Stereodivergent Strategy for Neurofuran Synthesis via Palladium-Catalyzed Asymmetric Allylic Cyclization: Total Synthesis of 7-epiST- $\Delta^{8}$-10-Neurofuran. J. Org. Chem. 2013, 78, 5556-5567.

(591) Vitanova, D. V.; Hampel, F.; Hultzsch, K. C. Linked Bis( $\beta$-diketiminato) Yttrium and Lanthanum Complexes as Catalysts in Asymmetric Hydroamination/Cyclization of Aminoalkenes (AHA). J. Organomet. Chem. 2011, 696, 321-330.

(592) Ren, X.; Guo, Q.; Chen, J.; Xie, H.; Xu, Q.; Lu, Z. Visible-Light Promoted Diastereodivergent Intramolecular Oxyamidation of Alkenes. Chem.-Eur. J. 2016, 22, 18695-18699.

(593) Zhu, J.; Liang, Y.; Wang, L.; Zheng, Z.-B.; Houk, K. N.; Tang, Y. Remote Ester Groups Switch Selectivity: Diastereodivergent Synthesis of Tetracyclic Spiroindolines. J. Am. Chem. Soc. 2014, 136, 6900-6903.

(594) Mei, L.-Y.; Wei, Y.; Tang, X.-Y.; Shi; M. Catalyst-Dependent Stereodivergent and Regioselective Synthesis of Indole-Fused Heterocycles Through Formal Cycloadditions of Indolyl-Allenes. J. Am. Chem. Soc. 2015, 137, 8131-8137.

(595) Xia, Q.; Ganem, B. Asymmetric Total Synthesis of (-)- $\alpha$-Kainic Acid Using an Enantioselective Metal-Promoted Ene Cyclization. Org. Lett. 2001, 3, 485-487.

(596) Harvey, J. S.; Malcolmson, S. J.; Dunne, K. S.; Meek, S. J.; Thompson, A. L.; Schrock, R. R.; Hoveyda, A. M.; Gouverneur, V. Enantioselective Synthesis of P-Stereogenic Phosphinates and Phosphine Oxides by Molibdenum-Catalyzed Asymmetric Ring-Closing Metathesis. Angew. Chem. Int. Ed. 2009, 48, 762-766.

(597) Le, C. M.; Menzies, P. J. C.; Petrone, D. A.; Lautens, M. Synergistic Steric Effects in the Development of a Palladium-Catalyzed Alkyne Carbohalogenation: Stereodivergent Synthesis of Vinyl Halides. Angew. Chem. Int. Ed. 2015, 54, 254-257.

(598) Carreras, J.; Livendahl, M.; McGonigal, P.R.; Echavarren, A. M. Gold(I) as an Artificial Cyclase: Short Stereodivergent Synthesis of (-)-Epiglobulol, (-)-4 $\beta, 7 \alpha$-Aromadendranediol and (-)-4 $\alpha$,7 $\alpha$ Aromadendranediol. Angew. Chem. Int. Ed. 2014, 53, 4896-4899.

(599) Zhao, Y.; Hu, Y.; Wang, H.; Li, X.; Wan, B. Transition-Metal Controlled Diastereodivergent Radical Cyclization/Azidation Cascade of 1,7-Enynes. J. Org. Chem. 2016, 81, 4412-4420.

(600) Lindsay, V. N. G.; Viart, H. M.-F.; Sarpong, R. Stereodivergent Intramolecular C(sp $\left.{ }^{3}\right)-H$ Functionalization of Azavinyl Carbenes: Synthesis of Saturated $N$-Heterocycles and Fused $N$ Heterocycles. J. Am. Chem. Soc. 2015, 137, 8368-8371.

(601) Liu, J.; Yan, J.; Qin, D.; Cai, Q. An Unexpected Inversion of Enantioselectivity in a CopperCatalyzed Intramolecular Desymmetric Aryl C-Coupling Reaction. Synthesis 2014, 46, 1917-1923. 
(602) Kobayashi, S.; Ishitani, H. Lanthanide(III)-Catalyzed Enantioselective Diels-Alder Reaction. Stereoselective Synthesis of Both Enantiomers by Using a Single Source and a Choice of Achiral Ligands. J. Am. Chem. Soc. 1994, 116, 4083-4084.

(603) Kobayashi, S.; Ishitani, H.; Hachiya, I.; Araki, M. Asymmetric Diels-Alder Reactions Catalyzed by Chiral Lanthanide(III) Trifluoromethanesulfonates. Unique Structure of the Triflate and Stereoselective Synthesis of Both Enantiomers Using a Single Chiral Source and a Choice of Achiral Ligands. Tetrahedron 1994, 50, 11623-11636.

(604) Ghosh, A. K.; Mathivanan, P.; Cappiello, J. Conformationally Costrained Bis(oxazoline) Derived Chiral Catalyst: A Highly Effective Enantioselective Diels-Alder Reaction. Tetrahedron Lett. 1996, 37, 3815-3818.

(605) Carbone, P.; Desimoni, G.; Faita, G.; Filippone, S.; Righetti, P. Chiral Lewis Acid Controlled Diels-Alder Reaction: Factors Affecting the Stereoselective Formation of Both Enantiomers in the Bis(Oxazoline)-Magnesium Catalyzed Process. Tetrahedron 1998, 54, 6099-6110.

(606) Wang, H.; Liu, X.; Xia, H.; Liu, P.; Gao, J.; Ying, P.; Xiao, J.; Li, C. Asymmetric Diels-Alder Reactions with Hydrogen Bonding Heterogeneous Catalysts and Mechanistic Studies on the Reversal of Enantioselectivity. Tetrahedron 2006, 62, 1025-1032.

(607) Quaranta, L.; Corminboeuf, O.; Renaud, O. Chiral Relay Effect: 4-Substituted 1,3-Benzoxazol-2(3H)-ones as Chiral Templates for Enantioselective Diels-Alder Reactions. Org. Lett. 2002, 4, 39-42.

(608) Park, D. H.; Kim, S. H.; Kim, S. M.; Kim, Y. D.; Kim, Y. H. A Lewis Acid Dependent Asymmetric Diels-Alder Process in the Cyclization of New Chiral Acrylamides with Dienes. Chem. Commun. 1999, 963-964.

(609) Seebach, D.; Dahinden, R.; Marti, R. E.; Beck, A. K.; Plattner, D. A.; Kühnle, F. N. M. On the TiTADDOLate-Catalyzed Diels-Alder Addition of 3-Butenoyl-1,3-oxazolidin-2-one to Cyclopentadiene. General Features of Ti-BINOLate- and Ti-TADDOLate-Mediated Reactions. J. Org. Chem. 1995, 60, 1788-1799.

(610) Altava, B.; Burguete, M. I.; Fraile, J. M.; García, J. I.; Luis, S. V.; Mayoral, J. A.; Royo, A. J.; Vicent, M. J. TADDOL-TiCl ${ }_{2}$ Catalyzed Diels-Alder Reactions: Unexpected Influence of the Substituents in the 2-Position of the Dioxolane Ring on the Stereoselectivity. Tetrahedron: Asymmetry 1997, 8, 2561-2570.

(611) Altava, B.; Burguete, M. I.; Escuder, B.; Luis, S. V.; Salvador, R. V. Polymer-Grafted TiTADDOL Complexes. Preparation and Use as Catalysts in Diels-Alder Reactions. J. Org. Chem. 1997, 62, 3126-3134.

(612) Altava, B.; Burguete, M. I.; Fraile, J. M.; García, J. I.; Luis, S. V.; Mayoral, J. A.; Vicent, M. J. How Important is the Inert Matrix of Supported Enantiomeric Catalysts? Reversal of Topicity with Two Polystyrene Backbones. Angew. Chem. Int. Ed. 2000, 39, 1503-1506.

(613) Altava, B.; Burguete, M. I.; García-Verdugo, E.; Luis, S. V.; Vicent, M. J. Functional Monolithic Resins for the Development of Enantioselective Versatile Catalytic Minireactors with Long-Term Stability: TADDOL Supported Systems. Green Chem. 2006, 8, 717-726.

(614) Sarotti, A. M.; Fernández, I.; Spanevello, R. A.; Sierra, M. A.; Suárez, A. G. $\pi$-Stacking Effect on Levoglucosenone Derived Internal Chiral Auxiliaries. A Case of Complete Enantioselectivity Inversion on the Diels-Alder Reaction. Org. Lett. 2008, 10, 3389-3392.

(615) Wang, C.; Jia, G.; Li, Y.; Zhang, S.; Li, C. $\mathrm{Na}^{+} / \mathrm{K}^{+}$Switch of Enantioselectivity in G-Quadruplex DNA-Based Catalysis. Chem. Commun. 2013, 49, 11161-11163.

(616) Li, Y.; Wang, C.; Hao, J.; Cheng, M.; Jia, G. Higher-Order Human Telomeric G-Quadruplex DNA Matalloenzyme Catalyzed Diels-Alder Reaction: An Unexpected Inversion of Enantioselectivity Modulated by $\mathrm{K}^{+}$and $\mathrm{NH}_{4}{ }^{+}$Ions. Chem. Commun. 2015, 51, 13174-13177.

(617) Feng, X.; Zhou, Z.; Zhou, R.; Zhou, Q.-D.; Dong, L.; Chen, Y.-C. Stereodivergence in AmineCatalyzed Regioselective [4+2] Cycloadditions of $\beta$-Substituted Cyclic Enones and Polyconjugated Malononitriles. J. Am. Chem. Soc. 2012, 134, 19942-19947. 
(618) Tawde, T. S.; Wagh, S. J.; Sapre, J. V.; Khose, V. N.; Badani, P. M.; Karnik, A. V. Reversal of Enantioselectivity Induced by the Achiral Part of an Organocatalyst in a Diels-Alder Reaction. Tetrahedron: Asymmetry 2016, 27, 130-135.

(619) Johannsen, M.; Jørgensen, K. A. Solvent Effects in Asymmetric Hetero Diels-Alder and Ene Reactions. Tetrahedron 1996, 52, 7321-7328.

(620) Ghosh, A. K.; Mathivanan, P.; Cappiello, J.; Krishnan, K. Asymmetric Hetero Diels-Alder Reactions of Danishefsky's Diene and Glyoxylate Esters Catalyzed by Chiral Bisoxazoline Derived Catalysts. Tetrahedron: Asymmetry 1996, 7, 2165-2168.

(621) Keller, F.; Weinmann, H.; Schurig, V. Chiral Polysiloxane-Fixed Metal 1,3-Diketonates (Chirasil Metals) as Catalytic Lewis Acids for a Hetero Diels-Alder Reaction- Inversion of Enantioselectivity upon Catalyst-Polymer Binding. Chem. Ber. Recueil 1997, 130, 879-895.

(622) Kobayashi, S.; Komiyama, S.; Ishitani, H. The First Enantioselective Aza-Diels-Alder Reactions of Imino Dienophiles on Use of a Chiral Zirconium Catalyst. Angew. Chem. Int. Ed. 1998, 37, 979-981.

(623) Kobayashi, S.; Kusakabe, K.; Komiyama, S.; Ishitani, H. A Switch of EnantiofacialSelectivities Using Designed Similar Chiral Ligands in Zirconium-Catalyzed Asymmetric Aza Diels-Alder Reactions. J. Org. Chem. 1999, 64, 4220-4221.

(624) Ishitani, H.; Kitazawa, T.; Kobayashi, S. Efficient Catalytic Enantioselective Mannich-Type Reactions Using a Zirconium-Bis(binaphthol)methane Complex. Tetrahedron Lett. 1999, 40, 2161-2164.

(625) Evans, D. A.; Johnson, J. S. Catalytic Enantioselective Hetero Diels-Alder Reactions of $\alpha, \beta$ Unsaturated Acyl Phosphonates with Enol Ethers. J. Am. Chem. Soc. 1998, 120, 4895-4896.

(626) Evans, D. A.; Johnson, J. S.; Olhava, E. J. Enantioselective Synthesis of Dihydropyrans. Catalysis of Hetero Diels-Alder Reactions by Bis(oxazoline) Copper(II) Complexes. J. Am. Chem. Soc. 2000, 122, 1635-1649.

(627) Evans, D. A.; Johnson, J. S.; Burgey, C. S.; Campos, K. R. Reversal in Enantioselectivity of tertButyl Versus Phenyl-Substituted Bis(oxazoline) Copper(II) Catalyzed Hetero Diels-Alder and Ene Reactions. Crystallographic and Mechanistic Studies. Tetrahedron Lett. 1999, 40, 2879-2882.

(628) Thorhauge, J.; Johannsen, M.; Jørgensen, K. A. Highly Enantioselective Catalytic Hetero Diels-Alder Reaction with Inverse Electron-Demand. Angew. Chem. Int. Ed. 1998, 37, 2404-2406.

(629) Tietze, L. F. Sterodivergent Hetero Diels-Alder Reactions of Chiral 1-Oxa-1,3-butadiene Through a Conformational Switch Induced by Lewis Acids. Chem. - Eur. J. 1996, 2, 139-148.

(630) Gohier, F.; Bouhadjera, K.; Faye, D.; Gaulon, C.; Maisonneuve, V.; Dujardin, G.; Dhal, R. Lewis Acid Tuned Facial Stereodivergent Hetero Diels-Alder Reactions Using $\beta$-Substituted $N$ Vinyloxazolidinones. Org. Lett. 2007, 9, 211-214.

(631) Huang, H.; Konda, S.; Zhao, J. C.-G. Diastereodivergent Catalysis Using Modularly Designed Organocatalysts: Synthesis of Both cis- and trans-Fused Pyrano[2,3-b]pyrans. Angew. Chem. Int. Ed. 2016, 55, 2213-2216.

(632) Kissane, M.; Maguire, A. R. Asymmetric 1,3-Dipolar Cyclodditions of Acrylamides. Chem. Soc. Rev. 2010, 39, 845-883.

(633) Nájera, C.; Sansano, J. M.; Yus, M. Metal Complexes Versus Organocatalysts in Asymmetric 1,3Dipolar Cycloadditions. J. Braz. Chem. Soc. 2010, 21, 377-412.

(634) Xing, Y.; Wang, N.-X. Organocatalytic and Metal-Mediated Asymmetric [3+2] Cycloaddition Reactions. Coord. Chem. Rev. 2012, 256, 938-952.

(635) Pellisier, H. Asymmetric Organocatalytic Cycloadditions. Tetrahedron 2012, 68, 2197-2232.

(636) Han, M.-Y.; Jia, J.-Y.; Wang, W. Recent Advances in Organocatalytic Asymmetric Synthesis of Polysubstituted Pyrrolidines. Tetrahedron Lett. 2014, 55, 784-794.

(637) Adrio, J.; Carretero, J. C. Recent Advances in the Catalytic Asymmetric 1,3-Dipolar Cycloaddition of Azomethine Ylides.Chem. Commun. 2014, 50, 12434-12446.

(638) Hashimoto, T.; Maruoka, K. Recent Advances of Catalytic Asymmetric 1,3-Dipolar Cycloadditions. Chem. Rev. 2015, 115, 5366-5412. 
(639) Sing, M. S.; Chowdhury, S.; Koley, S. Progress in 1,3-Dipolar Cycloadditions in the Recent Decade: an Update to Strategic Development Towards the Arsenal of Organic Synthesis. Tetrahedron 2016, 72, 1603-1716.

(640) Bdiri, B.; Zhao, B.-J.; Zhou, Z.-M. Recent Advances in the Enantioselective 1,3-Dipolar Cycloaddition of Azomethine Ylides and Dipolarophiles. Tetrahedron: Asymmetry 2017, 28, 876-899.

(641) Döndas, H. A.; Retamosa, M. de G.; Sansano, J. M. Current Trends towards the Synthesis of Bioactive Heterocycles and Natural Products Using 1,3-Dipolar Cycloadditions (1,3-DC) with Azomethine Ylides. Synthesis 2017, 49, 2819-2851.

(642) Gothelf, K. V.; Jørgensen, K. A. Catalytic Enantioselective 1,3-Dipolar Cycloaddition Reactions of Nitrones. Chem. Commun. 2000, 1449-1458.

(643) Gothelf, K.V.; Jørgensen, K. A. Transition-Metal Catalyzed Asymmetric 1,3-Dipolar Cycloaddition Reactions between Alkenes and Nitrones. J. Org. Chem. 1994, 59, 5687-5691.

(644) Gothelf, K. V.; Hazell, R. G.; Jørgensen, K. A. Control of Diastereo- and Enantioselectivity in Metal-Catalyzed 1,3-Dipolar Cycloaddition Reactions of Nitrones with Alkenes. Experimental and Theoretical Investigations. J. Org. Chem. 1996, 61, 346-355.

(645) Gothelf, K. V.; Hazell, R. G.; Jørgensen, K. A. Molecular Sieve Dependent Absolute Stereoselectivity in Asymmetric Catalytic 1,3-Dipolar Cycloaddition Reactions. J. Org. Chem. 1998, 63, 5483-5488.

(646) Desimoni, G.; Faita, G.; Mortoni, A.; Righetti, P. 1,3-Dipolar Cycloadditions Catalyzed by Bis(Oxazoline)-Magnesium-Based Chiral Complexes. The Importance of a $\mathrm{Mg}(\mathrm{II})$ Counteranion. Tetrahedron Lett. 1999, 40, 2001-2004.

(647) Crosignani, S.; Desimoni, G.; Faita, G.; Filippone, S.; Mortoni, A.; Righetti, P.; Zema, M. Chiral Amplification in Diels-Alder and 1,3-Dipolar Cycloaddition Catalyzed by Bis(Oxazoline)-Zn(II)-Based Chiral Complexes. Tetrahedron Lett. 1999, 40, 7007-7010.

(648) Kobayashi, S.; Kawamura, M. Catalytic Enantioselective 1,3-Dipolar Cycloadditions between Nitrones and Alkenes Using a Novel Heterochiral Ytterbium(III) Catalyst. J. Am. Chem. Soc. 1998, 120, 5840-5841.

(649) Kawamura, M.; Kobayashi, S. Switch of Enantiofacial Selectivity in Chiral Ytterbium-Catalyzed 1,3-Dipolar Cycloaddition Reactions. Tetrahedron Lett. 1999, 40, 3213-3216.

(650) Huang, Z.-Z.; Kang, Y.-B.; Zhou, J.; Ye, M.-C.; Tang, Y. Diastereoselectivity-Switchable and Highly Enantioselective 1,3-Dipolar Cycloaddition of Nitrones to Alkylidene Malonates. Org. Lett. 2006, 6, 1677-1679.

(651) Viton, F.; Bernardelli, G.; Kündig, E. P. Iron and Ruthenium Lewis Acid Catalyzed Asymmetric 1,3-Dipolar Cycloaddition Reactions between Nitrones and Enals. J. Am. Chem. Soc. 2002, 124, 4968-4969.

(652) Selim, K. B.; Martel, A.; Laurent, M. Y.; Lhoste, J.; Py, S.; Dujardin, G. Enantioselective Ruthenium-Catalyzed 1,3-Dipolar Cycloadditions between C-Carboalkoxy Ketonitrones and Methacrolein: Solvent Effect on Reaction Selectivity and its Rational. J. Org. Chem. 2014, 79, 3414-3426.

(653) Seerden, J.-P. G.; Scholte op Reimer, A. W. A.; Scheeren, H. W. Asymmetric 1,3-Dipolar Cycloaddition of Nitrones with Ketene Acetals Catalyzed by Chiral Oxazaborolidines. Tetrahedron Lett. 1994, 35, 4419-4422.

(654) Seerden, J.-P. G.; Scholte op Reimer, A. W. A.; Scheeren, H. W. Dramatic Solvents Effects on the Enantioselectivity of Chiral Oxazaborolidine Catalyzed Asymmetric 1,3-Dipolar Cycloadditions of Nitrones with Ketene Acetals. Tetrahedron: Asymmetry 1995, 6, 1441-1450.

(655) Zeng, W.; Chen, G.-Y.; Zhou, Y.-G.; Li, Y.-X. Hydrogen-Bonding Directed Reversal of Enantioselectivity. J. Am. Chem. Soc. 2007, 129, 750-751.

(656) Yan, Y.-X.; Peng, Q.; Zhang, Y.; Zhang, K.; Hong, W.; Hou, X.-L.; Wu, Y.-D. A Highly Enantioand Diastereoselective Cu-Catalyzed 1,3-Dipolar Cycloaddition of Azomethine Ylides with Nitroalkenes. Angew. Chem. Int. Ed. 2006, 45, 1979-1983. 
(657) Vivanco, S.; Lecea, B.; Arrieta, A.; Prieto, P.; Morao, I.; Linden, A.; Cossío, F. P. Origins of the Loss of Concertedness in Pericyclic Reactions Theoretical Prediction and Direct Observation of Stepwise Mechanisms in [3+2] Thermal Cycloadditions. J. Am. Chem. Soc. 2000, 122, 6078-6092.

(658) De Cózar, A.; Cossío, F. P. Stereocontrolled [3+2] Cycloadditions between Azomethine Ylides and Dipolarophiles: a Fruitful Interplay between Theory and Experiment. Phys. Chem. Chem. Phys. 2011, 13, 10858-10868.

(659) Conde, E.; Bello, D.; De Cózar, A.; Sánchez, M.; Vázquez, M. A.; Cossío, F. P. Densely Substituted Unnatural L- and D-Prolines as Catalysts for Highly Enantioselective Stereodivergent [3+2] Cycloadditions and Aldol Reactions. Chem. Sci. 2012, 3, 1486-1491.

(660) Maroto, E. E.; Filippone, S.; Suárez, M.; Martínez-Álvarez, R.; de Cózar, A.; Cossío, F. P.; Martín, N. Stereodivergent Synthesis of Chiral Fullerenes by [3+2] Cycloadditions to $\mathrm{C}_{60}$. J. Am. Chem. Soc. 2014, 136, 705-712.

(661) Filippone, S.; Maroto, E. E.; Martín-Domenech, A.; Suárez, M.; Martín, N. An Efficient Approach to Chiral Fullerene Derivatives by Catalytic Enantioselective 1,3-Dipolar Cycloadditions. Nat. Chem. 2009, 1, 578-582.

(662) Maroto, E. E.; De Cózar, A.; Filippone, S.; Martín-Domenech, A.; Suárez, M.; Cossío, F. P.; Martín, N. Hierarchical Selectivity in Fullerenes: Site-, Regio-, Diastereo-, and Enantiocontrol of the 1,3Dipolar Cycloaddition to $\mathrm{C}_{70}$. Angew. Chem. Int. Ed. 2011, 50, 6060-6064.

(663) Maroto, E. E.; Filippone, S.; Martín-Domenech, A.; Suárez, M.; Martín, N. Switching the Stereoselectivity: Fullero(Pyrrolidines) “a la Carte”. J. Am. Chem. Soc. 2012, 134, 12936-12938.

(664) Maroto, E. E.; Izquierdo, M.; Murata, M.; Filippone, S.; Komatsu, K.; Murata, Y.; Martín, N. Catalytic Stereodivergent Functionalization of $\mathrm{H}_{2} @ \mathrm{C}_{60}$. Chem. Commun. 2014, 50, 740-742.

(665) Kim, H. Y.; Shih, H. J.; Knabe, W. E.; Oh, K. Reversal of Enantioselectivity between the Copper(I)- and Silver(I)-Catalyzed 1,3-Dipolar Cycloaddition Reactions Using a Brucine-Derived Amino Alcohol Ligand. Angew. Chem. Int. Ed. 2009, 48, 7420-7423.

(666) Kim, H. Y.; Li, J.-Y.; Kim, S.; Oh, K. Stereodivergency in Catalytic Asymmetric Conjugate Addition Reactions of Glycine(ket)imines. J. Am. Chem. Soc. 2011, 133, 20750-20753.

(667) Li, J.-Y.; Kim, H. Y.; Oh, K. Brucine Diol-Copper-Catalyzed Asymmetric Synthesis of endoPyrrolidines: The Mechanistic Dichotomy of Imino Esters. Org. Lett. 2015, 17, 1288-1291.

(668) Li, J.-Y.; Kim, H. Y.; Oh, K. Enantiodivergent Brucine-diol-Catalyzed 1,3-Dipolar Cycloaddition of Azomethine Ylides with $\alpha, \beta$-Unsaturated Ketones. Adv. Synth. Catal. 2016, 358, 984-993.

(669) Arai, T.; Mishiro, A.; Yokoyama, N.; Suzuki, K.; Sato, H. Chiral Bis(imidazolidine)pyridine$\mathrm{Cu}(\mathrm{OTf})_{2}$ : Catalytic Asymmetric Endo-Selective [3+2] Cycloaddition of Imino Esters with Nitroalkenes. J. Am. Chem. Soc. 2010, 132, 5338-5339.

(670) Arai, T.; Yokoyama, N.; Mishiro, A.; Sato, H. Catalytic Asymmetric exo'-Selective [3+2] Cycloaddition of Imino Esters with Nitroalkenes. Angew. Chem. Int. Ed. 2010, 49, 7895-7898.

(671) Arai, T.; Tokumitsu, C.; Miyazaki, T.; Kuwano, S.; Awata, A. Catalytic Asymmetric [3+2] Cycloaddition for Stereodivergent Synthesis of Chiral Indolylpyrrolidines. Org. Biomol. Chem. 2016, 14, 1831-1839.

(672) Robles-Machin, R.; González-Esguevillas, M.; Adrio, J.; Carretero, J. C. Cu-Catalyzed Asymmetric 1,3-Dipolar Cycloaddition of Azomethine Ylides with $\beta$-Phenylsulfonyl Enones. Ligand Controlled Diastereoselectivity Reversal. J. Org. Chem. 2010, 75, 233-236.

(673) Antonchick, A. P.; Gerding-Reimers, C.; Catarinella, M.; Schurmann, M.; Preut, H.; Ziegler, S.; Rauh, D.; Waldmann, H. Highly Enantioselective Synthesis and Cellular Evaluation of Spiroindoles Inspired by Natural Products. Nat. Chem. 2010, 2, 735-740.

(674) Antonchick, A. P.; Schuster, H.; Bruss, H.; Schürmann, M.; Preut, H.; Rauh, D.; Waldmann, H. Enantioselective Synthesis of the Spirotryprostatin A Scaffold. Tetrahedron 2011, 67, 10195-10202.

(675) Cabrera, S.; Arrayás, R. G.; Carretero, J. C. Highly Enantioselective Copper(I)-FesulphosCatalyzed 1,3-Dipolar Cycloaddition of Azomethine Ylides. J. Am. Chem. Soc. 2005, 127, 16394-16395.

(676) Awata, A.; Arai, T. Catalytic Asymmetric exo'-Selective [3+2] Cycloadditionfor Constructing Stereochemically Diversified Spiro[pyrrolidin-3,3'-oxindole]s. Chem.-Eur. J. 2012, 18, 8278-8282. 
(677) Arai, T.; Ogawa, H.; Awata, A.; Sato, M.; Watanabe, M.; Yamanaka, M. PyBidine-Cu(OTf $)_{2}{ }^{-}$ Catalyzed Asymmetric [3+2] Cycloaddition with Imino Esters: Harmony of Cu-Lewis Acid and Imidazolidine-NH Hydrogen Bonding in Concerto Catalysis. Angew. Chem. Int. Ed. 2015, 54, 1595-1599.

(678) Xu, H.; Golz, C.; Strohmann, C.; Antonchick, A. P.; Waldmann, H. Enantiodivergent Combination of Natural Product Scaffolds Enabled by Catalytic Enantioselective Cycloaddition. Angew. Chem. Int. Ed. 2016, 56, 7761-7765.

(679) Potowski, M.; Bauer, J. O.; Strohmann, C.; Antonchick, A. P.; Waldmann, H. Highly Enantioselective Catalytic [6+3] Cycloadditions of Azomethine Ylides. Angew. Chem. Int. Ed. 2012, 51, 9512-9516.

(680) Potowski, M.; Antonchick, A. P.; Waldmann, H. Catalytic Asymmetric exo-Selective [6+3] Cycloadditions of Iminoesters with Fulvenes. Chem. Commun. 2013, 49, 7800-7802.

(681) Kanemasa, S.; Kanai, T. Lewis Acid-Catalyzed Enantioselective 1,3-Dipolar Cycloadditions of Diazoalkane: Chiral Ligand/Achiral Auxiliary Cooperative Chirality Control. J. Am. Chem. Soc. 2000, 122, 10710-10711.

(682) GarcíaRuano, J. L.; Peromingo, M. T.; Alonso, M.; Fraile, A.; Martín, M. R.; Tito, A. 1,3-Dipolar Cycloadditions of Diazoalkanes to Activated Sulfoxides: Influence of Lewis Acids. J. Org. Chem. 2005, 70, 8942-8947.

(683) GarcíaRuano, J. L.; Alonso, M.; Cruz, D.; Fraile, A.; Martín, M. R.; Peromingo, M. T.; Tito, A.; Yuste, F. Synthesis of Bicyclo[3.1.0]hexanones via 1,3-Dipolar Cycloaddition of Diazoalkanes to Homochiral $\alpha$-Sulfinyl-2-cyclopentenones. Tetrahedron 2008, 64, 10546-10551.

(684) Trost, B. M. [3+2] Cycloaddition Approaches to Five-Membered Rings via Trimethylenemethane and its Equivalents. Angew. Chem. Int. Ed. 1986, 25, 1-20.

(685) Trost, B. M.; Cramer, N.; Silverman, S. M. Enantioselective Construction of Spirocyclic Oxindolic Cyclopentanes by Palladium-Catalyzed Trimethylenemethane-[3+2]-Cycloaddition. J. Am. Chem. Soc. 2007, 129, 12396-12397.

(686) Rivinoja, D. J.; Gee, Y. S.; Gardiner, M. G.; Ryan, J. H.; Hyland, C. J. T. The Diastereoselective Synthesis of Pyrroloindolines by Pd-Catalyzed Dearomative Cycloaddition of 1-Tosyl-2-vinylaziridine to 3-Nitroindoles. ACS Catal. 2017, 7, 1053-1056.

(687) Qiao, S.; Wilcox, C. B.; Unruh, D. K.; Jiang, B.; Li, G. Asymmetric [3+2] Cycloaddition of Chiral $N$-Phosphonoyl Imines with Methyl Isocyanate for Accessing 2-Imidazolines with Switchable Stereoselectivity. J. Org. Chem. 2007, 82, 2992-2999.

(688) Palomo, C.; Aizpurua, J. M.; Ganboa, I.; Oiarbide, M. Asymmetric Synthesis of $\beta$-Lactams by Staudinger Ketene-Imine Cycloaddition Reaction. Eur. J. Org. Chem. 1999, 3223-3235.

(689) Singh, G. S. Recent Progress in the Synthesis and Chemistry of Azetidinones. Tetrahedron 2003, 59, 7631-7694.

(690) Palomo, C.; Aizpurua, J. M.; Ganboa, I.; Oiarbide, M. Asymmetric Synthesis of $\beta$-Lactams through the Staudinger Reaction and Their Use as Building Blocks of Natural and Unnatural Products. Curr. Med. Chem. 2004, 11, 1837-1872.

(691) Arrieta, A.; Lecea, B.; Cossío, F. P. Origins of the Stereodivergent Outcome in the Staudinger Reaction between Acyl Chlorides and Imines. J. Org. Chem. 1998, 63, 5869-5876.

(692) Banik, B. K.; Lecea, B.; Arrieta, A.; de Cózar, A.; Cossío, F. P. On the Stereodivergent Behavior Observed in the Staudinger Reaction between Methoxyketene and $(E)-N$-Benzylidenearyl Amines. Angew. Chem. Int. Ed. 2007, 46, 3028-3032.

(693) Jiao, L.; Liang, Y.; Xu, J. Origin of the Relative Stereoselectivity of the $\beta$-Lactam Formation in the Staudinger Reaction. J. Am. Chem. Soc. 2006, 128, 6060-6069.

(694) Taggi, A. E.; Hafez, A. M.; Wack, H.; Young, B.; Drury, W. J., III; Lectka, T. Catalytic Asymmetric Synthesis of $\beta$-Lactams. J. Am. Chem. Soc. 2000, 122, 7831-7832.

(695) Taggi, A. E.; Hafez, A. M.; Wack, H.; Young, B.; Ferraris, D.; Lectka, T. The Development of the First Catalyzed Reaction of Ketenes and Imines: Catalytic Asymmetric Synthesis of $\beta$-Lactams. J. Am. Chem. Soc. 2002, 124, 6626-6635. 
(696) Weatherwax, A.; Abraham, C. J.; Lectka, T. An Anionic Nucleophilic Catalyst System for the Diastereoselective Synthesis of trans- $\beta$-Lactams. Org. Lett. 2005, 7, 3461-3463.

(697) Hodous, B. L.; Fu, G. C. Enantioselective Staudinger Synthesis of $\beta$-Lactams Catalyzed by a Planar Chiral Nucleophile. J. Am. Chem. Soc. 2002, 124, 1578-1579.

(698) Lee, L. C.; Hodous, B. L.; Bergin, E.; Shih, C.; Fu, G. C. Catalytic Asymmetric Staudinger Reactions to Form $\beta$-Lactams: An Unexpected Dependence of Diastereoselectivity on the Choice of the Nitrogen Substituents. J. Am. Chem. Soc. 2005, 127, 11586-11587.

(699) Calter, M. A.; Tretyak, O. A.; Flaschenriem, C. Formation of Disubstituted $\beta$-Lactones Using Bifunctional Catalysis. Org. Lett. 2005, 7, 1809-1812.

(700) Takasu, K.; Ueno, M.; Inanaga, K.; Ihara, M. Catalytic [2+2]-Cycloaddition Reactions of Silyl Enol Ethers. A Convenient and Stereoselective Method for Cyclobutane Ring Formation. J. Org. Chem. 2004, 69, 517-521.

(701) Takasu, K.; Nagao, S.; Ueno, M.; Ihara, M. An Auxiliary Induced Asymmetric Synthesis of Functionalized Cyclobutanes by Means of Catalytic [2+2]-Cycloaddition Reaction. Tetrahedron 2004, 60, 2071-2078.

(702) Inanaga, K.; Takasu, K.; Ihara, M. A Practical Catalytic Method for Preparing Highly Substituted Cyclobutanes and Cyclobutenes. J. Am. Chem. Soc. 2005, 127, 3668-3669.

(703) Kurahashi, K.; Takemoto, Y.; Takasu, K. Room Temperature, Acid-Catalyzed [2+2]Cycloadditions: Suppression of Side Reactions by Using a Flow Microreactor System. ChemSusChem 2012, 5, 270-273.

(704) Arichi, N.; Hata, K.; Takemoto, Y.; Yamada, K.; Yamaoka, Y.; Takasu, K. Synthesis of Steroidal Derivatives Bearing a Small Ring Using a Catalytic [2+2]-Cycloaddition and a Ring-Contraction Rearrangement. Tetrahedron 2015, 71, 233-244.

(705) Micalizio, G. C.; Roush, W. R. A Three-Component Coupling Strategy for Tetrahydrofuran Synthesis: Application of the Diisopropyl Tartrate Modified (E)- $\gamma$-(Dimethylphenylsilyl)allylboronates as an $\alpha, \gamma$-Allyl Dianion Equivalent. Org. Lett. 2000, 2, 461-464.

(706) Tinsley, J. M.; Mertz, E.; Chong, P. Y.; Rang, R.-A. F.; Roush, W. R. Synthesis of (+)-Bullatacin via the Highly Diastereoselective [3+2] Annulation Reaction of a Racemic Aldehyde and a Nonracemic Allylsilane. Org. Lett. 2005, 7, 4245-4248.

(707) Tinsley, J. M.; Roush, W. R. Total Synthesis of Asimicin via Highly Stereoselective [3+2] Annulation Reactions of Substituted Allylsilanes. J. Am. Chem. Soc. 2005, 127, 10818-10819.

(708) Huh, C. W.; Roush, W. R. Highly Selective and Modular Syntheses of 10-Hydroxytrilobacin and Three Diastereomers via Stereodivergent [3+2]-Annulation Reactions. Org. Lett. 2008, 10, 3371-3374.

(709) Müller, J. M.; Stark, C. B. W. Diastereodivergent Reverse Prenylation of Indole and Tryptophan Derivatives: Total Synthesis of Amauromine, Novoamauromine, and epi-Amauromine. Angew. Chem. Int. Ed. 2016, 55, 4798-4802.

(710) Nagamoto, M.; Yamauchi, D.; Nishimura, T. Iridium-Catalyzed Asymmetric [3+2]-Annulation of Aromatic Ketimines with Alkynes via C-H Activation: Unexpected Inversion of the Enantioselectivity Induced by Protic Acids. Chem. Commun. 2016, 52, 5876-5879.

(711) Murakami, M.; Itami, K.; Ito, Y. Catalytic Asymmetric [4+1] Cycloaddition of Vinylallenes with Carbon Monoxide: Reversal of the Induced Chirality by the Choice of Metal. J. Am. Chem. Soc. 1999, 121, 4130-4135.

(712) Zhun, G.; Shi, M.-L.; He, Q.; Lin, W.-J.; Ouyang, Q.; Du, W.; Chen, Y.-C. Catalyst-Controlled Switch in Chemo- and Diastereoselectivities: Annulation of Morita-Baylis-Hillman Carbonates from Isatins. Angew. Chem. Int. Ed. 2016, 55, 2147-2151.

(713) Zhang, W.; Franzén, J. Diverse Asymmetric Quinolizidine Synthesis: A Stereodivergent One-Pot Approach. Adv. Synth. Catal. 2010, 352, 499-518.

(714) Zhang, W.; Bah, J.; Wohlfarth, A.; Franzén, J. A Stereodivergent Strategy for the Preparation of Corynantheine and Ipecae Alkaloids, Their Epimers and Analogues: Efficient Total Synthesis of (-)Dihydrocorynantheol, (-)-Corynantheol, (-)-Protoemetinol, (-)-Corynantheal, (-)-Protoemetine, and Related Natural and Nonnatural Compounds. Chem. - Eur. J. 2011, 17, 13814-13824. 
(715) Rana, N. K.; Huang, H.; Zhao, J. C.-G. Highly Diastereodivergent Synthesis of Tetrasubstituted Cyclohexanes Catalyzed by Modularly Designed Organocatalysts. Angew. Chem. Int. Ed. 2014, 53, 7619-7623.

(716) Martínez, J. I.; Villar, L.; Uria, U.; Carrillo, L.; Reyes, E.; Vicario, J. L. Bifunctional Squaramide Catalysts with the Same Absolute Chirality for the Diastereodivergent Access to Densely Functionalised Cyclohexanes through Enantioselective Domino Reactions. Synthesis and Mechanistic Studies. Adv. Synth. Catal. 2014, 356, 3627-3648.

(717) Ishikawa, H.; Sawano, S.; Yasui, Y.; Shibata, Y.; Hayashi, Y. Asymmetric One-Pot FourComponent Coupling Reaction: Synthesis of Substituted Tetrahydropyrans Catalyzed by Diphenylprolinol Silyl Ether. Angew. Chem. Int. Ed. 2011, 50, 3774-3779.

(718) Han, B.; Xie, X.; Huang, W.; Li, X.; Yang, L.; Peng, C. Organocatalytic Morita-Baylis-Hillman/Michael/Acetalization Cascade: Procedure-Controlled Diastereodivergence in the Asymmetric Synthesis of Fully Substituted Tetrahydropyrans. Adv. Synth. Catal. 2014, 356, 3676-3682.

(719) Li, N.; Chen, X.-X.; Song, J.; Luo, S.-W.; Fan, W.; Gong, L.-Z. Highly Enantioselective Organocatalytic Biginelli and Biginelli-Like Condensations: Reversal of the Stereochemistry by Tuning the 3,3'-Disubstituents of Phosphoric Acids. J. Am. Chem. Soc. 2009, 131, 15301-15310.

(720) Wang, S.; Xiao, J.; Li, J.; Xiang, H.; Wang, C.; Chen, X.; Carter, R. G.; Yang, H. Discovery of Temperature-Dependent, Autoinductive Reversal of Enantioselectivity: Palladium-Mediated [3+3]Annulation of 4-Hydroxycoumarins. Chem. Commun. 2017, 53, 4441-4444.

Table of Contents Graphic

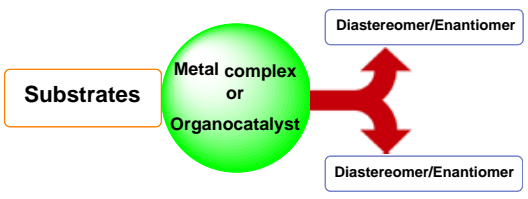

